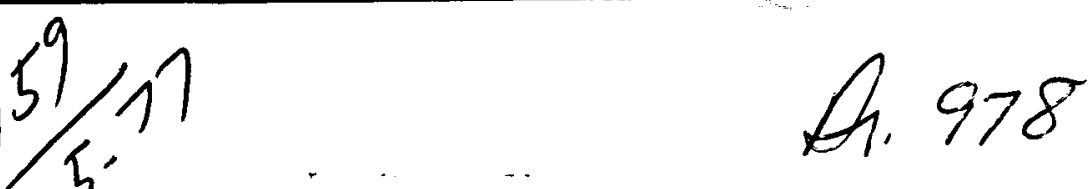

$5 / 5,11 \quad$ A. 1978

\title{
LBD 5299
}

\section{Energy and}

Environment

Annual Report 1975

Energy and Environment Division

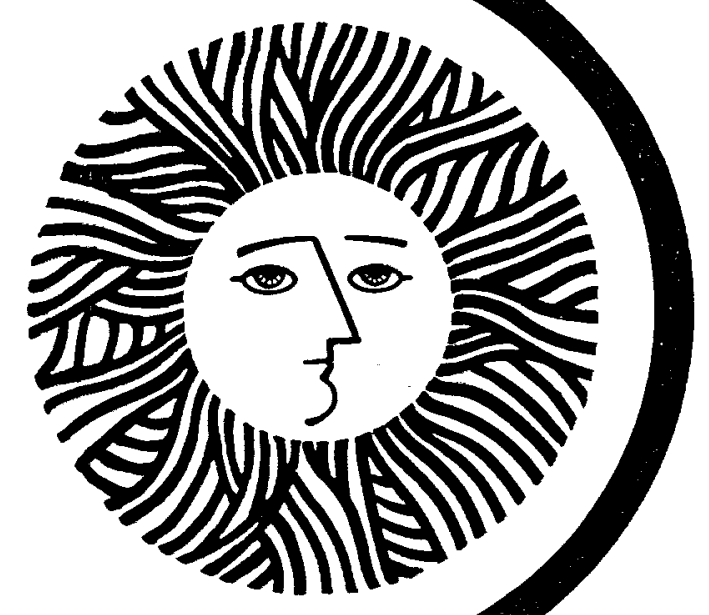

Lawrence Berkeley Laboratory

University of California 


\section{DISCLAIMER}

This report was prepared as an account of work sponsored by an agency of the United States Government. Neither the United States Government nor any agency Thereof, nor any of their employees, makes any warranty, express or implied, or assumes any legal liability or responsibility for the accuracy, completeness, or usefulness of any information, apparatus, product, or process disclosed, or represents that its use would not infringe privately owned rights. Reference herein to any specific commercial product, process, or service by trade name, trademark, manufacturer, or otherwise does not necessarily constitute or imply its endorsement, recommendation, or favoring by the United States Government or any agency thereof. The views and opinions of authors expressed herein do not necessarily state or reflect those of the United States Government or any agency thereof. 


\section{DISCLAIMER}

Portions of this document may be illegible in electronic image products. Images are produced from the best available original document. 


\section{LEGAL NOTICE}

This report was prepared as an account of work sponsored by the United States Government. Neither the United States nor the United States Energy Research and Development Administration, nor any of their employees, nor any of their contractors, subcontractors, or their employees, makes any warranty, express or implied, or assumes any legal liability or responsibility for the accuracy, completeness or usefulness of any information, apparatus, product or process disclosed, or represents that its use would not infringe privately owned rights.

Printed in the United States of America

Available from

National Technical Information Service

U.S. Department of Commerce

5285 Port Royal Road

Springfield, Virginia 22161

Price: Printed Copy $\$ 6.75$

Microfiche $\$ 3.00$ 


\title{
02304 \\ ENERGY AND ENVIRONMENT \\ ANNUAL REPORT
}

1975

\author{
Robert J. Budnitz \\ Acting Division Head \\ Melvin K. Simmons \\ Acting Deputy Head \\ Editorial Committee
}

Carl J. Blumstein, Chairperson

Nabil M. Amer

Charles W. Case

Yvonne L. Howell

Arlon J. Hunt
Edward P. Kahn

Wulf B. Kunkel

Donald J. Levy

Helen V. Michel

Amos S. Newton

Tecbnical Editing

Rudolf G. Barton

Loretta R. Lizama

Work done under U. S. Energy Research and Development Administration Contract No. W-7405-ENG-48

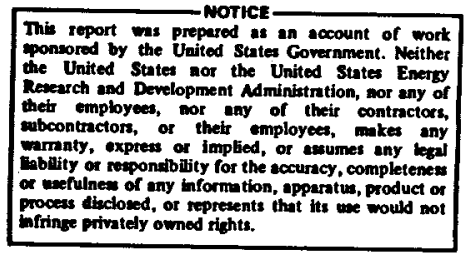

Energy and Environment Division

Lawrence Berkeley Laboratory

University of California

$\|^{20^{\circ}}$ Berkeley, California 94720 
$\mathcal{U}$

U 
CONTENTS

GEOTHERMAL AND GEOSCIENCES

Introduction

Geothermal Field Activities, North-Central Nevada

H.A. Wollenberg, T.V. McEvilly, and H.F. Morrison. ................

Mixed Explicit-Implicit Iterative Finite Element Scheme for the Diffusion Equation

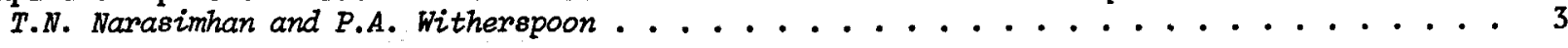

Reinjection in Geothermal Reservoirs

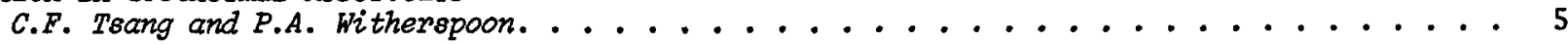

Numerical Simulation of Reservoir Compaction in Liquid Dominated Geothermal Systems

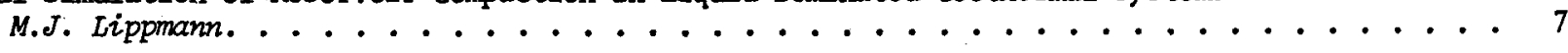

Development of a Multidimensional Model for Two-Phase Heat and Mass Transfer in Porous Media

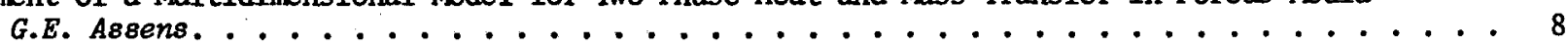

Thermodynamics of Geothermal Brines

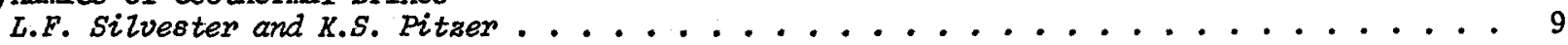

The Use of Program GEOTHM to Design and Optimize Geothermal Power Cycles

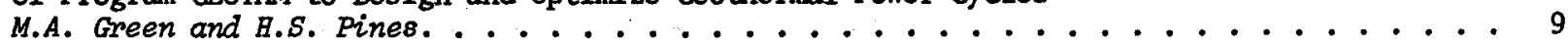

The Kinetics of Quartz Dissolution and Precipitation

J.A. Apps, E.L. Madsen and R.L. Hinkins. . . . . . . . . . . . . . 12

Physical Modeling of Geothermal Reservoirs

V.E. Schrock . . . . . . . . . . . . . . . . . . . . 15

Properties and Behavior of Rock-Fluid Systems at High Temperatures and Pressures

W.H. Somerton. . . . . . . . . . . . . . . . . 16

Results of Reservoir Evaluation Tests, 1975, Raft River Geothermal Field, Idaho

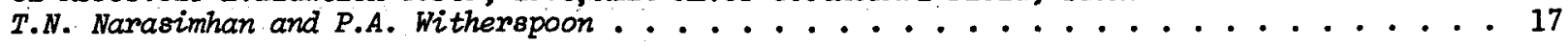

Radioactivity and Metal Mineralization

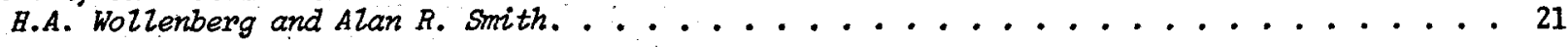

"Arms" and "Ceodose"

H.A. Wollenberg and J. Fitzpatrick . . . . . . . . . . . . . . . 22

Investigation of Radon-222 in Subsurface Waters as an Earthquake Predictor

A.R. Smith, H.R. Bowman, D.F. Mosier, F. Ascro, H.A. Wollenberg and Chi-Yu King. . . . . . 23

Sulfide Levels in Natural Waters: A Possible New Geothermometer

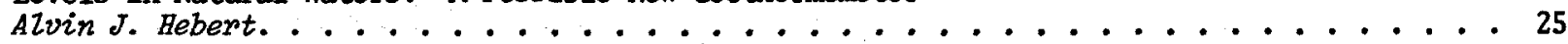

A Portable Field Water Sample Filtration Unit

Alvin J. Bebert and Gardener G. Young. . . . . . . . . . . . . . . . . . 27

Evidence in Support of a Homogeneous Source for Alkalic Basalts

H. Bowman, F. Ascro and I. Carmichael. . . . . . . . . . . . . . . . 28

High Temperature Properties of Silicate Liquids

I.S.E. Carmichael. . . . . . . . . . . . . . . . . . . 29

National Geothermal Information Resource (GRID)

S.L. Phillips, J.A. Fair, F.B. Henderson and S.R. Schuartz . . . . . . . . . 29

CONTROLLED THERMDNUCLEAR RESEARCH

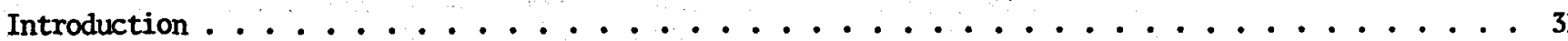

Plasma Theory

B.I. Cohen, G.L. Johnston, S. Johnston, A.N. Kaufman, M.A. Mostrom, D.R. Nicholson,

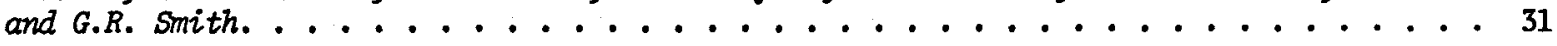

Plasma Production and Heating Experiments

C.F. Burrell, J.R. Cary, C.F. Chan, W.S. Cooper, W.B. Kunkel, and D.R. Pirkle. . . . . . 33

Atomic Physics Studies

K.H. Berkner, W.G. Graham, D. Leung, R.V. Pyle, S.E. Savas, A.S. Schlacter,

P.J. Schneider, and J.W. Stearns. . . . . . . . . . . . . . . 35 
Tormac Project

I.G. Brown, P.J. Channell, J. Coonrod, M. Greenwald, J. Harmer, W.B. Kunkel,

M.A. Levine, B. Myers, A.M. Sessler, R. Shaw, and W.I.B. Smith . . . . . . . .

Neutral-Beam Development and Technology

W.R. Baker, K.H. Berkner, W.S. Cooper, K.W. Ehlers, D.B. Hopkins, J.M. Haughian,

W.B. Kunkel, H.M. Ouren, B.A. Prichard, R.V. Pyle, and J.W. Stearns. . . . . . . .

SOLAR ENERGY

Introduction.

Solar Heating and Cooling of Buildings: Development of Control System and Ammonia-Water

Absorption Air Conditioner

Kim Dao, Melvin Simmons, Richard Wolgast, Eugene Binnall, Charles Dols,

Richard Shaw, Wigbert Siekhaus, James Dillworth, and Michael Wahlig. . . . . . . .

Measurement of Circumsolar Radiation

D.F. Grether, A.J. Bunt, and M. Wahlig. . . . . . . . . . . . .

Solar Cell Research

Wigbert J. Siekhaus, Yip Wah Chung, Wei-Jen Lo, Tom Kaminska, Chin An Chang,

and John MoLrue. . . . . . . . . . . . . . . . . . .

Photopotential Generation Catalyzed by Bacteriorhodopsin

Lester Packer, Paul Shieh, Tetsuya Konishi and Stephanie Tristram . . . . . . . . .

Biophotolysis Using Nitrogen-Starved Cultures of Anabaena cylindrica

Joseph C. Weissmon and John R. Benemann ....................

$\mathrm{H}_{2}$ Production by Photosynthetic Preparations: Studies with Heterocysts of Cyanobacteria

and a Coupled Chloroplast-Hydrogenase System

Lester Packer, Elisha Tel-Or, Ian Fry and George Papageorgiou . . . . . . . . . . .

The Nitinol Engine Project

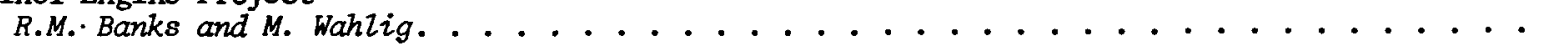

CHEMICAL PROCESS RESEARCH AND DEVELOPMENT

Introduction. . . . . . . . . . . . . . . . . . . . . . . . .

Selective Hydrogenation of Coal

A.T. Bell, E.A. Grens, E.E. Petersen, T. Vermeulen, K.H. Conklin, T.T. Derencsenyi,

G.P. Dorighi, D.C. Draemel, R.R. Holten, D.J. Madieros, and M. Seth. . . . . . .

Conversion of Coal to Clean Liquid and Gaseous Fuels

G.A. Somorjai, A.T. Bell, J.C. Smart, K.P.C. Vollhardt, M.J. Veraa, W.A. Bollinger, J.G. Ekerdt, C.S. Kellner, B.A. Sexton, C. Curtis, A. Sievert, K. Tanner,

W.G.L. Aalbersberg, A.J. Barkovich, R.L. Funk, R.C. Hillard III. . . . . . . . . .

The $\mathrm{SO}_{3}-\mathrm{SO}_{2}$ System for Chemical Storage of Thermal Energy

3 S. Lynn and A.S. Foss

INSTRUMENTATION DEVELOPMENT

Introduction. . . . . . . . . . . . . . . . . . . . . . . .

Origin and Control of the Dominant Impurities in High-Purity Germanium

E.E. HaZler, W.L. Hansen, G.S. Hubbard and F.S. Goulding. . . . . . . . . . . . .

The Survey of Instrumentation for Environmental Monitoring

D.A. Mack, N.M. Amer, P. Bhardwaja, R.J. Budnitz, C.D. Hollowell, M.J. Kland,

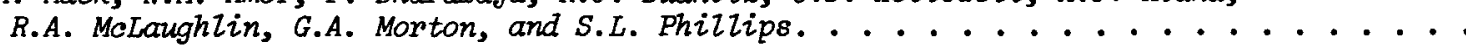

Identification of the Chemical Composition of Trace Elements by Extended X-Ray Absorption

in Fine Structure

A. Thompson, J. Jaklevic, F. Goulding, J. Kirby, M. Klein and A. Robertson. . . . . . .

Energy Dispersive X-Ray Fluorescence Spectrometry Using Pulsed X-Ray Excitation

J.M. Jaklevic, D.A. Landis, and F.S. Goulding . . . . . . . . . . . . . 79

Optimal Design Criteria for Millimeter-Wave Spectrometers

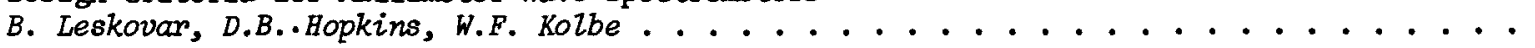

Construction and Performance Characteristics of a Simple $\mathrm{CO}_{2}$ Laser

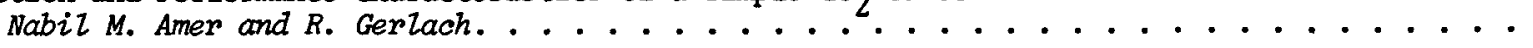

Large-Scale Measurement of Airborne Sulfur Particles Collected on Filters

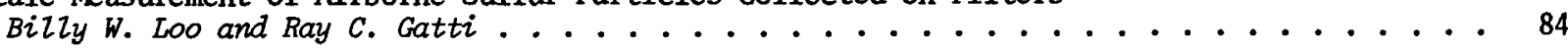

Modulated Fluorescence as a Possible In Situ Monitor for $\mathrm{NO}_{2}$

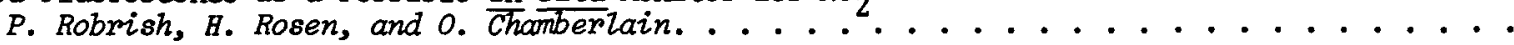


Evaluation of State of the Art Measurement and Instrumentation Techniques for Monitoring Plutonium and Uranium Particulates Released from Nuclear Facilities

A.V. Nero, D.A. Mack, and R.J. Budnitz . . . . . . . . . . . . .

Rubidium-Strontium Geochronology Maynard C. Michel. ......................

ENVIRONMENTAL RESEARCH

Introduction ...............................

Use of Charged Detergents and Hydrophobic Spin-Labels to Probe the Surface Structure of Liposomes and Mitochondria

Rolf J. Mehthorm, Alexandre Quintanitha and Lester Packer. . . . . . . . . . . . . . . . .

Effects of Visible Light and Oxygen of Human Diploid Cells and Mammalian Mitochondria

L. Packer, K. Fuehr, J. Walton, B. Aggarwal and Y. Avi-Dor . . . . . . . . . . . .

Estuarine Pollutant Availability Study

Victor C. Anderlini, John W. Chapman, Amos S. Newton, Robert W. Risebrough, Brian E. Cole Brock deLapp, Donald C. Girvin, Alfred T. Hodgson, Susan J. MeCormick, Lisa Nelback,

Robert K. Okazaki, MichaeZ H. Panietz, and Timothy Schmidt. . . . . . . . . .

Ecosystem Stability and Dynamics

John Harte and Donald Levy . . . . . . . . . . . . . . . . . . . . .

Environmental Assessment of Solar Energy Conversion

M. Davidson, D.F. Grether, M.W. Horovitz . . . . . . . . . . . . .

The Lake County Project - The Local Impacts of Geothermal Development at the Geysers

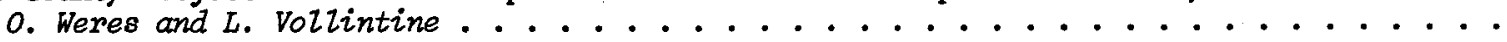

Combustion-Generated Indoor Air Pollution

Craig D. HolloweZl, Robert J. Budnitz, George D. Case, and Gregory W. Traynor. . . . . . .

Atmospheric Aerosol Research

T. Novakov, Principal Investigator; P.J. Bekowies, H.A. Brendel, S.G. Chang, M. Clemenson, R.L. Dod, R.D. Giauque, A.B. Harker, C. D. Hollowell, L. Hughes, S.S. Markowitz,

P.J. Pagni, H. Rosen, R.C. Schmidt, G.W. Traynor, J.R. Wallace, L. Wroth. . . . . . .

ENERGY ANALYSIS

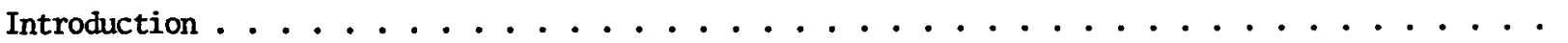

Impacts of A1ternative Electricity-Supply Systems for California

Williom E. Simi, Jayant A. Sathaye, Leonard Kunin, Henry Ruderman, Richard Sextro,

Peter Chan, and Mark Horovitz .....................

A Linear Economic Model of Fuel and Energy Use in the United States

Roger GLassey, Peter. Benenson, Benry Ruderman, Esther Schroeder, Barbar Kach,

and Peter Chan. ........................

Manpower Constraints on Energy Plant Construction and Operation

Peter Benenson, Henry Ruderman, Esther Schroeder, and Frederick Winyard. . . . . . . .

Interregional Energy Flows

Leonard Kunin.

Energy Budgets and Multipliers for the Rocky Mountain States Jayant A. Sathaye and Leonard Kunin. . . . . . . . . . . . . . . . . .

Estimates of 1972 U.S: Employment for Use in Input/Output Applications

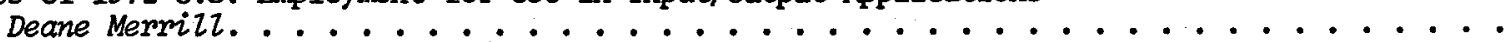

Probabilistic and Statistical Studies of Disease Effects of Pollutants

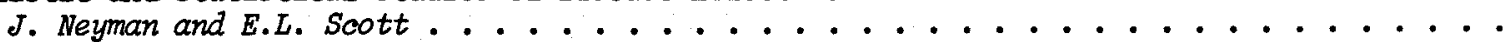

ENERGY USE AND CONSERVATION

Introduction . . . . . . . . . . . . . .............

Combustion Research

F. Robben, Y. Agrawal, T. Hadeishi and R. McLaughlin ..............

Energy and Buildings - A Report on the 1975 Berkeley Sumner Study

E.T. Dean and A.H. Rosenfeld .......................

Energy Conservation Assessment

S.M. Berman, C. Blumstein, P. Caesar, M. Davidson, E.P. Kahn, and A. Makhijani . . . . .

Investment Planning in the Energy Sector

E.P. Kahn, M. Davidson, A. Makhijani, P. Caesar, and S.M. Berman . . . . . . . . 
ENGINEERING SCIENCES

Introduction

Mass Transit Analysis

D. Theodore Scalise, AlZan A. Arthur, David Bereanai, J.G. Bolger, V.P. Elischer, D.M. Evans, Martin Graham, J. Gunn, Raymond Louis, Frank Olken, A. Roberts,

David B. Turner, Kenneth G. Wiley . . . . . . . . . . . . . . . . . . . . . . . 143

Roadway Power System for Ful1-Performance Electric Cars (ROLEC)

J.G. Bolger and D.T. Scalise . . . . . . . . . . . . . . . . . . . . . 145

Earthquake Prediction: In Situ Stress Monitoring - Resistivity

H.F. Morvison. . . . . . . . . . . . . . . . . . . . . . . . 147

Earthquake Reactor-Safety Research

M. Aslam, W.G. Godden, L. Ruby and D.T. Scalise. . . . . . . . . . . . . . . . . 147

SPECIAL RESEARCH PROJECTS

Introduction . . . . . . . . . . . . . . . . . . . . . . . 150

Mechanism of Sickle Cell Hemoglobin Modification by Ethyl Acetimidate - An Antisickling Agent

Lester Packer, Edurin N. Bymun and Gabriel Ogunmola . . . . . . . . . . . . . . . . 150

Applications of Scientific Techniques to Archeology

Erank Asaro and Helen V. Michel. . . . . . . . . . . . . . . . . . . 152

Resonant Raman Scattering from Trigonal Seleniun

Nabil M. Amer. . . . . . . . . . . . . . . . . . . . . . . 154

Tunable Laser Spectroscopy of $\mathrm{NO}_{2}$

P. Robrish and $H_{\text {. Rosen. }}{ }^{2}$. . . . . . . . . . . . . . . . . . . . . 156 


\title{
Geothermal and Geosciences
}

\author{
INTRODUCTION
}

The Energy and Environment Division's geothermal and geosciences program is concerned with the practical development of this energy source and with basic research in the geosciences to provide fundamental information for the development of more sophisticated energy systems in the future.

Several subjects of interest to geothermal development and the supporting basic science are included in the division program. A main effort is the program for the evaluation of techiques to locate and assess potential liquiddominated geothermal resource areas. Field activities in north central Nevada have employed a variety of geophysical, geological, and geochemical techniques for diagnostic evaluation.

Scientific and engineering projects include the formulation and improvement of numerical models of geothermal reservoirs, reservoir. compaction, reinjection of spent fluid, and twophase heat and mass transfer in porous media systems. Theoretical work is also being done to formulate equations predicting the thermodynamic properties of the mixed aqueous electrolytes present in geothermal brines. This information has been used in calculations aimed at the optimization of power cycles and will be of use to others in power plant design. The kinetics of silica dissolution and precipitation, directly related to scaling in geothermal power plants and the sealing of geothermal reservoir boundaries, is also being investigated. Physical models are being developed to study geothermal convection and reservoir dynamics, and test facilities are being designed to study the properties of rock-fluid systems related to geothermal use. This latter project will also be significant to the thermal recovery methods applied to the extraction of fossil fuels.

A brief reservoir assessment of the Raft River Geothermal Field in Idaho has been made using flow tests on existing wells. Some exploratory work has been done to correlate natural radioactivity with geothermal mineralization and rock type. Other field equipment has been built and tested.

The long range geosciences program includes geochemical studies of mantle source material, and the high temperature properties of silicate liquids relating to heat flow and other magmatic processes.

A project supporting the entire geothermal community is the National Geothermal Information Resource ( GRID), which serves as an information center, criticaily analyzing and evaluating data, and interfacing the information file with the user.

$$
1
$$

GEOTHERMAL FIELD ACTIVITIES, NORIH-CENTRAL NEVADA

\section{H.A. Wollenberg, T.V. MoEvilly, and H.F. Morrison}

A program for evaluation of techniques to locate and assess potential geothermal resourceareas is underway in north-central Nevada.

Geothermal field activities have included a preliminary electrical resistivity survey, reconnaissance geologic mapping and geochemical sampling of the Beowawe area, and extensive geological, geophysical, and geochemical surveys in Buffalo and Grass Valleys, encompassing Buffalo Valley Hot Springs and Leach Hot Springs, respectively. (See Fig. 1 for locations of these areas.) Surface surveys and heat flow measurements are complete in Buffalo Valley, and surface geophysical surveys are presently underway in Grass and Buena Vista Valleys, near Leach and Kyle Hot Springs. (A more detailed explanation of the program and descriptions of methods may be found in Refs. 1 and 2.)
Parameters that are diagnostic in evaluating and comparing geothermal areas were described in detail in Ref. 1. Temperature at depth within a hot spring system is estimated from silica and alkali-element abundances of the hot water. Conductive heat flow is calculated by combining the downhole thermal gradient with thermal conductivity measured on representative core samples. Electrical resistivity reconnaissance is done by bipole-dipole and telluric surveys, while more detailed resistivity data is obtained by the dipole-dipole method. Generally, hot water zones are associated with low resistivity. Microearthquakes (earthquakes with magnitudes « 1) are detected by very sensitive seismometers whose signals are radio-telemetered to a central data acquisition station. A microearthquake zone may indicate the presence of geothermal fluids in a fault zone. Electrical self-potential may 


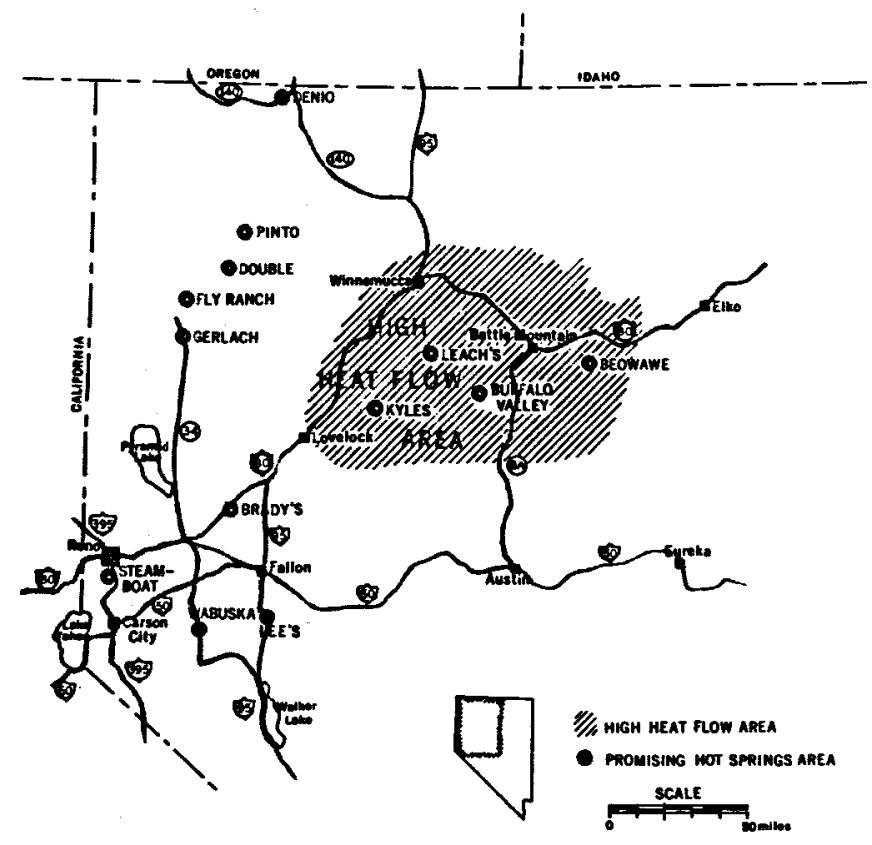

Hot Springs in Northwestern Nevada

Fig. 1. Location map of northwestern Nevada, showing prominent thermal spring areas within and outside of the Battle Mountain High heat flow region. (XBL 735-676) indicate upwelling thermal water as well as normal ground water flow. The nature of spring deposit material is a rough indication of the quality of a potential geothermal resource; generally deposition of silica indicates higher temperatures at depth than do calcium-carbonate deposits.

Important geophysical parameters at Buffalo, Grass, and Buena Vista Valleys are compared in Table 1. The tabulated results of Grass Valley surveys, the combination of electrical resistivity and teleseismic P-delay data suggests the presence of a capped, hot-water reservoir area north-northwest of Leach Hot Springs. Also of interest, two heat flow anomalies were discerned several kilometers southwest and south-southeast of the springs. 3 These are indicated by the 4.9 and 5.1 heat-flow-unit values on the accompanying map (Fig. 2). Neither one is associated with any surface hydrothermal manifestation, however both are significant conductive thermal anomalies. Therefore, it is planned to expand the heat flow coverage of southern Grass Valley, by drilling at least one more $\sim 50$ meter-deep gradient hole, to furnish data for a contour map of heat flow in the area.

Surface geophysical measurements in Buena Vista Valley indicated the presence of a large self-potential anomaly east of Kyle Hot Springs. The nature of this anomaly, whether it is primarily

Table 1. Comparison of important parameters at Buffalo, Grass, and Buena Vista Valleys.

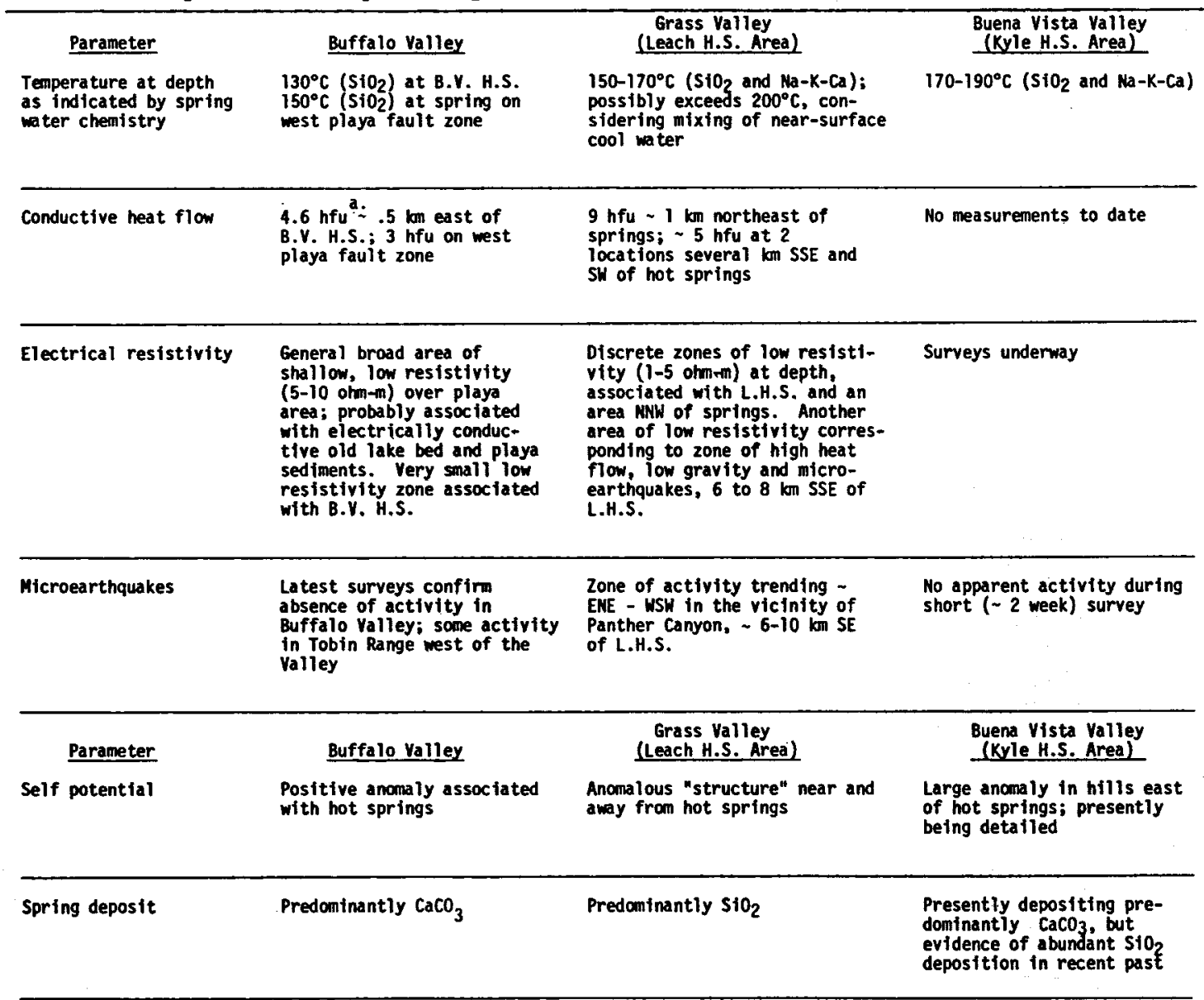

a. $1 \mathrm{hfu}=1 \mu \mathrm{cal} \mathrm{cm}^{-2} \mathrm{sec}^{-1}$ 
LEACH HOT SPRINGS QUAD

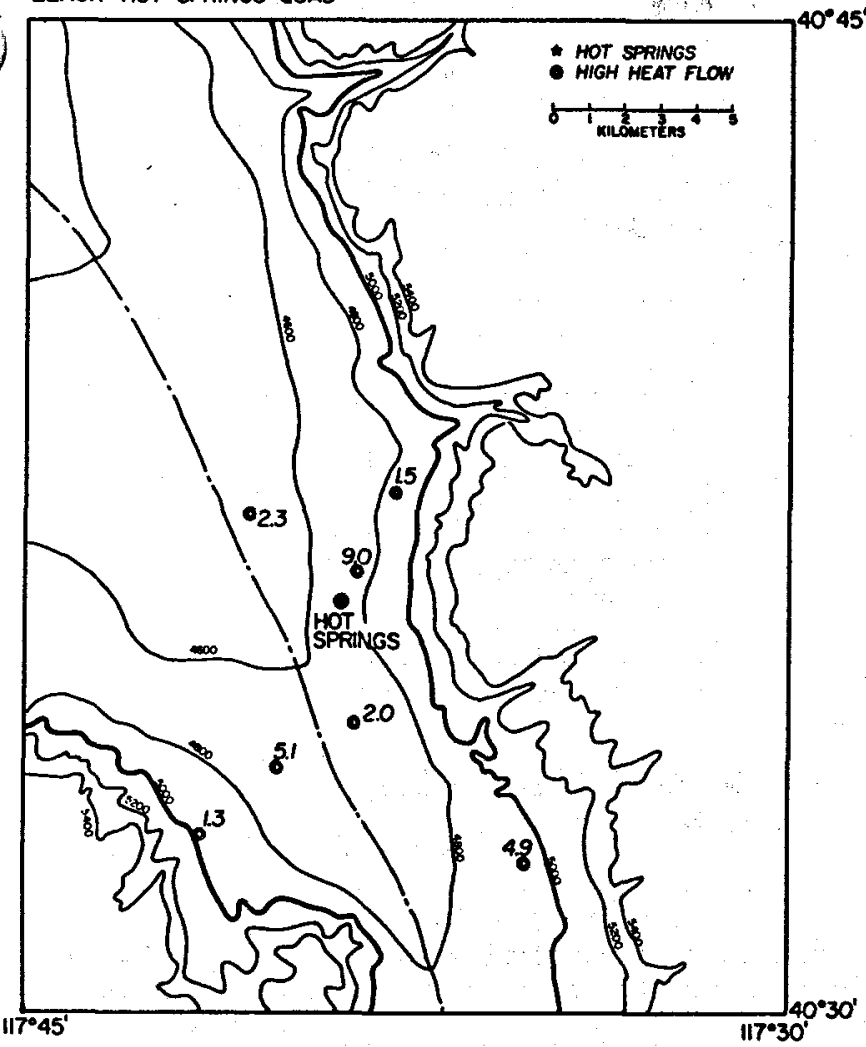

associated with geothermal activity, mineralization, or groundwater flow, will be examined by detailed geophysical, geological and geochemical surveys.

From inspection of Table 1 and the preceding paragraph, it is evident that, from existing data, Grass Valley offers a more promising geothermal resource area than does Buffalo Valley. Based on what we know now, if a geothermal resource does exist in Buffalo Valley, it is probably diffuse and of relatively low temperature, compared to a more wel1-defined, higher temperature geothermal area in Grass Valley.

\section{REFERENCES}

1. H.A. Wollenberg, F. Asaro, H. Bowman, T. McEvilly, F. Morrison and P. Witherspoon, Geothermal energy resource assessment, LBL Report UCID-3762 (1975).

2. Energy and Enviromment Annual Report, 1974, LBL-3253.

3. J.H. Sass, F.H. Olmsted, M.L. Sorey, H.A. Wollenberg, A.H. Lachenbruch, R.J. Munroe and S.P. Galanis, Jr., Geothermal data from test wells drilled in Grass Valley and Buffalo Valley, Nevada, U.S. Geol. Survey Open File Report 76-85; also LBL-4489 (1976).

Fig. 2. Topography (elevation in feet) and heat flow ( $\mu \mathrm{cal} \mathrm{cm}^{-2} \mathrm{sec}^{-1}$ ) in the vicinity of Leach Hot Springs, Nevada.

(CBB 762-1627)

\section{MIXED EXPLICIT-IMPLICIT ITERATIVE FINITE ELEMENT SCHEME FOR THE DIFFUSION EQUATION*}

\section{T.N. Narasimhan and P.A. Witherspoon}

\section{INTRODUCTION}

Geothermal reservoirs form complex physicochemical systems and their mathematical simulation is very much dependent on approximate numerical schemes. Hence, a significant part of the reservoir modeling efforts of the Geothermal Group is directed towards formulating and improving appropriate numerical models. In recent times, the finite element method (FEM) has evolved to become a powerful integral formulation for numerical analysis of the diffusion equation. It provides significant advantage, especially in handling complex geometry and general tensorial quantities such as anisotropy, stress, and dispersion. At the same time, the traditional finite difference approach, al though inconvenient for handling complex geometry or general tensors, possesses inherent advantages of mathematical simplicity and closeness to the physical approach. A.L. Edwards 1 of the Lawrence Livermore Laboratory incorporated an integrated form of the finite difference method (IFDM) ${ }^{2}$ into a powerful computer program called TRUMP for solving the heat transfer equation. This program employs a very efficient explicit-implicit approach combined with an iterative algorithm, thereby avoiding the need for inverting large, sparse matrices. Since it should be of great advantage to combine the special advantages of FEM (handling tensors) with those of the IFLM (mixed explicit-implicit approach, iterative solution), we examined the theoretical bases of the FEM and the IFDM and have successfully developed a mixed explicitimplicit, iterative FEM algorithm. This algorithm has been incorporated into a computer program called FLUMP (mnemonic for Finite element and tr(MP). This new program is suitable for solving the diffusion equation under complex geometry and with material properties, sources and boundary conditions varying with the dependent variable or with time.

An illustrative example of the saturated- 


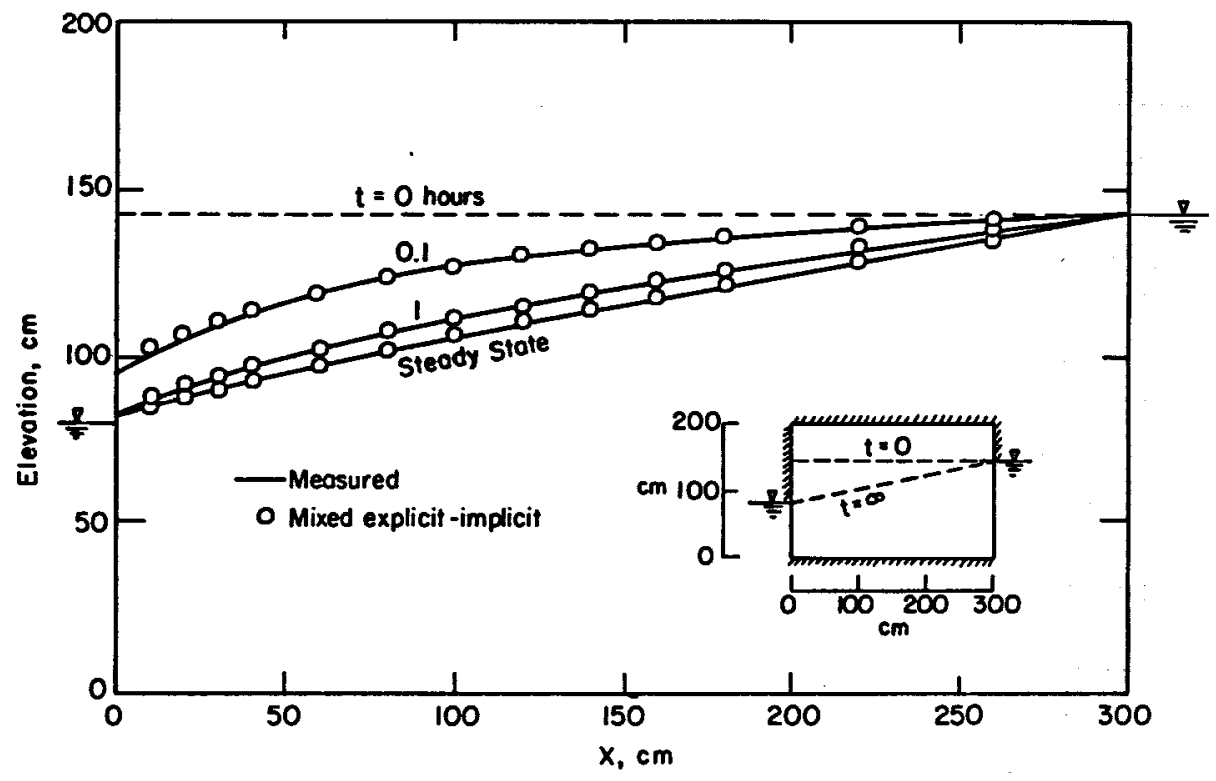

Fig. 1. Saturated-unsaturated flow problem; initial and boundary conditions and the evolution of water table.

(XBL 765-1912)

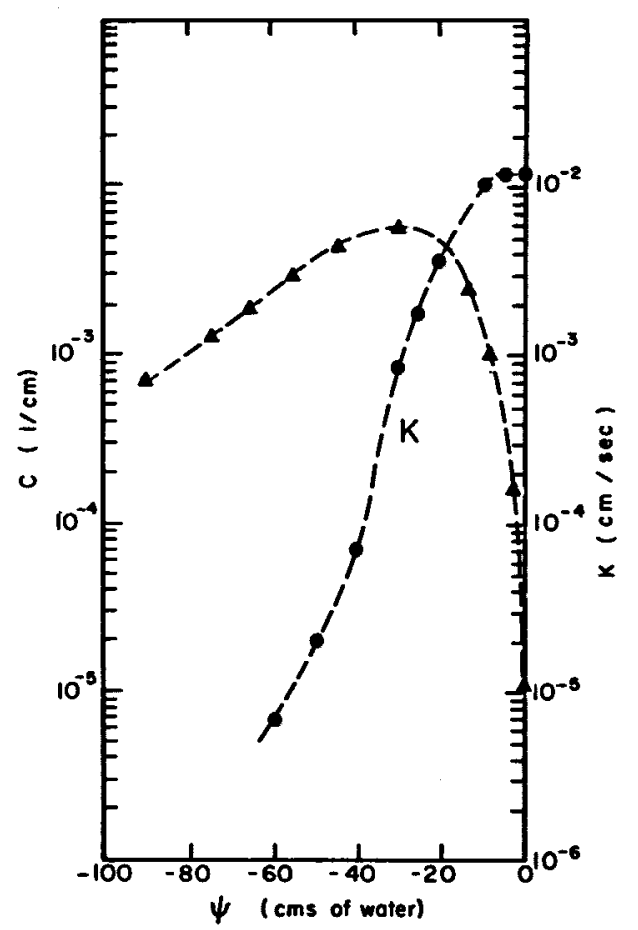

Fig. 2. Saturated-unsaturated flow problem; variation of material properties.

(XBL 764-2826)

unsaturated seepage of water in a sand box will show the effectiveness of this mixed explicitimplicit approach. For a detailed account of the theory the reader is referred to Refs. 3 and 4.

\section{ILLUSTRATIVE EXAMPLE}

This example is the nonlinear problem of saturated-unsaturated flow of water in a rectangular flow region. In addition to the role of gravity in governing the flow of water, the problem is complicated by the fact that both $k$ and $c$ are strong functions of the pressure head $\psi=\emptyset-z$, where $z$ is the elevation.

The problem pertains to a careful experimental investigation conducted by Vauclin et a1.5 and is governed by the equation

$$
\nabla \cdot k(\psi) \nabla[z+\psi]=c(\psi) \frac{\partial \psi}{\partial t} .
$$

The initial and boundary conditions of the problem are presented in Fig. 1.

The nonlinear functional dependence of $\mathrm{k}$ and c on $\psi$ are shown in Fig. 2. Also in Fig. 1 are shown the positions of the surface $\psi=0$ (the "water table") calculated at different instants of time compared with the experimentally observed positions. We see that the agreement is good. The cumulative amount of water depleted from storage within the flow region calculated for various instants of time is compared with the experimental observations in Fig. 3. Considering the complexities of controlling a saturatedunsaturated flow experiment, the discrepancies noted in Fig. 3 should be considered acceptable.

\section{PLANNED ACTIVITIES}

Further developmental work of the mixed explicit-implicit scheme is expected to follow two general directions. The first of these would be to incorporate the transport of heat or chemical species into the governing equation along with the introduction of thermal or chemical dispersion. Dispersion is known to be of tensorial nature, and the dispersion tensor generally rotates with time in response to changes in the velocity field. The FEM offers the most powerful way of handling general tensors. It is extremely difficult to handle the tensors in a general way with finite 


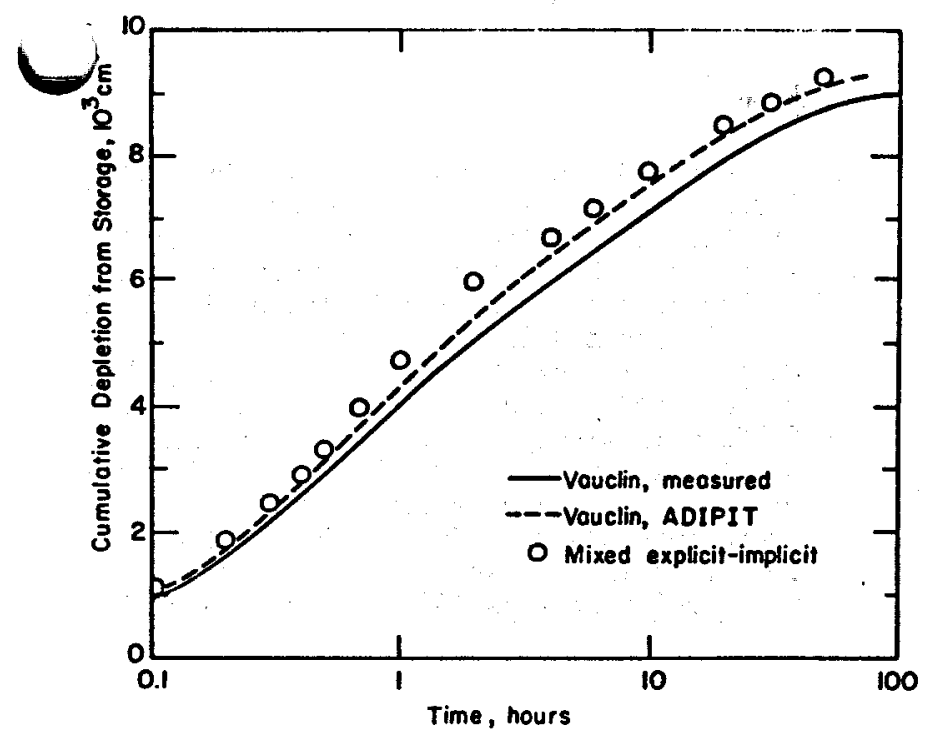

Fig. 3. Saturated-unsaturated flow problem; cumulative depletion from storage as a function of time.

(XBL 765-1910)

difference techniques. The second line of work would be to extend the FLIMP program to general three dimensions. Basically this extension would require an examination of the conductance matrix that would result from using three-dimensional brick elements and a study of their relation to diagonal dominance.

\section{FOOTNOTE AND REFERENCES}

* Condensed in part from LBL-4405 and -4406 .

1. A.L. Edwards, TRUMP: A Computer Program for Transient and Steady-State Temperature Distributions in Multidimensional Systems, DCRL-14754, Rev. 3, Lawrence Livermore Laboratory (1972).

2. T.N. Narasimhan and P.A. Witherspoon, Water Resources Res., 12, 57 (1976).

3. S.P. Neuman and T.N. Narasimhan, Int. J. Num. Meth. Eng., in press (1976).

4. T.N. Narasimhan, S.P. Neuman and A.L. Edwards, Int. J. Num. Meth. Eng., in press (1976).

5. M. Vauclin, G. Vachaud and J. Khanji, in Modeling and Simulation of Water Resource Systems, North Holland Publishing Co. (1975).

\section{REINJECTION IN GEOTHERMAL RESERVOIRS*}

\section{F. Trang and P. A. Witherspoon}

\section{INTRODUCTION}

Reinjection of used geothermal liquids into the producing reservoir has been proposed by many investigators as an effective way to avoid or alleviate the following problems normally associated with geothermal energy production:

(1) Disposal of used geothermal waters with minimum environmental impact.

(2) Land subsidence due to the reduced pressure in the reservoir upon fluid withdrawal.

(3) Depletion of fluid and heat from the reservoir.

However, reinjection creates a zone of relatively cold water around each injection well that grows with time and eventually will reach the production wells. After breakthrough occurs, the temperature of the produced water drops and drastically reduces the efficiency of the whole operation. It is thus important to design the system of production and injection wells in order to prevent injected water breakthrough before a specified time, and to maintain the temperature variations at the roduction wells after breakthrough within reasonable limits.

This project was started during 1975 and we have set ourselves the goal of calculating quantitatively the temperature decrease due to reinjection for a given system of production and injection wells.
Based on this, the physical basis for the optimization of fluid and heat recovery from geothermal reservoirs will be investigated, using as parameters the distribution of production and injection wells and their flow rates. This study, when coupled with a knowledge of above-ground utilization facilities, will afford a development of resource management strategies to optimize the economics of geothermal energy extraction.

\section{ANNUAL ACTIVITIES}

The project of calculating the temperature change in a system of production and injection wells was successfully completed. The calculation is based on a simple two-dimensional steady state mode 1 developed by A.C. Gringarten and J.P. Sauty of the Bureau de Recherches Geologiques et Minieres at Orleans, France. To illustrate the results, we display in Fig. 1 the simple case of a doublet of one production well and one injection well. The dotted lines represent the stream lines and the solid lines, the thermal fronts. In this particular case (with parameters given in the figure caption), the temperature at the production well stays at the reservoir temperature for 5.7 years, at which time the cold injected water reaches the production well by the shortest stream line. After this time, temperature begins to drop asymptotically to the injection temperature. One point to note is that the cold thermal front from the injection well advances toward the production well much more 


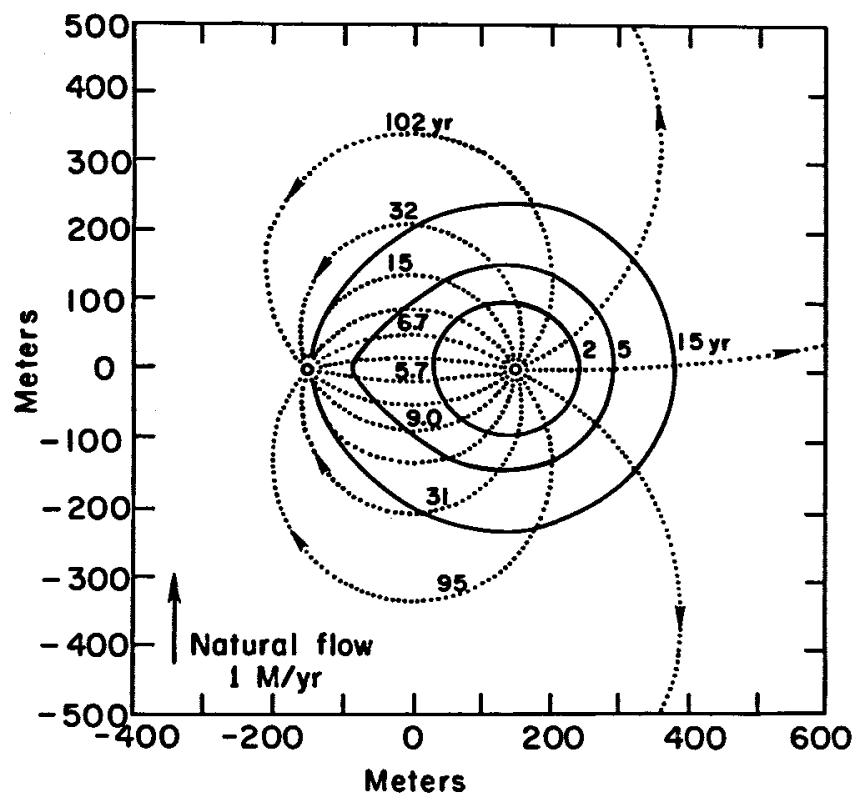

Fig. 1. A production-injection doublet. The dotted lines are streamlines and the solid lines are the thermal fronts. Numbers are given in years. The separation between the wells is 300 meters. A regional flow of 1 meter/ year is assumed in the positive- $Y$ direction. Heat capacity of the rock is assumed to be $0.5 \mathrm{cal} / \mathrm{cm}^{3} /{ }^{\circ} \mathrm{C}$ and its thermal conductivity $0.006 \mathrm{cal} / \mathrm{cm} / \mathrm{sec} /{ }^{\circ} \mathrm{C}$. Thickness of the aquifer is 100 meters with a permeability of 10\%. Flow rate of either well is 100 cubic meters/hour. Temperature of the reservoir is $70^{\circ} \mathrm{C}$ and the temperature of water reinjected is $40^{\circ} \mathrm{C}$. (XBL 7510-8598)

(a)

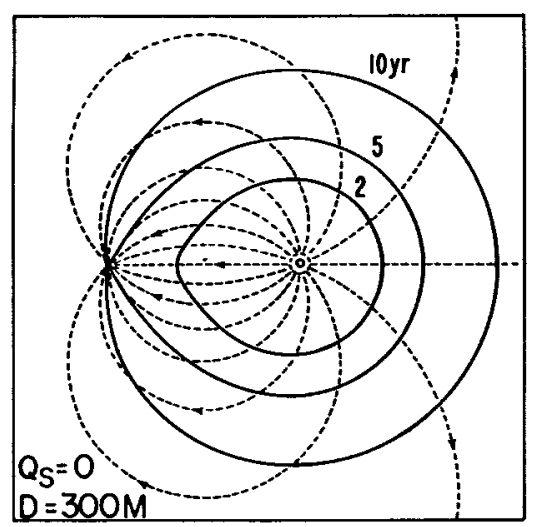

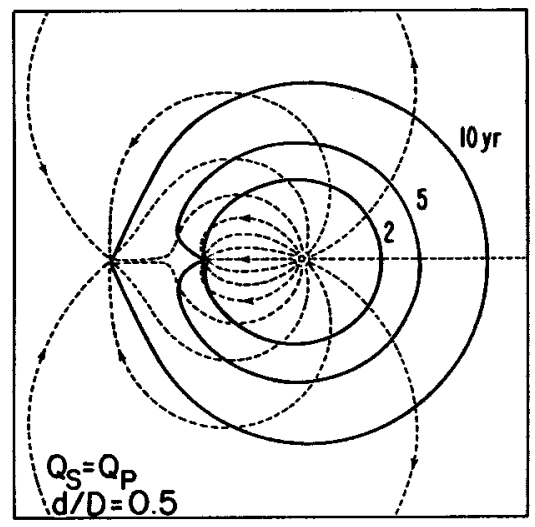

(b)

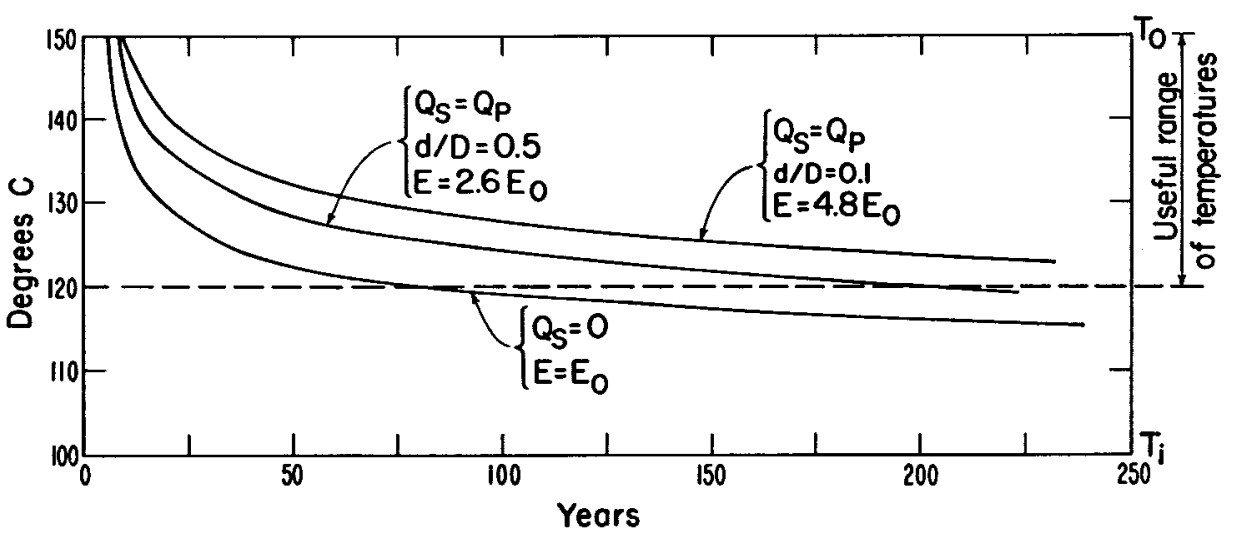

(c)

Fig. 2. The top half of the figure indicates the streamlines (dashed lines) and the thermal fronts (solid lines) of the doublet system with and without screening. The lower half shows the temperature at the production well as a function of time in three cases, (a) no screening well, (b) screening well halfway between production and injection wells at a flow rate equal to production, and (c) same as (b) but with screening well at $1 / 10$ the separation distance from the production well. The symbols $D$ and $d$ represent the distances from the production well to the injection well and to the screening well, respectively. 
slowly than the hydrodynamic front due to the heating of the injected water by the porous medium. The program developed is able to handle complicated systems of many production and injection wells.

In connection with the reinjection problem, we have developed a new concept (called the Screening Concept) of protecting the producing well from reinjection effects by means of one or more screening wells appropriately positioned in between. The basic idea is that by producing water from the screening we11s, one can intercept the cold water and put it back into the injection wells so that if the flow rate at the production well is $Q_{p}$, and that at the screening well is $Q_{S}$, the flow rate at the injection well will be $-\left(Q_{p}+Q_{S}\right)$. The physics of the idea have been worked out, and it is found that the time it takes for the cold, reinjected water to break through at the production well may be increased by as much as a factor of two. After breakthrough, the rate of temperature drop at the production well will be reduced,but not as drastically as when no screening wells are used.

Figure 2 illustrates a simple example of a doublet with and without the screening well. It can be seen from the top half of the figure that the effect of the screening well is to intercept the stream lines, thus pulling the thermal fronts toward it. The lower half of the figure illustrates the temperature curves. The energy extracted is proportional to the flow rate times the area under these curves above a certain given temperature below which the water will not be useful. To make a comparison we take a case of a doublet without screening in which the production and reinjection rates are each $Q$, to be compared with a case with screening in which the production and screening wells are each at a flow rate $Q$ and the injection at 2Q. It is found that the accumulative extracted energy of the system with the screening well (for the case where it is $1 / 10$ the doublet separation distance from the producing we11) after 50, 100 and $\infty$ years are respectively $1.6,2.0$ and 4.8 times larger than that for the unscreened system.

We have also made calculations on the same model for a system of many production and reinjection wells with screening wells in between. A gain in the energy extracted is also obtained, but the amount depends on the distribution of weils in each given case.

\section{PLANNED ACTIVITIES}

An economic feasibility study will be made on the reinjection problem as well as on the new screening concept.

Further physical basis of various means of optimizing fluid and heat recovery in a system of production and reinjection wells will be studied.

\section{FOOTNOTE AND REFERENCE}

${ }^{*}$ Based in part on the talk given by C. F. Tsang at the Workshop on Geothermal Reservoir Engineering, Stanford University, December 15-17, 1975.

1. A. C. Gringarten and J. P. Sauty, to be published, Journal of Geophysical Research.

\section{NUMERICAL SIMULATION OF RESERVOIR COMPACTION IN LIQUID DOMINATED GEOTHERMAL SYSTEMS}

\section{J. Lippmann}

\section{INIRODUCTION}

Geothermal systems are receiving increasing attention as an alternative source of energy. A large number of these systems are of the hot water reservoir type, which are dominated by circulating liquid that transfers most of the heat and largely controls subsurface pressures. During the exploitation of such geothermal systems (i.e., withdrawal of hot fluids) a reduction of pressures is inevitable, which, in some areas, may lead to land subsidence. For exarmle, this phenomenon has been observed 1 at the Wairakei Field, New Zealand, where over a ten year period ground subsidence reached up to 4.5 meters.

In addition to vertical ground movements, horizontal displacements are also observed. These effects may rupture the casings of the geothermal wells, reduce and/or reverse the slope of drainage and irrigation canals, and affect surface installations (e.g., buildings, roads, steam lines, etc.). One method of reducing ground movements is to reinject ${ }^{2}$ the used geothermal 1iquids into the reservoir to minimize pressure reductions.

The objective of this project, started in September 1975, is to develop computer programs to calculate the ground subsidence in liquid dominated geothermal systems. These codes will permit the prediction of how much and where subsidence might be expected in an area where geothermal fluids are produced. Also, by knowing the geologic characteristics of the geothermal system, the location of new production and reinjection wells can be optimized to minimize the environmental effects (subsidence) caused by geothermal energy development.

\section{ANNUAL ACTTVITIES}

A computer program was developed to simulate the effects of production as well as reinjection on the deformation of geothermal reservoirs. The program combines the numerical method of Sorey ${ }^{3}$ for mass and energy transport with another method by Narasimhan 4 for one-dimensional (vertical) con- 
solidation. The model is multidimensional and solves for reservoir deformation in liquid-dominated, heterogeneous, isotropic, non-isothermal systems. Deformation parameters may be non-1inear and non-elastic, while the thermal and hydraulic properties can be temperature and/or pressure dependent.

An integrated finite difference technique is used to solve the flow and energy equations, which are interlaced in time. Pore-pressures and temperatures are obtained, and the pressure changes are used to determine the volumetric and vertical deformations.

\section{PLANNED ACTIVITIES FOR NEXT YEAR}

1. The new program will be checked against available analytical and experimental results.

2. Attempts will be made to apply the program to real geothermal systems where reliable data are available.

3. The program will be combined with available finite element computer codes in order to calculate surface horizontal displacements.

4. New features will be added to the program (e.g., mesh generation and plotting routines).

\section{REFERENCES}

1. W.B. Stilwe11, W.K. Hall and J. Tawhai, Ground movement in New Zealand geothermal fields; presented at the Second United Nations Symp. on the Development and Use of Geothermal Resources, San Francisco, Ca., May 1975.

2. C. F. Tsang and P.A. Witherspoon, An investigation of screening geothermal production wells from effects of reinjection, presented at Geothermal Reservoir Engineering Workshop, Stanford, Ca. December 1975.

3. M.L. Sorey, Numerical modeling of liquid geothermal systems, Ph.D. thesis, University of California, Berkeley, 65 p. (1975).

4. T.N. Narasimhan, A unified numerical model for saturated-unsaturated groundwater flow, Ph.D. thesis, University of California, Berkeley, 244 p., (1975).

5. T.N. Narasimhan and P.A. Witherspoon, An integrated finite difference method for analyzing fluid flow in porous media, Water Resources Res., 12, pp. 57-64 (1976).

\section{DEVELOPMENT OF A MUTIDIMENSIONAL MODEL FOR TWO-PHASE HEAT AND MASS TRANSFER IN POROUS MEDIA}

\section{G. E. Assens}

\section{INTRODUCTION}

One-phase geothermal systems can be simulated on a computer by use of programs such as SCHAFF. 1 However, in many instances, both liquid water and water vapor are present in a geothermal reservoir; one must even expect that a pure liquid system will eventually evolve into a two-phase system as production goes on.

The drastic changes in the fluid properties as well as the transfer of energy involved at flashing or condensation make the simulation of a two-phase system considerably more difficult than that of a pure liquid or pure vapor system.

The development of a computer program able to handle two-phase flow of heat and mass in one to three dimensions was started in 1973 by Thomas J. Lasseter ; ${ }^{2}$ it incorporates many of the features of the program TRIMP. 3 During calendar year 1974, the program was applied to some test problems that revealed the necessity of additional development.

\section{ANNUAL ACTIVITIES}

Calendar year 1975 was planned to be devoted to a thorough checkup of the program SHAFT. A glossary of terms used in its programing was established.
Several errors were corrected, and that part of the progran that reads in properties of the fluid and of the porous matrix and transforms them into a table was remodeled.

\section{PLANNED ACTIVITIES FOR 1976}

1. Implementation of dynamic storage.

2. Provision for anisotropic media.

3. Insertion of the explicit-implicit mixed mode.

4. Simulation of the behavior of liquid/vapor dominated systems.

\section{REFERENCES}

1. M.L. Sorey, Numerical Modeling of Liquid Geothermal Systems, Ph.D. thesis, University of California (1975).

2. T. J. Lasseter and P.A. Witherspoon, The Numerical Simulation of Heat and Mass Transfer in Multidimensional Two-Phase Geothermal Reservoirs, LBL-3261 (1975).

3. A. L. Edwards, TRUMP: A Computer Program for Transient and Steady State Temperature Distributions in Multidimensional Systems, UCRL-14754, Lawrence Livermore Laboratory (1972). 


\title{
THERMODYNAMICS OF GEOTHERMAL BRINES
}

\author{
Leonard F. Silvester and Kenneth S. Pitzer
}

The objective is a set of equations predicting the various thermodynanic properties of mixed aqueous electrolytes with particular attention to the temperatures, pressures, and components present in geothermal brines. The properties considered include Gibbs energy, enthalpy, heat capacity, and density, and will provide the basis for both solubility calculations and various heat transfer or heat balance calculations. The results are to be summarized in a form for convenient engineering use in geothermal plant design. This work was initiated through a program under the Materials and Molecular Research Division (LBL) program.

In 1975 the equations developed previously were fitted to osmotic and activity coefficient data, enthalpy data, and heat capacity data on aqueous sodium chloride solutions from various sources. The thirteen parameter equation reproduces the osmotic coefficient data to \pm 0.005 over the composition range $0-6 \mathrm{M}$ and temperature range $0-300^{\circ} \mathrm{C}$, enthalpy data to \pm 5-10 cal/mole for compositions of $0-5 \mathrm{M}$ at temperatures from $25-100^{\circ} \mathrm{C}$, and heat capacity data to $\pm 0.5 \mathrm{cal} /{ }^{\circ} \mathrm{K}$ for compositions of $0-2 \mathrm{M}$ at temperatures from $25-200^{\circ} \mathrm{C}$. Details of the data treatment and results in the form of tabulated values of the total Gibbs energy, enthalpy, and heat capacity, plus partial molal and excess thermodynamic quantities of sodium chloride solutions for compositions of $0.6 \mathrm{M}$ at $25^{\circ} \mathrm{C}$ intervals from $0-300^{\circ} \mathrm{C}$ along with the same quantities in graphical form for compositions of $0-6 \mathrm{M}$ at temperatures of $100-350^{\circ} \mathrm{C}$ are given in Ref. 1.

Current activities planned in 1976 include: 1) extension of the methods used in treating $\mathrm{NaCl}$ solutions to the available thermal data on $\mathrm{Na}_{2} \mathrm{SO}_{4}$, and $\mathrm{MgCl}_{2}$ solutions, 2) analysis of volumetric $\mathrm{NaCl}$ solution data, and 3) design and initial construction of a micro-flow high-temperature, highpressure calorimeter-densimeter.

In the future the methods used in treating $\mathrm{NaCl}$ solutions will be extended to other salts of geochemical interest. First, calculations will be carried out for pure solutions of each relevant component. Later the available data for mixtures will be considered. The micro-flow calorimeterdensimeter will be used to obtain needed thermal and volumetric data first on pure solutions and later for mixtures.

\section{REFERENCE}

1. L.F. Silvester and K.S. Pitzer, LBL-4456 (1976).

THE USE OF PROGRAM GEOTHM TO

DESIGN AND OPTIMIZE GEOTHERMAL POWER CYCLES

M. A. Green and H. S. Pines

\section{PROPERTIES OF CONCENTRATED SALINE BRINES}

Some geothermal fields in the Imperial Valley of California are characterized by extremely high concentrations of dissolved salts. These geothermal waters, which can be found at temperatures as high as $360^{\circ} \mathrm{C}$, have dissolved solid concentrations as high as 350,000 parts per million. The predominant constituent of these brines is sodium chloride; substantial quantities of magnesium chloride are also found. Experimental thermodynamic data on concentrated brines is lacking. A couple of crude brine models exist but they are not accurate enough. The chemistry of brine solutions is complicated even for the simplest of brine; $\mathrm{NaCl}$ in water. Since $\mathrm{NaCl}$ is a major component of geothermal brines, xur model is based on ionic NaCl solution. The hodel, which has good theoretical backing, was developed by Silvester and Pitzer.

The model, which has many terms, shows good agreement with measured data. It was designed for use up to $300^{\circ} \mathrm{C}$ at concentrations up to 6 molar $(259,621 \mathrm{ppm})$ and was extended for use to about $330,000 \mathrm{ppm}$ ( 8.43 molar) and for lower concentrations it can be used up to $360^{\circ} \mathrm{C}$. The model was changed to include the saturated pressure and saturated liquid specific volume. Increased concentrations of $\mathrm{NaCl}$ result in an increased boiling point temperature at a given pressure. The vapor that is in equilibrium with the brine (when the concentration is greater than zero) is superheated vapor. The temperature and pressure are the same as the liquid phase. The enthalpy, $\mathrm{H}$, entropy, $\mathrm{S}$, and specific volume, V, of the gas are found using the Keenan and Keyes equation of state. Table $I$ is a short $T, P, V, H, S$, table for brine with concentrations of $0,100,000$ and $200,000 \mathrm{ppm}$ of $\mathrm{NaCl}$. Figure 1

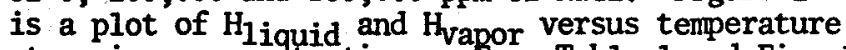
at various concentrations. From Table 1 and Fig. 1 one can see that the enthalpy (and internal space energy) is less for brine than for pure water. This plays an important role in determining the performance of a geothermal power plant which runs on 
Table 1. A short table of saturated liquid pressure, enthalpy, entropy, and specific volume as a function of temperature and concentration for sodium chloride-water brines. a

\begin{tabular}{|c|c|c|c|c|c|}
\hline $\begin{array}{l}\text { Concentration } \\
\text { (ppm) }\end{array}$ & $\begin{array}{c}\mathrm{T} \\
\left({ }^{\circ} \mathrm{C}\right)\end{array}$ & $\stackrel{\mathrm{P}}{(\mathrm{bar})^{\mathrm{b}}}$ & $\underset{\left(\mathrm{Jg}^{-1}\right)}{\mathrm{H}}$ & $\left(\mathrm{Jg}^{-1{ }^{\circ}} \mathrm{K}^{-1}\right)$ & $\left(\mathrm{cm}^{3} \mathrm{~g}^{-1}\right)$ \\
\hline 0 & 50 & 0.123 & 104.4 & 0.336 & 1.012 \\
\hline 0 & 100 & 1.013 & 314.2 & 0.939 & 1.044 \\
\hline 0 & 150 & 4.758 & 527.3 & 1.474 & 1.090 \\
\hline 0 & 250 & 39.73 & 980.5 & 2.425 & 1.251 \\
\hline 0 & 300 & 85.81 & 1239.1 & 2.886 & 1.404 \\
\hline 100,000 & 50 & 0.115 & 92.2 & 0.336 & 0.944 \\
\hline 100,000 & 100 & 0.946 & 280.2 & 0.877 & 0.968 \\
\hline 100,000 & 150 & 4.448 & 469.8 & 1.353 & 1.002 \\
\hline 100,000 & 200 & 14.53 & 661.8 & 1.779 & 1.047 \\
\hline 100,000 & 250 & 37.14 & 862.8 & 2.177 & 1.105 \\
\hline 100,000 & 300 & 80.12 & 1106.4 & 2.610 & 1.183 \\
\hline 200,000 & 50 & 0.110 & 85.8 & 0.335 & 0.910 \\
\hline 200,000 & 100 & 0.902 & 264.9 & 0.850 & 0.930 \\
\hline 200,000 & 150 & 4.249 & 444.6 & 1.301 & 0.958 \\
\hline 200,000 & 200 & 13.90 & 625.3 & 1.702 & 0.993 \\
\hline 200,000 & 250 & 35.59 & 815.4 & 2.079 & 1.033 \\
\hline 200,000 & 300 & 77.01 & 1065.3 & 2.522 & 1.076 \\
\hline
\end{tabular}

aapor values are superheated steam. See the pure water steam tables at the $\mathrm{P}$ and $\mathrm{T}$ of the liquid phase.

$\mathrm{b}_{1}$ bar $=10^{5} \mathrm{~N} \mathrm{~m}^{-2}$.

Imperial Valley brines.

To illustrate the use of concentrated saline brines in program GEOTH, 2 a simple bi-fluid cycle is used (as an example, see Fig. 2). The brine enters the heat exchanger at $280^{\circ} \mathrm{C}$ and 90 bar. Air enters an air cooled condenser at $25^{\circ} \mathrm{C}$. The secondary working fluid is isobutane. The turbine inlet temperature is $220^{\circ} \mathrm{C}$ at a pressure of $50 \mathrm{bar}$. The pump inlet temperature is $36^{\circ} \mathrm{C}$ at a pressure of 5.2 bar. The pinchpoint temperature difference is $10^{\circ} \mathrm{C}$ in the brine heat exchanger and $5^{\circ} \mathrm{C}$ in the air cooled condenser. The efficiencies of the turbine, pump and fan are $80 \%, 75 \%$, and $50 \%$ respectively. Generator and mutor efficiencies are assumed to be 98\%. The net power from the plant is set at $10 \mathrm{MW}$.

Two kinds of brine to isobutane heat exchangers are illustrated. The first is a simple tube shell heat exchanger. This kind of heat exchanger is subject to fouling-it is doubtful if the average $\mathrm{U}$ factor would ever be over $567 \mathrm{~W} \mathrm{~m}^{-2}{ }^{\circ} \mathrm{K}^{-1}$ (100 Btu hr-1 $\mathrm{ft}^{-2} \circ \mathrm{F}^{-1}$ ). The second type of heat exchanger is a four stage flashing cascade heat exchanger. 3 In each stage the brine is flashed; the vapor is scrubbed by the returning condensate (the vapor is de-superheated in the process): then the vapor is condensed on tubes that carry the isobutane. The condensate returns to the brine; the brine leaves the stage at the same concentration it entered the stage. The brine temperature is determined by the stage pressure; the condensing temperature at the tube surface is the condensing temperature of pure water at the stage pressure. Whenever the concentration is greater than zero, the condensing temperature on the tube is lower than the brine temperature. This has the effect of increasing the pinchpoint temperature difference without decreasing the tube surface area. The potential advantage of the cascade heat exchanger is reduced fouling and increased $U$ factor. The 


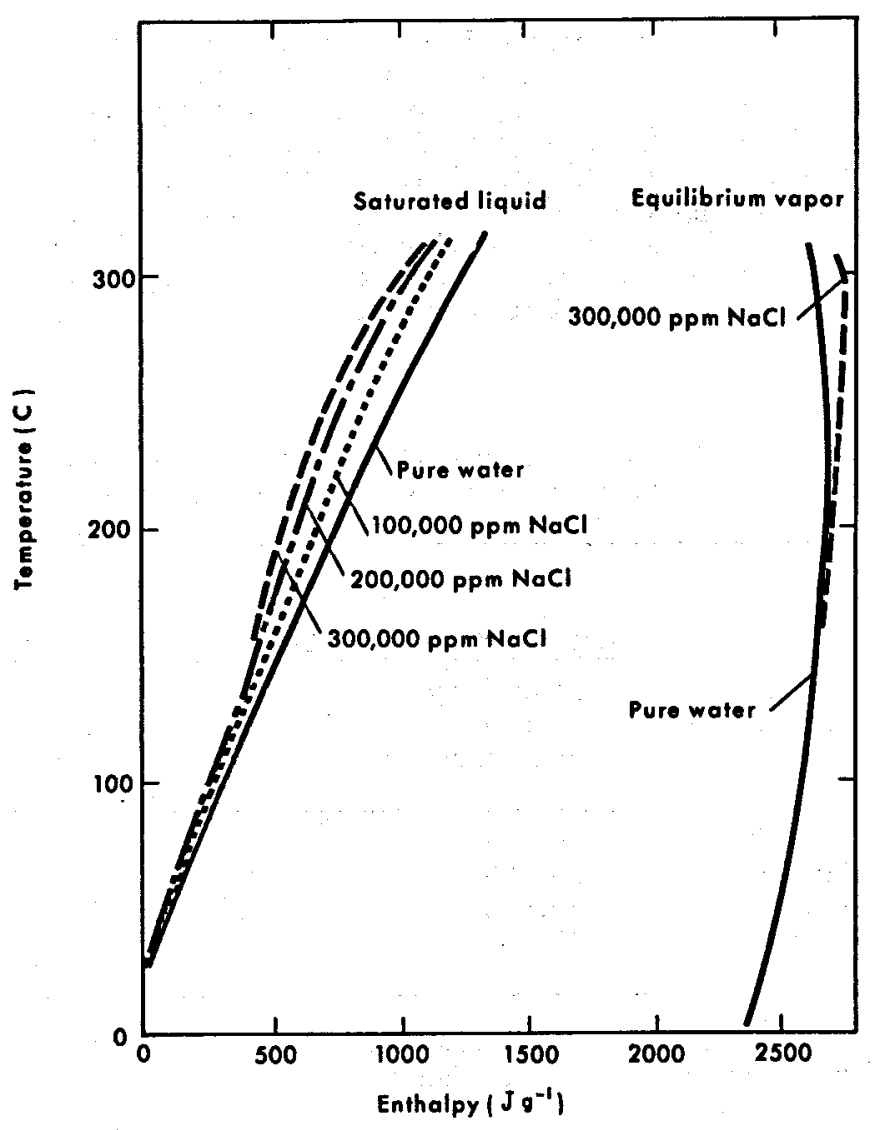

Fig. 1. The enthalpy of a saline brine and steam that is in equilibrium with the brine as a function of temperature and concentration.

(XBL 762-2243)

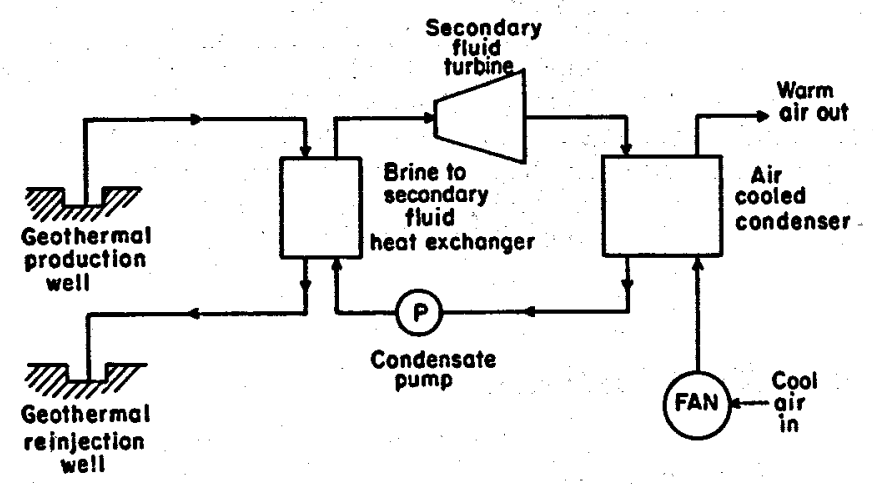

Fig. 2. A simple bi-fluid geothermal power plant cycle with an air cooled condenser. The brine to secondary heat exchanger may be either a tube and shell type or a cascade type.

(XBL 7410-4450)

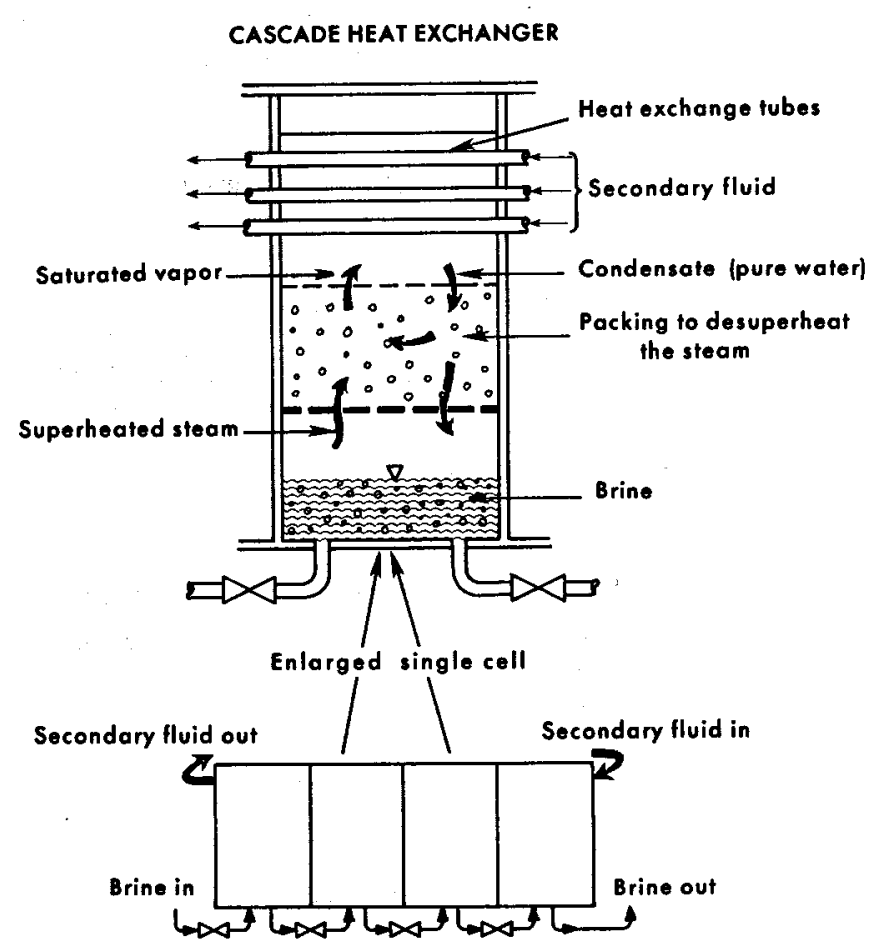

Fig. 3. A schematic diagram of a four stage cascade heat exchanger; detail of the stage is shown.

(XBL. 762-2242)

$\mathrm{U}$ factor in this heat exchanger could be as high as $3402 \mathrm{~W} \mathrm{~m}^{-2} \mathrm{~K}^{-1}\left(600 \mathrm{Btu} \mathrm{hr}-1 \mathrm{ft}^{-2}{ }^{\circ} \mathrm{F}^{-1}\right)$. This could result in a reduced cost power plant.

Figure 3 is a schematic view of a four stage cascade heat exchanger. Table 2, which is divided into two parts, shows the temperature and pressure within the cascade heat exchanger stages as a function of brine concentration. Table 3 compares the performance of a simple bi-fluid cycle using the cascade heat exchanger with the cycle using the ordinary tube and shell heat exchanger.

The cases shown in Table 3 show how the computer handles $\mathrm{NaCl}$ solutions as a wọking fluid. The cascade heat exchanger and tube type heat exchanger involve no change in brine concentration. Some processes such as flash tanks and two-phase turbine expanders will involve changing the concentration of the brine by the removal of water to the gas phase. GEOTHM contains some iterative subroutines which will handle the changing brine concentration problem. Multiple flash cycles have been modeled on GEOTHM for proposed power plants at Niland and Heber in the Imperial Valley. This has been made possible by the development of a saline brine properties deck. 
Table 2. State points within a four-stage brine to isobutane cascade heat exchanger for brine concentrations of 0 and $200,000 \mathrm{ppm}$.

A. FOUR STAGE CASCADE HEAT EXCHANGER

\begin{tabular}{cccccc}
$\begin{array}{c}\text { Stage } \\
\text { number }\end{array}$ & $\begin{array}{c}\text { Brine } \\
\mathrm{P} \\
(\mathrm{bar})\end{array}$ & $\begin{array}{c}\text { Brine } \\
\mathrm{T} \\
\left({ }^{\circ} \mathrm{C}\right)\end{array}$ & $\begin{array}{c}\text { Condensate } \\
\mathrm{T} \\
\left({ }^{\circ} \mathrm{C}\right)\end{array}$ & $\begin{array}{c}\text { Isobutane } \\
\text { temperature }\end{array}$ \\
\hline 1 & 27.95 & 230.0 & 230 & 163 & 220 \\
Inlet & Outlet \\
2 & 12.54 & 190.0 & 190 & 135 & 163 \\
3 & 4.76 & 150.0 & 150 & 95 & 135 \\
4 & 1.43 & 110.0 & 110 & 40 & 95
\end{tabular}

Brine concentration

Inlet brine enthalpy

Inlet brine temperature

Brine to isobutane flow ratio

Pinch point delta $\mathrm{T}$

\section{B. FOUR STAGE CASCADE HEAT EXCHANGER}

\begin{tabular}{cccccc}
\hline $\begin{array}{c}\text { Stage } \\
\text { number }\end{array}$ & $\begin{array}{c}\text { Brine } \\
\mathbf{P} \\
\text { (bar) }\end{array}$ & $\begin{array}{c}\text { Brine } \\
\mathrm{T} \\
\left({ }^{\circ} \mathrm{C}\right)\end{array}$ & $\begin{array}{c}\text { Condensate } \\
\mathrm{T}\end{array}$ & \multicolumn{2}{c}{$\begin{array}{c}\text { Isobutane } \\
\text { temperature }\end{array}$} \\
\hline 1 & 27.95 & 239.0 & 230 & 166 & 220 \\
Inlet & Outlet \\
\hline 2 & 12.54 & 197.5 & 190 & 136 & 166 \\
3 & 4.76 & 156.4 & 150 & 96 & 136 \\
4 & 1.43 & 115.4 & 110 & 40 & 96 \\
\hline
\end{tabular}

Brine concentration

Inlet enthalpy

Inlet temperature

Brine to isobutane flow ratio

Pinch point delta $\mathrm{T}$

The net power yields shown in Table 3 are interesting because the four stage cascade heat exchanger does not fare well thermodynamically as compared to the tube and she11 heat exchanger. Increasing the number of stages will improve the efficiency considerably. In both cases the 300,000 ppm brines cannot be cooled below $158^{\circ} \mathrm{C}$ without the $\mathrm{NaCl}$ coming out of solution. The cases in Table 3 are idealized but they show that salts in the water in a geothermal field will have a detrimental effect on plant performance aside from the obvious fouling and corrosion problems found in systems using saline brines.

Table 3. The net electrical energy per unit we11 flow as a function of brine concentration.

Net electrical energy yield (W hr/ $\mathrm{kg}$ )

Brine concentration cascade

Tube and she11 $280^{\circ} \mathrm{C}$

0.832

$10^{\circ} \mathrm{C}$ heat exchanger heat exchanger

\begin{tabular}{rll}
\hline 0 & 26.673 & 35.145 \\
100,000 & 23.189 & 30.968 \\
200,000 & 20.995 & 28.398 \\
300,000 & $15.066^{\mathrm{a}}$ & $\cdots \cdots$
\end{tabular}

aThis is a three stage heat exchanger, the fourth stage would fill up with salt.

$\mathrm{b}_{\mathrm{NaCl}}$ would cane out of solution plugging the heat exchanger.

\section{THE KINETICS OF QUARTZ DISSOLUTION AND PRECIPITATION \\ J. A. Apps, E. L. Madsen and R. L. Hinkins}

Geothermal brines contain silica which is normally saturated with respect to quartz at the temperature of the enclosing rock formation. This silica can precipitate when the brine is cooled, either in the geothermal power plant or in the formation in which the brine is subsequently injected. Convection of the brine within the geothermal reservoir also results in dissolution, transport and precipitation of silica, leading eventually to formation sealing at the reservoir boundaries.
At the present time none of these processes have been adequately investigated. It is not known whether the potential problems caused by such processes will be severe or inconsequential. However, their importance to utilities planning to exploit geothermal power is very great. The management of a utility does not wish to be in the position of having made substantial investments in plant construction only to discover that the plant is subject to repeated fouling, or that the injection wells are continually plugging and require expen- 
sive rehabilitation.

In order to provide quantitative answers to these problems, this project has been initiated in 1975 specifically to investigate the kinetics of silica dissolution and precipitation.

The objectives of the project are: (1) to determine what deleterious effects will result from rapid withdrawal of hot water and reinjection of spent fluids in a convecting reservoir, and (2) to formulate equations to predict the rate of scaling in geothermal power plant components.

\section{ANNUAL ACTIVITIES}

During 1975 the principal effort involved the acquisition of data on quartz and amorphous silica dissolution in the aqueous phase, development of a theory to explain the observed dissolution behavior, and evaluation of the data in terms of the theoretical mechanism. As a result of this study, further tasks are planned for 1976 to refine our knowledge of kinetic mechanisms of silica dissolution and precipitation and to verify these mechanisms through experiment. A detailed description follows:

\section{Theory}

Using absolute rate theory, ${ }^{1}$ an a priori assumption is made that the mechanism of silica deposition involves the following reaction:

$$
\mathrm{SiO}_{2}+2 \mathrm{H}_{2} \mathrm{O} \underset{\mathrm{k}_{\mathrm{b}}}{\stackrel{\mathrm{k}_{\mathrm{f}}}{\rightleftharpoons} \stackrel{\mathrm{k}_{\mathrm{f}}}{\leftrightarrows} \mathrm{Si}(\mathrm{OH})_{4}}
$$

where $\frac{k_{f}}{k_{b}}=k_{f}^{*}, k_{b}^{*}$

and $\mathrm{K}_{\mathrm{f}}^{*}=\frac{\left[\mathrm{C}^{*}\right]}{\left[\mathrm{SiO}_{2}\right]\left[\mathrm{H}_{2} \mathrm{O}\right]^{2}} \quad ; \mathrm{K}_{\mathrm{b}}^{*}=\frac{\left[\mathrm{C}^{*}\right]}{\left[\mathrm{Si}(\mathrm{OH})_{4}\right]}$

$C^{*}=$ the activated complex

$\mathrm{K}_{\mathrm{f}}{ }^{*}$ and $\mathrm{K}_{\mathrm{b}}{ }^{*}=$ the equilibrium constants relating

the activated complex with the reactants and product respectively.

$$
\text { Now } \begin{aligned}
& \frac{\mathrm{dn}_{f}}{\mathrm{dt}}=\frac{k \mathrm{kT}}{\mathrm{h}}\left[\mathrm{C}^{*}\right] \mathrm{A \sigma} \\
& =\frac{\mathrm{kkt}}{h} \mathrm{~K}_{\mathrm{f}}^{*}\left[\mathrm{SiO}_{2}\right]\left[\mathrm{H}_{2} \mathrm{O}\right]^{2} \mathrm{A \sigma}
\end{aligned}
$$

$K=$ transmission coefficient

$\mathrm{n}=$ moles of $\mathrm{SiO}_{2}$ going into solution

$k=$ Boltzmann's constant

$\mathrm{T}=$ Absolute temperature

$h=$ Planck's constant

$A=$ Surface area

$\sigma=$ Surface site density

$$
\text { let } \frac{\mathrm{kkT}}{\mathrm{h}} \mathrm{K}_{\mathrm{f}}^{*}=\mathrm{k}_{\mathrm{f}}^{\prime}
$$

where $k_{f}^{\prime}$ is the specific forward rate constant.
Substituting (5) in (4)

$$
\frac{d n_{f}}{d t}=k_{f}^{\prime} \mathrm{A \sigma}\left[\mathrm{SiO}_{2}\right]\left[\mathrm{H}_{2} \mathrm{O}\right]^{2}
$$

by analogy

$$
\frac{d n_{b}}{d t}=k_{b}^{\prime} \operatorname{A\sigma }\left[\mathrm{Si}(\mathrm{OH})_{4}\right]
$$

Net rate of change

$$
\frac{\mathrm{dn}_{f^{-}}-\mathrm{dn}_{\mathrm{b}}}{d t}=k_{f}^{\prime} \mathrm{A \sigma}\left[\mathrm{SiO}_{2}\right]\left[\mathrm{H}_{2} \mathrm{O}\right]^{2}-k_{b}^{\prime} \mathrm{A \sigma}\left[\mathrm{Si}(\mathrm{OH})_{4}\right]
$$

By assuming the activities of the silica and water to be unity, and the activity coefficient of $\mathrm{Si}(\mathrm{OH})_{4}$ to be unity, Eq. (8) can be integrated and reorganized to yield:

$$
\frac{n}{m}=\frac{n_{0}}{m} e^{-\frac{-A \sigma k_{b}^{\prime} t}{m}}+\frac{k_{f}^{\prime}}{k_{b}^{\prime}}\left(1-e^{\frac{-A \sigma k_{b}^{\prime} t}{m}}\right)
$$

where $m=$ mass of solvent

$$
\mathrm{n}_{\mathrm{o}}=\text { initial mass of } \mathrm{Si}(\mathrm{OH})_{4}
$$

\section{Data Aquisition and Evaluation}

The available literature on the kinetics of dissolution and precipitation of quartz and amorphous silica in the aqueous phase was reviewed. Unfortunately, few studies have been made which present information of sufficient quality to evaluate using Eq. (9). Most of the data involve the dissolution or precipitation of quartz in water. Some data are available covering the kinetics of dissolution of quartz in sodium hydroxide or sodium chloride solutions. Evaluation of sodium hydroxide data was not carried out, because of the need to interpret the data in terms of additional dissolved silica species. Only one experiment on the precipitation of amorphous silica was useable. 2

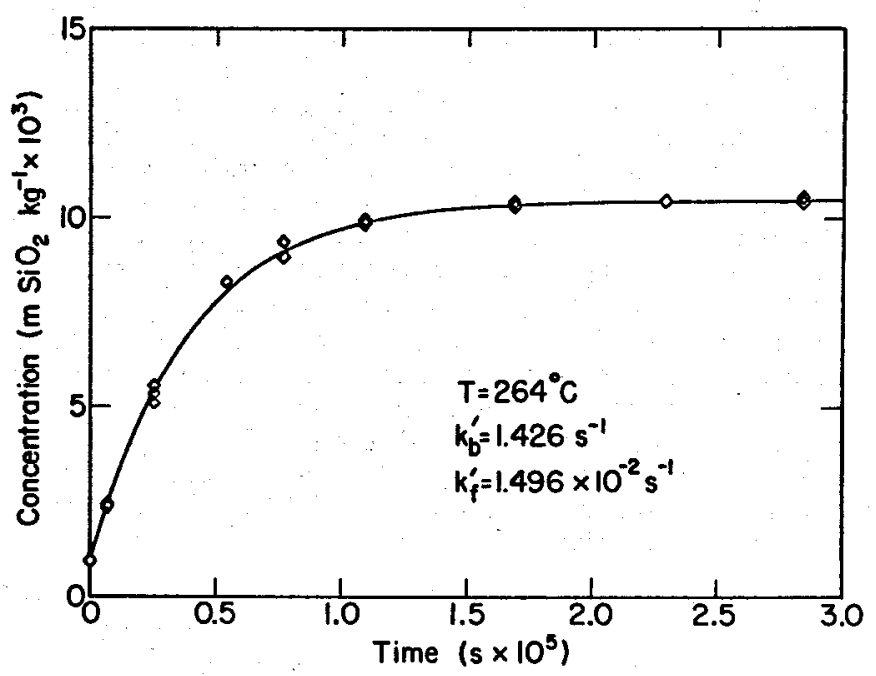

Fig. 1. The dissolution of quartz in water at $264^{\circ} \mathrm{C}$. The data are from Kolvoord and Yorgason ${ }^{3}$. The solid line represents the best fit to the data using Eq. 9.

(XBL 767-3228) 


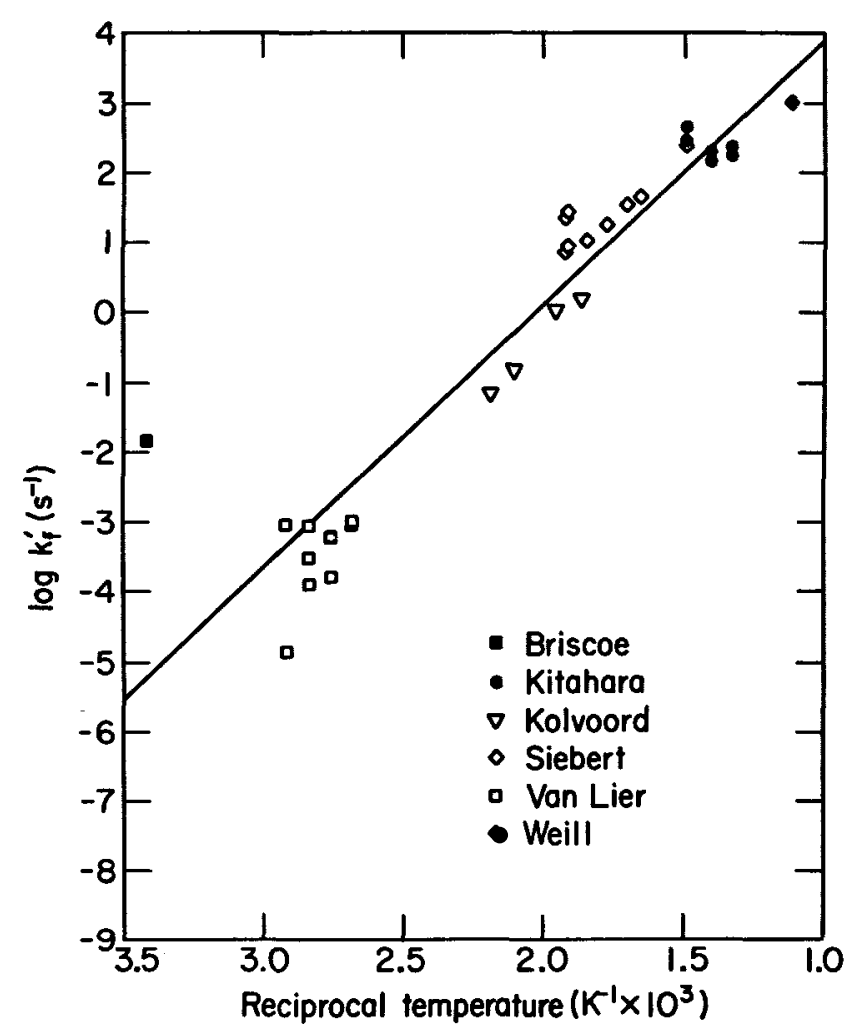

Fig. 2. The logarithm of the specific forward rate constant, $k f$ for the dissolution of quartz in water, plotted against the reciprocal of the absolute temperature. The values $k_{f}$ were calculated from data by Briscoe et al., 4 Kitahara, 5 Kolvoord and Yorgason, 3 Siebert et al., 6 Van Lier et al. 7 and Weill and Fyfe.'8

(XBL 767-3230A)

Equation 9 was fitted to selected experimental data in which $\mathrm{kf}, \mathrm{kb}$ and $n_{0}$ were variable, and values of $k_{f}^{\prime}$ and $k_{b}$ and $n_{0}$ obtained. An example, of an excellent fit, using data of Kolvoord and Yorgason 3 is given in Fig. 1. Arrhenius plots of $\mathrm{k}_{\mathrm{f}}^{\mathrm{k}}$ and $\mathrm{kb}$ for quartz dissolution or precipitation in water were made of data from several sources, 3-8 as illustrated in Figs. 2 and 3 . A straight line, fitted to the data yields activation energies of 21.8 and $17.2 \mathrm{kcal} / \mathrm{mole}$ respectively for the dissolution and precipitation reactions. The computed equilibrium constants assuming $\mathrm{k}_{\mathrm{f}}^{\prime} / \mathrm{k}_{\mathrm{b}}^{\prime}=\mathrm{K}$, for reaction (1) are shown in Fig. 4. The computed values for $k_{b}$ and $k_{f}^{\prime}$ from the $25^{\circ} \mathrm{C}$ data of Briscoe et al. ${ }^{4}$ do not correspond to the observed trends established by higher temperature experiments. This suggests that other mechanisms of silica dissolution and precipitation may prevail at lower temperatures. A possibility is the adsorption of a monolayer of $\mathrm{SiO}_{2}$, as has been suggested by Stöber. 9

The striking feature of quartz dissolution and precipitation kinetics is the large rate constant change between $25^{\circ} \mathrm{C}$ and $350^{\circ} \mathrm{C}$. Data for the dissolution and precipitation of quartz in $\mathrm{NaCl}$ solutions were insufficient to justify detailed analysis.

However, they do suggest that the presence of $\mathrm{NaCl}$ in solutions up to $0.1 \mathrm{~mole} / \mathrm{kg}$ does not affect the rate of quartz dissolution by much more than a

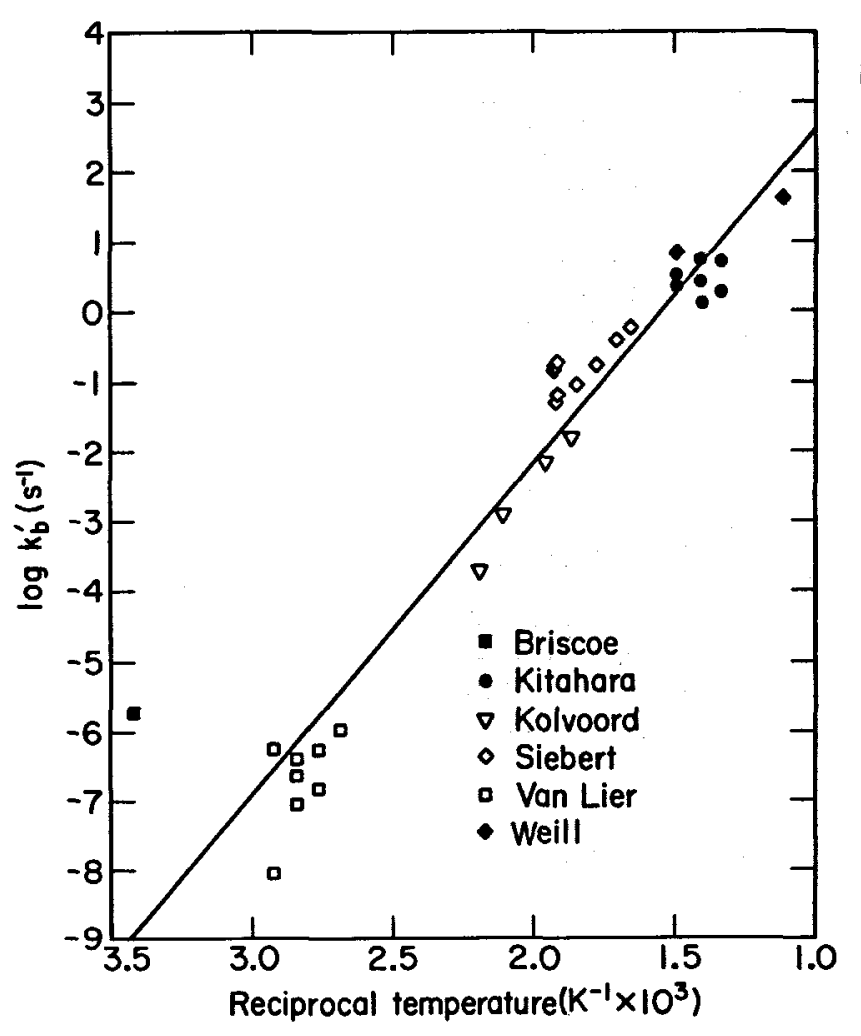

Fig. 3. The logarithm of the specific back rate constant, $\mathrm{kb}_{\mathrm{b}}^{\prime}$, for the dissolution of quartz in water, plotted against the reciprocal of the absolute temperature. The values of $\mathrm{kb}$ ' were calculated from data by Briscoe et a1. 4 Kitahara, 5 Kolvoord and Yorgason, 3 siebert et al., 6 Van Lier et al. 7 and Weill and Fyfe.8

(XBL 767-3232A)

factor of 10. At higher temperatures, the effect may be even less.

Because only one set of data on amorphous silica dissolution is available, no information could be obtained regarding the variation in rate of amorphous silica precipitation as a function of temperature.

\section{Conclusions}

1. The equation developed (Eq. 9) on the a priori assumption that the mechanism of silica dissolution and precipitation is represented by the reaction

$$
\mathrm{SiO}_{2}+2 \mathrm{H}_{2} \mathrm{O}=\mathrm{Si}(\mathrm{OH})_{4}
$$

is consistent with most quartz dissolution and precipitation data. However, the actual reaction may be more complicated.

2. A1though there are sufficient data to observe the trends in quartz dissolution and precipitation kinetics, the data are too variable in quality and insufficient in quantity to estimate the rates accurately.

3. Data describing quartz dissolution in $\mathrm{NaCl}$ solutions are insufficient to draw any firm conclusions regarding the influence of $\mathrm{NaCl}$ concentration on quartz dissolution kinetics. 


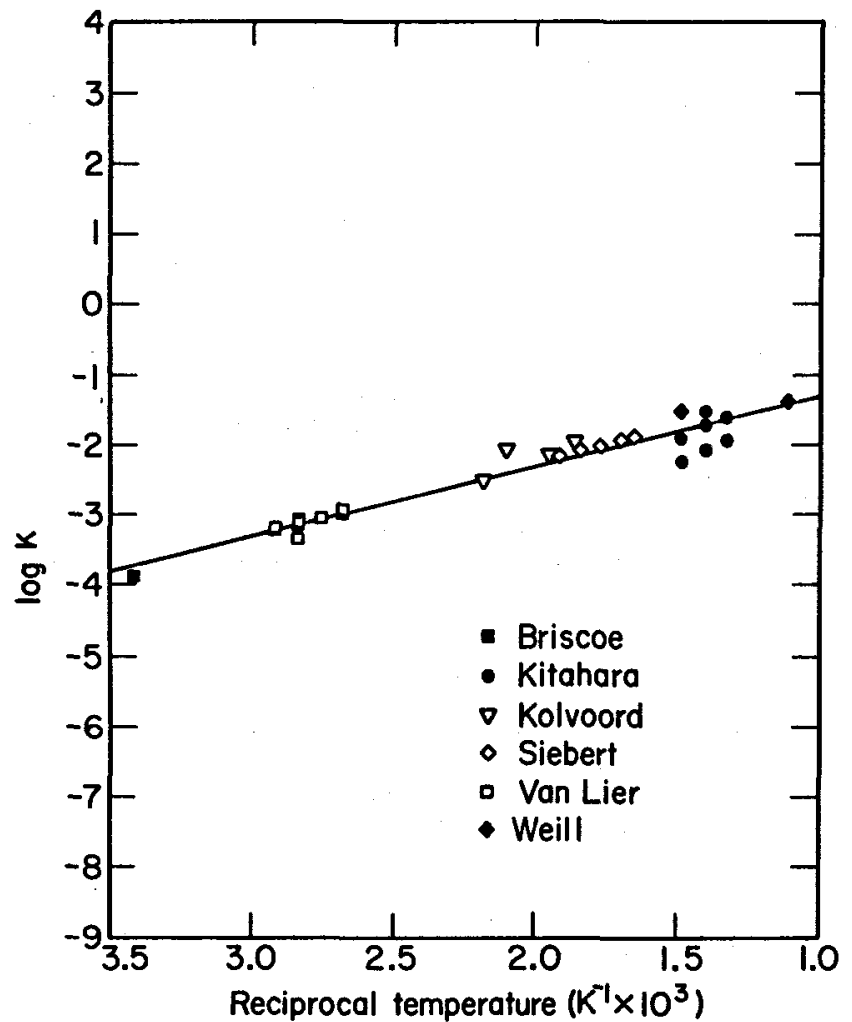

Fig. 4. The logarithm of the equilibrium constant $K$, for the dissolution of quartz in water, plotted against the reciprocal of the absolute temperature. The values of $K$ were calculated from data by Briscoe et a1., 4 Kitahara, 5 Kolvoord and Yorgason, 3 Siebert et al., 6 Van Lier et al. 7 and Weill and Fyfe. 8

(XBL 767-3231A)

4. No information is available that permits evaluation of the precipitation or dissolution of amorphous silica over the range of temperatures of interest.

5. The large changes in the rates of quartz dissolution kinetics are reflected in the use of the silica geothermometer in spring waters to determine temperatures at depth. It is evident that the so-called quartz geothermometer must in fact register temperatures at which the rate of equilibration of the dissolved silica with the environment becomes slow with respect to the cooling rate of the water.

6. At $25^{\circ} \mathrm{C}$ and below, quartz dissolution rates become so slow, as to be almost impossible to determine experimentally. It is possible that other mechanisms, such as adsorption tend to interfere.

\section{REFERENCES}

1. S. Glasstone, K.J. Laidler and H. Eyring, The Theory of Rate Processes, McGraw Hill Book Company, New York (1941).

2. R.K. Iler, J. Colloid Interface Science, 43, 399 (1973).

3. R. Kolvoord and K. Yorgason, "Quartz solubility kinetics", Kennecott Exploration, Inc., Exploration Services Department, Geochemical Research and Laboratory Division report, (November, 1971).

4. H.V.A. Briscoe, J.W. Mathews, P.F. Holt and P.M. Sanderson, Trans. Inst. Mining Met., 46, 291 (1937).

5. S. Kitahara, Rev. Phys. Chem., Japan, 30, 122 (1960).

6. H. Siebert, W.V. Youdelis, J. Leja and E.0. Lilge, "The Kinetics of the Dissolution of Crystalline Quartz in Water at High Temperatures and High Pressures", Unit Process in Hydrometallurgy, The Metailurgical Society of A.I.M.E. Conference, 24, 284 (1963).

7. J.A. Van Lier, P.L. de Bruyn and J. Th. G. Overbeek, J. Phys. Chem. 64, 1675 (1960).

8. D.F. Weill and W.S. Fyfe, Geochim. Cosmochim. Acta, 28, 1.243 (1964).

9. W. Stöber, "Formation of Silicic Acid in Aqueous Suspensions of Different Silica Modifications", Equilibrium Concepts in Natural Water Systems, Advances in Chemistry Series, 67, 161, American Chemical Society (1967).

\section{PHYSICAL MODELING OF GEOTHERMAL RESERVOIRS}

\section{E. Schrock}

In this program we are conducting laboratory experiments on thermal convection in porous media. These experiments serve two important purposes in the total research on reservoir dynamics:

(1) They provide reliable experimental results for use in testing and validating numerical computational methods and computer codes.

(2) They provide the basis upon which model laws can be studied and verified. Eventually, when we have refined the method sufficiently, experiments aimed at directing physical modeling of actual reservoirs may prove to be a useful tool.

To accomplish these objectives, it is necessary to experiment with a variety of geometries, medium characteristics and thermal and hydrodynamic boundary conditions. Our first series of experiments have been carried out in a sand bed in a 
rectangular enclosure $1.1 \times 1.85 \times 1.2$ meters. Water is pumped out of the sand bed through a horizontal porous ceramic tube, cooled by a controlled amount in an external heat exchanger and then reinjected into the sand bed through a second parallel porous tube. This configuration was chosen as a simple rough simulation of geothermal extraction and reinjection. It was designed to produce two-dimensional fields of fluid motion and temperature for comparison with 2-D codes. Initially the sand bed was isothermal. The boundaries of the box were impermeable and were maintained adiabatic. The results of such an experiment reveal the interplay between the forced convection (due to pumping) and natural convection brought about by fluid denisty changes. Tests have been made for a range of parameters such as pumping rate, temperature difference and sand bed permcability such that data have been obtained for cases ranging from strong to negligible natural convection.

The temperature distributions that develop are important in gaining an understanding of the effectiveness of energy remova1. Although the tests so far conducted are very simple we think this approach will eventually lead to an understanding of more complex systems of wells and the abilit to design optimum energy extraction methods. A paper based upon some results is currently in press. 1

A second series of experiments is now underway to study the effects of heating the bottom of the bed and cooling the top. Convection cells are being studied in the absence of pumping and this will be followed by superposition of the two. When these tests are completed we plan to carry out experiments in anisotropic media with horizontal stratification of permeability.

A portion of the research is aimed at identifying scaling laws for designing and interpreting model experiments.

\section{REFERENCE}

1. V.E. Schrock and A.D.K. Laird, "Physical Modeling of Combined Forced and Natural Convection in Wet Geothermal Formations," $\mathrm{J}$. of Heat Transfer (1976). In press.

PROPERTIES AND BEHAVIOR OF ROCK-FLUID SYSTEMS AT HIGH TEMPERATURES AND PRESSURES

W. H. Somerton

\section{INTRODUCTION}

Objectives of this project are to develop methods of and equipment for the measurement of properties and behavior of rock-fluid systems at environmental conditions of pressure, temperature and fluid saturation that may be encountered in geothermal power development and thermal recovery methods applied in the extraction of fossil fuels. These data are needed in the interpretation of well logs in relation to zonal evaluation, in the study of fluid and heat flow in relation to subsurface reservoir management, and in prediction of well bore stability and possible surface effects associated with fluid withdrawal from the reservoir.

Methods of measuring the desired properties and equipment capable of making such measurements to temperatures as high as $200^{\circ} \mathrm{C}$ and pressures of 1000 bars were developed through earlier projects.1,2 Support for this project from the Lawrence Berkeley Laboratory began in FY 1974. Design work is in progress on a new test facility capable of measuring al1 the desired properties concurrently on the same test specimen to temperatures as high as $500^{\circ} \mathrm{C}$ and pressures to 1250 bars. Design problems and materials limitations have necessitated modifications in measuring methods and the development of new methods in some cases. The present test facilities are being used for testing these methods and for a contimuing program of obtaining data on a widening variety of rock-fluid systems within the limits of the equipment.

\section{ACTIVITIES DURING 1975}

Design of the new test facility was completed to the point where actual construction will be necessary before final design details can be worked out. New features of the apparatus include an internal split-cylinder radiant test specimen heater, water cooling in regions of measuring components, seals, and signal lead outlets, an axial cylindrical electrode for resistivity and themal conductivity measurement, and use of alumina $\mathrm{AD}-85$ for transmitting sonic signals and to provide electrical and thermal insulation. Actual construction is awaiting funding from sources as yet unknown.

A new laboratory in Room 120 Hesse Ha11 has been partitioned and provided with services for the apparatus.

Wave velocity studies have shown that temperature decreases both shear and dilatational wave velocities and that the ratio of the two velocities $\mathrm{V}_{\mathrm{S}} / \mathrm{V}_{\mathrm{d}}$ is a good indicator of vapor-1iquid contacts at any pressure and temperature. This is due to the fact that liquid saturation affects the velocities in opposite directions. Knowledge of both shear and dilatational velocities improves the accuracy, of intrinsic strength estimations that are used in borehole stability studies.

The effects of temperature on decreasing permeability and increasing formation resistivity factor 
have been confirmed. Plugging problems in perme-

bility measurements have been virtually eliminated

y improved water treatment and handling and by use of ultra fine filters. The cylindrical electrode method, using the metal sheathing as the outside electrode, has given good results although further testing is needed.

Thermal expansion tests on dry rocks from a geothermal test well showed the strong effect of quartz content. Low quartz content samples showed about half the thermal expansion of quartz-rich specimens. Thermal expansion tests on liquid saturated specimens are now in progress. Application of the present test methods for both thermal expansion and compressibility in the new apparatus must await construction and preliminary testing to determine suitable reference locations.

Thermal conductivity tests were run on the suite of samples from a geothermal test well mentioned above. The steady state apparatus was used for these tests. The strong effect of quartz content mentioned above was also noted in these measurements. Work on the thermal conductivity model was nearly completed. Good agreement between model and test results was obtained, minimizing the importance of including a liquid-solid contact resistance term in the model. Work was continued on the measurement of liquid-solid contact resistivity. Although experimental results indicated the existence of such a resistance, the possible presence of a thin film of adsorbed air on the solid surface or a small amount of gas coming out of solution from the liquid upon transient heating, could not be disproved.

\section{PLANS FOR 1976}

Further progress on the design and construction of the new apparatus must await location of a funding source. In the meanwhile the pressure vessels and the pressure console used with the present apparatus will be moved into the new laboratory.

The Arenberg pulse generator will be repaired and modified to greatly increase the strength of sonic signals. Shear and dilatational transducers will be stacked on the alumina $\mathrm{AD}-85$ loading cylinders and velocity measurements of fluid saturated rocks will be continued.

Permeability, formation resistivity factor, thermal expansion and compressibility testing will be resumed with the relocated and redesigned test apparatus. Hopefully some geothermal testwell cores will become available for testing.

Thermal conductivity testing will be continued using the existing steady-state apparatus. The prediction model will be completed and tested against al1 experimental results. Further development of the needle probe method of measuring thermal conductivity will be a priority matter. Manufacture of the probe, the outer stainless steel sheathing of which will serve as the resistivity electrode, may present some problems, but a commercial manufacturer will be consulted.

Work on the liquid-solid contact resistivity problem will be completed early this year. Work will be resumed on determination of conductive heat transfer coefficients for liquids flowing in porous media.

\section{REFERENCES}

1. W.H. Somerton, Deformation Moduli of Water Bearing Formations at Elevated Temperatures, California Water Resources Center, Contrib. No. 146 (1974).

2. W.H. Somerton, Thermal Properties of Hydrocarbon Bearing Rocks at High Temperatures and Pressures, Final Report American Petroleum Inst. Research Project 117 (1973).

RESULTS OF RESERVOIR EVALUATION TESTS, 1975

RAFT RIVER GEOTHERMAL FIELD, IDAHO

T. N. Narasimhan and P. A. Witherspoon

\section{INTRODUCTION}

On a request from the Idaho National Engineering Laboratory (INEL), the Lawrence Berkeley Laboratory Geothermal Group was asked to provide assistance in assessing the size and producing capabilities of the geothermal reservoir in the Raft River geothermal area near Malta in southeastern Idaho. Between January and August, 1975, INEL had drilled two geothermal wells 4990 and 5990 feet deep respectively and successfully tapped artesian water at approximately $295^{\circ} \mathrm{F}$. The aim of the LBL project was to conduct flow tests on the two wells and make preliminary estimates on the reservoir parameters and geometry to help in further exploration and development of the reservoir.

\section{RESERVOIR ASSESSMENT}

\section{Location and Geology}

The Raft River Valley is located in southeastern Idaho (Fig. 1), about 160 miles northwest of Salt Lake City. The valley, which historically used to be at the intersection of the Humboldt River Trail to California and the Oregon Trail, is henmed on the west, south and east by hill ranges and is open towards the north. The valley is about 15 miles wide in an east-west direction, is over 40 miles long and is at an elevation of about 4800 feet while the surrounding mountains rise to over 9000 feet. 


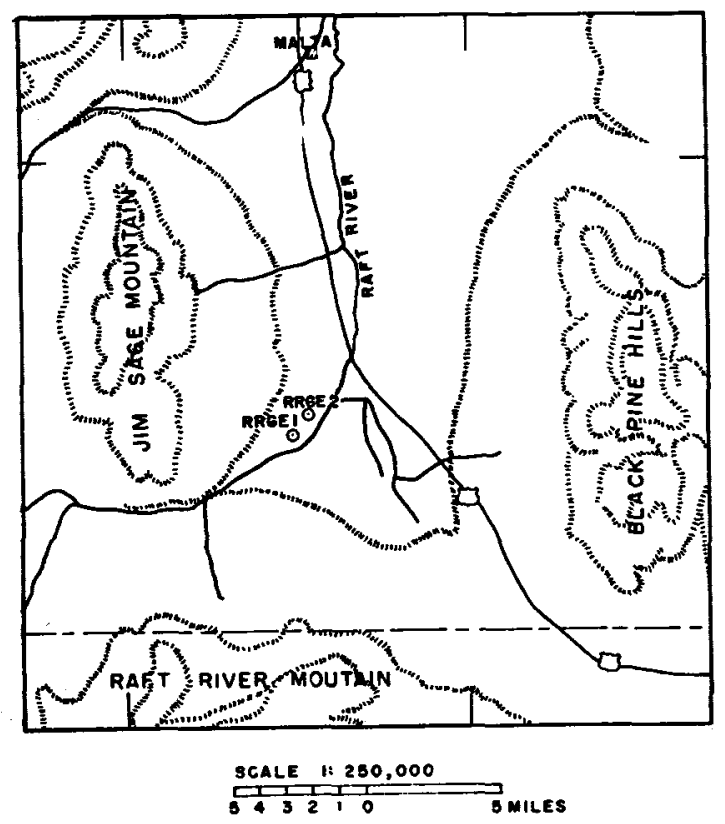

Fig. 1. Location map of Raft River Valley Geothermal Field, Idaho.

(XBL 764-2839)

Table 1

\begin{tabular}{|c|c|c|}
\hline & RRGE 1 & $\underline{\text { RRGE } 2}$ \\
\hline Total depth, feet & 4,989 & 5,988 \\
\hline $\begin{array}{l}\text { Casing } \\
\text { Diameter, in. } \\
\text { Depth, ft. }\end{array}$ & $\begin{array}{l}13-3 / 8 \\
3,624\end{array}$ & $\begin{array}{l}13-3 / 8 \\
4,227\end{array}$ \\
\hline $\begin{array}{l}\text { Uncased hole } \\
\text { Diameter, in. } \\
\text { Depth range, ft. }\end{array}$ & $\begin{array}{l}20 \\
3,624-4,989\end{array}$ & $\begin{array}{l}12-1 / 4 \\
4,227-5,988\end{array}$ \\
\hline $\begin{array}{l}\text { Major Production, } \\
\text { Depth range }\end{array}$ & $3,624-4,200(?)$ & $4,227-5,000$ \\
\hline $\begin{array}{l}\text { Reservoir } \\
\text { Temperature, } \\
{ }^{\circ} \mathrm{F}\left({ }^{\circ} \mathrm{C}\right)\end{array}$ & $\approx 294(146)$ & $\approx 294(146)$ \\
\hline We11 Head Pressure & $\approx 150 \mathrm{psi}$ & $\approx 150 \mathrm{psi}$ \\
\hline
\end{tabular}

Geologically, the Raft River Valley is located close to the junction of two great geological structures, namely, the Basin and Range province on the south and the Snake River Plain on the north. The valley itself is a north-south trending structural depression which is considerably faulted. The floor of the valley is apparently a westward-tilted down-faulted block. 1

The structural depression or the "graben" of the valley is filled with sediments ranging in age from Tertiary (the Salt Lake formation) to Quaternary (the Raft River formation), with a total thickness of about 6000 feet. The Salt Lake formation comprises sandstones, silts and tuffs while the Raft River formation consists of over 1000 feet of alluvial, fluvioglacial and lacustrine silts, sands and gravels. The floor of the depression containing the sediments is formed by a precambrian quartz monzonite, an igneous rock chemically allied to granite.

\section{Description of Wells}

The two geothermal wells, RRGE 1 and RRGE 2 are located in Section 23, Township 15 South, Range 26 East. The distance between the wells is approximately 4000 feet with RRGE 2 being northeast of RRGE 1. The completion data for the two wells are given in Table 1.

Careful geological study of the drilling data indicated that RRGE 1 penetrated a fault zone between 4250 and 4630 feet below ground level. No such fault zone could be recognized within RRGE 2 down to a depth of 5988 feet.

\section{Reservoir Tests}

The basic aims of the reservoir test were to evaluate the permeability and storage parameters of the reservoir and to decipher reservoir geometry. The tests essentially consisted in producing fluid from one of the wells at controlled rates and over prolonged periods of time and observing the water pressure changes in the producing well itself or in the other well. Since the wells have positive well head pressures (artesian), controlled flow can be achieved by carefully opening the well head valve. Also, since the theory of the tests is based on a single, homogeneous fluid, the flow had to be so controlled that there can be no flashing or formation of a vapor phase within the well.

Since the wells are about 4000 feet apart, the pulse introduced at one well can create only very small pressure changes in the observation well. A key piece of equipment in the tests was therefore a very sensitive quartz crystal pressure gage that can be conveniently lowered into the well to any desired depth and which communicates with a surface recording equipment through a conductor cable. The pressure gage has a resolution of $0.01 \mathrm{psi}$ (over the range $0-10,000 \mathrm{psi}$ ) and can withstand temperatures of up to $300^{\circ} \mathrm{F}$.

Details of the Tests

In all, three tests were conducted between September 12 and November 7,1975 . Two of these were production well tests in which the pressure gage was positioned in the production well within the producing interval. This was necessary in order to ensure a fairly stable temperature environment for the pressure gage, which is temperature sensitive. The production well tests were both of short duration and lasted less than 30 hours. After shutting down production, pressure buildup within the well was monitored for approximately 24 hours.

The third test was an interference test in which fluid was produced from RRGE 2 and the pressure changes were monitored in RRGE 1 . Since the latter was quiet and fluid filled, it was not essential to position the instrument close to the 
Table 2

\begin{tabular}{|c|c|c|c|c|c|c|c|c|}
\hline \multirow[b]{2}{*}{ Test No. } & \multirow[b]{2}{*}{ Description } & \multirow{2}{*}{$\begin{array}{l}\text { Duration } \\
\text { Hours }\end{array}$} & \multicolumn{2}{|c|}{ Production } & \multicolumn{2}{|c|}{ Pressure Gage in } & \multicolumn{2}{|c|}{ Maximum Pressure drop } \\
\hline & & & We11 No. & Flow Rate & Well No. & Depth, feet & We11 No. & $\Delta \mathrm{p}, \mathrm{psi}$ \\
\hline 1 & Short Term Test & 15 & RRGE \#2 & $225 \mathrm{gpm}$ & RRGE \#2 & 5200 & RRGE \#2 & 39 \\
\hline 2 & $\begin{array}{c}\text { Long Term Test } \\
\text { on RRGE \#2 }\end{array}$ & $615-1 / 2$ & RRGE \#2 & $400 \mathrm{gpm}$ & RRGE \# 1 & 1000 & RRGE \#1 & 3.6 \\
\hline 3 & $\begin{array}{c}\text { Short Term Test } \\
\text { on RRGE \#1 }\end{array}$ & 30 & RRGE \#1 & $26 \mathrm{gpm}$ & RRGE \#1 & 4700 & RRGE \# 1 & 1.1 \\
\hline
\end{tabular}

bottom of the we11. The interference test, which provides data on a large portion of the reservoir between the two wells, was of a long duration. During this test, RRGE 2 was flowed for approximate$1 y 26$ days.

The specificiations of the tests conducted are given in Table 2 .

\section{RESULTS AND INTERPRETATION}

Before we present and discuss the results it is pertinent to briefly state the theoretical bases of interpretation. The analytical model used for interpretation is that of transient potential distribution around a continuous line source (axisymmetric system) in an infinite, homogeneous, horizontal slab of finite thickness. By the principle of superposition and using image concepts, the model can be extended to non-infinite or bounded systems. Details of this interpretative technique can be found in Witherspoon et al. 2 or Ferris et a1.3 The aim of interpretation is to determine the reservoir permeability, transmissivity, $\mathrm{kH}$, the reservoir capacity, $\phi \mathrm{cH}$ and decipher the existence of barriers or other boundaries. The transient pressure change around a line source in an initially static system is given by the relation 2 (often called the Theis 4 equation)

$$
\Delta P=\frac{q \mu P_{D}}{(v) k H}
$$

where $\Delta P$ is pressure change, $q$ is constant flow rate, $\mu$ is fiuid viscosity, $P_{D}$ is dimensionless pressure, $V$ is a constant depending on units used, $\mathrm{k}$ is permeability and $\mathrm{H}$ is reservoir thickness. Moreover, $\mathrm{P}_{\mathrm{D}}$ is given as an integral of the dimensionless time $t_{D}$

$$
P_{D}=1 / 2 \int_{u}^{\infty} \frac{e^{-u}}{u} d u
$$
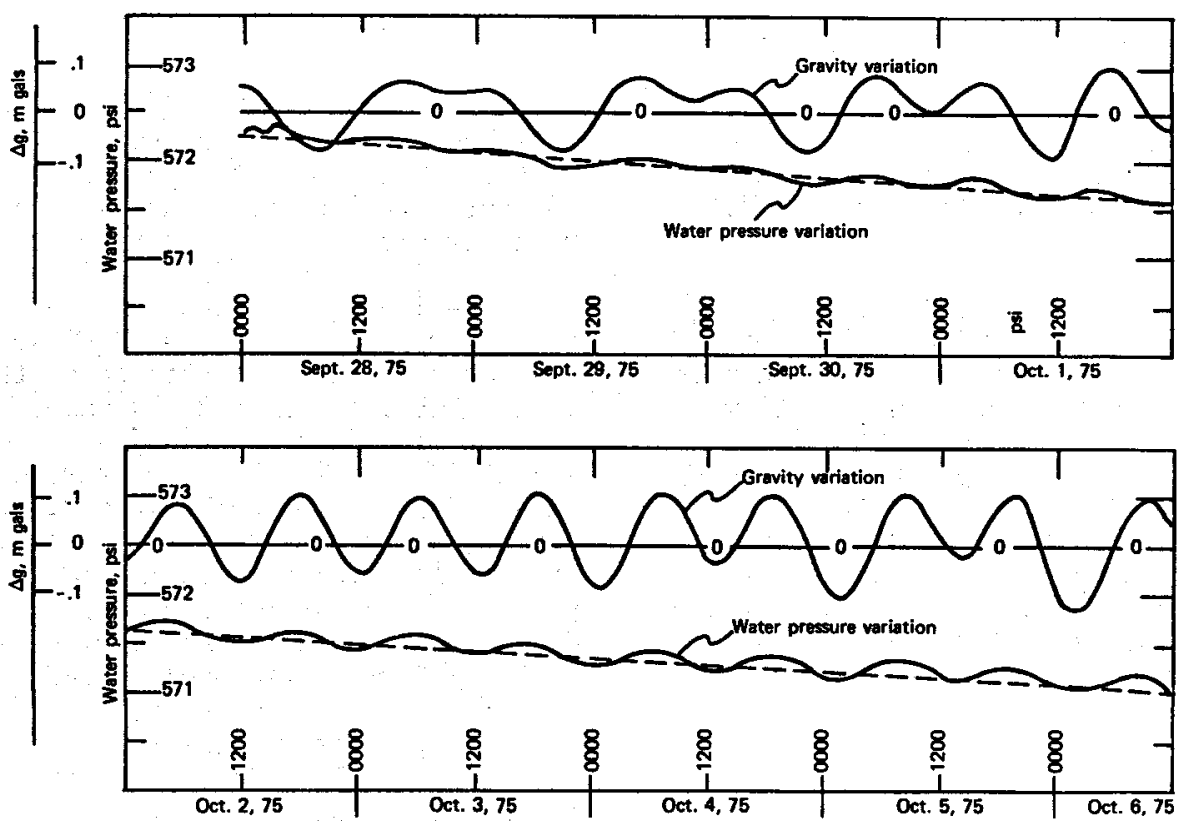

Fig. 2. Effect of earth tides on water pressure in RRGE 1, Raft River Valley, Idaho. 
where $u=1 / 4 t_{D^{*}}$. The dimensionless time $t_{D}$ is given by

$$
\mathrm{t}_{\mathrm{D}}=\frac{\mathrm{kt}}{\phi \mu \mathrm{Cr} \mathrm{r}^{2}}
$$

in which $t$ is time, $\phi$ is porosity, $c$ is compressibility of the porous medium and $r$ is the radius to the point of observation. In order to evaluate $\mathrm{kH}$ and $\phi \mathrm{CH}$ using (1), (2) and (3) a technique of curve matching 2,3 is conventionally used by plotting the pressure drawdown data on log-log paper. Or, for $t_{D} \gg 1$, an asymptotic approximation may be used in which drawdown is plotted as a function of the $\log$ of time. In addition to obtaining $\mathrm{kH}$ and $\phi \mathrm{cH}$, the departure of the plotted data from the type curve in the $\log -\log$ plot or the occurrence of breaks in the straightline segments of the semi log plot would indicate the presence of barrier or leaky boundaries in the system.

Influence of Earth Tides on Fluid Pressures A significant fact that came to light during the interference test was that the fluid pressures in the reservoir respond systematically to the changes in the earth's gravitational field induced by the paths of the moon and the sun. The field data indicated regular oscillations with a period varying from 10 to 12 hours and with amplitudes of up to 0.1 psi about the mean.

For a proper evaluation of the pressure data, the gravitational changes at the location of RRGE 1 over the period of the test was calculated by $\mathrm{Dr}$. Howard Oliver of the U.S. Geological Survey, Menlo Park, California. Figure 2 is a comparison between the calculated gravity variation and water pressure variation in RRGE 1 between September 28 and October 6, 1975. It can be seen from the figure that there is good correlation between gravity change and pressure change. Careful examination of the data showed that the troughs and crests of the water pressure wave tend to lead the corresponding features of the gravity wave in general by 15 to 45 minutes. As we will see from the subsequent section on interpretation, due consideration had to be given to the tidal influence before the interference data could be analyzed.

\section{The Production Tests}

As seen from Table 2, two production tests were conducted, one each on RRGE 1 and RRGE 2. Both these tests were of relatively short duration. The test on RRGE 2 indicated a $\mathrm{kH}$ of about 47,500 millidarcy 5 feet and a $\phi \mathrm{cH}$ of about $3 \times 10^{-2} \mathrm{psi}^{-1}$. The data indicated the probable existence of a barrier boundary very close to the well. The production test on RRGE 1 indicated a $\mathbf{k H}$ of about 110,000 millidarcy feet and a $\phi \mathrm{cH}$ of about $2 \times 10^{-2}$ $\mathrm{psi}^{-1}$ in the vicinity of that well. In both these cases, the computed values of $\phi \mathrm{cH}$ are uncertain due to ambiguities in defining the effective well radius.

\section{The Interference Test}

As can be seen from Fig. 2, the interference test data were considerably masked by pronounced earth tide effects. As a first approximation to eliminating the tidal effects and aiding in the interpretation, advantage was taken of the fact

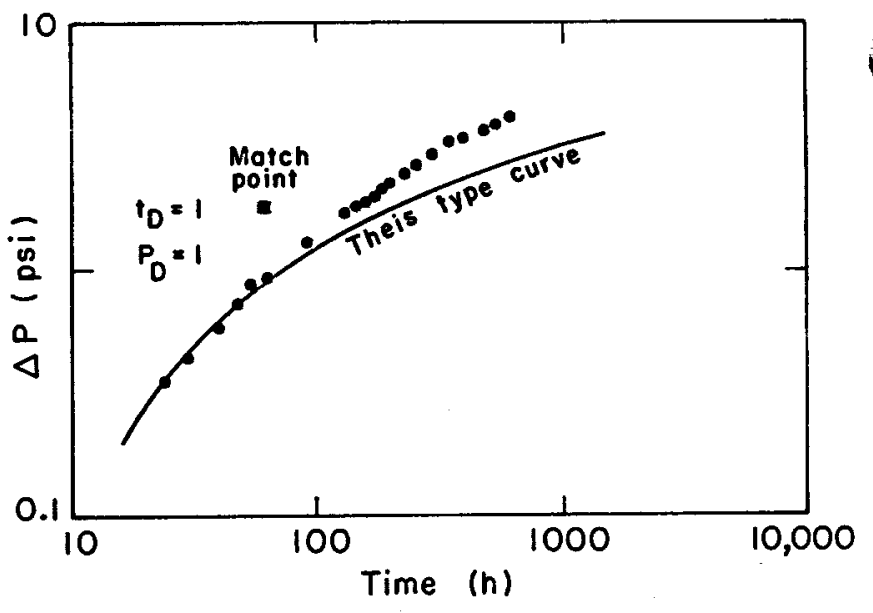

Fig. 3. Interference test, RRGE 1: $\log -1 \circ g$ plot of time vs. drawdown.

(XBL 764-2838)

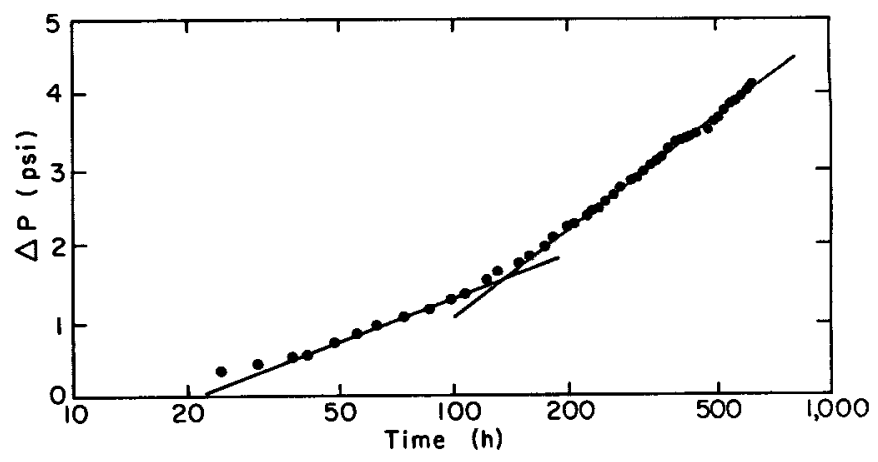

Fig. 4. Interference Test, RRGE 1: semi-1og plot of time versus drawdown.

(XBL 764-2835)

that the gravity and pressure waves are almost in phase. Hence only the pressure readings at those instants of time when gravity change was zero were used for plotting and interpretation.

The $\log -\log$ and the semi-log plots of time versus drawdown are shown in Figs. 3 and 4 . It was computed that the portion of the reservoir between RRGE 1 and RRGE 2 has an overall $\mathrm{kH}$ of about 193,000 millidarcy feet and a $\phi \mathrm{cH}$ of about $1.1 \times 10^{-3} \mathrm{psi}^{-1}$. The departure of the observed data from the type curve in Fig. 3 after about 100 hours and the prominent break in the slope of the straightline segment in Fig. 4 both suggest the presence of a barrier boundary. Calculations indicate that the existing barrier is equivalent to an "image" production we11 located about 11,000 feet from RRGE 1. However, with only two wells available, it is impossible to uniquely locate this image well and precisely fix the position of the harrier boundary.

Conclusions

The calculated value of nearly $200,000 \mathrm{milli}$ darcy feet for $\mathrm{kH}$ from the interference test sug- 
gests that the geothermal reservoir in the Raft jiver Valley is significantly permeable and hence nerits further exploration and study. The indication of barriers suggests that in certain directions the reservoir may be limited. However, the continuity of the reservoir between RRGE 1 and RRGE 2 over a distance of 4000 feet indicates that the reservoir is of considerable dimensions. In : order to be able to effectively define the location and orientation of barriers or other boundaries, it will be necessary to have one or more suitably located additional we11s.

\section{PLANNED ACTIVITIES}

Further exploratory work is being actively carried out in the Raft River Valley by the INEL. A third well, RRGE 3 is expected to be drilled by the end of April 1976, at a location about 1.2 miles southeast of RRGE 1 , towards the center of the valley.

The LBL Geothermal Group is in close touch with the INEL and more production and interference tests are proposed to be carried out between May and July 1976, in order to gain in improved knowledge of the promising geothermal reservoir.

\section{REFERENCES}

1. S.G. Spencer, Environmental Report: Deep Geothermal Test Wells in the Raft River Valley, Aerojet Nuclear Co., ANCR 1204, (Jan. 1975).

2. P.A. Witherspoon, I. Javande1, S.P. Neuman, and R.A. Freeze, Interpretation of Aquifer Gas Storage from Water Pumping Tests, American Gas Association, New York, (1967).

3. J.G. Ferris, D.B. Knowles, R.H. Brown and R.W. Stallman, Theory of Aquifer Tests, Water Supply Paper 1536-E, U.S. Geological Survey, (1962).

4. C.V. Theis, Trans. Am. Geophys. Union, 16, 519, (1935).

5. 1 millidarcy $=9.67 \times 10^{-7} \mathrm{~cm} / \mathrm{sec}$.

\section{RADIOACTIVITY AND METAL MINERALIZATION}

\section{H. A. Wollenberg and AZan R. Smith}

The natural terrestrial radiation environment is dominated by the gamma-radioactivity from $238 \mathrm{U}$, $232 \mathrm{Th}$, and $40 \mathrm{~K}$ in rocks and soil. The radioelements are concentrated according to the magmatic differentiation series, being of lowest abundance in ultramafic and basic igneous rocks and greatest in felsic rocks such as granite and rhyoiite.

The terrestrial gamma-ray field can be measured by sensitive scintillating detectors, based on a crystal of thallium-activated sodium iodide (NaI(TX)). NaI (TX) values are normally read as counts per second, directly convertible to microroentgens per hour. More advanced systems incorporate a scintillation detector connected to a portable single-channel pulse-height analyzer which can resolve the different gamma-ray energies characteristic of the radioelements. This type of system permits assay, on the outcrop, of the U, $T h$, and $K$ contents of the material beneath or surrounding the detector. The advantages of field assays may be outweighed by the long time required to obtain a statistically significant number of counts to properly analyze the individual radioelements. A portable gama-rate-meter may be more useful, in that nearly continuous measurements of the total gamma-ray field are an excellent parameter for control of sampling. Samples are then analyzed in the laboratory for their radioelement contents.
There is a possibility that ganma radiometry may be useful as a guide to metal mineralization. The relationship between field gamma-radioactivity and mineralization in metal deposits was demonstrated by Moxham et al. 1 and more recently by Davis and Guilbert. 2 In traverses of porphyryand vein-type deposits in Arizona they observed increased count rates from potassium associated with hydrothermal alteration minerals (sericite, chlorite) in country rock of the ore zones. The main variations were in $K$. Uranium and thorium were relatively constant, indicating no correlation with alteration zones. Thus, total gamma-radioactivity reflecting potassium may be indicative of mineralization.

This was tested in the Sunmer of 1975 by surface and underground traverses of altered and unaltered areas of country-rock rhyodacite at the mines of Cia. Minas Buenaventura at Julcani, Peru, where the principal product is silver. Instrumentation consisted of a 3-in. diameter by 3-in. thick NaI (Tl) detector, coupled to a count-rate meter. Readings were made continuously as the party walked along the underground drifts and on the surface. Counting rates underground varied from 500-600 counts per second in unaltered rhyodacite, to over 1100 counts per second in areas of alteration surrounding the ore vein. Surface readings, significantly lower than underground 
because of geometry factor, showed similar relative differences in counting rates between altered and unaltered areas. In most cases, the ore bearing veins themselves were of relatively low radioactivity in contrast to the altered wall rock.

Samples were taken, and analyzed by 1aboratory gamma spectrometry, to see which radioelements reflect hydrothermal alteration in the Julcani area.

When $K$ content is compared with the gamma-ray counting rate, there is a positive correlation for surface and underground samples, while there are no apparent correlations between $U$ and Th content and counting rates; indicating that variations in gamma radioactivities at Julcani reflect, primarily, variations in $\mathrm{K}$ content of the rocks. Therefore, results of radioelement analyses corroborate the supposition that high radioactivities reflect the relative enrichment in potassium associated with zones of hydrothermal alteration associated with ore mineralization.

\section{REFERENCES}

1. R.M. Moxham, R.S. Foote and C.M. Bunker, Gamma-ray spectrometer studies of hydrothermally altered rocks, Econ. Geol., 60 , no. 4, pp. 653-671 (1965).

2. J.D. Davis and J.W. Guilbert, Distribution of the radioelements potassium, uranium, and thorium in selected porphyry copper deposits, Econ. Geol., 68, no. 2, pp. 145-160 (1973).

"ARMS" AND "GEODOSE"

$H$. A. Wollenberg and $J$. Fitzpatrick

In support of the on-going surveys by the Aerial Radiological Measurement System (APMS), operated by EGEGG Inc. for the Division of Operational Safety of ERDA, we conducted radiogeologic studies of five reactor sites in the eastern U.S. Sites covered in brief reports to EG\&G were:

Big Rock Point, on the east shore of Lake Michigan;

Crystal River, on the Gulf Coast of Florida; Edwin I Hatch, on the Altamaha River, Baxley, Georgia;

Scriba, New York, on Lake Ontario; and Three Mile Island on the Susquehanna River, Pennsylvanía.

The objective of the studies was to furnish information on the geologic setting of the sites that would influence planning and interpretation of the aerial gamma-radiometric surveys. Relative radioactivities were predicted on the basis of the distribution of rock and soil types. Flight lines were oriented to best take advantage of the lithologic and structural "grain" of the site regions. Follow-up studies will compare gammaaeroradiometric patterns with site-region geology.

To facilitate the prediction and follow-up comparison of rock types with observed radioactivities, a project* called GEODOSE was started in the latter half of 1974. The objective of the GEODOSE project is to characterize rock and soil types by their natural gamma-ray exposure rates. The geochemical literature is being searched for data on $\mathrm{K}, \mathrm{U}$, and Th contents of earth materials, and these data are categorized by rock type, based on standard petrologic classifications. A data bank is being assembled, with incorporation of radio-element information on the igneous rocks essentially completed. Metamorphic and sedimentary rock data are presently being compiled. The data are treated to produce computer plots of histograms of $\mathrm{K}, \mathrm{U}$, Th contents, calculated gamma-

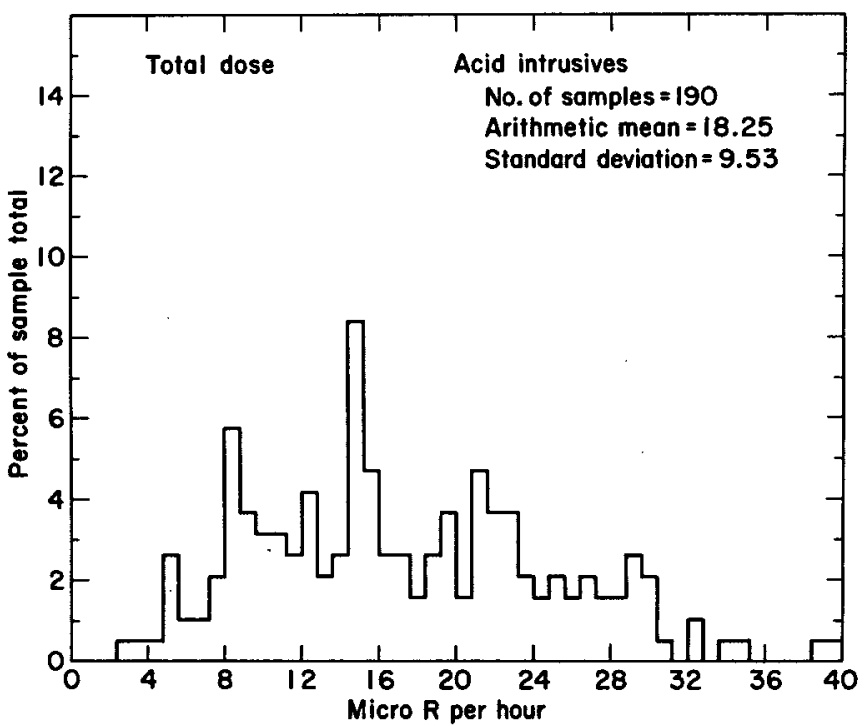

Fig. 1. Frequency distribution of total ganma-ray exposure rates for acidic intrusive rocks. (XBL 755-3015)

ray exposure rates, and radiogenic heat production for given rock types. Accompanying these plots will be means, standard deviations, and ranges of the parameters. Initial processing of data for igneous rocks indicates that alkaline and peralkaline terranes have generally the highest gammaray exposure rates, and suggests that alkaline and peralkaline rocks are probably associated with most of the broad areas of highest natural radioactivity. A summary report was prepared 1 and presented at the International Symposium on Areas of High Natural Radioactivity, Pocos de Caldas, Brazil in June 1975. Figure 1, from that report, illustrates the frequency distribution of gammaray exposure rates for acidic intrusive rocks. 
FOOTNOTE AND REFERENCE

*Project funded by EG\&G Inc.

1. H.A. Wollenberg and J. Fitzpatrick, Status of the GEODOSE Project, LBL-3646 (1975).

INVESTIGATION OF RADON-222 IN SUBSURFACE WATERS AS AN EARTHQUAKE PREDICTOR

A. R. Smith, H. R. Bowman, D. F. Mosier, F. Asaro, H. A. Wollenberg and Chi-Yu King*

\section{INTRODUCTION}

Recent reports of Chinese and Russian earthquake-prediction efforts 1 indicate that radon-222 in subsurface waters may respond to changes in earth strain which precede earthquakes. Such changes are also reflected in some characteristics of crustal rocks within and near epicentral areas, for example: variations in the ratios of compressional and shear wave velocities, and apparent electrical resistivity. It is postulated that dilatancy of rocks in response to strain may enhance release of $222 \mathrm{Rn}$ from the rocks into the ground water regime. Thus the onset of seismic activity may be preceded by an increase in the radon content of well and spring waters in the vicinity.

We summarize here the initial progress in a collaborative effort between the University of California Lawrence Berkeley Laboratory (LBL) and the National Center for Earthquake Research of the United States Geological Survey (USGS) at Menlo Park. The intent of this work is to develop a compact high-precision real-time continuous monitor system for field measurement of $222 \mathrm{Rn}$ in subsurface waters, an instrument that could serve as a prototype for a network of such stations. Data from a group of these stations could then be coordinated with other geophysical information related to earthquake precursors, to be used in earthquake prediction.

Although the work described here focuses on the $\gamma$-ray spectrometric method, application of $\alpha$-particle detection to this purpose is also being vigorously pursued, especially by the USGS side of the collaboration, as reported at the December meeting of the American Geophysical Union in San Francisco.2

\section{THE CONTINUOUS MONITORING TEST FACILITY AT LBL}

The prototype continuous monitoring station has been in operation at LBL since April 1975, functioning as a test facility to evaluate the measurement technique and system performance. A $\gamma$-ray spectrometer counting the $\gamma$-rays emitted by the daughters of $222 \mathrm{Rn}$ provides a stable and rugged system with the capability for continuous monitoring at high sensitivity.

The LBL system includes a NaI(T⿱) crystal detector located centrally in a tank through which radon-bearing water flows, a multichannel pulseheight analyser (PHA) with digital gain stabilization, and peripheral units that record digital quantities derived from the gamma spectrum. A tank size of 3- to 6-ft diameter and equal height is adequate; a 3-in diameter by 3-in thick NaI(Tl) crystal provides ample sensitivity for the relatively low-activity LBL water (100 to $300 \mathrm{c} / \mathrm{min})$ depending upon selection of the energy interval. The channels are integrated over selected energy regions at $10 \mathrm{~min}$ intervals and recorded. Precision of $1 \%$ or better is easily attained by summing several such intervals, permitting measurement of a few percentage change in $222 \mathrm{Rn}$ abundance in a small fraction of a day.

The water source for the LBL station is from a horizontal hole drilled approximately $400-\mathrm{ft}$ into the hillside for slope-stabilization purposes. The drain hole provides a constant flow rate of about $1.5 \mathrm{gal} / \mathrm{min}$ with a $222 \mathrm{Rn}$ content of $\sim 300$ $\mathrm{pCi} /$ ititer. The daughter products of $222 \mathrm{Rn}$ decay (the source of the observed $\gamma$-radiation) are greatly depleted in water that flows from this hole. Note that it is not practical to measure ${ }^{222} \mathrm{Rn}$ directly by $\gamma$-detection, but rather by detection of $\gamma$-rays from the two daughters $214 \mathrm{~Pb}$ and $214 \mathrm{Bi}$. The effective half-1ife for ingrowth of these daughters is approximately 40 minutes; thus our $222 \mathrm{Rn}$ measurement technique must take into account a period of time lasting as long a 5 hours in which the daughters and 222Ra may not be in equilibrium. This is the case for $222 \mathrm{Pn}$ entering the detection volume without any daughters, i.e., $222 \mathrm{Rn}$ that is either just created or freshly stripped of the daughters. This nonequilibrium problem may be expected as a general characteristic of natural aquifers, 3 and must be taken into account when designing flow-through measurement systems.

\section{THE DISCRETE SAMPLING PROGRAM}

Discrete sampling of well water is an integral part of the program ranging in application from reconnaissance studies in new areas, through longterm support in the vicinity of a continuous monitoring station, to exclusive use in long-term surveillance in a study area. These samples consist of groups of 2 to $7500-\mathrm{ml}$ capacity polyethylene bottles. The container was chosen for its inherent low radioactive content, acceptable 
small loss of radon by diffusion through the container walls and purity with respect to trace element contamination.

All discrete samples are transported to LBL and are assayed for $222 \mathrm{Rn}$ by $\gamma$-ray spectrometry, assuming equilibrium with the daughters, where we utilize a high-sensitivity $\mathrm{NaI}(\mathrm{T} \ell$ ) system located in the Low Background Counting Facility and the Health Physics Department. The low background counting enviromment is necessary for the successful measurement of the small 222Rn family activities present in most water samples.

THE OROVILLE AREA: STUDIES DURING AN AFTERSHOCK SEQUENCE

An earthquake of Richter magnitude 16 occurred near Palermo in the Oroville area of the Sierra Nevada foothills (about 120 miles northeast of San Francisco) on August 1, 1975. We established a discrete sampling program for water wells on August 5, and a continuous monitoring station at one well at the Palermo Elementary School on August 27-28. We sought to take advantage here of the large number of aftershocks in an effort to establish whether our techniques could demonstrate a relationship between $222 \mathrm{Rn}$ levels in well waters and seismic events, given the particular geologic terrain of the Sierra foothills and a limited selection of already existing relatively shallow wells in the epicentral area.

At present we obtain samples from six wells, four in the Sierra foothill metavolcanic bedrock formations and two in the Tertiary sedimentary deposits of the eastern margin of the Sacramento Valley. Sampling (on at least a daily basis) is done almost entirely by the local residents, without whon this part of the study would be impossible.

From mid-September onwards, intervals between aftershocks lengthened sufficiently so that we could begin to attenpt correlations between changes in

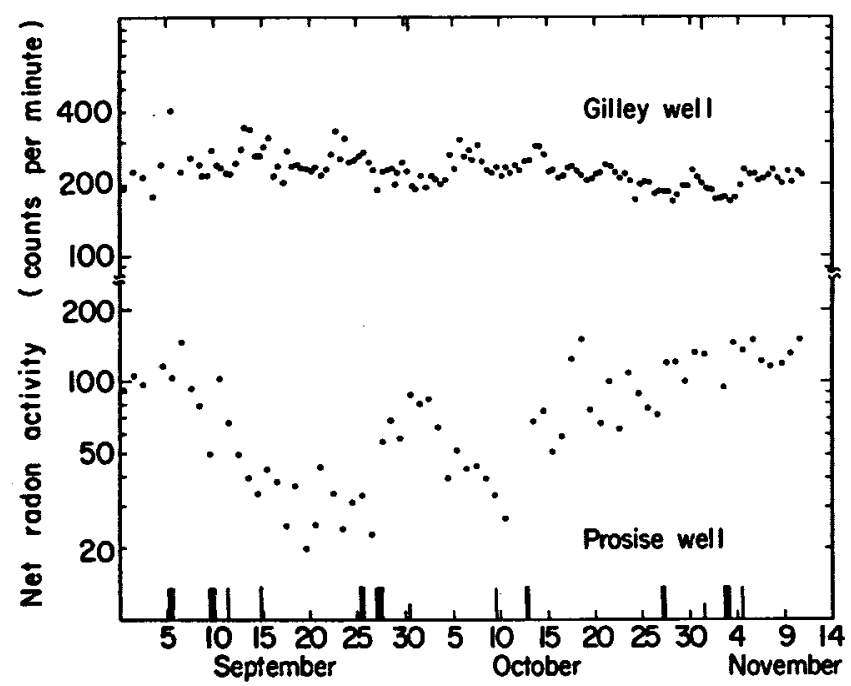

Fig. 1. Results of ${ }^{222} \mathrm{Rn}$ measurements in the discrete sampling program from two bedrock wells in the Oroville area.

(XBL 7511-9520)
222Rn levels and seismic activity. Data from two of the bedrock wells for this period are shown in Fig. 1, where the 1aboratory analyses are expressed in terms of decay-corrected $222 \mathrm{Rn}$ counting rates. The occurrence times of aftershocks with Richter magnitudes greater than 2 are indicated along the base line of Fig. 1 as the tallest vertical bars.

Some preliminary comments are given here to illustrate the current understanding of the data from the discrete sampling program. The two wells (Fig. 1) exhibit marked changes in radon abundance as a function of time, but in contrasting patterns. For example, the low/high/low structures in Gilley well data from the periods September 21-24 and October 4-8 may be related to the aftershock sequences of September 25-27 and October 10-13. The high/low structures in the Prosise well data may be related to these same aftershocks, although the time scale appears to be quite different. The Gilley well data also show a general decrease in $222 \mathrm{Rn}$ levels throughout this period, in a continuation of a trend observed since early August, and generally following the decrease in frequency of aftershocks. Data from the Gilley and Prosise wells show less structure with passage of time from September to mid November.

There appears to be some general correlation between aftershock activity and the radon abundances in the bedrock wells; however, the aftershocks may still be too closely spaced in time to permit distinction between precursor and response signals. On the other hand, a well drilled into the Tertiary sediments at the Palermo School (data not shown here) has a much more constant radon level varying less than $25 \%$ during the same period. The discrete sampling program must continue into the period when the region is again seismically inactive, so as to establish base levels and normal variation patterns for $222 \mathrm{Rn}$ content of these wells. Only then can the present data be completely evaluated to disclose which features were most probably related to seismic activity.

The continuous monitoring system at the Palermo School well was set up to measure the abundance of $222 \mathrm{Rn}$, using the school storage tank. Although discrete sampling had indicated the radon abundance in the water was nearly constant with time, marked variations in the apparent $222 \mathrm{Rn}$ abundance were noted with the continuous system. These variations correlated closely with water usage patterns. For example, during periods of high use, the mean residence time in the storage tank was too short to achieve equilibrium between $222 \mathrm{Rn}$ and daughters, and the observed count rate dropped considerably. An acoustic pickup was installed on the water system to indicate periods of pump operation, to permit possible recovery of the true radon abundance values from the data. The need for close control of water-flow patterns in such a continuous radon monitoring system was clearly demonstrated by this experience.

\section{THE HOLLISTER AREA}

In March 1975, preliminary survey in the 
Hollister area of central California (about 100 iiles south of San Francisco) disclosed the presence of $222 \mathrm{Rn}$ in appreciable quantity at severa1 wells; two wells at one site sustain considerable artesian flow during part of the year and also show relatively high $222 \mathrm{Pn}$ content, approximately $800 \mathrm{pCi} / 1$ iter. This site is in a zone of the San Andreas fault that is presently undergoing active tectonism in the form of abundant micro- and macro-earthquake activity and observable fault creep. It was chosen as the initial field station, to be occupied in the summer of 1975. However, occurrence of the earthquake near Oroville, California on August 1, 1975 caused postponement of establishment of a station in the San Andreas Fault zone until early Novenber 1975.

The Hollister area is especially well suited for the 222Rn study, not just because there are frequent small earthquakes, but primarily because here there are extensive instrumentation networks measuring geophysical parameters for the same purpose-sensing earthquake precursor signals. Thus the possibility exists to establish correlations between the $222 \mathrm{Rn}$ method and several other methods, an opportunity not available in the Oroville area.

\section{NEVADA TEST SITE STUDIES}

A program of ground radon monitoring at the ERDA Nevada Test Site was started in September 1975 as an extension of the joint USGS-LBL study, in cooperation with Lawrence Livermore Laboratory (LLL) Test Division, the LLL Hazards Control Department, and the ERDA Nevada Operations Office. The purpose is to see if ground $222 \mathrm{Rn}$ emanation is enhanced by the shock of underground explosions.

Two types of monitoring are employed. In one, discrete water samples are obtained at least weekly from deep wells that penetrate alluvium and sedimentary formations in the Yucca Flats area; these samples are sent to LBL for $222 \mathrm{Rn}$ assay by $\gamma$-spectrometry. The second method employs radon alpha-track detectors, buried $1 / 2$ meter deep in alluvium at distances ranging from 300 to 5000 meters from one particular shot point. The track detectors are changed weekly by LLL Hazards Control personnel. These detectors are provided, etched, and counted by Terradex, Inc. in Walnut Creek, California.

At the end of calendar 1975, neither the we11 samples nor the track detectors have indicated any clear response to the ground shocks associated with a number of underground explosions. However, the test shot for which the detector array was initially deployed was postponed until early 1976. The program is continuing to include this particular test, after which a report will be written to discuss the results of this study.

\section{SUMMARY}

Initial progress in the $\gamma$-ray detection method includes the following items. We have verified the major design features of a field station that performs continuous high-precision $222 \mathrm{kn}$ monitoring in water. We have established a long-term locally supported discrete well-water sampling program in the Oroville area during the period of aftershock activity, to investigate the link between $222 \mathrm{Rn}$ levels and the ongoing seismic activity. Investigation is underway at the Nevada Test Site to determine whether there is a variation in ground radon abundance that may be linked to shock waves from large underground explosions.

\section{FOOTNOTE AND REFERENCES}

*National Center for Earthquake Research, USGS, Menlo Park, Ca.

1. Frank Press, "Earthquake Prediction," Scientific American, 232, No. 5, pp. 14-23, (1975).

2. Chi-Yu King, "Radon Emanation Along an Active Fault," American Geophysical Union Transactions, 56, №. 12, (1975).

3. Allan B. Tanner, "Radon Migration in the Ground, A Review," and "Physical and Chemical Controls on Distribution of Radium-226 and Radon-222 in Ground Water Near Salt Lake City, Utah," Chaps. 9 and 13 in The Natural Radiation Environment, edited by J.A.S. Adams and W.M. Lowder (University of Chicago Press, 1964).

\title{
SULFIDE LEVELS IN NATURAL WATERS:
}

A POSSIBLE NEW GEOTHERMOMETER

\author{
Alvin J. Hebert
}

A previously described non-dispersive soft -ray fluorescence spectrometer 1 has been used to determine sulfide levels in natural waters. $2 \mathrm{~A}$ modification of the original technique has allowed a factor of five improvement in reproducibility. The technique is applicable when the waters con- tain species other than sulfides, (such as sulfate), which do not produce observable interferences.

Experiments are performed with a highly homogeneous group of simultaneously silver plated copper disks that have been punched from the same 


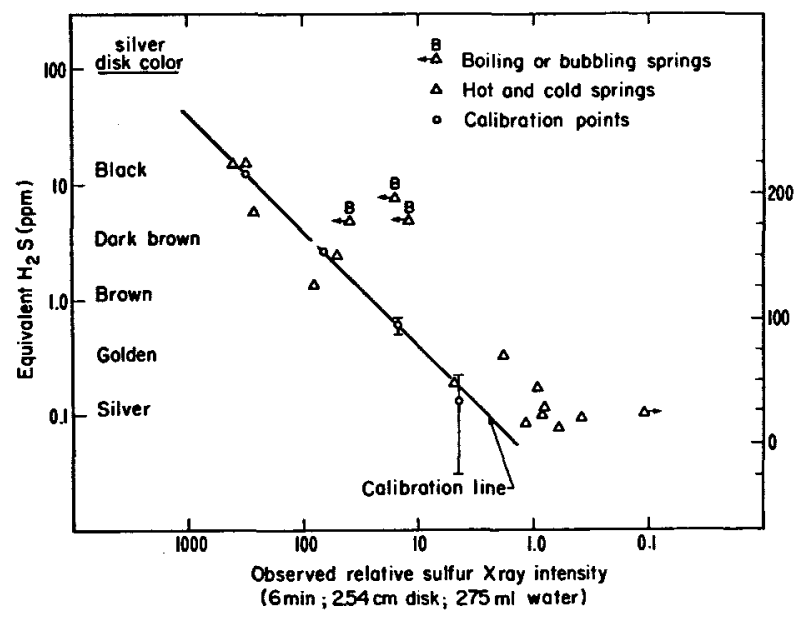

Fig. 1. Observed sulfur X-ray Intensities versus equivalent $\mathrm{H}_{2} \mathrm{~S}$ and also versus a linear Fournier-Truesdell (Na, K, Ca) geothermometer temperature or downwell reading $\left({ }^{\circ} \mathrm{C}\right)$.

(XBL 763-2527)

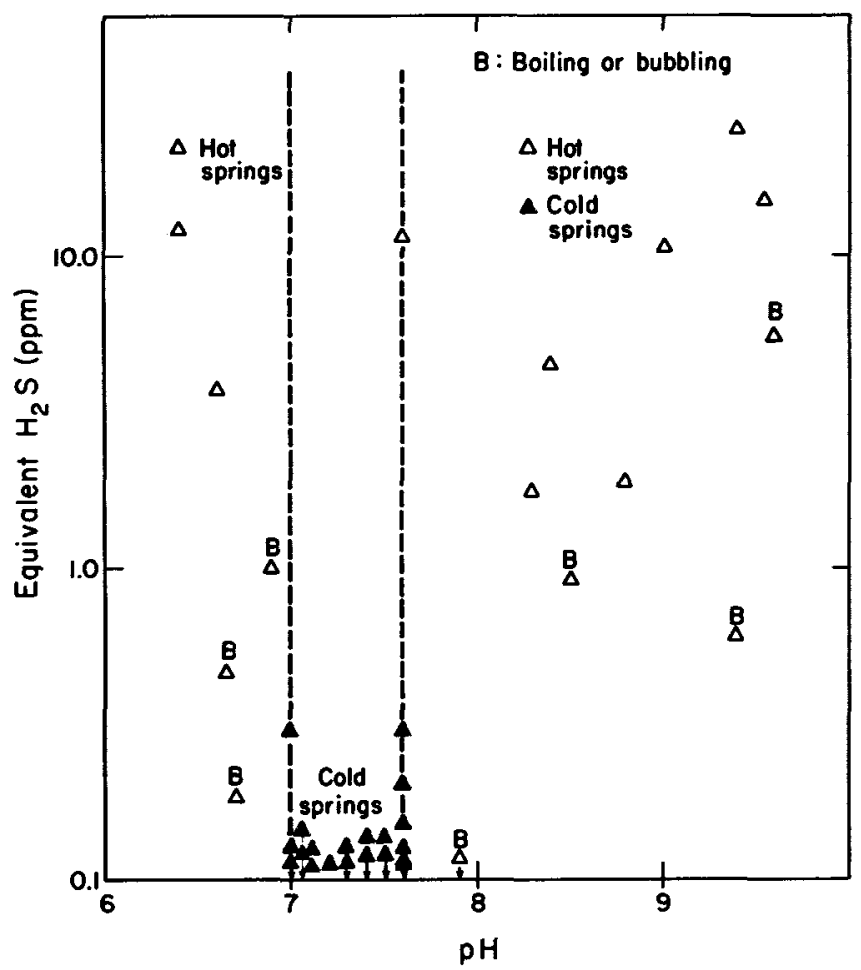

Fig. 2. A plot of $\mathrm{pH}$ versus Equivalent $\mathrm{H}_{2} \mathrm{~S}$ (ppm) for several North-Central Nevada springs.

(XBL 763-2528)

sheet of copper foil. The disks are stored together in a plastic bag before use along with those to be used for calibration in the lab at the end of collecting each series.

The disks are brought into contact with water. After several hours, or days under actual field conditions, the aqueous sulfide level decreases by orders of magnitude. The water is weighed and may be used to calibrate the analysis or as a check for the effects of other species with respect to a distilled water calibration. Calibration disks are obtained by spiking distilled water samples with aliquots of a saturated solution of hydro gen sulfide in distilled water, and introducing a silver disk for one day.

A typical calibration line is shown in Fig. 1 along with a plot of Fournier-Truesdell geothermometer $^{3}$ (Na, K, Ca) temperatures calculated for some natural waters. A relative color scale is also indicated. It appears that when a hot spring has a flowrate of a few liters per minute or more, and if the water hasn't boiled or bubbled, good agreement with observed downwell or $\mathrm{Na}, \mathrm{K}$, $\mathrm{Ca}$ geothermometer temperature calculations are obtained. Boiling or bubbling causes outgassing and lowered sulfide levels in most cases. It also appears that a set of calibration point disks could be made and used for semi-quantitative sulfide determinations by visual comparisons. This would eliminate the need for soft $X$-ray fluorescence analyses.

Sensitivities for color-tone comparison experiments are expected to be of the order of $0.1 \mathrm{ppm}$ at the $0.2 \mathrm{ppm}$ level and a few ppm at the $10 \mathrm{ppm}$ level. The use of multiple disks would maintain good sensitivity where the sulfide levels exceed $10 \mathrm{ppm}$.

Figure 2 is a plot of sulfide levels versus $\mathrm{pH}$. Although the data appear scattered, it is important to note that all cold springs, as determined by the $\mathrm{Na}, \mathrm{K}, \mathrm{Ca}$ geothermometer, plot in the $\mathrm{pH} 7$ to 7.6 range with sulfide levels at less than $0.2 \mathrm{ppm}$ equivalent $\mathrm{H}_{2} \mathrm{~S}$.

Both Fig. 1 and Fig. 2 indicate that the sulfide levels and the $\mathrm{pH}$ of natural springs may be of value in assessing the geothermal potential of an area.

\section{REFERENCES}

1. A.J. Hebert and K. Street, Jr., A Nondispersive Soft X-ray Fluorescence Spectrometer for Quantitative Analysis of the Major Elements in Rocks and Minerals, Anal. Chem., 46, 203 (1974).

2. H. Bowman, A. Hebert, H. Wollenberg, and F. Asaro, A Detailed Chemical and Radiometric Study of Geothermal Waters and Associated Rock Formations, with Environmental Implications, presented at the Second International Conference on Nuclear Methods in Environmental Research, University of Missouri, Columbia, Missouri, July 29-31, 1974; H.R. Bowman, A.J. Hebert, H.A. Wollenberg and F. Asaro, Trace, Minor and Major Elements in Geothermal Waters and Associated Rock Formations (NorthCentral Nevada), presented at the Second United Nations Symposium on the Development and Use of Geothermal Resources, San Francisco, California, May 20-29, 1975.

3. A.J. Hebert and H.R. Bowman, Nondispersive Soft X-ray Fluorescence Analyses of Rocks and Waters, presented to the Second United Nations Symposium on the Development and Use of Geothermal Resources, San Francisco, California, May 20-29, 1975. 


\section{A PORTABLE FIELD WATER SAMPLE FILTRATION UNIT*}

AZvin J. Hebert and Gardener G. Young

The need for a lightweight back-packable filtration unit that could easily be cleaned without cross-contamination at the part-per-billion level while allowing rapid filtration of boiling hot and sometimes muddy water has given rise to the units shown on Fig. 1.

Field and 1aboratory tests indicate essentially no changes in carbonic acid or bicarbonate or carbonate ion concentratons when filtrations are done at approximately $1 / 3$ atmosphere even though the $\mathrm{pH}$ value may range from 3.2 to 9.4 .

Filtrations are performed by pouring the solution into the funnel or by placing a piece of tygon tubing down a we11 and drawing the water up and through the filter. Boiling water has been drawn up old geothermal well pipes as high as 5 meters in this manner. Filtration usually takes less than one or two minutes per $500 \mathrm{ml}$ bottle when the water is not too muddy. During the filtration process, it is important that bubbles not be allowed in the input line, otherwise the unit will cease functioning properly until it is reopened and a new filter inserted.

Figure 2 shows the machined groove pattern of the main body teflon plate. These grooves are "round-bottomed" to facilitate cleaning. The tygon lines are connected to this plate with a small nyIon nipple and viton 0 -ring to ensure vacuum integrity. A viton 0 -ring is placed in the large outer groove. A standard Millipore acetate filter (variety HAWP-142, 0.45 micron, $142 \mathrm{~mm}$ plain white filter sheet) is laid over the groove plate and viton 0 -ring prior to each clamp down and filtration. No separate filter support is necessary.

The eight central holes shown in Fig. 2 lead to the nylon nipple connector which fits into a mating recess on the other side. The main body

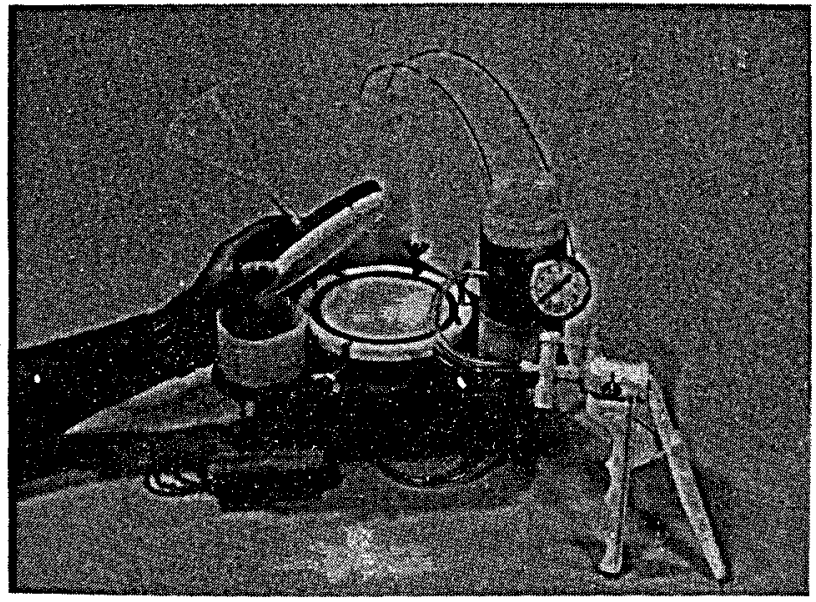

Fig. 1. Portable field water filtration unit. (BBC 7410-7355) teflon plates are backed by $1.27 \mathrm{~cm}$ thick aluminum plates.

The hand pump is manufactured by the Nalgene Labware Division of NALGE Sybron Corporation.

The legs on the unit are easily removed for packing. The end of the tie down bolts are expanded so that the brass butterfly nuts will not fall off.

The aluminum tubing sleeve shown in Fig. 1 on one of the $500 \mathrm{ml}$ Nalgene bottles guards against bottle collapse when filtering very hot water. The snap-in nipples for line connections to the lid of the bottle were machined from nylon.

Total unit weight without lines or bottles is $3.5 \mathrm{~kg}$.

\section{FOOTNOTE}

* Reference to this device has been made in the publications listed below.

H.R. Bowman, A.J. Hebert, H. Wollenberg, and F. Asaro, A Detailed Chemical and Radioactive Study of Geothermal Waters and Associated Rock Formations with Environmental Implications. Paper delivered at the University of MissouriColumbia, Conference on Nuclear Methods in Environmental Research, July 29, 1974.

H.R. Bowman, A.J. Hebert, H. Wollenberg, and F. Asaro, Trace Minor and Major Elements in Geothermal Waters and Associated Rock Formations (North Central Nevada), presented at the Second United Nations Symposium on the Development and Use of Geothermal Resources, San Francisco, May, 1975.
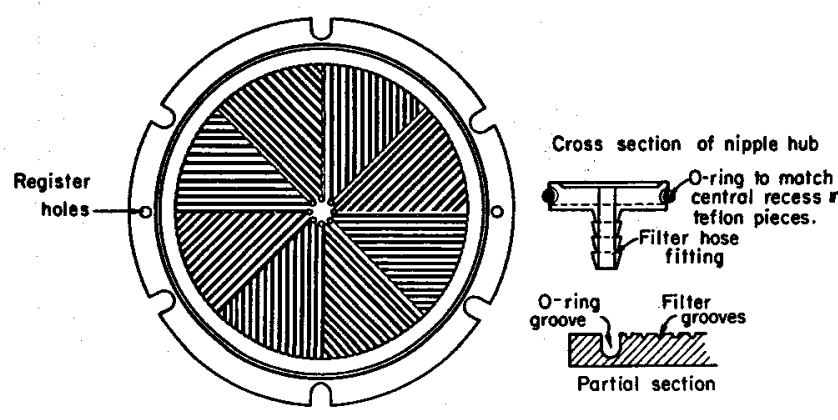

Fig. 2. Main body teflon plate. (XBL 758-3817) 
Alvin J. Hebert and Harry R. Bowman, Nondispersive Soft X-ray Fluorescence Analyses of Rocks and Waters, in the proceedings of the Second United Nations Symposium on the Development and Use of Geothermal Resources, San Francisco, May, 1975.
Harold A. Wollenberg, Sampling Hot Springs for Radioactive and Trace Elements, presented at the U.S. Environmental Protection Agency Workshop on Sampling Geothermal Effluents, Las Vegas, NV, October 20-21, 1975.

EVIDENCE IN SUPPORT OF A HOMOGENEOUS SOURCE FOR ALKALIC BASALTS

H. Bowman, F. Asaro and I. Carmichael

Alkalic basalt lavas (basalt lavas with substantial amounts of the alkaline elements) are generally thought to be generated through partial melting of the upper mantle. Bacon and Carmichael ${ }^{1}$ have shown that one of the lavas of these studies is consistent with this picture and could have equilibrated with mantle material at depths of approximately $75 \mathrm{~km}$. Precise trace element measurement on these lavas and similar lavas from widely separated parts of the world suggest that these lavas were generated under similar conditions from sources of similar composition. A concurrent study of the large crystals (megacrysts) and mantle fragments included in some of these lavas has demonstrated that there are a number of elenents in these lavas which can be used to test for the chemical composition of the source material in the mantle. Figure 1 shows a plot of two of these elements $\mathrm{Ta}$ and $\mathrm{U}$ from these widely separated geological environments. These data include over 20

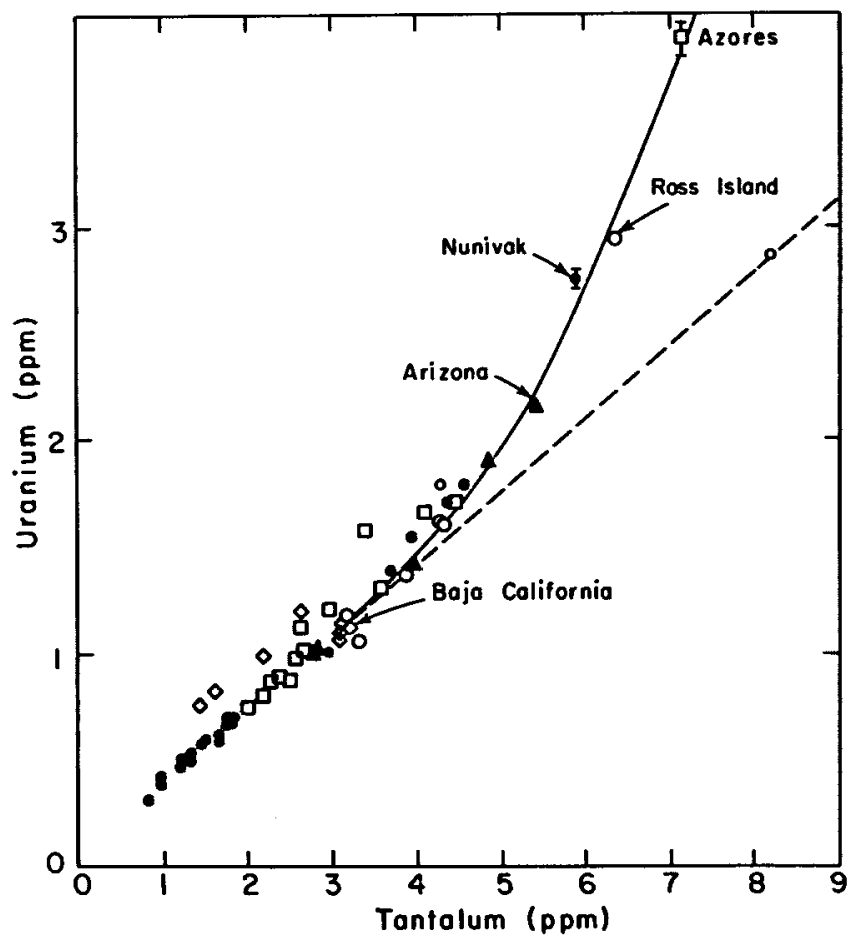

Fig. 1. Concentration of $\mathrm{U}$ vs $\mathrm{Ta}$ in alkalic basalt lava samples from the Azores, Nunivak Island, Ross Island, Arizona, and Baja California.

(XBL 767-3070)
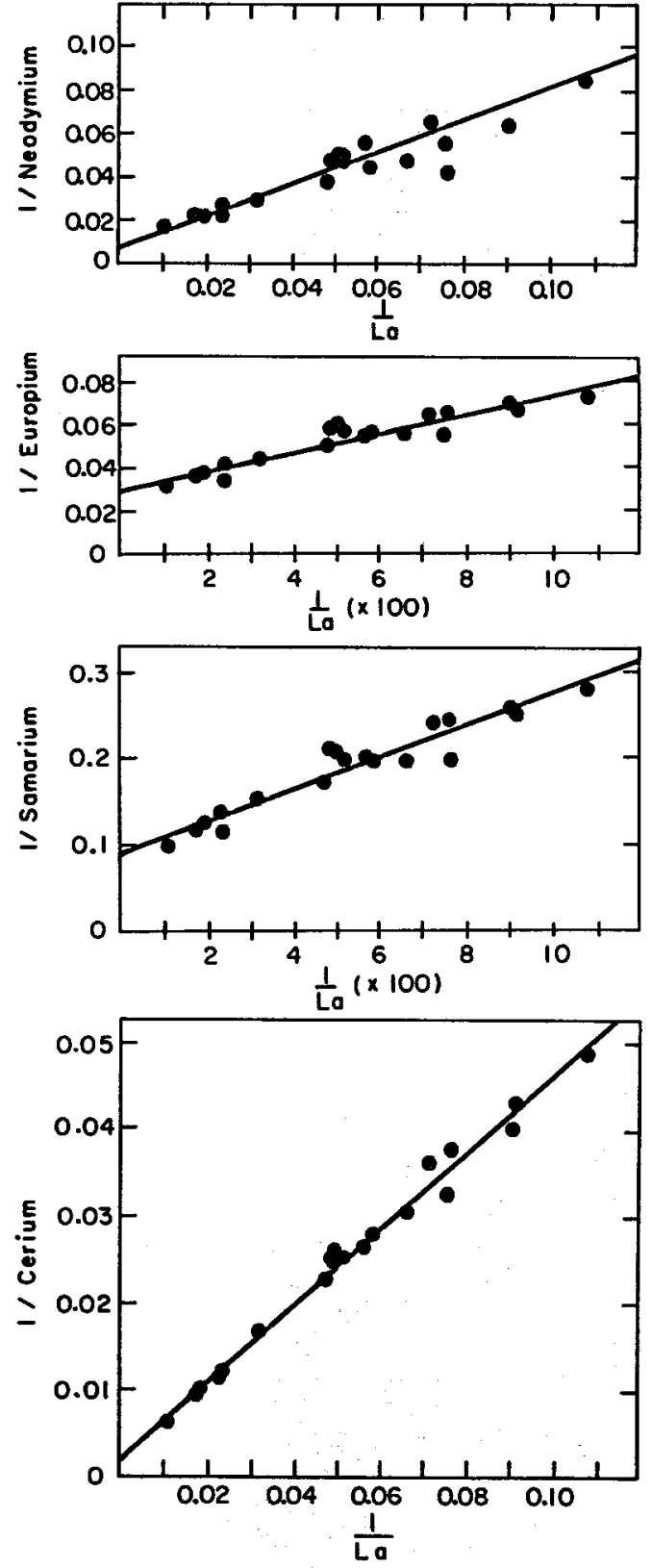

Fig. 2. Reciprocal concentration plots of several trace elements relative to reciprocal La concentration in alkalic basalt lavas.

(XBL 767-3071) 
amples from Nunivak Island, off the Alaskan jast, erupted over a 6 month period, 15 historic cruptions from the Azores in the central Atlantic Ocean, a dozen eruptions in the southwestern U.S. and a series of samples from Ross Island, Antarctica. The genera1 coherence between $\mathrm{Ta}$ and $\mathrm{U}$ and other trace and minor elements suggests a common source and generation process. In Fig. 2 we have plotted some of these element abundances relative to $\mathrm{La}$ as reciprocal concentrations to show how these data can be used to test for the composition of the mantle. If only a small fraction of the source material is melted and it is in equilibrium with the residual crystalline material then one can show that a plot such as Fig. 2 should produce straight lines with slopes related to the ratios of the mantle concentrations of these elements to the reciprocal mantle concentration of $\mathrm{La}$. The vertical intercepts are related to the overall elemental partitioning coefficients between the melt and the residual minerals in the mantle.

The equation is:

$$
\frac{1}{c_{y}}=\frac{c_{x_{0}}}{c_{y_{0}}} \frac{1}{c_{x}}+\frac{\left(k_{y}-K_{x}\right)}{c_{x_{0}}}
$$

where $\mathrm{C}_{\mathrm{y}}$ is the abundance of the trace element, $\mathrm{y}$, in the melt; $C_{y_{0}}$ and $C_{x_{0}}$ are the weighted averages of the abundance of $y$ and $x$ in the mantle associated with the melt. $C_{x}$ is the abundance in the melt of a specific trace element $x$ (chosen for its low partition coefficient), $K_{y}$ and $K_{x}$ are weighted averages of the partition coefficients for the trace elements $y$ and $x$ between the mantle and melt. The equation presumes the averaged partition coefficients are essentially constant for the various melts.

\section{REFERENCE}

1. C.R. Bacon and I.S.E. Carmichael, Contr. Mineral. and Petrol. 41, 1-22 (1973).

HIGH-TEMPERATURE PROPERTIES OF SILICATE LIQUIDS*

I. S. E. Carmichael

The heat content of a series of silicate liquids covering the whole known range of those found in nature has been measured by drop calorimetry in the temperature range 1200-1650K. From these and other published data, values of the partial molar heat capacities of $\mathrm{SiO}_{2}, \mathrm{TiO}_{2}, \mathrm{Al}_{2} \mathrm{O}_{3}$, $\mathrm{Fe}_{2} \mathrm{O}_{3}, \mathrm{FeO}, \mathrm{MgO}, \mathrm{CaO}, \mathrm{Na}_{2} \mathrm{O}$ and $\mathrm{K}_{2} \mathrm{O}$ have been derived, and apart from $\mathrm{Fe}_{2} \mathrm{O}_{3}$ show no compositional dependence. There is also no temperature dependence of the partial molar heat capacities in this temperature range.

\section{FOOTNOTE}

*Submitted to the Phil. Trans. of Royal Soc. of London, Series A (1976), LBL-5238 (1976).

\section{NATIONAL GEOTHERMAL INFORMATION RESOURCE (GRID)}

\section{S. L. Phillips, J. A. Fair, F. B. Henderson and S. R. Schwartz}

The main objective of GRID as an information analysis center is to provide critically evaluated and analyzed data to geothermal specialists (See Fig. 1). Our principal activities covered the following four areas: (1) compilation of geothermal exploration publications for the years 1974-1975; (2) compilation and evaluation of the thermodynamic and PVIX properties of $\mathrm{NaCl}$ solutions; (3) environmental aspects of geothermal utilization, and (4) adaptation of the Berkeley-Data Base Management System (BKY-DBMS) computerized data system for storage and retrieval of GRID information. A portion of these activities are described in more detail in Refs. 1-4.

As a pilot study, a report on the status of geothermal subsidence data was issued. The review is intended to provide quick referral to data of interest for the researcher engaged in assessing the effects of subsidence as related to the utilization of geothermal energy. It also contains a section noting either inadequate or currently unavailable data for topics that need further research, for example:

1. Development of a model that includes the important parameters for predicting geothermal subsidence: (a) rate of aquifer compaction as a function of fluid removal, (b) rate of land subsidence as a function of aquifer compaction, (c) effect of aquifer depth on land subsidence, (d) effect of recharge rate on aquifer compaction, (e) the relationships between aquitard structure and compaction.

2. Laboratory testing of rocks obtained at 


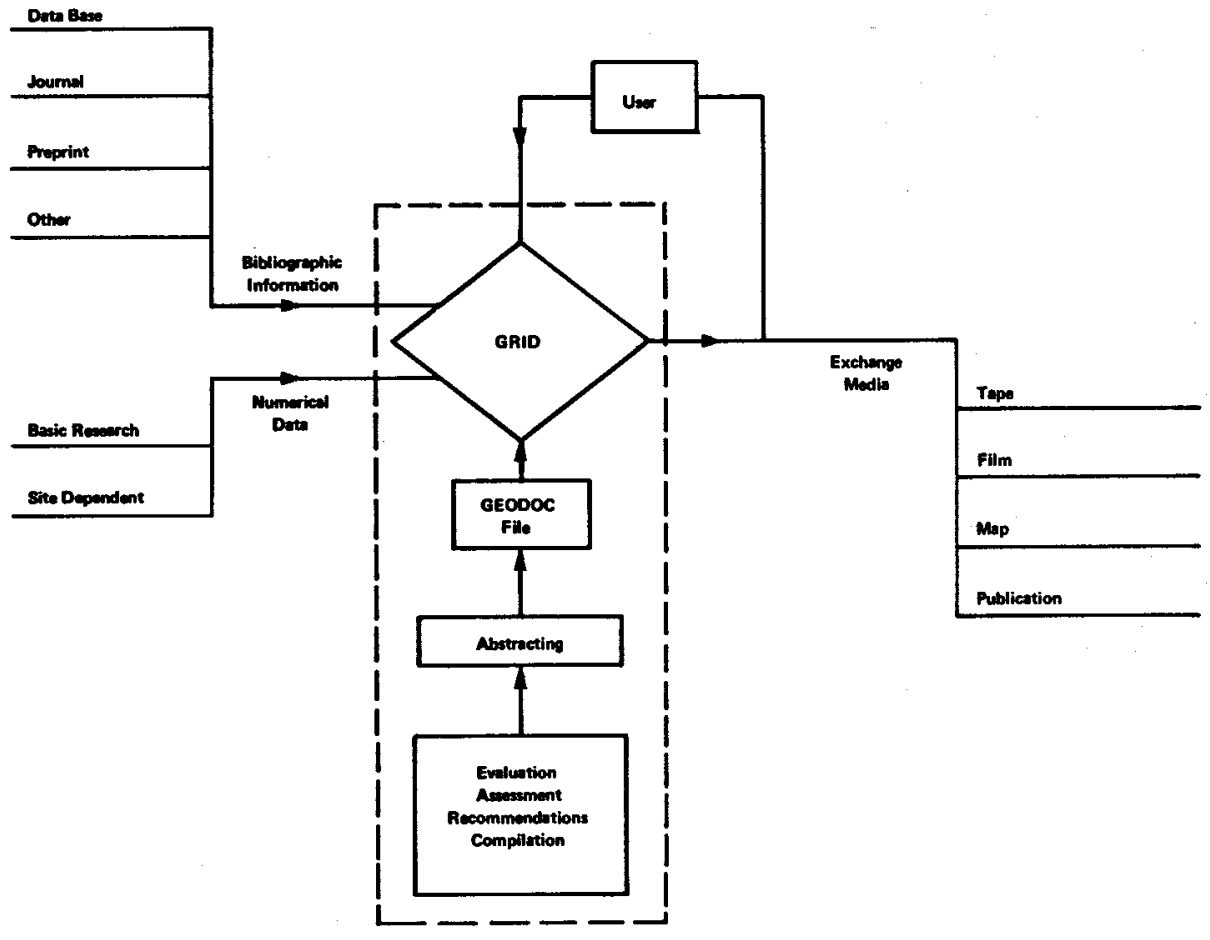

Fig. 1.

(XBL 7610-4873)

depth to measure the rock compaction as a function of important parameters (e.g., porosity, water content, temperature, pressure). The tests will provide site-dependent data on compaction and the likelihood of subsidence, and can also be used to test the applicability of mathenatical models. 3. Development of rock-sampling techniques at depth.

\section{REFERENCES}

1. S.L. Phillips, J.A. Fair, F.B. Henderson III, and S.R. Schwartz, Review of Geothermal Subsidence, LBL 3220, (1975).
2. S.L. Phillips, J.A. Fair, F.B. Henderson, and T.G. Trippe, The National Geothermal Informmation Resource, LBL 4420, (1975).

3. J.J. Herr, S.L. Phillips, S.R. Schwartz and T.G. Trippe, Standards for Multilateral and Worldwide Exchange of Geothermal Data, UCID-3792, (1975).

4. T. Trippe, V. White, S.L. Phillips, F. Henderson and S. Schwartz, GEODOC - The Document File for the National Geothermal Information Resource, LBL-4432, (1975). 


\author{
INTRODUCTION
}

The Plasma Physics and Controlled-Fusion Research Program at the Lawrence Berkeley Laboratory is divided into five projects:

1. Plasma Theory

2. Plasma Production and Heating Experiments

3. Atomic Physics Studies

4. Tormac Project

5. Neutral-Beam Development and Technology

All of these are parts of the coordinated program of ERDA's Division of Magnetic Fusion Energy (DMFE, formerly DCTR), although some of the problems studied in Plasma Theory are also of particular interest to the Division of Laser Fusion and a major portion of the Tormac Project is currently supported by the Electric Power Research Institute.

The projects are listed above in the order of increasing magnitude, as regards manpower and budget at LBL. By far the largest is the Neutral-Beam Development and Technology Project. It is carried out as a joint program with the Lawrence Livermore Laboratory, and in fact it is administered through LLL and handled as a subcontract to LBL. We are including it here for completeness sake, but we are restricting our report to the work done at LBL. only very brief summary statements are made because the detailed description of this project is included in the annual report on Magnetic Fusion Energy issued by LLL.

Present and future large Tokamak experiments in the U.S. as well as abroad, and the current and future mirror confinement experiments at Livermore depend on powerful and intense, low-divergence beams of neutral hydrogen (or deuterium) atoms for the production of their hot plasma. It is therefore not surprising that the neutral-beam injector problem has been given the highest priority within the Development and Technology category of the MFE program. The LLL/LBL joint project is one of the two major efforts in this area sponsored by ERDA (the other is located at the Oak Ridge National Laboratory).

The remaining four projects listed above have the following unifying features: both Plasma Production and Heating Experiments and Atomic Physics Studies are largely serving the function of basic support for the Neutral-Beam work. The first is concerned primarily with the improvement of our understanding of ion sources and similar matters of relevance to the neutral-beam injectors, while the second is aimed at supplying missing data on fundamental processes, such as certain chargetransfer cross sections or negative-ion production mechanisms.

A substantial fraction of the Plasma Theory, on the other hand, is carried out in support of the Tormac Project. The latter is our only direct contribution to the magnetic confinement problem in controlled-fusion research. The Tormac, short for toroidal magnetic cusp, is one of several so-called "alternate concepts." The latter are being worked on in the United States as back-ups, in case unexpected difficulties arise in the mainline developments of Tokamaks or magnetic mirrors, or in case a new concept is found to lead to a superior or more economical fusion reactor.

The Tormac Project originated at the Air Force Cambridge Research Laboratory several years ago, but was transferred in 1974 to LBL where it is being jointly supported by ERDA and by EPRI.

B. I. Cohen,* G. L. Johnston, $\dagger$ S. Johnston, A. N. Kaufman, M. A. Mostrom, \& D. R. Nicholson, ll G. R. Smith

\section{INTRODUCTION}

Our theoretical studies have been largely directed toward developing a fundamental understanding of the nonlinear interactions associated with plasma waves. Such waves may represent radiation injected into a plasma for the purpose of heating a magnetically confined plasma or for compressing an ummagnetized plasma or they may arise spontaneously as instabilities. The basic processes to be studied are the interactions of waves with each other and with particles, in plasmas that may be magnetized and/or nonumiform. The problems studied in 1975 are as follows, with detailed discussion following:

- Stochastic particle motion in the field of a single wave oblique to a magnetic field.

- The ponderomotive-potential method generalized to three-wave interactions in a magnetized plasma. 
- The evolution of parametric instabilities propagating perpendicular to a density gradient.

- The effect of weak spatial modulation on the development of parametric decay.

- Effects of particle trapping by a wave excited by nonlinear beats.

- Mathematical techniques for solving wave equations in nonumiform media.

STOCHASTIC PARTICLE MOTION IN THE FIELD OF A SINGLE WAVE OBLIQUE TO A MAGNETIC FIELDl (G. R. Smith and A. N. Kaufman)

A new effect in particle dynamics is being analyzed: stochastic acceleration by a single wave. Earlier studies had dealt with stochastic acceleration by a spectrum of waves, or with trapping of resonant particles by a single wave. We have chosen for study a single electrostatic wave propagating at $45^{\circ}$ to a uniform magnetic field. In such a wave there is a discrete set of resonant parallel velocities: $v_{n}=(\omega-n \Omega) / k_{z}$, where $\left(\omega, k_{z}\right)$ are the wave frequency and parallel wave number, $\Omega$ is the gyrofrequency, and $\mathrm{n}$ is any integer. The resonances have a width (in velocity) proportional to the square root of wave amplitude. At large enough amplitude, these resonances overlap, and particles tend to move stochastically from one resonance zone to another.

The motion in velocity space resembles a diffusion process. Numerical solution of the particle trajectories leads to criteria for stochasticity, and to diffusion rates. Elementary theories have been developed to interpret these results, and to obtain an expression for the diffusivity.

The phenomenon studied here may be expected to appear when coherent waves are used to heat a confined plasma, if the wave anplitude is sufficiently large. The basic ingredient is the coupling of two degrees of freedom by the wave, and the overlapping of resonances.

THE PONDEROMOTIVE-POTENTIAL METHOD GENERALIZED TO THREE-WAVE INTERACTIONS IN A MAGNETIZED PLASMA2 (S. Johnston and A. Kaufman)

The three-wave process occurs in problems of parametric instability and in plasma turbulence. A deep intuitive understanding of such processes was achieved several years ago, through the concept of ponderomotive force (the nonlinear force on a particle arising from the beating of two waves). This concept led to a unified treatment of nonlinear processes in uniform unmagnetized plasmas, when one of the three interacting waves was of relatively low frequency. We wished to extend this concept in several directions: arbitrary ordering of frequencies, magnetized plasma, and nonuniform medium. Our aim has been to obtain an expression for the three-wave coupling coefficient, which is concise and of great generality, and which manifests the symmetry implying the Manley-Rowe relations.

The method used was a canonical transformation of the particle Hamiltonian, which removed the first-order interaction of non-resonant particles. The ponderomotive forces then appear as a new non- linear term in the transformed Hamiltonian.

The desired expression has been obtained; it reduces to previously known results. We intend to apply it to problems of practical interest.

THE EVOLUTION OF PARAMETRIC INSTABILITIES PROPAGATING PERPENDICULAR TO A DENSITY GRADIENT ${ }^{3}$ (M. A. Mostrom)

The effect of plasma nonumiformity on the development of three-wave processes is important, because the resonance condition can be satisfied only over a limited region. Ordinarily one uses WKB methods for waves in nonuniform media, but they become invalid near turning points. For the parametric decay instability in (at least) two dimensions, the growth rate is a maximm when the decay products remain in the resonance region, i.e., near their turning points. Hence more sophisticated techniques are required to determine the maximal growth.

The model problem studied, the decay of an electromagnetic wave, propagating along the plasmia density gradient, into a cold-plasma Langmuir oscillation and a side-scattered electromagnetic wave, is representative of problems arising in connection with heating of confined plasma, or with laser penetration of plasmas.

The mathematical analysis concerns the study of the Green's function, i.e., the space-time response of the nonumiform, pumped plasma to an infinitesimal localized disturbance. We find that the response consists of two distinct parts:

(1) wave-packets which propagate, are refracted, and grow only while they remain in the resonance zone; (2) growing eigenmodes which are confined to the resonance zone. In practical cases, the latter terms (which were known earlier) are dominated by the former. Explicit expressions are obtained for the exponential growth, and for the saturation time due to refraction, in terms of the pump strength, scale length, and characteristic frequencies.

EFFECT OF WEAK SPATIAL DENSITY MODULATION ON THE DEVELOPMENT OF PARAMETRIC DECAY 4 (D. R. Nicholson)

The decay of a pump wave into two other waves is a prevalent plasma process: it may be desired, as a means of depositing radiation energy into the plasma; or it may be undesirable, reflecting such energy out of the plasma. Since a theoretical analysis of this process necessarily involves idealizations, it is essential to study the sensitivity of theoretical conclusions to such models. In the 1974 Reports, we discussed our discovery that a slight amount of long-wave spatial turbulence, superposed on a density gradient, could drastically affect the temporal development of the decay, i.e., it could replace the convective growth (characteristic of the gradient) with absolute growth (characteristic of a uniform plasma). This work led to two studies in 1975:

1. Since this effect of the turbulence seemed to depend primarily on its magnitude and scale length, it appeared that the randomess of the density perturbations was an inessential aspect. Hence we studied the effect of a coherent (sinusoida1) 
density modulation on the decay instability, and indeed found that it could produce absolute growth, with modulation wavelength replacing turbulent scale length as the important parameter. 2. The work discussed above being numerical, we wished to formulate a model amenable to analytic solution, so as to gain a deeper and broader understanding. For this we chose sinusoidal modulation of uniform-density plasma, and obtained explicit expressions for its reduction of the temporal and spatial growth rates of the decay instability.

EFFECTS OF PARTICLE TRAPPING BY A WAVE EXCITED BY NONLINEAR BEATS ${ }^{5}$ (B. I. Cohen and A. N. Kaufman)

In 1972 we began a study of the possibility of heating a plasma by beating two lasers to excite a damped plasma mode. If the latter wave becomes large enough to trap particles, the dynamics of the three-wave process is radically changed. (Previous studies of three-wave interactions had used a simple phenomenological damping coefficient.) In 1975, we undertook detailed computer simulation and analytic treatment of the trapping phenomenon, which heretofore had been thoroughly studied only for free waves:

1. To abstract the essence of the effect, we replaced the nonlinear effects of the two primary electromagnetic waves by a driving beat wave, which resonantly excited a Langmuir wave, and the latter then trapped electrons. The bouncing of these electrons produced oscillations in the magnitude and phase of the Langmuir wave. These were observed by simulation, and interpreted in terms of the nonlinear frequency shift and nonlinear damping of the corresponding normal mode.

2. Returning to the three-wave problem, the effect of electron trapping by the third wave on the amplitudes of the two primary waves was studied by simulation. The main effect observed was the heating of the electron velocity distribution, causing the Langmuir wave to be so heavily damped that the wave interaction became induced scattering of the two primary waves.

MATHEMATICAL TECHNIQUES FOR SOLVING WAVE EQUATIONS IN NONUNIFORM MEDIA (George L. Johnston)

Work is continuing on the development of a method for obtaining integral representations of particular integrals of some inhomogeneous secondorder linear ordinary differential equations. It is anticipated that the method will have applications to a wide range of problems in theoretical physics, engineering, and applied mathematics. Application to two problems of plasma dynamics and controlled thermonuclear research has already been made: electrostatic linear mode conversion in a parabolic density profile and resistive internal kink modes.

Recent work includes the establishment of the relationship between the method and the theory of integral representations of homogeneous secondorder linear ordinary differential equations and the determination of the class of equations to which the method can be applied. A paper titled "Integral Representations of Particular Integrals of Some Inhomogeneous Linear Ordinary Differential Equations" is being prepared for publication as an LBL report and submission to The Journal of Mathematical Physics.

\section{FOOTNOTES AND REFERENCES}

*Present address: Lawrence Livermore Laboratory

+Present address: Research Lab of Electronics, M.I.T.; permanent address: Physics Department Sonoma State College.

†Present address: Columbia University

§Present address: Princeton Plasma Physics Laboratory.

\|Present address: Dept. of Astrophysics and Geophysics, University of Colorado.

1. Condensed from Phys. Rev. Lett. 34, 1613 (1975), and from Diffusion Due to a SingTe Wave in a Magnetized Plasma, Lawrence Berkeley Laboratory Report LBL-5200 (1976).

2. Condensed from Generalized Ponderomotive Force and Three-Wave Interactions, Lawrence Berkeley Laboratory Report LBL-5201 (1976).

3. Condensed from Raman Side Scatter Instability in a Non-Uniform Plasma, Lawrence Berkeley Laboratory Report LBL-4487 (1976).

4. Condensed from Phys. Fluids 19, 889 (1976) and from Parametric Instabilities in Inhomogeneous Plasma, Lawrence Berkeley Laboratory Report L.BL-3267 (1975).

5. Condensed from Resonant Excitation of Nonlinear Plasma Waves. Lawrence Berkeley Laboratory Report LBL-3250 (1975) and from Theoretical Studies of Some Nonlinear Laser-Plasma Interactions, Lawrence Berkeley Laboratory Report LBL-3291 (1975).

\title{
PLASMA PRODUCTION AND HEATING EXXERIMENTS
}

\author{
C. F. Burrell, J. R. Cary, C. F. Chan, W. S. Cooper
}

W. B. Kunkel, and D. R. Pirkle

\section{INTRODUCTION}

Most of the work on plasma production at LBL is concerned, in one way or another, with our ion source and neutral-beam development program. Many questions have arisen about the characteristics and the optimization of the existing positive-ion sources. Our objective is to obtain a clear and 
unambiguous picture of the physical conditions and of the processes controlling the properties of these discharges. This involves careful diagnostic studies with probes and with spectroscopic techniques in the discharge as well as in the extracted beam. The development of understandable simplified models is also helpful, even if extensive numerical computations are eventually required. Two successful ongoing studies are reported here.

Other efforts, such as the search for better negative-ion sources, or attempts to increase the gas efficiency of sources, have not progressed very far and will be reported on at a later date. Similarly, the continuing work with laser-ionized deuterium pellets discussed at length in the 1974 Annual Report is omitted here because the effort has been small and has consisted primarily of an overhaul of the instrumentation.

\section{A COMPUTER MDDEL FOR THE LBL PLASMA ION SOURCE}

We are developing a computer model of the plasma ion source used in our neutral beam systems. The objectives of this effort are 1) to provide certain calculated plasma parameters, very difficult to measure experimentally, as input for the WOLF program (used for optimization of the ion accelerating structure), and 2) to provide a solid theoretical basis for optimization of the performance of the plasma ion source. With adequate understanding of the operation of the ion source, we should be able to improve the gas efficiency, the electrical efficiency, and possibly the fraction of atomic $\left(\mathrm{D}^{+}\right)$ions produced.

The ion source is a low-pressure, high current, low voltage arc discharge between hot tungsten filaments and an anode. The model is an extension of work by Self, 1 and includes recycling of ions and neutrals at the walls. The problem is assumed to be one-dimensional. Input parameters are the dimensions of the discharge chamber, the input flux of neutral atoms (no molecules are included), the velocity of the neutral atoms, the transparency of the accelerating structure for ions and neutrals, and the peak electron density. After simultaneous solution of the equation of motion for ions, Poisson's Equation and the local ionization rate equation with particle conservation, the model provides a calculated potential distribution through the plasma, the self-consistent electron temperature, and the available ion current density at the wall of the discharge chamber.

One prediction of the model, at first surprising, is that of a unique relationship of the electron temperature to the peak electron density, once all other parameters are specified. Experimental verification of this prediction has been complicated by the recent experimental indication that the electron velocity distribution is non-Maxwellian, although it can be well represented by two Maxwellians; the model is being modified to include this fact.

We already have useful predictions from the model-for instance, by making the plasma source deeper, we should be able to inprove the gas efficiency. We plan to include power balance in the model at a future stage in its development to reduce the number of input parameters that have to be specified from experimental observations, and to try to use the model to predict the ion temperature (which presently limits our beam divergence) and the ionic composition.

NEW DIAGNOSTIC TECHNIQUES FOR HIGH-POWER NEUTRAL BEAMS

Many existing and planned Controlled Thermonuclear Fusion experiments use beams of neutral atoms to replenish particle and energy losses from a magnetically confined plasma. Measuring the properties of these beams is becoming a very difficult problem because of increasingly high beam power densities. For example, the TFIR Tokamak, now in the final design stage, will employ four Neutral Beam Systems, each capable of $60-\mathrm{A}$ pulses (ions plus neutrals) at $120 \mathrm{keV}$, with a pulse duration of $0.5 \mathrm{sec}$; where the beams are focused we expect the peak power density to exceed $100 \mathrm{~kW} / \mathrm{cm}^{2}$, sufficient to melt tungsten in a few milliseconds.

An ideal monitoring system would not interfere with the beam, would not be destroyed by the beam, and would be capable of measuring the divergence, aiming direction, and the energy distribution of the beam.

We are investigating promising spectroscopic techniques for measuring all these beam properties. We produce beams of neutral atoms by first accelerating ions (a mixture, in our case, of $\mathrm{D}^{+}, \mathrm{D}_{2}^{+}$, and $\mathrm{D}_{3}^{+}$ions), and then permitting them to capture

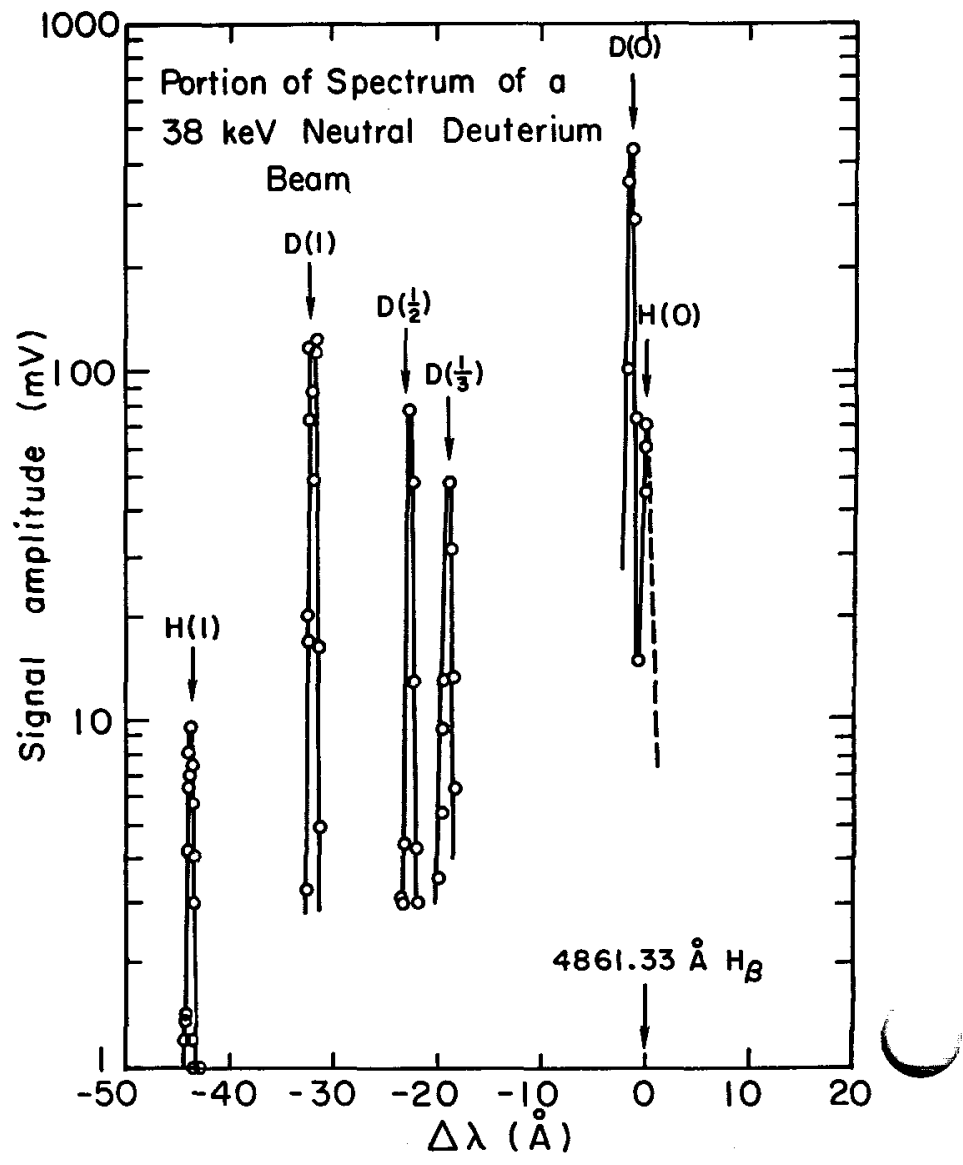

Fig. 1.

(XBL 758-3772) 
an electron from a background deuterium gas in a charge-exchange ce11. Many of the neutral atoms in the beam are in excited states and radiate, either because the electron is captured into an excited state, or because of collisional excitation from the ground state. It appears that spectroscopic analysis of this radiation can provide us with an adequate beam diagnostic technique.

Figure 1 shows a portion of the spectrum from a 38-keV deuterium beam, looking upstream. All spectral lines shown originate from the same atomic transition $(n=4+n=2)$ in either hydrogen or deuterium. The two lines labeled $D(0)$ and $H(0)$ result from excitation of the background gas; the hydrogen is present as an impurity in the Laboratory's deuterium supply. These lines do not coincide because of the isotope shift of $1.3 \AA$. The other lines originate from the moving beam atoms, and are Doppler-shifted toward the blue (we are looking upstream). The lines labeled $\mathrm{H}(1)$ and $D(1)$ are from neutral hydrogen and deuterium, respectively, which resulted from acceleration of $\mathrm{H}^{+}$ and $\mathrm{D}^{+}$ions. We also accelerate $\mathrm{D}_{2}^{+}$and $\mathrm{D}_{3}^{\dagger}$ ions from the source plasma; these break up and capture electrons in the neutralizer and produce the lines labeled $D(1 / 2)$ and $D(1 / 3)$, with Doppler shifts $1 / \sqrt{2}$ and $1 / \sqrt{3}$ that of the full energy $D(1)$ line. Calculated Doppler shifts are indicated by the arrows above the spectral lines.

A quantitative measurement of the relative abundances of the species $D^{+}, D_{2}^{+}$, and $D_{3}^{+}$is of considerable interest to the plasma experimenter who uses the neutral beam. Preliminary results of the relative abundances of these species, deduced from these data with the aid of a computer model including electron capture, ionization, excitation, and deexcitation are in reasonable agreement with measurements made by momentum se- lection of the remaining ions with a bending magnet.

In Figure 1, the line widths are entirely instrumental. We have measured the line width of the line labeled D(1) with a scanning Fabry-Perot interferometer, and found the full width at half maximum to be about $0.1 \AA$, corresponding to an energy spread in the beam of only $250 \mathrm{eV}$ out of (in that case) $24 \mathrm{keV}$. We believe this energy spread is due mainly to energy loss of the beam in the neutralizer gas, which could provide a means of measuring the neutralizer target thickness.

If we look across the beam rather than along it, we measure the component of velocity perpendicular to the beam direction, a measurement equivalent to measuring the beam divergence. Although this measurement is complicated by the presence of the several species of neutrals with different velocities in the beam, it appears to be possible to measure the beam divergence this way. Similar analysis might permit the measurement of the beam aiming direction, although this is less certain.

This work is continuing and will be first used as a diagnostic tool on Test Stand III, our new facility nearing completion. This facility will be able to produce $120-\mathrm{keV}, 15-\mathrm{A}, 0.5-\mathrm{sec}$ deuterium beam pulses, with peak power densities in the beam of about $20 \mathrm{~kW} / \mathrm{cm}^{2}$. We will monitor the beam with a vidicon tube attached to a spectrometer; on-line computer analysis of the data will provide the capability of feed-back control of the beam, if necessary.

\section{REFERENCE}

1. S. A. Self, Phys. Fluids, 6, 1762 (1963).

\section{ATOMIC PHYSICS STUDIES}

K. H. Berkner, W. G. Graham, D. Leung, R. V. Pyle, S. E. Savas, A. S. Schlachter, P. J. Schneider, and J. W. Stearns

\section{PRODUCTION AND DESTRUCTION OF $\mathrm{H}^{-}$}

The atomic physics section of the CTR group is continuing to study the formation and destruction of $\mathrm{H}^{-}$ions ${ }^{1}$, a topic of considerable interest to the neutral-beam injection program.

\section{FORMATION OF $\mathrm{H}^{-}$BY BACKSCATTERING FROM CLEAN METALLIC SURFACES}

$\mathrm{H}^{-}$sources, which feature a plasma discharge in a hydrogen and cesium atmosphere with a $\sim 700 \mathrm{~K}$ metal cathode, have recent $1 y$ produced current densities up to $3.7 \mathrm{~A} / \mathrm{cm}^{2}$. 2,3 To investigate the fundamental processes which lead to such large negative-ion yields we measured the backscattered negative ions from "dirty" and cesiated $\mathrm{Cu}$ and $\mathrm{Mo}$ surfaces bombarded by $\mathrm{H}_{3}^{+}$and $\mathrm{D}_{3}^{+}$ions. Preliminary results indicate that for cesiated $\mathrm{Cu}$ and $\mathrm{Mo}$ the negative fractions (expressed as a fraction of the number of incident protons or deuterons, i.e., $\mathrm{H}_{3}^{+}$ represents three incident protons) rise from $i 3 \%$ at $4.5 \mathrm{keV}$ to $26 \%$ at $0.5 \mathrm{keV}$ incident energy per proton. "Dirty" $\mathrm{Cu}$ and $\mathrm{Mb}$ give values of $1.2 \%$ and 0.68 , respectively, which are fairly constant over this energy range. Work to investigate the collection efficiency for the negative ions and the effect of heating the cesiated surface is now underway.

\section{LOW-ENERGY SURFACE-SCATTERING EXPERTMENT}

The backscattering experiment mentioned above is limited to incident ions with energies greater than $\sim 500 \mathrm{eV} /$ nucleon. Hiskes ${ }^{3 \mathrm{a}}$ has developed a theoretical model (which may explain the $\mathrm{H}$ production in the Dimov source 2,3 ) in which $\mathrm{H}^{-}$may be produced by several-eV $\mathrm{H}$ atoms impinging on a hot 


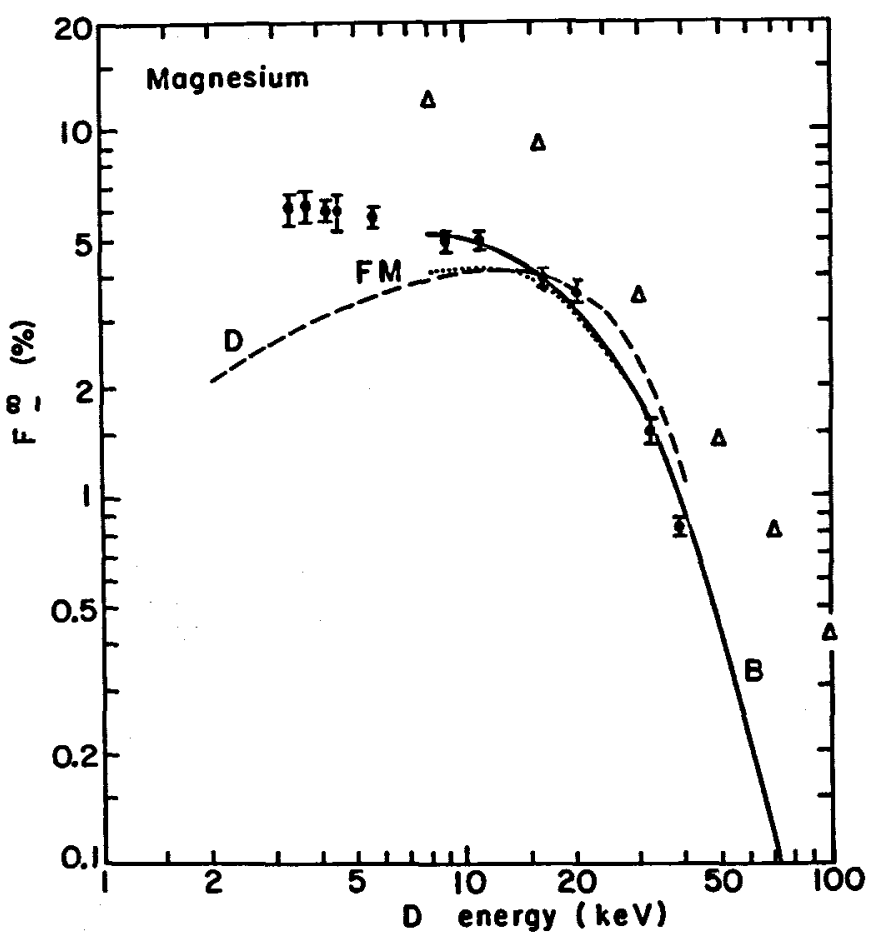

Fig. 1. Thick-target yield of $\mathrm{D}^{-}$ions produced in a Mg-vapor target.

(XBL 7610-4146)

tungsten surface covered with a fraction of a monolayer of cesium. To test this theory a low-energy surface-scattering experiment has been designed and will be constructed during the next year. In the first experiments an atomic-hydrogen beam produced in a hot tungsten tube will bombard a hot, cesiated tungsten surface. The backscattered $\mathrm{H}^{-}$ will be analyzed with a quadrupole mass spectrometer.

\section{CHARGE EXCHANGE OF $\mathrm{H}^{+}$BEAMS IN METAL VAPORS}

As part of a continuing effort to explore negative-ion production by charge transfer in metal vapors we have measured the equilibrium charge fractions that result when 3.3- to 35-keV deuterium beams emerge from "thick" targets of $\mathrm{Mg}$ vapor. Preliminary results for the equilibrium $\mathrm{D}^{-}$fraction, $F_{-}$, are shown in Figure 1 along with other measurements reported in the literature. ${ }^{4}$ Work in this energy range is continuing with $\mathrm{Sr}$ and $\mathrm{Ba}$ targets.

At energies below several $\mathrm{keV}$ the largest $\mathrm{D}^{-}$ yield reported to date has been in Cs vapor 8 (218 at $21.5 \mathrm{keV}$ ); however, other authors have reported lower values, and, in particular, recent results reported by Cisneros, et al. 9 are less than half those reported in Ref. 8. The scatter in these various results is thought to arise from the difficulty of (a) calibrating secondary-emission detectors (used to detect $D^{8}$ ) at low energies and (b) assuring collection of all beam particles under large-angle multiple-scattering conditions. To resolve these discrepancies we are constructing experimental apparatus with a duoplasmatron ion source, a large-aperture Cs oven, large acceptanceangle Faraday cups for the detection of charged particles, and a large acceptance-angle pyroelec- tric wafer for the detection of the $D^{0}$. This experiment is expected to be operational by June of 1976 .

\section{$\mathrm{H}^{-}$INTERACTIONS WITH A PLASMA}

The plasma target to be used in converting 100 - to $150-\mathrm{keV} \mathrm{H}^{-}$ions to neutral atoms is sti11 being developed, Work is continuing in producing a dense (n1 $10^{15}$ ions $/ \mathrm{cm}^{2}$ ) hydrogen plasma in a pulsed, high-current, hot-cathode arc discharge in a magnetic field.

H AND $\mathrm{H}_{2}$ PRODUCTION IN COLLISIONS OF 5- TO 30-keV $\mathrm{H}_{2}^{+}$IONS ${ }^{2}$ WITH XENON GAS

The difficulty in analyzing the neutral products of collisions has meant that very few measurements of individual neutral particle production have been reported, especially at low energies. We

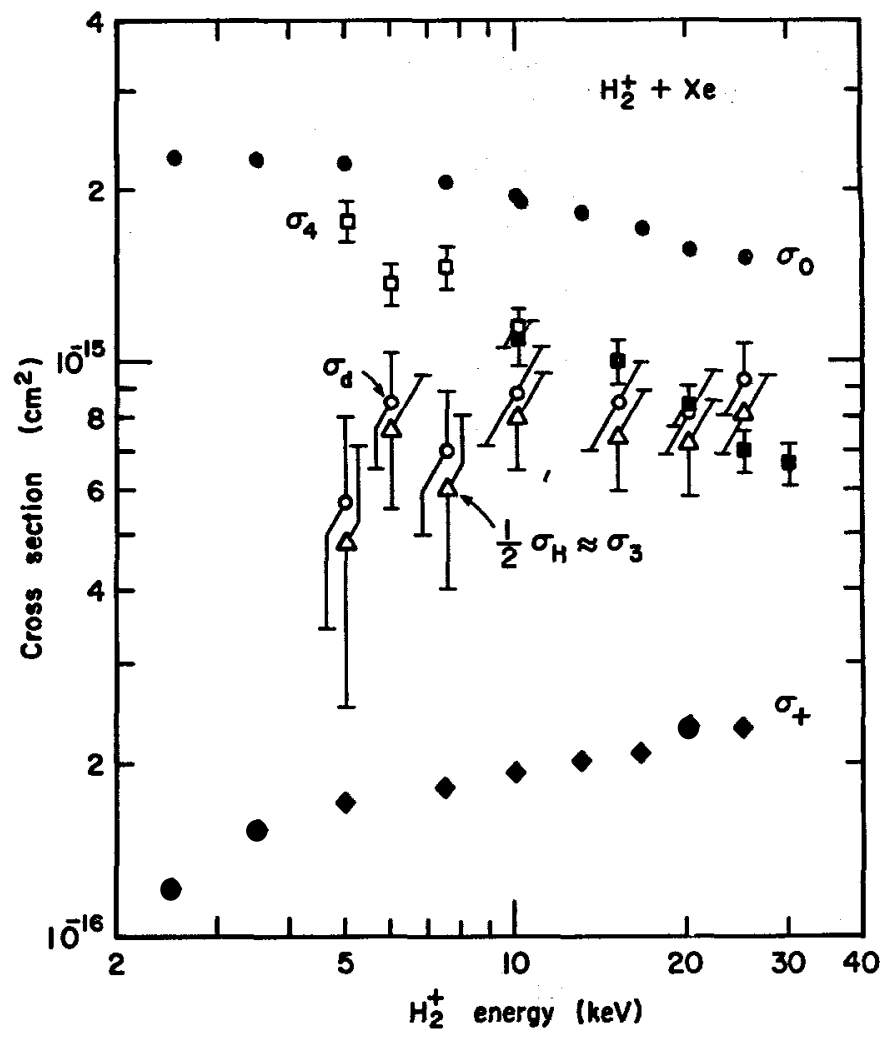

Fig. 2. Cross sections for collisions of $\mathrm{H}_{2}^{+}$with Xe gas. Present results: $\square \mathrm{H}_{2}{ }^{2}$ production, $\sigma_{4}$ (open squares indicate a $\mathrm{D}_{2}^{+}$incident ion plotted at the equivalent $\mathrm{H}_{2}{ }^{+}$energy. This substitution makes extension to low energies more convenient); 0 , dissociation, od; $\Delta$, one-half the $\mathrm{H}$-atom production, $1 / 2 \sigma_{\mathrm{H}}$, which is approximately the cross section for $\mathrm{H}_{2}{ }^{+} \rightarrow \mathrm{H}+\mathrm{H}, \sigma_{3}$. Also shown: $\mathrm{Q}$, total neutral-power production, $\sigma_{0} ;$, proton production, $\sigma_{+}$(from Morgan, et al.12). Error bars on $\sigma_{4}$ indicate the relative uncertainties; those on $\sigma_{\mathrm{d}}$ and $1 / 2 \sigma_{\mathrm{H}}$ indicate the absolute uncertainties.

(XBL 763-2364) 
have measured the cross section for $\mathrm{H}_{2}$ production in collisions of 5- to $30-\mathrm{keV} \mathrm{H}_{2}^{+}$ions with $\mathrm{Xe}$ gas.

$\mathrm{H}_{2}^{+}$ions were extracted from an electron bombardment source. After acceleration and momentum analysis a beam of about $10^{-16} \mathrm{~A}$ was passed through a target gas cell containing xenon gas. On leaving the gas cell the charge component of the beam could be deflected electrostatically if desired. The beam was then detected using a thin-window proportional counter provided by Barnett 10 after a design by McClure.11

The entrance slit of the detector was narrow $(0.025 \mathrm{~mm})$ and could be moved horizontally at a constant rate of $6 \mathrm{~mm} /$ minute. In sweeping horizontally across the beam the transverse spreading of the undissociated fragnents could be observed. "Half-energy" dissociated products could be discriminated out by pulse height analysis while the narrow slit ensured that "large angle" scattered hydrogen atoms produced by dissociative single electron capture were not detected simultaneousiy.

Figure 2 shows some of the measured cross sections for $\mathrm{H}_{2}$ production. Absolute values of the cross sections have standard deviations of \pm 158 , while reproducibility of the data is within $\pm 10 \%$. Also shown are values of the total neutral power measured in this laboratory by Morgan 12 using a pyroelectric detector. The cross sections for total hydrogen atom production were calculated using the relationship

$$
\left(\sigma_{\mathrm{N}}-\sigma_{\mathrm{H}_{2}}\right) \times 2=\sigma_{\mathrm{H}}
$$

with uncertainties of \pm 228 .

\section{REFERENCES}

1. Energy and Environment Annual Report 1974, Lawrence Berkeley Laboratory Report LBL-3253 (1975).
2. Y.I. Belchenko, G.I. Dimov, and V.G. Dudnikov, Nucl. Fusion 14, 113 (1974).

3. K. Prelec and Th. Sluyters, in Sixth Symposium on Engineering Problems of Fusion Research, San Diego, Calif. (1975).

3a. J.R. Hiskes, A. Karo, and M. Gardner, A Mechanism for Negative Ion Production in the Surface-Plasma Negative Hydrogen Ion Source, Lawrence Livermore Laboratory Report UCRL-76650 (1976).

4. R.A. Baragiola, E.R. Salvatelli, and E. Alonso, Nuc1. Instrum. Methods 110, 507 (1973).

5. B.A. D'yachkov and V.I. Zinenko, Sov. At. Energy 24, 16 (1968).

6. B.A. D'yachkov, V.I. Zinenko, and M.A. Pavlii, Sov. Phys.-Tech. Phys. 16, 1868 (1972).

7. A.H. Futch, Jr. and K.G. Moses, Bull. Am. Phys. Soc. 11, 344 (1966) and private communication.

8. A.S. Schlachter, P.J. Bjorkholm, D.H. Loyd, L.W. Anderson, and W. Haeberli, Phys. Rev. 177, 184 (1969).

9. C. Cisneros, I. Alvarez, C.F. Barnett, and J.A. Ray, Phys. Rev. A 14, 76 (1976).

10. C.F. Barnett, J.A. Ray, J.C. Thompson, Oak Ridge National Laboratory Thermonuclear Division, Semiannual Progress Report, ORNL-3652, p. 78 (1964).

11. G.W. McClure, Phys. Rev. 130, 1852 (1963).

12. T.J. Morgan, K.H. Berkner, W.G. Graham, R.V. Pyle, and J.W. Stearns, Phys. Rev. A 14, 664 (1976).

\section{INTRODUCTION}

Tormac (toroidal magnetic cusp) is a magnetic configuration for confining a fusion plasma. It is an alternate concept. The primary concept is Tokamak, and using Tokamak the U.S. should produce a break even fusion reactor by 1984 . Unfortumately, there are indications that Tokamak may not be suitable as a commercial reactor. It is pulsed; it has a minimm size larger than most currently used power installations and it requires magnetic fields of size and intensity awesome to contemplate and expensive to build. Tormac as an alternate concept looks more economically practical as well as technically simpler. If Tormac works, it can be operated in steady state, it can be of relatively small size and it requires magnetic fields of a size and intensity currently obtainable with superconductors.

To determine if the Tormac concept can be made into a fusion reactor, LBL has embarked on an experimental and theoretical program to test the several postulates about plasma behavior on which it is based. These include questions of MDD stability, collisionless containment of particles and wave heating of plasma.

\section{EXPERIMENTAL TORMAC STUDIES}

The experimental Tormac is a toroidally symmetric "bicusp" with an internally trapped mag- 


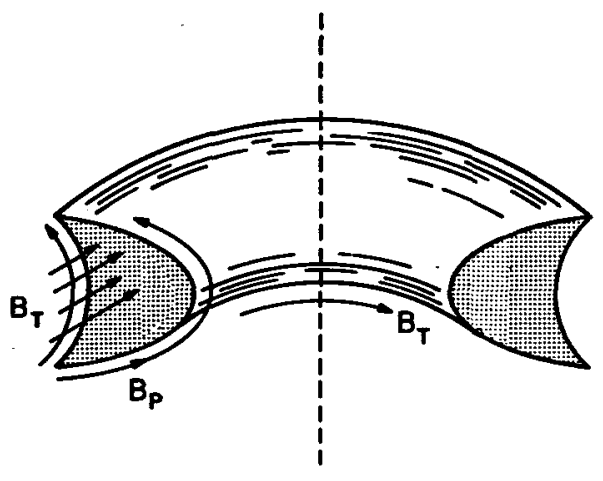

Fig. 1. Drawing of the toroidal bicusp indicating plasma contained in the shaded area. The absolute minimu- $B$ is made up using a combination of toroidal $\mathrm{B}_{\mathrm{T}}$ and poloidal Bp field. It should be noted that a region of magnetic field internal to the plasma exists with a toroidal magnetic field only.

(XBL 751-2085A)

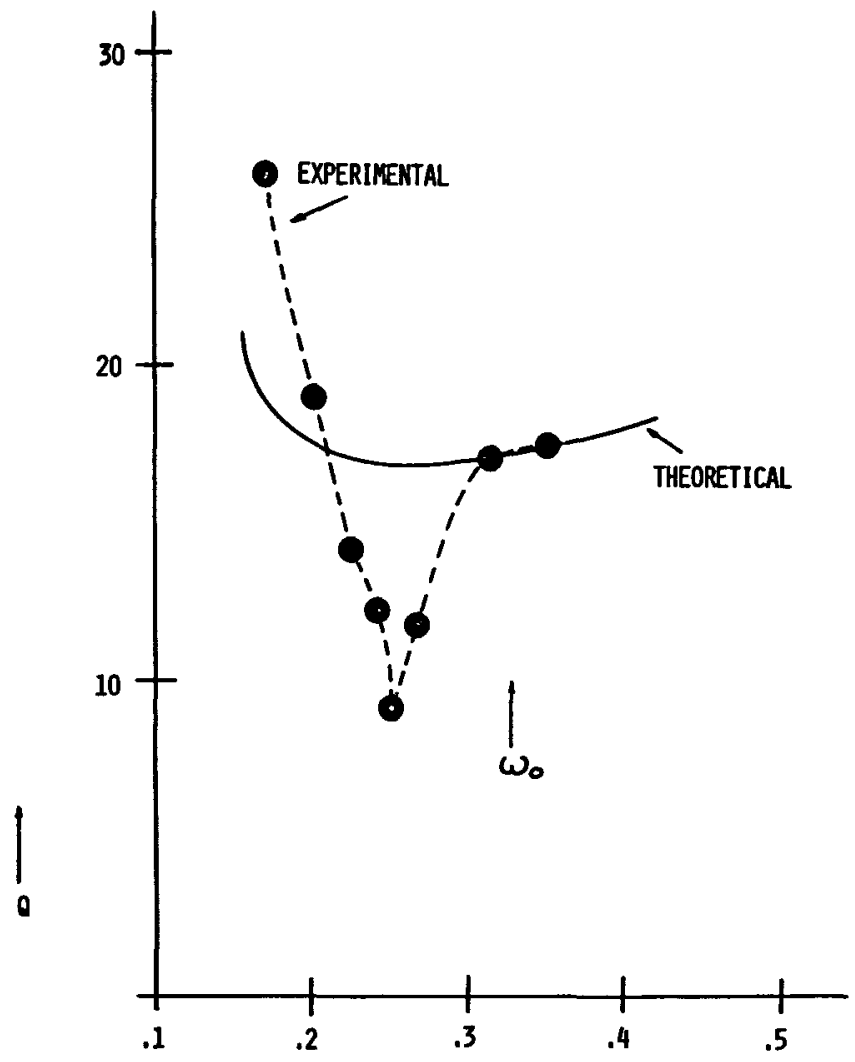

FREQUENCY $($ MHZ $) \longrightarrow$

Fig. 2. Predicted and measured absorption curve for shaker heating.

(XBL 764-1174)

netic field (Fig. 1).1 Two systems have been built to test the properties of Tormac. The smaller apparatus, Tormac IV, is designed to test MHD stability and wave heating in the collisional regime. The larger device, Tormac $\mathrm{V}$, is designed to give information about the containment of par-

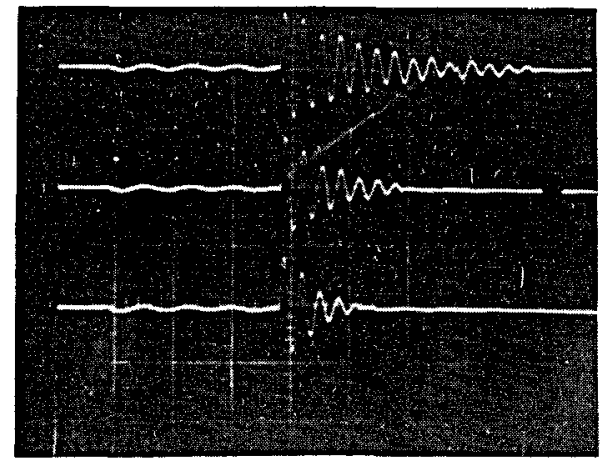

No gas

$10 \mu \mathrm{sec} / \mathrm{cm}$

Fig. 3. "Shaker" heating current waveform.

(XBB 757-5544)

ticles at high temperature, in the "collisionless" regime.

Tormac IV is a $50-\mathrm{cm}$ toroid producing a plasma with a $35-\mathrm{cm}$ major diameter and an $10-\mathrm{cm}$ minor diameter. The plasma is collisional, has a time constant of 50 to $100 \mu \mathrm{sec}$, temperatures of 5 to $50 \mathrm{eV}$ and densities of $10^{15}$ to $10^{16}$ particles $/ \mathrm{cm}^{3}$. To heat the plasma, "shaker" heating has been used.

Shaker heating represents a new method of heating a plasma by wave trapping. A magnetosonic wave is launched into a plasma by coupling to the plasma in such a way that principal modes of the plasma are not directly excited. The wave is then scattered into the several normal modes of the system and decoupled from the driving system. Thus, to the extent to which the wave no longer couples to the driver, the energy is trapped in the plasma. Once wave energy is trapped in the plasma, damping of the waves can occur through wave steepening or through coupling to unstable longitudinal modes. These, as well as other mechanisms, have been suggested for turning wave energy into plasma heat.

The shaker heating is applied using a single turn coil so that the plasma forms the secondary to a transformer in which the shaker coil is the primary. The heating is applied by ringing down a charged capacitor through the shaker coil.

Using the concept that the wave energy launched into the plasma is totally absorbed, an effective $Q$ for the shaker coil can be calculated. $\omega_{0}$ represents a characteristic frequency of oscillation of the plasma in the external magnetic field. In Figure 2 the solid line represents a calculated $Q$ for the coil. The dots represent data points with a plasma present. One notes a resonant-type response in the experimental data. This resonance indicates that the total absorption assumption is violated so that the theory gives only an approximate answer. However as non-linear effects are emphasized by larger wave amplitudes and higher frequencies the theory should hold more exactly. 
In Figure 3 a plot of the damping of the current in the shaker heating circuit is shown with and without plasma. From the damping in this system $20 \%$ of the capacitor energy or 27 , joules is being absorbed when the plasma is present showing that the damping is plasma dependent. Based on these encouraging results, a pulsed generator is now under construction that will be able to deliver kilojoules of energy to the plasma with efficiencies of over $50 \%$.

Tormac IV has also been used to test the MHD stability of the bicusp. To do this a new technique of radiation reconstruction similar in principle to classical tomography has been developed. Spectral line broadening of the $\mathrm{H}_{\beta}$ line of hydrogen can be used to determine plasma density. Radiative reconstruction determines the spectral line shape emitted from each point in the plasma thereby determining the density as a function both of time and of position. To do this, light as a function of position, direction, wavelength and time is measured around the outside of the plasma vessel. A high resolution monochromator was modified into a sixteen channel polychromator, each channel separated by a few tenths of an angstrom. Light is brought to the monochromator using a light pipe with a small $\left(5^{\circ}\right)$ acceptance cone. The light pipe was then moved on successive tests around the outside of the plasma. The data were then analyzed to give the density plot shown in Figure 4. With these density plots as a function of position and time it is shown that the plasma in a bicusp is MHD stable.

\section{TORMAC RECONSTRUCTION PLOT}

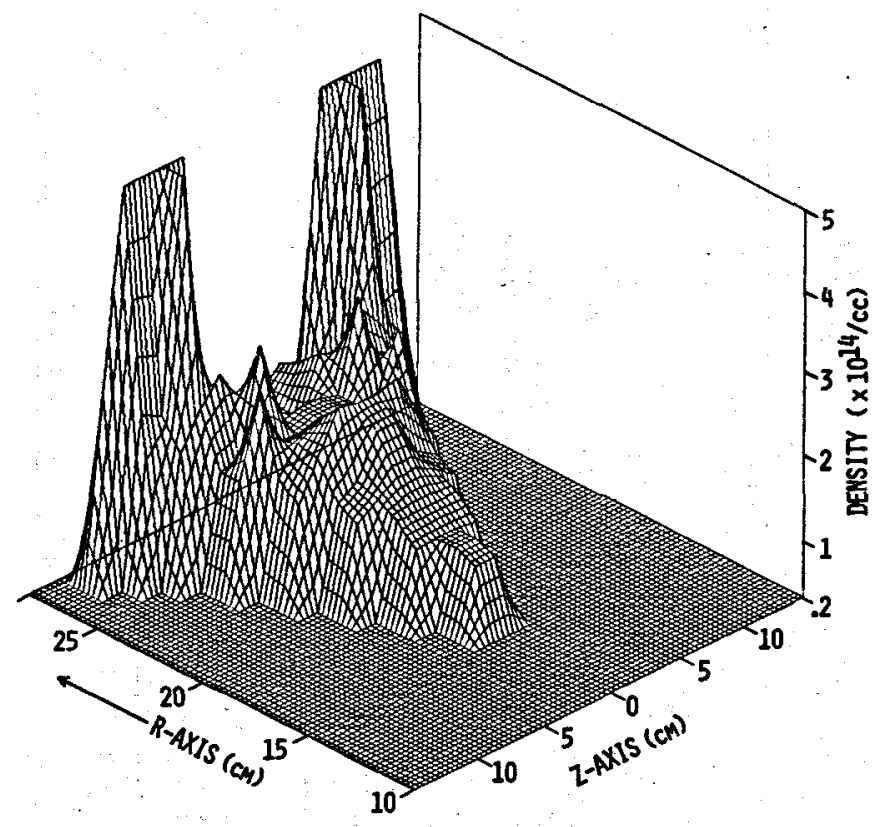

Fig. 4. Tormac IV density profile obtained from Stark effect in Hydrogen- $\beta$. Data are analyzed using computer-aided reconstruction of radiation source similar to tomography. Compare density profile to plasma shape shown in Fig. 1.

(XBL 767-8562)

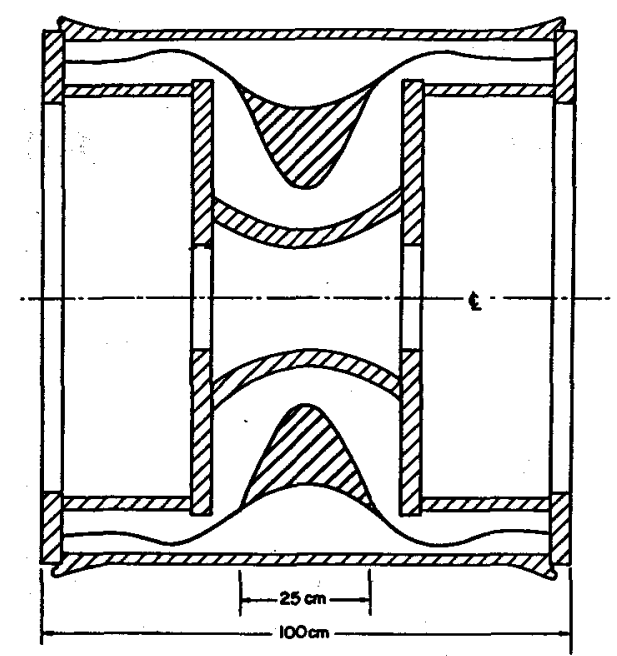

Fig, 5. Tormac $V$ showing placement of plasma in glass vessel.

(XBL 757-8562)

During the past year a difficult construction program has been carried out to build Tormac V. One of the more difficult tasks was the assembly of the Pyrex chamber. The pieces of glass necessary for this device were so large that they were ordered from France and England as no oven in the U.S.A. could produce them. To assemble the device one operation required the machining of a glass cylin$\operatorname{der} 1 \times 1 \mathrm{~m}$ to within $\pm 0.005 \mathrm{~cm}$ in length. This task was successfully accomplished in the LBL machine shops.

To power Tormac V a set of four separate fastpulsed capacitor banks have been constructed. Also constructed is a pulsed oscillator which is designed to deliver five cycles of $500 \mathrm{kHz}$ power at $10^{8}$ watts. The Tormac $\mathrm{V}$ experiment is designed to produce a toroidal bicusp. A cross section of the 1 -meter Pyrex chamber is shown in Figure 5 . The experiment is designed to test the sheath stability of Tormac. Unlike Tormac IV, where the peak of the cusp magnetic field is on the wa11, in Tormac $V$ the peak of the cusp and mirror reflection points are produced in free space, thus eliminating the. contact of the plasma with the wall. Unfortunately, at the same time it eliminates the sheath back pressure along field lines which gave Tormac IV its long-time containment properties.

The lack of containment along magnetic field lines in the Tormac $V$ configuration means the plasma must be heated very rapidly. For it is only in the high temperature, long mean free path regime that the mirror containment of particles along magnetic field lines prevents the free-flowing or very rapid loss of plasma to the walls.

To rapidly heat Tormac $V$, a three-phase system has been designed. This system would combine the shock heating and adiabatic compression method used for $\theta$-pinches with the shaker heating developed in Tormac IV. The power of this method has been illustrated in Tormac IV, where with shock and compression alone the plasma is heated to only $5 \mathrm{eV}$, 
and with shaker heating a $5-\mathrm{eV}$ plasma can be heated to $10 \mathrm{eV}$. On the other hand, when shock and shaker heating is used during the compression phase of the plasma, the combination of shock, shaker, and compression brings the plasma to $40 \mathrm{eV}$.

Tests have begun on Tormac $\mathrm{V}$ and during the next year it is hoped that temperatures in excess of $400 \mathrm{eV}\left(4 \times 10^{6}{ }^{\circ} \mathrm{K}\right)$ can be reached so that some of the problems of collisionless containment of plasma in Tormac can be determined.

\section{TORMAC THEORY}

As discussed already in the 1974 Annual Report, plasma confined in the Tormac configuration can be made to rotate about the minor axis so that the guiding-center drifts do not carry the particles to the boundary layer even in a purely toroidal field $B(R)$. In this way the anisotropy in velocity space caused by losses at the cusps will not be rapidly commmicated to the interior. Rotation can be enhanced and controlled, in principle, by neutral injection with angular momentum about the minor axis. The properties and stability characteristics of such a state have been theoretically investigated. The conditions for a stationary state are shown to require that the number of particles on a flux tube remains constant, yielding the relation $n(R) R / B(R) \approx$ constant. The equilibrium properties of the plasma had been derived on the basis of a single-fluid MHD model with a scalar pressure. In an upgraded analysis ${ }^{2}$ the plasma is treated as consisting of two fluids with negligible centrifugal effects and interspecies interactions, where electrons are considered as isotropic with the equation of state $d / d t\left(P_{e^{n}}{ }^{-\gamma}\right)=0$, while the ions are anisotropic and obey the Chew-Goldberger-Low equations. The equilibrium solutions $n(R)$ are found to satisfy the conditions of marginal stability against flute modes, just as in the previous case. This is understandable because the assumed slow rotation represents exactly the idealized interchange process.

A second problem tackled during 1975 addresses the sheath structure from the point of view of Vlasov theory.

The stability of the sheath is of paramount importance for Tormac. Because the shear and inhomogeneity, scale lengths are not large compared to an ion gyroradius, the usual $10 \mathrm{w}-\beta$, weak inhomogeneity theories cannot be applied. Thus we have begun an investigation of linear Vlasov stability for high- $\beta$, strongly inhomogeneous plasmas.

In order to do linear Vlasov theory, one must know the particle orbits and the equilibrium distribution function. We have developed a formalism for generating a large class of exact VlasovMaxwel1 equilibria with sheared magnetic fields. 3 The equilibrium quantities vary in one direction, and the magnetic field has components in the two orthogonal directions. This machinery allows us to generate model equilibria for Tormac and we are in the process of constructing various models.

\section{TORMAC REACTOR SCALING STUDIES}

An analysis and survey of the characteristics of a (thermonuclear) fusion reactor whose plasma is confined in a Tormac configuration has been carried out (see Figure 6). Scaling laws and detailed design parameters have been developed. A toroidal bicusp with a "hole size" two ion gyroradii wide is assumed. For a Maxwellian D-T plasma the resulting density confinement time product is given by

$$
\text { nt } \simeq 5 \times 10^{10} \mathrm{r}_{\mathrm{p}} \mathrm{T} \mathrm{B} \mathrm{cm}^{-3} \mathrm{sec} \text {, }
$$

where $r_{p}$ is the plasma minor radius $(m), T_{j}$ the ion temperature (keV), and $B$ the magnetic field strength at the plasma surface (kG). This scaling relationship allows for achievement of breakeven for quite modest values of $r_{p}, T_{i}$ and $B$.

More detailed calculations show that a Tormac reactor can have $\mathrm{Q}_{\mathrm{E}}$ values $\left[\mathrm{Q}_{\mathrm{E}}=\right.$ gross power generated (electric)/circulating power (electric)] of from 2 to about 10 for very reasonable sizes and field strangths. Of considerable interest to the electric power producing industry is the fact that one can design for plants whose net power out is in the range 50 to $500 \mathrm{MN}_{\mathrm{e}}$ and whose reactor parameters remain appealing. A set of curves, for a chosen set of parameters, showing output power as a function of plasma size is shown in Figure 6 . In general, the minimum size of high- $\beta$ reactors of a given output is dictated by the maximm permissible wall loading.

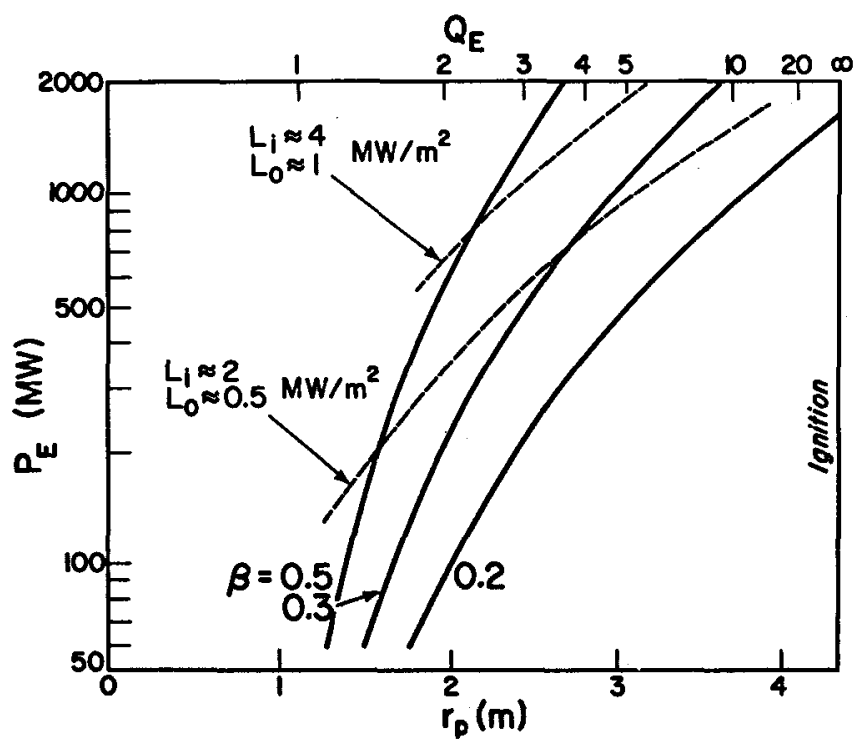

Fig. 6. Net electric power out $\left(\mathrm{P}_{E}\right)$ vs. plasma minor radius $\left(r_{p}\right)$ for several plasma betas. $L_{i} o$ are neutron wall loadings at the inner (i) and outer (o) walls. (Parameters: thermal efficiency $=0.5$, injection efficiency $=0.7, \alpha$-particle heating efficiency - 0.8; plasma aspect ratio $=3$, vessel aspect ratio $=1.8$; electron temperature $=20 \mathrm{keV}$, ion temperature $=40 \mathrm{keV}$; magnetic field strength $=20 \mathrm{kG}$.) (XBL 771-224) 
Our conclusion is that, although our results are of a preliminary nature in the sense that we have not carried out detailed engineering studies, the Tormac scaling yields reactor designs whose critical parameters are considerably more attractive than those of other proposed reactor schemes.

\section{FOOTNOTES AND REFERENCES}

*This research funded in part by the Electric Power Research Institute.

tVisiting scientist, permanent address, University of Sydney, Sydney, Australia.
1. M.A. Levine, I.G. Brown, and C.C. Gallagher, Plasma Containment in a Toroidal Bicusp (TORMAC), Lawrence Berkeley Laboratory Report LBL-3262, to appear in Plasma Physics, Supplement (Pergamon Press, 1976).

2. J.H. Hammer and W.B. Kunke1, Phys. Fluids $\underline{19}$, 438 (1976).

3. P.J. Channe11, Exact Vlasov-Maxwell Equilibria with Sheared Magnetic Fields, Lawrence Berkeley Laboratory Report LBL-4640 (1976), to appear in Phys. Fluids.<smiles>CC</smiles>

\title{
NEUTRAL-BEAM DEVELOPMENT AND TECHNOLOGY*
}

\author{
W. R. Baker, K. H. Berkner, W. S. Cooper, K. W. Ehlers, D. B. Hopkins, \\ J. M. Haughian, W. B. Kunkel, H. M. Owren, \\ B. A. Prichardt, R. V. PyZe, and J. W. Stearns
}

The objective of this program is the development of the technology and hardware required for neutral-beam-heating of plasmas in CTR confinement devices and fusion reactors. There is close cooperation and interaction with fusion experimenters at Livermore, Oak Ridge, Princeton, and General Atomic.

The pulsed-beam development in 1975 mainly was oriented toward the near-term programmatic goal of developing an $80 \mathrm{MN}$ (total), $120 \mathrm{keV} \mathrm{D}$ injection system for the Tokamak Fusion Test Reactor to be built at Princeton. This power will be divided among 12 modules, packaged (in groups of three) in four 9-m long, cryogenically-pumped injection lines. A conceptual design of this system was completed in September 1975.1

The plasma source and accelerator structure designs are extrapolations of our previously described $15 \mathrm{~A}$ to $75 \mathrm{~A}, 2 \mathrm{O} \mathrm{keV}$ and $40 \mathrm{keV}, 10$-msec pulse-length injectors. ${ }^{2}$ The main development effort on the plasma source, which must be uniform within a few percent in space and time over a $10 \times 40 \mathrm{~cm}$ area has been toward extending the pulse length from $10 \mathrm{msec}$ to $0.25 \mathrm{sec}$; the technique for accomplishing this has been demonstrated on a plasma source with $20 \%$ of the required extraction area.

The design effort on the accelerator structure was of two kinds:

(1) A computational effort based on our meager knowledge of electrical breakdown near intense ion beams and other operating experience. The shapes and potentials of the four electrodes were obtained with the optimizing program WOLF. 3

(2) A mechanical design incorporating molybdenum, multi-aperture (slotted) electrodes capable of holding tolerances of about $0.05 \mathrm{~mm}$ during severe thermal cycling. Tests with $20 \mathrm{kV}$ to $40 \mathrm{kV}$ structures have shown that this should be possible, but the extrapolation to $120 \mathrm{kV}$ operation must be recognized as very large.
To permit testing of high-powered neutralbeam systems we have designed and started construction of a large new facility, to be operational in the Sumner of $1976.4,5$

Since gas to present injection systems is pulsed on for only $0.5 \mathrm{sec}$, at intervals of 1 minute or more, it is convenient to use a largevolume vacuum chamber to handle the peak gas load by volume expansion into a sphere with a volume of 172,000 liters; the system will be pumped down to its base pressure between pulses by titanium sublimation pumps.

The power supplies and their controls are of a new LBL design which should make it possible to control the large power precisely enough that good beam optics are maintained, and spark damage is kept to an acceptable level.

The system will be capable of complete computer control, using techniques developed by the LBL Real Time Systems Group and Draper Laboratories.

Basic information for this development activity is provided by research carried out in the Plasma Production and Heating and the Atomic Physics programs, as described in preceding subsections of this report.

\section{FOOTNOTES AND REFERENCES}

*This work is part of a program of neutral-beam development being conducted jointly by the Lawrence Livermore and Lawrence Berkeley Laboratories. It also has been reported in the LLL report series UCRL-50002.

tPermanent address: Princeton Plasma Physics Laboratory.

1. TFTR Neutral Beam Injection System Conceptual Design, Lawrence Berkeley Laboratory Report LBL-3296 (1975). 
2. W.R. Baker, et al., "Intense-Neutral-Beam Research and Development," in Plasma Physics and Controlled Nuclear Fusion Research 1974, (IAEA, Vienna, 1975), Vo1. I, p. 329.

3. W.S. Cooper, et al., "Computer-Aided Extractor Design," in Proc. of the Second Symposium on Ion Sources and the Formation of Ion Beams, Berkeley, California (1974), p. II-1, LBL-3317.

4. J.M. Haughian, et al, , "Test Facility for the Development of $150-\mathrm{keV}$, Multi-Megawatt Neutral Beam Systems," in Sixth Symposium on Engineering Problems of Fusion Research, San Diego, Calif. (1975), LBL-4427. 


\title{
INTRODUCTION
}

The National Solar Energy Program conducted by ERDA has experienced a dramatic increase in effort in the past few years. This growing involvement is based on the premise that solar energy can play a significant role in the nation's energy budget.

A parallel growth has occurred in the solar energy program in the Energy and Environment Division. Current projects within the Division provide technological support for programs such as the solar heating and cooling of buildings and solar thermal collection for large scale electric power production. The Division is also involved in basic research efforts in support of the solar program: material studies of inorganic-photovoltaic materials for solar cells, the exploration of the use of biological materials for photovoltaic cells, and hydrogen production by biological systems. Another rather unusual project involves the development of an engine for direct conversion of low temperature solar derived heat to mechanical energy.

The project on the solar heating and cooling of buildings has as a major goal the development of a control system to take maximum advantage of the available solar resource. The controller uses state-of-the-art electronic techniques to provide prepackaged control functions that can be tailored to various climates and personal prefer- ences. The circumsolar telescope project has developed an instrument to measure the detailed profile of the sun. This is an important consideration in the solar thermal-electric program since these measurements are necessary to the determination of the amount of solar radiation lost from a concentrating collector system.

The solar cell research program is investigating the properties of polycrystaline silicon to find ways of reducing the cost of photovoltaic conversion. The effort is directed toward understanding impurity-induced surface states and their origin during the production of the cell. The possibility of large scale photovoltaic production by biological systems is also being studied by using photosensitive lipid membranes that contain chlorophy11.

The use of biological systems to produce hydrogen with sunlight is being explored. The functions of certain types of algae can be modified in order to frustrate the usual photosynthetic behavior and divert it to the production of hydrogen. The shape memory effect in a nickel-titanium alloy (Nitinol) is being used to convert small temperature differentials (near ambient temperature) into mechanical work. Engines have already been built and tested utilizing solar heated water and a more powerful engine of new design is being constructed.

SOLAR HEATING AND COOLING OF BUILDINGS:

DEVELOPMENT OF CONTROL SYSTEM AND AMDONIA-WATER ABSORPTION AIR CONDITIONER

\author{
Kim Dao, Melvin Simnons, Richard Wolgast, Eugene Binnall \\ Charles Dols, Richard shaw, Wigbert Siekhaus, James Dillworth, Michael Wahlig
}

\section{INTRODUCTION}

One of the primary tasks of this project has been the development of an inexpensive electronic control system that will be capable of operating a solar combined heating and cooling system in an optimized or near-optimized way. This controller is the equivalent for solar energy heating and cooling systems of the simple thermostat control used for conventional heating and cooling systems. An experimental solar heating and cooling system has been designed and constructed that allows testing and exercise of the controller. This solar heating and cooling system will also function as an experimental simulator facility to test the performance of various control algorithms for a variety of input meteorological conditions and output load demands.
A second, equally important part of this project is the development of an amonia-water $a b$ sorption air conditioner that will provide the cooling function of the solar combined heating and cooling system. This air conditioner is being designed for operation at temperatures less than $200^{\circ} \mathrm{F}$, so that moderate-cost flat plate collectors may be used. It uses air cooling of the condenser and absorber coils, as water cooling towers appear to be unfeasible for residential use.

\section{CONTROLLER}

The block diagram of the control system is shown in Fig. 1. The main input signals to the electronic controller circuit are provided by temperature sensors and the room thermostat. The temperature sensors are remotely located at criti- 


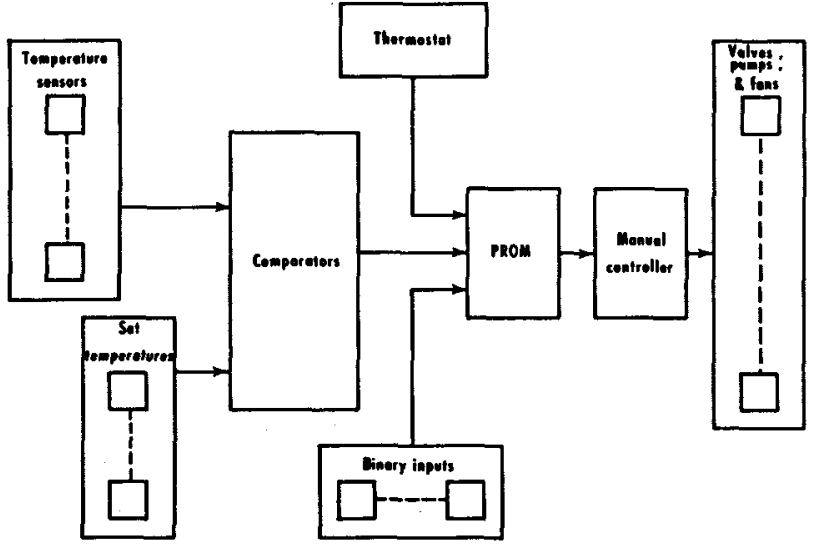

Fig. 1.

(XBL 757-3600)

cal (in terms of decision-making) locations in the solar heating and cooling system. For example, a sensor in the fluid return line from the heating coil will permit a decision whether to direct this flow to the storage tank or to the collectors. A new type of temperature sensing devicel consisting of an integrated circuit packaged in a small (TO-46) can provided the input to the controller. The large output levels (10 millivolts per ${ }^{\circ} \mathrm{C}$ ) of this device reduce the possibility of noise interference encountered with a thermocouple or thermistor.

The first stage of the controller circuit consists of a set of comparators in which the input analog signals from the temperature sensors are inter-compared, or are compared with pre-set temperature levels. These set temperatures are dialed-in on potentiometers mounted on the front panel of the controller chassis; they are used to supply specific temperatures (such as the maximum desired storage tank temperature) to the comparator circuits. The output of each comparator circuit is a binary signal that indicates which of the two inputs is the greater, allowing for the manually adjustable offset and hysteresis levels built into each comparator.

The heart of the controller circuit consists of one or more Programmable Read-Only Memory (PROM) modules. These modules contain the operating algorithm - the set of rules that determines the mapping of input to output signals. Besides the binary output signals from the comparators, there are two other sources of binary signals that serve as inputs to the Prass. The first of these is the room thermostat.

The thermostat will have four set points, indicating five states. These indicate that the building requires 1) heating, 2) boosted heating, 3) cooling, 4) boosted cooling, or 5) nothing. "Boosted" means that the solar system itself cannot satisfy the load demand, and so auxiliary fuel must be used. The thermostat will provide this information to the PROM via three binary signals. The circuitry used in the thermostat will be similar to that used in the comparators, and the thermostat development will be a part of this project.
Another source of binary signals arises from remote devices that communicate to the PROM binary state information that is relevant to the decisionmaking process. For example, one binary signal would indicate whether a flow-actuated switch is on or off, and another binary signal would tell whether or not the temperature has exceeded the thermostat set point in the first hot water tank.

The algorithm programmed into the PROMs translates the set of input binary signals into a corresponding set of output binary signals that actuate the output devices; namely, solenoid valves for fluid flow through the collector and load loops, solenoid valves for gas flow to the auxiliary heaters, pumps for the water circulation loops, and fans for air movement across heat exchanger coils.

The main concept governing the operation of a solar heating and cooling system should be minimization of cost. Usually this is synonymous, once the size of the solar system is fixed, with minimizing the amount of conventional fuel used as back-up to the solar system. It is not always obvious which algorithm will achieve this goal, and it is entirely possible that different algorithms will be optimal for different climatic regions and different load patterns. As the algorithms are contained in the modular, easily-replaceable PROMs, this controller design not only allows experimental flexibility, but also ensures that the basic controller can be used in different climatic and load situations, merely by plugging in different PROMs. A particular residential system in a given climatic region would only employ a single algorithm, and thus, in general, would only require a subset of the temperature sensors and control valves used in our generalized experimental system.

The controller fabrication should be completed and the unit under test by the end of FY76. It will then be used as an integral part of the experimental solar heating and cooling system in the investigation of the value of competing control algorithms. The development of an electronic thermostat that will use circuitry similar to that used in the controller will be underway before the end of FY76. This unit will provide input levels to the controller, and will function as a necessary part of the overail control subsystem.

Consultants will be used to obtain advice and criticisms on this controller and its prospects for leading to a commercially acceptable model.

\section{HEATING AND COOLING SYSTEM}

The installation of the experimental solar heating and cooling system has been completed, and debugging has proceeded using a manual control mode. Minor inprovements were made to the initial design of the roof manifolding in order to balance flows through the individual collectors. 2 During this process we discovered what we believe is a serious flaw in the design of the flow ports on the PPG collectors for our type of installation. The current design permits the formation of vapor pockets in the outflow channels from the collectors, making it difficult or impossible to achieve steady, 
balanced flow from all the individual collectors. Investigation of this effect is continuing.

The basic operation of the pseudo-collector has been verified; in this mode its output fluid temperature duplicates that coming from the real collectors. Some improvements are being made in the time response of the pseudo-collector, and also in the magnitude of the output temperature excursions when the pseudo-collector is cycled on and off.

A11 of the thermocouples have been installed throughout the system for temperature monitoring, as planned, and the readout system, consisting of panel meters and chart recorder, is fully operational. Aluminum coupons have been installed in the collector loop for corrosion monitoring (these will be periodically removed and weighed accurately), and periodic monitoring of the water $\mathrm{pH}$ level has been initiated.

The operation of the pseudo-load system has been checked out successfully, using hot water pumped from the storage tank through the heating coil. This system simulates the air flow through the forced-air ducts of a building heating or cooling system.

Studies are underway, beginning with the domestic hot water system, to determine the effects of different control algorithms on the amount of auxiliary fuel required for system operation. The methods and costs for fully automating this experimental system, thus producing an experimental simulation facility, are being determined. Information is also being gathered to ensure that the benefits of operation of this facility will justify the costs of constructing and operating it.

\section{AIR CONDITIONER}

The design of the air conditioner (Fig. 2) has been guided by the operating parameters of the experimental system. Since the system is intended to test control algorithms for residential systems, the cooling capacity of the air conditioner was chosen to be three tons. The heat source for the unit is water, either directly from the solar collectors or indirectly via the storage tank. The temperature available to heat the generator is assumed to be less than $212^{\circ} \mathrm{F}$. We have decided to adopt as a further design constraint the assumption that only dry air cooling of the condenser and absorber will be used since it was felt that wet cooling towers were impractical for residential use. Thus, significantly higher temperatures will occur at the condenser and absorber than if a wet cooling tower were used. The combination of low generator temperature $\mathrm{T}_{\mathrm{G}}$ and high condenser/absorber temperatures, $T_{C}$, can prevent operation of an absorption refrigeration cycle. Assuming no pressure drops in the system except those across the expansion valves, the cutoff temperature is $188^{\circ} \mathrm{F}$ for $\mathrm{T}_{\mathrm{C}}=110^{\circ} \mathrm{F}$ and an evaporating temperature, $\mathrm{T}_{\mathrm{E}}$, jequal to $45^{\circ} \mathrm{F}$. Thus the system is operating near the cut off when $\mathrm{T}_{\mathrm{G}}$ is low (of the order of $200^{\circ} \mathrm{F}$ ). Another problem is encountered because the usual design point of a vapor compression unit is a $125^{\circ} \mathrm{F}$ condensation temperature, and an outside air temperature of $95^{\circ} \mathrm{F}$. Such a high condenser temperature would made the design of a solar driven

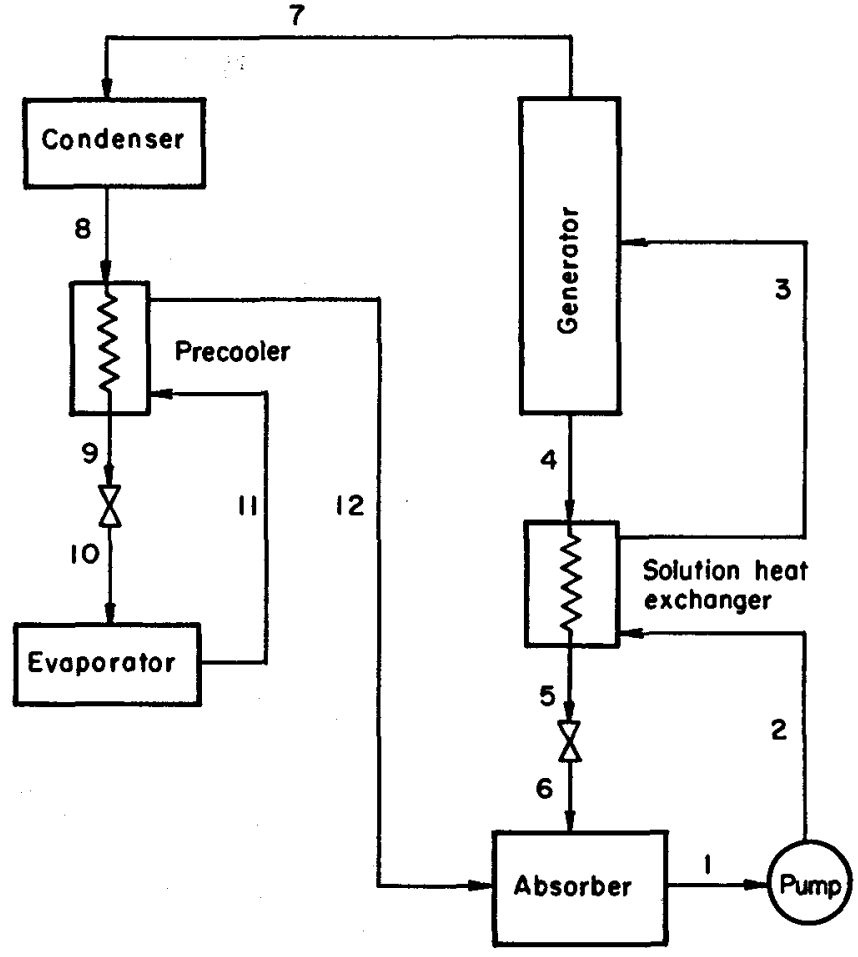

Fig. 2

(XBL 753-2462)

unit essentially impossible, so some means must be found to reduce this value. Factors to be considered are: how close to the cut off will the system work satisfactorily and what economic tradeoffs are involved in increasing the heat exchanger size. Both of these strategies are being explored.

A gas-fired, air cooled amonia-water absorption chiller was used as a starting point for fabrication of a solar-driven unit. The unit obtained was an Arkla model ACB-60, which had a rated capacity of five tons. Three major modifications were necessary to the gas-driven unit. First, the gasfired generator had to be replaced by a water heated generator. This required replacement of the generator rather than just modification. Second, because the temperatures available in the generator did not allow large changes in the ammonia concentration of the solution passing through the generator, more solution had to be pumped for each pound of refrigerant that was to be generated. Two more solution pumps identical to the original one were added, for three times the original capacity. Third, because the solution-cooled absorber would no longer be effective in preheating the solution entering the generator, a liquid-liquid heat exchanger (the preheater) had to be added.

The generator design decided upon is a packed tower counter-flow heat exchanger (Fig. 3). The strong solution drips down through the steel pa11ring packing, making contact with a solar-heated hot water coil with a surface area of $36 \mathrm{ft}^{2}$. The hot water within the coil and the hot vapor generated outside the coil flow upward, counter current to the solution. The preheater and rectifier are she11-and-tube, one-pass, counterflow heat exchangers of surface area $22 \mathrm{ft}^{2}$ and $2.4 \mathrm{ft}^{2}$ respective$1 y$. 


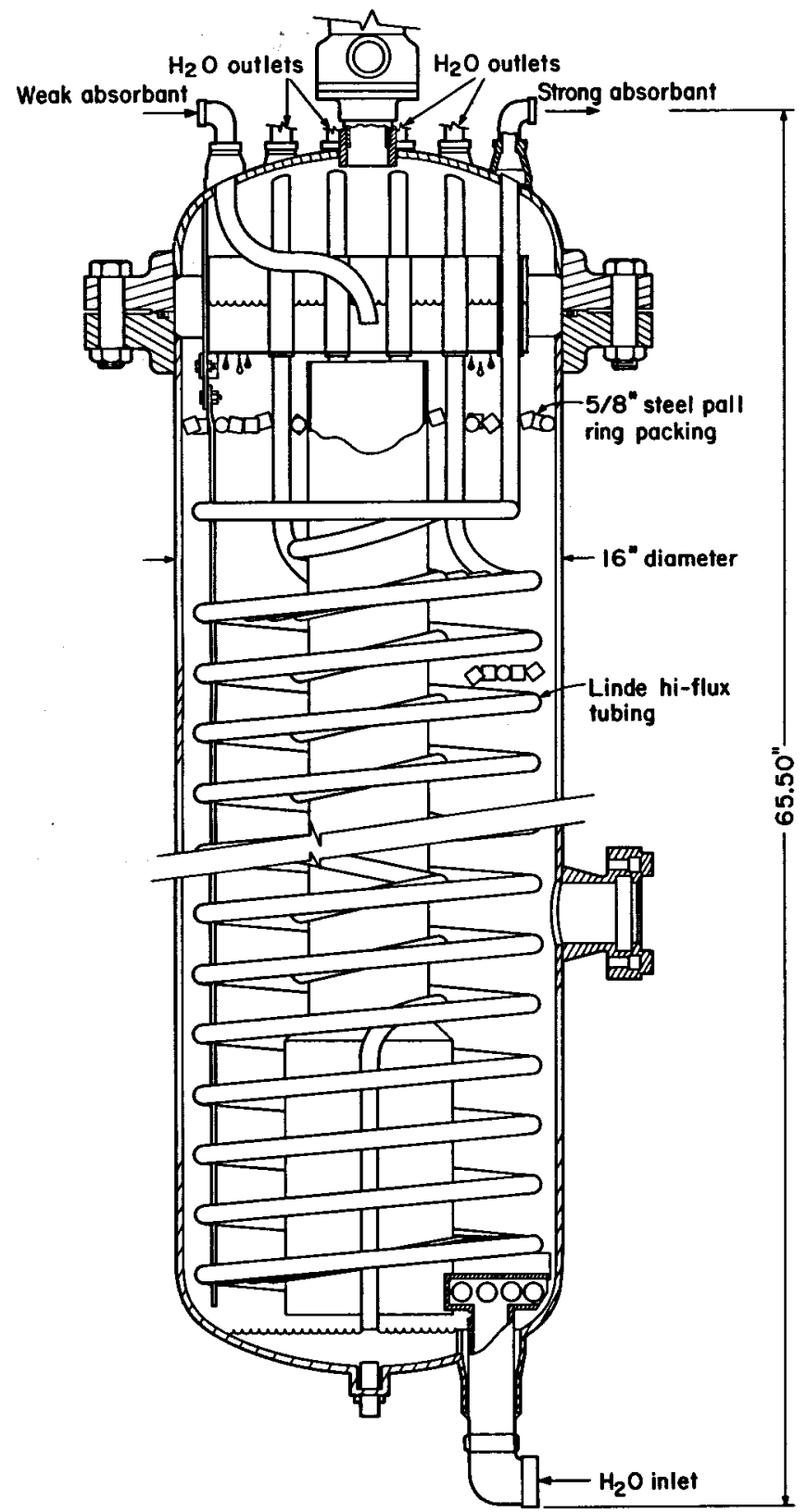

The generator fabrication is nearly complete. Fabrication and assembly of the remainder of the modified chiller is proceeding in parallel. Following completion of the assembly phase, an extensive test program will be conducted to determine the stability of operation near cut-off conditions, and to determine experimentally the accuracy of calculated operating temperatures and coefficient of performance.

\section{PYRANOMETER NETWORK}

Work on the solar insolation measurement network is continuing and the interface circuits (between the pyranometers and the PGGE tape recorders) have been completed. The interface-recorder units are presently undergoing qualification tests in a temperature-controlled chamber to ensure operation over the full range of temperature conditions expected in the field.

At the request of PGEE, the locations of the six measurement stations have been modified. The presently planned locations are LBL, Santa Rosa, Salinas, Red Bluff, Bakersfield, and the FresnoDavis area.

As part of the pyranometer tests, output data tapes were successfully processed through the PGE्E computer facility in San Francisco, with the data then being forwarded to LBL.

\section{REFERENCES}

1. The temperature sensor is type LX 5700A, manufactured by National Semiconductor.

2. The collectors were purchased from PPG Industries, and contain an aluminum roll-bond absorber plate, painted flat black.

Fig. 3

(XBL 757-3558)

\title{
MEASUREMENT OF CIRCUMSOLAR RADIATION
}

\author{
D. F. Grether, A. J. Hunt, and M. Wahlig
}

\section{INTRODUCTION}

This project is concerned with the detailed measurement of the character of the solar flux that reaches the surface of the earth. This information is necessary in order to make accurate predictions of the performance of focusing solar collector systems for prevailing weather conditions at various geographic locations. These measurements consist of angular profiles of the solar and circunsolar (the small angle region around the sun) radiation as a function of wavelength, and the total hemispherical and normal incident flux.
Current research projects on the conversion of solar energy to thermal energy for the generation of electricity involve focusing mirrors to concentrate the incident energy upon a receiver. The use of such collectors results in high-temperature operation of the receiver, allowing a conversion efficiency from heat to electricity comparable to that of conventional generating stations.

Past calculations of the performance of focusing collector systems have assumed that the 
solar flux is uniform and is contained within the $1 / 2^{\circ}$ angle subtended by the sun. The second assumption would be justified if the only effect of the atmosphere were to absorb the incident sunlight. However, this is not the case. The sunlight is scattered by molecules (Rayleigh Scattering) and by atmospheric aerosols. While the molecular scattering is nearly isotropic, aerosols having dimensions greater than the wavelength of light scatter the sunlight predominately through small angles, resulting in circumsolar radiation (also referred to as the solar aureole). Pyrheliometers, the standard instruments used to measure the "direct" solar flux, typically have a $6^{\circ}$ field of view. The circumsolar radiation is included in the measurement of the pyrheliometers, thus. over-estimating the amount of energy that would be collected by focusing devices. Prior to this project, virtually no quantitative data existed on the amount of circumsolar radiation and its dependence on atmospheric conditions. The instrumentation for acquiring such data was also lacking.

\section{CIRCUMSOLAR TELESCOPE}

A "scanning telescope" has been constructed to make the circumsolar measurements. The instrument scans through a $6^{\circ}$ arc with the sun at the center, and measures the brightness of the solar and circumsolar radiation as a function of angle. The scan takes one minute of time, with a digitization of the brightness every $1.5^{\prime}$ of arc. Within $0.5^{\circ}$ on either side of the center of the sun, an aperture of size $1.5^{\prime}$ of arc is used. Outside this region (i.e., in the region of relatively low circumsolar levels) the aperture is increased to $5^{\prime}$ of arc in order to increase the signal level by roughly a factor of ten. A set of measurements consists of one scan at each of ten "filter positions". There are eight optical filters that divide the solar spectrum into eight bands of approximately equal energy content, one open (or "clear") position, and one opaque position. The latter is used to monitor the detector noise. An Active Cavity Radiometer ${ }^{1}$ (a type of pyrheliometer) provides an absolute calibration for the telescope as we11 as the usual normal incident measurement. Two pyranometers are used, one mounted in the conventional horizontal position, and one tracking the sun.

A portable (or prototype) instrument has been constructed and tested, and has been making measurements at Berkeley for some time. This instrument will be used to make measurements in a variety of atmospheric conditions and geographic locations. Three "fixed-site". instruments are under construction and will soon be deployed at locations of interest to solar thermal conversion.

Figure 1 is a photograph of the completed instrument. The telescope proper (the large, cylindrical tube at the top of the assembly) is connected by a pivot point and a scanning mechanism to a reference platform (the rectangular tube about the same length as, and directly under, the telescope). A solar tracker keeps the reference platform pointed at the center of the sum to within $\pm 0.5^{\circ}$ of arc. The pyrheliometer is mounted inside the reference platform. The sumtracking pyranometer is mounted on a rack at the front of

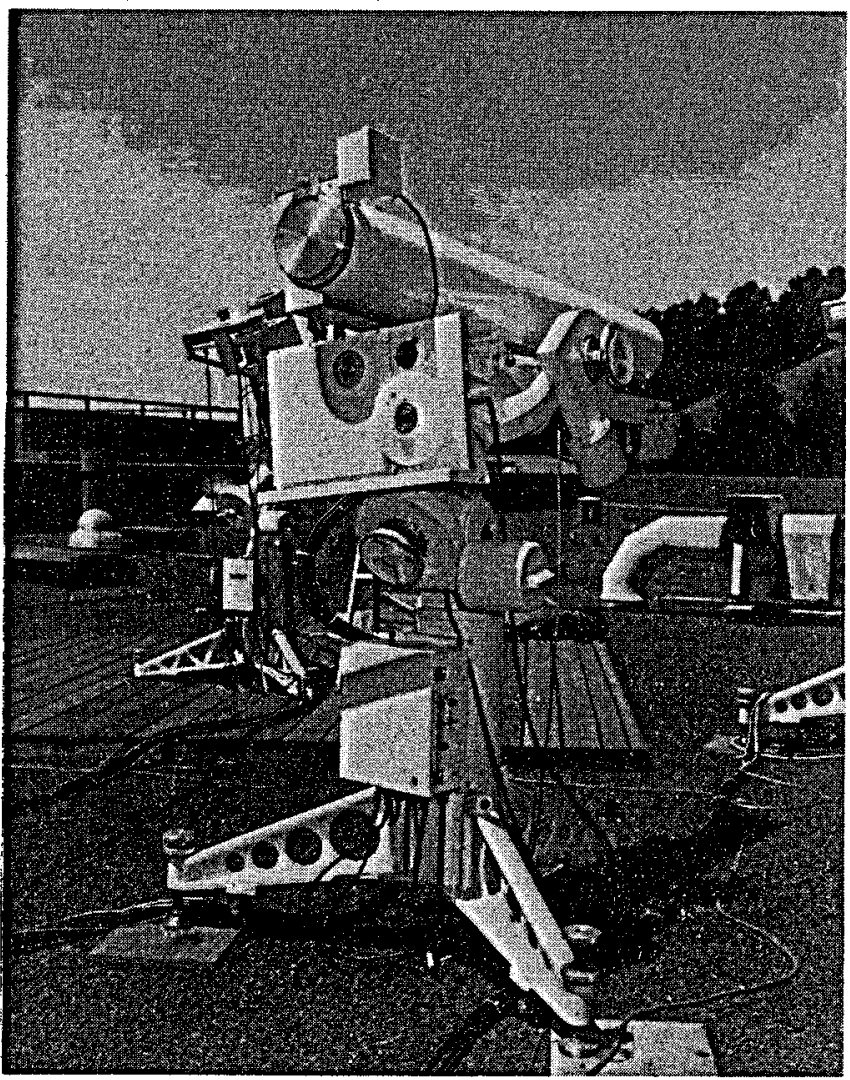

Fig. 1.

(XBB 764-3819)

the platform. The telescope and associated equipment are connected by cables to an electronic cabinet (not shown) that controls the instrument and records the output of the telescope, pyrheliometer, and pyranometers on magnetic tape.

Figure 2 shows computer generated graphs of two sample scans for the clear filter. Consider the upper graph. The dots are the individual scan digitizations. The scan started at $+3^{\circ}$, crossed the sun near $\theta=0$, and ended at $-3^{\circ}$. (The discontinuities at roughly one solar radius on either side of the sun are artifacts introduced by the change in aperture size). The computer code proceeds as follows. A search is made for the peak point of the sun. The two "edges" of the sun are then empirically defined as the angles at which the brightness is down by 1.5 orders of magnitude from the peak. These edges are indicated on the graph by the two vertical, dotted lines. The center of the sun is then taken as the angle midway between the two edges, and is indicated by the solid vertical line. An effective radius is then defined as the true radius of the sun ( $\left(16^{\prime}\right.$ of $\operatorname{arc}$ ) plus the radius of the detector aperture ( 0.8 of arc). Effective radii are indicated by the solid vertical lines that are at the top and bottom of the graph and that are nearly coincident with the edges of the sun. The brightness is then integrated from the center of the sun to the effective radius to give the intensity of the solar radiation, and integrated from the effective radius to the end of the scan to give the intensity of the circumsolar radiation. The ratio of circumsolar to solar radiation is then calculated and is given 

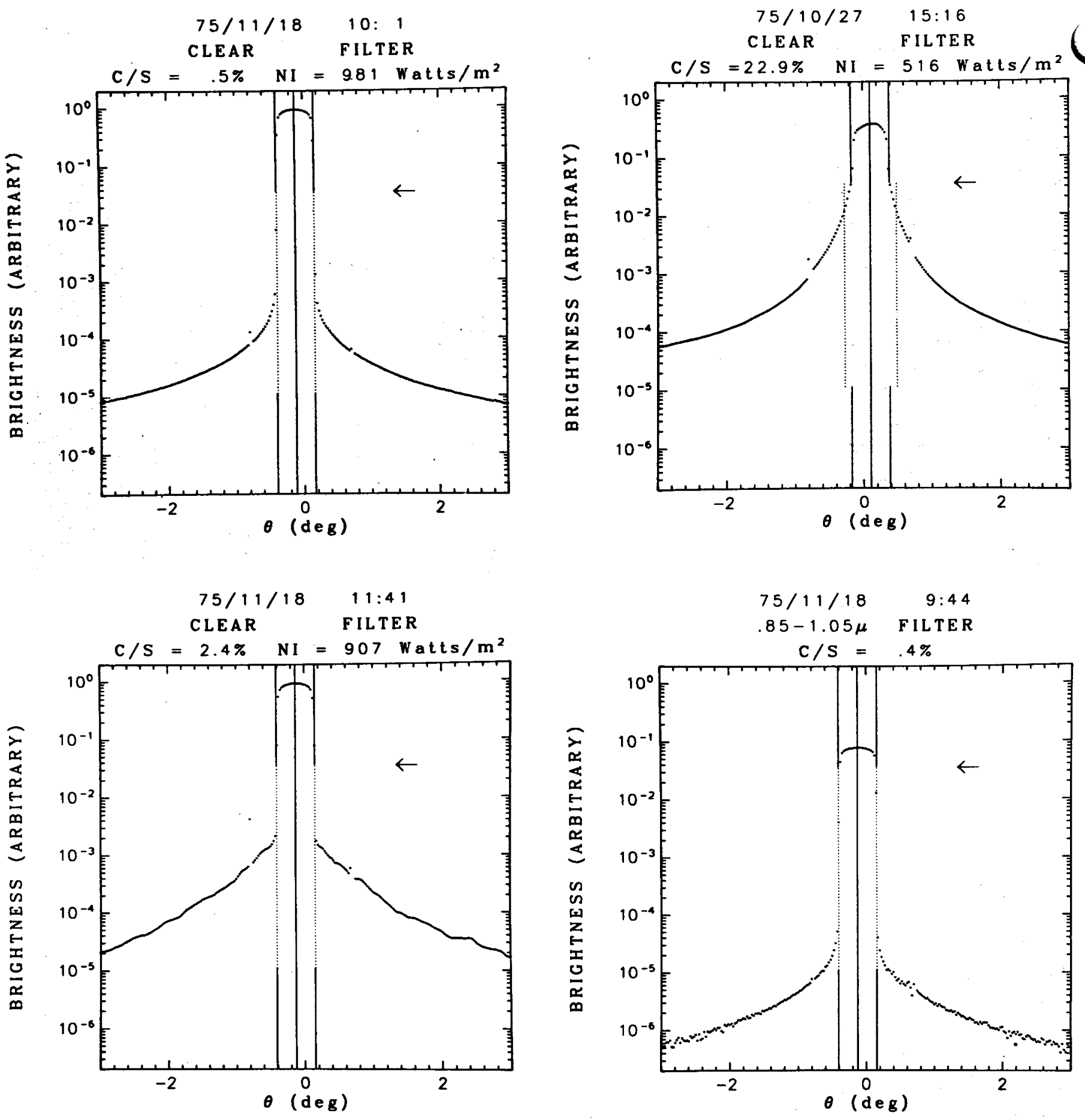

Fig. 2.

(XBL 762-570)

Fig. 3.

(XBL 762-569)

at the top of the graph $(\mathrm{C} / \mathrm{S}=)$. Also indicated is the normal incidence measurement provided by the pyrheliometer $(\mathrm{NI}=)$ ). This particular scan is typical of a clear Berkeley winter day, with a circumsolar to solar ratio of only . 58 and a normal incidence value of 981 watts/square meter.

The bottom graph of Fig. 2 and the top graph of Fig. 3 are samples of scans with higher levels of circumsolar radiation. The latter scan is probably an extreme case, but does show that the circumsolar radiation can be at a high level when

the normal incident radiation is still within the anticipated operating range of a solar thermal power plant. The bottom graph of Fig. 3 and the top graph of Fig. 4 show sample scans for two of the optical filters. The filter bandwidths are given in the graphs. The optical filters lower the overall intensity by as much as a factor of 20 , and for low levels of circumsolar radiation (as in the bottom graph of Fig. 3) the random component of the detector and electronics noise introduces noticeable fluctuations in the scan digitizations. This noise is, however, sufficiently 


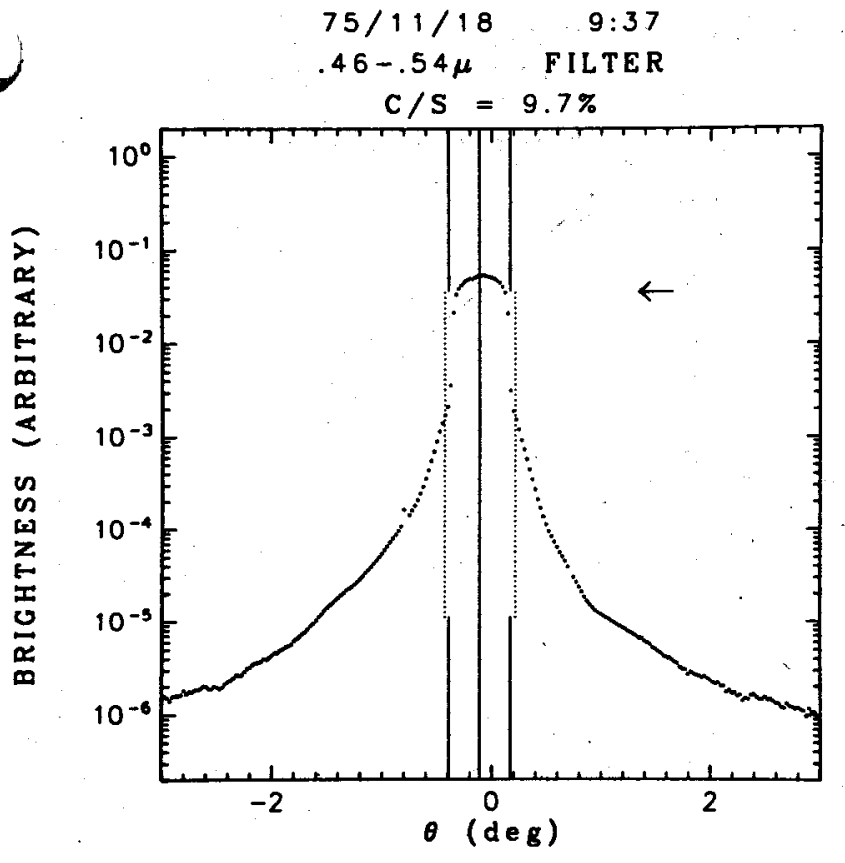

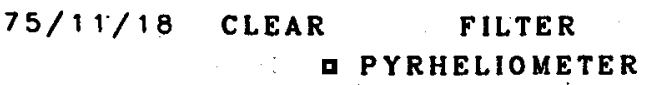

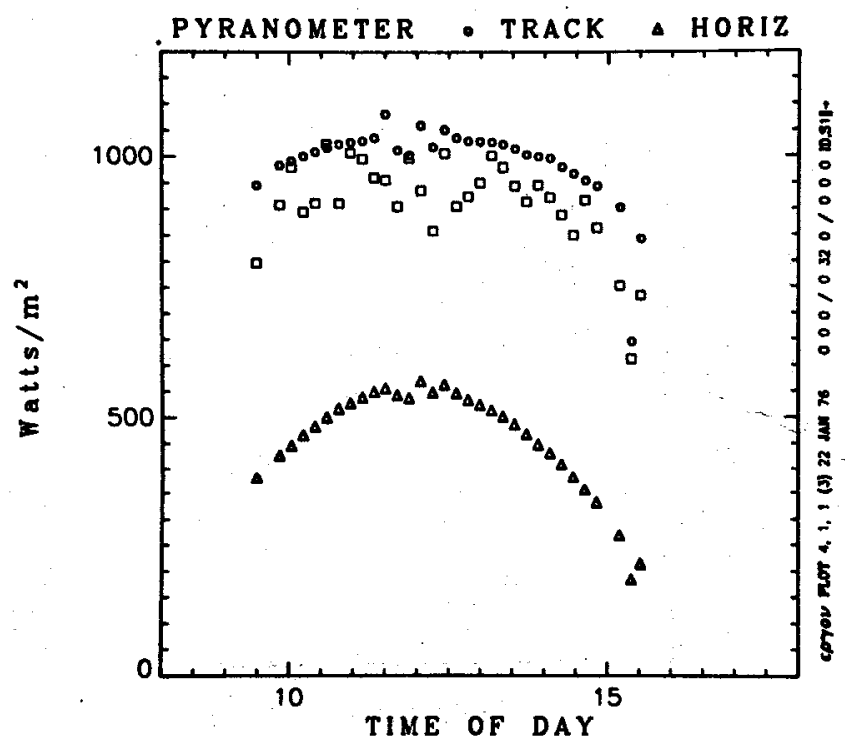

Fig. 4.

(XBL 762-568).

small so as not to significantly affect the quantities of interest, such as the ratio of circumsolar to solar radiation.

The bottom graph of Fig. 4 displays, plotted as a function of time of day, the output of the pyrheliometer (squares), the sun-tracking pyranometer (circles) and the horizontal pyranoneter (triangles). These data are for a day of quite high solar radiation levels, but with fluctuations around 1200 hours resulting from some atmospheric disturbance. The graphs in Fig. 5 show the ratio of circumsolar to solar radiation for the same day, for the clear filter and a selected optical

\section{$75 / 11 / 18$ CLEAR - FILTER}

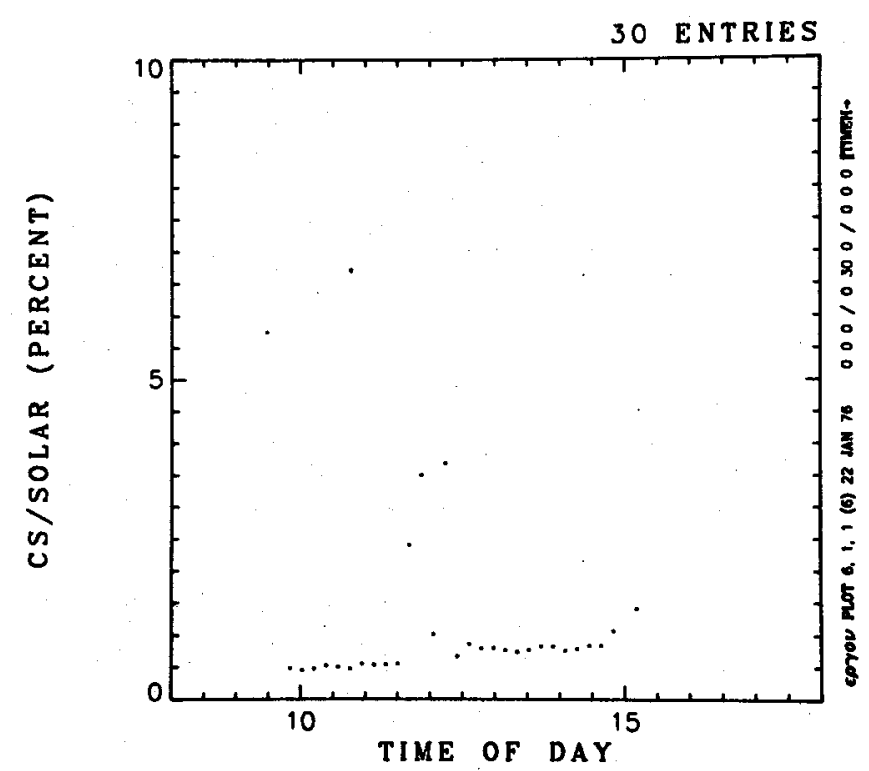

$75 / 11 / 18 \quad .62-.72 \mu \quad$ FILTER

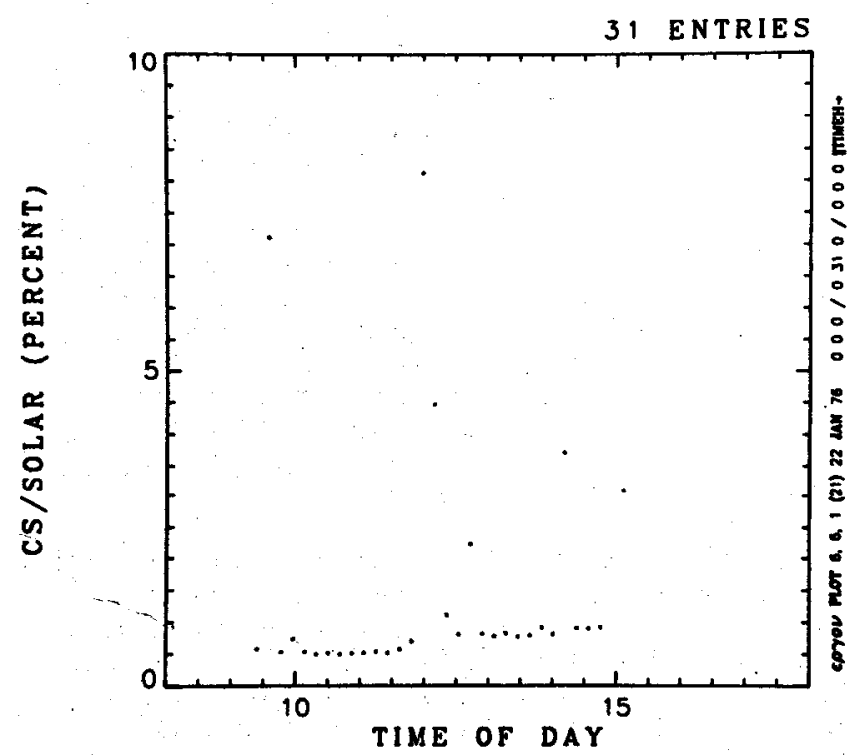

Fig. 5.

(XBL 762-567)

filter. While this ratio is quite small for most of the day, it rises to as much as $5 \%$ for the clear filter, and 7-88 for the optical filter, during the time of the atmospheric disturbance.

One of the checks of the quality of the data concerns the linearity of the output of the detector and electronics as a function of the input light leve1. To investigate the linearity, we have made measurements with the telescope pointed at the center of the sun, and the incident light level systematically reduced by placing a series of neutral density filters over the front of the telescope. The density of each filter as a func- 
tion of wavelength was measured on a Cary 17. Using these measurements, and taking into account the nominal shape of the solar spectrum, an effective density was calculated for each neutral density filter, for each optical filter passband. The output of the telescope versus the effective density is shown in Fig. 6 for the clear filter and for a selected optical filter. If the system were exactly linear, then all of the points would lie on the straight lines. The agreement with the data is considered satisfactory.

\section{FUTURE WORK}

The main thrust of the work is to finish the de-bugging and to deploy the remaining three instruments. Necessary operational improvements are being incorporated into these telescopes as they are being tested. All the telescopes are completed mechanically, and satisfactorily carry out the sun-tracking operation. The final operational checkout is underway and is accomplished by running each telescope side-by-side with the prototype telescope until all bugs in the new telescope have been corrected. This final stage is being carried out sequentially for the three telescopes, and each telescope will be deployed as it completes its test period.

The instruments will be initially placed at three sites; one is near the 5 megawatt (thermal) solar test facility at Albuquerque, N.M.; a second is at the site of a total energy system pilot plant at Ft. Hood, Texas. The third, located at China Lake, Ca., will be relocated at the site of a 10 megawatt (electric) Central Receiver pilot plant. These telescopes are capable of unattended operation for up to a week, although they typically receive a daily inspection during the work week. The instruments will withstand most weather conditions. During the night the solar trackers rum backwards and automatically initiate operation at the beginning of each day. The data are recorded on magnetic tape and processed weekly at the LBL computer center.

As the data base grows, it will become possible to identify "typical" days for each site as a function of the season. Such a day would consist of a number of solar profiles from dawn to dusk. These data can be used as input to power tower simulation programs and will provide a valid statistical basis for predicting solar power plant performance. In addition to the circumsolar data, the solar profiles contain enough detail to give quantitative information on the limb darkening of the sum, a parameter that may be important in some power tower applications.

Further work will concentrate on trying to match certain types of solar profiles and circumsolar/solar ratios with the output of a simpler instrument such as a sunphotometer, a turbidity measuring device, or a sky coronameter ${ }^{2}$ that compares off axis light with direct sunlight. If such correlations turn out to be meaningful, the circumsolar measurements could be expanded to additional sites, using the simpler instruments.
DATA FOR $17 / 12 / 75$

CLEAR

FILTER

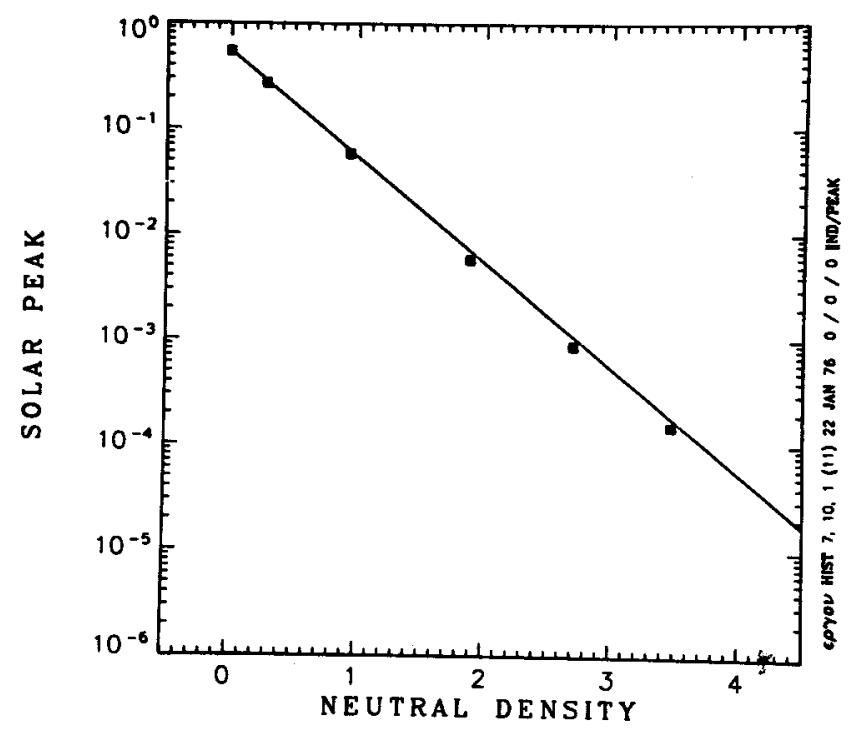

DATA FOR $17 / 12 / 75$

$.62-.72 \mu \quad$ FILTER

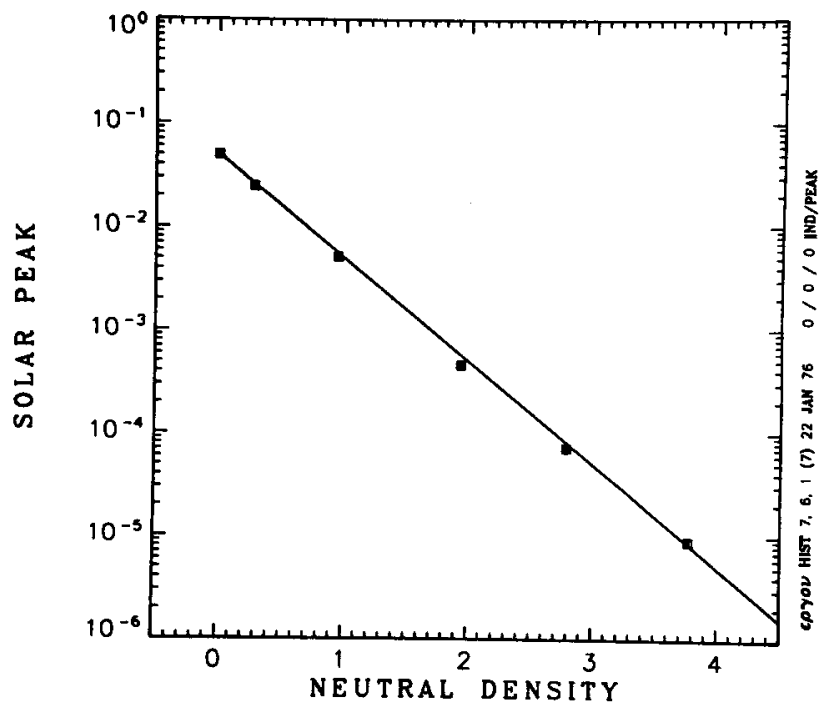

Fig. 6 .

(XBL 762-566)

All the telescopes will be furnished with automatic weather equipment to measure wind direction and velocity, relative humidity and barometric pressure. These data will be recorded on magnetic tape along with the circumsolar measurements. This has a lower priority than the development of the telescopes and will be added later in the year.

\section{REFERENCES}

1. R.C. Willson, Applied Optics 12810 (1973).

2. G.E. Shaw and C.S. Deehr, J. of Applied Meteorology 141203 (1975). 


\section{INTRODUCTION}

The objective of this research is to accelerate the development of economical photovoltaic solar energy conversion. The problem areas investigated are those where the application of recently developed surface science techniques either promise improvement in the production of the raw material (silicon) and the cell (thin polycrystalline films with large grains) or allows determination of the phenomena that determine lifetime and efficiency of polycrystalline solar cells (impurity segregation, effect of surface structure and impurities on electronic states).

THE EFFECT OF SURFACE STRUCTURES AND IMPURITIES ON ELECTRONIC SURFACE STATES

Electron loss spectroscopy (ELS) was used to determine the electronic surface states of the $\mathrm{Si}(111)$ surface as a function of surface order and surface impurities. Surface order was determined by low energy electron diffraction (LEED) and varied by temperature treatment or argon ion bombardment. Impurities deposited onto the surface under UHV conditions were acetylene, oxygen and aluminum.

Acetylene and oxygen were adsorbed on both the disordered and ordered silicon surfaces of (111) orientation. The ELS data suggest that electrons in the silicon-oxygen chemisorption bond are more tightly bound on the disordered than on the silicon (111) surface with a (7x7) surface structure.

The adsorption of acetylene produces two electron loss peaks $(7.0$ and $8.4 \mathrm{eV}$, respectively) on the disordered silicon surface. In the Si(1ii)(7x7) surface acetylene adsorption gives rise to only one strong electron loss peak at $7.3 \mathrm{eV}$ and a weak shoulder at $8.4 \mathrm{eV}$. In addition the dangling bond state transition of $\mathrm{Si}$ is completely removed on the disordered surface by the adsorption of .25 monolayer of $\mathrm{C}_{2} \mathrm{H}_{2}$, while it is still present on the Si(111)- (7x7) surface even after saturation. These results indicate that the adsorption of acetylene on the Si(111)-(7x7) surface takes place on preferred sites, arising mainly as a result of the overlap between the silicon surface dangling bond orbitals and the $\pi$ orbitals of the $\mathrm{C}_{2} \mathrm{H}_{2}$ molecules. They support the conclusion that on the (111) - (7x7) surface the acetylene molecule predominantly sits between two silicon atoms in a bridge position (Fig. 1b). On the disordered surface, however, most of the adsorbed acetylene molecules are 10cated on top of the silicon surface atoms (Fig. 1a).

The adsorption of aluminum on the (111) silicon surface introduces changes in the ELS spectrum which show that the interaction of $\mathrm{Al}$ with $\mathrm{Si}$ is strongly dependent on the Si surface temperature. At temperatures below $300^{\circ} \mathrm{C}$ and a coverage of about 0.5 monolayer the absorbed A1 is uniformly distributed over the surface, and the ELS and LEED data show that a Al-Si surface phase ( $\alpha-S i(111)-7-A 1)$

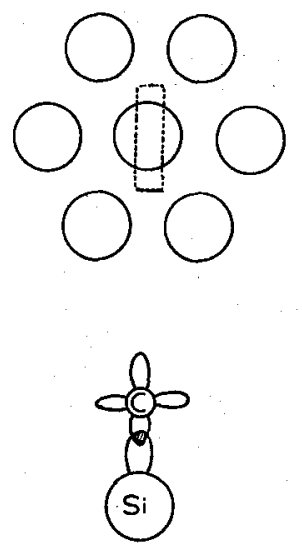

(A)

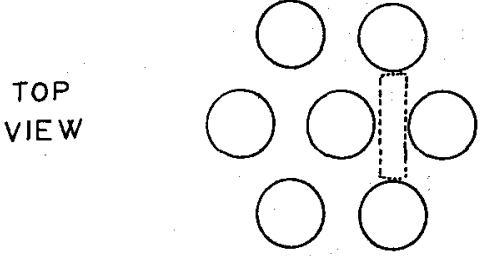

SIDE

VIEW

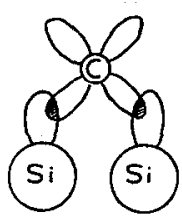

(B)
Fig. 1. The positions of adsorbed acetylene molecules on (a) a disordered and (b) an ordered Si surface. (XBL 7512-10067)

is formed. At temperatures above $600^{\circ} \mathrm{C}$ and a coverage between 0.5 and 1 monolayer a different Al-Si surface phase is formed $(\gamma-S i(111)-7-A 1)$, as shown by the observed changes in the ELS spectrum and the LEED pattern. Further, it is observed that under this condition Al coalesces on the Si surface as evidenced by the appearance of strong Al bulk and surface plasmons. The results show that the ELS technique can be used to study the initial stages in the formation of a metal-semiconductor contact which is of utmost importance in Schottkybarrier type devices.

Electron loss spectroscopy does not generally determine the absolute location of or the electron population in electronic states. We therefore want to complement it with UV photoelectron spectroscopy and have designed and are building a high intensity UV light source, differentially pumped, to retain UHV conditions at the target during electron loss as well as photoelectron spectroscopy.

\section{IMPURITY SEGREGATION}

During the past year we have examined polycrystalline silicon fracture characteristics and Auger analyzed some of these surfaces after fracture in ultra high vacum to determine impurity segregation onto grain boundaries. 'To facilitate better examination of the samples after fracture we have built a new pumping station and installed a Scanning Auger Microprobe with secondary electron detection capability.

our intent in examining polycrystalline silicon fracture surfaces was to determine if impurities do in fact segregate to grain boundaries as suggested by some authors. 1 If the experiment is to succeed the silicon must fracture along the grain boundary. We therefore tried fracturing polycrystalline sili- 
con in the following four ways: 1) shear fracture at room temperature, 2) shear fracture at liquid nitrogen temperature, 3) thermal stress, and 4) rapid bending at elevated temperature. The resulting surfaces were examined with a scanning electron microscope. Micrographs indicated that all surfaces appeared to give predominately transgranular fracture. Point Auger analysis of such surfaces did not indicate the presence of inpurities even when the doping level was a high as $10^{19}$.

It is possible however that some small percentage of the fracture surface is intergranular and that only by good fortume would a point by point analysis land on such an intergranular surface. We therefore built a new pumping station and installed a Scanning Auger Microprobe with secondary electron detection capability. This instrument gives a physical image as well as an image of the impurity distribution of the fractured surface. We now have a spatial resolution capability of 1 micron and an analytical capability of 1 part in 1000. This will enable us to look in situ at fracture surfaces, pick out likely looking fracture regions, and Auger analyze them to look for evidence of segregation. We will be pursuing this course of research in the future.

In addition to the above approach, we will, in the next year, add two additional efforts. For the first, we have prepared one series of arsenic and one series of boron doped silicon single crystal samples. Boron and arsenic represent opposite segregation tendencies according to theories of segregation based on heats of sublimation.2 The doped silicon wafers will be cleaned in ultra high vacunm and annealed to establish an equilibrium surface concentration which will be Auger analyzed. This will permit a check on the segregation theory. Electron loss spectra will also be employed to study the surface states resulting from impurity segregation and to determine surface crystallinity.

Our second approach will involve diffusing materials such as nickel into polycrystalline silicon. Nickel rapidly diffuses in silicon especially along grain boundaries, and it has low solubility so it will tend to remain in the grain boundary region. The presence of nickel may weaken the grain boundary so a larger percentage of intergranular fracture will occur. These surfaces can be Auger analyzed using the nickel as an indicator of an intergranular region.

\section{CATALYSIS OF CHEMICAL VAPOR DEPOSITION (CVD)}

Both the deposition rate and the activation energy of Si-CVD processes are strongly affected by impurities present at the silicon surface. In general, a p-type dopant impurity increases the deposition rate. Since both $\mathrm{SiH}_{4}$ and $\mathrm{SiCl}_{4}$ are
Table 1. Predicted and observed dopant gas effects on the deposition rates of silicon and carbon chemical vapors ${ }^{a}$

\begin{tabular}{lcccc}
\hline & $\mathrm{SiH}_{4}$ & $\mathrm{SiCl}_{4}$ & $\mathrm{CH}_{4}$ & $\mathrm{CCl}_{4}$ \\
\hline $\begin{array}{l}\text { Bonding } \\
\text { Characters }\end{array}$ & $\mathrm{Si}^{+}-\mathrm{H}^{-}$ & $\mathrm{Si}^{+}-\mathrm{Cl}^{-}$ & $\mathrm{C}^{-}-\mathrm{H}^{+}$ & $\mathrm{C}^{+}-\mathrm{Cl}^{-}$ \\
$\mathrm{B}_{2} \mathrm{H}_{6}$ & $\mathrm{I}$ & $\mathrm{I}$ & $\mathrm{D}$ & $\mathrm{D}$ \\
& $\mathrm{Ia}$ & $\mathrm{I}^{\mathrm{b}}$ & $\mathrm{D}$ & \\
$\mathrm{PH}_{3}$ & $\mathrm{D}$ & $\mathrm{D}$ & $\mathrm{I}$ & $\mathrm{I}$ \\
$\mathrm{AsH}_{3}$ & $\mathrm{D}$ & & & \\
& $\mathrm{D}$ & $\mathrm{D}$ & $\mathrm{I}$ & $\mathrm{D}$ \\
\hline
\end{tabular}

${ }^{\mathrm{a}} \mathrm{I}$ and $\mathrm{D}$ indicate an increase and decrease, respectively, in deposition rate. The first row for each dopant gas is for the predicted effects, the second row for the observed effects.

${ }^{b_{F}}$.A. Cotton and G. Wilkinson, Advanced Inorganic Chemistry, Second Ed., John Wiley and Sons, New York, (1966), p. 466.

tetrahedral molecules carrying a slight negative excess charge on the hydrogen atoms, it is tempting to propose that the p-dopant forms a charge-layer at the surface which attracts such molecules whereas n-dopants form a repulsive layer. Table 1 shows that a prediction of this proposed model, i.e., the reduction of the deposition rate of diamond from $\mathrm{CH}_{4}$, a molecule with a slight positive excess charge on the hydrogen in the presence of $\mathrm{B}_{2} \mathrm{H}_{6}$, is verified by the literature cited.

To test the predicted influence of surface charges induced by electric fields on the deposition rate in silicon CVD processes, a CVD reactor has been modified such that external electric fields of variable strength and polarity can be applied during deposition.

\section{REFERENCES}

1. M.E. Cowher and T.0. Sedwick, J. Electrochem. Soc., Solid State Science and Technology, 11, 1565-1659 (1972).

2. Overbury, Bertrand, Somorjai, 'The Surface Composition of Binary Systems, Predictions of Surface Phase Diagram of Solid Solutions", LBL-2746 (1974). 
PHOTOPOTENTIAL GENERATION CATALYZED BY BACTERIORHODOPSIN

Lester Packer, Paul Shieh, Tetsuya Konishi and Stephanie Tristram

Among the various ways that biological systems can be utilized for solar energy purposes, one particular approach appears attractive: to devise artificial systems patterned after biological energy transduction. Accordingly, we have undertaken to study ways to stabilize planar membranes containing pigments which can act as sensitizers in order to generate photo-induced electrical potentials.

Lipid membranes that contain chlorophyl1 are photosensitive. 1 If a photosensitive membrane separates solutions of different redox potential upon illumination it can cause charge movement, thus producing a photopotential. It is believed that such redox reactions occur at each membrane solution interface. We have undertaken to incorporate photosensitive catalysts into planar membranes which separate a two-compartment photovo1taic cell. Our aim is to develop an in vitro system capable of converting solar into electrical energy. and/or develop a biochemical fuel for photolysis of water into $\mathrm{H}_{2}$ and $\mathrm{O}_{2}$.

Our studies this year concerned incorporation of bacteriorhodopsin (BR), a photosensitive catalyst, into a planar membrane, and its stabilization and orientation. This was followed by an investigation of the stability, electrical characteristics, and photovoltage generation in polymer stabilized planar membranes in the presence of BR.

The source of the photocatalyst is Halobacterium. Transferring these bacteria to ordinary water causes them to burst, releasing a "purple membrane", which contains BR in an aggregated state. ${ }^{2}$ The purple membrane preparation possesses a light driven $\mathrm{H}^{+}$ pump activity and a stable structure, composed of a two dimensional array of hexagonal units which aggregate into the patches that are rigid and stable, even if dried in vacuo. 3 Thus it seems ideally suited for use in a biosolar energy conversion system amenable to the approaches of enzyme technology, i.e., ordering and stabilizing the pigment containing patches on a surface or in the plane of an artificial membrane.

The stability of BR in purple membrane fragments has been examined with regard to thermal, oxidative, and light induced damage. Heat treatment for 80 minutes at $80^{\circ} \mathrm{C}$ caused the loss of $36 \%$ of its initial light induced $\mathrm{H}^{+}$activity (when the treated BR was incorporated into egg lecithin 1iposomes). The peak height of the chromophore at 570 $\mathrm{nm}$, which represents the Schiff base of the pigment2 between retinaldehyde and the protein of BR decreased to $34 \%$ of its original absorbance, i.e., "bleaching" was parallel. Treatment of $\mathrm{BR}$ at $50^{\circ} \mathrm{C}$ for 80 minutes resulted in no bleaching and $\mathrm{H}^{+}$ activity in liposomes was only slightly lowered.
The correlation between bleaching and stabilization of protein structure is being studied using bifunctional crosslinking reagents, like glutaraldehyde, which protect against bleaching of BR when it is kept at $80^{\circ} \mathrm{C}$ in the light, protecting illuminated BR against temperature stress. We have also found BR unable to catalyze a light and oxygen dependent peroxidative destruction of lipids when it is incorporated in liposomes. Apparently its organization in the "patch" prevents it. 4

We are seeking simple ways to order the photosensitive component at a surface or in the plane of a membrane, in order to obtain efficient energy transfer and to prevent back reactions which can dissipate the energy and have been experimenting with vesicular membranes, liposomes formed of known lipids, cell envelope vesicles from Halobacterium, and with artificial planar membranes (see below). We have utilized the rate and extent of light induced $\mathrm{H}^{+}$ion uptake (BR in reconstituted 1iposomes) ${ }^{5}$ or extrusion (BR in cell vesicles) as a means to assess the orientation of $\mathrm{BR}$ in the membrane.

Alkaline earth metal bivalent cations stimulated light induced $\mathrm{H}^{+}$activity especially by $\mathrm{Ca}^{2+}$ $\mathrm{Co}^{2+}>\mathrm{Mn}^{2+}$ and $\mathrm{Ni}^{2+}$ in $\mathrm{BR}$ liposomes formed by sonication in their presence, probably resulting from effects on the membrane surface causing a more uniform asymmetric organization of $B R$ in the membrane plane. A dose response curve correlating the stimulative effect of $\mathrm{Ca}^{2+}$ on $\mathrm{H}^{+}$pump activity showed that $\mathrm{Ca}^{2+}$ concentrations (between 50-100 mM) were required for maximal stimulation (Fig. 1). $\mathrm{H}^{+}$pump activity in $\mathrm{H}$. Halobium envelope vesicles, where the direction of light induced $\mathrm{H}^{+}$activity is opposite, 6 i.e., extrusion, is inhibited by $\mathrm{Ca}^{2+}$.

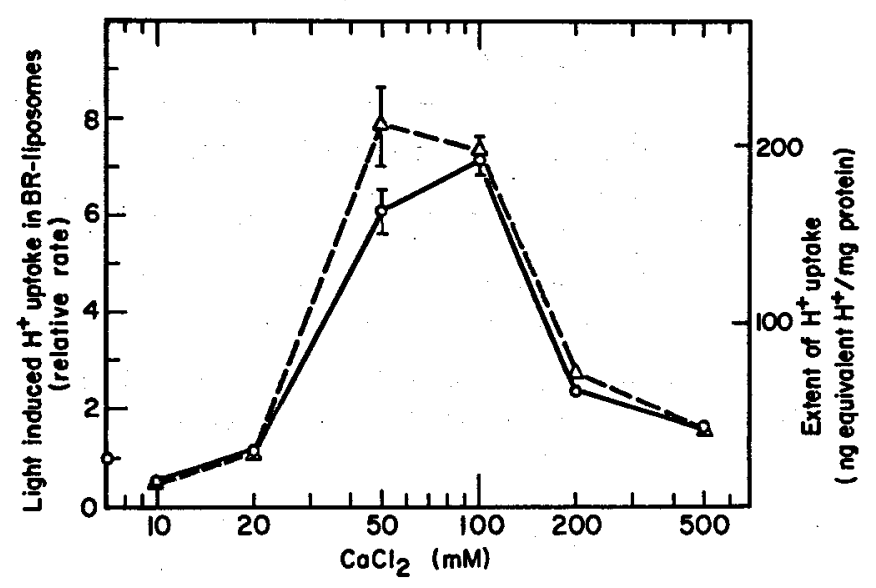

Fig. 1. Effect of $\mathrm{Ca}^{2+}$ on light induced $\mathrm{H}^{+}$activity in BR 1iposomes. 
STABILITY, ELECTRICAL CHARACTERISTICS, AND PHOTOVOLTAGE GENERATION IN POLYMER STABILIZED PLANAR MEMBRANES IN THE PRESENCE OF BACTERIORHODOPSIN

A planar membrane was formed by painting a solution of $0.2 \mathrm{mg}$ oxidized cholesterol $/ \mathrm{ml}$ octane over an orifice of about $2 \mathrm{~mm}$ diameter in the wall of a small teflon cup, located inside a larger cup to form two aqueous compartments; electrical contact was established by immersing a pair of calomel electrodes into each aqueous compartment. 7 The membrane surface was illuminated with $80 \mathrm{~W} / \mathrm{cm}^{2}$ from a 350 watt tungsten filament source.

The electrical properties of planar membranes prepared with various concentrations of polystyrene are summarized in Fig. 2. As polystyrene concentration increases there is a fall in capacitance, rise in resistance, increase in calculated membrane thickness, and increased stability to membrane breakdown by applied voltage. Increasing membrane thickness with higher polystyrene concentration causes the electric field strength (which is a function of breakdown voltage divided by thickness) to pass through a maximum at about $100 \mathrm{mg} / \mathrm{ml}$. Membranes prepared with $100 \mathrm{mg} / \mathrm{ml}$ polystyrene when maintained in the dark, are stable for at least 4-5 hours (Table 1). It was impossible to sustain photovoltage generation by $\mathrm{BR}$ in a membrane that was not stabilized with polymers. Similar results were obtained for a planar membrane made with 47 $\mathrm{mg} / \mathrm{ml}$ polyacrylamide in the membrane forming solution.
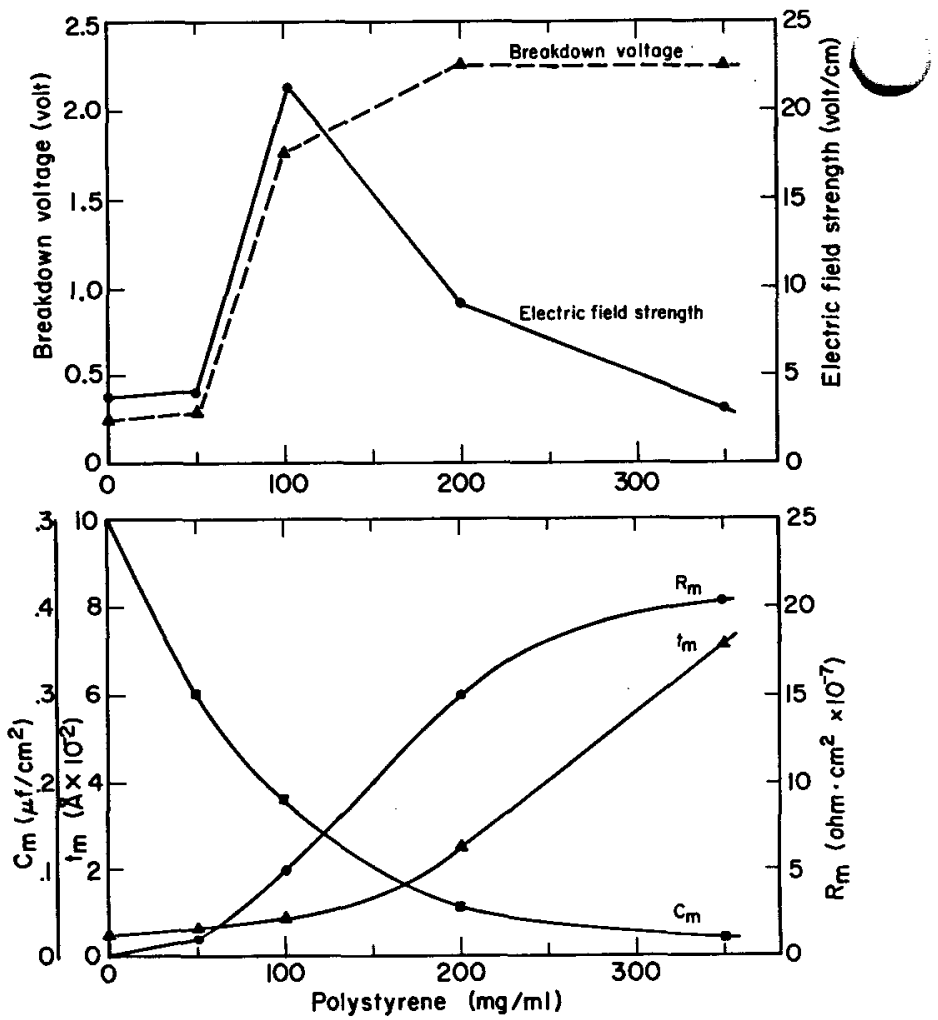

Fig. 2. Effect of polystyrene on the electrical properties of planar membranes.

(XBL 764-5401)

Table 1. Effect of Bacteriorhodopsin And Polystyrene On The Stability of A Black Lipid Planar Membrane.

\begin{tabular}{|c|c|c|c|c|}
\hline \multirow[b]{2}{*}{ Planar membrane } & \multirow{2}{*}{$\begin{array}{l}\text { Membrane } \\
\text { resistance } \\
\left(\mathrm{ohm} / \mathrm{cm}^{2} \text { ) }\right.\end{array}$} & \multicolumn{2}{|c|}{ Lifetime } & \multirow{2}{*}{$\begin{array}{c}\text { Breakdown } \\
\text { voltage } \\
\text { (applied volts }\end{array}$} \\
\hline & & $\begin{array}{l}\text { Dark } \\
\text { (hours) }\end{array}$ & $\begin{array}{l}\text { I1luminated } \\
\text { (hours) }\end{array}$ & \\
\hline Contro1 $^{\mathrm{a}}$ & $5-8 \times 10^{6}$ & 0.50 & $0.16-0.31$ & $0.20-0.25$ \\
\hline $\mathrm{BR}^{\mathrm{b}}$ & $5-6 \times 10^{5}$ & 0.16 & $0.10-0.20$ & $0.15-0.20$ \\
\hline Polystyrene ${ }^{c}$ & $5-6 \times 10^{7}$ & $3-5$ & $0.31-0.80$ & $1.50-2.10$ \\
\hline $\mathrm{BR}+$ polystyrene & $2-4 \times 10^{6}$ & $3-5$ & $0.30-0.80$ & $1.30-1.80$ \\
\hline
\end{tabular}

$\mathrm{a}_{0.2} \mathrm{mg}$ oxidized cholestrol/ml octane.

$\mathrm{b}_{5} \mathrm{mg}$ BR (as purple membrane fragments)/ml added to a.

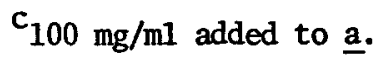

Photo-induced potentials were recorded (Fig. 3) with $\mathrm{BR}$ incorporated within the planar membrane. Illumination results in a potential change of about $30 \mathrm{mV}$ which reaches saturation in about 3 seconds; when the light is switched off, the potential decays to its dark level in 4 seconds. In the presence of $\mathrm{Ca}^{2+}$ the photopotential is about twice as large.

$\mathrm{Ca}^{2+}$ ions are known to reduce the electrostatic repulsion of charged phospholipid head groups and to promote vesicle fusion. Skulachev showed that $\mathrm{Ca}^{2+}$ permitted BR containing liposomes to interact with a black lipid planar membrane (e.g., smaller than $200 \AA$ ) and photopotential development.8

With $B R$ in the planar membrane and in liposome in $1 \mathrm{mM} \mathrm{CaCl} 2$ in the outer compartment, illumination causes a $150 \mathrm{mV}$ photopotential. The rise in potential peaks at $150 \mathrm{msec}$, then decays to a steady-state level at $110 \mathrm{mV}$; the signal decays to its original dark level when light is removed. In this case, 

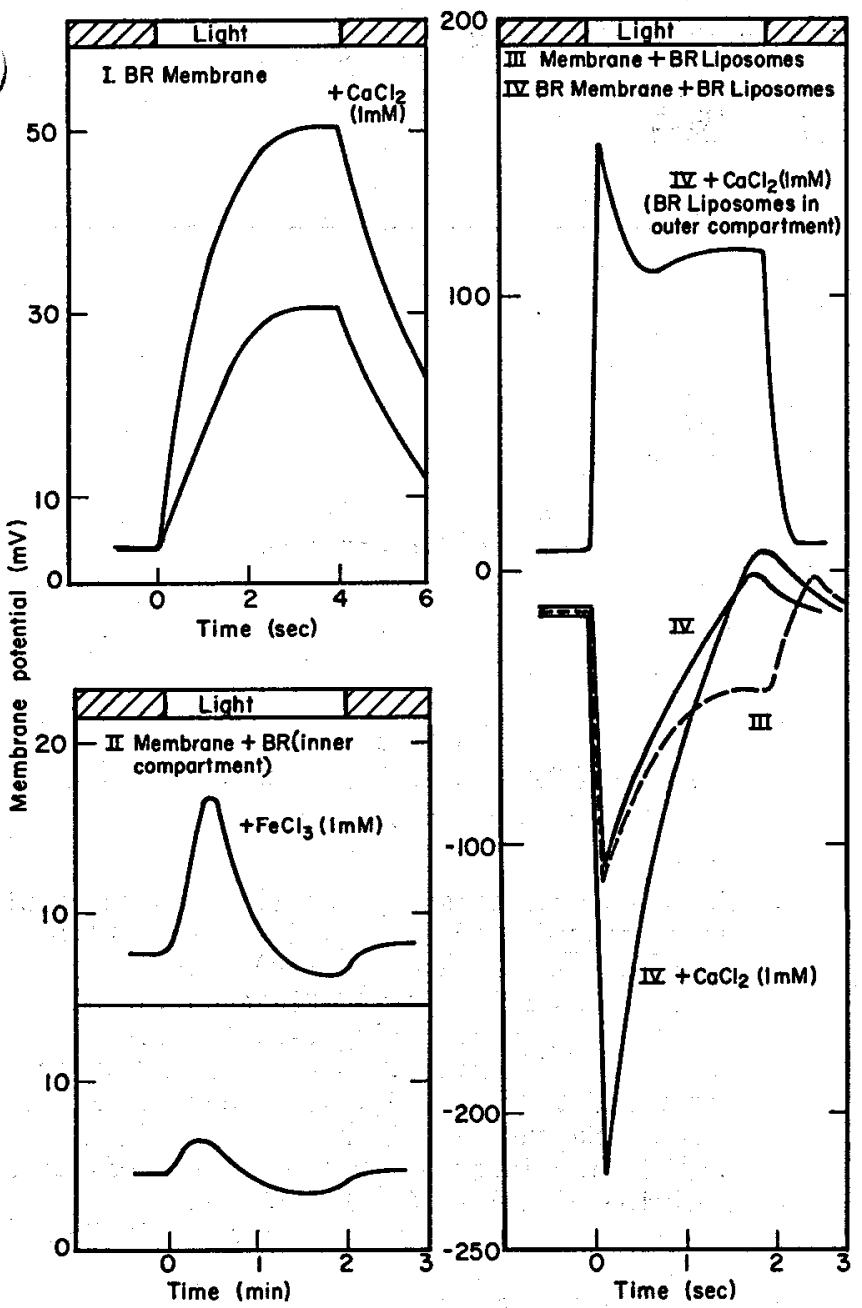

Fig. 3. Time course of a photoresponse of a polymer stabilized planar membrane containing rhodopsin.

(XBL 764-5400)

the photoresponse was dependent upon the time of dark incubation, with the maximm photoresponse occurring after 60 minutes of dark preincubation.

Some experiments were performed to determine the effect of applied fields on photopotentials. A linear increasing relationship between the photopotential and the applied field was found when purple membrane fragments were located in one aqueous compartment and absent in the planar membrane (Fig. 4). A maximm photoresponse of $500 \mathrm{mV}$ was obtained when the applied field was up to 1.5 - 2 volt. At zero applied field a photopotential of $25 \mathrm{mV}$ was observed.

Since no photopotential occurred in any of our studies in the absence of purple membrane fragments and the photovoltaic action spectrum of polymer incorporated membrane closely coincided with the adsorption spectrum of the purple membrane fragments, the implication is that $B R$ is catalyzing charge transfer across the planar membrane.

Summarizing, this year we have shown that bacteriorhodopsin is a good candidate for the development of a biological solar cell. We have further shown that black lipid planar membranes prepared

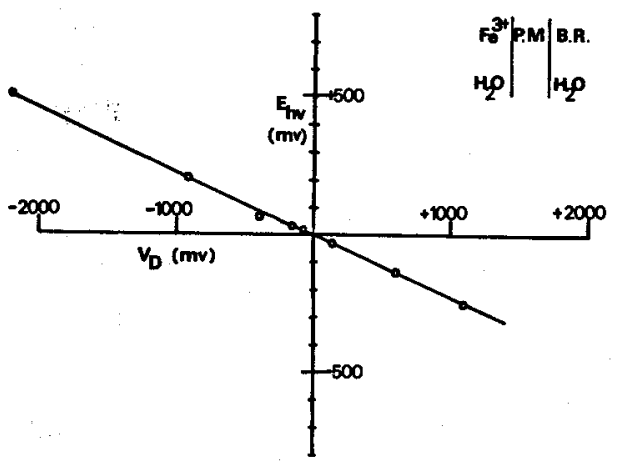

Fig. 4. Relation between the photoresponse of the polymer incorporated planar lipid membrane and applied electrical field.

(XBL 762-550)

in the presence of polymers show great stability to applied electric fields and have long useful lifetimes, even in the presence of light, extending their applicability in biological research and for development of a useful photovoltaic device. 9,10

\section{ACKNOWLEDGEMENTS}

We thank Janos Llanyi of NASA, Ames Research Center, for providing us with purple membrane preparations and envelope vesicles and for discussion of this research.

\section{REFERENCES}

1. H.T. Tien, J. Phys. Chem., 72, 4512 (1968).

2. D. Osterhelt and W. Stoeckenius, Nature N.B. 233, 149-152 (1971).

3. A.E. Blaurock and W. Stoeckenius, Nature N.B. 233, 152-155 (1971).

4. K.W. Novikov, A.A. Shvedove, V. Ye. Kagan, Yu P. Kozolov, and M.A. Ostrovskii, Biofizika 19 , 280-284 (1974).

5. R. Lozier, R. Bogomolni, and W. Stoeckenius, Biophysical J. 15, 955-962 (1975).

6. R.E. MacDonald and J.K. L1anyi, Biochemistry 14, 2882 (1975).

7. P. Shieh and H.T. Tien, J. Bioenergetics $\underline{6}$, 45-55 (1974).

8. L.A. Drachev, A.A. Jasaitis, A.D. Kaulen, A.A. Kondrashin, E.A. Liberman, L.B. Nemeck, S S.A. Ostroumov, A. Semenov, A. Yu, and V.A. Skulachev, Nature 249, 321 (1974).

9. P. Shieh and L. Packer, Biophysical J. 16, 22a (1976).

10. L. Packer, T. Konishi and P. Shieh Abstract Bacteriorhodopsin-catalyzed Photopotential Generation across Planar Membranes, International Congress of Photobiology, Rome, Italy. August 29-September 3. (1976). 
BIOPHOTOLYSIS USING NITROGEN-STARVED CULTURES OF Anabaena cylindrica

Joseph C. Weissman and John R. Benemann

Biophotolysis, the light-driven biological decomposition of water into hydrogen and oxygen, has thus far been demonstrated only with Anabaena cylindrical. ${ }^{1}$ This filamentous nitrogen-fixing, blue-green algae contains two types of cells: the common vegetative cells and the infrequent (about one in twenty) heterocysts which are characterized by a larger size, heavier wall, and less pigmentation. In the vegetative cells, photosynthetic oxygen production and $\mathrm{CO}_{2}$ fixation take place; the heterocysts are the site of $\mathrm{N}_{2}$ fixation. 2,3 The oxygen-sensitive nitrogenase reaction is protected in the heterocysts against photosynthetic and ambient oxygen. Since the nitrogenase enzyme evolves $\mathrm{H}_{2}$ in the absence of molecular $\mathrm{N}_{2}$ (or other reducible substrates such as acetylene), it is possible to use these algae for biophotolysis by the simple expedient of incubating them under an inert gas such as argon.

The requirements for development of a technically feasible system of biophotolysis are 1) sustained hydrogen and oxygen evolution for many weeks, 2) a $2: 1$ ratio of hydrogen to oxygen, 3) high specific rates of hydrogen production and high photosynthetic efficiencies and 4) no limitations to scale-up of the system. Previously we demonstrated hydrogen production for about three hours with $2 \mathrm{mI}$ of cultures with a $\mathrm{H}_{2}$ to $\mathrm{O}_{2}$ ratio of 1:7. We have now shown that nitrogen-starved cultures, of up to 2 liter volume, will produce hydrogen and oxygen for at least two weeks $\mathrm{(H}_{2}: \mathrm{O}_{2}$ $1.7: 1-4.0: 1$ ) with a minimm photosynthetic efficiency of 0.48 .

Anabaena cylindrica cultures (Strain 629) were grown as previously described 2 on Allen and Arnon media 4 in 2-liter and $250 \mathrm{ml}$ bottles at $2 \times 10^{4}$ to $4 \times 10^{5} \mathrm{ergs} / \mathrm{cm}^{2} / \mathrm{sec}$. After appropriate cell densities were reached, argon (with $3 \% \mathrm{CO}_{2}$ ) was substituted for air (with $0.38 \mathrm{CO}_{2}$ ) in sparging the cultures. The cultures turned yellow, increased heterocyst frequencies two or three-fold (to 10 to 15\% of total cells), and exhibited increased nitrogenase activity. Hydrogen production was measured by sampling at the effluent tube from the cultures (using gas chromatography to measure the hydrogen). As shown in Fig. 1, a high peak in hydrogen production is reached after the first day of sparging with argon; hydrogen production achieves, thereafter, a lower, steadier rate, which is maintained through periodic additions of small amounts of ammonium chloride. If the photosynthetic oxygen inhibitor DOMU is added, hydrogen evolution rapidly falls to zero. Oxygen can also be measured in the effluent gas (see Table 1); the ratio of $\mathrm{H}_{2}: \mathrm{O}_{2}$ being 4:1 in completely nitrogen-starved cultures and 1.7:1 when small amounts of ammonium are added. The limiting factor of hydrogen production rates (e.g., the decline after the first day of high rates) are filament breakage (which was shown to affect heterocyst function ${ }^{2}$ and reducant supply to the heterocyst.

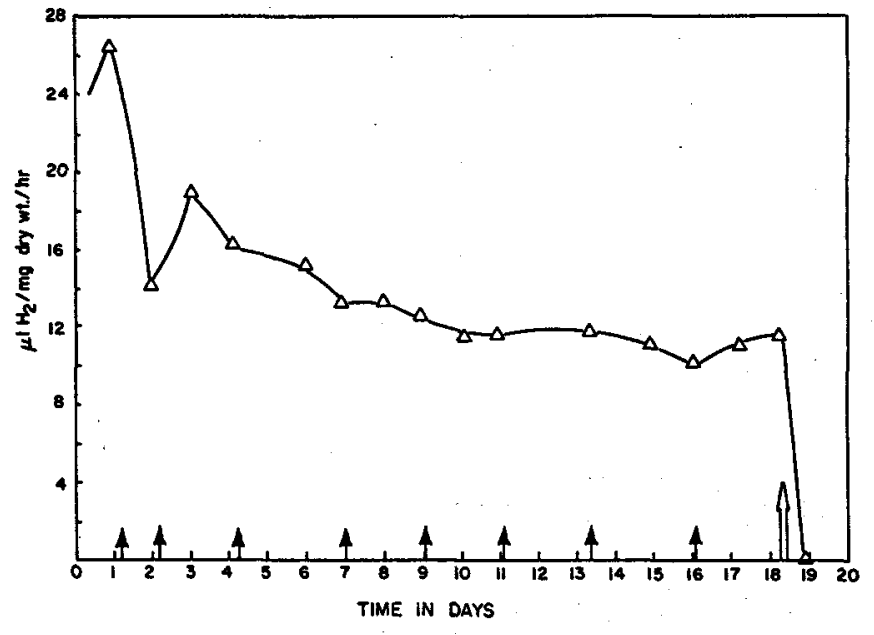

Fig. 1 Hydrogen production by two liter cultures of Anabaena cylindrica under low light intensity $\left(2.0 \times 10^{4} \mathrm{ergs} / \mathrm{cm}^{2} / \mathrm{sec}\right)$. Culture density was initially $60 \mathrm{Klett}$ units and ranged down to 50 during experiment. Chlorophyll A content: $5.6 \mu \mathrm{g} / \mathrm{mg}$ dry wt on day 0 and $6.7 \mu \mathrm{g} / \mathrm{mg}$ dry wt on day 1 . Small arrows indicate addition of $1.0 \times 10^{-4} \mathrm{M}$ $\mathrm{NH}_{4} \mathrm{Cl}$. The large arrow (19th day) indicates addition of $2.1 \times 10^{-5} \mathrm{M} \mathrm{DOMU}$.

(XBL 771-7283)

It appears likely that strains of heterocystous blue-green algae, specifically selected and genetically improved for the task, could be used to develop a biophotolysis system that could sustain efficient conversion of sunlight energy to hydrogen fuel. The economics and practicality of such systems remains to be determined.

\section{ACKNOWLEDGMENTS}

We gratefully acknowledge Professor W.J. Oswald for his advice and support. We wish to thank Richard Stover and Leon Kochian who assisted in media preparation. This work was supported in part by an ERDA Grant and HEW Training Grant

PHS \# 5 T01 GM00829-15.

\section{REFERENCES}

1. J.R. Benemann and N.M. Weare, Science 184, 174-175 (1974).

2. J.R. Benemann and N.M. Weare, Arch. Microbiol. 90, 323-332 (1972).

3. W.D.P. Stewart, P. Rowell, and E. Tel-Or, Biochem. Soc. Trans. 555 Meeting, ABERYSTWYTH 3, 357 .

4. M.B. Allen and D.I. Arnon, P1. Physiol. 32, 366-512 (1955). 
Table 1. Simultaneous oxygen and hydrogen evolution by a $250 \mathrm{ml}$ culture of Anabaena cylindrica under high light intensity $\left(4.0 \times 10^{5} \mathrm{ergs} / \mathrm{cm}^{2} / \mathrm{sec}\right)$.

\begin{tabular}{|c|c|c|c|c|c|c|}
\hline Day $^{a}$ & $\begin{array}{l}\text { Addition of } \\
\mathrm{NH}_{4} \mathrm{Cl} \\
\text { (final conc.) }\end{array}$ & $\frac{\mathrm{ml} \mathrm{H}_{2}}{\ell-\mathrm{hr}}$ & $\frac{\mathrm{ml} \mathrm{O} \mathrm{O}_{2}}{\ell-\mathrm{hr}}$ & $\begin{array}{l}: \mathrm{H}_{2} \text { in } \\
\text { gas phase }\end{array}$ & $\begin{array}{l}\mathrm{SO}_{2} \text { in } \\
\text { gas phase }\end{array}$ & $\frac{\mathrm{H}_{2}}{\mathrm{O}_{2}}$ \\
\hline 1.2 & -- & 27.6 & 6.9 & .96 & .24 & 4.0 \\
\hline 1.8 & $\ldots$ & 22.8 & 5.7 & .80 & .21 & 3.8 \\
\hline 2.1 & $5.0 \times 10^{-4} \mathrm{M}$ & -- & --- & -- & $\cdots$ & $\cdots$ \\
\hline 2.8 & -.- & 23.3 & 13.7 & .74 & .42 & 1.8 \\
\hline 2.9 & $4.0 \times 10^{-4} \mathrm{M}$ & $\cdots$ & $\cdots$ & $\cdots$ & $\cdots$ & -- \\
\hline 3.9 & -.- & 15.4 & 11.9 & .40 & .31 & 1.3 \\
\hline 4.9 & -- & 15.0 & 13.6 & .51 & .45 & 1.1 \\
\hline
\end{tabular}

aay 0 coincides with switching from air/ $/ \mathrm{CO}_{2}$ to $\operatorname{argon} / \mathrm{CO}_{2}$.

$\mathrm{H}_{2}$ PRODUCTION BY PHOTOSYNTHETIC PREPARATIONS:

STUDIES WITH HETEROCYSTS OF CYANOBACTERIA

AND A COUPLED OHLOROPLAST-HYDROGENASE SYSTEM

Lester Packer, Elisha Tel-Or, Ian Fry and George Papageorgiou

\section{INTRODUCTION}

It is possible to divert the normal pathway of photosynthesis to produce molecular $\mathrm{H}_{2}$ gas as follows:

$$
\mathrm{H}_{2} \mathrm{O} \underset{1 / 2^{\dagger} \mathrm{O}_{2}}{\stackrel{\text { sunlight }}{\longrightarrow}} 2 \mathrm{H}^{+}+2 \mathrm{e}^{-} \stackrel{\text { hydrogenase }}{\longrightarrow} \mathrm{H}_{2}
$$

In fact, some organisms do this naturally since they already have the machinery for such a process. The process of photosynthesis, or artificial systems patterned after it, might be utilized to develop a stable photochemical system to photolyze water into $\mathrm{H}_{2}$ and to create and maintain electrochemical gradients. Two in vitro systems were investigated: isolated heterocysts of cyanobacteria (also known as blue-green algae) and chloroplasts plus hydrogenase components of photosynthetic organisms are being examined with regard to stability and efficiency of $\mathrm{H}_{2}$ production.

A scheme showing the basic components of in vitro and in vivo systems for $\mathrm{H}_{2}$ generation is given in Fig. 1. In these systems light drives the biophotolysis of water; reducing a low potential electron acceptor. In energy production systems the low potential reductant is converted into $\mathrm{H}_{2}$ gas either by manipulating the metabolic machinery, or by foupling the system with hydrogenase catalyst.

\section{$\mathrm{H}_{2}$ PRODUCTION IN CYANOBACTERIAL HETEROCYSTS}

Cyanobacteria, primitive photosynthetic micro-organisms existing under extreme nutritional limitation possess a unique self-sufficient system for fixation of atmospheric $\mathrm{CO}_{2}$ and $\mathrm{N}_{2}$. They employ water photolysis as the means to generate reductant and chemical energy (ATP), utilized by metabolic processes which reduce the $\mathrm{CO}_{2}$ and $\mathrm{N}_{2}$ to carbohydrate $\mathrm{n}\left(\mathrm{CH}_{2} \mathrm{O}\right)$ and $\mathrm{NH}_{3} .1$

$\mathrm{N}_{2}$-fixation is mediated by the enzyme nitrogenase, a molybdo-iron-sulphur protein inactivated by $\mathrm{O}_{2}$. Since the photosynthetic apparatus produces $\mathrm{O}_{2}$, it is essential to eliminate interaction of the $\mathrm{N}_{2}$-fixing system with $\mathrm{O}_{2}$. C Cyanobacteria contain a certain percentage of specialized cells (named heterocysts), which allow it to overcome this difficulty.

Heterocysts are morphologically and biochemically different from vegetative celis (Figure 2). They are protected by a thickened wall and are metabolically dependent upon neighboring vegetative cells maintaining a narrow channel, or pore, through which metabolite transport can occur. Their internal biochemical activities are also modified; $\mathrm{O}_{2}$ evolution and $\mathrm{CO}_{2}$ fixation are absent, but other parts of the photosynthetic electron transport activities are retained, allowing light energy to continue forming ATP; they are found to possess most of the $\mathrm{N}_{2}$-fixation activity. 2,3

The occurrence of a $\mathrm{H}_{2}$ metabolism (mediated by hydrogenase) in cyanobacterja was established in 1964 by Fujita and Hattori. 4 . It was assumed that the physiological role of hydrogenase was to enploy $\mathrm{H}_{2}$ as an electron donor resulting in the fixation of $\mathrm{CO}_{2}$ (Ref. 4) 


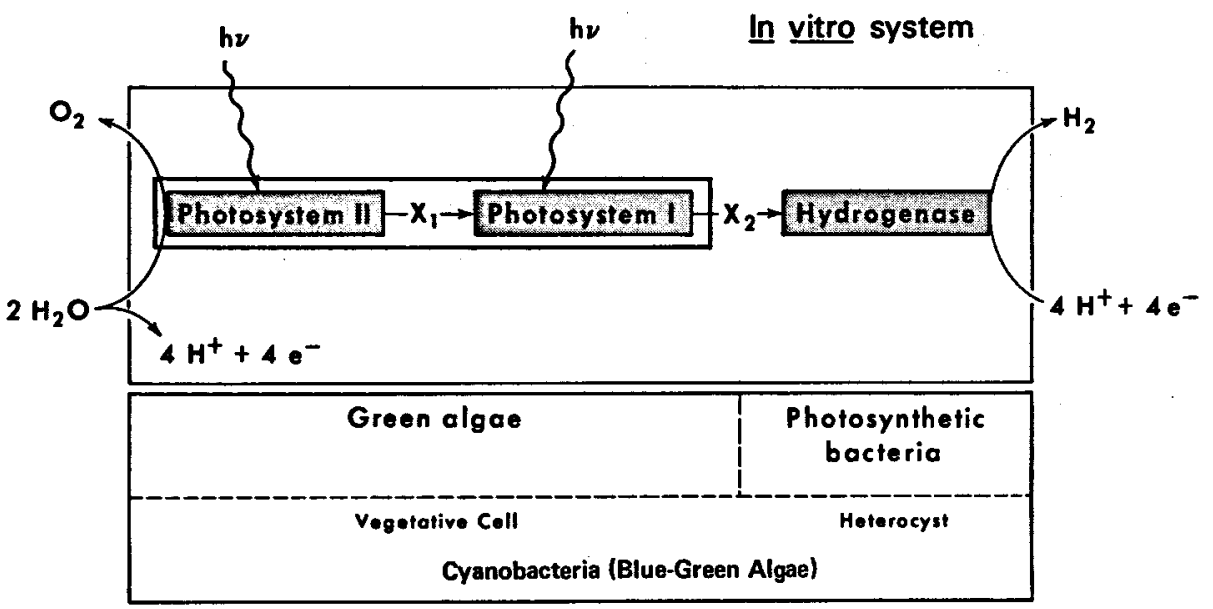

In vivo system

Fig. 1. Scheme for biological solar energy conversion for $\mathrm{H}_{2}$ generation. In vitro systems, solid lines; in vivo systems, dashed lines. $\overline{X_{I}}$ designates electron carriers linking the photosystens together. For the in vitro system, $X_{2}$ designates a low potential electron acceptor. For the in vivo system, $x_{2}$ designates reduced carbon compounds excreted by algae. (XBL 758-3709)

$$
\mathrm{nH}_{2}+\mathrm{nCO}_{2} \stackrel{\mathrm{h} \nu}{\rightarrow} \mathrm{n}\left(\mathrm{CH}_{2} \mathrm{O}\right)+\mathrm{n} / 2\left(\mathrm{O}_{2}\right) \text {. }
$$

Hydrogenase catalyzes a readily reversible reaction,

$$
\mathrm{H}_{2} \rightleftharpoons 2 \mathrm{H}^{+}+2 \mathrm{e}^{-}
$$

which may be driven by the photosynthetic apparatus.

Low potential reductants can also be converted to $\mathrm{H}_{2}$ by nitrogenase. Under conditions of nitrogen deficiency, this enzyme catalyzes $\mathrm{H}_{2}$ production irreversibly by an energy dependent (ATP) mechanism.

$$
2 \mathrm{H}^{+}+2 \mathrm{e}^{-}+5 \mathrm{ATP} \rightarrow \mathrm{H}_{2}+5 \mathrm{ADP}+5 \mathrm{P}_{\mathbf{i}} \text {. }
$$

This process is energy-consuming, unfavored physiologically and inefficient, requiring nitrogen starvation, but is observed when cyanobacteria are flushed with a noble gas.5

If the importance of photobiological production of $\mathrm{H}_{2}$ by cyanobacteria is to be evaluated, it is necessary to isolate and characterize the process in heterocysts which are the most likely site of $\mathrm{H}_{2}$ production.

Annual Activities. We have developed a procedure for isolation of heterocysts from Nostoc muscorum and have undertaken studies to characterize their capacity to produce $\mathrm{H}_{2}$ (Table 1 ).

$\mathrm{H}_{2}$ production requires an electron donor. We have selected for initial studies two electron donors: dithinonite $\left(\mathrm{Na}_{2} \mathrm{~S}_{2} \mathrm{O}_{4}\right)$ which donates electrons via methylviologen-a light independent reaction, and dithiothreitol (DTT) which reduces the methylviologen utilizing the photosynthetic electron transport chain-a light dependent reaction. One problem is the permeability of the reducant into the heterocysts. However, we found that the anionic detergent sodium-dodecylsulphate (SDS) stimulated
$\mathrm{H}_{2}$ evolution two to four fold when dithionite served as electron donor, but not when DTT was the electron donor. Our results indicate that the channels at each end of a heterocyst possess a permeability barrier limiting entry of the reducant. The rates of $\mathrm{H}_{2}$ production with both electron donors was in the range $20-50 \mathrm{ml} \mathrm{H} / \mathrm{mg} \mathrm{Ch} 1 / \mathrm{hr}$ in a constant rate over $10 \mathrm{hr}$. Hence isolated heterocysts are an efficient and stable system for studying $\mathrm{H}_{2}$ production. Therefore, we began a more detailed study of factors affecting $\mathrm{H}_{2}$ production. Distribution of $\mathrm{H}_{2}$ production activity was studied after mechanical rupture of the heterocysts revealing that the light-dependent $\mathrm{H}_{2}$ production requires the particulate fraction of the heterocysts, while the dithionite reaction requires the soluble fraction, indicating that two routes of $\mathrm{H}_{2}$ production might be present.

Incubation of intact or ruptured heterocysts, followed by anaerobic incubation through the assay phase, showed that $\mathrm{O}_{2}$ did not suppress the activity irreversibly. $\mathrm{N}_{2}$ which is known to inhibit $\mathrm{H}_{2}$ production in vivo, 5 did not affect $\mathrm{H}_{2}$ production in the heterocysts. $\mathrm{CO}$, a potent inhibitor of $\mathrm{N}_{2}$ fixation and hydrogenase greatly inhibited the production of $\mathrm{H}_{2}$ with both electron donors. Exogenous ATP did not stimulate the activity very markedly, and inhibitors of ATP formation did not affect the rate of $\mathrm{H}_{2}$ production. Isolated heterocysts were found to have even higher rates of $\mathrm{H}_{2}$ uptake than production. 6

The further understanding of the mechanism of $\mathrm{H}_{2}$ production in terms of the contribution to this activity by hydrogenase and nitrogenase is of paramount importance for optimizing the efficiency of $\mathrm{H}_{2}$ production by cyanobacteria and their eventual feasibility for large scale use for energy purposes. 


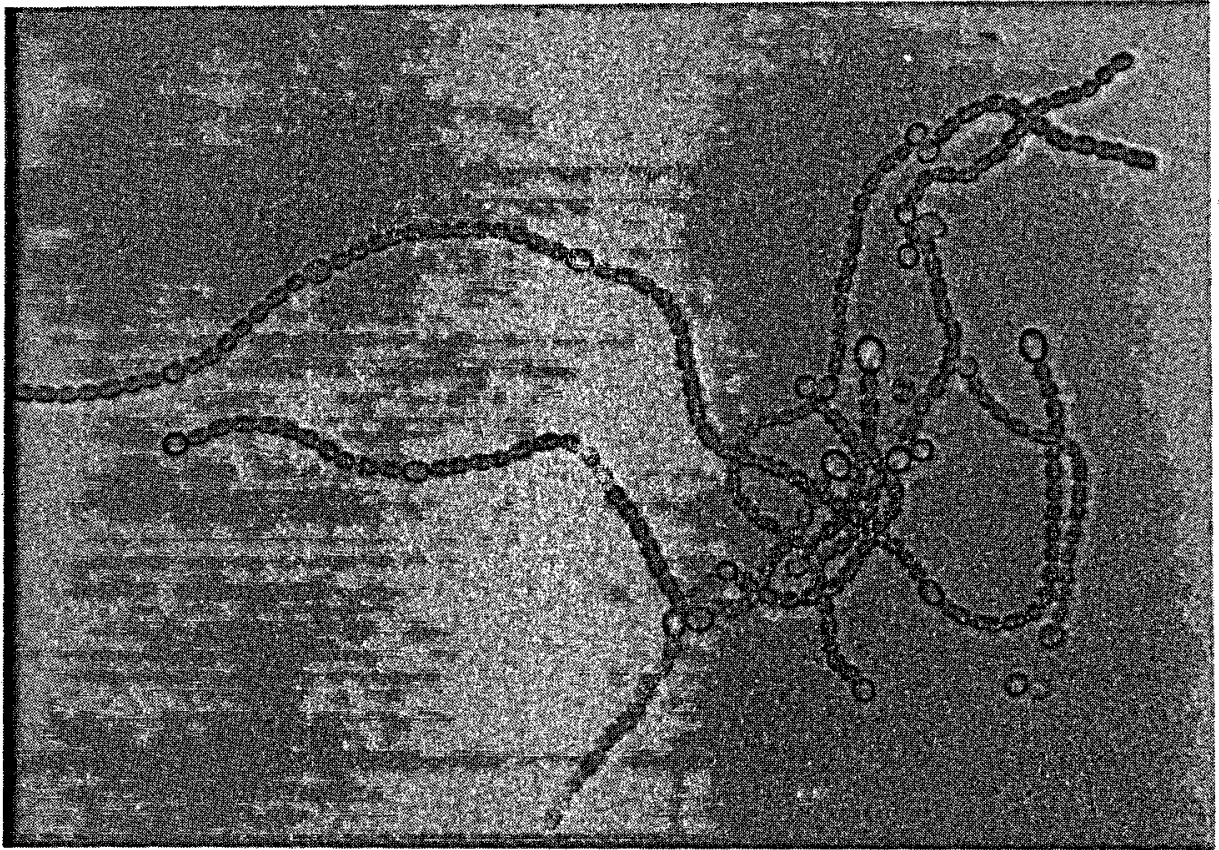

(A)

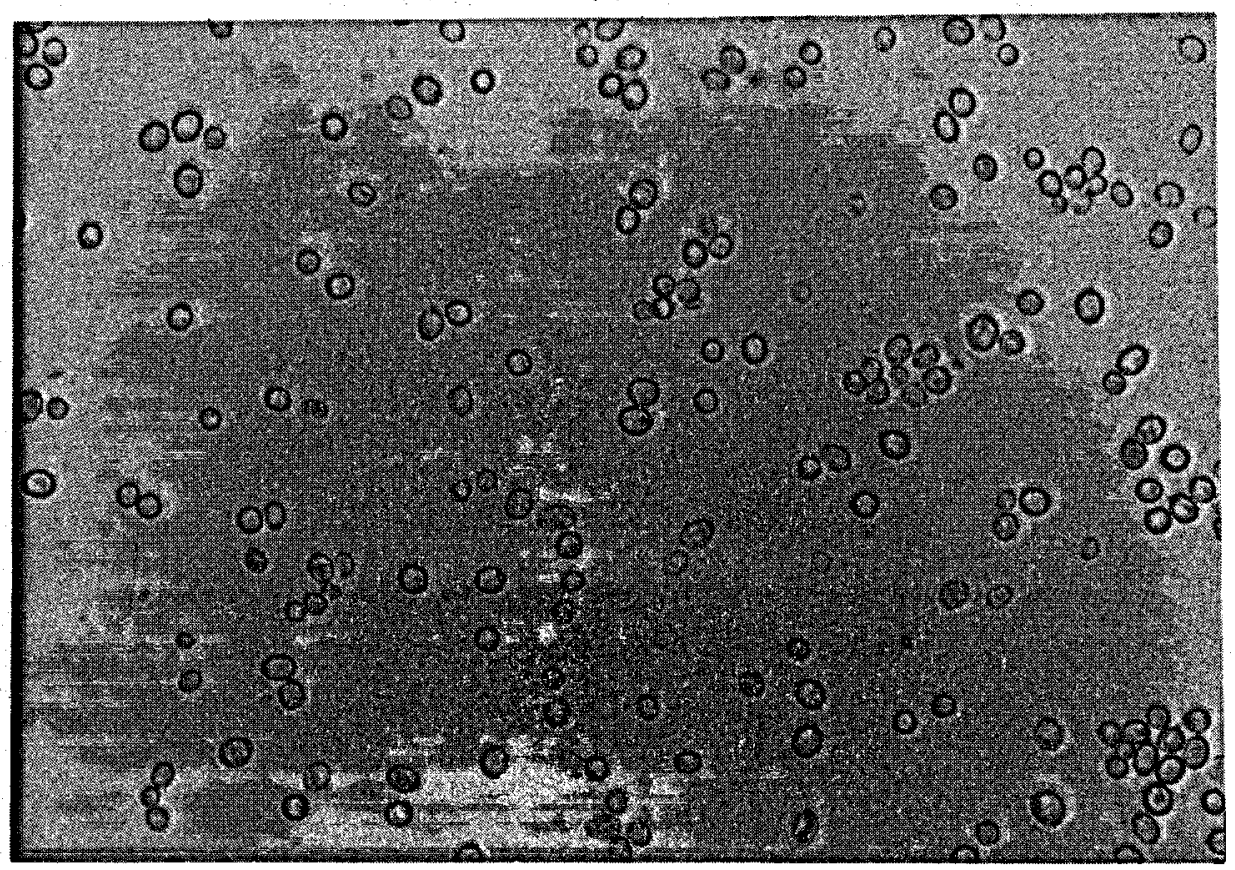

(B)

Fig. 2. Light and Electron Microscopy of Nostoc muscorum, and isolated heterocyst preparation. (A) N. muscorum filaments. The heterocysts located between the vegetative cells are clearly larger. (B) Heterocyst preparations. Heterocysts isolated from filaments show a high degree of purity.

(XBB 771-196 and 771-195) 
Table 1. Some properties of the $\mathrm{H}_{2}$ producing system in isolated heterocysts of Nostoc muscorum

\begin{tabular}{|c|c|c|c|}
\hline \multirow{3}{*}{ Expt. } & \multirow{3}{*}{ Test System } & \multicolumn{2}{|c|}{ Reductant } \\
\hline & & Dithionite & Dithiothreitol \\
\hline & & \multicolumn{2}{|c|}{ Activity (percent of control) } \\
\hline & \multicolumn{3}{|l|}{ Intact Heterocysts } \\
\hline 1 & light & 100 & 100 \\
\hline 2 & dark : & $90-100$ & 0 \\
\hline 3 & light $+2.5 \mathrm{mM} \mathrm{SDS}$ & $200-350$ & 0 \\
\hline 4 & light under $\mathrm{N}_{2}$ & $105-150$ & $110-130$ \\
\hline 5 & 1ight $+10^{-5} \mathrm{M}$ FCCP & 85 & 90 \\
\hline \multirow[t]{2}{*}{6} & light $+10^{-4} \mathrm{M} \mathrm{DCCD}$ & 100 & 100 \\
\hline & Fragmented Heterocysts & & \\
\hline 7 & light & $100-200$ & $60-80$ \\
\hline 8 & $\begin{array}{l}\text { light, preincubated } 1 \mathrm{hr} \text {. } \\
\text { under air. }\end{array}$ & $90-105$ & 90 \\
\hline 9 & light (or dark) +0.1 atm $C O$ & $10-20$ & $0-10$ \\
\hline \multirow[t]{2}{*}{10} & light $+5 \mathrm{mM}$ ATP & 100 & $110-140$ \\
\hline & Supernatant of Fragmented Hetero & & \\
\hline 11 & light & $60-80$ & $0-20$ \\
\hline
\end{tabular}

$\mathrm{H}_{2}$ PRODUCTION BY A CHLOROPLAST - FERREDOXIN HYDROGENASE COUPLED SYSTEM

These experiments attenpted to reconstruct in vitro, a photochenical system for $\mathrm{H}_{2}$ production using biological components and light to produce reductants (like reduced ferredoxin) which hydrogenase uses to convert protons into $\mathrm{H}_{2}$.

$$
\begin{aligned}
& 2 \text { ferredoxin }+\mathrm{H}_{2} \mathrm{O} \text { - Solar radiation } \\
& 1 / 2 \mathrm{O}_{2}+2 \mathrm{H}^{+}+2 \text { ferredoxin }(-1) \\
& 2 \text { ferredoxin }{ }^{(-1)}+2 \mathrm{H}^{+} \stackrel{\text { Hydrogenase }}{\longrightarrow} \\
& \mathrm{H}_{2}+2 \text { ferredoxin }
\end{aligned}
$$

The feasibility of coupling the reducing power of isolated chloroplasts to soluble bacterial hydrogenases was first demonstrated by Arnon et al., ? and has been studied elsewhere. $8,9,10,11$ bue to the oxygen lability of hydrogenase, the activity of the chloroplast-ferredoxin-hydrogenase system was short-lived, (15-20 minutes). However, Rao et al. 10 included an oxygen trap (glucose plus glucose oxidase) in the reaction mixture and showed that in an oxygen-free environment at $20^{\circ} \mathrm{C}$, the in vitro illuminated chloroplast plus hydrogenase system functioned for six and a half hours at a continuous rate of about 10 moles $\mathrm{H}_{2} / \mathrm{mg}$ chlorophyl1/hour.

Anmual Activities

During the past year our efforts have been directed at (1) setting up a suitable apparatus for $\mathrm{H}_{2}$ evolution studies and (2) optimizing the $\mathrm{H}_{2}$ production system for longer functional life and higher efficiency.

The reaction is carried out in airtight vials, purged with high purity $\mathrm{N}_{2}$. During the reaction, the photosynthetically produced $\mathrm{O}_{2}$ is removed by means of a glucose - glucose oxidase trap. The vials are incubated in a specially constructed shaker (Fig. 3) which provides for sample illumination and temperature regulation ( $W$. Worthington collaboration). The reaction is monitored by withdrawing aliquots from the gas phase of the vials, and injecting them to a Varian Aerograph Model 920. $\mathrm{H}_{2}$ is estimated from the recorded signals by reference to a calibration curve.

In the $\mathrm{O}_{2}$-free environment of the vials, and at $15^{\circ} \mathrm{C}$, isolated spinach chloroplasts can perform 


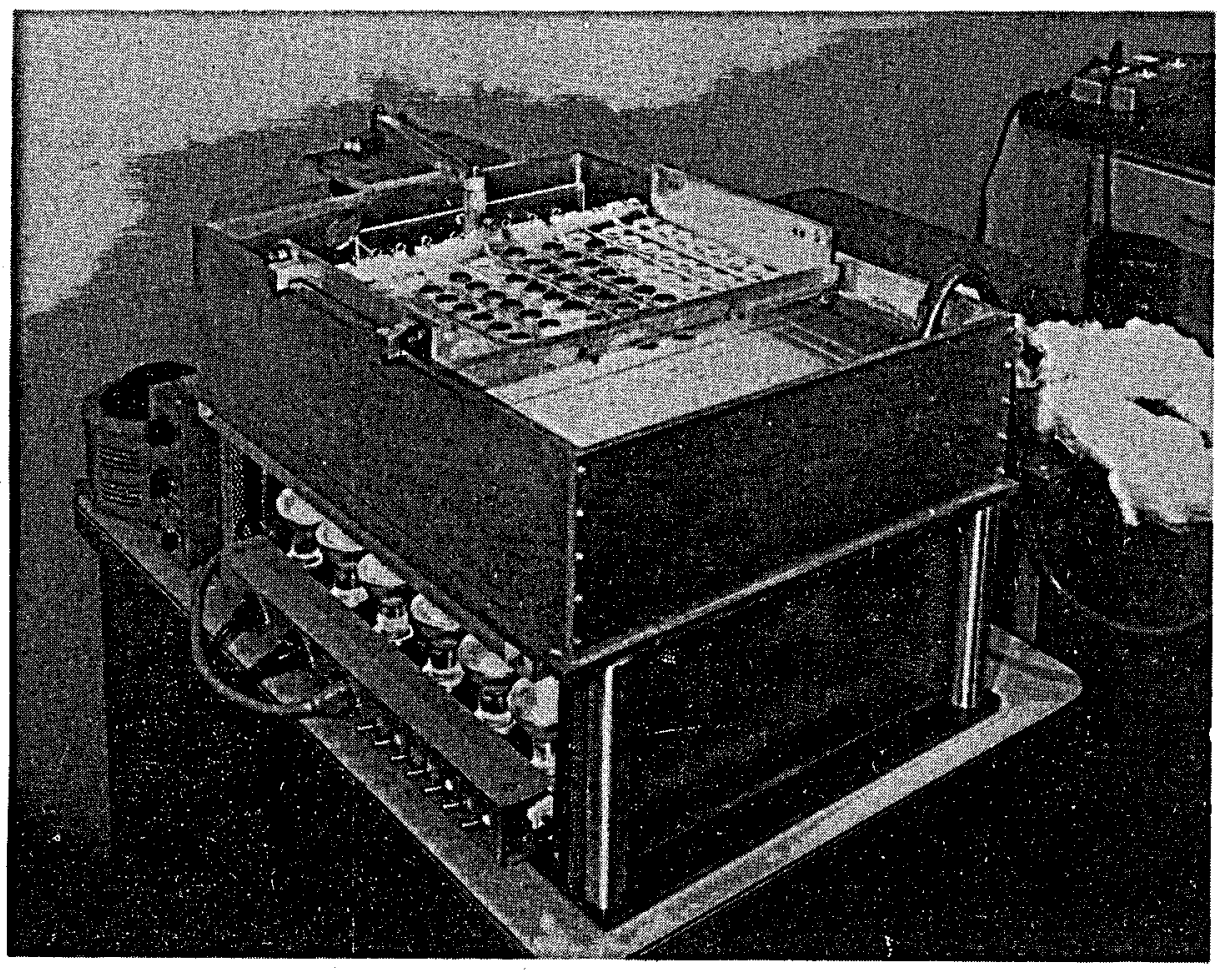

Fig. 3. Apparatus for Photochemical Studies of Hydrogen Production System. A specially designed shaking apparatus was constructed to provide illumination and temperature control of samples.

(CBB 761-279)

photosynthetic production of $\mathrm{H}_{2}$ for more than 24 hours, although after the first 8 hours the rate is considerably diminished. At $30^{\circ} \mathrm{C}$, the reaction can proceed up to 6-8 hours. This activity far exceeds that of isolated chloroplasts in an aerobic environment. 10,11

Various reaction parameters were studied to extend the functional life of the coupled chloroplast-ferredoxin-hydrogenase system, and to enhance its efficiency. The reaction operates optimally at $\mathrm{pH}$ 7.0. The free fatty acid scavenger, bovine serum albumin at a concentration of $0.5 \mathrm{mg} / \mathrm{ml}$ prolonged the activity and enhanced the rate of $\mathrm{H}_{2}$ production, whereas at a higher concentration $(3 \mathrm{mg} / \mathrm{ml})$ was slightly inhibitory. Butylated hydroxytoluene, a common antioxidant, was found to be inhibitory. No improvement was also detected when a cyclic electron transport catalyst was included in the reaction system in order to dissipate the unused excitation overload.

Thus far the efficiency of the coupled system is generally low. $\mathrm{H}_{2}$ production rates are 25-30 umoles $\mathrm{H}_{2} / \mathrm{mg} \mathrm{Chl} . \mathrm{hr}$, i.e. about $10 \%$ of the usual photosynthetic rates of isolated chloroplasts. The reaction may be diffusion limited, since it relies on ferredoxin to relay chloroplast electrons to the hydrogenase-proton couple.

Two aspects of this in vitro photochemical system for $\mathrm{H}_{2}$ production require further study: stabilizing the water splitting reaction of photosynthesis, and increasing the efficiency of the interaction of photo-chemical reductants with a stabilized hydrogenase.

A stabilized hydrogenase catalyst or suitable artifical substitute will be important in the eventual construction of a biological fuel cell.

\section{REFERENCES}

1. G.E. Fogg, W.D.P. Stewart, P. Fay and A.E.

Walsby, The Blue-Green Algae, Academic Press (London), 1973.

2. E. Te1-Or and W.D.P. Stewart, Biochem. Biophys . Acta. 423, 189-195 (1976).

3. E. Te1-Or and W.D.P. Stewart, Nature 258, 715-716 (1975).

4. Y. Fujita, H. Ohama and A. Hattori, Plant and Ce11 Physiol. $\underline{5}, 305-314$ (1964).

5. J.R. Benemann and N.M. Weare, Science 184, 174-175 (1974).

6. E. Te1-Or and L. Packer, Hydrogen metabolism in isolated heterocysts of Nostoc muscorum, presented at the VIIth International Congress on Photobiology, Rome, Italy, August 29 Sept. 3, 1976.

7. D.I. Arnon, A. Mitsui and A. Paneque, Biochem. Biophys. Res. Commn. 64, 355-359 (1961). 
8. A. Ben Amotz and M. Gibbs, Biochem. Biophys. Res. Commun. 64, 355-359 (1975).

9. J.R. Benemann, J.A. Berenson, N.0. Kaplan and M.D. Kamen, Proc. Nat. Acad. Sci. (U.S.), pp. 2317-2320 (1973).
10. K.K. Rao, L. Rosa and D.0. Hall, Biochem. Biophys. Res. Commun. 68, 21-27 (1976).

11. L. Packer, I. Fry, S. Sarma and K. Rao, Stability of an in vitro. Photochemical System for $\mathrm{H}_{2}$ Evolution, Biophys. J. 16, 132a (1976).

THE NITINOL ENGINE PROJECT

\author{
R. M. Banks, M. Wahlig
}

\section{INTRODUCTION}

Thermally induced martensitic phase transformation in the nicke1-titanium alloy "55 Nitinol"1 produces an energetic Shape Memory Effect at nearambient temperature thresholds. The transformation, resulting in changes of the internal crystal structure of the material, is accompanied by related changes in many physical properties of the alloy. Among these is a readily detectable contrast in the elastic properties of the two phases. When cooled below the threshold temperature (determined by the composition of the material), Nitinol exhibits a copper-like ductility and may easily be deformed by stress. Heating a cooled and deformed specimen above the threshold causes a reversion to the original internal structure, and thus to the shape it had before deformation. The purpose of the Nitinol Engine Project is to investigate the feasibility of applying this material to the conversion of low-temperature heat (such as that provided by solar collectors) into useful mechanical energy.

A prototype Nitinol heat engine has been operated at LBL since 1973. Producing about 1 watt of mechanical power at the present time, the prototype has made $>22 \times 10^{6}$ cycles without deterioration of the original working elements. The engine runs on a temperature difference of $\sim 25^{\circ} \mathrm{C}$, and is normally operated at the temperatures available in hot and cold tap water.

\section{HISTORY DEPENDENCE OF NITINOL SHAPE MEMORY EFFECT}

Many factors in the thermal and mechanical history of a Nitinol specimen will have an effect on its shape memory properties. Of particular significance to heat engine applications is the development of an automatic shape change on cooling as well as heating, after a period of continuous cycling in an engine. This effect (which we have called "double training") contributes to a progressive increase in the net output of a Nitinol engine running at uniform thermal conditions. This effect has been observed in the increased performance with time of the prototype Nitinol engine.

Wire diameter (itself a feature of mechanical history as a result of drawing) is another factor in the work-output potential of Nitinol. Among the commercially available materials used in engine development at LBL, the optimum size has proven to be 0.020 in. $(0.5 \mathrm{~mm})$ diameter wires, in which recovery stresses of 110,000 psi have been observed. During the past year, we have confirmed that wires of this diameter are capable of work output, after 1800 cycles, in excess of 1 watt per gram of Nitinol. The progressive increase in net work as a function of cyclic history is detailed in the project TestBed report, 2 covering much of the engine development work of the past year.

\section{THE TEST-BED ENGINE}

The Nitinol Test-Bed Engine, designed both to evaluate the work output potential of Nitinol and later to be run as an engine, used 0.020 in. diameter wires in linear tension between two non-parallel rotating disks (Fig. 1). The machine was designed to be capable of varying the percent elongation and speed of the cycle, but not the rate of relieving stresses built up within the Nitinol wires upon heating. While work output achieved during this experiment was encouraging, problems were encountered in the cyclic lifetime of the wires; they progressively and irreversibly elongated during testing and eventually failed.

Study of oscillograph tracings of stresses developed in the Nitinol wires during cycling in the Test-Bed typically showed a peak just after heat was applied to the wire. As this was also the point in the cycle at which the wires had the least mechanical advantage (ability to relieve stress by doing work on the system), the wires were repeatedly subjected to rather sudden stress concentrations with each cycle. In addition, correlations were observed between the locations of certain fracture points and the position of water-spray jets by which the wires were heated, indicating that thermal uniformity on heating was inadequate in this device.

Data from the Test-Bed experiment confirmed that the work-output potential for Nitinol heat engines justified further investigation of this approach to thermal energy conversion. However, these data also indicated that several mechanical and thermal cycle problems had to be overcome.

\section{DESIGN CRITERIA FOR THE NEXT PHASE}

Preliminary testing is now underway to evaluate a new engine concept intended to overcome the problems encountered in the Test-Bed by 1) ansuring thermal uniformity of the wires on heating and cooling, and 2) providing flexibility in the mechanical cycle that allows immediate relief of stresses that build up in the wires upon heating. The mechanical criterion necessitated abandoning the kinematics 

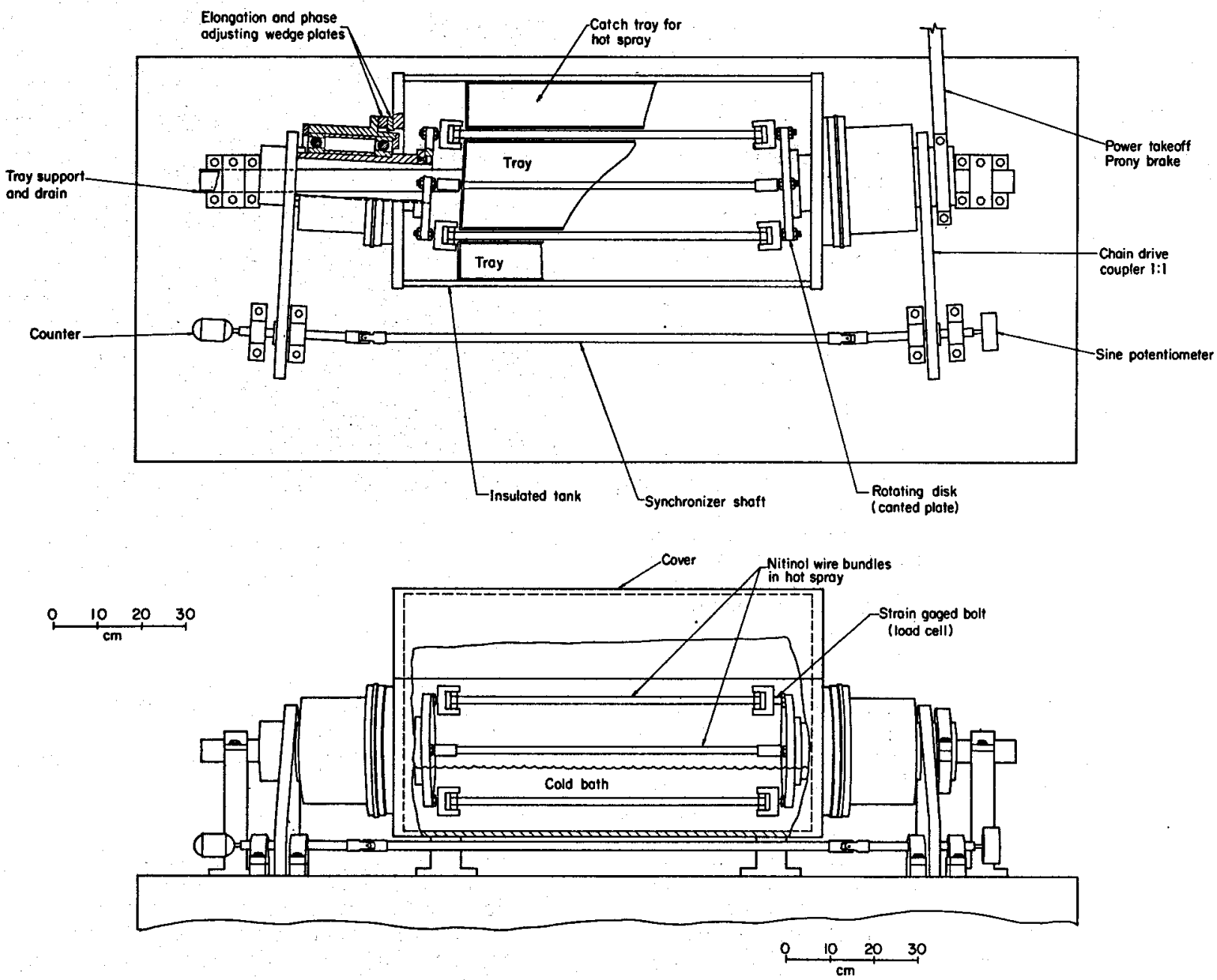

Fig. 1. Top and side views of the Nitinol Test-Bed Engine utilizing wires in linear tension between two non-parallel rotating disks.

(XBL 752-2312)

of rotary motion, represented by the crankshaft of the prototype and the "wobble plate" principle of the Test-Bed, because the rate of dimensional change in these systems is sinusoidal, providing the least leverage for the Nitinol wires at the point in the cycle when they presumably need it most.

In the new design, designated the Nitinol CamTrack Engine, sets of wires are held in linear tension between two moving trolleys on continuous tracks (Fig. 2). The spacing between the tracks ("Cam-tracks") varies at different points of the cycle; when it is increasing (tracks diverging), the Nitinal wires are elongated during the colddeform part of the cycle. Conversely, when the wires are heated, their axial contraction begins at a point where the tracks start to converge. Thus the contraction of the wires drives the trolleys forward on the track by the component of force perpendicular to the Nitinol wires. Heating and cooling of the wires is accomplished by complete immersion in two concentric semi-cylindrical baths.

By this design it is expected that the rate at which the tracks converge during the power stroke may essentially be matched to the rate of stress

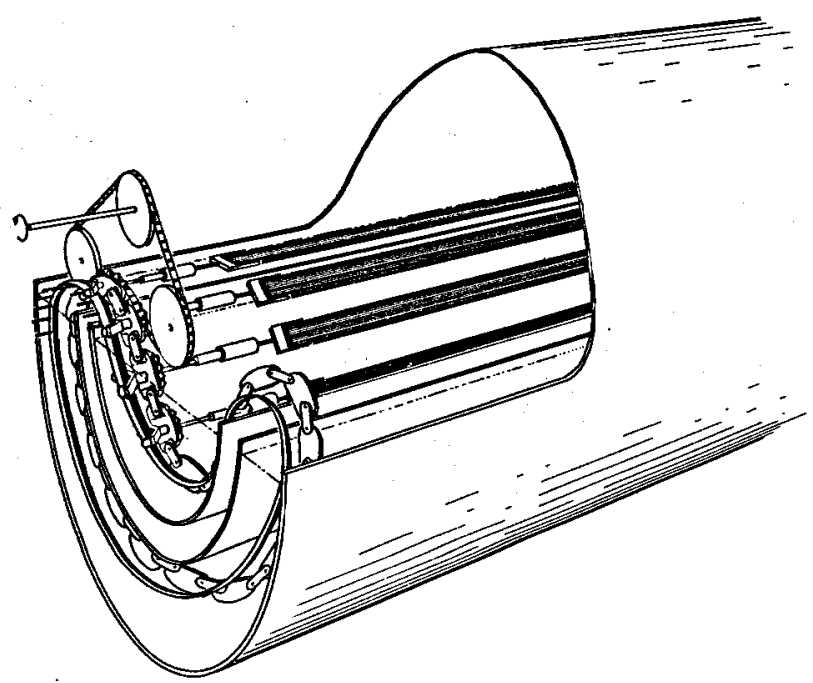

Fig. 2. Cut away view of the Nitinol Cam-Track Engine.

(XBL 767-3131) 
development in the Nitinol wires on heating, thereby distributing stress more uniformly during this part of the cycle. Moreover, the concentric configuration of the water baths allows for a significantly slower rate of deformation on cooling than the rate of shape recovery on heating, as the length of track passing through the cold bath is more than twice the length of track in the hot bath. Since Nitinol rejects heat on deformation (an exothermic reaction), time must be allowed for dissipation of this heat as the wire is stretched.

The Cam-Track prototype design provides for twenty sets of Nitinol wires (a total of 4800 feet) linked together on a continuous track that will be precision machined on a computer-operated mill. Assuming a work output of approximately 500 in-1b. / in $3 /$ cycle for the Nitinol (at 2.78 deformation), a cycling rate of $60 \mathrm{cpm}$, and parasitic losses of $50 \%$ for mechanical and hydraulic friction, the net output of the prototype is estimated to be approximately $0.5 \mathrm{HP}$. A further feature of the design is the ability to scale-up in power without increasing the size of the machine or the number of moving parts, but simply by the addition of more Nitinol wires.

Test work has begun on a typical single wire set comprising 80 three-foot lengths of 0.020 in. diameter Nitinol wire (Fig. 3). This set has been cycled > 1000 times at $3.4 \%$ deformation and has developed no detectable permanent elongation. Recovery stresses, measured at $\sim 350 \mathrm{lb}$ (net) in the first test, were absorbed by compression springs to provide mechanical compliance in the system. In the next phase of testing the springs will be eliminated, and the mechanical cycle changed to simulate the proposed configuration of this prototype. If the results of this phase are satisfactory, construction of the machine itself will begin.

Testing of the Cam-Track Nitinol engine, in addition to determing the mechanical performance

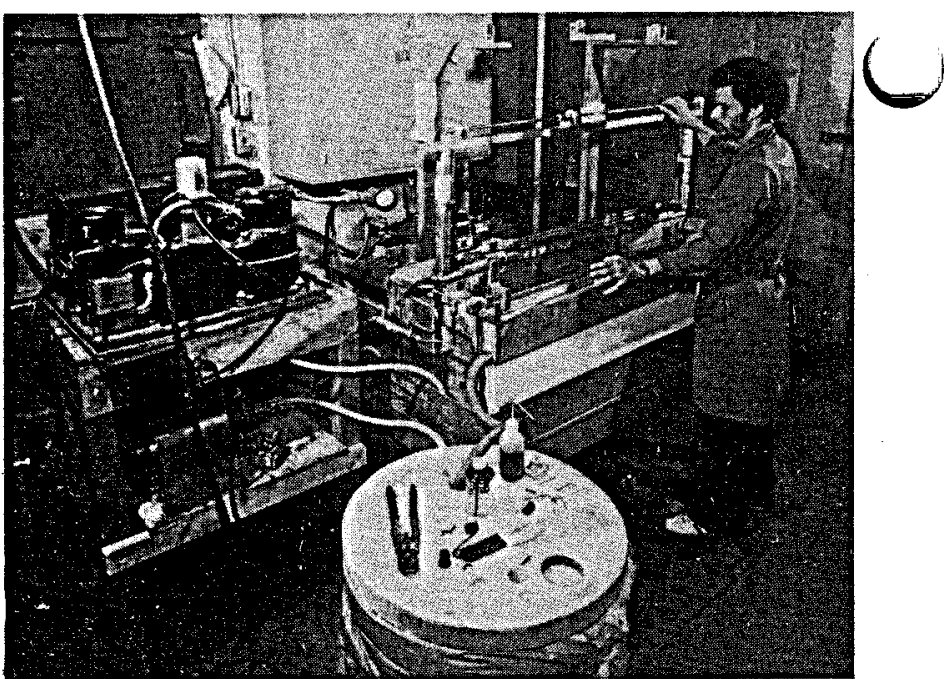

Fig. 3. View of the Cam-Track test chamber.

(CBB 7611-10019)

of the device in the laboratory, will include measurement of its thermal efficiency under conditions approximating a realistic installation.

\section{REFERENCES}

1. The alloy is nominally 55\% Nickel by weight with the balance titanium; it takes its name from the chemical initials of the two elements and of the Naval Ordnance Laboratory (Silver Spring, Maryland) where the shape memory effect was first observed.

2. H. P. Hernandez et al., Nitinol Engine Project Test Bed; Lawrence Berkeley Laboratory Report UCID-3739 (1975). 


\title{
$2^{2308}$ \\ Chemical Process Research and Development
}

\author{
INTRODUCTION
}

In the area of chemical process development, research on processes that might result in the development of substitute fuels to replace petroleum and natural gas has been emphasized. The limited reserves of petroleum and natural gas make the development of improved processes for the liquifaction and gasification of coal increasingly attractive. Ultimately, the depletion of petroleum and natural gas reserves will require the use of solid fossil fuels. Many experts, however, argue that even now petroleum should be conserved for petrochemical raw material purposes and our energy needs be satisfied from other sources such as solid fossil fuels, solar energy and nuclear energy.

Processes for the liquifaction and gasification of coal are not new, but processes that have operated commercially in the past are not economically competitive within the present energy price structure. Part of this economic disadvantage arose from the extreme conditions of pressure and temperature that these processes required. We are studying the fundamental processes involved in the liquifaction and gasification of coal to determine whether milder operating conditions might not befeasible. Studies of various types of catalysts that might lead to liquifaction at low pressures and low temperatures, and on the development of some new and unusual catalysts are being conducted. These studies are being made with model compounds as we11 as with bituminous and sub-bituminous coals.

Short term energy storage is an important means of increasing the efficiency of present energy systems by providing a means of smoothing peak loads with respect to minimum loads. Chemical systems are one means of energy storage. Such systems must operate in a completely reversible series of chemical reactions at temperatures convenient for the storage and subsequent release of thermal energy. A study of the $\mathrm{SO}_{2}-\mathrm{O}_{2}-\mathrm{SO}_{3}$ system for energy storage has been started and a preliminary design study for use of that system to store energy has been made.

Studies have continued on the enzymatic conversion of cellulose to glucose and the fermentation of the resulting glucose to ethanol. Utilization of a large part of the biomass that is presently wasted might well be developed into an attractive energy source.

\section{SELECTIVE HYDROGENATION OF COAL}

\author{
A.T. BeIZ, E.A. Grens, E.E. Petersen, T. Vermeulen, \\ K.H. Conklin, T.T. Derencsenyi, G.P. Dorighi, D.C. DraemeZ, \\ R.R. Holten, D.J. Madieros, M. Seth
}

\section{INTRODUCTION}

Existing technology for coal liquefaction is based on thermal decomposition of coal, that is pyrolysis, with subsequent hydrogenation of initial pyrolysis products. This thermal bond-breaking is nonselective. It results in a wide range of products including methane and is thus not efficient in hydrogen utilization. Moreover, the relatively severe conditions involved lead to difficult materials and processing problems. This project is directed toward establishing the basis for converting coal to liquid products in the heavy oil range by selective scission of certain bond types in the coal structure at temperatures below the onset of coal pyrolysis. The band scission is to be achieved by hydrogenolysis, dealkylation, or hydrolysis with homogeneous catalysts. Bonds to be attacked are those linking conjugated hydroaromatic groups in the coal, including aliphatic bridges and, especially in sub-bituminous coals, noncarbon linkages such as ether, carboxylic, and disulfide bridges. The use of homogeneous catalysts in a fluid reaction medium permits access to reaction sites in the extensive interior surface of the coal.
The investigation encompasses the salient features in the interaction of coals with inorganic and organic reaction media, the effects of homogeneous catalysts in promoting hydrogenation in such media at moderate temperatures, and the action of potential catalyst species in breaking specific bond types in model compounds representative of certain aspects of coal structure. Consideration of the interaction of the physical structure of the coal with the distribution of the reactions involved and the influence of transport phenomena in this structure on the reaction rates is an essential feature of this study.

This project was initiated in January, 1974. During that year 1aboratory equipment and procedures were developed and preliminary studies conducted into the interaction of sub-bituminous coal with organic solvents and zinc chloride melts, and into hydrogenolysis of a range of mode1 compounds. 


\section{ANNUAL ACTIVITIES}

The work planned for 1975 included investigation of coal interaction with hydroaromatic solvents representative of coal derived liquids and with inorganic liquid "melts" such as zinc chloride, phosphoric acid, and sodium hydroxide. The effects of these interactions on coal pore structure were also to be studied. At the same time hydrogenolysis of model organic compounds was planned to guide the selection of homogeneous catalysts. The coal used in all of this work was from the Roland Seam of the Wyodak mine in Gillette, Wyoming, a representative western, sub-bituminous coal.

INTERACTION OF COAL WITH ORGANIC SOLVENTS (Grens, Draeme1, Dorighi)

The solubility of coal in benzene, tetralin, and phenol at temperatures from $150^{\circ} \mathrm{C}$ to $350^{\circ} \mathrm{C}$ was examined in an apparatus providing for extraction of the coal in pure refluxed solvent under closely controlled temperature. 1 Below $350^{\circ} \mathrm{C}$ no hydrogen donation by tetralin took place (as verified by spectrometry), but coal solubility in tetralin increased about threefold as temperature increased from $150^{\circ} \mathrm{C}$ to $300^{\circ} \mathrm{C}$ as shown in Table 1 .

Table 1. Properties of tetralin extracts of Roland seam coal. ( $4 \mathrm{hr}$ extraction time, $\sim 70 \%$ completion)

\begin{tabular}{ccccc}
$\begin{array}{c}\text { Extraction } \\
\text { Temperature } \\
\left({ }^{\circ} \mathrm{C}\right)\end{array}$ & $\begin{array}{c}\text { Extract } \\
\text { Yield } \\
\text { (dry basis) }\end{array}$ & $\begin{array}{c}\text { Extract } \\
\text { Yield } \\
\text { (DAF basis) }\end{array}$ & $\begin{array}{c}\text { Extract } \\
\text { H/C } \\
\text { Ratio }\end{array}$ & $\begin{array}{c}\text { Extract } \\
\text { Nr Average } \\
\text { Mole Wt }\end{array}$ \\
\hline 150 & $3.6 \%$ & $4.2 \%$ & 1.42 & 880 \\
200 & $5.5 \%$ & $6.4 \%$ & 1.42 & 780 \\
250 & $7.2 \%$ & $8.4 \%$ & 1.35 & 780 \\
300 & $14.3 \%$ & $16.6 \%$ & 1.32 & 510 \\
$350^{*}$ & $26.4 \%$ & $30.7 \%$ & 1.18 & 490 \\
& & & &
\end{tabular}

*Above coal pyrolysis temperature

Over this temperature range the $\mathrm{H} / \mathrm{C}$ ratio $(1.07$ in the coal) of the dissolved material decreased gradually with increasing temperature (extent of dissolution) as did the number average molecular weight of this material. These results suggest that under conditions leading to greater coal solubility (higher temperature, better solvents) the dissolved materials include groups with lower hydrogen content, but that larger fragments further break down to yield smaller dissolved species. Moreover, the low $\mathrm{H} / \mathrm{C}$ ratio in the dissolved material under some circumstances points to the opportunity for achieving liquid products at very moderate hydrogen expenditures.

The materials extracted into aromatic and hydroaromatic solvents under these mild conditions have relatively little aromatic content. For example a proton NMR spectra of $200^{\circ} \mathrm{C}$ tetralin extract (Fig. 1) reveals an aromatic hydrogen content of only $9 \%$ (solvent refined coal has 30-50\%) with $15 \%$ a hydrogen, 58\% $\beta$ hydrogen, and $18 \% \gamma$ hydrogen.

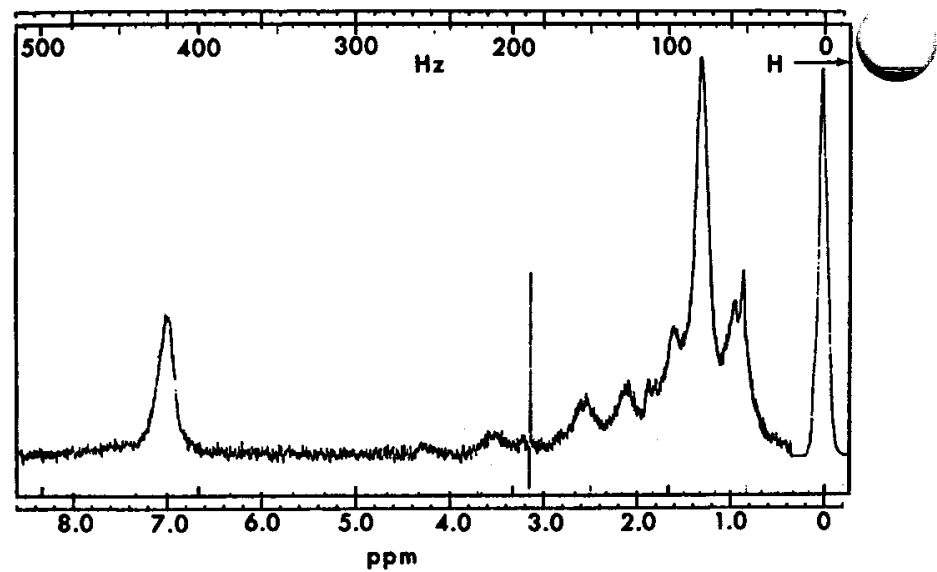

Fig. 1. Proton nuclear magnetic resonance spectrum of the extract from Roland seam coal using tetralin at $200^{\circ} \mathrm{C}$ for 4 hours. (TMS reference and deuterated toluene solvent.)

(XBL 758-1909)

This work is being extended to examine the interactions with other solvents, particularly polar compounds (e.g., pyridine) and solvent combinations.

INTERACTION OF COAL WITH INORGANIC LIQUIDS

(Vermeulen, Derencsenyi, Seth, Holten)

Metal-halide salts, above their melting points, or liquefied by small admixtures of water, have been shown to be effective dispersants of coal. Zinc chloride was found to give moderate increases in the benzene extractibility of coal by treatment under $\mathrm{H}_{2}$ at $200^{\circ} \mathrm{C} .{ }^{2}$ Experiments have now been conducted to examine phosphoric acid and sodium hydroxide, both of which are as good as, or better than, zinc chloride in regard to dispersal of the raw coal.

Phosphoric acid, along and with additives, was studied in a series of experiments. Carbon tetrachloride extractibles, in the treated coals, were found to increase with severity of treatment. Additives, tested individually in the phosphoric acid, included $\mathrm{AlCl}_{3}, \mathrm{NiCl}_{2}, \mathrm{CoCl}_{2}, \mathrm{CuCl}_{2}$, and $\mathrm{ZnCl}_{2}$. None of these influenced the results significantly. In experiments of up to 4 hours duration, extractibility was found to reach a maximum at 30 minutes, declining slightly thereafter. The decline may be due to extraction directly into the treating liquid; this possibility is being investigated. The work with phosphoric acid, and that with zinc chloride, is being extended to temperatures of $250^{\circ}$ to $300^{\circ} \mathrm{C}$. Whereas zinc chloride appears to possess Lewis-acid. activity, phosphoric acid functions as a Brönsted acid.

Sodium hydroxide, also studied at $200^{\circ} \mathrm{C}$, has been found to produce more extensive chemical degradation of coals. As much as $12 \%$ of the treated coal remains dispersed and filterable when the solution is neutralized to $\mathrm{pH} 7$. Although $\mathrm{CCl}_{4}$ extractibles were only about $1 \%$, subsequent extraction with 2-methy1-4-pentanone (MIBK) 
yielded $5 \%$ extract with $\mathrm{NaOH}$ alone and $9 \%$ when sodium acetate or sodium phosphate was also present.

\section{CHANGES IN PORE STRUCTURE OF COAL WITH PROGRESSIVE EXTRACTION \\ (Madieros, Petersen)}

Reliable techniques were sought to determine experimentally the total surface area of coal as received and after progressive processing. A knowledge of total surface area is informative to assess where reactions are occurring within the coal and to learn whether transport processes are limiting overall reaction rates.

The Brunauer-Emmett-Teller (BET) method of determining surface areas was used to obtain apparent surface areas of two coals, Wyodak mine (Roland Seam) sub-bituminous coal and Illinois No. 6 high sulfur bituminous coal, and of two reference materials, $4 \mathrm{~A}$ and $5 \mathrm{~A}$ linde molecular sieves. ${ }^{3}$ Four gases, $\mathrm{CO}_{2}$ and $\mathrm{CF}_{4}$ at $196^{\circ} \mathrm{K}$ and $\mathrm{N}_{2}$ and $A$ at $77^{\circ} \mathrm{K}$, were used to determine apparent surface areas on the above substances. High areas were obtained with $\mathrm{CO}_{2}$ and much lower areas were obtained with the remaining gases. The $\mathrm{CO}_{2}$ areas of Roland Seam and Illinois No. 6 were 106 and $227 \mathrm{~m}^{2} / \mathrm{g}$, respectively. A comparison of these results with similar measurements on $4 \mathrm{~A}$ and $5 \mathrm{~A}$ Linde sieves suggests that the structures of Roland Seam and Illinois No. 6 coals resemble $4 \mathrm{~A}$ and $5 \mathrm{~A}$ Linde sieves, respectively. That is, the structure suggests a macropore system serving as main arteries into the macro-coal particles and a micropore system, less than $12 \AA$ in diameter, into the microparticles.
This structure raises questions about the accessibility of reactants into the microparticles through the micropores. Accordingly, a study was made of the total surface areas of Roland Seam Coal $\left(\mathrm{CO}_{2}\right.$ adsorption at $\left.196^{\circ} \mathrm{K}\right)$ progressively extracted with organic solvents in the work cited in a previous section. Although the final surface areas depend upon extraction time, temperature and the chemical nature of the solvent used, the overall result of significance to this study was that the total surface area increased with the degree of extraction. On Fig. 2 this result is demonstrated by plotting total surface area versus extraction yield for benzene, tetralin, phenol and hexane solyents. The highest surface area is about $265 \mathrm{~m}^{2} / \mathrm{g}$ as compared to an initial area of $106 \mathrm{~m}^{2} / \mathrm{g}$. It appears that the area is going through a maximum value at $32 \%$ extraction with tetralin at $350^{\circ} \mathrm{C}$. However, the important result is that the areas increase with the degree of extraction, which establishes that a substantial fraction of the micropore structure is accessible to these solvents.

In summary, the accomplishments of this work are that the surface areas of coal can be measured using $\mathrm{CO}_{2}$ at $196^{\circ} \mathrm{K}$ and that this technique can be used to monitor changes in pore structure of coal with progressive processing. The use of different adsorbates has established the similarity of coal to molecular sieves in that they have a macropore and micropore structure, the latter being smaller than $12 \AA$. The technique was used to show that total surface areas of coal increase with progressive extraction thereby establishing the accessibility of solvents to the microporous structure of coal.

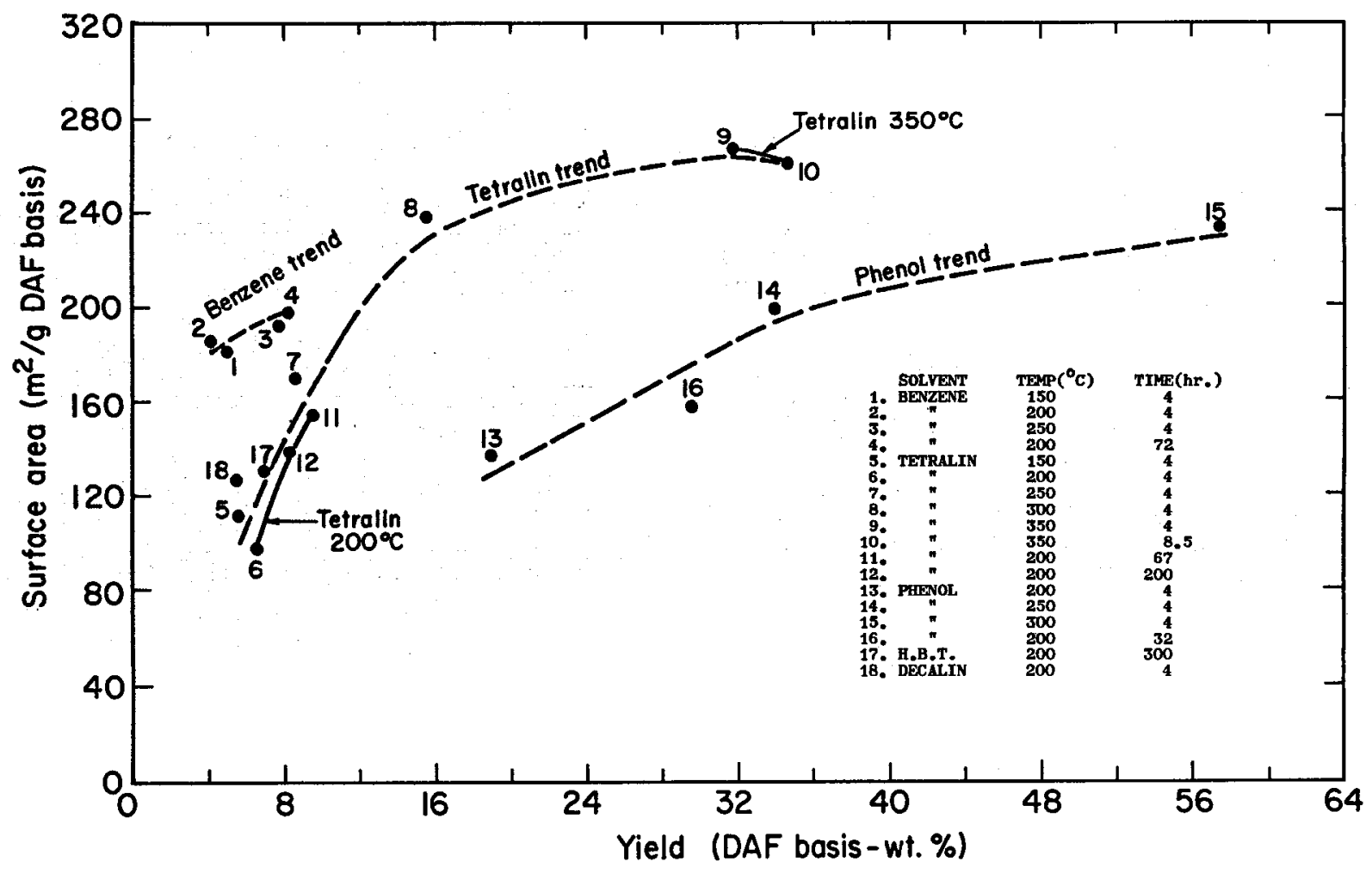

Fig. 2. Coal residue surface area as a function of extract yield. (XBL 7510-7730) 
CATALYTIC HYDROGENATION AND HYDROGENOLYSIS OF ORGANIC STRUCTURES RESEMBLING THOSE FOUND IN COAL (Be11, Conk1in)

For this work model organic compounds, containing structures similar to those found in coal, were studied to determine the ability of catalysts to activate particular types of reactions. By using mode1 compounds rather than coal, it is possible to identify reaction products and thereby determine the reaction pathway. The particular classes of reactions being studied include hydrogenation, hydrogenolysis, cracking, desulfurization, and denitrogenation.

In a study completed recently the hydrogenation 4 and hydrogenolysis of bibenzyl was investigated. This compound was chosen to represent the aliphatic bridges which hold the aromatic and hydroaromatic portions of coal together. When coal is liquefied, these bridging structures must be cleaved and hydrogen added to terminate the opened ends of the bridge. The extent to which coal liquefaction catalysts promote the cleavage of aliphatic bridges has not been established and was the subject of this investigation.

An initial screening of catalysts was conducted in a magnetically stirred autoclave operated at $325^{\circ} \mathrm{C}$ and 3500 psig. It was found that cobalt molybdate on silica-promoted alumina, tungsten disulfide, lithium chloride, stannous chloride promoted with ammonium chloride, stannous chloride, zinc chloride, antimony trichloride, and p-toluene sulfonic acid were all inactive for promoting the hydrogenolysis or hydrogenation of bibenzyl. Under the same conditions, ferric chloride caused bibenzyl to undergo a disproportionation reaction. Only aluminum chloride, a strong Lewis acid, was found to bring about both hydrogenolysis and hydrogenation of bibenzyl.

More extensive investigation of the catalytic properties of aluminum chloride revealed that it caused not only the reactions of bibenzyl but also those involving benzene, the solvent used in most of the experiments. The reaction products were found to contain primarily benzene, ethylbenzene, toluene and a tarry substance. Lesser amounts of cyclohexanes, other alkylbenzenes, and diphenyl were also found. Similar products were produced by reacting benzene in the absence of bibenzyl. However, careful examination of the results showed that both toluene and ethylbenzene were formed directly from bibenzyl, and that bibenzyl appears to inhibit the reactions involving benzene. From a study of the effects of hydrogen pressure, it was established that the conversion of bibenzyl and the yields of toluene and ethylbenzene do not depend on the pressure of gaseous hydrogen. The formation of these products can in fact be achieved without gaseous hydrogen using only the hydrogen available in the organic materials. By contrast, the yields of cyclohexane derivatives are strongly affected by the hydrogen pressure, leading to the conclusion that gaseous hydrogen is involved in the formation of these products.

Studies with different catalyst-to-bibenzyl ratios revealed that the extent of bibenzyl conversion is reduced when smaller amounts of catalyst are used. Furthermore, low catalyst loadings appear to reduce significantly the extent to which benzene is reacted and the tarry substance is formed. These results suggest that future work should be done with low loadings of catalyst to avoid the interfering effects of solvent reaction.

Several mechanisms have been proposed to explain the formation of the tarry substance as well as toluene and ethylbenzene, the major reaction products. A key step in all of these mechanisms is the protonation of the bibenzyl which subsequently leads to either a symmetric or unsymmetric cleavage of the aliphatic bridge between the phenyl groups.

In continuing studies, the effect of bridge length and the nature of the aromatic groups at the bridge ends are being investigated. A separate set of experiments are being initiated to determine what effects the inclusion of oxygen and sulfur have on the nature and ease of bridge cleavage.

\section{REFERENCES}

1. D.C. Draemel and E.A. Grens, Action of Solvents on Sub-bituminous Coal Below Pyrolysis Temperature, Lawrence Berkeley Laboratory Rpt. LBL-4434 (1975).

2. T.T. Derencsenyi and T. Vermeulen, Interaction of a Sub-bituminous Coal with Molten Zinc Chloride Solutions, Lawrence Berkeley Laboratory Rpt. LBL-3265 (1975).

3. D.J. Madieros and E.E. Petersen, Changes in the Pore Structure of Coal with Progressive Extraction, Lawrence Berkeley Laboratory Kpt. LBL-4439 (1975).

4. K.H. Conklin and A.T. Be1l, Hydrogenation, Cracking and Hydrogenolysis of Bibenzy 1 in a High-Pressure Stirred Autoclave, Lawrence Berkeley Laboratory kpt. LBL-4440 (1975). 


\author{
G.A. Somorjai, A.T. Beil, J.C. Smart, K.P.C. Vollhardt, \\ M.J. Veraa, W.A. Bollinger, J.G. Ekerdt, C.S. Kellner, \\ B.A. Sexton, C. Curtis, A. Sievert, K. Tanner, W.G.L. Aalbersberg, \\ A.J. Barkovich, R.L. Funk, R.C. Hilzard III
}

\section{INTRODUCTION}

This project is directed at developing novel and effective catalytic approaches to the conversion of coal to liquid and gaseous fuels. To accomplish this objective we are examining the performance of a variety of catalysts and relating their activity and selectivity to their physical and chemical characteristics. Efforts are also being made to synthesize new homogeneous and heterogeneous catalysts.

During the past year significant progress has occurred in the following areas: 1) studies of coal gasification catalyzed by salts of alkalai metals; 2) investigation of the interactions of carbon monoxide and hydrogen with the surfaces of methanation catalysts, 3) synthesis of nove1 transition metal complex catalysts of potential use for coal liquefaction and hydrocarbon synthesis, and 4) studies of the interactions between transition metal complex hydrogenation catalys ts and aromatic structures resembling those found in coal.

\section{CATALYZED COAL GASIFICATION}

(A.T. Bel1 and M.J. Veraa)

Coal gasification processes presently under development operate at temperatures between 800 and $1000^{\circ} \mathrm{C}$ and at pressures up to $100 \mathrm{~atm}$.

These conditions place severe demands upon the materials which must be used for the construction of gasification reactors. As a result there is significant incentive to find low cost catalysts which might be used to achieve suitable gasification rates at lower temperatures. The objective of this project has been to screen several candidate catalysts and to perform in-depth studies of the most effective one.

Samples of a sub-bituminous Western coal (Wyodak Corp. Roland seam) were ground to $-65+325$ mesh and impregnated with aqueous solutions of either $\mathrm{LiCl}, \mathrm{NaCl}, \mathrm{KCl}, \mathrm{RbCl}, \mathrm{CsCl}, \mathrm{K}_{2} \mathrm{CO}_{3}$, or $\mathrm{KOH}$. The same number of moles of catalyst per gram of coal was used for each catalyst. After drying, the coal was pressed into a $1 / 2$ inch diameter pellet and charred in a flowing stream of helium at $900^{\circ} \mathrm{C}$ for $7 \mathrm{~min}$. The char pellet was then suspended from the beam of a microbalance. Gasification of the char was accomplished by passing a helium-steam mixture past the pellet, which was heated in a furnace. The gasification products were analyzed by gas chromatography.

Figure 1 illustrates the rates of carbon gasification as a function of the percent fixed carbon gasified (PFCG), in the absence of a catalyst and in the presence of different catalysts. It is apparent that the alkali chlorides will behave in a similar manner. These catalysts cause

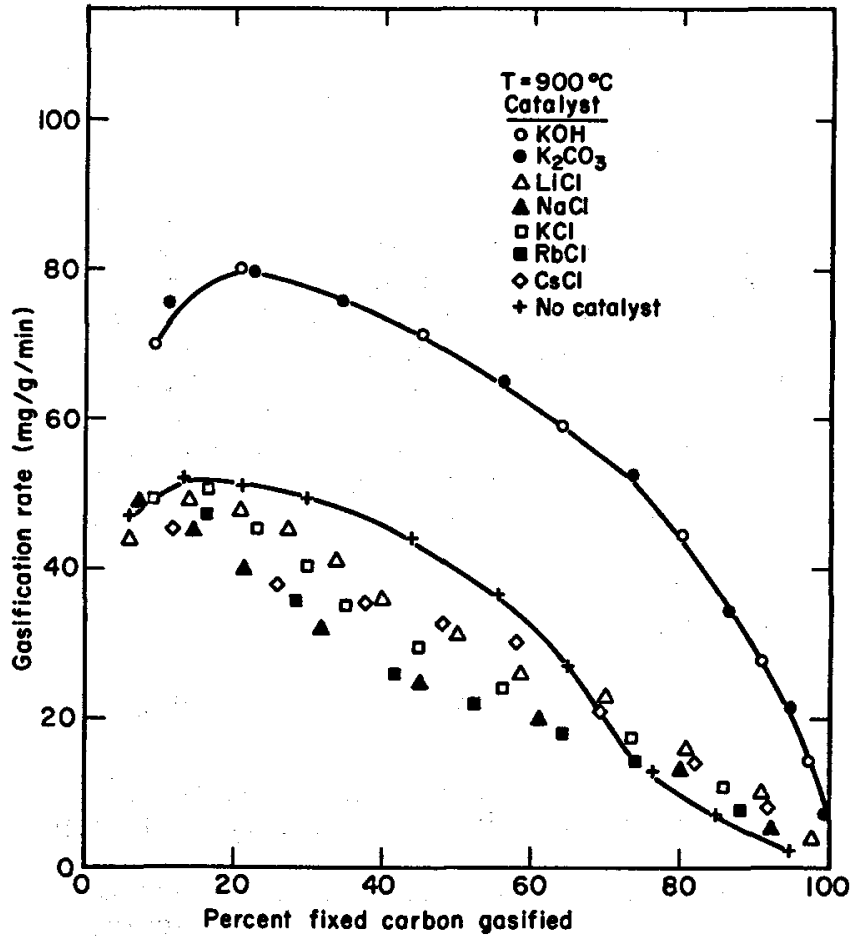

Fig. 1

(XBL 764-5424)

a retardation in the gasification rate for low PFCG, relative to that obtained without catalyst. An accelerating effect is achieved, however, during the period when the last $20 \%$ of fixed carbon is gasified. By contrast both $\mathrm{K}_{2} \mathrm{CO}_{3}$ and $\mathrm{KOH}$ significantly accelerate the gasification rate throughout the gasification process. Furthermore, it is noted that both catalysts behave identically. This observation is due to the fact that $\mathrm{KOH}$ is rapidly converted to $\mathrm{K}_{2} \mathrm{CO}_{3}$ in the presence of $\mathrm{OO}_{2}$.

Investigations of the effect of $\mathrm{K}_{2} \mathrm{CO}_{3}$ loading on gasification rate revealed that the rate increased with loading. The greatest increase was obtained during the gasification of the last $20 \%$ or so of the fixed carbon.

The effects of gasification temperature were also explored. For temperatures between 600 and $700^{\circ} \mathrm{C}$ gasification was not influenced by mass transfer inside the char pellet. Activation energies of $49.1,44.8$ and $39.1 \mathrm{kcal} / \mathrm{mole}$ were measured for samples containing 0,5 , and $10 \% \mathrm{~K}$ as $\mathrm{K}_{2} \mathrm{CO}_{3}$. At temperatures above $700^{\circ} \mathrm{C}$ gasification was increasingly limited by internal mass transfer.

The distribution of accumulated gaseous products is shown in Table 1 as a function of temperature and whether or not $\mathrm{K}_{2} \mathrm{CO}_{3}$ was used as 
Table 1. Distribution of accumulated gaseous products.

\begin{tabular}{rrrrrrr}
\hline & \multicolumn{2}{c}{$\mathrm{CO} /\left(\mathrm{CO}_{2}+\mathrm{CO}_{2}\right)$} & \multicolumn{2}{c}{$\mathrm{H}_{2} / \mathrm{CO}$} & \multicolumn{2}{c}{$\mathrm{CH}_{4} /\left(\mathrm{CO}+\mathrm{CO}_{2}+\mathrm{CH}_{4}\right)$} \\
$\mathrm{T}\left({ }^{\circ} \mathrm{C}\right)$ & uncat. & cat. & uncat. & cat.* & uncat. & cat. \\
\hline 700 & .107 & .159 & 18.2 & 11.9 & .0044 & .0019 \\
800 & .295 & .467 & 6.3 & 3.8 & .0100 & .0048 \\
900 & .556 & .836 & 2.9 & 1.9 & .0106 & .0063 \\
\hline
\end{tabular}

*5 wt: $\mathrm{K}\left(\mathrm{K}_{2} \mathrm{CO}_{3}\right)$

a catalyst. For both the catalyzed and uncatalyzed samples, an increase in temperature causes an increase in the yield of $\mathrm{CO}$ relative to $\mathrm{CO}_{2}$, a decrease in the ratio of $\mathrm{H}_{2}$ to $\mathrm{CO}$, and an increase in the yield of $\mathrm{CH}_{4}$. At a given temperature the use of $\mathrm{K}_{2} \mathrm{CO}_{3}$ as a catalyst causes an increase in the production of $\mathrm{CO}$, a decrease in the ratio of $\mathrm{H}_{2}$ to $\mathrm{CO}$, and a decrease in the yield of $\mathrm{CH}_{4}$. These trends indicate that catalyzed gasification favors the production of $\mathrm{CO}$ at the expense of the production of $\mathrm{H}_{2}$ and $\mathrm{CH}_{4}$.

\section{CATALYTIC SYNTHESIS OF METHANE}

(A.T. Be11, W.A. Bollinger, J.G. Ekerdt, C.S. Kellner)

The synthesis of methane from mixtures of carbon monoxide and hydrogen is an important step in the conversion of coal to synthetic natural gas. The development of catalysts resistant to deactivation by carbon deposition and poisoning by sulfur compounds is a major objective of current catalytic development programs. Our study is contributing to this effort through an investigation of the mechanism of methanation and an identification of the relation between catalyst structure and composition and catalys activity. To this end studies are being conducted on the adsorption of carbon monoxide and hydrogen on ruthenium, a particularly active methanation catalyst, and apparatus is being constructed to study the mechanism of methanation on ruthenium and other catalysts.

Studies of the kinetics and equilibria of hydrogen adsorption on a silica supported ruthenium catalyst have been conducted using gravimetric techniques. These investigations were conducted over a pressure range of 2 to 200 torr and for temperatures between 20 and $250^{\circ} \mathrm{C}$. For coverages approaching a monolayer the adsorption kinetics can be fitted by an Elovich equation. The activation energy associated with this equation varies linearly with coverage from 1 to $7.0 \mathrm{kcal} /$ mole at monolayer coverage. The adsorption isotherms were characterized by an initially curved segment which then joined a linearly increasing segment at higher pressures. The latter segment was observed at temperatures as high as $250^{\circ} \mathrm{C}$ and is indicative of the presence of physisorption. Estimated heats of adsorption varied between 4.9 and $3.0 \mathrm{kcal} / \mathrm{mole}$ for surface coverages between 0.46 and 1.0 .

To investigate the nature of the adsorbed hydrogen, thermal desorption spectra were taken.
An example is shown in Fig. 2. It is evident from this figure that two major and at least three minor forms of adsorption exist. The binding energies associated with these states are currently under investigation.

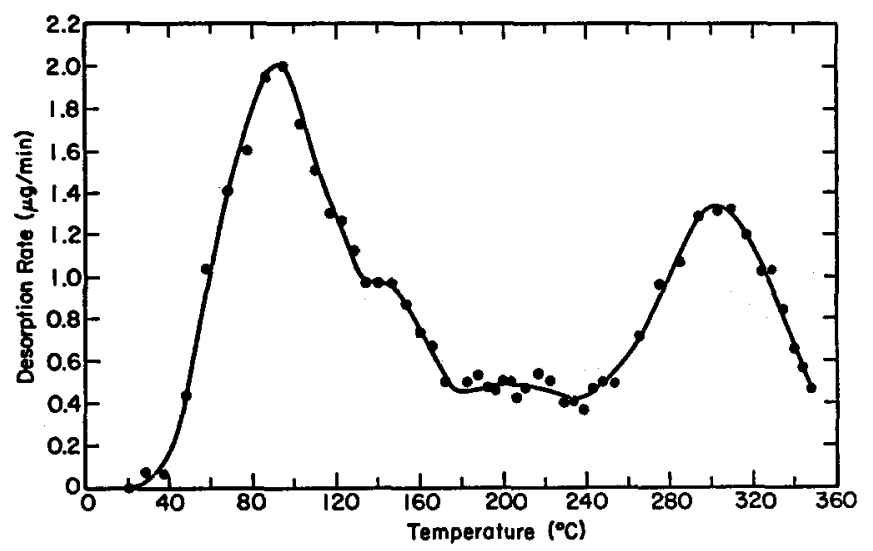

Fig. 2

(XBL 771-246)

Studies of carbon monoxide adsorption were also conducted, using techniques similar to those employed in studying hydrogen. Carbon monoxide chemisorption was considerably more rapid than that of hydrogen and precluded accurate measurement of its rate. The isotherms for carbon monoxide approached saturation at low pressures and showed no evidence of physisorption. A comparison of the saturation coverages obtained with hydrogen and carbon monoxide led to the conclusion that the ratio of $\mathrm{CO} / \mathrm{H}$ at saturation is 0.33 . This ratio is substantially lower than that reported earlier (up to 2) and suggests that a portion of the ruthenium surface sites is covered by carbon formed through a disproportionation of the adsorbed carbon monoxide. Up to $80 \%$ of the original hydrogen capacity can be restored, however, by mildly oxidizing and then reducing the catalyst. Further investigations of the deposition of carbon are now being pursued since this process is an important one for deactivating methanation catalysts.

Construction is being completed of a specially designed apparatus to be used for investigating the mechanism by which methane is synthesized from carbon monoxide and hydrogen. The central component of this apparatus is a cell which allows infrared spectra of the adsorbed species present on the catalyst surface to be recorded under reaction conditions. Through the use of this apparatus it will be possible to obtain both reaction rate data and information regarding the presence and interaction of species on the catalyst surface. These data will be used to learn more about the reaction pathways leading to methane.

HIGH-PRESSURE, LOW-PRESSURE STUDIES OF THE CARBON MONOXIDE-HYDROGEN REACTION ON TRANSITION METAL SURFACES

(G.A. Somorjai and A. Sexton)

The $\mathrm{CO}-\mathrm{H}_{2}$ reaction on transition metals is an important method of synthesizing organic fuels 
(alkanes, alkenes and alcohols). The normal synthesis conditions on industrial catalysts range from 1-100 atmospheres pressure and temperatures up to $500^{\circ} \mathrm{C}$. In order to study this reaction on well defined transition metal surfaces, an apparatus has been constructed which incorporates a high pressure isolation cell into the usual UHV apparatus. This enables the circulation of gases over the sample at pressures up to 100 atmospheres, and analysis of the reaction products with gas chromatography. After reaction the sample is exposed to vacuum and analyzed with Auger spectroscopy, LEED, and thermal desorption. Low pressure catalysis $\left(10^{-4}-10^{-8}\right.$ torr) can also be studied, with the mass spectrometer as a detector. A feature of this apparatus is a manipulator which allows $180^{\circ}$ rotation of the sample and direct-current heating. This is necessary in the cleaning and analysis of the sample surface.

Preliminary studies on the surfaces of iron, cobalt and rhodium at 1 atm. pressure have shown that they are catalytically active in the range $200-500^{\circ} \mathrm{C}$ and $\mathrm{C}_{7}-\mathrm{C}_{5}$ species are formed. The relative activities are in the order $\mathrm{Fe}>\mathrm{Co}>\mathrm{Rh}$. At lower pressures, $\left(10^{-4}\right.$ torr $)$ no products were detectable. The life of the catalyst sample in the high pressure chamber is shortened by the transfer of sulphur impurity from the stainless steel walls. Auger electron spectroscopy studies indicated that the active, methane producing rhodium surface is covered with a monolayer of carbonaceous residue.

Studies are presently underway to investigate the influence of pressure, temperature, gas composition and surface composition on the reaction on $\mathrm{Fe}, \mathrm{Co}, \mathrm{Rh}$ and $\mathrm{Ru}$ foils. These studies will be extended to include surface structure information on single crystals over the next 12 months.

\section{THE SYNTHESIS AND EVALUATION OF NOVEL CATALYSTS FOR COAL CONVERSION \\ (James C. Smart, Calvin Curtis, Allen Sievert and Kylan Tanner)}

A11 aspects of coal conversion will require effective catalysts for the hydrogenation and hydrogenolysis of covalent bonds. In an effort to catalyze such reactions in coal and related compounds, we have prepared a variety of soluble Group VIII metal carbonyl cluster compounds. These will be impregnated into the coal structure in an effort to depolymerize coal, with a minimum consumption of hydrogen, to yield liquid products. These products will be separated by high pressure liquid chromatography and characterized by a variety of standard techniques.

We are exploring the use of supported metal clusters in Fischer-Tropsch synthesis of alcohols and liquid hydrocarbons from $\mathrm{CO}-\mathrm{H}_{2}$ mixtures. We have designed and are constructing a vacuum/flow cell that will allow observation of a thin sample disc through salt plate and quartz windows. This cell will be used to photochemically activate silica and alumina supported metal cluster compounds at low pressure or in a gas flow, and to simultaneously monitor their infrared spectra. These studies, in conjunction with product analysis, and chemisorption studies will aid in determining what features of the metal clusters are involved in catalysis at the molecular level. In addition, comparisons can be made between these discrete, we11 defined cluster systems and related, highly dispersed metal catalysts.

Another aspect of our work has focused on the development of binuclear compounds with bridging organic ligands to yield novel homogeneous catalysts that will activate and combine sma11 unsaturated molecules. Utilizing the bridging bicyclopentadienide ligand, we have developed efficient syntheses of complexes of the following structural type:

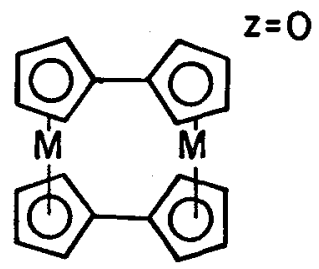

These thermally stable complexes of $\mathrm{V}^{\mathrm{II}}$, Cr II MoII are coordinatively unsaturated and will rapidly' coordinate small unsaturated molecules such as carbon monoxide. Our studies to date have focused on the $\mathrm{MbIII}$ complex, since there is precedent for activation (oxidative addition) of hydrogen as we11 as coordination of carbon monoxide in the "parent" mononuclear dicyclopentadienyl molybdenum. We have found that solutions of our dinuclear complex readily react with carbon monoxide at one atmosphere and room temperature to first yield a monocarbonyl complex and then a dicarbonyl complex. Infrared spectra indicate that the carbon monoxide of the monocarbonyl is bonded in a bridging manner, which is unprecedented in molybdenum carbonyl chemistry. Initial reactions with hydrogen at low pressure to yield a metal hydride have been unsuccessful, and studies at higher pressures are underway.

We have also developed alternative routes to the hydride and alkyl complexes, and we plan to react these with carbon monoxide and hydrogen in an effort to produce alcohols. We are also preparing the more reactive $\mathrm{T} i$ and $\mathrm{V}$ group derivatives, and a variety of other bridging ligands to control the geometry and electronic structure of these complexes.

STUDIES OF THE TRANSITION METAL CATALYZED HYDROGENATION OF BENZENOID AROMATICS

(K.P.C. Vollhardt,W.G.L. Aalbersberg, A.J. Barkovich, R.L. Funk, R.L. Hillard III)

The hydrogen content of coal is about $5 \%$ by weight while that for gasoline is about $14 \%$. Regardless of the technical process used to convert coal to a usable liquid fuel, hydrogen will have to be added to increase its hydrogen to carbon ratio. Chemically this problem translates into a need for a better understanding of the mechanism of polycyclic hydrocarbon hydrogenation and the development of nove1, more efficient and specific catalysts to effect hydrogenation and hydrogenolysis.

The aim of various ongoing projects is threefold: first the mechanism by which carbo- and 
heterocyclic aromatics are hydrogenated is being investigated using specifically constructed activated polycycles; second, novel catalysts are being constructed to specifically enable benzenoid hydrocarbon hydrogenation and ring-opening processes; third, basic research is aimed at elucidating the electronic influence of metals and their ligands on the stability and reactivity of remote bonds in an effort to understand the long range effects of transition metal complexation.

\section{Activated Benzenoids}

Several activated polycyclic hydrocarbons have been constructed, utilizing noyel synthetic routes developed in our laboratory. 1,2 These include 2, 3:6,7-dicyclobutabiphenylene, 3 1,2-cyclopropa-4,5cyclobutabenzene, ${ }^{4}$,3-cyclobutatriphenylene, and 2,3:6, 7:10,11-tricyclobutatriphenylene. 5 These hydrocarbons reveal remarkable reactivity with respect to catalytic hydrogenation which can be rapidly effected at normal pressures and ambient temperatures. This, of course, strikingly contrasts with the drastic conditions required to hydrogenate benzene, triphenylene and other polycyclic benzenoids. The above compounds are ideal model systems for an investigation of the mechanism of catalytic hydrogenation of aromatic molecules, and efforts are currently directed towards that goal. Routes to the corresponding heterocyclic analogs are also being explored. In addition, the potential carcinogenic hazard of these molecules is being tested.

\section{Nove1 Catalysts}

A polystyrene polymer-supported half-sandwich cobalt complex has been synthesized to evaluate its catalytic potential in $\mathrm{C}-\mathrm{C}$ and $\mathrm{C}-\mathrm{H}$ bond making and breaking processes. A completely novel ligand for the synthesis of tailored trinuclear metalclusters, 1,5,9-cyclododecatrine, has been synthesized. 6 This ligand is currently being tested as a matrix for heteronuclear cluster formation which might provide uniquely designed catalysts fd benzene hydrogenation and ring opening processes.

Effect of Transition Metal Complexation on the Stability of Remote Bonds

The new anti-irontricarbonyl complexes of bicyclo [4.2.1]-nonatrienone and-nonadienone have been synthesized, and the chemical reactivity of the remote ketone functionality is being explored and compared with the uncomplexed systems. We have observed unusual reactivity in reduction and oxidation reactions, changes in stereoselectivity and interesting trends in physical properties. Kinetic data on various functional group transformations are currently being assembled, but interpretation of the data will have to await further studies.

\section{REFERENCES}

1. W.G.L. Aalbersberg, A.J. Barkovich, R.L. Funk, R.L. Hillard III, and K.P.C. Vollhardt, J. Amer. Chem. Soc. 97, 5600 (1975).

2. R.L. Hillard III and K.P.C. Vollhardt, Angew. Chem., 87, 744 (1975); Angew. Chem. Internat. Edit., 14,712 (1975).

3. R.L. Hillard III and K.P.C. Vollhardt, J. Amer. Chem. Soc., 98, 3579 (1976).

4. C.J. Saward and K.P.C. Vollhardt, Tetrahedron Lett., 4539 (1975).

5. R.L. Funk and K.P.C. Volthardt, Angew. Chem., 88, 63 (1976); Angew. Chem. Internat. Edit., $\underline{15}, 53(1975)$.

6. A.J. Barkovich and K.P.C. Vollhardt, J. Amer. Chem. Soc., 98, 2667 (1976).

\section{THE $\mathrm{SO}_{3}-\mathrm{SO}_{2}$ SYSTEM FOR CHEMICAL STORAGE \\ OF THERMAL ENERGY}

S. Lynn and A.S. Foss

\section{INTRODUCTION}

Energy storage is of interest when either the source or the demand for energy is intermittent or widely variable. The first is the case for solar energy, wind, etc. And the second is usually the case for the power demand of most electric utilities, which varies with the time of day, the day of the week, and the season of the year. In the former case energy storage is essential if power is to be available at the time it is needed, rather than at the time it is provided by nature. In the second case energy storage offers a potential method of utilizing low-cost nuclear energy to provide a larger fraction of the daily power cycle than is presently practical.
There are a large number of approaches to the problem of energy storage. The solutions have been demonstrated on a commercial scale in some cases, but in others are still problematical. They include pumped water, compressed air, electric batteries, flywheels, magnetic fields, latent heat, and chemical systems. The last four mentioned are among the newer methods and are still in an early stage of development. Of these, the first three suffer the inherent disadvantage of a steady rate of dissipation of the stored energy. Chemical energy storage systems, on the other hand, are potentially stable under storage conditions. 
Chemical storage of thermal energy depends upon absorbing energy at high temperature by means of an endothermic reaction. The reaction products are cooled by heat exchange with the reactants and stored at ambient temperature. The heat is finally released by carrying out the reverse, exothermic reaction, again utilizing heat exchange to maximize the heat release in the reaction zone. To be effective, a reaction system must be reversible (with negligible by-products), require catalysis for both the endothermic and exothermic reactions, and have a relatively high heat of reaction. Additional questions involving cost, corrosivity, and toxicity must also be considered. The number of reaction systems meeting the essential criteria is severely limited. The $\mathrm{SO}_{3}-\mathrm{SO}_{2}-\mathrm{O}_{2}$ system is one of them.

The objective of this project is to design technically feasible flowsheets for energy storage using the sulfur oxide system and to estimate the thermal efficiency and equipment size which would result. From the analysis of the flowsheet, large sources of inefficiency and capital investment can be identified, and alternative designs can be proposed. Although the sulfur oxide system is being used as the mode1, many of the design features developed are expected to be applicable to other systems as well.

Work on this project started in the fall of 1974. However, funding was not obtained until the fall of 1975. The flowsheet shown in Fig. 1 is the first to be analyzed.

\section{ANNUAL ACTIVITIES}

Complete material and energy balances have been done on an expanded version of the Fig. 1 flowsheet. (To aid in visualization, a number of minor but essential processing steps are not shown in this diagram.) Equipment sizes have been estimated and rough approximations of equipment costs have been made. The thermal efficiency for this flowsheet, defined as the ratio of useful heat recovery to the sum of heat input and the thermal equivalent of work input, would be about $50 \%$. An analysis of major heat losses and equipment costs has led to flowsheet modifications which should increase thermal efficiency to about $80 \%$ and substantially reduce the cost of the equipment. The modifications consist primarily of operating the system at a nearly constant pressure, except for frictional drop due to flow, and making use of

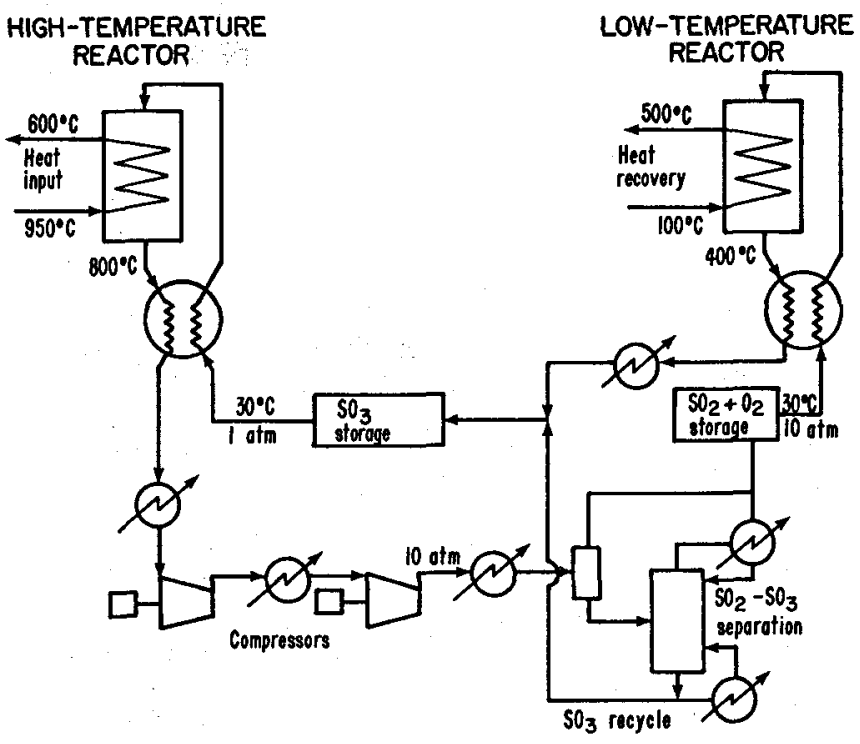

Fig. 1. Chemical energy storage flow chart.

(XBL 771-241)

waste heat from the power plant to which this system would be coupled. Further work on this revised flowsheet is planned.

An alternative chemical system for energy storage uses methane, water, carbon monoxide, carbon dioxide, and hydrogen. The steam reforming of methane is the endothermic, high-temperature reaction. Work has started on flowsheet construction for this system. The objective is not only to determine its potentiality but also to compare it with the $\mathrm{SO}_{3}-\mathrm{SO}_{2}-\mathrm{O}_{2}$ system for advantages and disadvantages.

\section{PLANNED ACTIVITIES FOR NEXT YEAR}

In addition to the continuation of the projects as indicated above, a thorough search for altemative chemical energy storage systems will be undertaken. This will include open, noncyclic systems in addition to closed, cyclic systems of the type which are presently under study. In the former type, a cheap, low-grade fuel such as waste cellulose would be converted to a high-grade fuel such as methane through the application of heat. Candidate reactions for this type of system have not yet been identified. 


\title{
Instrumentation Development
}

\author{
INTRODUCTION
}

Energy production and utilization result in the emission of pollutants that are potentially harmful to man and to other forms of life. It is desirable to provide means to measure these pollutants, to understand their fate, and to assess their effects on health and other biological activities such as agriculture.

In order to control these pollutants, adequate methods for monitoring the level of various substances often present at low concentrations are required. The Energy and Environment Division instrumentation development program is directed toward meeting these needs. Emphasis is on the development of physical methods (as opposed conventional chemical analysis techniques). The advantages, now widely recognized, include ease of operation coupled with minimal sample preparation. In some instances physical methods provide multi-parameter measurements, and they often provide the only means of achieving the sensitivity necessary for the detection of trace contaminants.

Among the areas in instrumentation now being investigated are laser opto-acoustic spectroscopy, $\mathrm{x}$-ray fluorescence spectroscopy, optical fluorescence spectroscopy, liquid crystal gas detectors, advanced forms of atomic absorption spectroscopy, electro-analytical chemistry, and mass spectroscopy.

\section{ORIGIN AND CONTROL OF THE DOMINANT IMPURITIES IN HIGH-PURITY GERMANIUM*}

\section{E. E. Haller, W. L. Hansen, G. S. Hubbard and F. S. Goulding}

\section{INTRODUCTION}

High-purity germanium single crystals suitable for large-volume thick radiation detectors have been grown in two 1aboratories over the last few years. 1,2 Experience in our laboratory is that purification, and to some degree crystal growth, have not hitherto been well controlled processes. Spontaneous appearance of contaminants, lack of purity of the starting material and deviation from 'normal' segregation of some impurities are serious problems. While we have reduced spontaneous contamination by maintaining a clean small working area and by working quickly with standardized procedures, the removal of impurities contained in the starting material has presented difficulties. Unfortunately, the quality of raw germanium as purchased from supplies is not under our control, and maximum impurity levels are not specified even in 'high-grade' material. It has therefore been necessary for us to adopt extra purification steps to reduce all impurities to the necessary low levels.

The well-established multi-pass zone purifica$\operatorname{tion}^{3}$ method is therefore used on germanium prior to our growing single crystals by the Czochralski technique. However, we have found that zonepurification using standard techniques rarely achieves the desired purity levels in the refined material that is to be used for crystal pulling. This failure of zone refining has led to our detailed studies of the segregation behavior of impurities in Czochralski single crystal growth in a very clean crystal puller. These studies have elucidated the mechanisms responsible for the poor results in zone-refining and have suggested better techniques which we are able to apply successfully.

\section{IMPURITY SEGREGATION DURING CZOCHRALSKI SINGLE CRYSTAL GROWTH AND ZONE PURIFICATION}

The value of the segregation coefficient $K$ of an impurity $(K=$ concentration in solid/concentration in liquid) tells directly how easily an impurity can be removed from a semiconductor by melting and recrystallization. Most impurities in $\mathrm{Ge}$ have a value of $\mathrm{K}$ between $10^{-3}$ to $10^{-5}$ (i.e.s. a very small number). Such impurities are easily removed. Our experiments indicate that neither of the acceptor elements boron or aluminum follow the expected behavior under our typical refining and growing conditions. We show that this complication is caused by these elements forming complexes with oxygen which do not segregate (i.e., $\mathrm{K}=1$ ).

Using a Czochralski crystal puller 4 together with analytical methods such as conductivity, Ha11-effect measurements and Phototherma1 Ionization Spectroscopy (PTIS) ${ }^{5}$ we have studied the formation of the complexes and have found ways to reduce their effect.

Edwards 6 showed in 1963 that, under suitable conditions, Al can form an electrically inactive complex with oxygen. In his work, carbon crucibles were employed and the oxygen was introduced in the ambient gas. In our case, we believe that the quartz crucible releases oxygen at the meltcrucible interface. An oxygen concentration of $5 \times 10^{13} \mathrm{~cm}^{-3}$ as determined by li-precipitation 
experiments 7 is typical in crystals grown from uartz crucibles. We will call such Ge 'oxygenrich'. The mechanism proposed by us to explain our results involves the formation of the Al-0 complex in the melt in the 'oxygen-rich' situation. We postulate that this Al-0 complex does not segregate between the liquid and solid Ge, so its concentration is the same in the solid and liquid. In the final crystal a small fraction of the A1-0 complex is assumed to dissociate to provide the elemental $\mathrm{Al}$ observed by electrical or PTIS measurement.

The behavior of boron in $\mathrm{Ge}$ also depends very much on the presence of oxygen as was shown by the investigations of Edwards.8 We have not studied boron to the same extent as aluminum since it is not usually present at high concentrations in our starting material. In contrast to $\mathrm{Al}$, we find a reduction of the boron concentration during multigeneration crystal growth in bare quartz crucibles that are etched in between successive use. Regrowing an oxygen rich boron-contaminated crystal under oxygen poor conditions does not significantly increase the boron concentration. This observation is in contrast to the results obtained by Edwards, who, after leaching oxygen from his system, found that the boron dopant again became an electrically active acceptor. The conflict can be resolved by assuming that the quartz crucibles in our experiments form stable boron-silicate glass layers at the melt-crucible interface. The etching of the crucible between successive crystal growing operations then removes the boron-contaminated layer and leads to a reduction in concentration. Edwards' experiments were carried out in graphite susceptors where no glass formation takes place.

The experiments discussed show that formation of an A1-O complex and the associated nonsegregating behavior of $\mathrm{Al}$ can be circumvented by using oxygen-poor conditions or by the addition of an appropriate oxygen getter (e.g., silicon). The next logical step in the development of a purification process is the application of these results to zone purification. The selection of a clean container for the germanium bar is more difficult than for the crystal puller since a material must be found to which $\mathrm{Ge}$ does not adhere. (The difference in thermal expansion coefficients demands that: the Ge bar be allowed to slide in its container.) Tests with bare carbon boats and carbon boats coated with pyrolitic carbon showed that boron and phosphorous, in substantial quantities, were present in the refined bars. Therefore, a better choice for a boat material seens to be quartz with a carbon smoke layer providing a suitable sliding surface. Tests show that such boats are satisfactory only if the Al concentration in the initial material is very low. For typical initial material, which contains high levels of $\mathrm{Al}$, an almost constant $\mathrm{Al}$ concentration is observed along the length of the refined polycrystalline bar. This is similar to the situation in single crystals grown from quartz crucibles.

In an attempt to improve the barrier between the quartz boat and the molten zone, and thereby to reduce the oxygen concentration, we have coated quartz boats with a pyrolitic-carbon film on top of the smoke layer. Bars refined in such boats exhibit some Al segregation but the effective segregation coefficient is still rather close to 1 . We observe that the pyrolitic carbon layer may itself be a source of boron causing an average boron concentration $\simeq 10^{11} \mathrm{~cm}^{-3}$.

The failure of pyrolitic-carbon coatings to provide the ideal oxygen poor conditions required in the zone refiner and their effect in introducing boron led to a search for alternatives. The best solution has proved to be a quartz smoke layer on the quartz boat produced by burning silane $\left(\mathrm{SiH}_{4}\right)$. The results obtained with this type of coating are striking, the impurity concentration being in the $10 \mathrm{w} 10^{10} \mathrm{~cm}^{-3}$ range over $90 \%$ of the polycrystalline bar. The dominant impurity is Al. Very little boron can be detected even when initial material containing boron is used. Phosphorous appears only in the very tail of the bar. The flat concentration profile suggests oxygen rich conditions and complex formation. This is confirmed by growing crystals from this starting material under oxygen rich and oxygen poor conditions.

Single crystals grown from such polycrystalline germanium usually have net-impurity concentrations below $2.5 \times 10^{10} \mathrm{~cm}^{-3}$ in more than $80 \%$ of their volume (see Fig. 1). An obvious concern is that the presence of silicon or $\mathrm{Si}_{\mathrm{x}}-\mathrm{O}_{\mathrm{y}}$ in the germanium might cause trapping in detectors. However, $1 \mathrm{~cm}$ thick detectors fabricated from these single crystals exhibit no visible trapping in $60 \mathrm{Co}$ spectra indicating that the effect of the silicon is negligible.

\section{CONCLUSIONS}

These experiments with single crystal growth and zone purification show that it is possible to create conditions which substantially reduce the levels of electrically-active impurities in germanium. An oxygen poor environment or the addition of silicon as an oxygen getter prevents complex formation with the associated non-segregating behavior (particularly of Al).

We have shown that zone-refining carried out in quartz boats coated with quartz-smoke produced adequate low impurity levels. If the raw germanium

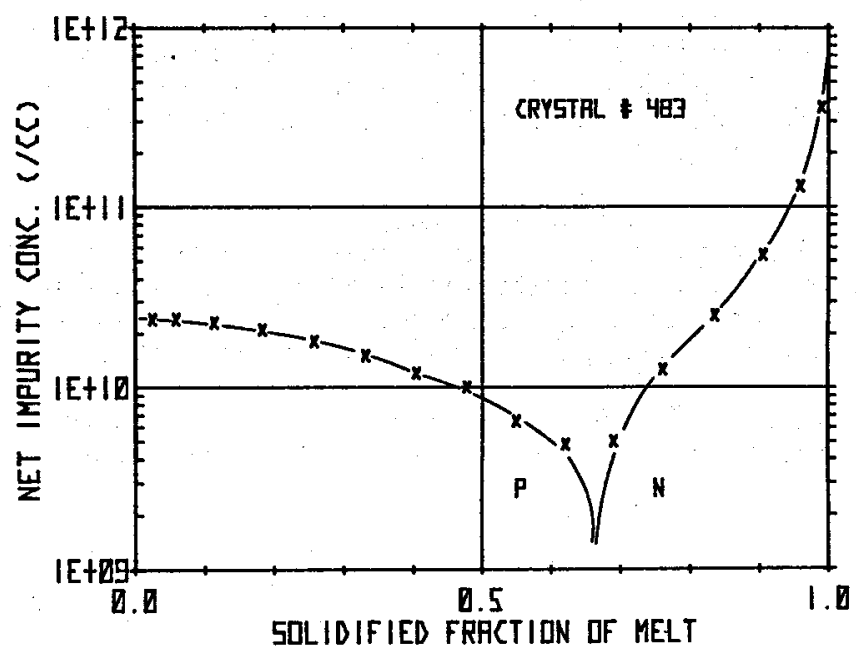

Fig. 1.

(XBL 7511-9010) 
is very impure, a two step process, first zone-refining in a quartz boat coated with pyrolitic carbon or in a graphite boat, and then refining in a quartz boat coated with quartz smoke, will reduce all impurity concentrations to low levels. Using this method it is possible to use any commercially available intrinsic ( $40 \Omega \mathrm{cm})$ germanium for starting material which means a substantial reduction in initial material cost.

The lower limit of the impurity concentration in our high-purity single crystals is set by the phosphorous released from quartz crucibles. Suprasil crucibles contribute the lowest phosphorous contamination (about $2 \times 10^{10} \mathrm{~cm}^{-3}$ near the seed end of the crystal). Detector-grade single crystal can be grown in one pull from suitably zone-refined material and no compensating impurities need be added to the melt.

\section{ACKNOWLEDGEMENTS}

We would like to thank Professor P.L. Richards and his colleagues at U.C. Berkeley for the use of the Fourier Transform Spectrometer in our PTIS studies. R.C. Cordi and R.H. Pehl are responsible for data on detector performance. Special thanks also go to A.J. Travendale of the Australian Atomic Energy Commission and his colleagues for the Spark Source Mass Spectroscopy results on a number of $\mathrm{Ge}$ samples.
FOOTNOTE AND REFERENCES

* Condensed from LBL-4273.

1. R.N. Hall, IEEE Trans. Nuc1. Sci., NS-21, No. 1, 260 (1974), also: Proc. of the Twelfth Int. Conf. Physics of Semiconductors, Stuttgart, 363 (1974).

2. W.L. Hansen and E.E. Ha1ler, IEEE Trans. Nuc1. Sci., NS-21, No. 1, 251 (1974).

3. W.G. Pfann, Zone Melting, John Wiley \& Sons (1966).

4. W.L. Hansen, Nucl, Instr. and Methods, 94, 377 (1971).

5. E.E. Haller, W.L. Hansen and F.S. Goulding, IEEE Trans. Nuc1. Sci., NS-22, No. 1, 127 (1975). also: IEEE Trans. Nucl. Sci., NS-21, No. 1, 279 (1974).

6. W.D. Edwards, J. App1. Physics, 34, 2497 (1963).

7. R.J. Fox, IEEE Trans. Nuc1. Sci., NS-13, No. 3, 367 (1966).

8. W.D. Edwards, J. Appl. Physics, 39, 1784 (1968).

\title{
THE SURVEY OF INSTRUMENTATION FOR ENVIRONMENTAL MONITORING*
}

\author{
D. A. Mack, N. M. Amer, P. Bhardwaja, R. J. Budnitz, \\ C. D. Hollowell, M. J. Kland, R. A. MeLaughlin, G. A. Morton, S. L. Phillips
}

In response to the need for up-to-date in information on pollution monitoring systems technology, Lawrence Berkeley Laboratory publishes the survey entitled Instrumentation for Environmental Monitoring (LBL-1) in a loose-leaf format that facilitates frequent up-dating. LBL-1 consists of four volumes: 1. Air (Parts 1 and 2), 2. Water, 3. Radiation, and 4. Biomedical. The contents of the most recent up-dates of these volumes are summarized in Table 1 and described in greater detail in a brochure published by the Technical Information Department. 1

Since its inception LBL-1 has enjoyed recognition both in the United States and abroad as a source book for monitoring information in the four areas covered by its volumes, over 3000 copies of which have been purchased to date by both private and public laboratories and other agencies. In addition, some 450 copies have been distributed to emviromment scientists in government agencies and 1aboratories.

The LBL-1 survey has also proven to be a useful pedagogic tool in the envirommental monitoring field where textbook materials are as yet limited.
To be most useful, sophisticated monitoring equipment must be provided with reliable sampling materials, and techniques as well as primary standardization and calibration methods. Therefore, the principle focus of the past year has been in this area of instrument calibration, sampling, and standardization of analytical techniques, and also in the identification and evaluation of standards, both primary and derived, their sources and avai1ability as they relate to the contents of the Air and Water volumes primarily. The up-dating of all four volumes of LBL-1 will be continued in the coming year.

\section{FOOTNOTE AND REFERENCE}

* Research supported by the National Science Foundation, Research Applied to National. Needs and the U.S. Energy Research and Development Administration.

1. Available from the Technical Information Department, Lawrence Berkeley Laboratory, University of California, Berkeley, CA 94720. 
Table 1. Pollutants and parameters covered in Survey of Instrumentation for Environmental Monitoring.

\begin{tabular}{|c|c|c|c|}
\hline $\begin{array}{l}\text { Vol. I } \\
\text { AIR }\end{array}$ & $\begin{array}{l}\text { Vol. II } \\
\text { WATER }\end{array}$ & $\begin{array}{l}\text { Vo1. III } \\
\text { RADIATION }\end{array}$ & $\begin{array}{l}\text { Vo1. IV } \\
\text { BIOMEDICAL }\end{array}$ \\
\hline $\begin{array}{l}\mathrm{SO}_{2} \text { (updated) } \\
\mathrm{NO}^{2} \text { and } \mathrm{NO}_{2} \\
\text { Oxidants } \\
\mathrm{CO} \\
\text { Hydrocarbons } \\
\text { Particulates } \\
\text { Mass } \\
\text { Size } \\
\text { Opacity } \\
\text { Composition } \\
\text { Mercury } \\
\text { Asbestos } \\
\text { Beryllium } \\
\text { Lead } \\
\text { Noise } \\
\text { Instrument } \\
\text { Calibration } \\
\text { Sanpling } \\
\text { Techniques }\end{array}$ & $\begin{array}{l}\text { Metals } \\
\text { Nitrogen } \\
\text { Phosphorus } \\
\text { Sulfur } \\
\text { Biochemical Oxygen } \\
\quad \text { Demand } \\
\text { Chemical Oxygen } \\
\text { Demand } \\
\text { Dissolved Oxygen } \\
\text { Total Organic Carbon } \\
\text { Pesticides } \\
\text { Phenolics } \\
\text { Petrochemicals } \\
\text { Oil and Grease } \\
\text { pH, Alkalinity, } \\
\text { Acidity } \\
\text { Radiation } \\
\text { Turbidity } \\
\text { Temperature } \\
\text { Instrument } \\
\text { Calibration }\end{array}$ & $\begin{array}{l}\text { Alpha Radiation } \\
\text { Beta Radiation } \\
\mathrm{X} \text { and Gamma } \\
\text { Radiation } \\
\text { Gamma Spectroscopy } \\
\text { Neutrons } \\
\text { Personnel Dosimetry } \\
\text { Radionuclides } \\
\text { Tritrium } \\
\text { Krypton-85 } \\
\text { Strontium-89 and -90 } \\
\text { Iodine-129 and -131 } \\
\text { Radon-222 and its } \\
\text { daughters } \\
\text { Radium } \\
\text { Uranium } \\
\text { Plutonium } \\
\text { Microwaves } \\
\text { Lasers } \\
\text { U1traviolet }\end{array}$ & $\begin{array}{l}\mathrm{CO} \\
\mathrm{SO}_{2} \\
\text { Asbestos } \\
\text { Mercury } \\
\text { Cadmium } \\
\text { Lead } \\
\text { Pesticides } \\
\text { Radiation }\end{array}$ \\
\hline
\end{tabular}

IDENTIFICATION OF THE CHEMICAL COMPOSITION OF TRACE ELEMENTS BY EXTENDED X-RAY ABSORPTION FINE STRUCTURE

A. Thompson, J. Jaklevic, F. Goulding,

\section{INTRODUCTION}

A non-destructive, sensitive technique to identify the chemical form of various elements which are present in trace amounts in a sample would provide a very powerful research tool for many applications. A possible technique for doing this, which we have been investigating, utilizes the variation of the K X-ray photoelectric cross section near the absorption edge. Very near the absorption edge the cross section for a given element is influenced by the valence of the atom and further above the edge up to about $500 \mathrm{eV}$ the cross section can have a fine structure which is dependent on the position and identity of the atom's nearest neighbors.

Studies of this extended $X$-ray absorption fine structure (EXAFS) have undergone a renaissance recently with development of high-intensity, tunable $X$-ray beam lines using synchrotron radiation from electron storage rings. Using the $\mathrm{X}$-ray beam line at the Stanford Synchrotron Radiation Project (SSRP) on the SPEAR storage ring at the Stanford Linear Accelerator we have made a preliminary study of the zinc EXAFS spectra of several samples which had zinc concentrations of about $5 \mu \mathrm{g} / \mathrm{cm}^{2}$ and obtained some very promising results.

\section{CURRENT ACTIVITIES}

In Fig. 1 the basic layout of our experiment is presented. Synchrotron radiation from SPEAR is incident on a channel cut double crystal monochromator which produces a $20 \mathrm{~mm}$ by $0.5 \mathrm{~mm} X$-ray beam with an intensity of between $10^{8}-10^{10}$ photons/sec in an energy bandwidth of $1 \mathrm{eV}$. By rotating the monochromator crystal the beam energy is easily varied from about $5 \mathrm{keV}$ to over $20 \mathrm{keV}$. The experiment consists of sweeping the incident beam over the absorption edge in steps of about $3 \mathrm{eV}$ and at 


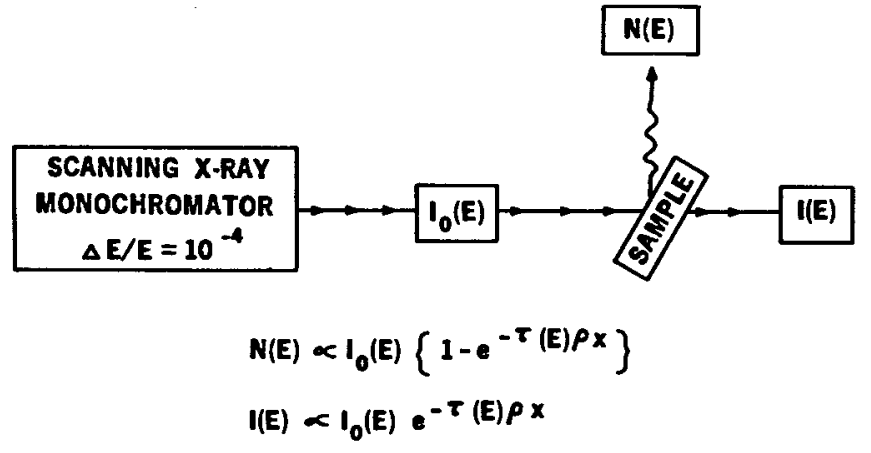

Fig. 1. Schematic layout of the EXAFS experiment. $I_{0}(E)$ and $I(E)$ are measured with ionization chambers and N(E) is measured with a Si(Li) detector. The formulas give the dependence of $N(E)$ and $I(E)$ on the incident flux $I_{0}(E)$, photoelectric cross section $\tau(E)$, density $\rho$ and thickness $x$.

(XBL 764-1102)

each energy measuring the incident beam $I_{0}(E)$, the transmitted beam I(E) and the fluorescent $x$-ray yield N(E). An ionization chamber which absorbs about $10 \%$ of the incident flux is used to measure $I_{p}(E)$. I (E) is measured with a large ionization chamber and $N(E)$ is determined with a high resolution Si(Li) detector.

The variation of the photoelectric cross section $\tau(E)$ is calculated from the formulas given in Fig. 1. The measurement using both ion chambers is very good for concentrated samples but for diluted samples the fluorescent detector is much better. Since we are primarily interested in samples with only small concentrations we have concentrated on optimizing the fluorescent detector system with the use of X-ray filters and special electronics.

To illustrate the potential of this technique we have made a study of the zinc absorption edge with three different pollution filters which had zinc concentrations of $3.8,0.9$ and $5.6 \mu \mathrm{g} / \mathrm{cm}^{2}$. These samples were selected from a St. Louis Air Pollution Study to represent three different conditions. The first sample is of small particles $(<2.4 \mu \mathrm{m})$ from an urban site, the second is of small particles from a rural site and the third is of large particles $(>2.4 \mu \mathrm{m})$ from an urban site. Figure 2 shows the fluorescent EXAFS spectra we obtained from the three samples. All the data was taken in about three hours of beam time. From the figure it can be seen that both the small particle samples (a) and (b) have similar spectra whereas the large particle sample (c) is somewhat different from the other two. Figure 3 shows the comparison of the first sample EXAFS spectra with standard EXAFS spectra from zinc sulfate and zinc metal. The sample spectra were also compared to the spectra of $\mathrm{ZnO}, \mathrm{ZnS}$ and $\mathrm{ZnCl}_{2}$ and the agreement with $\mathrm{ZnSO}_{4}$ was much better than the other compounds. This preliminary study then indicates that the zinc present on the small particle urban and rural samples is primarily zinc sulfate and the coarse particle zinc is probably partially zinc sulfate with some other additional component.

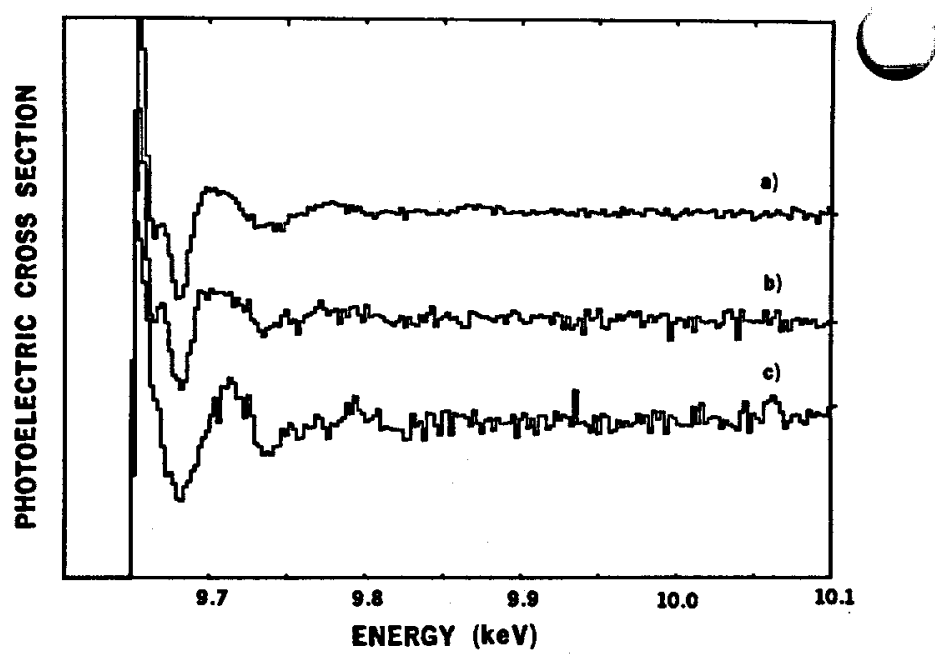

Fig. 2. Plot of the fine structure in the zinc photoelectric cross section as a function of incident photon energy near the K X-ray edge. Curve a) is with a sample containing $3.8 \mu \mathrm{gg} / \mathrm{cm}^{2}$ of small particle $(<2.4 \mu \mathrm{m})$ zinc, curve $b$ ) is with one containing $0.9 \mu \mathrm{g} . \mathrm{cm}^{2}$ of small particle zinc and curve c) is with a sample of $5.6 \mu \mathrm{gg} / \mathrm{cm}^{2}$ large particle $(>2.4 \mu \mathrm{m})$ zinc. Curves (b) and (c) have been translated vertically to improve the comparison of the three samples.

(XBL 764-1101)

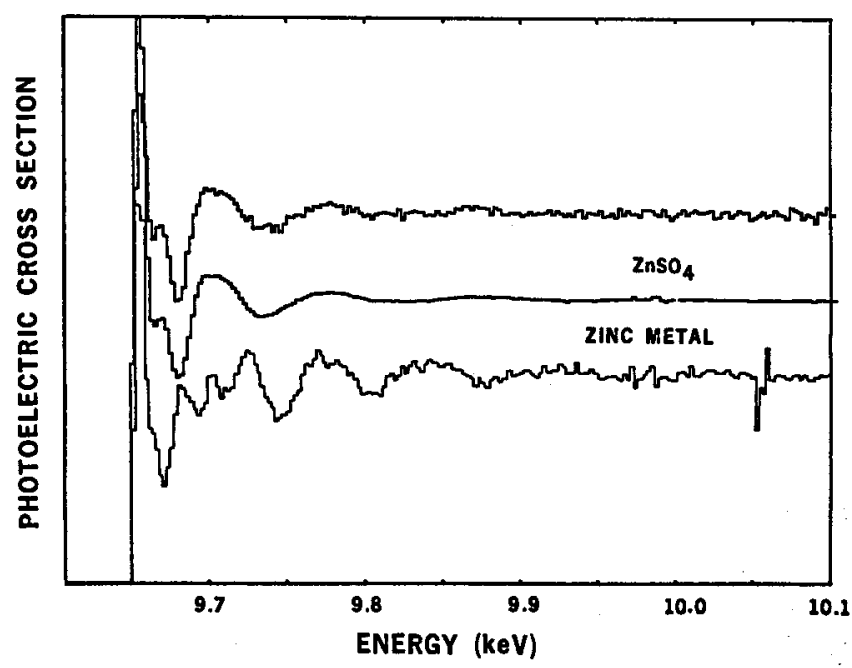

Fig. 3. Plot of zinc photoelectric cross section against incident photon energy. The top curve is the same as curve (a) of Fig. 2 and the two bottom curves are comparison curves for standards of zinc sulfate and zinc metal.

(XBL 771-7308)

\section{SUMMARY}

Our preliminary measurements of the EXAFS spectra of several low concentration samples of 
zinc have shown that this is a very promising technique to identify the chemical form of elements which are present in only trace amounts. This technique has the advantages that it is non-destructive, it measures the properties of the bulk material and not only the surface atoms and it can measure individual elements in a complicated sample. There is no doubt that more careful analysis and comparisons of spectra used for the identification of the compounds will allow the analysis of more complex mixtures of compounds than those considered here. As the technique is improved it should find possible applications in many fields. Some of the immediate fields which come to mind are envirommental monitoring, geology and biochemistry.

\title{
ENERGY DISPERSIVE X-RAY FLUORESCENCE SPECTROMETRY USING PULSED X-RAY EXCITATION*
}

\author{
J. M. Jaklevic, D. A. Landis and F. S. Goulding
}

\section{INTRODUCTION}

Semiconductor spectrometer systems intended to operate at high counting rates typically use a pileup rejection circuit to prevent distortion of the pulse-height spectrum due to interference between successive pulses. These circuits measure the interval between successive pulses and reject those which fail to satisfy certain criteria.

Figure $1(A),(B)$ and (C) show the timing sequence of pulses that accompany the detection of an $x$-ray in the detector. The fast output indicates the time of arrival of an event and is used to detect the occurrence of pile-up. The slow output has been filtered to achieve a symmetric Gaussian shape with peaking time of $\tau_{p}$. Associated with the detection of the event is a dead time $\tau_{d} \approx 2 \tau_{p}$ during which pileup may occur. Typical values for the dead time vary from 20 to $70 \mu s$.

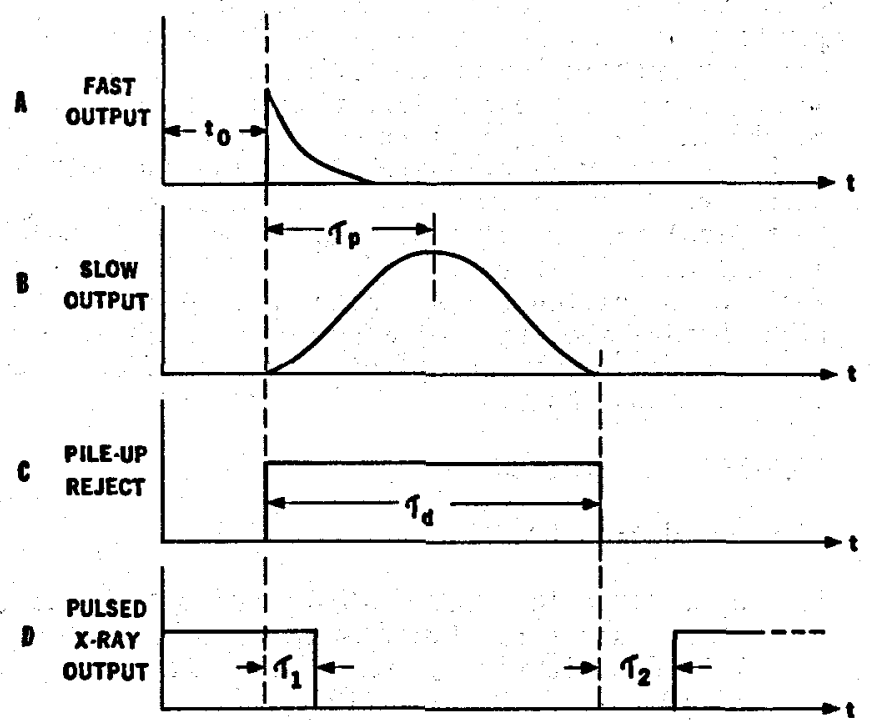

Fig. 1. Timing sequence of $x$-ray spectrometer outputs.

(XBL 756-1684)

Figure 2 (b) shows the rate of the non-pile-up output pulses as a function of input counting rate. The scale is in units of $\left(1 / \tau_{d}\right)$. The curve can be

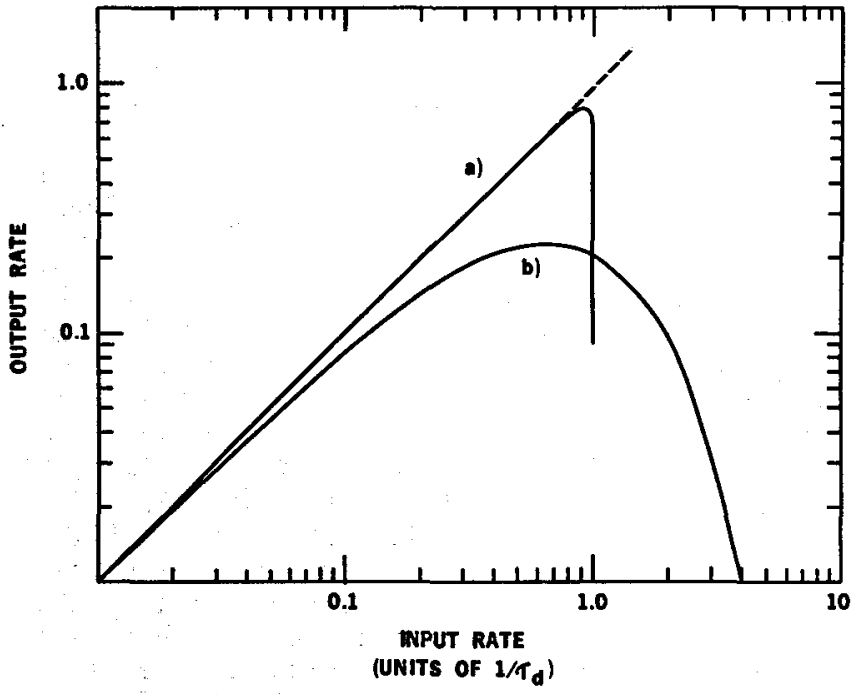

Fig. 2. Output rate vs input rate.

(XBL 756-1685)

derived from elementary probability considerations assuming a random arrival distribution and is represented by the equation:

$$
N_{0}=N \exp ^{-N \tau}
$$

where $\mathrm{N}_{0}$ and $\mathrm{N}$ are the average output and input counting rates and $\tau$ is the dead time of the pileup circuit. Since most circuits reject a pulse if it is preceded by an earlier pulse in a time less than $\tau_{\mathrm{d}}$ or if it is followed by one in less than $\tau_{p}$, the curves assume $\tau=3 \tau_{\mathrm{d}} / 2$. The maximum possible output rate achieved under such conditions is less than $1 / 4 \pi \mathrm{d}$.

The pulsed X-ray tube that we have developed overcomes this limitation by eliminating the possibility of a further event, arriving during the time $\tau_{\mathrm{d}}$ taken to process a pulse. Figure 3 is a schematic diagram of the simplest method of pulsed excitation. A discriminator on the fast output of the detector senses the arrival of an event and initiates turning off the pulsed $X$-ray tube for an interval equal to the time $\tau_{d}$ required to fully process the first pulse. Ideally no pile-up can occur and the output rate is equal to the input rate up to a maximum input rate of $1 / \tau$. At this point the events are essentially periodic. 


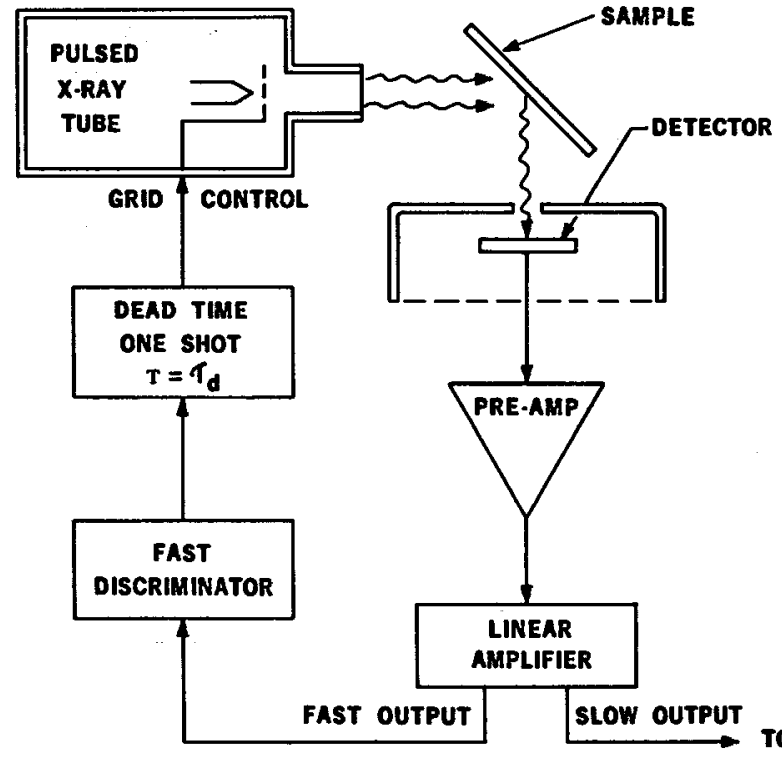

Fig. 3. Schematic of a pulsed excitation system.

(XBL 756-1686)

In actual systems there is a slight delay in sensing the event and turning off the tube $\left(\tau_{1}\right.$ in Fig. 1(D)) resulting from the finite propagation time of the signal from the detector to the X-ray tube grid. During this period, pile-up can occur at the higher counting rates. However, since this time interval is typically small $(<300 \mathrm{~ns})$ the pile-up is almost negligible. The curve in Fig. 2(a) shows the non-pile-up output of a pulsed system. The slight curvature near the limiting rate of $1 / \tau_{\mathrm{d}}$ is a result of the pile-up at higher rates. If the input rate is further increased after achieving a periodic rate at $N=1 / \tau_{d}$, all the additional events are pile-up pulses and the output of undistorted pulses drops rapidly to zero.

\section{RESULTS}

The pulsed X-ray tube was adapted to an earlier design of excitation system using $\mathrm{Ti}, \mathrm{Mo}$ and $\mathrm{Tb}$ as secondary fluorescers. 3 This range of exciting energies allows the analysis of elements ranging from $\mathrm{Al}$ to $\mathrm{Ba}$ in the periodic table. The semiconductor spectrometer was a guard-ring type 4 with energy resolution of $185 \mathrm{eV}$ at $5.9 \mathrm{keV}$ using a Gaussian shaper peaking at $17 \mu \mathrm{s}\left(\tau_{\mathrm{p}}\right.$ in Fig. 1).

The control circuits were adjusted to correspond to this processing time of $\tau_{d}=50 \mu \mathrm{s}$ and a maximum duty factor corresponding to $10 \mu \mathrm{s}$ maximum pulse width. The minimum on time setting was $4 \mu \mathrm{s}$ corresponding to 58 pile-up probability during the turn-off delay time. This corresponds to an instantaneous counting rate at the minimm duty cycle condition of $10^{5} \mathrm{cts} / \mathrm{sec}$. The maximum anode dissipation was $100 \mathrm{~W}$ when achieving the peak counting rate $(20 \mathrm{kc} / \mathrm{s})$ on samples whose mass exceeded 4 $\mathrm{mg} / \mathrm{cm}^{2}$.

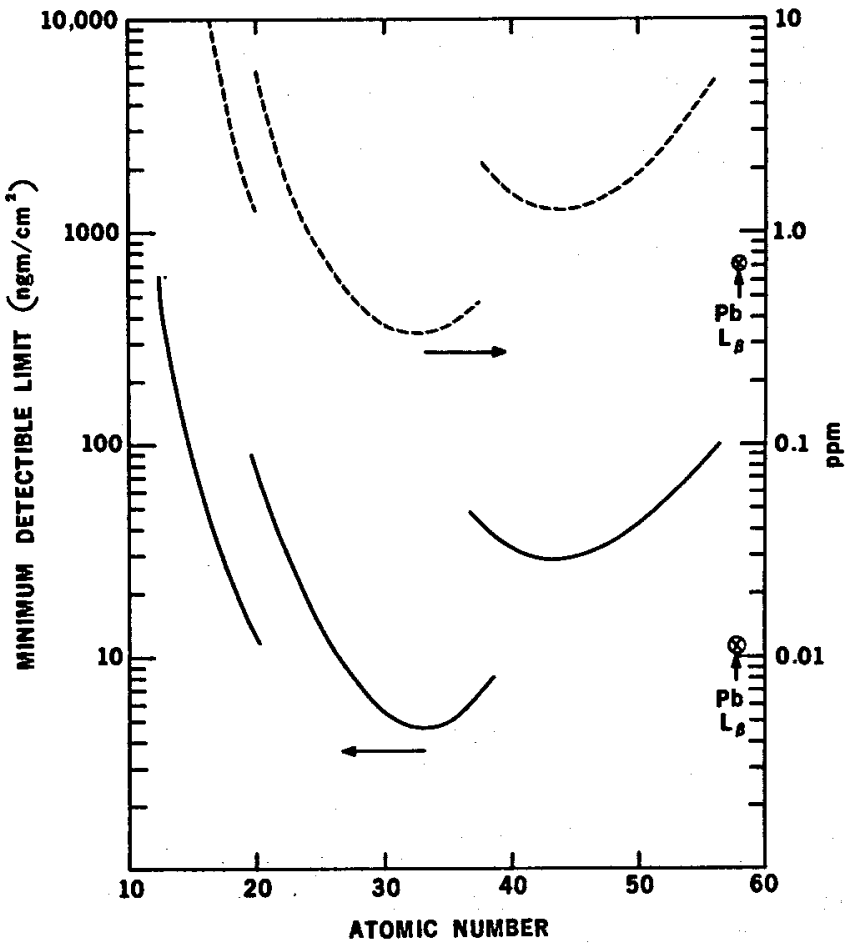

Fig. 4. Detectibility limits using pulsed $x$-ray tube. The solid curves assume a $5 \mathrm{mg} / \mathrm{cm}^{2}$ membrane type filter and refer to the left ordinate. The dashed curves assume a $30 \mathrm{mg} / \mathrm{cm}^{2}$ biological specimen and refer to the right ordinate.

(XBL 757-1840)

Measurements have been made using a number of specimen types in order to verify the performance of the system. Measured detectibility limits are shown in Fig. 4. These results represent the quantity of each element required to produce a number of counts in the characterisitic peak equal to $3 \sigma$, where $\sigma$ is the rms deviation in the number of counts in the background inmediately beneath the peak. An analysis time of $100 \mathrm{sec}$ is assumed. Results are given for two substrates corresponding to typical biological specimens $\left(30 \mathrm{mg} / \mathrm{cm}^{2}\right)$ and air pollution filters $\left(5 \mathrm{mg} / \mathrm{cm}^{2}\right)$. The dashed curves are the biological specimens and refer to the right hand scale; the solid curves were obtained with $5 \mathrm{mg} / \mathrm{cm}^{2}$ membrane filters and are referred to the left hand scale.

\section{FOOTNOTE AND REFERENCES}

${ }^{*}$ Condensed from LBL-4248

1. J.M. Jaklevic, F.S. Goulding and D.A. Landis, 'High Rate X-ray Fluorescence Analysis by Pulsed Excitation", IEEE Trans. Nucl. Sci. NS-19, No. 3, 392-395 (1972).

2. F.S. Goulding and J.M. Jaklevic, "Development of Air Particulate Monitoring Systems". EPA Environmental Monitoring Series Report \#EPA650/4-74-030 (1974). 
3. F.S. Goulding, J.M. Jaklevic and B.W. Loo, "Fabrication of Monitoring System for Determining Mass and Composition of Aerosol as a Function of Time", EPA Technology Series Report \#EPA-650/2-75-048 (1975). LBL Report UCID-3767.
4. J.M. Jaklevic and F.S. Goulding, "Semiconductor Detector X-ray Fluorescence Spectrometry Applied to Environmental and Biological Analysis" IEEE Trans. Nuc1. Sci. NS-19, No. 3, 384-391 (1972).

\title{
OPTIMAL DESIGN CRITERIA FOR MILLIMETER-WAVE SPECTROMETERS
}

\author{
B. Leskovar, D. B. Hopkins, W. F. Kolbe
}

The millimeter-wave high resolution spectrometer consists of a mechanically and electrically tumable microwave generator (energy source), a high- $Q$ cavity absorption cell, a low-noise mícrowave receiver with an automatic frequency control device, and a data processing system. The sensitivity of the millimeter-wave spectrometer is essentially determined by the total noise introduced during generation of the microwave radiation, the absorption process in the resonant cavity, and the detection and amplification of a spectral line signal. A simplified diagram of the system with all relevant noise sources is shown in Fig. 1. The total noise of the spectrometer includes the AM and FM noise side-bands of the microwave oscillator, thermal radiation noise of the sample absorption cavity, and noise of the microwave receiver.

The AM noise characteristic of the carrier is usually defined by the double-side band AM noise power to carrier power ratio in a bandwidth $B$, measured at a distance $f_{m}$ from the carrier, (N/C) Similarly, the FM noise of the carrier is defined ${ }^{2}$ as the rms noise deviation, $\Delta f_{m m s}$, measured in a bandwidth $B$ at the output of an FM discriminator with a microwave oscillator at its input. Generally,

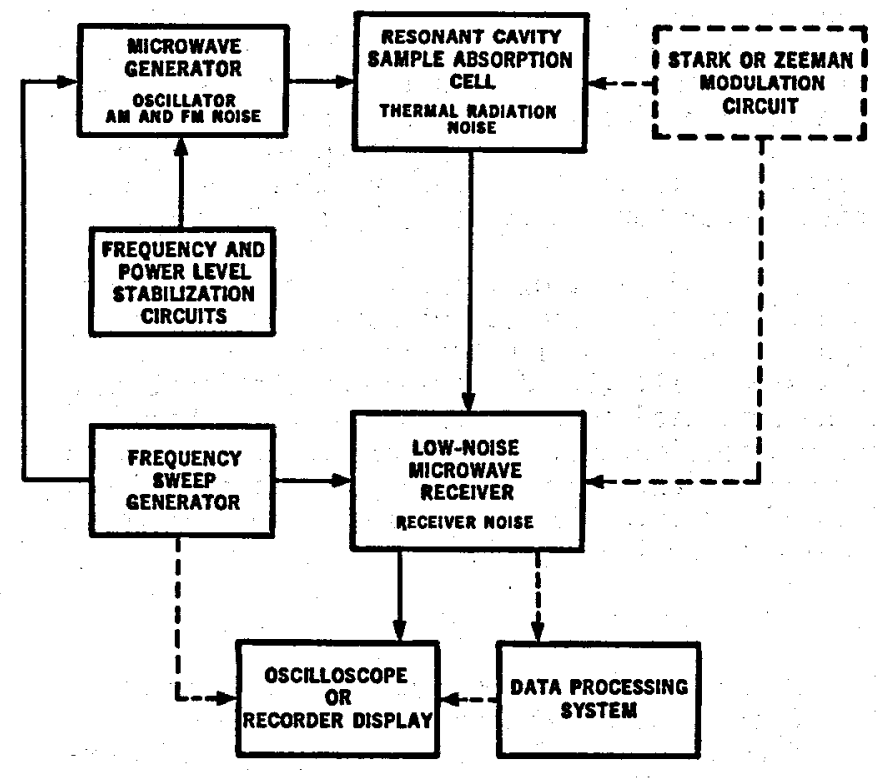

Fig. 1. Typical Millimeter-wave spectrometer system with noise sources.

(XBL 756-1495) accurate measurements of AM and FM source noise at carrier frequencies above about $18 \mathrm{GHz}$ are rarely made. From device manufacturers' comments and limited measurements2-5 together with our calculations and measurements, at 10,28 and $35 \mathrm{GHz}$, an estimate of source noise to be expected at higher frequencies can be made. The $\mathrm{AM}$ and $\mathrm{FM}$ noise performance of microwave sources were averaged, normalized, and extrapolated to frequencies of 100 $\mathrm{GHz}$ for klystron sources and to $90 \mathrm{GHz}$ for a Gunn oscillator. (Because of finite inter-valley transfer time, the GaAs Gunn oscillator loses its differential negative mobility at about $90 \mathrm{GHz}$.) The source AM noise characteristics were normalized and extrapolated according to the expression:

$$
(\mathrm{N} / \mathrm{C})_{\mathrm{DSB}}^{\mathrm{AM}}=\left(\mathrm{kTB} / \mathrm{P}_{\mathrm{o}}\right) /\left[(\mathrm{S} / 2)^{2+}\left(\mathrm{Q}_{\text {ext }} \mathrm{f}_{\mathrm{m}} / \mathrm{f}_{0}\right)^{2}\right]
$$

where $k$ is Boltzmann's constant, $T$ is the effective temperature of the device, $B$ is the bandwidth, $P_{0}$ is the device output power, $S$ is the saturation factor given by Edson; 6 and Qext is the external circuit quality factor. The source FM noise characteristics were normalized, and extrapolated according to the equation:

$$
\Delta f_{r m s}=\left(f_{o} / Q_{\text {ext }}\right)\left[\mathrm{kTB} / \mathrm{P}_{0}\right]^{1 / 2}
$$

The results are presented in Fig. 2 for free rumning oscillators, without external stabilization. On the basis of $A M$ and FM noise alone, solid-state Gunn oscillators presently appear to be superior to reflex klystrons, up to frequencies of approximately $35 \mathrm{GHz}$. Above this frequency the reflex klystrons, and particularly the two-cavity klystrons, are superior to Gunn devices.

The equivalent noise temperature contribution, $T_{e f f}$, of the microwave source to the total noise temperature of the spectrometer at the input of the detector has been calculated by means of eq. (1) derived from the consideration given in Ref. 1 .

Using a $100 \mathrm{~mW}$ reflex klystron source in the spectrometer, with $f_{Q}=70 \mathrm{GHz}, Q_{O}=2 \times 104$, $f_{m}=10 \mathrm{kHz}$, it can be seen from Fig. 2 that $\Delta \mathrm{f}_{\mathrm{rms}}=18 \mathrm{~Hz}$ and $(\mathrm{N} / \mathrm{C}) \mathrm{AM}=-125 \mathrm{~dB}=3.16 \times 10^{-13}$. Equation (1), from Ref. 1 , gives, for $S=10^{-9}$, $\xi=10^{-2} \mathrm{rad}, B_{S}=100 \mathrm{~Hz}, P_{0}=10^{-4} \mathrm{~W}$, and $\Gamma^{2}=$ $\left(Q_{o} f_{m} / f_{0}\right)^{2}$, the value of the equivalent noise temperature $\mathrm{T}_{\text {eff }} \approx 382^{\circ} \mathrm{K}$.

Thermal radiation noise of the absorption cell is characterized by the effective noise temperature 


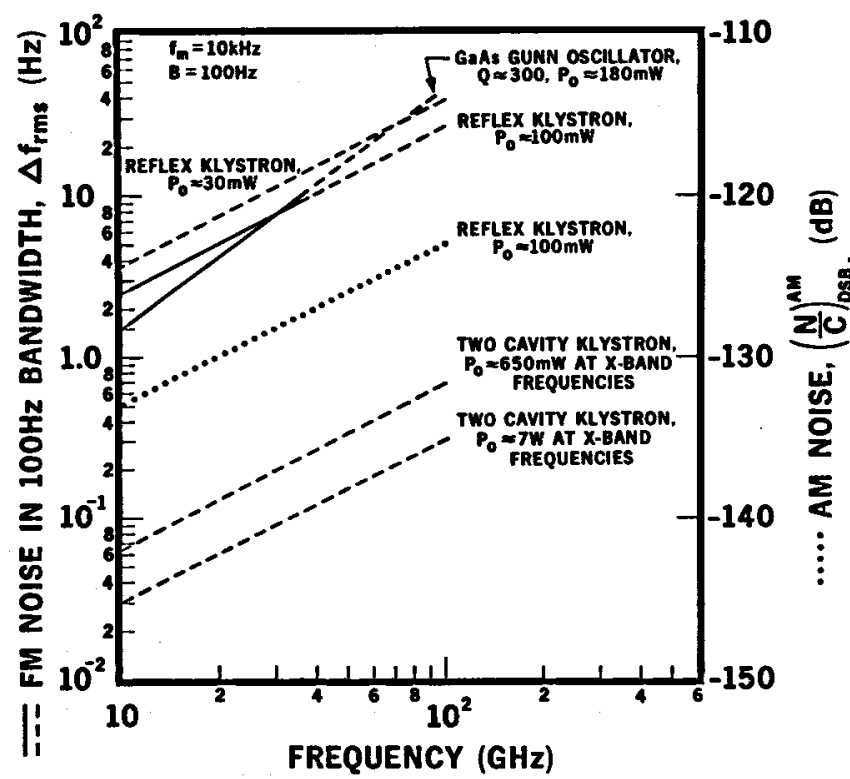

Fig.,2. AM and FM noise of the microwave source as a function of frequency.

(XBL 756-1493)

of the resonant cavity, which is typically $\mathrm{T}_{\mathrm{a}} \approx$ $300^{\circ} \mathrm{K}$ for a high sensitivity spectrometer.

Microwave receiver noise is characterized by the effective input noise temperature $T_{e}$, defined as $T_{e}=P_{n} / K B_{r}$, where $P_{n}$ is the available noise power. Figure 3 shows the measured overall effective input noise temperature and noise figure as

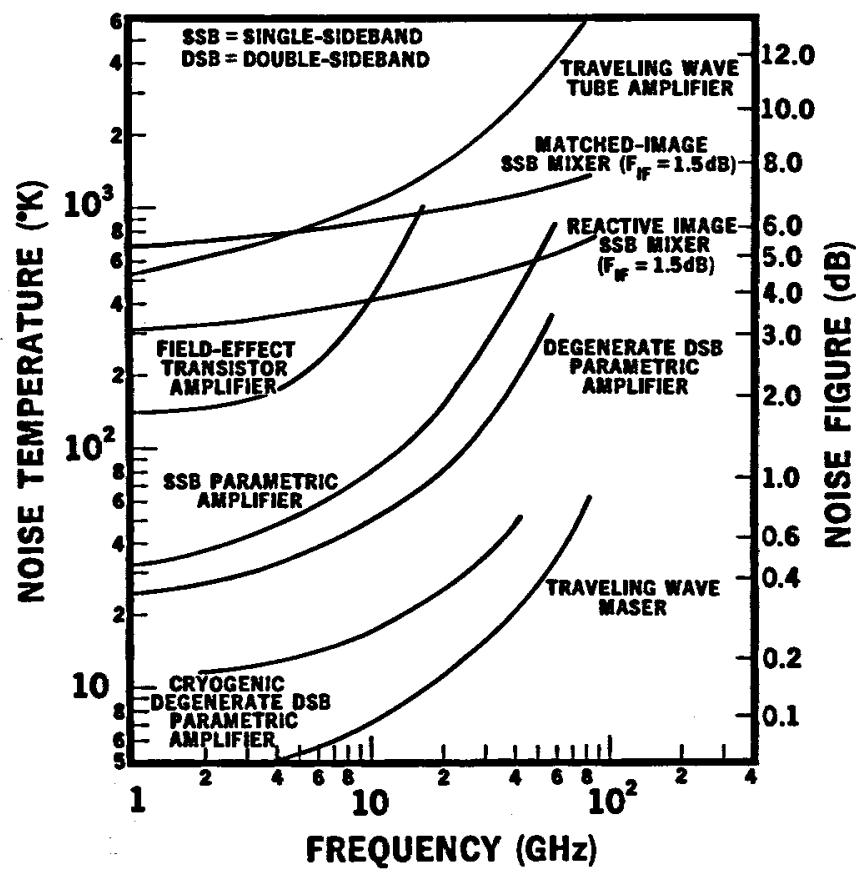

Fig. 3. Effective noise temperature and noise figure as a function of frequency.

(XBL 956-1494) functions of input signal frequency for the microwave receiver, using as the front end the traveling wave tube amplifier, the SSB mixer, the fieldeffect transitor, noncryogenic and cryogenic parametric amplifiers, and a traveling wave maser. ${ }^{7}$ It can be seen from the figure that at $70 \mathrm{GHz}$ the receiver has effective noise temperatures of $500^{\circ} \mathrm{K}$ and $700^{\circ} \mathrm{K}$, achieved with the double-sideband parametric amplifier and the single-sideband mixer front ends, respectively.

For the detection of very weak absorption lines, or for a reduction in the measuring system response time, the spectrometer sensitivity is considerably increased by the use of Stark or Zeeman modulation of the electromagnetic field, followed by phase-sensitive detection. A phase-sensitive detector, as a cross-correlator, utilizes amplitude and phase information of an input signal, offering a considerable gain in the output signal-to-noise ratio, compared with a conventional envelope detector. With properly optimized operating conditions, a phase-sensitive detector has excellent performance having low nonlinearity, wide dynamic range, and bandwidth under almost any signal-tonoise ratio conditons. 8

A comparison of the microwave source noise with the absorption cell noise and the microwave receiver noise shows that the microwave source noise contributes significantly to the total spectrometer sensitivity. Furthermore, it can be seen from comparison of $T_{e}$ with the effective noise temperatures of the microwave source and sample absorption cell, $\mathrm{T}_{\text {eff }}$ and $\mathrm{T}_{\mathrm{a}}$, respectively, that in the case where $T_{e} \ll T_{a}$, the sensitivity of the millimeter-wave measuring system cannot be significantly improved, since the sample absorption cell would radiate thermal noise into the receiver, making $\mathrm{T}_{e}$ comparable with the absorption cell temperature $\mathrm{T}_{\mathrm{a}}$. Similarly, for $\mathrm{T}_{\mathrm{e}}=\mathrm{T}_{\mathrm{a}} \ll \mathrm{T}_{\mathrm{eff}}$, the source noise temperature would dominate the total noise temperature of the measuring system. Consequently, in millimeter-wave measuring system applications, the optimum tradeoff between the sensitivity, complexity, reliability and cost occurs at a level of receiver effective noise temperature $\mathrm{T}_{\mathrm{e}}$ comparable to the sample absorption cell noise temperature $T_{a}$ and the effective microwave source noise temperature $T_{\text {eff }}$; i.e., $T_{\text {eff }} \approx T_{a} \approx T_{e}$.

It is of particular interest now to further investigate the influence of noise sources in a high-sensitivity millimeter-wave spectrometer. This study will give direction for the developnent of an optimized spectrometer with the multiparameter detection capability, particularly suitable for measurements of weak absorption lines and studies of the molecular properties of short-lived transient chemical species.

\section{REFERENCES}

1. B. Leskovar, D.B. Hopkins, and W.F. Kolbe, Optimal Design Criteria for Millimeter-wave Spectrometers, Proceedings of the 5th International Microwave Conference, Hamburg, West Germany, Sept. 1-4, 1975, pp. 228-232.

2. M. Ohtomo, Experimental Evaluation of Noise Parameters in Gunn and Avalanche Oscillators, 
IEEE Trans. in Microwave Theory and Techniques, MTT-20, No. 7, p. 425, 1972.

3. T.G. Ruttan, High Frequency Gumn Oscillators, IEEE Trans. on Microwave Theory and Techniques, MTT-22, No.2, pp. 142-144, Feb. 1974.

4. Dr. Berin Fank and Mr. James Hice, Varian Associates, Palo Alto, California, Private Communication, June 1975.

5. S.L. Johnson, B.H. Smith, D.A. Calder, Noise Spectrum Characteristics of Low-Noise Microwave Tubes and Solid-State Devices, Proc. of the IEEE, 54, No. 2, pp. 258-265, Feb. 1966.
6. J. Josenhaus, Noise Spectra of Read Diode and Gunn Oscillators, Proc. of the IEEE, Vol. 54, No. 10, pp. 1478-1479, Oct. 1966.

7. H.C. Okean, P.P. Lombardo, Noise Performance of $M / W$ and $M M-W a v e$ Receivers, Microwave Journal, Vo1. 16, No. 1, pp. 41-50, Jan. 1973.

8. B. Leskovar, Generalized Analysis of PhaseSensitive Detection Circuit Operating Characteristics at the Signal Detection in the Presence of Noise, IEEE Trans. on Instrumenta tion and Measurements, Vol. IM-21, No. 1, pp. 15-24, Feb. 1972.

\title{
CONSTRUCTION AND PERFORMANCE CHARACTERISTICS OF A SIMPLE $\mathrm{CO}_{2}$ LASER
}

\author{
Nabiz M. Amer and R. Gerlach
}

In this report we describe the construction and characteristics of a very simple $\mathrm{CO}_{2}$ laser design, built entirely from readily available parts and involving no glass blowing. This laser produced an output power of 5 watts with no wavelength selective optics, and up to 2 watts gratingtuned.

The discharge tube is a 23-in. length of $1 / 2$ in. outer diameter, $3 / 32$ in. wall pyrex tubing. Attached to the ends of this type are two $1 / 2$ in. stainless steel swage-type male run tees which serve both as electrodes and as Brewster window mounts. The male pipe thread portions of these tees have been sawed off at Brewster's angle, and the $32 \mathrm{~mm}$ diam. $\times 4 \mathrm{~mm}$ thick $\mathrm{NaCl}$ windows are glued on with epoxy. Teflon ferrules are used with the swage fittings.

A water cooling jacket covers most of the length of the discharge tube. This cooling jacket consists of a 13 in. length of rigid transparent plastic tubing, 3/4 in. 0.d., 1/16 in. wall; two $3 / 4$ in. swage-type tees; and two $3 / 4 \times 1 / 2$ in. swage-type bored-through reducers. A distance of about 3/4 in. is maintained between the cooling jacket and the electrodes to prevent arcing.

Gas was flowed into the laser at the anode end and out at the cathode end through $1 / 2$ in. o.d., 1.16 in. wall polyethylene tubing. The gas mixture used was $70 \% \mathrm{He}, 20 \% \mathrm{CO}_{2}$, and $10 \% \mathrm{~N}_{2}$, and slow flow was maintained using a $0.9 \mathrm{cfm}$ vacurm pump.

The cavity consisted of a flat dielectriccoated $\mathrm{ZnSe}$ mirror having a $95 \%$ reflectivity at $10 \mu \mathrm{m}$ and a flat dielectric-coated copper maximm reflector for $10 \mu \mathrm{m}$. For grating-tuned operation, the maximm reflector was replaced by a $150 \mathrm{line} / \mathrm{mm}$ grating blazed for $10 \mathrm{~mm}$. The output mirror was mounted on a piezoelectric translator for cavity length tuning. No difficulty in aligning the laser was encountered due to the marginally stable resonator, but the mode pattern was poor. If, however, a 6 meter radius of curvature gold-coated mirror was used in place of the maximm reflector, a very

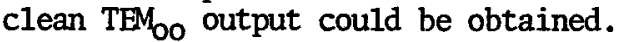

A $15 \mathrm{kV}, 20 \mathrm{~mA}$ dc high voltage supply was used for most of our investigation. The discharge was stabilized by use of a $100 \mathrm{k} \Omega$ ballast resistor, and later by use of an electronic current regulator.

There was no apparent problem due to cathode sputtering causing deposits on the Brewster window. However, it was necessary to keep the current low enough to prevent the discharge from extending too close to the window on the cathode end.

The maximum output power we observed from this laser was 5 watts. We believe that somewhat higher power could be achieved by use of higher pressure and current, since power was still increasing at the highest pressure (50 torr) and current (20 mA) we tried. We were limited in current by the power supply rating, and in pressure by the increase in voltage with pressure, also reaching the limit of the power supply ratings.

The efficiency of this laser, defined as optical ouput power divided by electrical input power, was about $3 \%$ and depended only very weakly on either pressure or current. It is for this reason that we believe the laser is capable of much higher output power, since we have not yet reached the point where output power begins leveling off as a function of input power.

When operated with a grating for line selection, this laser oscillated on 29 lines in the $10 \mu \mathrm{m}$ band, including $P(10)$ through $P(38)$ and $R(10)$ through $R(34)$, at pressure 45 torr and current $20 \mathrm{~mA}$. The highest power obtained on any transition was 2.0 watts on $P(20)$, while the highest power on an Rbranch transition was 1.2 watts on $R(18)$. No effort was made to obtain transitions in the $9 \mu \mathrm{m}$ band.

An electronic current regulator/modulator has been built for use with $\mathrm{CO}_{2}$ and $\mathrm{CO}$ lasers. Due to the large negative dynamic resistance of the glow 
discharge, becoming increasingly negative at higher pressures and lower currents, it is necessary to place a large positive resistance in series in order to stabilize the discharge. At high pressures the necessary resistance is so large that power dissipation and voltage drop in an ordinary passive resistor become a source of extreme inconvenience. A very high dynamic resistance can be achieved for a much smaller voltage drop and power dissipation by using an electronic regulator. Our regulator is similar in principal to that described by Posakonyl through differing in many details. This device makes it possible to reach much higher pressures and lower currents than we could attain with the $100 \mathrm{k} \Omega$ balast resistor, and since the voltage drop across the regulator can be as small as 700 volts, more of the supply voltage can be dropped across the discharge.

This device also has the capability of modulating current when provided an input from an oscillator. Modulation at frequencies from $100 \mathrm{~Hz}$ to $10 \mathrm{kHz}$ is readily achieved. This capability is important since the lasers with which this regulator will be used are to be used for our program of optoacoustic spectroscopy, in which an intensity-modulated laser beam is passed through a gas cell containing the sample, and pressure fluctuations caused by absorption of the modulated beam are detected by a microphone in the cell. Use of a mechanical chopper to produce the intensity modulation of the light has several disadvantages: mechanical choppers produce a great deal of vibration, from which the opto-acoustic cell would have to be isolated; they have poor phase and frequency stability; and finally, it is difficult to build a chopper that has as high a frequency as a resonance of an opto-acoustic ce11, typically several $\mathrm{kHz}$. A1l of these problems should be solved by using modulation of the laser discharge current to produce the necessary intensity modulation.

\section{REFERENCE}

1. Posakony, Rev. Sci. Inst. $\underline{43}, 270$ (1972).

LARGE-SCALE MEASUREMENT OF AIRBORNE SULFUR PARTICLES COLLECTED ON FILTERS*

Billy W. Loo and Ray C. Gatti

As greater use is made of sulfur-containing fossil fuels to meet the nation's energy requirements, it will be necessary to monitor the sulfur concentration in atmospheric aerosols more extensively. Wet chemical procedures are presently used to measure sulfates extracted from glass fiber filters used to collect particles from the atmosphere. $\mathrm{X}$-ray fluorescence (XRF) is a powerful technique that can be automated to analyze large numbers of samples nondestructively. Since no sample preparation or extraction is needed for the XRF method, a more rapid analysis at a reduced cost is possible.

For the XRF analysis of light elements such as sulfur, a potential difficulty lies in the attenuation of the characteristic $x$-rays emitted from the particles. Such attenuations may occur within the particle (particle size effect), the aerosol deposition layer (layer effect) and the filter matrix in which the particle may be imbedded (matrix effect). The validity of the method is then to be evaluated in light of the desired goal of achieving an error of under $10 \%$ on sulfur measurements.

It is well established that urban aerosols tend to have a bimodal mass distribution according to their particle sizes. The coarse particle mode has a mass median diameter of $8 \mu \mathrm{m}$ or larger and consists mainly of mechanically generated aerosols. The fine particle mode has a mass median diameter of typically $0.3 \mu \mathrm{m}$ and consists of primary and secondary combustion products. Previous measurements indicate that most sulfur particles are submicron in size. The analysis is much facilitated by the use of dichotomous air samplers in which the two modes of the bimodal distribution are collected on separate filters. Since the particle size and layer corrections are typically 18 and
$5 \%$ respectively for heavily loaded filters of up to $200 \mu \mathrm{g} / \mathrm{cm}^{2}$, the problem is practically reduced to that of determining the filter penetration effect.

The attenuation of sulfur $x$-ray by the filter medium is very large for glass fiber or Watman 41 filters. Previous estimates of the matrix effect, based on indirect measurements, for cellulose ester membrane filters, however, have ranged from $22 \%$ to less than 5\%. The purpose of the present study is to measure this effect more definitively for 1.2- $\mu \mathrm{m}$ pore size cellulose ester filters that are being extensively employed in the sampling network of the St. Louis Regional Air Pollution Studies.

\section{DIRECT MEASUREMENTS}

Particles of $\mathrm{K}_{2} \mathrm{SO}_{4}$ and $\mathrm{CuSO}_{4} \cdot 5 \mathrm{H}_{2} \mathrm{O}$ in the range of $0.05 \mu \mathrm{m}$ to $2.0 \mu \mathrm{m}$ were generated in aerosol form, at the University of Minnesota Particle Technology Laboratory, and subsequently deposited on test filters. Similar 0.3- $\mu$ m aerosols were deposited on the surface of Nuclepore polycarbonate filters with straight $0.1-\mu \mathrm{m}$ holes such that the particles were known to be on the surface.

These aerosol samples were analyzed under a helium atmosphere using an energy dispersive $x$-ray spectrometer with a pulsed $x$-ray source. Accurate calibration was first performed for $\mathrm{Cu}$. The samples deposited on the surface of Nuclepore filters were then used to calibrate the system for $S$ and $K$, taking the stochiometric ratios of $S / C u$ and $S / K$ to be 0.505 and 0.410 respectively. The measured $S / K$ and $S / C u$ ratios of each test specimen relative to those of the surface reference samples immediately yielded the attenuation factors $A(D)$ as a 
Table 1. Results of direct measurements on sulfur $x$-ray attemuation by filter matrix.

\begin{tabular}{|c|c|c|c|c|c|c|}
\hline \multirow{2}{*}{$\begin{array}{l}\text { Filter } \\
\text { type }\end{array}$} & \multirow{2}{*}{$\begin{array}{c}\text { Particle } \\
\text { Diam., D } \\
\text { (um) }\end{array}$} & \multicolumn{3}{|c|}{ Observed } & \multirow{2}{*}{$\begin{array}{l}\text { Corrected } \\
\mathrm{S} / \mathrm{K}\end{array}$} & \multirow{2}{*}{$A(D)^{c}$} \\
\hline & & $\underset{\left(\mu \mathrm{g} / \mathrm{cm}^{2}\right)}{\mathrm{c}^{2}}$ & $\underset{\left(\mu \mathrm{g} / \mathrm{cm}^{2}\right)}{K}$ & $\mathrm{~S} / \mathrm{K}$ & & \\
\hline \multicolumn{7}{|c|}{$\mathrm{K}_{2} \mathrm{SO}_{4}$ Particles: } \\
\hline $\mathrm{CE}$ & 2.0 & 1.91 & 4.66 & $0.409 \pm 0.004$ & 0.411 & $1.01 \pm 0.01$ \\
\hline $\mathrm{CE}$ & 0.3 & 17.48 & 42.84 & $0.408 \pm 0.004$ & 0.408 & $0.97 \pm 0.01$ \\
\hline CE & 0.1 & 18.66 & 45.54 & $0.410 \pm 0.004$ & 0.410 & $1.00 \pm 0.01$ \\
\hline $\mathrm{CE}$ & 0.05 & 19.16 & 49.47 & $0.387 \pm 0.002$ & 0.387 & $0.95 \pm 0.01$ \\
\hline NP & 0.3 & 12.94 & 31.56 & $0.410 \pm 0.004$ & 0.410 & $1.00 \pm 0.01$ \\
\hline \multicolumn{7}{|c|}{$\mathrm{CuSO}_{4} \cdot 5 \mathrm{H}_{2} \mathrm{O}$ Particles: } \\
\hline $\mathrm{CE}$ & 0.41 & 7.98 & 15.93 & $0.501 \pm 0.008$ & 0.508 & $1.01 \pm 0.02$ \\
\hline $\mathrm{CE}$ & 0.41 & 2.76 & 5.38 & $0.513 \pm 0.009$ & 0.505 & $1.00 \pm 0.02$ \\
\hline CE & 0.41 & 2.76 & 5.55 & $0.497 \pm 0.011$ & 0.489 & $0.97 \pm 0.02$ \\
\hline $\mathrm{CE}$ & 0.07 & 3.31 & 6.69 & $0.495 \pm 0.007$ & 0.493 & $0.98 \pm 0.02$ \\
\hline $\mathrm{NP}$ & 0.41 & 3.88 & 7.69 & $0.505 \pm 0.010$ & 0.505 & $1.00 \pm 0.02$ \\
\hline
\end{tabular}

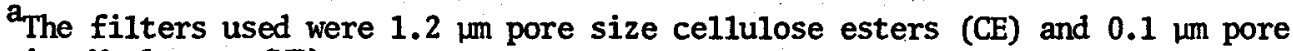
size Nuclepore (NP).

Differential layer corrections were made for submicron particles and individual particle size corrections were made for the $2 \mu \mathrm{m}$ particles.

$\mathrm{C}_{\text {Attenuation due to filter media determined assuming } A(D)}=1$ for the $0.1 \mu \mathrm{m}$ pore diameter Nuclepore filter.

function of particle size. Results of mean values together with standard deviations of four repeated measurements on each sample are summarized in Table 1.

\section{SEM OBSERVATIONS}

Extensive measurements have been made by $\mathrm{T}$. Hayes using a scanning electron microscope equipped with an XRF spectrometer. In order to determine the particle penetration profile in the filter, a cellulose ester sample with 0.3- $\mu \mathrm{m} \mathrm{K}_{2} \mathrm{SO}_{4}$ particle deposition has been cooled to liquid nitrogen temperature and cracked to obtain a clean cross-sectional fracture. Figure 1 illustrates the five $(5 \mu \mathrm{m} \times 5 \mu \mathrm{m})$ zones over which XRF signals have been integrated. Zones 1 and 2 are on the slanted surface (Y-Z plane) of the filter. Zone 3 stradales the top edge and spanning 2.5 um into the filter, while 4 and 5 are successively deeper zones of 5 $\mu \mathrm{m}$ depths. The rapid disappearance of the $S$ and $K$ signals at even a few microns below the surface varifies that particles are mostly captured on or above the top layer of fiber structure in the filter.

\section{CONCLUSION}

For ambient sulfur particles in the range of $0.05 \mu \mathrm{m}$ to $1.0 \mu \mathrm{m}$, the filter penetration correction to $x$-ray measurement can simply be represented by $3 \pm 3 \%$. It has become clear that for the cellulose ester filters used, particle deposition occur mostly on the surface rather than penetrating

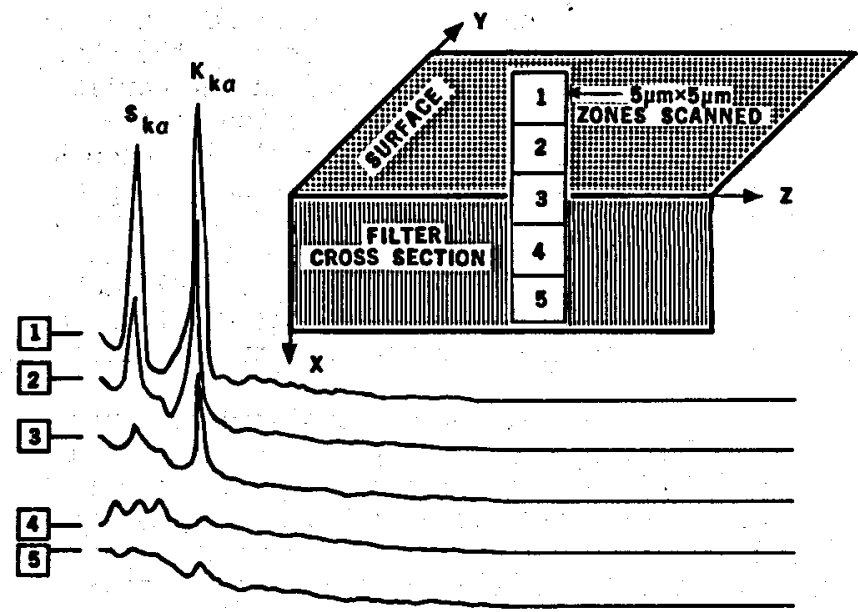

Fig. 1.

(XBL 766-8157)

exponentially into the matrix as suspected previous$1 y$, in which case the required matrix correction would exceed $20 \%$ based on filter efficiency measurements.

We have found that the use of dichotomous air samplers to be very useful in removing the complex large-particle interference problem. The use of surface samples of submicron $\mathrm{CuSO}_{4} \cdot 5 \mathrm{H}_{2} \mathrm{O}$ and $\mathrm{K}_{2} \mathrm{SO}_{4}$ aerosols have also proved to be reliable means for 
accurate sulfur calibration purposes in a XRF spectrometer.

\section{FOOTNOTE}

"Condensed from "Absorption Corrections for Submicron Sulfur Collected in Filters", coauthored with B.Y.H. Liu and C.S. Kim of the University of Minnesota and T.G. Dzubay of EPA, Research Triangle Park, North Carolina, in X-Ray Fluorescence Methods for Analysis of Environmental Samples, T.G. Dzubay, Ed. To be published by Ann Arbor Science, 1976. LBL report LBL-4833.

\title{
MODULATED FLUORESCENCE AS A POSSIBLE IN SITU MONITOR FOR $\mathrm{NO}_{2}$
}

\author{
P. Robrish, H. Rosen, and O. Chamberlain
}

Recently, the successful operation of an ambient $\mathrm{NO}_{2}$ monitor based on laser excited broadband molecular fluorescence has been reported.1 In order to avoid fluorescence from aerosols, however, it was necessary to filter the incoming air stream. This filtering procedure could change the measured $\mathrm{NO}_{2}$ concentration either because of adsorption directly on the filter or on particulates trapped by the filter. We would like to suggest an alternate procedure which has the virtue that it requires no processing of the air sample and can monitor $\mathrm{NO}_{2}$ and particulates simultaneously. In addition, this scheme may also prove useful for in situ monitoring of $\mathrm{NO}_{2}$ in catalytic and combustion studies.

We have investigated the excitation spectrum of the $v_{2}$ fluorescence mode of $\mathrm{NO}_{2}$ and have found several narrow spectral regions in which this reemission mode is sharply enhanced. 2 Following the work of Tucker et al.1, we have looked at broad-band fluorescence from $1 \mathrm{~mm}$ of $\mathrm{NO}_{2}$ in 1 atmosphere of $\mathrm{N}_{2}$ as we tumed a laser over the sharp excitation lines which were found in our earlier study. We find substantial modulation of the broad-band fluorescence. This modulation occurs when the laser is tuned over a very narrow spectral region and is characteristic of $\mathrm{NO}_{2}$. Since particulate fluorescence is expected to change quite slowly with laser frequency, we feel that we have found the basis for an ideal way to separate $\mathrm{NO}_{2}$ fluorescence from particulate fluorescence.

The experimental set up consisted of a pressure tumed nitrogen punped dye laser with a bandwidth of $0.04 \mathrm{~cm}^{-1}$ and an average power of $0.15 \mathrm{~mW}$. The sample cell was filled with $1 \mathrm{~mm}$ of $\mathrm{NO}_{2}$ and 1 atm of $\mathrm{N}_{2}$. Fluorescence was detected at right angles to the laser beam direction by a photomaltiplier. The signal was integrated with a gated electrometer that we developed. 3 A liquid filter of $\mathrm{Na}_{2} \mathrm{Cr}_{2} \mathrm{O}_{7}$ was used to eliminate background at the laser frequency.

We have observed the broad-band fluorescence as we tuned the laser over the $Q(15), Q(13)$ and $Q(11)$ rotational lines of $X+2_{B_{1}}$ transition in $\mathrm{NO}_{2}$ located near $4545 \mathrm{~A}$. Figure 1 (a) shows the behavior of this fluorescence as a function of laser frequency and compares it to the $v_{2}$ excitation spectrum of $1 \mathrm{~mm}$ of $\mathrm{NO}_{2}$ in vacunm shown in Fig. 1(b). The figure clearly shows modulation of the broad-band fluorescence as the laser is tuned over these sharp transitions. A background

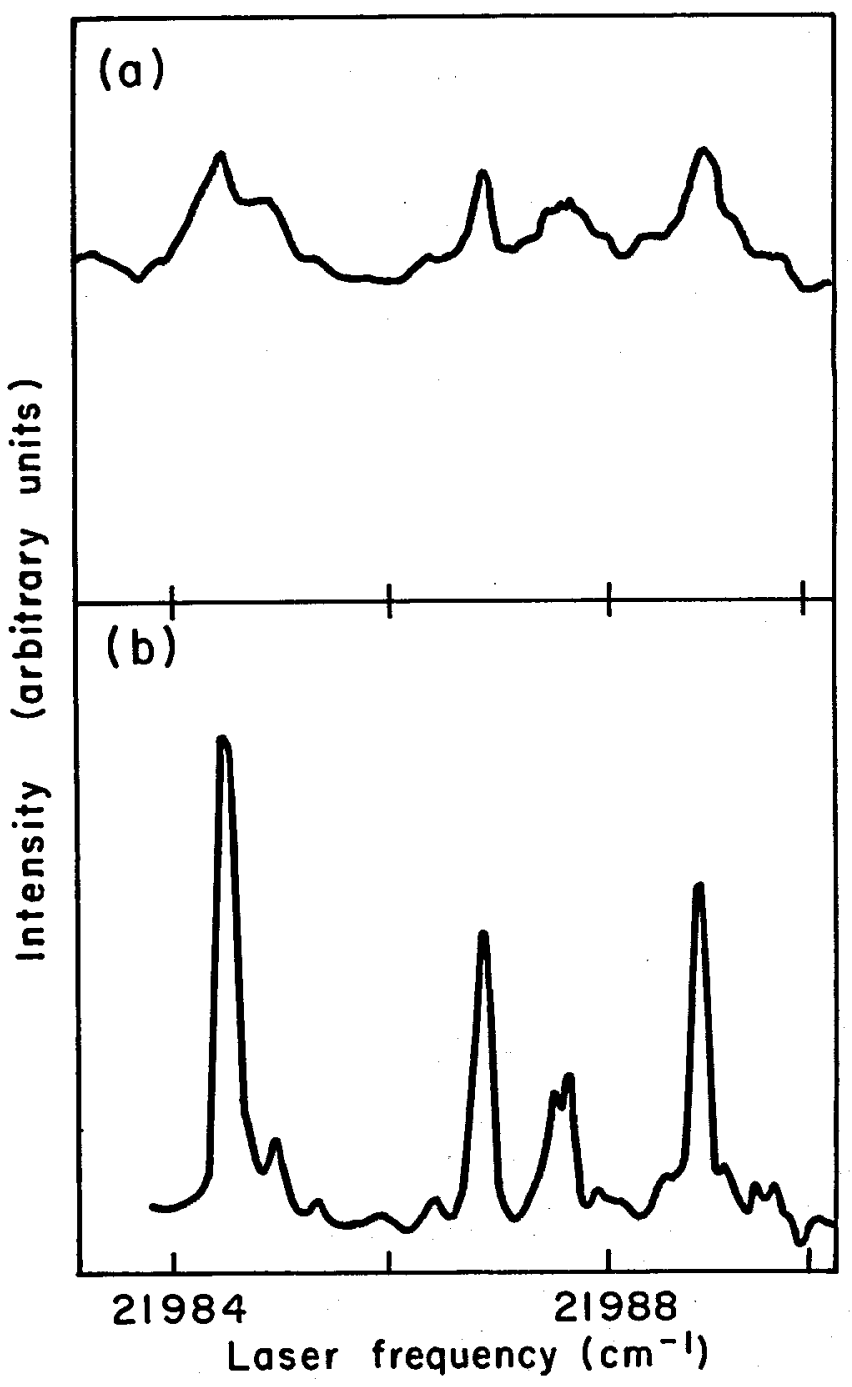

Fig. 1. (a) Intensity of broad-band fluorescence of $1 \mathrm{~mm}$ of $\mathrm{NO}_{2}$ in $1 \mathrm{~atm}$. of $\mathrm{N}_{2}$ as a function of laser frequency.

(b) Intensity of $v_{2}$ fluorescence for $1 \mathrm{~mm}$ of $\mathrm{NO}_{2}$ as a function of laser frequency.

(XBL 7511-9531)

check with the cell filled with 1 atm of $N_{2}$ gave a signal < 18 of the signal shown in Fig. 1(a). The modulation that we have observed is large enough to allow one to use it easily to separate 
aerosol fluorescence from that of $\mathrm{NO}_{2}$ in ambient air, provided that the signal from aerosols is comparable to that from $\mathrm{NO}_{2}$ and there is little additional background in the measuring apparatus. Gelbwachs and Birnbaum 4 have shown that, at least for some spectral regions, the aerosol fluorescence and that of $\mathrm{NO}_{2}$ are comparable in intensity, and Tucker et al. $1^{2}$ have operated an apparatus that has very low background fluorescence. Our measurements therefore show that the use of a tunable source may allow measurement of both aerosols and $\mathrm{NO}_{2}$ simultaneously at ambient levels with no processing of the polluted air.

\section{FOOTNOTE AND REFERENCES}

* Research supported by the National Science Foundation from RANN Division and the U.S. Energy Research and Development Administration.

1. A.W. Tucker, M. Birnbaum and C.L. Fincher, App1. Opt. 14, 1418 (1975).

2. P. Robrish and H. Rosen, Chem. Phys. Letts. 37, 156 (1976).

3. H. Rosen, P. Robrish and G.J. de Vries, Rev. Sci. Instrum. 46, 115 (1975).

\author{
EVALUATION OF STATE OF THE ART MEASUREMENT AND INSTRUMENTATION TECHNIQUES \\ FOR \\ MONITORING PLUTONIUM AND URANIUM PARTICULATES RELEASED FROM NUCLEAR FACILITIES
}

\author{
A. V. Nero, D. A. Mack, and R. J. Budnitz
}

\begin{abstract}
Uranium and plutonium, two basic materials of both commercial and military power, are both recognized to pose important radiological dangers to man. Accordingly, standards have been established defining limits to the amount of these materials that may be ingested by humans, and - considering the specific pathways for uptake - to the amount that may be present in any environmental medium. Regardless of the details of these limitations, it is important that means be available for monitoring both the amounts of these materials that actually escape into the environment and the concentrations in which they are present at any specific site. The purpose of this study is to evaluate the state of the art of instrumentation for monitoring release of plutonium and uranium particulates into the environment, to compare the capabilities of this instrumentation with the needs as represented by current standards, and to discuss the possible ways to improve the manner in which these needs are met. We are evaluating the types of instrumentation that are currently employed at nuclear facilities, those that are planned for new facilities, developments that are now taking place in considering in each case how instrumental sensitivity and versatility compares with
\end{abstract}

the requirements imposed by current 1 imits on releases and concentrations.

The primary component of this work has been to examine the actual instrumental techniques that are available for plutonium and uranium monitoring. The emphasis is on techniques that depend for their ultimate measurement capability on the detection of radiation from the radioactive decay of these two elements. Air monitoring has been examined most carefully, although attention is also given to monitoring in soil, water, and biological media. In addition to decay monitoring, atomic and nuclear activation electrochemistry, and mass measurement, are examined as they may be applied to monitoring. In every case, the particular circumstances in which the monitoring will be performed-whether, for example, at the stack on a routine basis or in the field after a major release-are considered in evaluating the value of applying any specific technique.

A report on this work, giving evaluations and recommendations, will be available during the summer, 1976.

\title{
RUBIDIUM-STRONTIUM GEOCHRONOLOGY
}

\author{
Maynard C. Michel
}

As part of the LBL geoscience program on the properties of the earth's mantle, we have developed the capability of moderately high precision $\mathrm{Rb}-\mathrm{Sr}$ dating and the associated techniques. The basic problem was to adapt the existing five-foot radius isotope separator as a high precision mass spectrometer using simultaneous collection to eliminate the effect of ion source intensity fluctuation on the quality of the data. We have succeeded in achieving a precision in isotopic ratios (using the customary internal standard technique) of a little better than 1 part in $10^{4}$ with samples of $\mathrm{Sr}$ small enough to be of use in most geological work. Associated with this, another mass spectrometer has been adapted to modest precision $(0.3 \%$ standard deviation) isotope dilution analysis of $\mathrm{Rb}$. The necessary chemical techniques, which are fairly standard, have also been developed and 
demonstrated to give blanks of $\mathrm{Rb}$ that are satisfactory for the level of precision being attained in this work.

The use of simultaneous collection to measure isotope ratios to high precision is not new but, because of physical size limitations in conventional mass spectrometers, has not been applied outside the low mass region. Partly because of its size, the isotope separator at LBL is well adapted to this use and, as expected, the only new problem introduced by its use was increased difficulty in suppression of secondary electrons. These, because of the necessarily high beam energy (40 keV), are more numerous and energetic than in conventional instruments, and simultaneous collection is very sensitive to a differential loss of secondary electrons between collectors. In contrast, the conventional mass spectrometer measures a11 isotopes sequentially in the same collector, thus avoiding such problems. Proper design of the collectors, and the availability of new Co-Sm magnetic alloys have made a simple collector of the type shown in Figure 1 quite satisfactory. As shown, the ribbon shaped beams enter a stack of three broad slits, larger in all dimensions than the beam, and designed to pass all of the beam, including the exponential tails down to the $10^{-4}$ to $10^{-5}$ level of intensity. This ensures that small movements of the beam relative to these collimators. will not cause significant differential collection yields of the isotopes even if the peaks are asymmetric and the collimators are not exactly spaced properly for the beams, conditions almost impossible to avoid in practice. The magnetic field produced by the collector magnets $M$, combined with electrostatic repulsion from the collimator plates, suppress most secondary electrons. Other details not whown in Fig. 1, eliminate effects such as secondary ions and the multiple reflections of energetic ions or neutrals.

The other major problem was to supply an ion source capable of relatively high beam currents ( $10^{-10}$ amperes, made necessary only by the particular commercial readout system we used for reasons of economy), and giving the appropriate sized effective source for the ion-optical system.

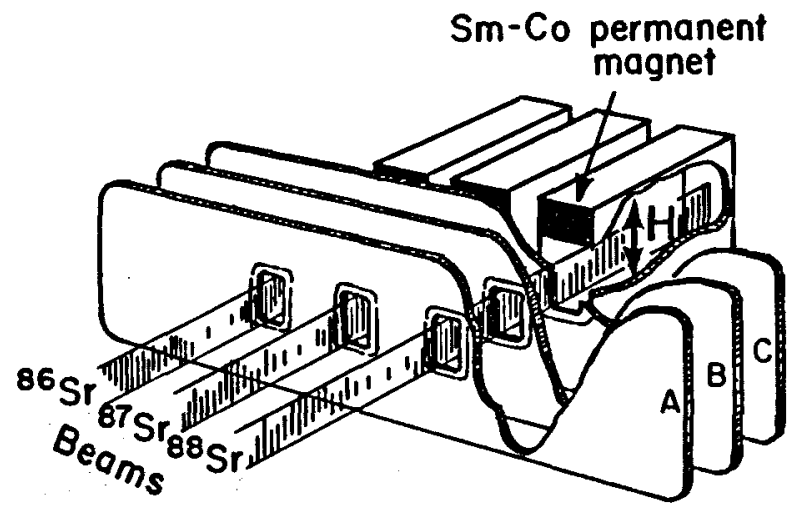

Fig. 1. Simultaneous collector for strontium isotopes.

(XBL 772-437)
The separator directly images the source (which is the $0.75 \mathrm{~mm}$ hole shown in Fig. 2) at the collector using no defining slits. Thus, there must be no ion emission from any part of the ion source radially outside the hole or the resolution will be destroyed. This requirement can be met in a thermal ion source of this type by appropriate choice of materials, because of the extreme sensitivity of thermal ionization efficiency to work function: The sample is placed in the rhenium lined cavity and heated by direct resistive heating by current passed through the concentric tubes. The efficiency, based on calculations and experiments here and elsewhere is higher than open ribbon sources common in conventional mass spectrometers and may exceed that of the so-called "veefilament" source. Best estimates place the average efficiency near $10-20 \%$ for $\mathrm{Sr}$. The ion optical transmission of the separator is nearly $100 \%$ efficient for ions emitted from the source, so the efficiency of the entire system is quite high and probably equals or exceeds that of any other instrument in use for this purpose. An added benefit of the high ion transmission is that the usual instrumental man discriminations common to man spectrometers will be reduced since they often result from differential transmission effects on different ion-optical paths.

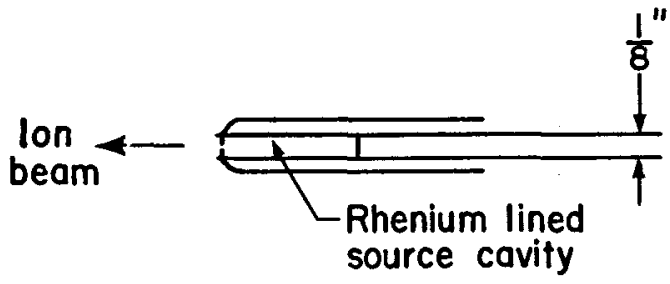

Fig. 2. Thermal ion source for strontium. (XBL 772-438)

Readout of the beam currents has been done in a relatively crude way and can clearly be improved. At present, each output is fed through a high value resistor which is part of a current amplifier-feedback system of high stability. A built-in constant-current source is used to adjust the gain. of the two systems to be identical to about 1 part in $10^{5}$. Then the two outputs from the collector (e.g. $87 \mathrm{Sr}$ and $86 \mathrm{Sr}$ ) are fed to a commercial digital multimeter in the voltage ratio mode and the isotopic ratio is read directly. By successive measurements of the $87 / 86$ and $86 / 88$ ratios, proper correction can be made for isotopic fractionation and/or any other linear discrimination of the system as long as such changes are slow. The simultaneous nature of the individual measurements is not absolute since the conmercial digital multimeter does sample sequentially, so improvements can easilty be made at some expense.

Even so, the performance has been rather good and is clearly limited by the readout system, giving results slightly better than the multimeter manufacturers' specifications would suggest. For 
oxample, in a single rin of the NBS standard stronjium reference material, we measured the $87 / 86$

ratio throughout the life of the $2 \mu \mathrm{g}$ sample, such that the uncorrected ratio varied by about $1 \%$. The internally corrected ratio averaged $0.71027 \pm$ .00005 . The certified value from NBS is $0.71014 \pm$ .0002 although we believe the NBS number may be more accurate than the precision limits indicate. The difference is a little puzzling but the reproducibility is quite satisfactory, and it is common practice to normalize to some standard for interlaboratory comparisons.

Future modifications to the readout system should allow determination of the ultimate precision possible by this technique. It would seem reasonable to achieve a precision of $1 / 10^{5}$ or better with little modification and perhaps, if no ion optical limitation exists, an even better precision. 


\title{
22310 \\ Environmental Research
}

\author{
INTRODUCTION
}

The environmental program at LBL is a broad one that includes the study of a diverse spectrum of systems of all sizes. They range from tiny mammalian mitochondria to the atmosphere. In most cases, the impacts considered are those associated with energy use and production. They range from physical-chemical-biological to socio-economic. Many of the projects will ultimately provide information or methodologies that will aid in making siting decisions, decisions between alternate technologies and fuels, the reclamation of already effected systems, and providing strategies for mitigating future environmental degragation. The paragraphs below briefly describe the various environmental projects.

The cell membrane project is involved in the role of membranes in damage and protection of cells against environmental effects. In particular, this work seeks to determine how and where various pollutants act on membrane structures.

The ecosystem stability and dynamics project involves perturbation and other experiments on freshwater microbiomes in order to study their nutrient regeneration and stability properties with the goal of developing stability indicators. This work is also coordinated with theoretical analysis of general ecosystem dynamics so that results may be extended to other ecosystems and to new experiments designed.
The pollutant availability study is designed to study what factors determine the uptake of trace metals by invertebrates in the San Francisco Bay after dredging and subsequent spoiling operations.

The environmental assessment of solar energy conversion is concerned with socio-economic and climatological effects of large scale implementation of solar conversion technologies.

The Lake County geothermal study is concerned with the socio-economic effects of development of the Geysers geothermal steam field on the surrounding community.

The indoor air pollution project is a study of the contaminents generated by indoor combustion such as cooking on a gas stove. This direct source of pollution has been previously neglected in studies of air quality.

The atmospheric aerosol project is divided into three major areas of research: chemical characterization of atmospheric aersols, studies of specific reaction mechanisms, and field studies. These research areas are designed to elucidate the nature of atmospheric particulates as well as to determine the role of catalytic gas-particulate reactions in determing atmospheric chemistry.

USE OF CHARGED DETERGENTS AND HYDROPHOBIC SPIN-LABELS

TO PROBE THE SURFACE STRUCTURE OF LIPOSOMES AND MITOCHONDRIA

Rolf J. Mehlhorm, Alexandre Quintanitha and Lester Packer

This project involves the role of membranes in damage and protection of cells against enviromental effects. We want to characterize the immediate and long term effects of pollutants on cells with a view to gaining insight into where and how they act, and how to protect against the damage they create. In particular, we initially want to characterize the effects of gaseous pollutants on biological membranes. Our working hypothesis is that damage that ultimately leads to the loss of membrane linked functions has significant structural manifestations. Once a quantitative relationship between induced membrane structural anomalies and loss of specific membrane linked functions is established, then the effects of pollutants on membrane function can be quantitatively determined by structural analysis. Using treatment of mitochondrial membranes with intermediate to high concentrations of ionic detergents as a well defined method for inducing membrane disruption, we have established a quantitative correlation between loss of energy linked functions and membrane structure. It was found that complete inactivation of respiratory functions was attended by very slight but measurable alterations of structure as determined by spin-labeling criteria of microviscosity. The general conclusion from these studies was that the major role of ionic detergents in the loss of respiration was, in fact, a charge effect rather than a membrane fluidity effect. 1

To investigate the possibility that pollutant action at the membrane level involves charge modifications, we developed a new, highly sensitive, spin labei assay for membrane specific charge.2' To accomplish this, spin labels of the electrically charged structures as shown in Fig. 1 were synthesized. In a suspension of membranes of virtually any given concentration, one of these spin labels will display spectral features arising from both the membrane and aqueous phases. The degree of partitioning between these two phases can be de- 


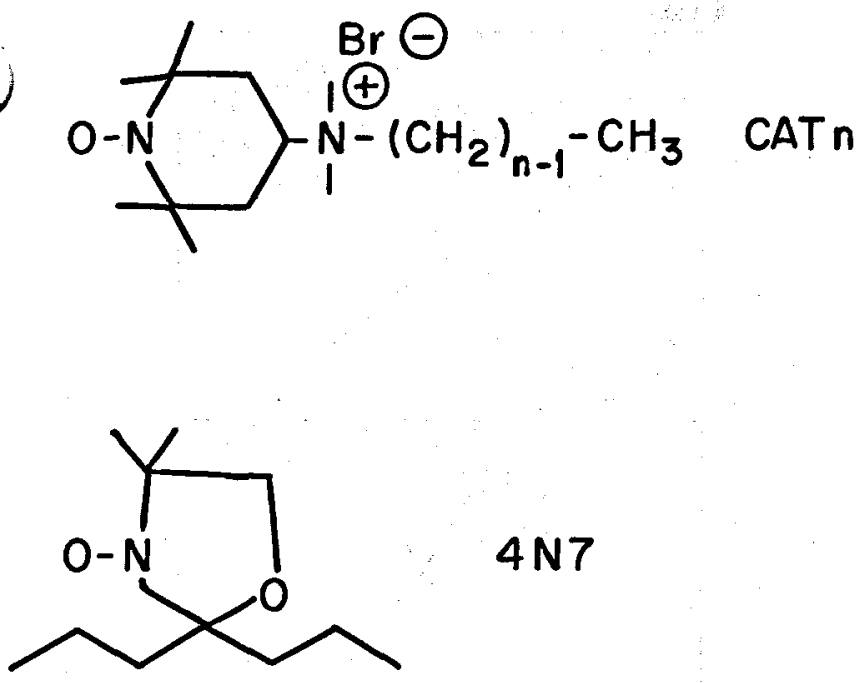

Fig. 1. $\mathrm{n}=1,4,6,8,10,12,16,20$.

(XBL 762-5173)

duced readily from spectral measurements, and is a function of the membrane charge, concentration, ionic composition of the aqueous phase and of membrane hydrophobic components. To establish a quantitative correlation between membrane charge and partitioning, lipid vesicles (prepared from electrically neutral egg lecithin) were used as model membrane systems. Their charge was modified by ionic detergent additions and spectral measurements of CAT10 partitioning were carried out. The results are plotted in Fig. 2 . The aqueous fraction of CAT10 relative to that in membrance is seen to be highly sensitive to membrance charge; indeed, the magnitude of the effect is seen to be comparable to observed functional changes at similar detergent levels in mitochondrial nembranes. 3 By comparison, the electrically neutral spin label, 4N7, exhibits partition properties which are considerably less sensitive to detergent additions or changes in membrane charge state. Partitioning of spin labels like $4 \mathrm{~N} 7$ is considered to reflect the state of membrane fluidity. 4

It also seemed to us that membrane structure seen with electron microscopy might provide an important assay system for pollutant damage. We, therefore, developed a computer-linked method-for obtaining aggregation parameters to describe particle patterns observed in freeze fracture electron micrographs of biological membranes. Upon completing this work we concluded that the method did not confer any substantial advantages over visual characterizations of particle pattern classes.

A frequently imvoked mode1 of membrane damage involves unsaturated lipids. Such damage is initiated by reactive free radical molecules and is propagated through lipid peroxides in the presence if oxygen. We are currently investigating the role free radical molecules in membrane damage. In rticular, we have been studying the disposition of endogenous free radical species generated within the mitochondrial electron transport chain. To this end, the spin label CAT16 was used as a free radical "trap" whose electron paramagnetic reso-

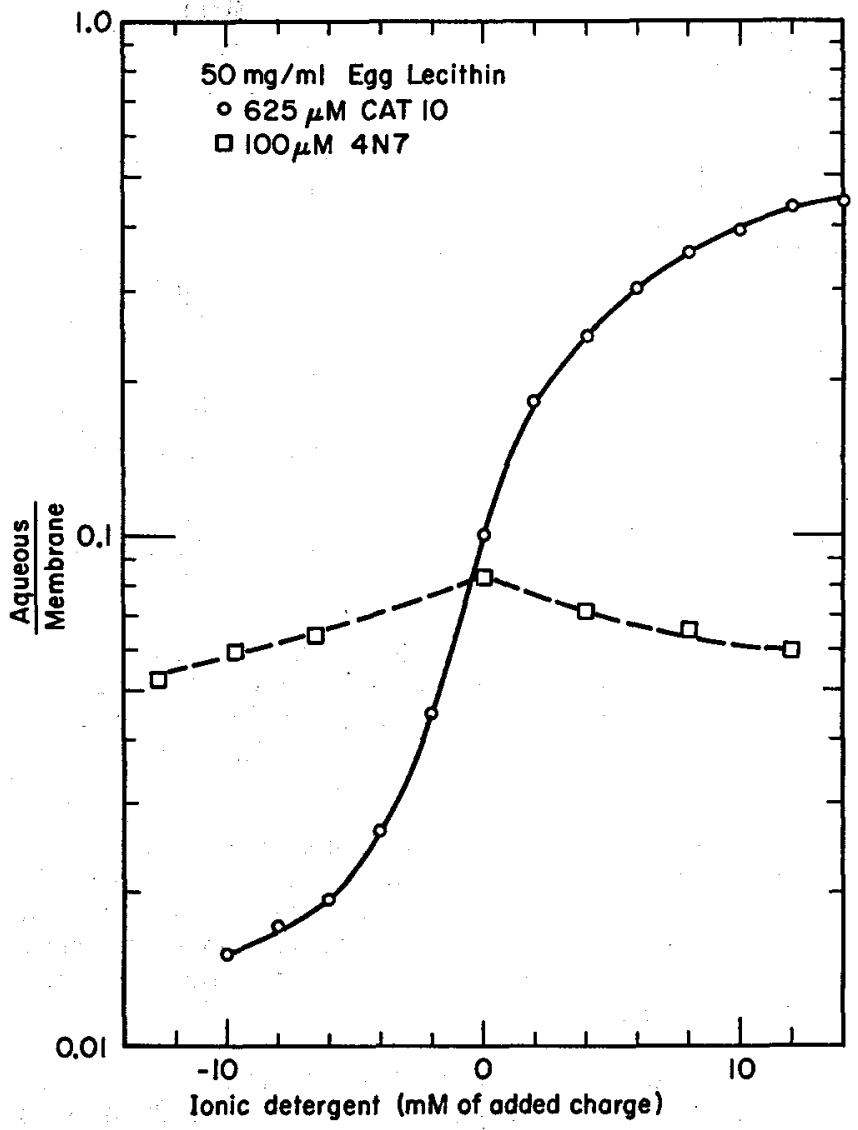

Fig. 2. Partitioning changes in the spin labels CAT10 and $4 N 7$ as a function of the charge of model membrane vesicles.

(XBL .762-5175)

nance (EPR) signal is lost upon combining with free radical intermediates in mitochondrial respiration. A comparison of CAT16 reduction by the respiratory chain with the reduction rates of a variety of other spin label molecules revealed that CAT16 suffered the most rapid signal loss during substrate oxidation (Fig. 3) as compared to other spin label molecules. Since this molecule is location specific, our results imply that maximal generation of endogenous free radicals occurs at the hydrophobic-hydrophilic membrane interfaces. 5

Studies of the effects of low concentrations (<20 nmoles/mg) of cationic detergent on mitochondrial membrane protein showed that all assayed mitochondrial functions were unaltered. (This is shown in Fig. 4 for different respiratory substrates.) Above these low concentrations, a slight enhancement of respiratory rates was observed and respiratory control was lost. This probably corresponds to the loss of oxidative phosphorylation, generally considered one of the most labile membrane linked functions.

To understand the meaning of changes in the functional characteristics of mitochondria it was important to localize our spin labeled compound with respect to the mitochondrial respiratory chain. Using mitochondrial membranes in their usual configuration and in an inside-out configuration (submitochondrial preparation) and a variety of res- 


\begin{tabular}{|c|c|c|c|}
\hline Lobel & Structure & $\begin{array}{l}\text { Reduction } \\
\text { Rote } \\
1=2 \text { inmoles/ } \\
\text { moprotein/min. }\end{array}$ & Type of Signol \\
\hline TEMPO & & $0.52 \div 0.01$ & \\
\hline TEMPOL & & $0.22 \pm 0.01$ & \\
\hline TEMPAMINE & & $0.19 \pm 0.01$ & \\
\hline CAPRATE & & $0.21 \pm 0.05$ & \\
\hline CDTAB & & $1.00 \pm 0.05$ & \\
\hline
\end{tabular}

Fig. 3. Comparison of rates of reduction of different spin labeled molecules during succinate oxidation and their signals. (CAT16 $=$ CDTAB)

(XBL 7510-7772)

piratory substrates and inhibitors we have been able to localize the CAT16 molecule (at concentraions of $<20$ moles/mg mitochondrial protein), in the ubiquinone region between the rotenone and the antimycin A inhibitory sensitive sites of the mitochondrial respiratory chain. ${ }^{6}$

It is planned to apply the recently developed assays of membrane charge to pollutant treated mitochondrial membranes. EPR techniques will be used to identify free radicals in membranes. Chemical and low temperature trapping techniques will be employed to observe free radicals arising in membrane systems exposed to ambient environmental factors and pollutants.

We intend to use the EPR assay of CATn for pollutant (or otherwise) altered mitochondrial membranes to identify modifications in the membrane function as well as to study some protective mechanisms of damage to such membranes.

\section{REFERENCES}

1. R. J. Mehlhom and L. Packer, Biochim. Biophys. Acta 423, 382-397 (1976).

2. R. J. Mehlhom and L. Packer, Biophys. J. $\underline{16}$, $194 a$ (1976).

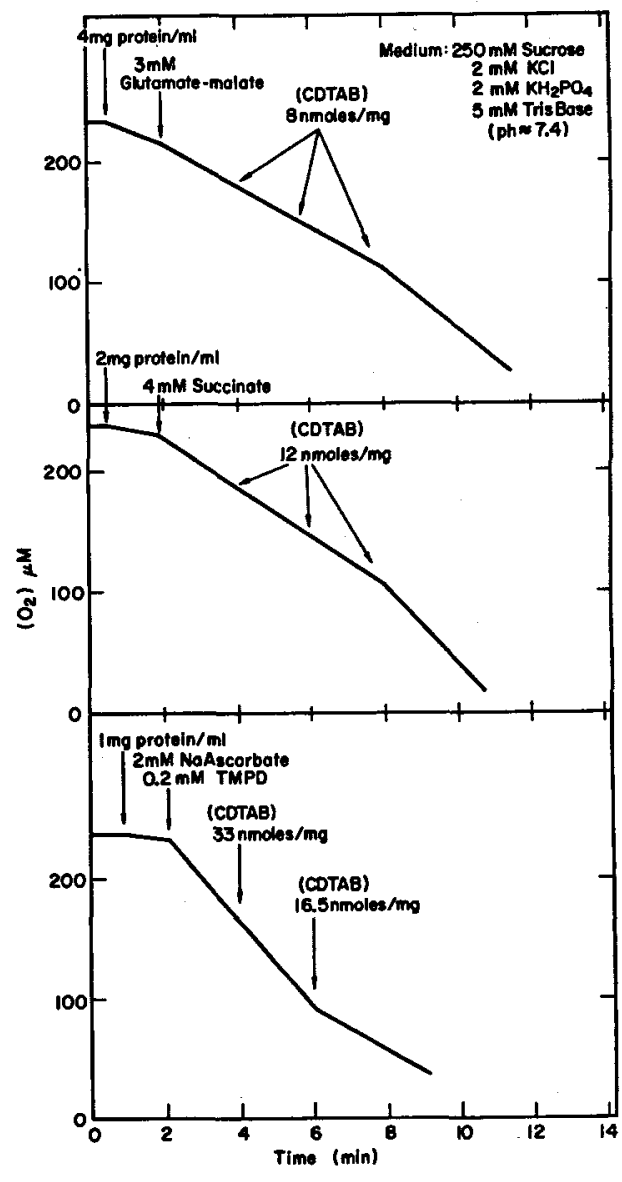

Fig. 4. CAT16 titration of mitochondrial respiration for different substrates. (CAT16 $\equiv$ CDTAB)

(XBL 762-5179)

3. R. J. Mehlhom and L. Packer, Biophys. J. 16, 613-625 (1976).

4. E. J. Shimshick and H. M. McConne11, Biochemistry 12,2351 (1973).

5. A. T. Quintanilha and L. Packer, Biophys. J. 16, $102 a$ (1976).

6. A. T. Quintanilha and L. Packer (Proc. Natl. Acad. Sci, February 1977).

\title{
EFFECTS OF VISIBLE LIGHT AND OXYGEN ON HUMAN DIPLOID CELLS AND MAMMALIAN MITOCHONDRIA
}

\author{
L. Packer, K. Fuehr, J. Walton, B. Aggarwal and Y. Avi-Dor
}

Membranes lie at the interface between the external and internal cellular environment and, as such, may afford a means to quench or sequester damaging envirommental substances on the one hand, or serve to cause the spread of damage on the other. Many agents that cause cell damage, such as nonionizing radiation and free radical forming sub- stances (e.g., ozone, nitrogen dioxide, peroxyacetyl nitrate, and oxygen), need to cross membranes and may react with them causing oxidative damage. To understand these phenomena we need precise answers to questions such as: 'What is damaged ?" "How do the reactions occur?" "How could protective mechanisms be devised?" 
The growth and viability of normal human diploid cells in culture, and the activity of mammalian mitochondria are two of the test systems that we are employing. Each is designed to enable us to ask and try to answer certain aspects of the above mentioned questions. Thus far, our experiments have mainly involved studies of the effect of visible light and oxygen on these two systens.

Normal human diploid cells in culture like those of the WI-38 strain, manifest a finite lifespan in vitro. It is not known whether lifespan limitation is genetically programmed or environmentally limited, or due to a combination of both effects. Therefore, we are investigating the effect which certain factors in the environment have on the short term and long term growth and viability of these cells. We intend to learn how we can control the cellular environment, particularly by manipulating nutrition to optimize the ability of cells to survive in a given environment.

We have found that visible light causes photokilling of human cells which is oxygen dependent and which can be protected by d1- $\alpha$-tocopherol (vitamin E). The effect of near ultraviolet and visible light on WI-38 human lung cell viability is illustrated in Fig. 1. Cells in culture were killed by light doses ranging from 2-10 × 105 $\mathrm{mW} / \mathrm{cm}^{2}-\mathrm{hr}$. Interestingly, there was an inverse correlation between culture age and photosensitivity. This finding, that younger cells are more sensitive to photokilling than older cells, is reminescent of the greater susceptibility of young cells to

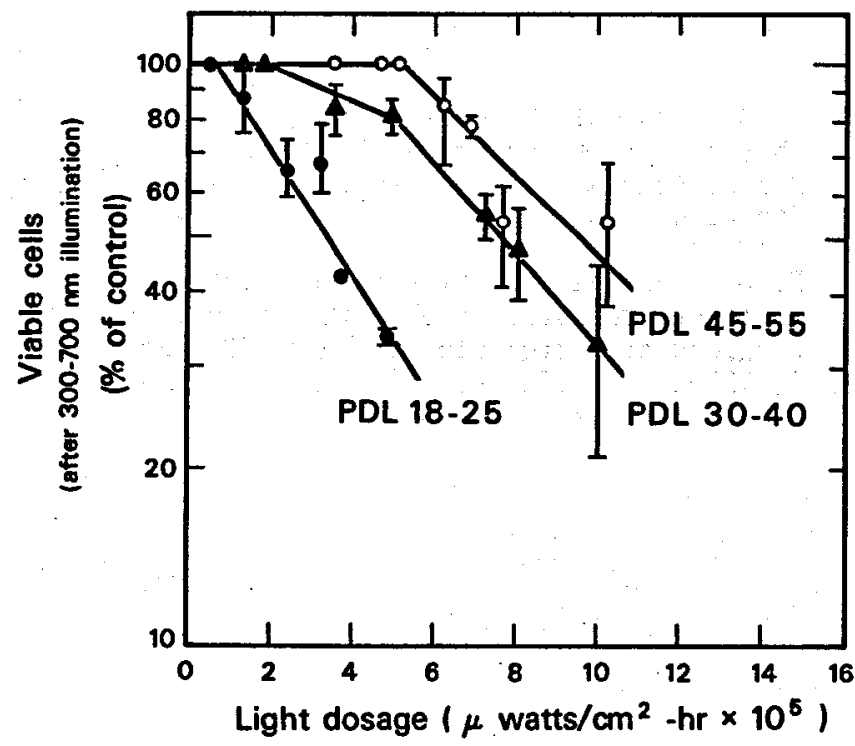

Fig. 1. The effect of near ultraviolet and visible light on the viability of the human WI-38 cells-dose response. Note the inverse relation between culture age and photosensitivity; the dose required for damage was greatest for late passage cells. However, the slope of the survival curves was nearly the same for all passage levels.

(XBL 763-5244) the damaging effects of ionizing radiation. The photokilling does seem to be related to the cells capacity for DNA systhesis in either younger or older cells. Anti-oxidants added to the growth medium such as d1- $\alpha$-tocopherol (vitamin E), butylated hydroxy toluene and ascorbic acid were found to afford the cells protection against light damage. We also found a direct relation between the amount of riboflavin in the growth medium and increasing photo-sensitivity (or increasing photokilling). Furthermore, products of lipid peroxidation could be detected in cell homogenates that were irradiated in the presence of riboflavin. Riboflavin is known to sensitize the photo-oxidation of many compounds in vitro. Our evidence indicated that intracellular flavins may be the photo-sensitizers which increase susceptibility to damage from visible light.

In summary, normal human cells in culture irradiated by visible light appear to be a useful system for investigating the mechanisms of photo-induced oxidative damage. The results of this study raise the question of the importance of routinely incorporating anti-oxidants into the culture medium. Future experiments could involve manipulation of cell nutrition, in particular antioxidants and 1ipid composition, to optimize the ability of the cells to survive damage due to a given dosage of visible 1ight. Also electron microscopy could be further used to determine the intracellular localization of damage.

Our other experimental system used is manmalian mitochondria. Mitochondrial pigments are responsible for the absorption of light in the visible region in the mammalian cell. Thus a study of visible light damage to mitochondria contributes to the elucidation of the mechanism of the photo-killing in cultured human cells. Manmaliam mitochondria are good models for these studies, because they are readily available in amounts necessary for experiments. In vitro, they exhibit the property of energy coupling, i.e., they are able to maintain ionic and electrical gradients which provide a sensitive test of functional integrity. Furthermore, their inner membranes contain a high concentration of endogenous free radicals arising from the biological oxidation catalysts. This is a result of the high concentrations of unsaturated fatty acids in their membrane phospholipids, as well as the relatively high concentrations of the natural anti-oxidants vitamin $E$ and the quinones. This is useful, because the unsaturated fatty acids provide potentially vulnerable sites in the membrane.

So far, we have found that the mechanism of visible light damage to mitochondria is oxidative and requires an activated species of oxygen $\mathrm{CO}_{2}^{-}$ or singlet oxygen). As to the location of the damage, we have found that the enzymatic activities of proteins localized on the membrane surface are more readily impaired by visible light than are the hydrophobic or integral proteins of the membrane. We have also implicated flavin photo-sensitized reactions and damage to flavin enzymes as important in the early stages of damage. Later stages of damage involve lipid peroxidation and/or cross linking of membrane components. This causes further amplification of the damage resulting in marked morphological changes. Some typical results are shown below. 
Figure 2 shows that exposure of mitochondria to visible light in the presence of riboflavin resulted in an initial loss of respiratory control, followed by inhibition of electron transport. Damage to the unsaturated fatty acids of the membrane phospholipids was also indicated. No inhibition of cytochrome c oxidase activity or ATPase activity occurred. Visible light damaged the mitochondrial membranes more in the presence of active photo-sensitizers (endogenous flavins), than in their absence. All of those effects of visible light were only found in the presence of oxygen. Figure 3 shows photomicrographs of the damage seen after light exposure to mitchondria in vitro and in intact cells.

Future studies will include precise determinations of the light spectrum which most effectively disrupts membrane function and manipulation of the lipid composition of the membranes. Membrane composition can be altered by growing animals (rats) in the presence of specific lipids and fat and water soluble anti-oxidants in their diet. An attempt can then be made to optimize the ability. of the isolated mammalian mitochondria of the animal to be protected against visible light damage.

The systems investigated thus far are considered mainly as models for elucidation for the "nature of damage", i.e. the identification of factors involved in sensitization and protection against damage induced by visible light and $\mathrm{O}_{2}$. In the future, the information so gained could be applied to a study of the factors which protect organs such as the skin and the retina from harmful effects of sunlight.

\section{REFERENCES}

1. O. P. Pereira, J. R. Smith, and L. Packer, Photochem. Photobiology 24, 237-242 (1976).

2. 0. P. Pereira, Riboflavin Sensitized Visible Light Effects on Human Diploid Fibroblasts in Culture, (Master's thesis), University of California, Berkeley.

3. B. B. Aggarwal, Y. Avi-Dor, H. M. Tinberg, and L. Packer, Biochem. Biophys. Res. Commn. 69, 362-368 (1976).

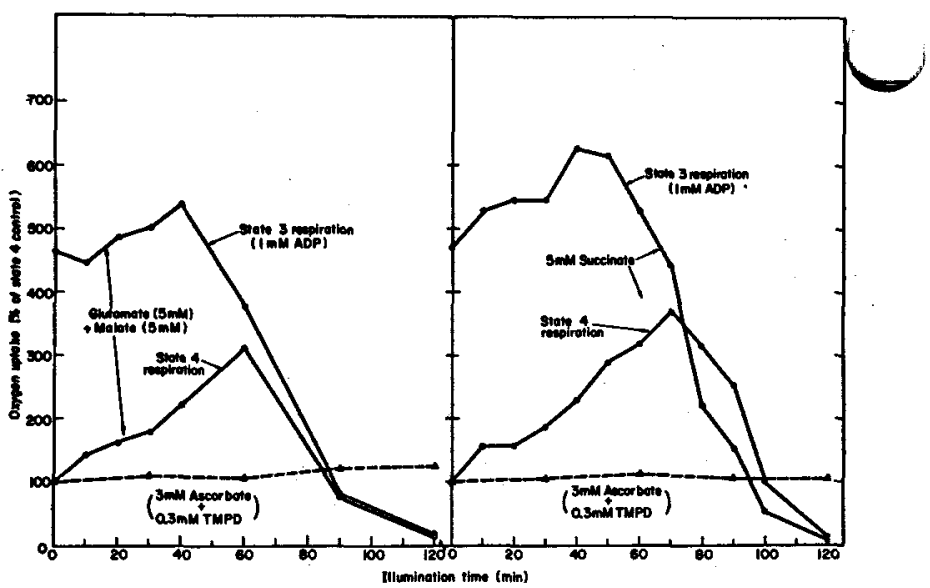

Fig. 2. Effect of illumination with visible light on respiration of rat liver mitochondria. Note that the first parameter effected is the release of coupling of the substrates, glutamate-malate (left hand) and succinate (right hand) respiration as shown by the increase in the basal level of respiration. This release of the coupling of respiration indicates a loss of energy conservation capacity. With further illumination both the basal and coupled respiration is inhibited.

Under similar conditions cytochrome c oxidase and ATPase activity is unaffected. The results implicate damage to dehydrogenases and damage to the structural integrity of the membrane, but not to catalytic activity of proteins buried in the hydrophobic membrane interior. For details see Ref. 3 .

(XBL 7512-9231)

4. B. B. Aggarwa1, Y. Avi-Dor and L. Packer, Abstract presented at the American Society of Biological Chemists Meeting, June 6-10, 1976, San Francisco, CA.

5. B. B. Aggarwa1, Y. Avi-Dor, and L. Packer, Abstract presented at the 7th Intermational Congress of Photobiology, August 29 - September 3, 1976, Rome, Italy. 


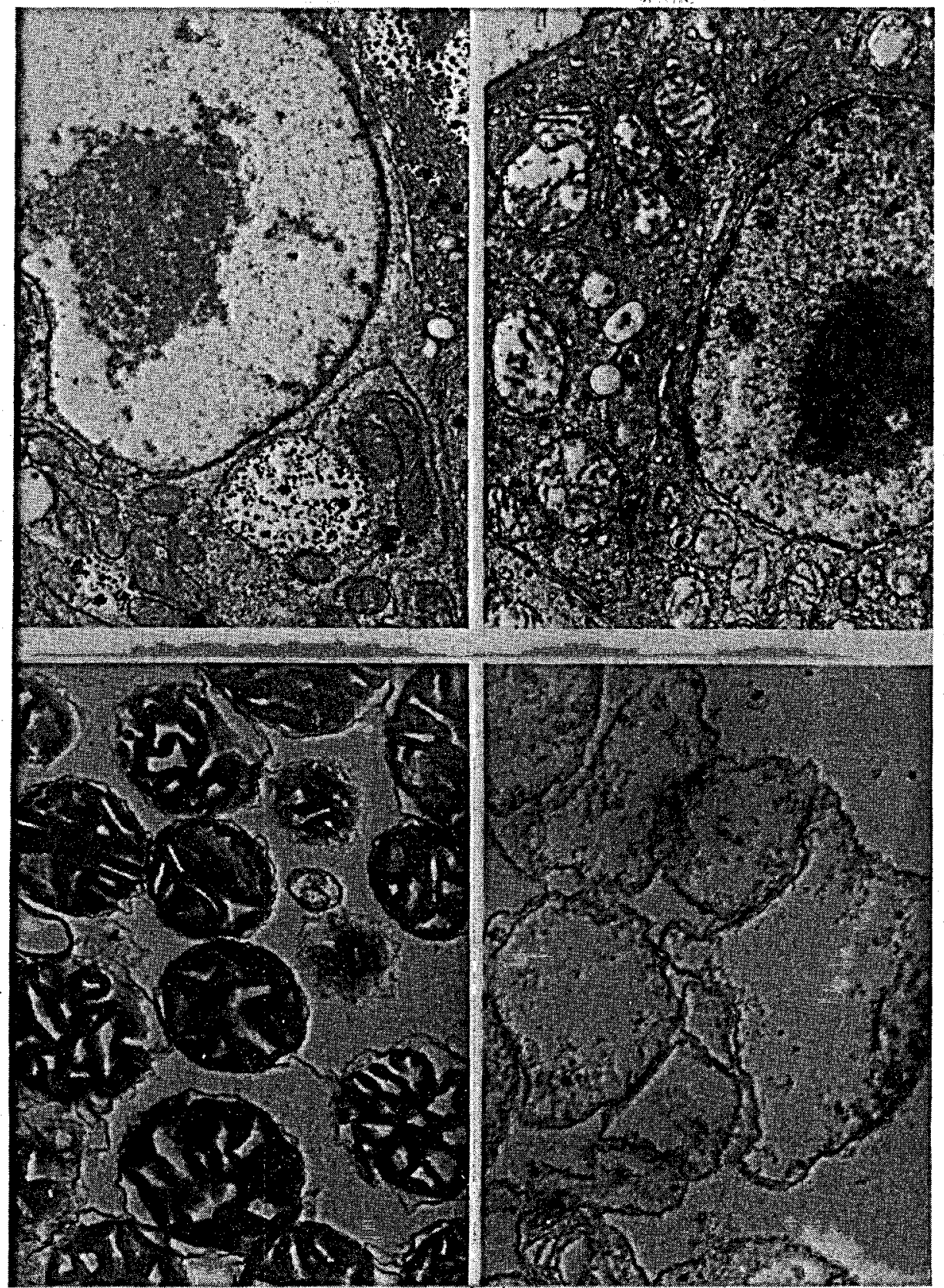

(A)

(B)

Fig. 3. Effect of visible light treatment on mitochondrial morphology. Illumination with visible light causes damage to the structure of mitochondria. (A) in situ experiment with illuminated hepatcytes. Showing mitochondrial morphological changes in early stage of light exposure. (Upper left control; upper right, light exposed.)

(B) Isolated rat liver mitochondria exposed for 5 hours to visible light as in Fig. 2 shows swelling, derangement of inner membrane structure and loss of matrix material. (Lower left, control; lower right, light exposed.)

(XBB 771-202) 


\section{ESTUARINE POLLUTANT AVAILABILITY STUDY* ${ }^{\dagger}$}

Victor C. Anderlini, John W. Chapman, Amos S. Newton, Robert W. Rosebraugh, Brian E. Cole, Brock deLapp,

Donald C. Girvin, Alfred T. Hodgson, Susan J. McCormick, Lisa Nelback, Robert K. Okazaki, Michael H. Panietz, Timothy Schmidt

The pollutant availability study was designed to determine which factors effect the availability of trace elements to invertebrates in central San Francisco Bay, and specifically to determine whether the spoiling of dredged materials results in an increase in the availability of trace elements to the invertebrate fauna in the spoil area. Spoiling is the disposal of dredge material in the water column in an area removed from the area being dredged. In this study, a change in availability is defined as a change in a trace element concentration in an invertebrate species. In central S.F. Bay, the normal spoil site is near Alcatraz Island. . For the work described here, an experimental spoil site was established near Buoy 6 , east of Angel Island. For a comparison of the effects of spoiling with those of a large point source of pollution, a study site near the East Bay Municipal Utility District sewer outfall (EBMD Site) was established. Figure 1 shows the location of the two sites in central S.F. Bay. The insets on Fig. 1 show the arrangements of sampling stations at each respective site.

The trace elements $\mathrm{Ag}$, $\mathrm{As}, \mathrm{Cd}, \mathrm{Cr}, \mathrm{Cu}, \mathrm{Fe}$, $\mathrm{Hg}, \mathrm{Mn}, \mathrm{Ni}, \mathrm{Pb}, \mathrm{Se}$, and $\mathrm{Zn}$, and the chiorinated hydrocarbons DDT, DDD, DDE, and PCB (as Aroclor 1254) were determined in sediments, suspended sediments, settled particulates, selected benthic invertebrates and mussel transplants at each spoil site station before during and after the disposal at the spoil site center of $10,000 \mathrm{~m}^{3}$ of polluted sediments from the Oakland Inner Harbor. Dissolved $\mathrm{Cd}, \mathrm{Cu}$, and $\mathrm{Pb}$ were determined in water samples collected during and after the experimental spoiling operation. The water quality parameters, temperature, salinity dissolved oxygen, nitrate, ammonia nitrogen, and $\mathrm{pH}$ were monitored before, during and after the spoiling operation. Similar measurements were made at stations at the EBMUD site.

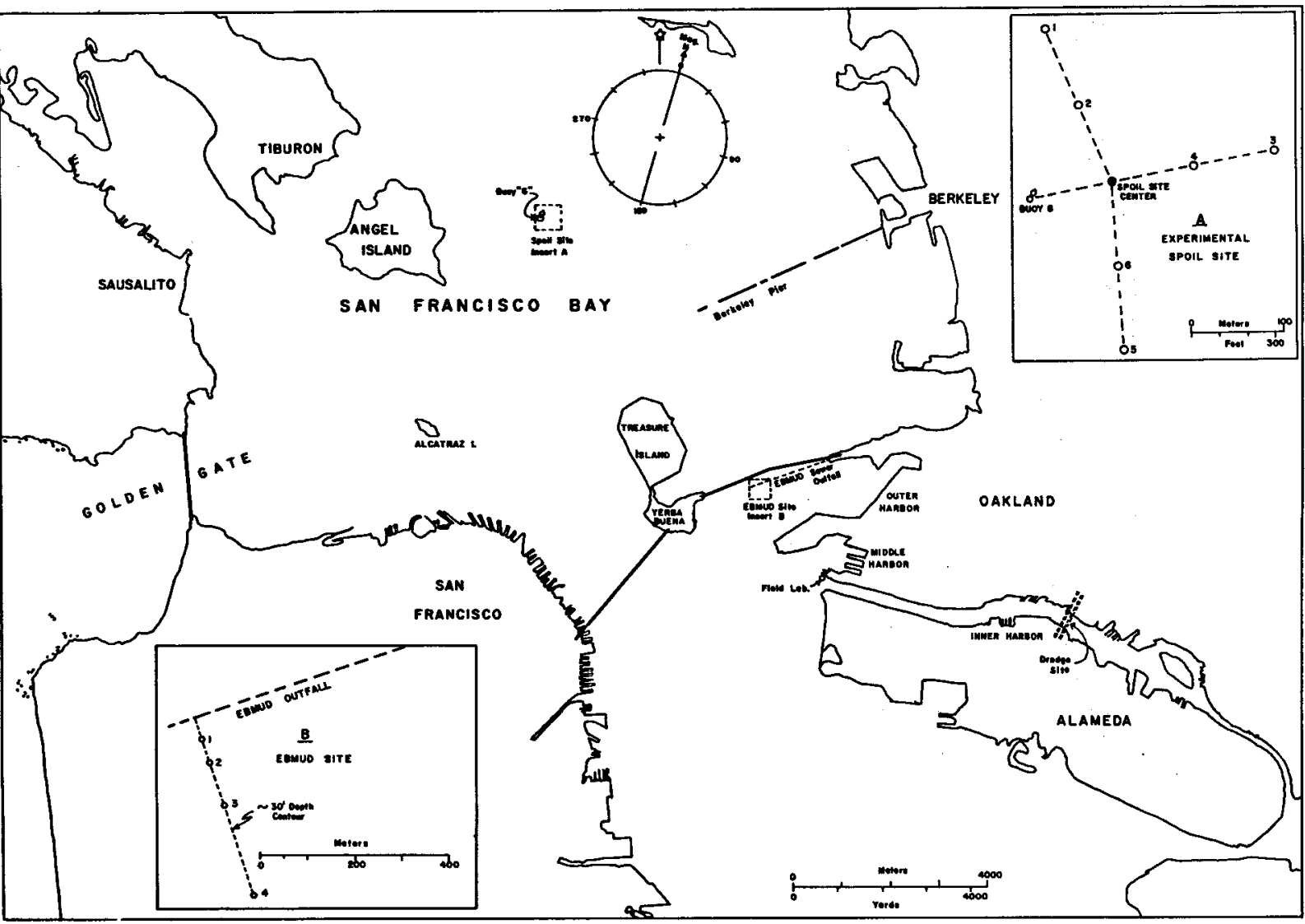

Fig. 1. Map of experimental study areas in San Francisco Bay. Insets show the experimental Spoil Site area and the EBMD Site area. The Field Laboratory Site and the experimental Dredge Site are indicated. 
The pathways by which estuarine invertebrates ccumulate trace elements were investigated in a laboratory study in which the clam, Macoma Nasuta; the worm, Pectinaria californiensis; and the mussel, Mytilus edulis, were exposed to a sea water control (2.7\% salinity) and three added concentrations of $\mathrm{Cd}, \mathrm{Hg}$, and $\mathrm{Pb}$ in sea water. The influence of suspended particulates was studied in a field experiment in which selected invertebrates were exposed in situ to altered concentrations of suspended particulates.

Six stations were established at the spoil site as shown in Fig. 1, inset A. The inner stations were $100 \mathrm{~m}$ from the spoil site center and the outer stations $200 \mathrm{~m}$ from that center. At each station the local invertebrate populations were sampled by divers using a water suction dredge. In addition, three sets of Mytilus edulis transplants (from Berkeley Pier) in Nytex bags with four bags of transplants in each set were arranged at each station as shown in Fig. 2. At each station, settling tubes

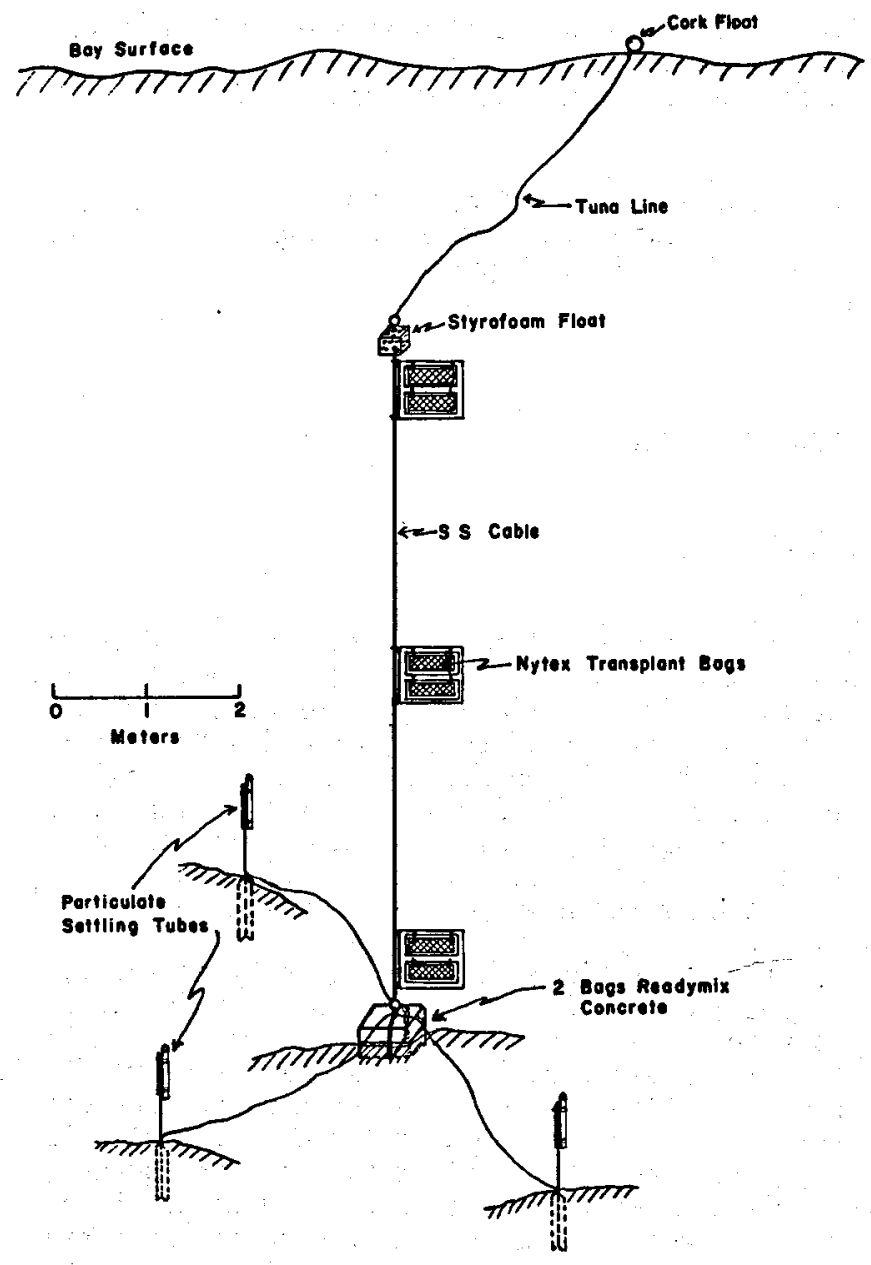

Fig. 2. Diagram of one station at the Spoil Site or the EBMD Site illustrating the arrangement of transplant bags and particulate settling tubes.

(XBL 771-7227) were installed to collect settled particulates from the water column. Stations at the EBMUD site were arranged similarly. Spoil site stations were sampled one month before, immediately after, and one month after the experimental spoiling operation in which $10,000 \mathrm{~m}^{3}$ of polluted sediment from Oakland Inner Harbor were disposed at the spoil site center in 7 dredge-hopper loads over a 38 hour period.

The results of the study showed spoiling to produce no significant effect on the trace element or the chlorinated hydrocarbon content of the $M$. edulis transplants or of the invertebrates in the 1ocal area (specifically $\underline{M}$. nasuta, $P$. californiensis, and S. elongata). An increase in the concentrations of $\overline{\mathrm{Cu} \text { and } \mathrm{Fe}}$ in the surface sediments at the spoil site inner stations was observed after the spoiling operation. In water samples collected at the spoil site center during the spoiling operations, transient increases in the concentrations of chlorinated hydrocarbons and of dissolved $\mathrm{Cd}, \mathrm{Cu}$, and $\mathrm{Pb}$ were observed, but these increases lasted for less than 1.5 hours. Dissolved oxygen decreased momentarily and nitrate and ammonia nitrogen increased momentarily during each spoiling operation. These changes lasted less than 5 minutes. At the EBMD site, the clam, $M$. nasuta had higher concentrations of all 12 trace elements than did the clams at the spoil site. Trace element concentrations in the worms, P. californiensis, Glycera americana and Glycera robusta were approximately equal at the two sites.

In sediments, $\mathrm{PCB}$ and $\mathrm{DDE}$ concentrations were higher at the EBMUD site than at the spoil site, while DDD concentrations were about equal at the two sites.

Trace element concentrations in mussel transplants at the spoil site, EBMD site, and Berkeley Pier (the source of all mussel transplants) were about equal. Concentrations of DDD and PCB increased through time at all three locations with the largest increases occurring at the EBMUD stations.

The results of the laboratory study of the uptake of metals from sea water showed the following: a) Mercury was accumulated from sea water at all added concentrations by $M$. nasuta, $P$. californiensis, and $\underline{M}$. edulis; b) Little, if any lead was accumulated by $\underline{\underline{F}}$. californiensis for all $\mathrm{Pb}$ concentrations added, while the control worms desorbed lead during the 15 day experiment. $M$. nasuta absorbed $\mathrm{Pb}$ at the highest added concentration, but no change was observed in control clams or in clams in low concentration tanks. $M$. edulis absorbed $\mathrm{Pb}$ from all added concentrations, but the control mussels showed a desorption of $\mathrm{Pb}$; and c) Cadmium concentrations decreased in the worm, P. californiensis, at all concentrations tested. The clam, M. nasuta, accumulated $\mathrm{Cd}$ at high and medium concentrations, while control clams showed a constant Cd content. $M$. edulis in control tanks showed no accumulation of cd, whereas the accumulation of $\mathrm{Cd}$ in these mussels was observed to be linear with time for all concentrations of $\mathrm{Cd}$ added.

The results of the field experiment showed that a factor of two change in concentration of suspended particulates in water produced no change in the concentration of the twelve trace elements 
studied in the mussel, M. edulis, the oyster, Crassostrea gigas, or the clam, $\underline{M}$. nasuta.

In order to design a sampling strategy taking into account organism size and to evaluate the effect of internal deposits of sedimentary material on our results, two subsidiary experiments were performed. An investigation of the effect of invertebrate size on trace element concentration showed that: (1) in $\mathrm{M}$. nasuta, the elements $\mathrm{Ag}, \mathrm{As}$, $\mathrm{Cu}, \mathrm{Fe}, \mathrm{Hg}, \mathrm{Mn}$, Ni, and $\mathrm{Zn}$ correlate with size; (2) in P. californiensis concentrations of the elements $\mathrm{Ag}, \mathrm{As}, \mathrm{Hg}, \mathrm{Se}$, and $\mathrm{Zn}$ correlate with size, and (3) in M edulis, concentrations of the elements $\mathrm{Cd}, \mathrm{Cu}, \mathrm{Hg}, \mathrm{Mn}, \mathrm{Ni}$, and Se correlate with size. (These correlations were statistically significant at $p<0.05, p<0.01$, or $p<0.001 \mathrm{de}-$ pending on the metal tested.) As a result of the above internal metallic concentrations depending on organism size, a single size class of each particular invertebrate was taken for analysis, if possible, in the pollution availibility studies. Where the animals were not of sufficient abundance so a single size class could be isolated, an attempt was made to analyze a uniform mix of animal sizes.

Internal deposits of sedimentary material, possibly containing high concentrations of metals, were removed during a purge study which used $M$. nasuta and $\mathrm{P}$. californiensis as test organisms. These tests demonstrated that three days purging in water from the area in which organisms were collected with daily water changes gave optimal results, as measurements of the organisms' trace element content usually did not change after three days. A few exceptions were found, e.g., in P. californiensis, but a still larger decrease occurred between 6 and 12 days of purging. In the pollution availibility studies, most of the invertebrates were purged for three days, but some, such as the seapen, Stylatula elongata and the amphipod, Ampelisca milleri do not survive under purge conditions.

From the results of this study and the previous study at Mare Island Strait 1 it is concluded that dredging and spoiling produce little immediate effect and no lasting effects on the trace element content or the chlorinated hydrocarbon content of the invertebrates in the immediate and adjacent areas involved.

\section{FOOTNOTES AND REFERENCE}

*Condensed from LBL report no. UCID-3818, March 1976. This research was supported by the U.S. Army Corps of Engineers.

${ }^{T}$ This research was a collaborative study between LBL Energy and Environment Division and the Bodega Bay Institute of Pollution Ecology.

1. V.C. Anderlini, J.W. Chapman, D.C. Girvin, A.S. Newton and R.W. Risebrough, LBL report no. UCID-3666, Sept. 1974.

\title{
ECOSYSTEM STABILITY AND DYNAMICS
}

\author{
John Barte and Donald Levy
}

Technological and social changes in the next decades will frequently result in human activities making significant impact upon surrounding ecosystems. It is the long range goal of this program to understand ecosystem dynamics with a specific goal of developing reliable ecosystem stability indicators. These indicators, measured in an undisturbed ecosystem, are designed to predict whether the ecosystem will respond gently or violently to a specified and possibly large perturbation. This approach differs substantially from most previous attempts to assess human impact on ecosystems which usually concentrate on developing techniques that indicate the extent to which a system has already been disturbed by human activity. Our approach includes theoretical investigation of ecosystem properties and laboratory investigation of aquatic microbiomes ranging in size up to 1000 liters.

Persistent nontrivial freshwater aquatic microbiomes have been established in our laboratory and procedures for measuring and testing the theoretically suggested stability indicators are being carried out. Initial applications of our work to energy technologies will focus on coal gasification and liquefaction and geothermal technologies.
Work is also planned on developing a detection system for measuring trace gas $\left(\mathrm{H}_{2} \mathrm{~S}, \mathrm{~N}_{2} \mathrm{O}\right)$ transfer rates from/into soil and water surfaces. Subsequent applications of this system will be an investigation of the question of whether intensive fertilizer use will increase $\mathrm{N}_{2} \mathrm{O}$ production rates enough to ultimately alter stratospheric ozone levels and to the assessment of background $\mathrm{H}_{2} \mathrm{~S}$ production rates near potential geothermal sites.

The theoretical work, using analytic (e.g., Liapunov) and computer techniques to study various ecosystem models, has identified several potential stability indicators. 1-3 The models used are simple enough to allow salient relationships between ecosystem parameters to be abstracted, yet realistic enough to suggest the orientation of subsequent experimental work. One of these indicators is a combination of parameters related to the bacterial carrying capacity and bacterial action on detritus, 1,2 In a number of models, this indicator is linearly related to the ratio of the change in bacterial acitivity to the change in decomposable matter $(\triangle B / \Delta D)$, where $B$ is a measure of the activity of the bacterial community and $D$ represents the decomposable organic matter that provides an energy and carbon source for bacteria. Large values of $\Delta B / \Delta D$ 
indicate instability, small values stability.

There is an intuitive basis for believing that this quantity might be a stability indicator. If the decomposers are not saturating their resources of decomposable substrate, but are limited by another mechanism (e.g., grazing, temperature, surface area), then a perturbation of the system (which eventually will be expressed by a change in decomposable substrate) may not affect the activity of decomposers. Nutrient recycling activity then remains fairly undisturbed and the system as a whole does not change drastically. The converse also is true. A large change in bacterial activity would suggest that decomposable resources are limiting and that the system is less stable than a sma1l change in bacterial activity would suggest.

A practical experimental method for determination of this indicator immediately presents itself. Replicate samples of the system under study are removed and half of these samples are subjected to an addition of organic matter; the ratio of the change in bacterial activity in treated subsamples to that in the controls after equilibration serves as a measure of $\Delta B / \Delta D$. Measurements of this quantity are now being carried out in our laboratory.

The other stability indicator studied theoretically uses the time distribution for an initial c14 pulse injected into the inorganic carbon pool of an ecosystem to cycle back through various intermediate pathways and re-enter this pool. 3 our theoretical work has defined an index which can be used to correlate, in a number of cases, the shape of this transit time distribution with the systems' stabi1ity properties. In other work, a method has been developed for reducing a large class of integrodifferential equations with time-delays to purely differential equations, which are then ammenable to our basic techniques of stability analysis. Theoretical work looking for new stability indicators will continue.

We have established four aquatic microbiomes, housed in a refrigerated room where their temperature is maintained at $18 \pm 1^{\circ} \mathrm{C}$. A bank of eight high-output fluorescent lights on a controlled light-dark cycle illuminates each tank. The microbiomes have a diverse microbial community (bacteria, several dozen species of algae and protozoa), a zooplankton community (rotifers, copepods, cladocerans, and ostracods), a benthic community of chironomid larvae, and small mosquito fish. Results to date indicate that these systems persist for a long period of time and that the values of various biological and chemical parameters in these microbiomes are similar to those found in many natural aquatic systems (Fig. 1). Current1y, the facilities are being expanded to include a number of microbiomes ranging in size from 15 to 200 1iters. The four large 1000-1iter microbiomes serve as both experimental systems and as sources of inoculum for the smaller systems. We use the smaller tanks to 1) test effects of size and shape in the replicability and persistance of the microbiomes, 2) evaluate the effects of two bottom substrates (silica gravel versus 258 dolomite + $75 \%$ silica grave1), and 3) evaluate the effects of various aeration techniques.

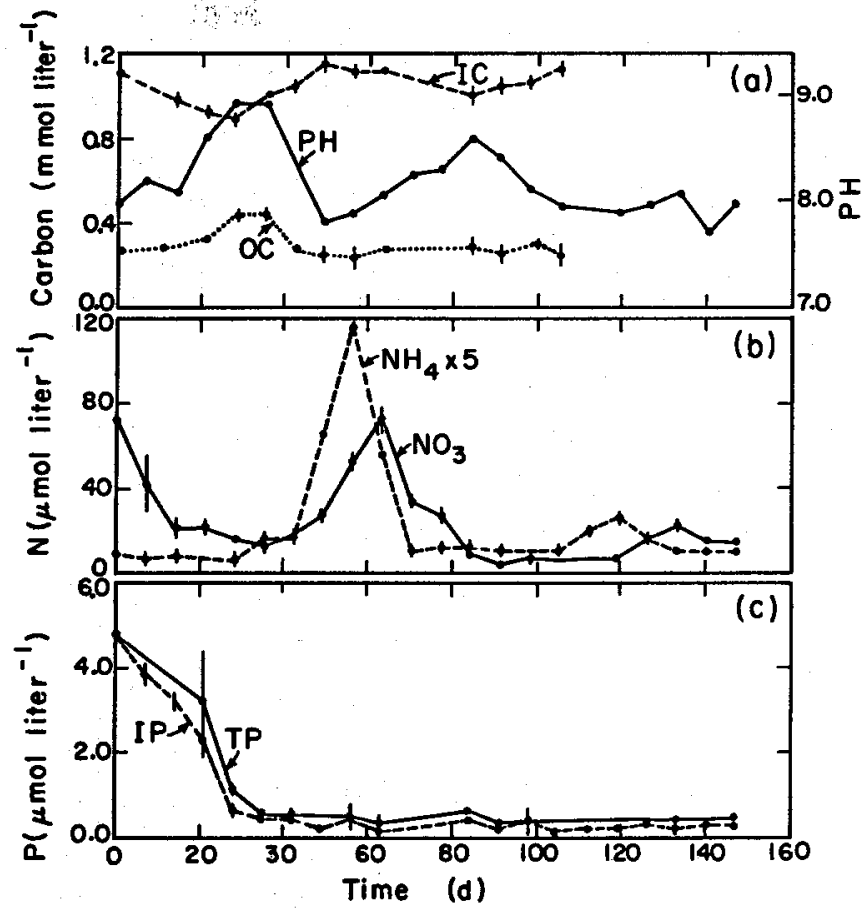

Fig. 1(a). Chemical measurements from System I. Vertical lines indicatd the standard error for duplicate measurements. (a) $\mathrm{pH}$, inorganic carbon (IC), and organic carbon (OC); (b) $\mathrm{NH}_{4}$ and $\mathrm{NO}_{3}+\mathrm{NO}_{2}$; (c) inorganic phosphorus (IP) and total phosphorus (TP). (XBL 771-120)

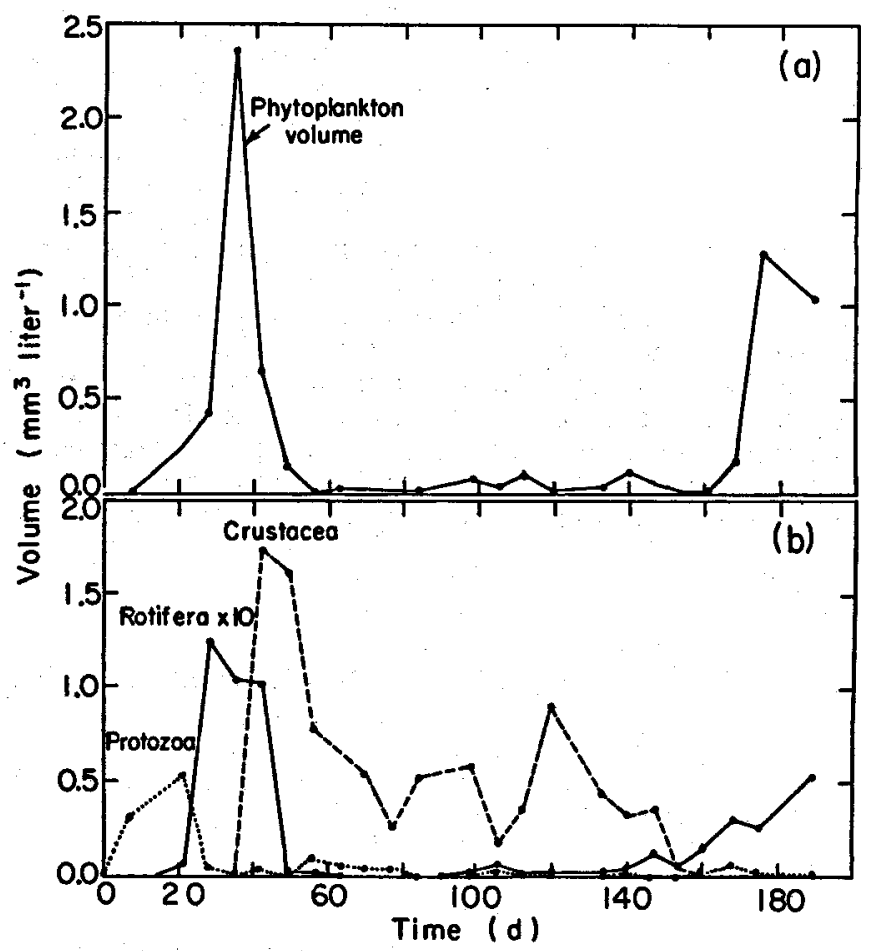

Fig. 1(b). (a) Total volume of phytoplankton in system I; (b) total volume of protozoa, rotifera, and crustacea in system $I$. (XBL 771-119) 
The stability indicator related to bacterial carrying capacity and action on detritus $(\mathrm{AB} / \triangle \mathrm{DD})$ is currently being measured. This involves the addition of several types of detrital spikes composed of either autoclaved bacteria (E. coli), algae, or mixture of organic carbon compounds to selected aliquots. The bacterial responses to this spike are then measured by plate counts and comparison of dark to light $\mathrm{CO}_{2}$ evolution rates. Other parameters measured in these experiments are phosphatase activity, florescence, phosphate levels, nitrate-nitrite levels, and ammonia levels. These allow for additional correlations with stability properties as well as provide additional information as to how a pulse in the detrital compartment is transmitted through the system.

Perturbation experiments will be carried out on five different sets of microbiomes in order to test the stability indicators. Each set will consist of 4 microbiomes. The systems will differ in their nitrogen and phosphorous levels and will include a broad range of trophic states found in nature. A number of biological and chemical parameters will be monitored in the systems, and their response to perturbations will be correlated with values of the bacterial response stability indicator measured prior to the perturbations. Two of the replicates in each set of microbiomes will serve as controls. The following parameters are monitored during experiments, (some routinely, others occasionally): $\mathrm{pH}$, temperature, dark and light $\mathrm{CO}_{2}$ evolution, chlorophy11, primary productivity, ATP, organic and inorganic carbon, nitrogen, phosphorus, bacterial counts, activities of selected enzymes associated with decomposition, and occasional taxonomy. The perturbations studied will include the addition of different concentrations of a mixture representative of coal gasification and liquefaction effluents. In addition to providing a test of our indicators, such a perturbation provides the advantage of direct assessment of the impact of this type of effluent on a functioning, persistent ecosystem (albeit, relatively sma11). This reflects the in situ response of organisms more closely than methods which use pure cultures of organisms in a medium conditioned by their presence alone, as is usually done. The broad range of parameters monitored during experiments will provide new useful information about aquatic ecosystem dynamics, particularly decomposerdetritus interactions, as well as enable us to determine new correlations of stability properties with a wide range of parameters. In the future, reliable methods for treating samples and measuring the bacterial response stability indicator in the field will be developed and tested both for aquatic and terrestrial ecosystems. Terrestrial field testing will first be done on a set of replicate soil patches (each about $10 \mathrm{~m}^{2}$ ). Later, measurement of the indicators in selected soil patches in the vicinity of the Geysers geothermal area will be made.

We are also developing an in-situ detector of trace gas transfer rates over soil and water surfaces, sensitive to levels of $\sim .01 \mathrm{ppm}$. The device will consist of a transparent chamber placed over a selected patch of soil or water. The chamber is linked to a gas reservoir which will receive the net excess or produced trace gas. Gas analysis will be done using standard chromatographic detection systems. Once operational, the device will be used to:

1. Measure natural background trace gas emission rates at geothermal sites (in particular $\mathrm{H}_{2} \mathrm{~S}$ ). This will allow impact studies to rigorously compare conditions before and after construction of geothermal facilities.

2. Determine the increase in $\mathrm{N}_{2} \mathrm{O}$ production rates due to intensive application of nitrogenous fertilizers. Significant increases in $\mathrm{N}_{2} \mathrm{O}$ production could result in significant depletion of stratospheric ozone.

\section{REFERENCES}

1. J. Harte and D. Levy, "On the Vulnerability of Ecosystems Disturbed by Man," Report of the Plenary Sessions of the First International Congress of Ecology, The Hague, Netherlands, Edited by W. H. Van Dobben and R. H. LoweMcConne11, Dr. W. Junk B. V. Publishers, 1975, pp. 208-224.

2. M. Dudzik, J. Harte, D. Levy, and J. Sandusky, "Stability Properties of Ecosystem Nutrient Cycles," LBL-3238, 1975 (to be published in Ecology).

3. J. Harte and H. Morowitz, 'Nutrient Transit Time Diversity: A Measure of Ecological Organization and Stability," LBL-4441, 1975.

\title{
ENVIRONMENTAL ASSESSMENT OF SOLAR ENERGY CONVERSION
}

\author{
M. Davidson, D. F. Gnether, M. W. Horovitz
}

\section{INTRODUCTION}

In recent years there has been a rebirth of interest in solar energy conversion, and a rapid growth (albeit from a sma11 base) in Federal support for solar energy research and development. This interest resulted not only from the realization that solar energy could supplant diminishing sup- plies of fossil fuels, but also from the expectation that this form of energy would not have the environmental problems of conventional energy sources. Solar energy conversion includes solar thermal conversion (usually to electricity), photovoltaic conversion, wind energy conversion, bioconversion, heating and cooling of buildings, and industrial and agricultural process heat. of 
these, only bioconversion would generate air and vater pollution in a manner analogous to the use of fossil fuels. None of these conversion processes would have the problems associated with the radioactive materials of nuclear plants. However, especially if one takes environmental effects to include those of a socio-economic nature, the various solar technologies will impact the environment. Some of the effects will be similar to those of conventional energy sources, such as land and water use conflicts. Others will be fairly unique, such as the potential impacts on ecosystems and climate due to large arrays of wind turbines, solarthermal mirror fields, or ocean thermal plants.

The Federal solar energy program is currently in the development phase, and it will be some years before solar conversion receives widespread use. However, preliminary environmental assessments based on existing designs and development plans serve several purposes. The assessments could influence choices between alternative approaches to a given conversion process; could lead to modifications to mitigate the environmental impacts; or could contribute to the termination of the development of a technology that is judged to be environmentally unacceptable. Further, in future energy supply scenarios involving solar energy, a proper comparison between choices of alternate energy types should include the relative environmental impacts of the different types.

This project was initiated late in calender year 1975. However, as a precursor to the project, the laboratory coordinated the solar imput to the Balanced Program Plan (BPP) of the Division of Biomedical and Environmental Research of ERDA. This BPP was to identify and develop a research plan for investigation of environmental impacts associated with the energy technologies being pursued by ERDA. This effort will be discussed here as it provides a logical framework for presenting the initial work of the project.

\section{ANNUAL ACTIVITIES}

The solar input to the Balanced Program Plan 1 was a joint effort of the various ERDA laboratories. The effort was coordinated by $D$. Grether in association with M. Simons and J. Sandusky, all from LBL. The document describes the state of development of solar technologies; identifies potential environmental problems; suggests research that would lead to a quantitative understanding of these problems; and estimates the costs and time required for the research. The potential environmental effects can be grouped into four broad categories.

\section{Socio-economic Impacts of Wide-spread Utilization}

Solar energy conversion will (depending on the solar technology) use relatively greater quantities i land, materials, water and capital than convenlonal technologies. Solar technologies are usually only optimizable in certain geographical areas (e.g., solar thermal conversion to the semi-arid southwest), and demographic shifts may accompany utilization of solar energy. Energy used, materials consumed, and pollution generated during manufacture of solar devices must be accounted for in comparisons with conventional energy sources.

\section{Alterations of Ecosystems and Climate}

Solar energy facilities such as mirror fields, wind turbines, and ocean thermal plants, will alter the local ecosystem and micro-climate. Many such facilities may impact regional or global climate. The relatively unique aspects of most solar facilities indicate that these alterations will differ in kind and degree from those of conventional energy sources.

\section{Health and Ecological Effects of Effluents from Bioconversion Processes}

Conversion of organic wastes or especially grown material (bioproduction) will result in air and water pollution from effluents in much the same manner as for fossil fuels. These effluents will differ in kind from those of fossil fuels and thus have potentially different health and ecological effects.

\section{Health and Ecological Effects from Materials of Solar Devices}

Solar devices, such as those installed on residential or commercial buildings, will release working fluids, refrigerants, storage media and other materials to the environment. This release will result from erosion, corrosion, fire, and routine maintenance. To the extent that these materials are new; or would be released in greater quantities than at present, they may constitute a hazard.

The initial effort in the project has been to identify selected areas, from the above categories, that can be studied with existing techniques and methodologies. In order to focus the investigation, a particular solar technology was selected: the central receiver concept for solar thermal conversion to electricity. Current designs, while still evolving, are considered to have the basic properties of any future, cormercial power plants. From an environmental analysis point of view, central receiver facilities are reasonable models of solar conversion to study because (1) they would be large and therefore have the possibility of maximum impact and (2) their basic design is not likely to change much in the future, thereby rendering this work obsolete. The categories most relevant to solar thermal conversion are (1) and (2). For category (1), computer models were identified that could be used to estimate the impact on the economy of the relatively greater materials requirements of solar plants as compared to conventional power plants. complementary models give the labor requirements and residues (pollution) associated with the production of the materials. For category (2) a preliminary investigation was made into microclimatological models that could be used to study the local climate effects of a solar thermal plant.

\section{PLANNED ACTIVITIES}

ERDA is currently funding four design studies of the central tower concept. Estimates of materials requirements for the four designs, together with 
scenarios of market penetration of solar thermal conversion, will be used to provide input to the computer models referred to above. Based on experience with the central receiver concept, the methodology will be developed to have more detail and analytical depth in the models. The methodology will then be applied to other solar technologies.
Microclimatological effects will continue to be pursued. In addition, an attempt will be made to collaborate with existing atmospheric research institutions to investigate the regional and global implications of massive use of solar thermal conversion. These institutions have computer models that, while relatively crude, may be sensitive to large land areas modified by the power plants.

THE LAKE COUNTY PROJECT-THE LOCAL, IMPACTS OF GEOTHERMAL DEVELOPMENT AT THE GEYSERS

\author{
o. Weres and $L$. Volzintine
}

The geothermal steam field in the Sonoma portion of the Geysers KGRA (known geothermal resource area) is currently the largest geothermal energy development in the world ( $502 \mathrm{MW}$, and the only producing field in the U.S. The Lake County portion of this KGRA is now being prospected and appears to have an even greater potential than the area of original exploration, so that geothermal development in Lake County appears imminent. But local reactions to this prospect are deeply divided. On one hand Lake County is the poorest county in California on a per capita basis, and geothermal development would undoubtedly stimulate its economy. On the other hand, with one fourth of the population being retired and another apparently large segment having breadwinners who work outside the county, there is a large group of people who value environmental quality much more than they value economic growth. Tourism is a major industry in the county and could be seriously hurt by environmental degradation. Finally, the fears of all these people have been greatly aggravated by occasional incursions of hydrogen sulfide contaminated air from the Sonoma county geothermal fields (which lie very near the county line), and the noise of air drilling.

One results of this conflict is that the 1ocal govermment wants to make a serious effort to fully comprehend the implications of geothermal development and to develop a well thought out policy with attendant regulations. Unfortumately, their efforts have been greatly handicapped by lack of scientific expertise and money. This project serves the purpose of providing such specialized aid to Lake county as is possible at its level of funding, and represents the bulk of ERDA's effort at the Geysers.

During the past year, the main emphases of the project have been on the analysis of public opinion regarding geothermal development and on the local socio-economic impacts. The public opinion work is nearly completed and published, 1,2 and the socioeconomic analysis is in full swing. We have also pursued bio-environmental integrated assessment and analytic studies of the reservoir and plant cycle in order to determine their environmentally decisive attributes. Massive reports covering these areas are nearing completion and will be released shortly.

our study of attitudes towards geothermal development was based on a county-wide opinion poll, to which approximately $4 \%$ of the registered voters in
Lake County responded. The survey data were collected from a mail-out questionnaire prepared by students of the College of Natural Resources, University of California Berkeley, and sent out over the signature of Don Johnson, Lake County P1anning Director. The secondary analysis was done by us.

Table 1 depicts the raw percentages responses to the "policy and perception" questions. (For brevity, the responses to the purely demographic questions have been omnitted.) We combined some of the questions to produce two "indicies" of overall attitudes. The Go No-Go Index was constructed by averaging the responses to question (1) "County needs economic expansion," (9) "I will lease my land", and (10) "Exploratory wells should be drilled". The No Environmental Impacts Index was constructed by averaging the responses to questions (6) "No odor impacts," (7) "No noise impacts," and (12) "No visual impacts."

The main conclusions of our analysis were:

1) A large majority of the respondents are in favor of geothermal development provided that it is suitably regulated to minimize negative environmental impacts. Table 1 shows that $68 \%$ of the respondents felt that the county should Go ahead with development. However, this does not indicate anything about a need for regulation in the process. Question 5 showed that $67 \%$ felt that unregulated development was not compatible with Lake County's present economy. Furthermore, even among those respondents who were "Go" and also saw the econonic benefits of geothermal development to be greater than any environmental costs, $84 \%$ still favored close regulation (question 16).

2) The main determinants of the respondents' approval or disapproval of geothermal development are their expectations concerning environmental inpacts and economic benefits of development. These influences were remarkably strong. Figure 1 shows that of those who perceive no environmental impacts, more than $90 \%$ favor development, while for those who do see envirommental impacts, only 36\% favor development. The same sort of relationship occurred for those who do or do not expect economic benefit Used together, these two variables produce a treme dous division from $94 \%$ favoring development (those who see economic benefits and no envirommental impacts), to only $9 \%$ favoring development (those who see environmental impacts and no economic benefits). 
Table 1. Aggregated percentage tabulation of responses, total $\mathrm{N}=786$.

1. Lake County needs economic expansion

2. Geothermal development will increase jobs and tax revenues

3. Can trust geothermal companies

4. Economic benefits environmental costs

5. Non-regulated development is compatible with the present economy

6. Will be no odor impacts

7. Will be no noise impacts

8. I will not move because of geothermal development

9. I would lease my land

10. Exploratory wells should be drilled

11. Geothermal development will increase property values

12. Will be no visual impacts

13. Go No-Go Index

\section{N}

781

779

772

773

772

774

771

774

751

771

770

768

745

14. No envirommental Impacts Index

$\&$ Agree $\&$ No opinion $:$ Disagree

15. I have visited a geothermal facility: $N=764$

$$
\frac{\text { Yes }}{42.5} \quad \text { No }
$$

16. Lake County geothermal should be: $\mathrm{N}=751$

$\begin{array}{lr}\text { Prohibited } & 8.0 \\ \text { Closely Regulated } & 84.7 \\ \text { Unregulated } & 7.3\end{array}$

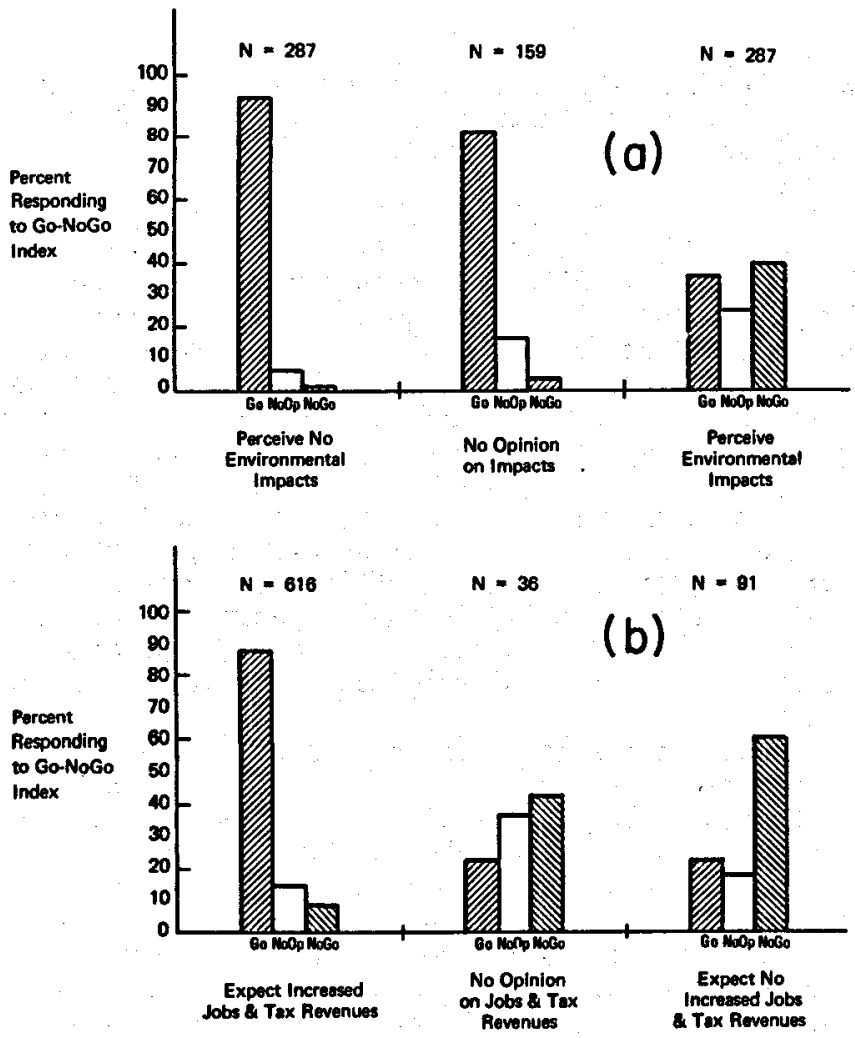

3) Pro- and anti-geothermal biases have a strong effect upon the perception of environmental impacts. This is demonstrated in Table 2 in which we present the relationship between a person's having visited a geothermal site, perception of environmental impacts, and Go No-Go attitude. It appears that those who favor development are reassured that it isn't environmentally disruptive when they visit the Geysers, while those who are undecided regarding development are likely to be displeased by what they see when they visit. The environmental perceptions of those who oppose development aren't affected by a visit as almost all of the opponents perceive development as being environmentally disruptive, regardless of having seen it or not.

4) Purely demographic characteristics of the respondents, such as employment status and years of residence in the county seem to have only iimited effects upon their attitudes toward geothermal development. A second report ${ }^{2}$ will show an analysis of responses to the same questionnaire responded to by $65 \%$ of the adults in the Cobb Valley, an area already impacted by $\mathrm{H}_{2} \mathrm{~S}$ emissions from the existing power plants in Somma County.

Fig. 1. (a) The relationship of the perception of environmental impacts and Go-NoGo index. (b) The relationship of the expectation of increased jobs and tax revenues and Go-NoGo index.

(XBL 764-1393) 
Table 2(a). Influence of visiting a geothermal facility upon the perception of envirommental impacts.

\begin{tabular}{lccc}
\hline & \multicolumn{3}{c}{ No Environmental Impacts } \\
& \& Agree & \& No Opinion & \& Disagree \\
\cline { 2 - 5 } Have visited & 43.3 & 17.6 & 39.0 \\
Have not visited & 35.2 & 26.5 & 38.3 \\
\hline
\end{tabular}

Table 2(b). Influence of visiting a geothermal facility upon the perception of environmental impacts, controlled for Go No-Go Index.

\begin{tabular}{|c|c|c|c|c|c|}
\hline $\begin{array}{l}\text { Controlled } \\
\text { response }\end{array}$ & $\begin{array}{l}\text { Visited } \\
\text { geo. facility }\end{array}$ & \& Agree & No opinion & : Disagree & $\begin{array}{l}\text { Correlation } \\
\text { coefficient }\end{array}$ \\
\hline \multirow{2}{*}{$\begin{array}{l}\text { "Go" } \\
N=485\end{array}$} & Yes & 62.2 & 20.1 & 17.7 & \multirow{2}{*}{.157} \\
\hline & No & 46.4 & 31.2 & 22.4 & \\
\hline \multirow{2}{*}{$\begin{array}{l}\text { No Opinion } \\
N=116\end{array}$} & Yes & 9.4 & 16.3 & 74.4 & \multirow{2}{*}{-.162} \\
\hline & No & 20.5 & 24.7 & 54.7 & \\
\hline \multirow{2}{*}{$\begin{array}{l}\text { "No Go" } \\
\text { N = } 117\end{array}$} & Yes & 0.0 & 3.6 & 96.4 & \multirow{2}{*}{-.137} \\
\hline & No & 3.2 & 4.8 & 91.9 & \\
\hline
\end{tabular}

Part of our Integrated Assessment of the Geysers KGRA will be an examination of the present and potential impacts of development upon the communities of Lake county. Industrialization can alter employment markets which, in turn, can have ramification for local economies, housing patterns, and the need for social services. Industrial development in rural areas has often been thought to be intrinsically beneficial, but as a result of recent environmental legislation (NEPA, CEQA, etc.) this assumption must be thoroughly analyzed. Because Lake County is a rural, sparsely populated and isolated county, the potential for large scale socio-economic disruption is great. Such "worst case" incidents stemming from energy development projects have been well documented in other rural areas (e.g., Sweetwater Co., Wyoming, 3 . Mercer Co., North Dakota). The likelihood of such a duplication occuring in Lake County will be examined.

We have chosen five main areas of potential econonic impacts for study. They are:

1) supply and demand of local geothermal labor markets with payroll and multiplier effects

2) public school districts

3) public protection

4) roads and highways

5) fiscal impacts (tax revenues, etc.)
We will chart the baseline cases for each section and then project the marginal impact of geothermal development expansion.

Geothermal development is a capital intensive venture. The specialized skills required are not found in the local Lake County employment market. Many of the drillers have immigrated from the oil and gas fields of southern California, Louisiana, and Texas. Local workers may pick up some peripheral contracts, but the bulk of the impact here will be on secondary employment in service industries.

Lake County is one of the few counties experiencing an increase in school enrollment, to the puzzlement of local school officials. Only a small portion of this, in the southern part of the county, can be attributed to geothermal operations at this time. This picture may change in the future.

To date the most direct impacts from geothermal development have been felt in the area of public protection. The social climate of Middletown, the largest town near the Lake County geothermal fields resembles, on a microcosmic scale; that of the stereotypical modern "frontier town". Local law enforcement time is disproportionately spent in the southern part of the county responding to physical altercations, disturbances, and odor complaints. 
The ultimate and biggest impact on the county fhould be, if development proceeds to the estimated $3000+M N$ potential, the massive generation of $\operatorname{tax}$ revenues. We feel there is the likelihood that the assessed valuation for tax purposes of geothermal power plants and steam fields may well exceed the assessed valuation of all other property and improvements in the county. One presently proposed $135 \mathrm{MW}$ geothermal power plant (P.G. and E. Geysers Unit 13) will produce the equivalent of $28 \%$ of all tax revenues now generated in the southern one third of the county. In addition to studying the implications of increased tax revenues, we also hope to look at land value changes, sociological trends, land use conflicts, leasing arrangements, steam pricing policies, capital investment requirements, and ultimate profitability of geothermal development in the Geysers KGRA.

The main environmental problems at the Geysers are, in order of decreasing importance: 1) $\mathrm{H}_{2} \mathrm{~S}$ emissions, 2) land surface modifications and erosion, and 3) drilling and well testing noise. The last is transitory and inevitable, and great advances have been made in minimizing it. The second is also to some degree inevitable, but it is clear that there is much room for improvement in field 1ayout and development plans. This improvement will probably come when local government and people fully realize that geothermal development can indeed be made much neater and demand this of the developers. Considerable improvement is already evident in the more recently developed areas.

There has also been some progress in dealing with the $\mathrm{H}_{2} \mathrm{~S}$ emissions but no particular technology has yet been proved to be completely satisfactory. Because of the relatively low temperature of the reservoir (approximately $238^{\circ} \mathrm{C}$ ) and the thermodynamics of the geothermal energy producing cycle, improved technology will be able to reduce $\mathrm{H}_{2} \mathrm{~S}$ emissions but not eliminate them.

\section{REFERENCES}

1. "Public Opinion Concerning Geothermal Development in Lake County, California". L. Vollintine and 0 . Weres, March 1976, LBL-4447.

2. "Public Opinion in Cobb Valley Concerning Geothermal Development in Lake County, California", L. Vollintine and 0. Weres, June 1976, LBL-5204.

3. Boom Town Growth Management: A Case Study of Rock Springs - Green River, Wyoming by John S. Gilmore and Mary K. Duff, Westview Press 1975.

\title{
COMBUSTION-GENERATED INDOOR AIR POLLUTION
}

\author{
Craig D. Hollowell, Robert J. Budnitz,
} George D. Case, and Gregory W. Traynor

Air pollution research has focused almost exclusively on pollution in outdoor air and has virtually neglected the indoor envirorment, despite the fact that the major proportion of the population spends far more time indoors than outdoors. Recent evidence1-8 however indicates that the levels of pollutants in the indoor environment can frequently exceed those levels that commonly occur in outdoor air. The inportance of indoor air pollution, only now being recognized, will ultimately have a large impact on the future design of epidemiological studies, on energy conservation strategies for buildings, and on the need for more stringent control of air pollution from indoor sources.

Most studies of indoor air pollution have been concerned with $\mathrm{SO}_{2}, \mathrm{CO}$ and/or total suspended particulate matter, and have assumed that the indoor levels arise from and are directly related to the outdoor levels, 1,2 Surprisingly little work has been done with respect to other potentially important indoor air pollution species, such as $\mathrm{NO}, \mathrm{NO}_{2}$, nitrates, sulfates, metals, organics, and the respirable fraction of the particulate matter. Even more surprisingly, indoor generated air pollution has been neglected in most indoor air pollution studies until quite recently.
A number of indoor sources may contribute appreciably to the total indoor air pollution, notably those associated with combustion (i.e., cooking, heating, and smoking), with aerosol spray can usage, with cleaning, and with food preparation. To date, there are a handful of documented studies that suggest that indoor sources may give rise to levels of pollutants in indoor air that greatly exceed typical levels in the outdoor enviromment. Although elevated levels of $\mathrm{NO}$ and $\mathrm{NO}_{2}$ have been observed during gas stove operation, the emissions from other combustion sources such as heating systems, water heaters, and clothes dryers have not been thoroughly investigated. More important is the fact that many nitrogen compounds, particulate as we11 as gaseous, were not considered in the gas stove studies, despite the recent demonstration that $\mathrm{NO}, \mathrm{NO}_{2}$, and $\mathrm{NH}_{3}$ can rapidly undergo catalytic oxidation or reduction to other important air pollution species such as nitrates, nitric acid, ammonium, and organic nitrogen compounds of the amino- and pyridino-type. 9

The work reported here is a field program undertaken in the summer and fall of 1975 to characterize gaseous and particulate air pollutants in six occupied homes with varying gas and electric cooking and heating configurations. 
Further work during this phase of the study will expand the field characterization work to more indoor sites of varying composition and will focus on laboratory controlled experiments which study emissions and control techniques of air pollutants from typical indoor combustion appliances.

The principal objective of the field study described here was to obtain quantitative data on the relationship of indoor to outdoor air pollutant concentrations as a function of gas cooking and heating appliance use. Six homes in Berkeley and Albany, California were selected for this study. All homes were fitted with gas heating systems, five with gas stoves, and one with an electric stove. Field measurements reported here were made under conditions simulating typical cooking and heating usage. Only the home with the electric stove was characterized during a cool season when the heating system is normally operated, and is therefore the only home to be evaluated for the effect of gas heating systems on indoor air pollutant levels. The major air pollutants that have been identified with gas combustion sources are carbon monoxide $(\infty)$, nitric oxide (NO), nitrogen dioxide $\left(\mathrm{NO}_{2}\right)$, and to a lesser degree, sulfur dioxide $\left(\mathrm{SO}_{2}\right)$ and particulates. The program also included measurement of ozone $\left(\mathrm{O}_{3}\right)$.

Figure 1 summarizes the average indoor/ outdoor data for gaseous air pollutant levels in the six homes under typical occupancy conditions of cooking and heating. The results clearly indicate high levels of $\mathrm{CO}, \mathrm{NO}$, and $\mathrm{NO}_{2}$ and elevated levels of $\mathrm{SO}_{2}$ from gas stoves, even during noncooking periods when pilot lights are probably the only major indoor source of these gases. The results are consistent with other studies 4-7 and show for the first time elevated indoor $\mathrm{SO}_{2}$ levels associated with gas stoves. A small increase in $0_{3}$ was observed during usage of the electric stove, but no elevated levels of the other gases were seen. Figure 1 also shows the high levels of $\mathrm{NO}$ and $\mathrm{NO}_{2}$ observed in the one home where the effect of a forced-air gas-fired furnace system was studied. The other five homes were not tested for the effect of gas heaters because the study was conducted in the warm summer months when the heaters are normally off. This study represents the first evaluation of the effects of heating systems on indoor air pollution levels. Previous studies $10-12$ have examined oil- and gas-fired residential and commercial heating systems; however, measurements were made of the flue gases to evaluate the contribution of heating systems to ambient outdoor air pollution and did not examine their effect on indoor air quality.

More detailed analyses of gaseous pollutant emissions from gas stoves were made in quasi-controlled experiments in some of the homes. Pans constructed of various materials (stainless steel with stainless steel, copper, and aluminum bottoms, iron, Pyrex, and Corning Ware Pyroceram) were used over one top gas burner to heat water. No appreciable differences in gaseous emissions were observed among the six pans tested. The effects of burner configurations on gaseous emissions were also ex-
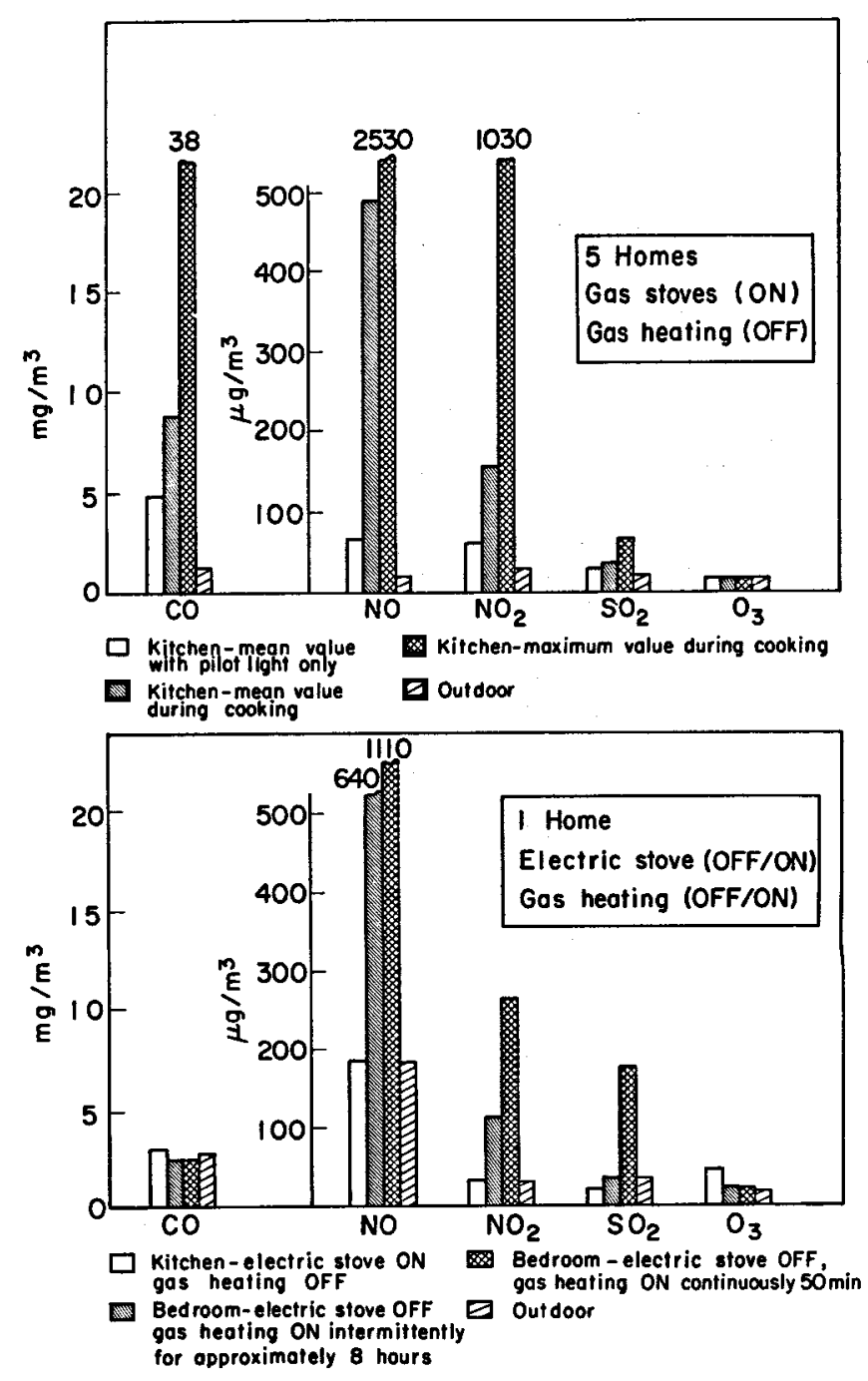

Fig. 1. Gaseous indoor and outdoor air pollutant levels observed at homes with varying cooking and heating configurations.

(XBL 7510-8561)

amined: (1) without the grate, (2) with the grate, and (3) with the grate plus a stainless steel pan containing water. CO emissions increased appreciably with two of the four stoves studied, when the pan was placed on the burner; the NO emissions were reduced by $50 \%$ from the same stoves when the pan was placed above the flame. This presumably indicates a reduction in oxygen availability and redistribution of flame energy with a resultant reduction in flame temperature. These conditions could give rise to increased $\mathrm{CO}$ production due to incomplete oxidation of $\mathrm{CO}$ to $\mathrm{CO}_{2}$ and reduced NO production in accordance with the high temperature dependent Zeldovich mechanism:

$$
\begin{aligned}
& \mathrm{N}_{2}+\mathrm{O} \rightarrow \mathrm{NO}+\mathrm{N} \\
& \mathrm{N}+\mathrm{O}_{2} \rightarrow \mathrm{NO}+\mathrm{O}
\end{aligned}
$$

The $\mathrm{NO}_{2}$ and $\mathrm{SO}_{2}$ levels were not appreciably affected by the burner conditions.

One of the homes was equipped with a ventilation fan above the stove and no exhaust duct 
from the oven to the outdoor air; the other homes, however, were fitted with an oven exhaust duct, but no fan. The effect of gas oven usage was studied in three homes and the results are summarized in Table 1. It is obvious that gas oven usage under poor ventilation conditions can result in elevated levels of $\mathrm{CO}, \mathrm{NO}, \mathrm{NO}_{2}$ and even $\mathrm{SO}_{2}$.

Table 1. Gaseous air pollutant levels observed in homes with gas ovens ${ }^{a}$ (Observed Levels $\pm 15 \%$ ).

\begin{tabular}{lrrrr}
\hline & \multicolumn{4}{c}{ Home numbers } \\
\cline { 2 - 5 } & \multicolumn{2}{c}{2} & 3 & 5 \\
\cline { 2 - 5 } Ventilation & $\begin{array}{r}\text { No duct, } \\
\text { conditions }\end{array}$ & $\begin{array}{c}\text { No duct, } \\
\text { fan off }\end{array}$ & $\begin{array}{c}\text { Duct, } \\
\text { fan on }\end{array}$ & $\begin{array}{c}\text { Duct, } \\
\text { no fan }\end{array}$ \\
$\mathrm{CO}\left(\mathrm{mg} / \mathrm{m}^{3}\right)$ & 23 & 6 & 2 & 7 \\
$\mathrm{NO}\left(\mu \mathrm{g} / \mathrm{m}^{3}\right)$ & 2000 & 1000 & 500 & 150 \\
$\mathrm{NO}_{2}\left(\mu \mathrm{g} / \mathrm{m}^{3}\right)$ & 850 & 350 & 150 & 95 \\
$\mathrm{SO}_{2}\left(\mu \mathrm{g} / \mathrm{m}^{3}\right)$ & 110 & 65 & $<25$ & $<15$ \\
\hline
\end{tabular}

Measurements in center of kitchen, approximately 1.5 meters above the floor, with the gas oven at $550^{\circ} \mathrm{F}$ for approximately 20 minutes.

The studies on gaseous pollutants clearly indicate elevated levels of $\mathrm{CO}, \mathrm{NO}$, and $\mathrm{NO}_{2}$ from gas combustion appliances. The duration of these elevated levels may be under one hour for gas stove usage, but may be continuous for gas-fired heating system usage in cool seasons. The levels of $\mathrm{NO}_{2}$ observed in the six homes all greatly exceed the Japanese 24-hour standard 13 and exceed the proposed one-hour U.S. standard.14 Certainly the high $\mathrm{NO}_{2}$ levels observed in this study deem that more attention be directed to the potential health impact of indoor combustion sources.

Ambient standards for NO have not been promulgated; however, a concurrent LBL study15 on the biotransformation of NO in blood indicates that the elevated NO levels observed in this study may have an inpact on human health similar to, and in addition to, that attributed to 00.16 Observed carbon monoxide levels exceeded the onehour standard in a few cases and may be of concern.

Figure 2 gives the mean indoor and outdoor particulate concentrations for $\mathrm{S}, \mathrm{Pb}, \mathrm{Zn}, \mathrm{Fe}$, and Ca from eight indoor/outdoor sample pairs collected during cooking in five homes with gas stoves. The results indicate an increase in indoor particulate sulfur during cooking while the indoor particulate levels for other species are comparable to or lower than the outdoor levels. No elevated sulfur levels, gaseous or particulate, were found in the home with the electric stove on.

Sulfur and lead aerosols are typically found in California urban air in the particle size range of less than $2 \mu \mathrm{m}$; iron and calcium aerosols are typically greater than $2 \mathrm{~mm}$ in size, and zinc is found in all size ranges. 17 The results shown

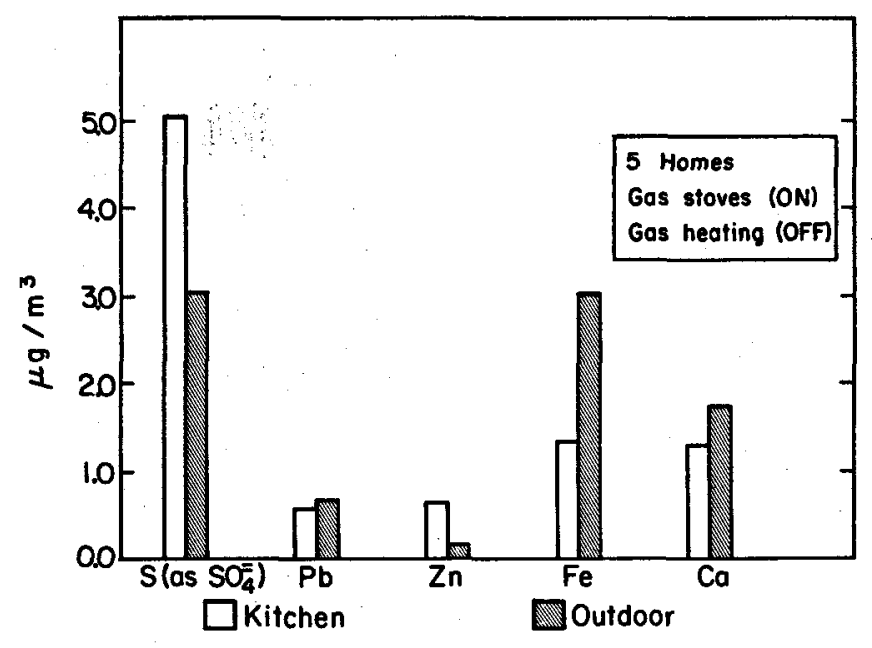

Fig. 2. Indoor and outdoor particulate air pollutant levels observed at five homes with gas stoves during cooking. Levels indicated represent average of eight indoor/outdoor sample pairs collected on cellulose filters and analyzed by $\mathrm{X}$-ray fluorescence analysis.

(XBL 7510-8559)

in Figure 2 are consistent with recent studies 18,19 which show particulate loading to be lower indoors than outdoors as a result of less penetration of large particles from the outside air, but with higher indoor levels of respirable particles $(<3.5 \mu \mathrm{m})$ due to indoor sources.

Figure 3 illustrates the elevated indoor particulate nitrogen levels observed in the home where the effect of the gas furnace was studied. The ESCA spectra illustrate the different nitrogen species $\left(\mathrm{NO}_{3} ; \mathrm{NH}_{4}{ }^{+}, \mathrm{N}_{\mathrm{x}}\right.$ [organic nitrogen])

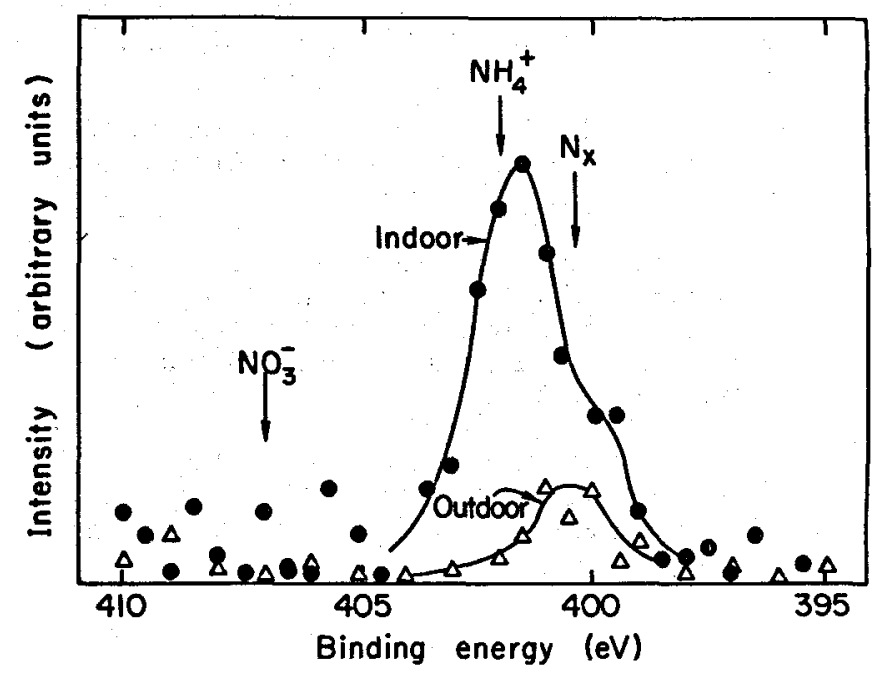

Fig. 3. Nitrogen (1x) photoelectron (ESCA) spectra of indoor and outdoor particulate samples taken at home with the gas furnace on. Individual peaks corresponding to $\mathrm{NO}_{3}{ }^{-}, \mathrm{NH}_{4}{ }^{+}$, and $\mathrm{N}_{x}$ (organic nitrogen species) are indicated. (XBL 7510-8560) 
sbserved indoors and outdoors; the peak areas are proportional to the relative atomic concentrations (as $\mathrm{N}$ ) of these species. Most of the indoor nitrogen is found as $\mathrm{NH}_{4}^{+}$and $\mathrm{N}_{\mathrm{x}}$; the outdoor nitrogen is found as $N_{x}$. In previous outdoor studies 9 $\mathrm{N}_{\mathrm{x}}$ was found to be either the dominant or at least major component of the total particulate nitrogen. The elevated levels of reduced nitrogen species should be particularly noted, because some of these (especially those labeled ' $\mathrm{N}_{\mathrm{x}}$ ' in Fig. 3, which are interpreted as $\mathrm{NH}_{2}$-type compounds) are species whose health implications deserve further study.

\section{FOOTNOTE AND REFERENCES}

${ }^{\star}$ Condensed from LBL-4416

1. F.B. Benson, J. J. Henderson, and D. E. Caldwe11, "Indoor-Outdoor Air Pollution Relationships: A Literature Review," Publication No. AP-112, U.S. Environmental Protection Agency, Research Triangle Park, N.C. (1972).

2. J.J. Henderson, F.B. Benson, and D.E. Caldwe11, "Indoor-Outdoor Air Pollution Relationships: Vol. II, An Annotated Bibliography," Publication No. AP-112b, U.S. Environmental Protection Agency, Research Triangle Park, N.S. (1973).

3. S.J. Penkala and G. de Oliveira, "The Simu1taneous Analysis of Carbon Monoxide and Suspended Particulate Matter Produced by Cigarette Smoking," Environ. Res. 9, 99 (1975).

4. A. Levy, R.W. Courant, D.W. Joseph, E.L. Merryman, and C.W. Spicer, "Evaluation of Measurement Methods for Indoor Levels of $\infty$ and $\mathrm{NO}_{\mathrm{x}}$," Battelle Columbus Laboratories, Columbus, OH 43201 (1974).

5. G.A. Lutz, R.E. Mitche11, R.W. Cote and M.D. Keller, "Indoor Epidemiology Study," Battelle Columbus Laboratories, Columbus, OH 43201 (1974).

6. W.A. Wade III, W.A. Cote and J.E. Yocum, "A Study of Indoor Air Quality," J. Air Pollut. Contr. Ass. 25, 933 (1975).

7. E. Schwarzbach, "Zur Frage der Entstehung von Stickstoffoxiden bei Erdgasfeuerungen (The Question of the Formation of Nitrogen Oxides During Burning of Natural Gas)," Staub-Reinhaltung der Luft 35, 134 (1975).

8. I. Anderson, G.R. Lundqvist and M $\$$ lhave, "Indoor Air Pollution Due to Chipboard Used as a Construction Material, "Atmos. Environ. 9, 1121
9. S.G. Chang and T. Novakov, "Formation of Pollution Particulate Nitrogen Compounds by NO-Soot and $\mathrm{NH}_{3}$-Soot Gas-Particle Surface Reactions," Atmos. Environ. 9, 495 (1975).

10. R.E. Barrett, D.W. Locklin, and S.E. Miller, "Investigations of Particulate Emissions from Oil-Fired Residential Heating Units," Enviromental Protection Agency Publication EPA-65-/2-74-026 (March 1974).

11. B. Miche1, M. Neufelder and D. Pruggmayer, 'Emission of Pollutants by Domestic Fireplaces, Exhaust Gas Measurements on Oil and Gas Heaters," Staub Reinhaltung der Luft in English, 34, 129 (1974).

12. K. Lützke, "Emissionen von Stickstoffoxiden aus Feuerungsanlagen: (Enissions of Nitrogen Oxide from Industrial and Domestic Combustion Installations) "Staub-Reinhaltung der Luft, 35, 127 (1975).

13. A. Ise, Materials Research and Process Development Department, Toyota Motor Co., Aichi, Japan, Private Communication (July 16, 1975).

14. U.S. Congress, Committee on Interstate and Foreign Commerce, Subcomittee on Health and the Environment, House of Representatives, "Clean Air Act Amendments - 1975, Hearings," (Washington, D.C., 1975).

15. G.D. Case, J.C. Schooley, and J. Dixon, "Uptake and Metabolism of Nitrogen Oxides in Blood," Report LBL-4417, Lawrence Berkeley Laboratory, University of California, Berkeley, California 94720 , presented at the 20th Annual Biophysical Society Meeting Feb. 25, 1976 in Seattle, WA.

16. D.M. Snodderly, "Biomedical and Social Aspects of Air Pollution," Adv. Environ. Sci. Technol. 3, 157 (1974).

17. R.D. Giauque, L.Y. Goda and R.B. Garrett, 'X-ray Fluorescence Analysis of ACHEX Aerosols', Report LBL-4414, Lawrence Berkeley Laboratory, University of California, Berkeley, CA. 94720 (October 21, 1975).

18. I. Anderson, "Relationships between Outdoor and Indoor Air Pollution," Atmos. Environ. 6, 275 (1972).

19. R.M. Lum and T.E. Graede1, 'Measurements and Models of Indoor Aeroso1 Size Spectra," Atmos. Environ. 7, 827 (1973). 


\title{
ATMOSPHERIC AEROSOL RESEARCH* ${ }^{*}$
}

\author{
T. Novakov, Principal Investigator, \\ P. J. Bekowiest, H. A. Brendel, S. G. Chang, \\ M. Clemenson, R. L. Dod, R. D. Giauque, \\ A. B. Harkerf, C. D. Holloweil, L. Hughes\$, \\ S. S. Markowitz, P. J. Pagnill., H. Rosen, \\ R. C. Schmidt, G. W. Traynor, J. R. Wallacel, L. Wroth
}

\section{INIRODUCTION}

Aerosol particles (also referred to as particulate matter or particulates) play a major role in the air pollution problem. They are responsible for reduced visibility, acid rain, and in certain size ranges, are deposited in the lungs where they can cause a variety of adverse health effects. These particles can consist of solid and/or liquid substances. Some of these, such as windblown dust, soot, and flyash, originate in sources outside the atmosphere and are known as "primary" pollutants. Others are formed directly in the atmosphere by reactions among the primary particulate and gaseous species and are known as "secondary" pollutants. The nature of both these primary and secondary particulate species, as well as the extent to which atmospheric chemistry is governed by photochemical gas-phase mechanisms or catalytic gas-particle mechanisms, is presently a very active area of research.

In order to devise an effective control strategy, the particles responsible for the adverse environmental and health effects must be selective1y identified and the process by which these species enter the atmosphere must be ascertained. In this regard one mist distinguish between two aspects of the analytical chemistry of atmospheric particulates. First we need to know the bulk chemical composition of any given sample of particulate matter. This is useful since some particles or some of their major bulk constituents may eventually be soluble in body fluids, so that practically their entire content can be toxicologically harmful. On the other hand, particle surfaces often are chemically dissimilar to the interiors of the particles. Particle surfaces carry microscopic layers of contaminants that make contact with lung membranes, leading to exposures that have little to do with the bulk composition of the particles. A situation intermediate between these two extremes exists, when the harmful contaminants cover the surface of extremely small particles (of the order of $100 \mathrm{~A}$ or less) which grow in the atmosphere as the result of coagulation. In this case the surface composition will be virtually undistinguishable from the bulk composition.

Most of the mass of atmospheric particulates consists of compounds of carbon, nitrogen, and sulfur. This illustrates the importance of pursuing earlier findings of the project that sulfur and nitrogen species, identical to those observed in the ambient air, can be synthesized in a few honphotochemical reactions involving $\mathrm{SO}_{2}, \mathrm{NO}, \mathrm{NH}_{3}$, and soot particles, which in many respects behave like activated carbon. The health effects of some of these species are unknown now. The carbonbound sulfate is one such species. Others are the three or more reduced nitrogen forms - amines, amides, and nitriles. The existence of these substances in the carbonaceous fraction of atmospheric particulates requires that the composition and chemical fate of particulates be looked at in a new light. Namely, our results strongly suggest that some of the sulfur and nitrogen forms are primary pollutants coming directly from sources, while others such as sulfate are essentially secondary pollutants but formed under catalytic action of chemically active primary particulates. This means that atmospheric sulfate pollution may be dominated, or significantly influenced, by processes of soot production, and that the chemical (and toxicological) properties of atmospheric particulates may depend on the soot-bearing surface and/or bulk sulfur and nitrogen species.

The research on this project has continued along two parallel and interrelated approaches. The first approach is to prepare an inventory of chemical species found in various ambient and source-enriched particulates, and to study the relationship between the particulate species and the concentrations of concomitant gaseous pollutants and meteorological parameters. The second approach is to conduct laboratory experiments to elucidate various gas-particle reactions and to assess the role and significance of these processes in atmospheric pollution.

The main research tool for the characterization of particulate samples has been X-ray photoelectron spectroscopy (ESCA, or Electron Spectroscopy for Chemical Analysis). The systematic chemical characterization of particulates by means of ESCA continued to be an ongoing activity during the past year's work on the project. Most of the samples used for this purpose were from St. Louis cobtained from Dr. T. Dzubay of EPA, Research Triangle Park, N.C.) and from the Caldecott Tunnel near LBL (collected by the project investigators). In addition to these, a number of other California samples were also analyzed. Also, during the past year the infrared and Raman scattering techniques have been incorporated into the program and have yielded some rather interesting results. The X-ray fluorescence technique has been used for elemental analysis, and preliminary measurements of the feasibility of using activation analysis for a determination of the light elements has been made. These instrumental techniques have been complemented by wet chemical and microanalytical methods. A field program was also initiated last year with the construction of a Mobile Atmospheric Research Laboratory (MARL) which is now ready to make comprehensive measurements of gaseous and particulate concentrations in a field situation. 
The present program can be divided into three main areas of research: chemical characterization of atmospheric aerosols, studies of reaction mechanisms, and field studies.

\section{CHEMICAL CHARACTERIZATION OF ATMOSPHERIC AEROSOLS}

Ammonium and Sulfate Species in Atmospheric Aerosols

The speciation of atmospheric aerosol particles is an important task because many of their environmental effects will depend on their specific chemical and physical states. Furthermore, it is important to determine the chemical compounds and species as they actually exist in aerosol form and not as they may appear in aqueous solutions. Most analyses of pollution aerosol particles, however, have employed wet chemical methods that only reflect the chemical composition in solution. Wet chemical methods are of no use for insoluble aerosol species; nondestructive physical methods are therefore preferred as a means for chemical characterization of collected particles. X-ray photoelectron spectroscopy (ESCA) is one such method. For example, the application of this method has helped to uncover the presence of significant concentratons of reduced nitrogen species of the amino type $\left(-\mathrm{NH}_{2}\right)$ in pollution aerosol particles. 1 These species are not soluble in water or solvents such as benzene, and therefore could not be detected by wet chemical methods.

ESCA analysis enables the possibility not only of detection of specific ions and functional groups but also of their mutual relationship. This is achieved by the measurements of the ESCA chemical shift augmented by the determination of relative concentrations and the study of the volatility properties of particulate species.

The capability of ESCA for a straight forward differentiation of different forms of atmospheric sulfates is illustrated in Fig. 1, where the nitrogen (1s) and sulfur (2p) spectral regions of two ambient samples are shown. (One was collected in West Covina, California, in the summer of 1973; the other, in St. Louis, Missouri, in the summer of 1975.) The peak positions corresponding to $\mathrm{NH}_{4}{ }^{+},-\mathrm{NH}_{2}$, and $\mathrm{SO}_{4}{ }^{--}$are indicated. The solid vertical bar indicates the ammonium peak intensity expected under the assumption that the entire sul fate is in the form of ammonium sulfate. Obviously, the observed ammonium content in the West Covina sample is insufficient to account for the sulfate by itself. This is in sharp contrast with the St. Louis sample where the observed ammonium intensity closely agrees with that expected for anmonium sulfate.

These results demonstrate that amnonium sulfate in the aerosols can easily be distinguished from other forms of sulfate such as the one found in the West Covina case. Wet chemical analyses, 2 however, performed on West Covina samples collected simultaneously with the ESCA samples resulted in ammonium concentrations substantially higher than those suggested by the ESCA measurements. This apparent discrepancy between the two methods was subsequently explained by the volatility of some ammonium species in the ESCA spectrometer vacurm. 3,4

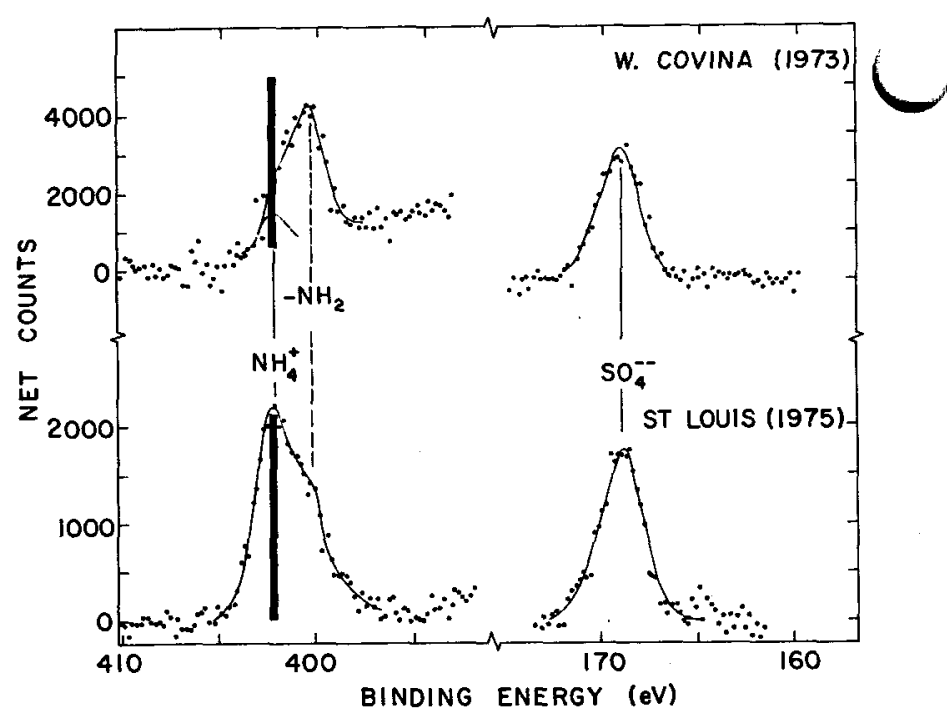

Fig. 1. Nitrogen (1s) and sulfur (2p) regions in $X$-ray photoelectron spectra of two ambient samples. The peak positions corresponding to $\mathrm{NH}_{4}{ }^{+},-\mathrm{NH}_{2}$, and $\mathrm{SO}_{4}{ }^{--}$ are indicated. The solid vertical bar represents the ammonium intensity expected under the assumption that the entire sulfate is in the form of ammonium sulfate. The difference in the relative anmonium content of the two samples is obvious. The sulfate and ammonium intensities in the St. Louis sample are compatible with ammonium sulfate. The ammonium content in the West Covina sample is insufficient to be compatible with ammonium sulfate. Both samples were exposed to the spectrometer vacuum for about one hour.

(XBL 763-2535)

That the volatile ammonium is not necessarily associated with sulfate or nitrate ions is illustrated by means of results represented in Fig. 2 . Here the changes in the nitrogen (1s) spectrum of a sample collected in a highway tunne $1^{5}$ are shown as a function of the sample vacuum exposure time. Obviously, the ammonium peak intensity decreases with the vacuum exposure time of the sample. The amino-type nitrogen species intensity remains constant, however. The amount of nitrate in this sample was negligible compared with ammonium. The maximum ammonium peak expected under the assumption that the entire sulfate is ammonium sulfate is indicated by the solid vertical bar in Fig. 2. It is obvious, therefore, that the counterions for this ammonium are neither nitrate nor sulfate. Figures 3 and 4 summarize the findings about ammonium volatility in the three samples discussed above as well as six samples from St. Louis.

The anions corresponding to the volatile amnonium species cannot be identified with certainty at this time. One possibility is that these species are produced by the adsorption of ammonia on fine soot particles to form carboxy1- and hydroxyl-ammonium complexes which have been shown to have volatility properties similar to those of ambient particulates. 3 Another possibility is that these species could be due to ammonium halides 


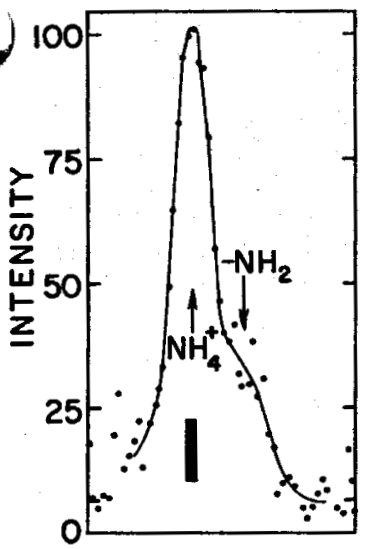

$30 \mathrm{~min}$.

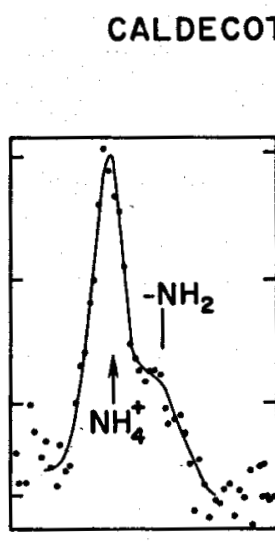

$90 \mathrm{~min}$.

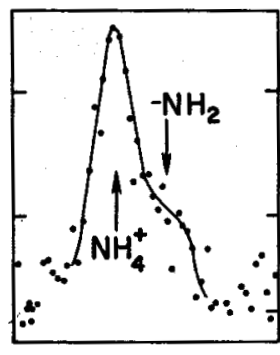

$150 \mathrm{~min}$.

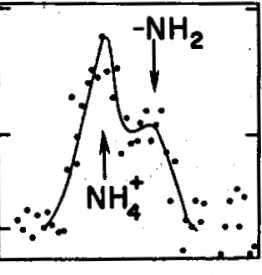

$170 \mathrm{~min}$.
VACUUM EXPOSURE TIME (min.)
Fig. 2. The variation in the observed ammonium peak intensity with a vacuum exposure for a sample collected in a highway tunnel. The decrease in the peak intensity is caused by the violatilization of the ammonium species present in the sample. The solid vertical bar represents the ammonium intensity expected under the assumption that the sulfate in this sample is in the form of ammonium sulfate. The ammonium in this sample is considerably in excess of that expected for ammonium sulfate or ammonium nitrate. (XBL 763-2537)

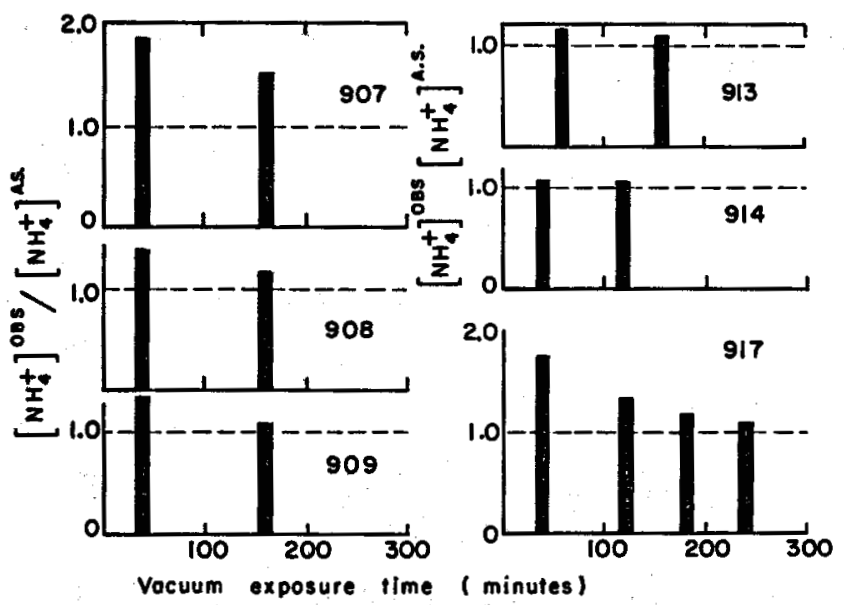

Fig. 4. Volatility properties of ammonium in six ambient St. Louis samples. The ratio of the observed ammonium peak to the one expected under the assumption that the entire sulfate in these samples is ammonium sulfate vs vacuum exposure time is shown. Note the cases of apparently stoichiometric ammonium sulfate (samples 913 and 914) and the cases where the volatile ammonium component is found in excess of that required for ammonium sulfate.

(XBL 766-3049)

which are also volatile in vacum. More experimental work is necessary in order to evaluate these as well as other possibilities for explaining the volatile ammonium species.

Application of Raman Scattering to the Characterization of Atmospheric Aerosol Particles

Preliminary studies are being carried out to determine the feasibility of characterizing particulate pollutants by means of Raman spectroscopy. 
We believe that this is the first attempt to apply this spectroscopic technique in this area of research. Our initial results indicate that physical structures similar to activated carbon are a major species and may be the dominant species in both source-enriched and ambient samples. The implications of this observation could be quite important in terms of atmospheric chemistry, health effects, weather modifications, and the effects of aerosols on the albedo.

In Fig. 5 we show the Raman spectrum obtained from automobile exhaust. Similar spectra were observed for diesel exhaust, activated carbon, and polycrystalline graphite. The major features of the Raman spectrum are two intense lines at $\sim 1350 \mathrm{~cm}^{-1}$ and $\sim 1600 \mathrm{~cm}^{-1}$. In the ambient samples, where the signal to noise is severely limited by the large fluorescence background, the only lines that were clearly seen above the noise level were also in this spectral region. In Fig. 6 we show the Raman spectrum for the various samples in the region between $1200-1700 \mathrm{~cm}^{-1}$.

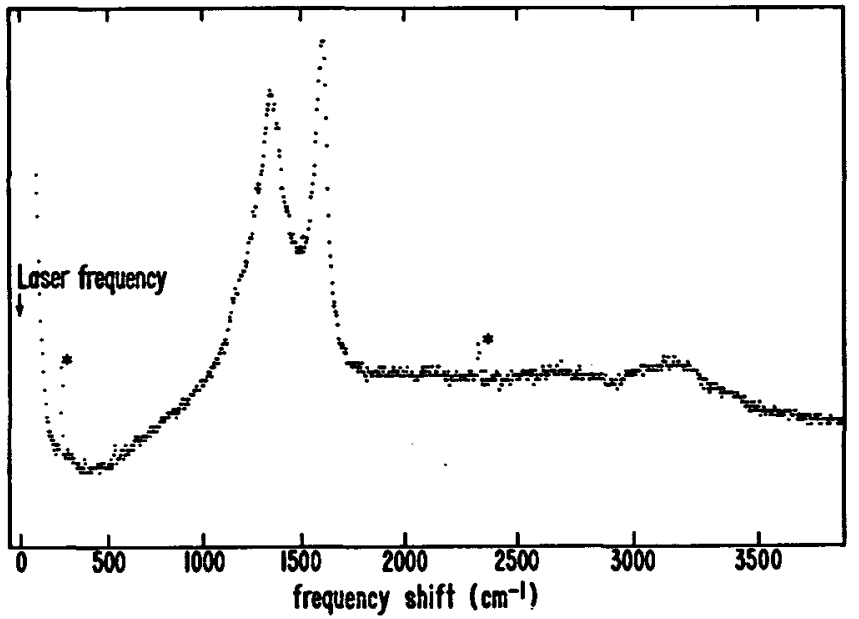

Fig. 5. Raman spectrum of automobile exhaust in the spectral region between 90 and 3830 $\mathrm{cm}^{-1}$. The sample was collected from 100 cold starts of a 1974 Pinto using leadfree gas and having no catalytic converter. The slit width for this scan was $3 \AA$. The lines identified with an asterisk are due to grating ghosts.

(XBL 767-3092)

Fig. 6. Raman spectra between 1200 and $1700 \mathrm{~cm}^{-1}$ of (a) ambient sample collected in 1975 as part of the RAPS program (the sample was collected on a dichotomous sampler and was in the small size range fraction); (b) automobile exhaust collected from 100 cold starts of a 1974 Pinto using leadfree gas and having no catalytic converter; (c) diesel exhaust; (d) activated carbon; and (e) polycrystalline graphite. The slit width for samples b-e was $3 A$; while for sample $a, 7 \mathrm{~A}$ slits were used to improve signal to noise.

(XBL 767-3091)
In perfectly ordered single crystal graphite specimens only the Raman mode near $1600 \mathrm{~cm}^{-1}$ is observed and from a group theoretical analysis has been assigned to the $\mathrm{K}=0, \mathrm{E}_{2} \mathrm{~g}$ phonons of the graphite lattice. 6 The mode hear $1350 \mathrm{~cm}^{-1}$ appears only in samples that are not perfectly ordered, and its intensity relative to that of the one near $1600 \mathrm{~cm}^{-1}$ varies inversely with the crystallite size, $\mathrm{L}_{a}$, as obtained from $\mathrm{X}$-ray data. ${ }^{5}$

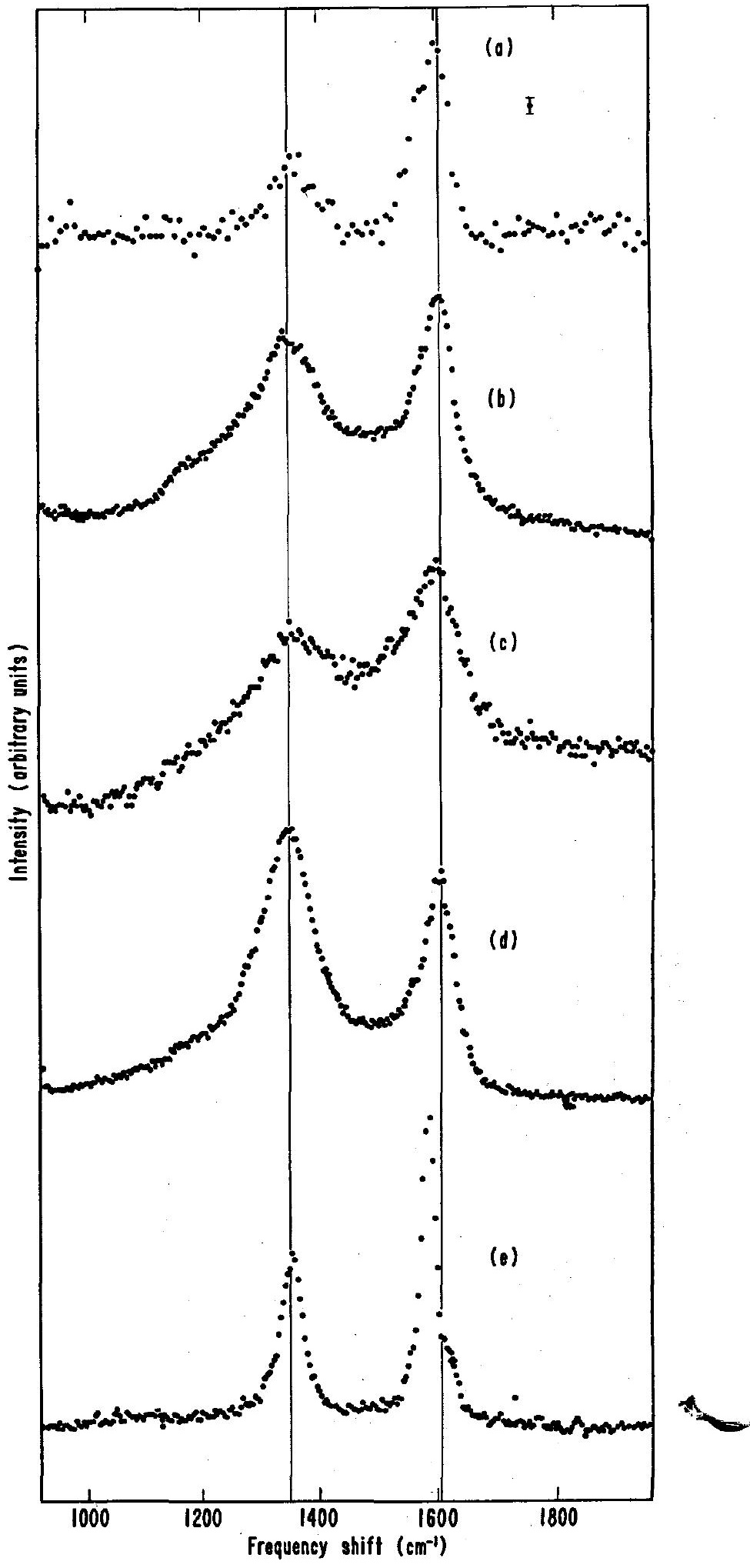


It is evident from Fig. 6 that the spectra of activated carbon, diesel exhaust, automobile exhaust, and ambient samples are very similar. The positions of the two Raman modes in these spectra are coincident to within $\pm 10 \mathrm{~cm}^{-1}$, which is the estimated error. Since the phonon frequencies are a sensitive probe of the lattice, we suggest that these spectra give strong evidence for the existence of physical structures similar to activated carbon in the samples studied.

Using the available results from the literature, we can estimate the crystallite sizes in the various samples from the intensity ratios of the two observed Raman modes. The ambient, diesel exhaust, and automobile exhaust particles appear to have roughly the same peak intensity ratio, yielding crystallite sizes between 50 and 100 '

our results indicate that physical structures similar to activated carbon are present in exhaust and ambient samples. The fact that the two observed modes are the dominant features of the Raman spectrum may indicate that "graphitic" soot is the major component in these samples. However, the large intensity of these modes may be due just to their large Raman cross section. Quantitative interpretation of these results will have to await a more detailed analysis.

Nuclear Activation Analysis for Low-Z Elements in Atmospheric Aerosol Particles

We have been investigating the feasibility of using activation analysis for a determination of the concentration of light elements, which comprise a major fraction of airborne particulates. our initial experiments involve the detection of elemental nitrogen using protons to induce the reaction ${ }^{14} \mathrm{~N}(\mathrm{p}, \alpha) \mathrm{C}^{11}$ and then monitoring the positron annihilation from $\mathrm{C}^{11}$. In these experiments there is no interference from carbon because of the high threshold $(20.3 \mathrm{MeV})$ for production of 11C from ${ }^{12} \mathrm{C}$.

A compound with a known amount of nitrogen, melamine $\left(\mathrm{C}_{3} \mathrm{~N}_{6} \mathrm{H}_{6}\right)$ was prepared by vacuum sublimation onto $\mathbf{0 . 0 0 1 - i n . ~ A 1 ~ f o i l s . ~ T h e ~ m e l a m i n e ~ t h i c k n e s s e s ~}$ for the various samples were either $1.5 \mathrm{mg} / \mathrm{cm}^{2}$ or $3.2 \mathrm{mg} / \mathrm{cm}^{2}$. Of this, 66.78 by weight is $\mathrm{N}$. A stack of these foils was irradiated for $3.8 \mathrm{~min}$ in the LBL 88-in. cyclotron at a beam intensity of $\sim 1.4 \mu \mathrm{A}$ of protons.

Radioactive ${ }^{11} \mathrm{C}$, a positron emitter with a half-life of $20.4 \mathrm{~min}$, was conveniently counted for its $511-\mathrm{keV}$ annihilation radiation by means of a $\mathrm{Ge}(\mathrm{Li})$ multichannel spectrometer. The decay of the integrated intensity in this peak for one of the target foils is shown in Fig. 7. The 20min half-life of ${ }^{11} \mathrm{C}$ is clearly observed for several decades. After the $1_{1} \mathrm{C}$ has decayed away, the decay curve indicates $511-\mathrm{keV}$ radiation from longer-1ived species, probably $15-\mathrm{hr} 24 \mathrm{Na}$ and 18-hr ${ }^{55} \mathrm{Co}$.

Measured count rates were corrected back to end-of-bombardment, $A_{0}$, converted to disintegration rates, $\mathrm{D}_{0}$, and the cross sections were calculated for each foil. The results are shown in Fig. 8.

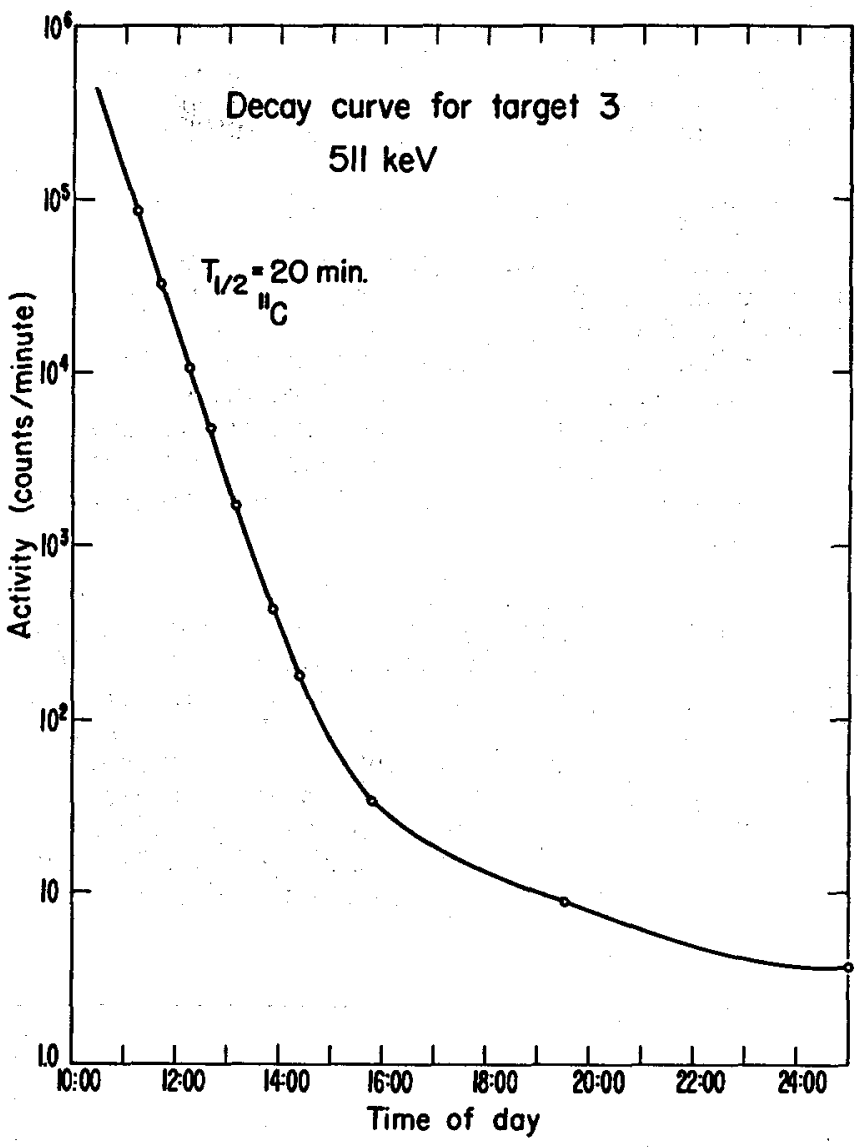

Fig. 7. Decay of the $511-\mathrm{keV}$ peak from ${ }^{11} \mathrm{C}$ produced by $12.3-\mathrm{MeV}$ protons.

(XBL 766-3053)

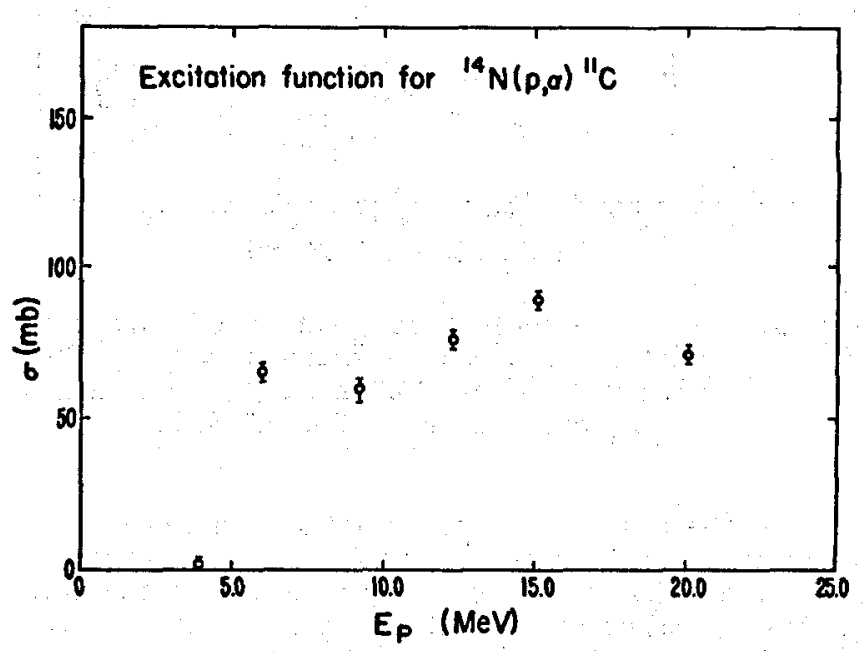

Fig. 8. Excitation function for the ${ }^{14} \mathrm{~N}(\mathrm{p}, \alpha)^{11} \mathrm{C}$ reaction.

(XBL 766-3051)

The measured count rates at a proton energy of $12.3 \mathrm{MeV}$ and a bombardment time of $3.8 \mathrm{~min}$ at a beam current of $1.4 \mu \mathrm{A}$ yield a sensitivity of $w 0.1 \mu \mathrm{g} / \mathrm{cm}^{2}$ for the detection of nitrogen, provided that other impurities do not give both a similar $\gamma$ energy and half-life to that of ${ }^{11} \mathrm{C}$. 
Therefore the $(p, \alpha)$ activation for $N$ seems promising. Irradiations of synthetic and actual aerosol filter samples will be carried out to further examine the proton activation procedure.

\section{Manganese Emissions from Combustors}

X-ray photoelectron spectroscopy has been used to analyze particulate exhaust collected from a model combustor burning JP-4 with 2-methy1 cyclopentadienyl manganese tricarbonyl (MM) added in concentrations ranging from 0 to 0.258 by volume. It is desired to determine the oxidation state and physical form of manganese after combustion as well as its effect on other particulate emissions.

Since the chemical shifts in manganese are very small, the multiplet splitting effect was employed here to determine the oxidation state. A photoelectron spectrum of the exhaust particles in the binding energy range 0 to $670 \mathrm{eV}$ is shown in Fig. 9. The major constituents are manganese, carbon, and oxygen, with trace amounts of nitrogen and sulfur. The oxidation state is made by examining the multiplet splittings of the $3 \mathrm{~s}$ electron level in spectra such as those shown in Fig. 10.

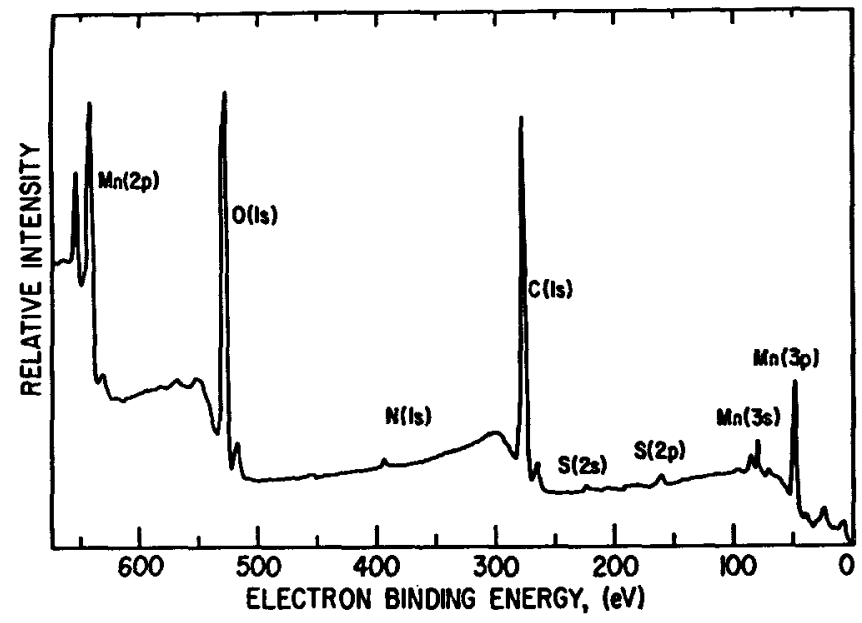

Fig. 9. X-ray photoelectron spectrum of particles emitted by a model combustor burning JP-4 with 0.25 (vol) MMT and collected on a Nucleopore filter. (XBL 767-8540)

On the basis of these splittings and on stoichiometric balance principles, it was determined that almost a11 the manganese was in the form of MnO. Furthermore, the ESCA measurements showed that the ratio of sulfur to carbon atoms increased from 0.047 for pure JP-4 fuel to 0.145 when the additive was used. It has been demonstrated that MT strongly influences the size distribution in the exhaust, producing many more small particles. The resulting increase in the surface area available for heterogeneous reactions could explain the increase in the sulfur-to-carbon ratio.

A neutron activation analysis of the exhaust samples was a1so obtained7 which indicated that all the manganese added to the fuel was enitted.

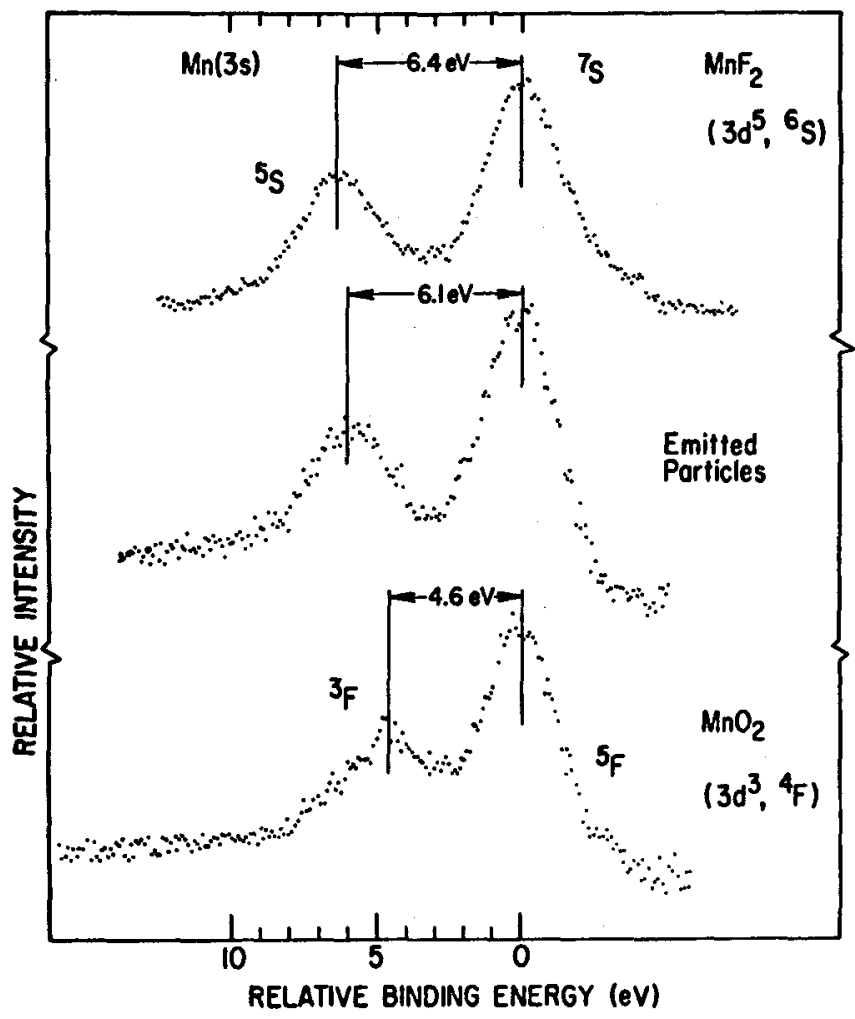

Fig. 10. Comparative $X$-ray photoelectron spectra of 3s core levels for $\mathrm{MnF}_{2}$, exhaust particles, and $\mathrm{MnO}_{2}$ showing the multiplet splitting of the $3 \mathrm{~s}$ level. It is concluded that $\mathrm{MnO}$ is the principal manganese oxide in the emitted particles.

(XBL 767-8541)

Knowing the amount of manganese emitted and the oxidation state as $\mathrm{MnO}$, we can calculate the mass of manganese monoxide emitted. Since the total mass of particulate emissions is also measured, the mass of carbon enitted is immediately obtained. The resulting emission indices are shown in Fig. 11 as a function of additive concentration. Smail amounts of the additive, less than 0.01 vol\%, decrease the emitted mass. However, at additive concentrations greater than 0.01 vols both the total mass and the mass of carbon emitted increase. The carbon emission index reaches a plateau as additive concentration increases at $11.7 \mathrm{~g} / \mathrm{kg}$ fuel above the no-additive level. A carbon deposition rate of $\sim 1.6 \mathrm{~g} / \mathrm{kg}$ of fuel has been reported for a similar combustor. 8 This leads to the conclusion that prohibition of carbon deposition within the combustion is the cause of the increased mass enission. Similar minimums in mass emissions at low additive concentrations have been observed for oil-burning furnaces 9 and power-generating turbines.10

\section{STUDIES OF REACTION MECHANISMS}

Infrared and Photoelectron Spectroscopic Study of $\mathrm{SO}_{2}$ Oxidation on Soot Particles

We have recently demonstrated that combustiongenerated air-suspendable carbonaceous (soot) 


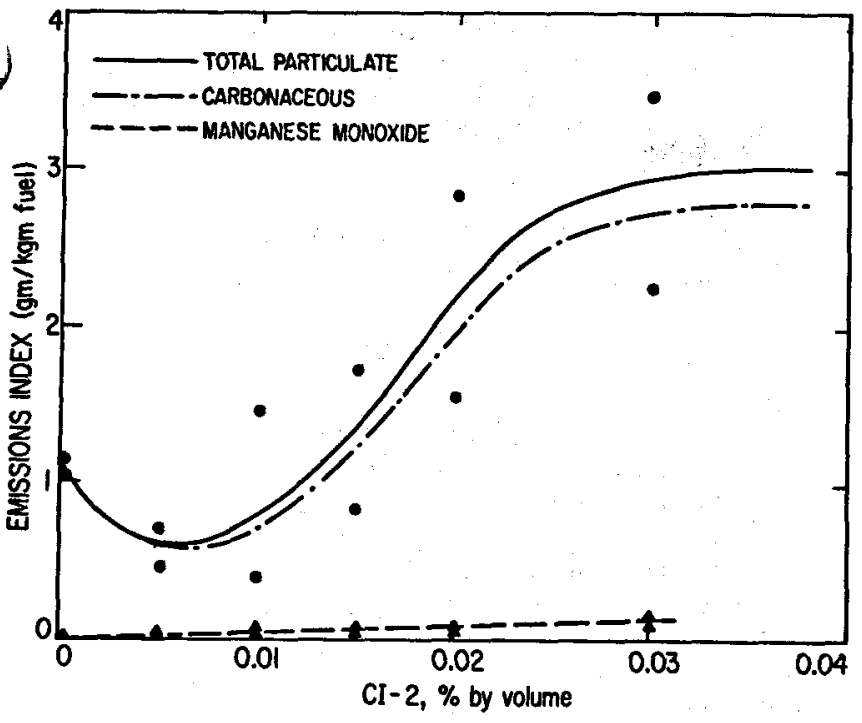

Fig. 11. Typical experimental results for the total particulate enission index. The solid line is an averaged total particulate EI. The carbonaceous EI is the difference between the total $\mathrm{EI}$ and the MnO EI.

(XBL 767-8539)

particles are an efficient catalyst for the oxidation of $\mathrm{SO}_{2}$ to sulfate and have suggested that this heterogeneous oxidation is important from the air pollution standpoint. ESCA and wet chemical methods were used to characterize the product of $\mathrm{SO}_{2}$ oxidation.

Interaction of $\mathrm{SO}_{2}$ with soot particles was studied either in a static regime, i.e., with soot particles precollected on a filter with subsequent exposure to $\mathrm{SO}_{2}$ at room temperature, or in a flow system by introducing $\mathrm{SO}_{2}$ downstream from a propane flame.

Photoelectron spectra representing the sulfur (2p) and carbon (1s) regions of propane soot particles produced by a Bunsen burner are shown in Fig. 12a. The $S(2 p)$ photoelectron peak at a binding energy of $169 \mathrm{eV}$ corresponds to sulfate. The C(1s) peak appears essentially as a single component line and corresponds to a mostly neutral charge state consistent with the soot structure. It is of interest to note that even the combustion of very low sulfur content fuels ( $0.005 \%$ by weight) results in the formation of easily detectable sulfate emission.

The specific role of soot particles as a catalyst for the oxidation of $\mathrm{SO}_{2}$ is demonstrated with the aid of Fig. 12a. Here we show the $S(2 p)$ and $C(1 s)$ photoelectron peaks of soot particles, generated in an analogous manner but exposed to additional $\mathrm{SO}_{2}$ in a flow system. A marked increase in the sulfate peak intensity relative to carbon is evident. The atomic ratios of sulfur to carbon in Fig. 12 are about 0.15 and 0.50 .

In order to understand the nature of the sulfate species formed on soot, infrared (IRS) spectra of the samples used for ESCA analyses (Figures 13a and 13b) were studied. The IRS spectra of these

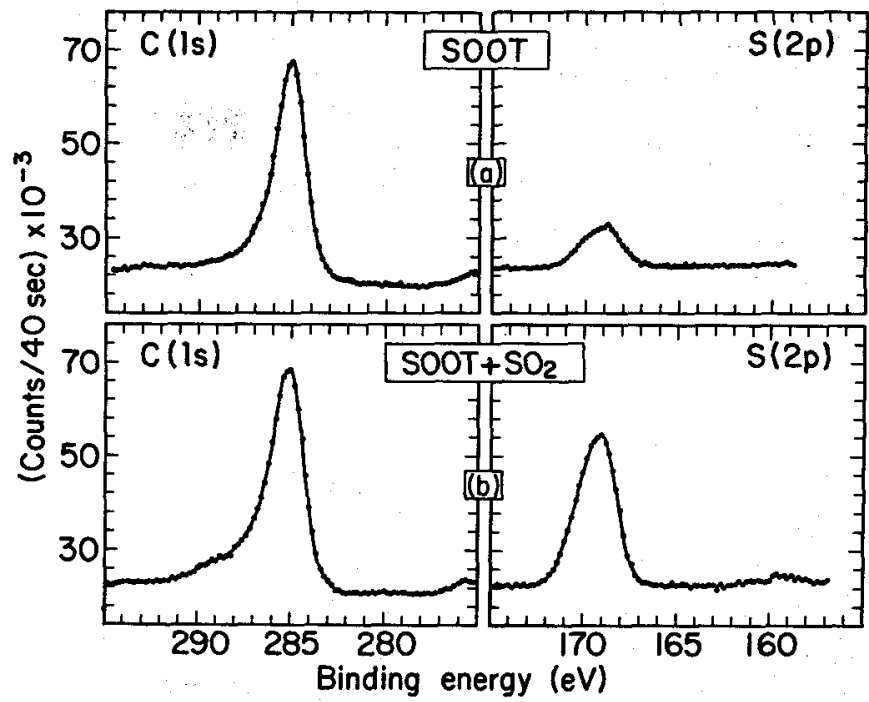

Fig. 12. Carbon (1s) and sulfur (2p) photoelectron spectrum of (a) soot particles produced by combustion of propane saturated with benzene vapor. (The sulfur content of this fuel is $0.005 \%$ by weight.); (b) soot particles generated in analogous manner to (a), but exposed to additional $\mathrm{SO}_{2}$ in humid air.

(XBL 758-3730)

samples, in the spectral region between 1500 and $500 \mathrm{~cm}^{-1}$, are shown in Fig. 13. The absorption

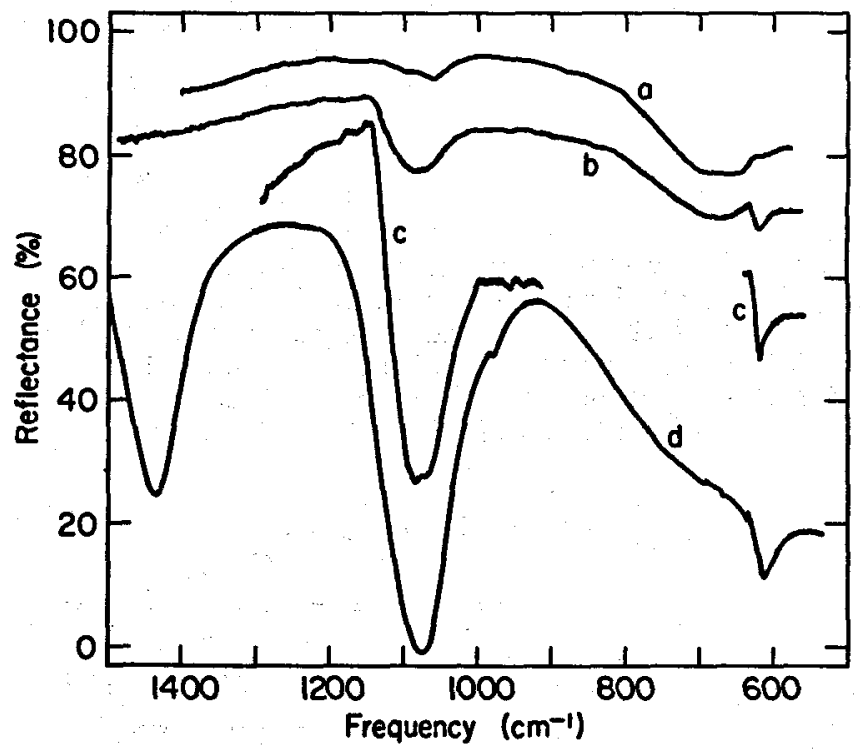

Fig. 13. Infrared (IRS) spectra (between 1500 and $500 \mathrm{~cm}^{-1}$ ) of (a) soot particles produced by combustion of propane saturated with benzene vapor; (b) soot particles generated in an analogous manner to (a), but exposed to additional $\mathrm{SO}_{2}$ in humid air; (c) soot particles as in (b) but expanded by a factor of 5 ; (d) aqueous solution of ammonium sulfate. (The adsorption band at $1400 \mathrm{~cm}^{-1}$ is due to the $v_{4}$ vibration of che $\mathrm{NH}_{4}^{-}$cation :)

(XBL 763-2367) 
band near $1400 \mathrm{~cm}^{-1}$ is due to an ammonium vibration while the bands near $1080 \mathrm{~cm}^{-1}$ and $615 \mathrm{~cm}^{-1}$ are due to the $v_{3}$ and $v_{4}$ vibrations of the sulfate ion. It is clear from the figure that the spectra of ammonium sulfate and sulfate associated with soot are very similar. In contrast, these spectra are dissimilar to those of a $1 \mathrm{~N}$ solution of $\mathrm{H}_{2} \mathrm{SO}_{4}$ and an aqueous solution of ammonium bisulfate shown in Fig. 14.

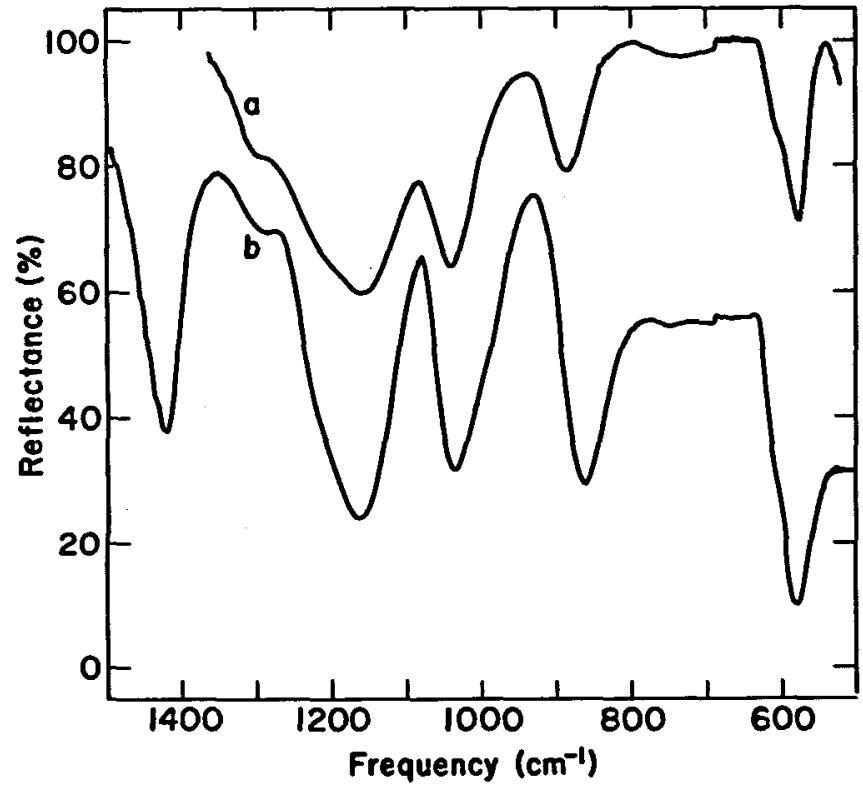

Fig. 14. Infrared (IRS) spectra of (a) $1 \mathrm{~N}$ sulfuric acid aqueous solution; (b) aqueous solution of ammonium bisulfate.

(XBL 758-3726)

The observed frequencies of different sulfate anions and their assigmment on the basis of a group theoretical analysis are listed in Table 1. An inspection of these data shows that the spectra of sulfate species produced by catalytic oxidation of $\mathrm{SO}_{2}$ closely match the spectrum of the sulfate anion $\mathrm{SO}_{4}^{--}$but not the hydrogen sulfate anion $\mathrm{HSO}_{4}^{-}$. This would indicate that oxidation of $\mathrm{SO}_{2}$ on soot particles results in the formation of sulfate salt-1ike species with tetrahedral structure. The soot particle surface must in that case provide relatively strong basic sites for the neutralization of sulfuric acid.

Possible Mechanism for the Catalytic Formation of Nitrates in the Atmosphere

Nitric oxide is the major nitrogen pollutant produced by the combustion of fossil fuels. In the atmosphere NO can be oxidized to nitrates which are viewed as an environmental hazard. Most proposed mechanisms 11 require sunlight to initiate the reaction. There are many indications, however, that nitrates can be produced in heterogeneous nonphotochemical reactions. We have confirmed the presence of substantial nitrate concentrations by means of infrared spectroscopy and ESCA in the following cases: (a) soot particles from propanebenzene combustion in air; (b) exhaust particulates from an internal combustion engine; (c) airborne particles collected in a highway tunnel; and (d) activated carbon exposed to NO in humid air. As a result of these experimental findings and other available information, we have been able to propose a nonphotochemical step-by-step mechanism for the formation of nitrates. It should be emphasized, however, that more detailed experiments are necessary in order to establish the relative importance of this mechanism.

Infrared measurements were obtained using a Perkin-Elmer 621 spectrometer. The spectra between 1450 and $1300 \mathrm{~cm}^{-1}$ for the various samples are shown in Fig. 15. In Fig. 15(e) the spectrum of ammonium nitrate is shown for comparison. In all cases an intense and sharp band is found at 1385 $\pm 5 \mathrm{~cm}^{-1}$. This band corresponds to the $v 3$ asymmetric stretching vibration of the $\mathrm{NO}_{3}^{-}$anion. Parallel measurements have been made using $X$-ray photoelectron spectroscopy (ESCA). These spectra verify the presence of nitrates in all the samples except the one taken in the Caldecott Tunnel where a large observed ammonium peak made the observation of the nitrate peak difficult.

Using some results of other workers, 12 we have been able to propose the following step-by-step mechanism for the formation of these nitrate species:

Table 1. Observed IRS fundamental vibrational frequencies of sulfate ions ions in the spectral region between 500 and $1500 \mathrm{~cm}^{-1}$.

\begin{tabular}{|c|c|c|c|c|c|c|}
\hline Origin & $\begin{array}{c}\text { Main } \\
\text { sulfur species }\end{array}$ & Pt. group & $v_{1}$ & $v_{2}$ & $v_{3}$ & $v_{4}$ \\
\hline $1 \mathrm{~N} \mathrm{H}_{2} \mathrm{SO}_{4}$ & $\mathrm{HSO}_{4}^{-}$ & $c_{3 v}$ & $885(5)$ & -- & $\begin{array}{l}1040(\mathrm{~S}) \\
1160(\mathrm{~S})\end{array}$ & $\begin{array}{l}578(\mathrm{~S}) \\
605(\mathrm{M})\end{array}$ \\
\hline $\begin{array}{l}\mathrm{NH}_{4} \mathrm{HSO}_{4} \\
\text { aqueous soln. }\end{array}$ & $\mathrm{HSO}_{4}^{-}$ & $c_{3 v}$ & $860(s)$ & - & $\begin{array}{l}1035(S) \\
1162(S)\end{array}$ & $\begin{array}{l}578(S) \\
605(\mathrm{M})\end{array}$ \\
\hline $\begin{array}{l}\left(\mathrm{NH}_{4}\right)_{2} \mathrm{SO}_{4} \\
\text { aqueous soln. }\end{array}$ & $\mathrm{SO}_{4}^{-}$ & $\mathbf{T}_{\mathbf{d}}$ & $978(W)$ & - & $1072(S)$ & $61.2(\mathrm{M})$ \\
\hline $\begin{array}{l}\text { soot }+\mathrm{SO}_{2} \\
\text { In humid alr }\end{array}$ & $\mathrm{SO}_{4}^{--}$ & $\mathbf{T}_{\mathrm{d}}$ & $960(W)$ & - & $1080(S)$ & $615(\mathrm{M})$ \\
\hline
\end{tabular}




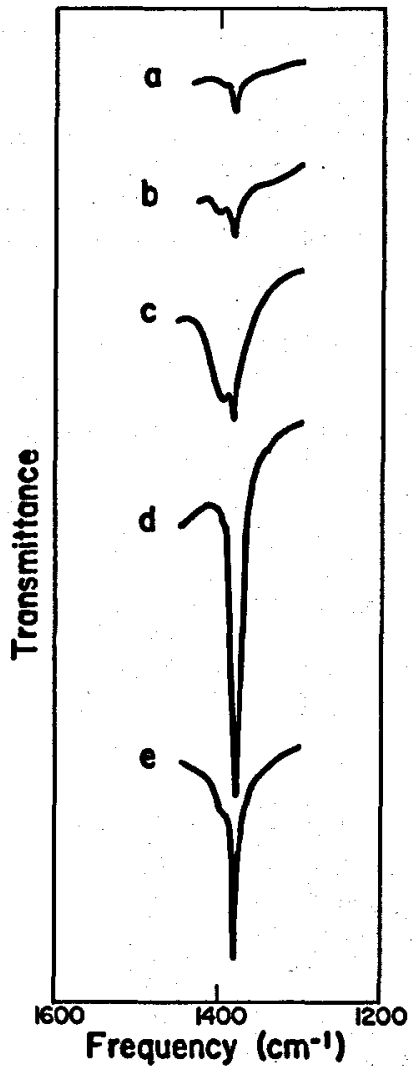

Fig. 15. Infrared spectra between 1450 and 1300 $\mathrm{cm}^{-1}$ of a) soot particles from propanebenzene combustion in air; b) exhaust particles from an internal combustion engine; c) airborne particles collected in a highway tunnel; d) activated carbon exposed to NO in humid air; and e) ammonium nitrate.

(XBL 764-2612A)

$$
\begin{aligned}
& \mathrm{H}_{2} \mathrm{O}+\mathrm{S} \Rightarrow \mathrm{H}_{2} \mathrm{O} \cdot \mathrm{S} \quad \text { fast equilibrium } \\
& \left.2 \mathrm{NO}_{2}+\mathrm{NO}_{2}+\mathrm{M}-\mathrm{N}_{2} \mathrm{O}_{4}+\mathrm{M}\right) \quad \text { fast equilibrium } \\
& \mathrm{N}_{2} \mathrm{O}_{4}+\mathrm{H}_{2} \mathrm{O} \cdot \mathrm{S}+\mathrm{S}+\mathrm{HNO}_{3} \cdot \mathrm{S}+\mathrm{HNO}_{2} \cdot \mathrm{S} \text { fast equilibrium } \\
& \mathrm{N}_{2} \mathrm{O}_{4}+\mathrm{HNO}_{2}+\mathrm{S} \rightarrow \mathrm{HNO}_{3} \cdot \mathrm{S}+\mathrm{N}_{2} \mathrm{O}_{3} \text { rate determining step } \\
& \mathrm{N}_{2} \mathrm{O}_{3}+\mathrm{M}=\mathrm{NO}+\mathrm{NO}_{2}+\mathrm{M} \text { fast equilibrium } \\
& \mathrm{H}_{2} \mathrm{O}+3 \mathrm{NO}_{2}+2 \mathrm{~S}-2 \mathrm{HNO}_{3} \cdot \mathrm{S}+\mathrm{NO} \text { overall reaction }
\end{aligned}
$$

Here $M$ represents any third body; and $\mathrm{HNO}_{3} \cdot \mathrm{S}$ represents the adsorbed $\mathrm{HNO}_{3} ; \mathrm{H}_{2} \mathrm{O} \cdot \mathrm{S}$, adsorbed water, etc.

\section{FIELD STUDIES}

Chemical and Physical Characterization of Vehicular Emissions

The chemical and physical properties of gases and particles emitted by highway vehicles have been characterized in a highway tunnel which represents for the purpose of this study a "real-world" nonphotochemical reaction chamber for vehicular emissions. Studies were carried out in the Caldecott Tunnel, a vented, three-bore, six-lane, 1100 meter-1ong tunnel on a major San Francisco Bay Area commuter route with a traffic flow of about $10^{5}$ vehicles per day.

Particulate lead in ambient air has often been used as a tracer for gaseous and particulate air pollutants from automotive sources. The specific purpose of these experiments was to study the carbon-to-lead ratio in order to assess the possibility of using lead as a tracer for particulate carbon from vehicles. It became immediately apparent that the $\mathrm{C} / \mathrm{Pb}$ ratio was extremely variable as the ratio was found to vary over almost an order of magnitude.

Experiments were conducted to study the causes of deviation in the $\mathrm{C} / \mathrm{Pb}$ ratio. During these experiments the $\mathrm{C} / \mathrm{Pb}$ ratio varied from 0.3 to 2.3 and was found to be highest during rush-hour and stalled-traffic periods. The $\mathrm{C} / \mathrm{Pb}$ ratios correlated well with diesel truck traffic density as shown in Fig. 16.

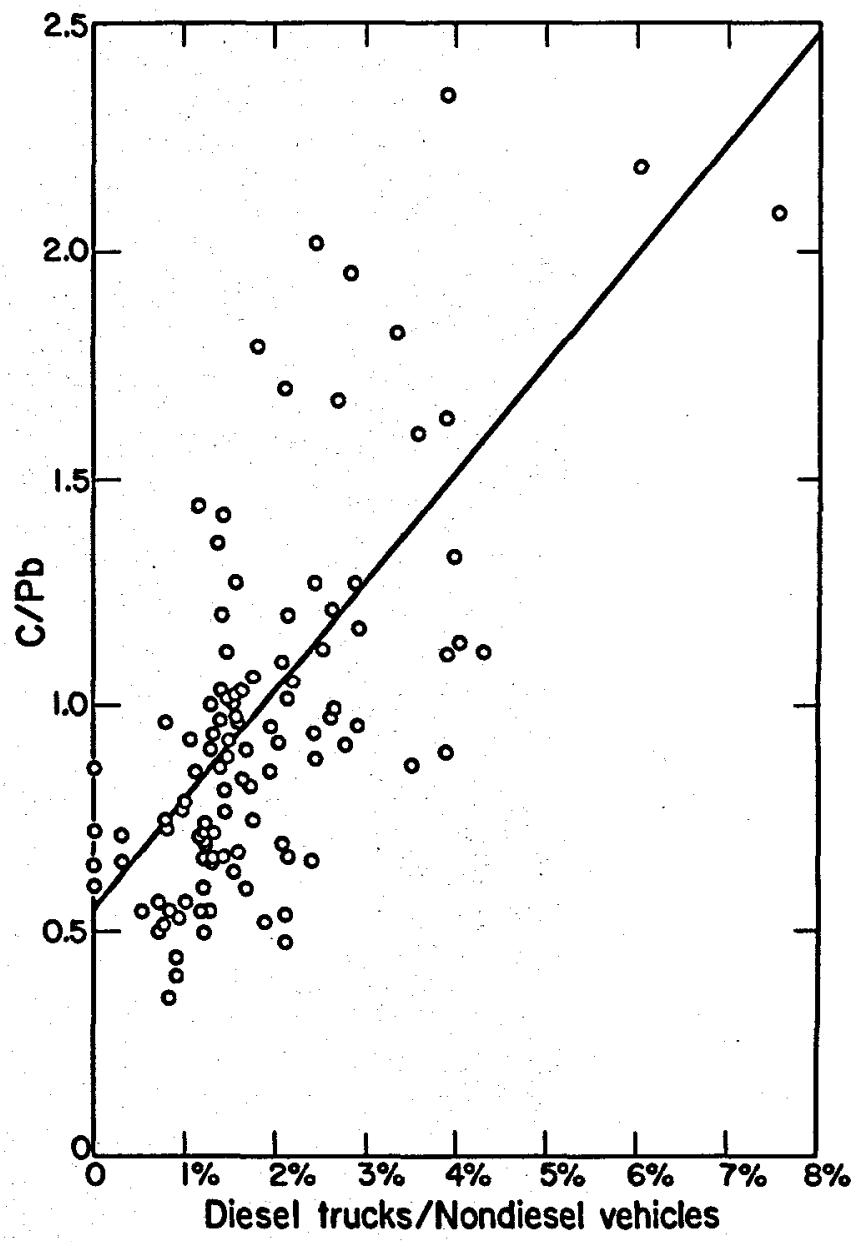

Fig. 16. Measured $\mathrm{C} / \mathrm{Pb}$ ratios as a function of percent of diesel trucks. The straight line is obtained by a least squares fit to the data and is represented by

$$
\begin{aligned}
\mathrm{C} . \mathrm{Pb}= & .55( \pm .11)^{+}+ \\
& 24( \pm 5)\left(\frac{\text { No. diesel trucks }}{\text { No. nondiesel vehicles }}\right)
\end{aligned}
$$

(XBL 766-2946) 
These experiments clearly show that vehicular emissions of carbon and lead vary considerably with traffic conditions. Our results indicate that $\mathrm{Pb}$ may be only marginally useful as a tracer for particulate carbon emitted by vehicles. The results also emphasize the importance of diesel trucks as a source of primary particulate carbon. An increase of the diesel fraction of the total vehicles from the current $2 \%$ to, for example, $10 \%$ would be equivalent to a sixfold increase in the number of nondiesel vehicles in terms of the primary carbon particulate loading.

Tunne1 roadway air containing fresh vehicular emissions was sealed in a $600-\mathrm{m}^{3}$ room for 2.5 hours and analyzed for particulate $\mathrm{C}, \mathrm{Pb}$, and $\mathrm{S}$ as well as $\mathrm{CO}$ and $\mathrm{SO}_{2}$. The experiment was repeated at relative humidities in the range of $65-100 \%$ and temperatures from $17^{\circ}$ to $22^{\circ} \mathrm{C}$. No change in concentration of the above species was observed in the 2.5-hour period. Further studies will follow the concentrations of these species over periods of 12 to 24 hours to study the oxidation of $\mathrm{SO}_{2}$ to sulfur-containing aeroso1. Recent work13-15 has reported this conversion rate in power plant plumes to be from $1.5 \% / \mathrm{hr}$ to $5 \% / \mathrm{hr}$, a rate which could be observed in our experiment only after many hours due to relatively low $\mathrm{SO}_{2}$ levels in the tunnel.

An electrical aerosol size analyzer (EASA) ${ }^{16}$ was used to measure the size distribution of fresh and aged aerosols at the tunnel roadway and in the sealed room filled with vehicular emissions. Surface distributions calculated from the EASA data showed two prominent modes: one at about $0.02 \mu \mathrm{m}$ and another at 0.1 to $0.2 \mu \mathrm{m}$. This bimodal distribution was observed to vary with time in the sealed room, filled initially with fresh vehicular emissions. The $0.02-\mu \mathrm{m}$ mode was found to decrease rapidly with time, while the 0.1-to-0.2 $\mu \mathrm{m}$ mode decreased at a much slower rate. A simple model

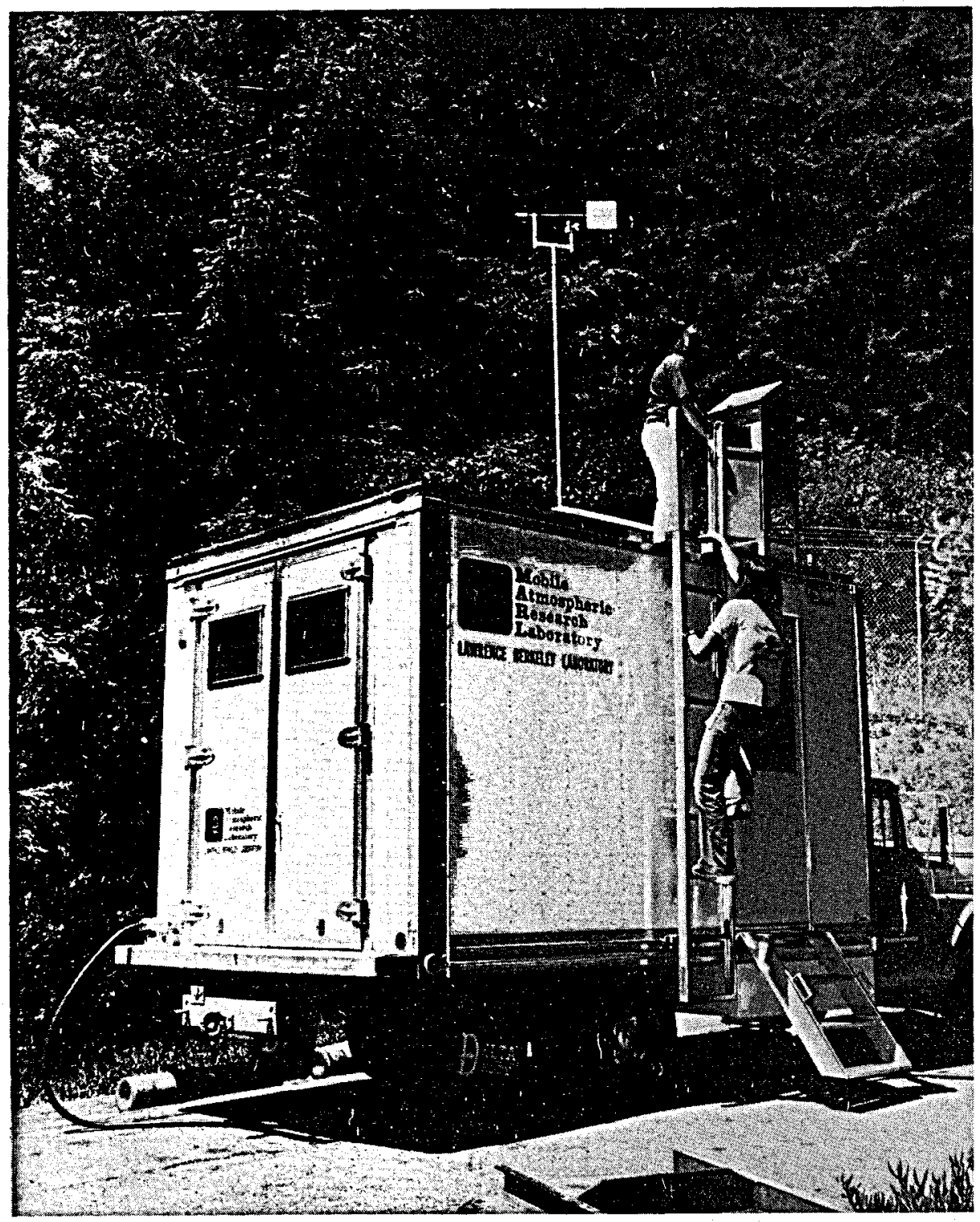

Fig. 17. Exterior view of Mobile Atmospheric Research Laboratory.

(CBB 766-5702) 
Table 2. Mobile Atmospheric Research Laboratory-parameters measured and instrumentation.

\begin{tabular}{|c|c|c|c|}
\hline Parameter Measured & Principle of Measurement & Manufacturer/Model & 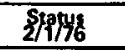 \\
\hline \multicolumn{4}{|l|}{ Sampling Equipment } \\
\hline Gases and Aer osols & $\begin{array}{l}\text { Aluminum and stainless steel rampling intake } \\
\text { and manifold to continuous monitoring instru. } \\
\text { ments and filter sampling equipment }\end{array}$ & LBL & In progress \\
\hline Gases & $\begin{array}{l}\text { Multi-point automatic olectronic sequential } \\
\text { sampler with Teflon sampling lines }\end{array}$ & LBL & Proposed \\
\hline \multicolumn{4}{|l|}{ Aerosols } \\
\hline \multirow{3}{*}{$\begin{array}{l}\text { Size fractioning aerosol filter } \\
\text { sampling equipment for } \\
\text { laboratory mass distribution } \\
\text { and chemical composition } \\
\text { analysis by } \beta \text {-gauge and XRF }\end{array}$} & $\begin{array}{l}\text { Automatic dichotomous virtual impactor } \\
(2.4 \mu \mathrm{m} \text { cut point) }\end{array}$ & LBL & Installed \\
\hline & $\begin{array}{l}\text { Manual dichotomous virtual impactor } \\
\text { (3.5 «m cut point) }\end{array}$ & Sierra Instruments 243 & Proposed \\
\hline & $\begin{array}{l}\text { 4-stage Lundgren impector with } \\
0.3,1,3 \text { and } 10 \mu \mathrm{m} \text { cut-offs }\end{array}$ & Sierra Instruments $\mathbf{4 2 2 0}$ & Proposed \\
\hline \multirow{2}{*}{$\begin{array}{l}\text { Total aerosol filter sampling } \\
\text { equipment for laboratory chem. } \\
\text { ical composition analysis by } \\
\text { XRF, ESCA, IR, combustion } \\
\text { techniques (for C/H/N enalysis) } \\
\text { and wet chemistry }\end{array}$} & Purnp with $\mathbf{4 7} \mathbf{~ m m}$ filter holder & 3 L8L Systems & Installed \\
\hline & High volume sampler & Sierra Instruments UItra-Vol & Installed \\
\hline \multicolumn{4}{|l|}{ Continuous Monitoring Instruments } \\
\hline \multicolumn{4}{|l|}{ Gases. } \\
\hline$\overline{\mathrm{SO}_{2}}$ & UV Fluorescence & Thermo Electron 43 & Installed \\
\hline $\mathrm{NO} / \mathrm{NO}_{2} / \mathrm{NO}_{x}$ & Chemiluminescence & Thermo Electron 140 & Installed \\
\hline $\mathrm{O}_{3}$ & UV Absorption & Dasibi 1003-AAS & Installed \\
\hline co & NDIR & Beckman 7000 & Installed \\
\hline Hydrocarbons & GC/FID & -- & Proposed \\
\hline $\mathrm{NH}_{3}$ & Chemiluminescence & Thermo Electron, LBL & Proposed \\
\hline $\mathrm{H}_{2} \mathrm{~S}$ & GC/FPD & Tracor $270 \mathrm{H}$ & Proposed \\
\hline \multicolumn{4}{|l|}{ Aerosols } \\
\hline Size $\left(0.01-1 \mu \mathrm{m}^{\prime}\right)$ & Condensation Nuciei Formation & $\begin{array}{l}\text { Environment/One } \\
\text { Rich } 100\end{array}$ & Installed \\
\hline$(0.01-1 \mu \mathrm{m})$ & Electrical Mobility Analysis & Thermo-Systems 3030 & Installed \\
\hline$(0.3-20 \mu \mathrm{m})$ & Optical Counting & Royco 225 & Proposed \\
\hline$b_{\text {scat }}$ & Nepholometry & $\begin{array}{l}\text { MRI } \\
\text { LBL }\end{array}$ & $\begin{array}{l}\text { Installed } \\
\text { In progress }\end{array}$ \\
\hline \multicolumn{4}{|l|}{ Zero, Span and Calibration Equipment } \\
\hline \multicolumn{4}{|l|}{ Gases } \\
\hline Zero/CO/NO/NO $/ 2 / \mathrm{SO}_{2}$ & $\begin{array}{l}\text { Scrubbers, Gas Cylinders, Dilution System, } \\
\text { and Permeation Tubes }\end{array}$ & Monitor Labs $\mathbf{8 5 0 0}$ & Installed \\
\hline $\mathrm{O}_{3} / \mathrm{NO}^{-\mathrm{NO}_{2}}$ & Gas Phase Titration & $--\quad \cdots$ & Proposed \\
\hline \multicolumn{4}{|l|}{ Meteorology Instruments } \\
\hline Wind Speed & Generator & $\begin{array}{l}\text { Young } 3 \text { Cup Anemometer } \\
\text { B101 }\end{array}$ & Installed \\
\hline Wind Direction & Potentiometer & Young Wind Vane 6301 & Installed \\
\hline Temperature & Thermistor & $\begin{array}{l}\text { Singer } 84 R \text { with } 2 \\
\text { remote sensors }\end{array}$ & Installed \\
\hline Dew Point & Thermoelectric Cooling/Resistance Measurement & $\begin{array}{l}\text { Singer } 84 R \text { with } 2 \\
\text { remote sensors }\end{array}$ & Installed \\
\hline Total Solar Radiation & Spectral Pyranometer-Thermopile & Eppley PSP & Proposed \\
\hline UV Radiation & UV Radiometer-Photcelectric Cell & Eppley TUVR & Proposed \\
\hline Pressure & Potentiometer & -- & Proposed \\
\hline \multicolumn{4}{|l|}{ Data Acquisition Equipment } \\
\hline \multirow[t]{2}{*}{ Analog Output } & Strip Chart Recording & Soltec 6.Pen Recorder & Installed \\
\hline & $\because$ & 3 Heathkit Recorders & Installed \\
\hline Automated Data Acquisition System & A/D Corverter; Magnetic Tape Rocorder & -- & Proposed \\
\hline
\end{tabular}


was used to show that coagulation of the aerosols was the dominant mechanism governing changes in the size distribution. This model successfully explained the observed time evolutions and the relative intensities of the two modes.

\section{Mobile Atmospheric Research Laboratory}

The Mobile Atmospheric Research Laboratory (MARL) has been designed to provide a comprehensive atmospheric characterization and monitoring 1aboratory for field air pollution studies. The laboratory is a self-contained unit capable of supporting field studies at almost any location and is readily movable to new sampling sites without requiring extensive time and effort.

MARL, which is shown in Fig. 17, contains the latest state-of-the-art air pollution monitoring equipment, meteorology instrumentation, calibration equipment, and data acquisition systems necessary for routine and fundamental research characterization and monitoring studies. The parameters measured and instrumentation are given in Table 2.

An automatic computer data acquisition and control system is currently being installed. Al1 of the continuous monitoring instrument outputs will be digitized and stored on magnetic tape. In addition, the computer system will synchronize the timing and control of the instruments so that samples are taken only after the individual systems are calibrated and stabilized.

Several programs currently in progress or proposed will utilize the Mobile Atmospheric Research Laboratory. Some of these programs are outlined below.

- The Atmospheric Aerosol Field Studies are designed to assess the role of heterogeneous gas-particle reactions in the formation and evolution of aerosol air pollution in ambient air, stationary source, and mobile source environments.

- The Indoor Air Pollution Study is designed to assess the extent of indoor-generated air pollution and conduct epidemiology studies on the disease effects of indoor pollutants.

- The Geothermal Field Study is designed to assess the emission of gaseous and aerosol air pollutants, their transport, and transformation from geothermal energy sites.

\section{FOOTNOTES AND REFERENCES}

*Shortened version of Atmospheric Aerosol Research Annual Report (1975-76), LBL-5214. Certain subsections have also been submitted for publication elsewhere as follows: "Application of Raman Scattering to the Characterization of Atmospheric Aeroso1 Paticles" to Nature (LBL-5228); 'Manganese Emissions from Combustors" in Chemosphere 6,339 (1975); and "Infrared and Photoelectron Spectroscopic Study of $\mathrm{SO}_{2}$ Oxidation on Soot Particles" accepted by J. Colloid Interface Sci. (1975), (LBL-4446).

+Present address: 2135 Essex St., Berkeley, CA 94705 .
FPresent address: Science Center, Rockwell Internationa1, Thousand Oaks, CA 91360.

\$NASA, Ames Research Center, Moffett Field, CA 90435

II Department of Mechanical Engineering, University of California, Berkeley, CA 94720.

TPresent address: CDM/Limnetics, 11485 w. 48th Ave., Wheat Ridge, CO 80033.

1. T. Novakov et a1., J. Colloid Interface Sci, 39,225 (1972).

2. C.W. Spicer, private communication.

3. S.G. Chang and T. Novakov, Atmos. Environ. 9, 495 (1975).

4. B.R. Appel et al., Intern. J. Environ. Anal. Chem. 4 , 169 (1976).

5. C.D. Hollowell et al., this report.

6. F. Tuinstra and J.L. Koenig, J. Chem. Phys. 53, 1126 (1970).

7. L. Hughes, "The Effect of a Manganese Fuel Additive on Air Quality," dissertation, Environ. Health Sci., School of Public Health, University of California, Berkeley, 1973.

8. E.S. Starkman, A.G. Cattaneo, and S.H. McAllister, Indust. Engr. Chem. 43, 2822 (1951).

9. G.B. Martin, D.W. Pershing, and E.E. Berkau, Office of Air Programs Publication No. AP-87, U.S. Environ. Protection Agency, Research Triangle Park, N.C. (1971).

10. L. Plonsker, E.B. Rifkin, M.E. Gluckstein, and J.D. Bailie, "Reduction of Gas Turbine Smoke and Particulate Emissions by a Manganese Fuel Additive", Ethyl Corp., Ferndale, Michigan . (1974).

11. D.F. Miller and C.W. Spicer, J. Air Poll. Control Assoc. 25, 940 (1975).

12. M.N. Rao and O.A. Hougen, A.I.Ch.E. Symposium Series 48, 110 (1952).

J.H. Smith, J. Phys. Chem. 69, 1741 (1947).

13. L. Newman, J. Forrest, and B. Manowitz, Atmos. Environ. 9, 969 (1975).

14. R.B. Smith and G.H. Jeffrey, Atmos. Environ. 9, 643 (1975).

15. K.T. Whitby, B.K. Cantre11, R.B. Husar, N.V. Gillani, J.A. Anderson, D.L. Blumenthal, and W.E. Wilson, Jr., Aerosol Formation in a Coal Fired Power Plant Plume (unpublished report, 1976).

16. B.Y.H. Liu, K.T. Whitby, and D.Y.H. Pui, A Portable Electrical Aerosol Analyzer for Size Distribution Measurement of Sub-Micron Aerosols, presented at the 66th Annual Meeting of the Air Pollution Control Association, Chicago, I1linois, June 24-28 (1975). 


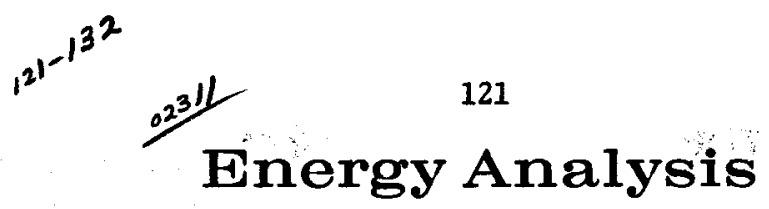

\section{INTRODUCTION}

Decisions made now will define our energy options - and their impacts - for years to come. Advancing energy technologies and fuel resources will play key roles in determining by what means energy may be supplied and consumed in the future. They cannot by themselves however tell use what choices will best serve the complex energy systems needed to supply the country's needs nor the furture costs to society and the environment.

To find the answers or at least to establish sound bases for rational energy policy development, responsible federal and state agencies are supporting wide-ranging research on the consequences of future energy alternatives.

At LBL analytical studies on the impacts of energy on society and the environment are centered in a multidisciplinary unit, called the Energy Analysis Program, within the Energy and Environment Division. Along with the other national laboratories, LBL participates in these nation-wide, integrated assessment programs for ERDA. Under these programs, the task of the LBL Energy Analysis Group is to assess the multifaceted impacts within California of alternative energy development paths.

The objectives of the ERDA regional energy assessment program are comprehensive. They call for analyses and projections of environmental, economic, health and social impacts of energy production and consumption by varied choices of technologies, including, of course, energy conservation and new technologies. To effectively serve the decision makers, such studies will need to highlight in quantitative terms the relative costs, risks, benefits, and trade offs among alternative energy technologies, and explore solutions to the constraints that may confront implementation of energy policy.

In its first year, the LBL energy impacts assessment studies have focused first on essential basic analyses whose results form a foundation for subsequent more specific impact assessments. These include quantitative characterization of certain aspects of the California economy as reflected in the production and interindustry flow of goods and energy to final demand, the state energy structure in terms of intra- and inter-regional energy flows, manpower constraints and requirements for energy facilities, energy systems modeling, and further acquisition of data.

While the studies conducted under the ERDA integrated assessment program are expected to extend over a span of several years, short-term studies are frequently conducted in response to urgent needs for information bearing on consequences of alternative energy policy choices. Among such studies conducted for ERDA are an analysis of some of the impacts of a nuclear moratorium on California, and an assessment of effects of future coal utilization in California.

Although the ERDA energy assessment program is the Group's major continuing assigment, related analytical studies are routinely performed for other agencies and institutions. Among present and recently completed projects are studies conducted for the Army Corps of Engineers, the Bureau of Mines, the National Science Foundation, and the Electric Power Research Insitutute.

Disciplines represented in the staff of the Energy Analysis Program include economics, physics, biophysics, engineering, systems analysis, computer sciences, and marine biology. As in other LBL activities, the competence of this program is greatly extended by the participation of outstanding authorities in many fields at the Berkeley campus of the University of California.

\section{IMPACTS OF ALTERNATIVE ELECTRICITY-SUPPLY SYSTEMS FOR CALIFORNIA}

William E. Sim, Jayant A. Sathaye, Leonard Kunin, Henry Ruderman, Richard Sextro, Peter Chan, and Mark Horovitz

We have examined a set of divergent electricitysupply futures - some with nuclear energy, others without - to identify and compare estimates of their consequences. While recognizing that a shift in major energy sources for the future can affect, to some degree, every segment of society, the economy, and the enviroment, we have confined our study primarily to an analysis of direct costs and pollutants associated with alternative means for supplying electricity to California, and of certain direct consequences of phasing out nuclear energy.
To assess the consequences of phasing out nuclear energy, we considered three mixes of fuels and technologies for supplying electricity to California; these bracket the full range of choices that appear possible for energy futures between 1976 and 1990. The first assumed no impediment to the expanded use and deployment of nuclear power plants and other technologies are included. In the second, nuclear power plants are phased out within 10 years, and the required new generating capacity is met primarily with coal-fired power 
Table 1. Assumed generating facilities additions, 1976-1900 (in $1000 \mathrm{MWe}$ ).

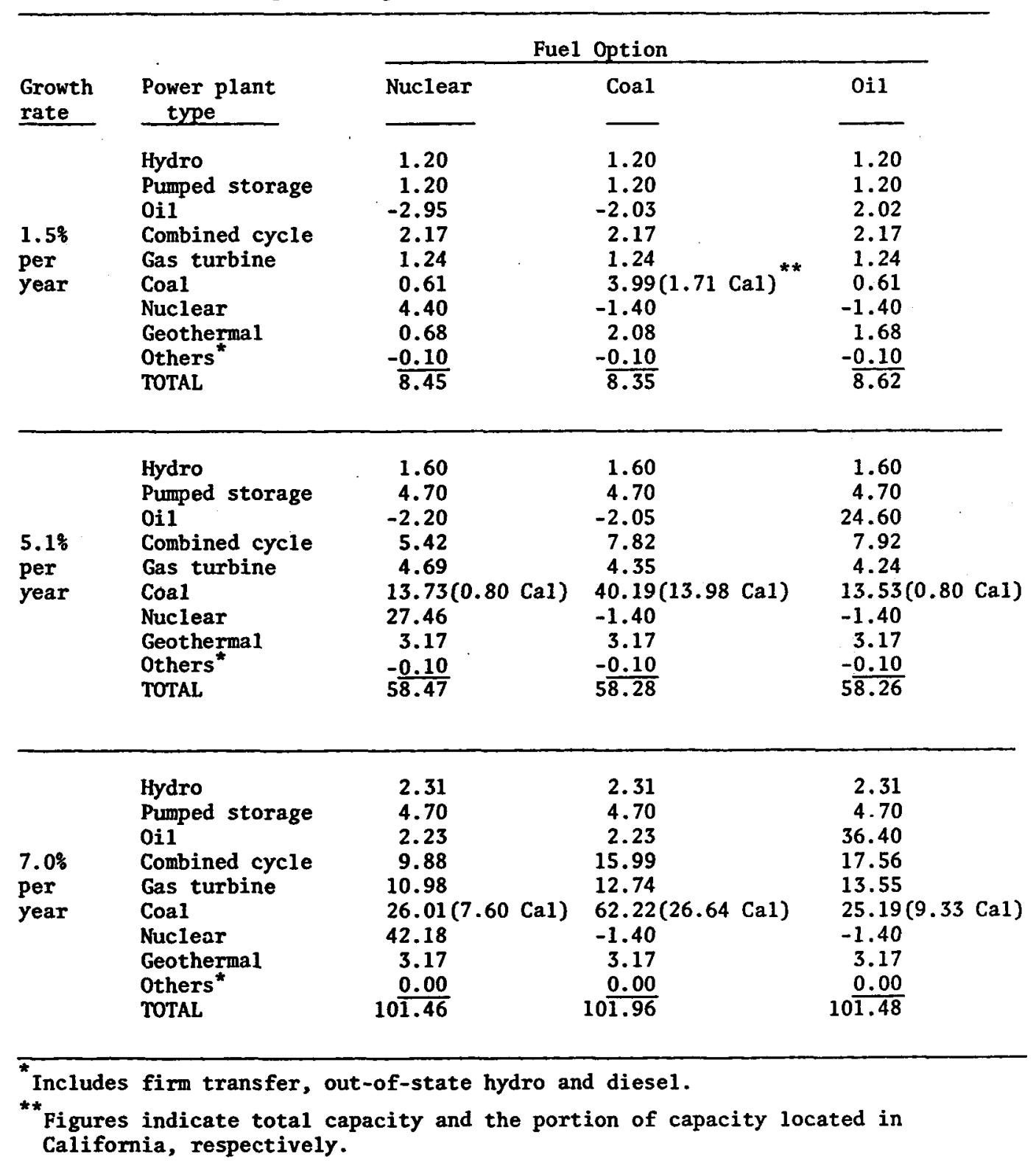

plants. Half the new coal-fired plant additions are placed in California; the others are located in unspecified locations in the southern mountain region, which consists of Arizona, Colorado, Nevada, New Mexico, and Utah. This division is arbitrary and is intended solely for the purpose of providing a basis for assessing economic and pollution effects in the states supplying coal and electricity generated from coal to California. In the third case, nuclear energy is phased out and new generating capacity is met primarily with oil-fired plants. All the oil-fired and nuclear power plants are placed in California for the three cases.

A11 three electricity systens begin with the facilities (mines, refineries, power plants, etc.) that already exist or are under construction in 1976. Facilities are then added and retired as needed in each system to meet peak-1oad capacity requirements plus $20 \%$ reserve capacity. For a summary view of the generating facilities that make up each of the electricity systems, Table 1 lists the total capacity of each type of facility to be added by 1990 .

Growth in generating capacity will also affect the relative magnitudes of the impacts produced by the three systems. Therefore, three arbitrary rates of growth $(1.5,5.1$ and $7 \%$ ) were adopted which bracket a range sufficiently wide to. emphasize, within the 15-year span under study, the potential differences among the fuel mixes that could emerge. 
With the aid of the Energy Supply Planning Model developed by the Bechtel Corporation, we determined, for each energy $\mathrm{mix}$ and for the three rates of growth, the annual requirements for construction and operation of al1 the facilities 10cated in the two regions and associated with production of electricity - from mine (or oil we11) to power plant. Costs (less fuels), manpower, materials, equipment, and resources required for construction and operation were computed for the resultant nine electricity-supply futures (called "scenarios" hereafter). Industrial casualties, quantities of fuels, and pollutants were derived from data in the Matrix of Environmental Residuals for Energy Systems (MERES) and other sources. It should be noted that since fuel prices could not confidently be forecast over the span of 15 years, the total cost of generating electricity was not determined.

\section{RESULTS}

In general, capital costs, manpower, materials, and other requirements for construction of al1 facilities, when combined for California and the southern mountain region are similar for the scenarios emphasizing nuclear and coal-fired plants. Requirements for the oil scenarios are somewhat lower.
Operating costs and manpower in California are essentially identical for all three fuel mixes. Differences in fuel costs, however, may introduce significant differences in economic effects. Thus if the price of oil per million Btu remains three to four times that for uranium, the cost of generating electricity in the $5.1 \%$ growth-rate oil scenario would exceed that for the nuclear scenario by several billion of dollars during the next 15 years.

Considering only construction and operating requirements, there is little evidence that California's economy would be affected more by one fuel mix than another, although some secondary and local impacts may differ. However, for the southern mountain region, the differences among the scenarios in their economic consequences are profound. This region clearly would derive greater income and employment from substitution of coal for uranium and oil.

Between 1976 and 1990, the total resources requirements-and therefore the direct economic impacts of California's future electricity supply system-depend far more profoundly on how fast the system grows than on the choice of technologies. Capital, manpower, and materials may differ by 10 to 158 in 1990 for the three extreme fuel mixes ex-

Table 2. Changes in airborne emissions 1976 to 199u (Ratio of estimated 1990 to 1976 quantities).

\begin{tabular}{|c|c|c|c|c|c|}
\hline \multirow[t]{2}{*}{ Growth Rate } & \multirow[t]{2}{*}{ Substance } & \multirow[t]{2}{*}{ Region ${ }^{*}$} & \multicolumn{3}{|c|}{ Generating Mix } \\
\hline & & & Nuclear & Coal & 0i1 \\
\hline $\begin{array}{l}1.5 \% \\
\text { per } \\
\text { year }\end{array}$ & $\begin{array}{l}\text { Particulates } \\
\text { Particulates } \\
\mathrm{NO}_{\mathrm{X}} \\
\mathrm{NO}_{\mathrm{X}} \\
\mathrm{SO}_{2} \\
\mathrm{SO}_{2} \\
3_{\mathrm{H}} \\
85_{\mathrm{Kr}} \\
131_{\mathrm{I}} \\
222_{\mathrm{Rn}}\end{array}$ & $\begin{array}{l}\text { CA } \\
\text { SM } \\
\text { CA } \\
\text { SM } \\
\text { CA } \\
\text { SM } \\
\text { CA } \\
\text { CA } \\
\text { CA } \\
\text { SM }\end{array}$ & $\begin{array}{l}1.03 \\
1.21 \\
0.99 \\
1.11 \\
0.95 \\
1.10 \\
2.36 \\
2.36 \\
2.36 \\
2.36\end{array}$ & $\begin{array}{l}1.34 \\
1.21 \\
1.28 \\
1.11 \\
1.26 \\
1.10 \\
0 \\
0 \\
0 \\
0\end{array}$ & $\begin{array}{l}1.34 \\
1.21 \\
1.31 \\
1.11 \\
1.29 \\
1.10 \\
0 \\
0 \\
0 \\
0\end{array}$ \\
\hline $\begin{array}{l}5.1 \% \\
\text { per } \\
\text { year }\end{array}$ & $\begin{array}{l}\text { Particulates } \\
\text { Particulates } \\
\mathrm{NO}_{\mathrm{X}} \\
\mathrm{NO}_{\mathrm{X}} \\
\mathrm{SO}_{2} \\
\mathrm{SO}_{2} \\
\mathrm{3}_{\mathrm{H}} \\
85_{\mathrm{Kr}} \\
131 \mathrm{I} \\
222_{\mathrm{Rn}}\end{array}$ & $\begin{array}{l}\text { CA } \\
\text { SM } \\
\text { CA } \\
\text { SM } \\
\text { CA } \\
\text { SM } \\
\text { CA } \\
\text { CA } \\
\text { CA } \\
\text { SM }\end{array}$ & $\begin{array}{l}1.40 \\
5.71 \\
1.29 \\
4.52 \\
1.22 \\
4.54 \\
6.88 \\
6.88 \\
6.88 \\
6.88\end{array}$ & $\begin{array}{l}2.63 \\
9.57 \\
2.06 \\
7.37 \\
1.86 \\
7.38 \\
0 \\
0 \\
0 \\
0\end{array}$ & $\begin{array}{l}2.19 \\
5.64 \\
2.07 \\
4.43 \\
1.99 \\
4.44 \\
0 \\
0 \\
0 \\
0\end{array}$ \\
\hline $\begin{array}{l}7.0 \% \\
\text { per } \\
\text { year }\end{array}$ & $\begin{array}{l}\text { Particulates } \\
\text { Particulates } \\
\mathrm{NO}_{\mathrm{X}} \\
\mathrm{NO}_{\mathrm{X}} \\
\mathrm{SO}_{2} \\
\mathrm{SO}_{2} \\
3_{\mathrm{H}} \\
85 \mathrm{Kr} \\
131_{\mathrm{I}} \\
222_{\mathrm{Rn}}\end{array}$ & $\begin{array}{l}\text { CA } \\
\text { SM } \\
\text { CA } \\
\text { SM } \\
\text { CA } \\
\text { SM } \\
\text { CA } \\
\text { CA } \\
\text { CA } \\
\text { SM }\end{array}$ & $\begin{array}{l}2.88 \\
6.86 \\
1.85 \\
5.33 \\
1.65 \\
5.33 \\
9.29 \\
9.29 \\
9.29 \\
9.29\end{array}$ & $\begin{array}{c}4.13 \\
12.3 \\
3.01 \\
9.43 \\
2.63 \\
9.42 \\
0 \\
0 \\
0 \\
0\end{array}$ & $\begin{array}{l}3.53 \\
6.07 \\
2.96 \\
4.76 \\
2.91 \\
4.76 \\
0 \\
0 \\
0 \\
0\end{array}$ \\
\hline
\end{tabular}

$C A=$ California; $S M=$ Southern Mountain 
amined here, but the differences may be as great as $1000 \%$ between growth rates of $7 \%$ and $1.5 \%$ per year. A difference of $1 \%$ in the rate of electricity consumption appears to have a more significant economic impact than varied choices of probable fuel mixes.

Residuals (pollutants) discharged to the enviromment are strongly dependent upon the choice of electrical supply system. For some pollutants, the differences in quantities produced by one scenario relative to another are comparable to the differences that would result from a change in growth rate of several percent.

Estimates of the principal residuals discharged to air, for 1990 relative to 1976 , are given in Table 2 for the three energy scenarios. In general, sulfur dioxide $\left(\mathrm{SO}_{2}\right)$, nitrogen oxides $\left(\mathrm{NO}_{\mathrm{X}}\right)$, particulates, and hydrocarbons discharged as pollutants in California throughout the next 15 years are comparable for the coal and oil scenarios, with both increasing rapidly after 1980 .

For the nuclear scenario, emissions of some chemical pollutants remain relatively constant; others increase slowly. On the other hand, tritium and krypton increase by an order of magnitude in the nuclear scenario for the high rate of growth and disappear in the coal and oil scenarios. However, radioactive emissions from nuclear power plants through 1990 appear to lie within the levels permitted by current federal regulations. Radioactive wastes produced in the nuclear scenario will require management and ultimate disposal in a manner that will ensure that none are released to the enviromment before they have decayed.

An estimated $\$ 2.3$ billion is presently invested in nuclear power plants in California. on completion of units still under construction, the total asset value will be somewhat greater. Writing off these assets raises questions of equity concerning how this economic loss would be shared. A likely recourse is the inclusion of this investment in the electricity rate base, amortized over a span of one or more decades. Tax levies are possible alternatives to rate adjustments. The consumer of electricity would bear the burden in either case.

Potential local economic impacts were briefly examined for each of the four counties containing nuclear facilities (Sacramento, San Diego, Humboldt, and San Luis Obispo), primarily to conpare employment for nuclear power plant construction and operation to the local labor market pool. In Sacramento County, where Rancho Seco I is in the operating phase, approximately 150 jobs are directly at stake. In San Diego County, the figure is 2000 jobs. Phasing out the small reactor in Humboldt County would involve the direct loss of about 25 jobs. In San Luis Obispo County, no local construction employment impact would be linked to a nuclear phase-out. The siting of new non-nuclear generating capacity in any of these regions would mean that negative local impacts due to a nuclear phase-out would be transient.

\title{
A LINEAR ECONOMIC MODEL OF FUEL AND ENERGY USE IN THE UNITED STATES
}

\author{
Roger Glassey*, Peter Benenson, Henry Ruderman,
} Esther Schroeder, Barbar Kach, and Peter Chan

\section{INTRODUCTION}

In this study we have developed a model with which to investigate the pattern of energy use in the United States and to examine the economic impacts of fuel and energy shortages.1 Work on this model began in January 1975 as a continuation of earlier work for the Electric Power Research Institute (EPRI).

Discussion with the staff of the energymodeling program at EPRI had revealed two questions that needed to be explored:

- What are the patterns of energy use in the U.S. economy?

- What are the economic consequences of energy shortages?

At that time, we were working with the 1963 national input-output (I-0) table, which was the most recent then available; we also had a linear programing code that had already been developed and tested. Consequently, we agreed to provide a preliminary analysis of these two questions before undertaking an in-depth study. The preliminary work was reported to EPRI in 1974.2
This first model contained 97 I-0 sectors measured in 1972 dollars, employment for each sector expressed in number of jobs, and the requirements by sector for five types of energy expressed in Btu's. The I-O and energy data enabled us to estimate the energy content of the outputs and inputs of each industrial sector. The data were then incorporated into a linear programing model in which gross national product, expressed as the summation of value-added, was maximized subject to constraints on industrial capacity and production, final demand, labor supply, and unemployment.

Although this model provided some interesting preliminary answers to the questions posed, it was limited in several important respects:

- It was based primarily on 1963 data.

- Fuel substitution possibilities by the electric utilities and other sectors were not included.

- The model formulation did not incorporate a distinction between peak power and total energy demand in the electric utilities sector, which makes it difficult 
to place an accurate upper limit on electrical generating capacity.

- Some of the data were too highly aggregated for the purpose of the study.

- The model was formulated on a gross-output rather than on a gross-domestic-output base; thus imports were aggregated with domestic output, resulting in an inflated picture of U.S. industrial output. Moreover, imports could not be regulated as a policy variable.

\section{THE PRESENT MODEL}

Our present work explores the two questions posed by EPRI in much greater detail with the use of a more refined model and a more current data base. An additional objective is to explore the extent to which input-output and linear programming techniques can be used to analyze energy problems.

A schematic diagram of the present model is shown in Fig. 1; the numbers within each box denote the dimensions of the matrix. In this new model, an updating process provides a more recent description than before of the technologies available to each industry. Also, it now permits fuel substitution in the iron and steel sector and in the electric utilities sector as the availabilities of energy sources change. This enables us to estimate the impact of energy shortages.

Several other features lend a further degree of realism to the new model:

- The distinction between peak power and total energy provides a more accurate estimate of the extent to which other sectors may draw on electricity for their
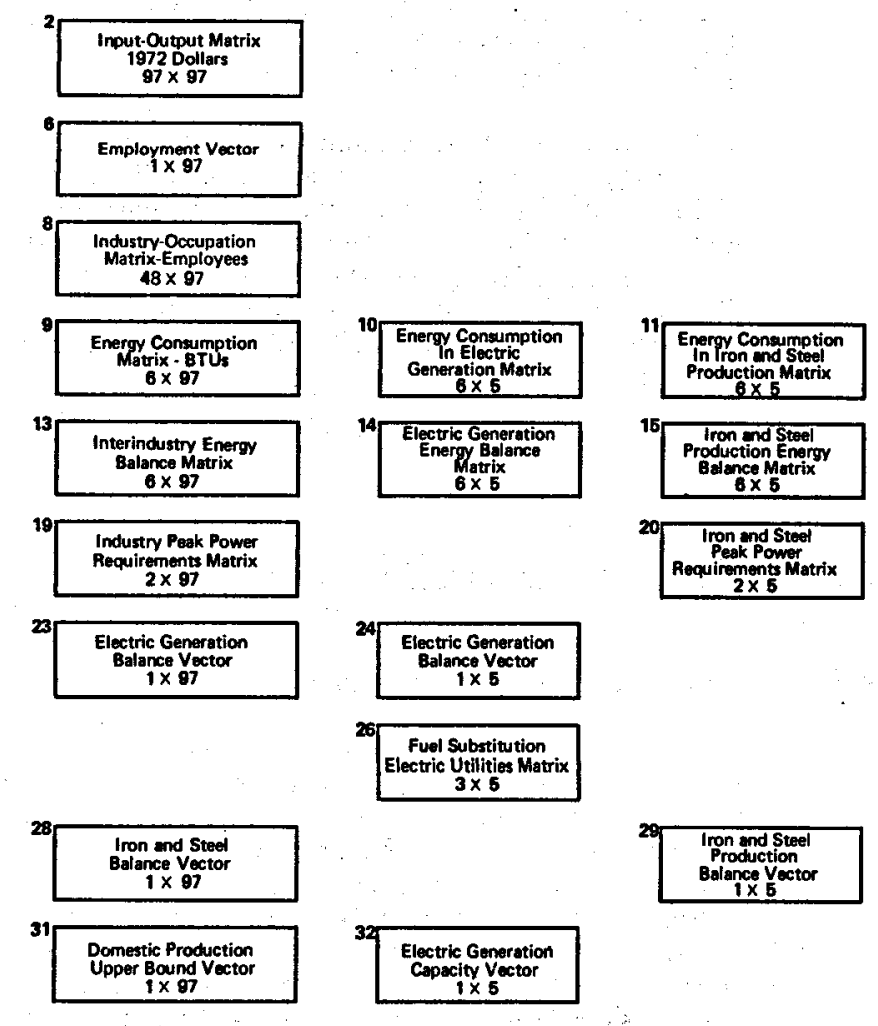

output.

- Increased detail in the energy sector permits us to trace changes in energy use more precisely.

- Occupational detail provides a finer picture of employment impacts.

- Separation of imports from domestic outputs renders the technical coefficients more accurate and helps to give a more precise picture of input requirements as output levels change in response to energy shortages; it also permits us to simulate energy shortages resulting from foreign or domestic trade policy.

\section{Application of the Present Model and Results}

We have constructed a set of scenarios in which we gradually tighten the constraint on each energy sector separately, using a parametric linear programming code written at LBL. In so doing, we have observed a general pattern in the behavior of the model components. First, the scarce energy resource is reduced to its final-demand lower bound, and then is increased until its upper bound on imports is reached. Next, fuel substitution in the electric-utilities and the iron-and-steel sectors takes place. Substitution continues until either the use of scarce fuel is reduced to zero or the substitute fuel is burned to the limit of fuel-burning capacity. Usually a fue1-substitution limit is reached, after which the fuel scarcity begins to make a noticeable impact on other sectors of the mode1. Sector output changes depend on the intensity with which the scarce fuel is used in production in a sector, and on the sector's contribution to GNP.
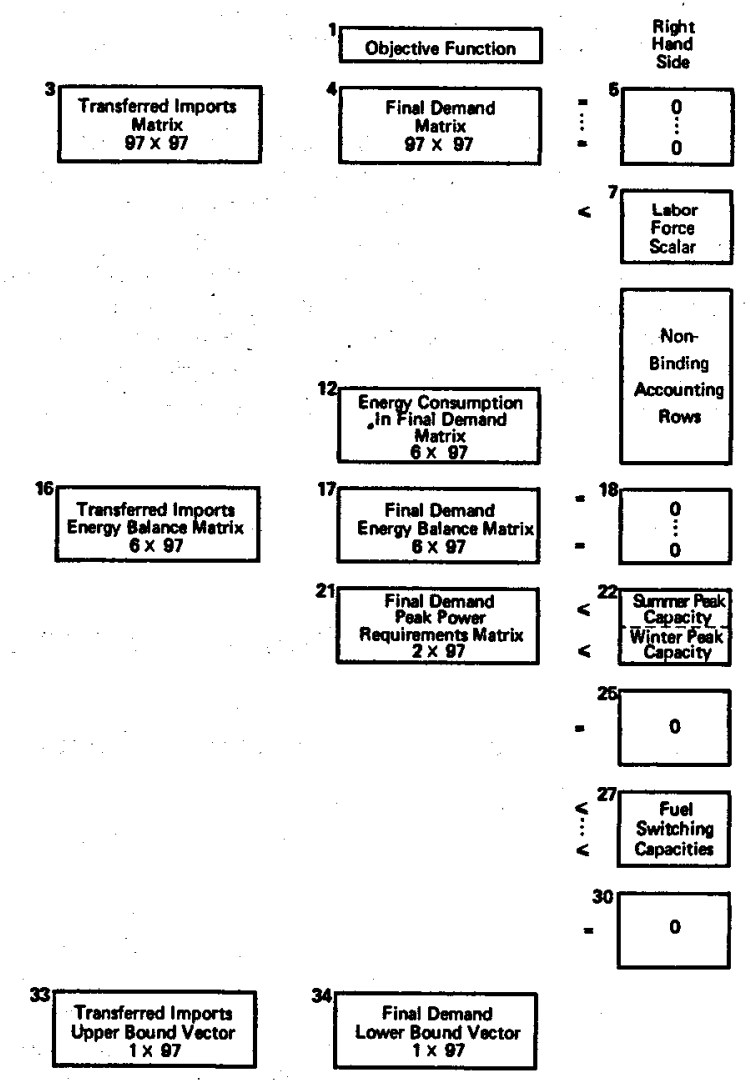

Fig. 1. Model components. (XBL 7512-9775) 
The order in which the constraints have an impact on the model variables is given in the table below. The measure of sensitivity is the inmediacy with which decreases in the constraint variable, (expressed as a percentage of that variable's upper bound) effect a downturn in GNP, employment, production in the steel and aggregate manufacturing sectors, and consumption of crude and refined petroleum.

Approximate decrease from capacity at which first

Parametric constraint downturn occurs (\&)

Electric generation

Petroleum refining

Crude petroleum and natural gas

Natural gas from utilities

Coal production

Petroleum imports

Although a decrease in electricity-generating capacity brings about a downturn in these variables sooner than any other capacity reduction, it is the reduction of coal and gas that effects the sharpest decreases - followed by crude petroleum, refined petroleum, petroleum imports, and electric-generating capacity.

Changes in fossil-fuel consumption and the attendant behavior of the shadow prices are determined by the links between the constrained variable and the other fuels. For example, the crude-petroleum constraint also constrains crude natural gas because these are treated as joint products in the model. Since these energy sources are inputs to the production processes for refined petroleum and natural gas from utilities, the latter two are

also constrained in this scenario; thus the shadow prices of all four fuels rise.

In the electric utilities sector, most fuel substitution takes place between gas and oil. When coal is constrained, however, gas is used in its place. Coal, hydroelectric, and nuclear power generation are always used to capacity due to their relatively low fuel prices.

Eventually, as the constraints are applied, the aggregate output in the major sectors of the model declines. Mining and transportation behave most uniformly across scenarios, declining gradually as the constraints are tightened. Output of the service industries increases slightly before finally declining, in every scenario except the electric-utilities case. This result is expected since wholesale and retail trade uses electricity much more intensively than any other type of energy.

Similarly, the agricultural sector increases its output before finally declining in those scenarios in which petroleum products are not constrained. This is a1so expected since agriculture relies relatively heavily on petroleum products and does not make extensive use of electricity or natural gas. Output from the manufacturing sector increases temporarily in the refined petroleum and petroleum imports scenarios. The manufacturing sector, which uses electricity relatively intensively, can therefore expand temporarily in the face of petroleum cutbacks.

\section{FOOTNOTE AND REFERENCES}

"University of California, Berkeley

1. C.R. Glassey, P. Benenson and H. Ruderman, "A Linear Economic Model of Fuel and Energy Use in the United States," LBL Report No. LBL 4442, December 1975.

2. C.R. Glassey, P. Benenson, D.W. Merril, E.M. Lofting, K. Ang, J.A. Sathaye, and E.H. Schroeder, "An Input-Output and Linear Programming Analysis of Fuel and Energy Use in the United States Economy," Preliminary Report to the Electric Power Research Institute, Report No. UCID-3732, July 19, 1974.

\title{
MANPOWER CONSTRAINTS ON ENERGY PLANT CONSTRUCTION AND OPERATION
}

\author{
Peter Benenson, Henry Ruderman, Esther Schroeder, Frederick Winyard
}

The objective of this work was to estimate whether or not there is sufficient manpower in all occupations to construct and operate the energy plants required by current energy-consumption projections. Because of the mobility of labor in the United States, the national and regional manpower situations must be considered together. To accormplish this, we use a two-region input-output (I-0) model in which one region is the region of interest and the other is the "rest of the U.S." This model takes into account the interconnectedness of all producing sectors and geographical regions of the U.S. economy. We then couple this with a linear programming (LP) model in which we maximize gross national product, subject to the constraints imposed by independently projected labor supply and 
industrial capacity. The model yields estimates of manpower requirements by industry and occupation, direct and indirect impacts on industrial production, and occupations in which stiortages may occur. From these results we assess the feasibility of the entire scenario. The model and results are described in detail in our LBL progress report, UCID-3760, 30 June 1975.

The basic model components described above were assembled from existing model formulations and published data. Work during this year has been devoted to improving the model by refining the formulation and updating the components. The objective of these improvements is to strengthen our ability to assess the impact of energy development by region and the importance of manpower constraints on projected development. The improvements were based upon experience acquired in earlier work and suggestions from persons doing energy modeling at other research centers.

The following tasks have been completed:

1. The model was disaggregated into separate sectors for electric utilities, gas utilities, and water and sanitary services. The manner in which electricity is actually generated in each region (either by coal, oil, gas, hydro, or nuclear) is reflected in the coefficients for each region.

2. The component of final demand for personal consumption expenditures was isolated from the other final demand vectors. This permits us to simulate policies such as taxes or subsidies designed to affect consumer expenditures.

3 . In conjunction with task 2 , the objective function of the linear programing model has been reformulated to maximize the sum of consumer expenditures (the component directly affected by the simulated policy) rather than the sum of valueadded, of which it is a part.

4. We have also isolated the profits component of value-added so that profits alone may be maximized. This is perhaps a more realistic formulation if we wish to focus on the production side of the economy, since the assumption of profit maximization is one possibility for describing producer behavior in the U.S. economy. As an alternative to this formulation, we retain the option to maximize value added (GNP).

5. Data have been acquired for projected energy supply constraints and projected and current capital requirements matrices.

6. We have discussed and formulated a manpower mobility model, but this has not yet been coupled with the main I-O and LP model.

To complete the improvements phase of this study, it will be necessary to update to 1972 the California and "rest of the U.S." input-output tables. These will be connected by 1972 trade flow data and will provide the most recent model that can be constructed with published census data, enabling us to model the economic interdependence between the two regions.

After the 1972 two-region model is constructed, three major tasks will be undertaken: we will develop scenarios that emphasize production in different energy sectors, we will calculate the direct capital and manpower requirements to construct the energy plants called for by each scenario, and we will increment the appropriate component of the two-region model by the magnitude of these requirements. For each scenario, we will then use the two-region model, in conjunction with a linear programming formulation, to estimate the total employment and production impacts and the occupations in which bottlenecks may occur.

\title{
INTERREGIONAL ENERGY FLOWS
}

\author{
Leonard Kunin
}

Originally, we planned to construct a comprehensive set of interregional flows of energy resources and energy-intensive commodities. From this there developed the complementary goal of setting up regional energy budgets that would account for the sources and uses of energy resources and commodities on a regional basis. We hoped to use this set of accounts with a variety of socioeconomic indicators to test the correlation of regional energy-resource development with other types of regional economic activity, levels of personal income, and health and welfare indices of various kinds.

Construction of the kind of framework we envisage requires collecting large quantities of data on energy-related commodity flows. These data come from many sources and are extremely uneven in their coverage and reliability. The need to evaluate the data carefully has led to a rescoping of our plan of work.

At present we are assessing flows of energy embodied in commodities produced by manufacturers. The coverage of these commodity flows will be the most comprehensive and reliable possible, and results achieved will stand on a reliably sound empirical base. We are focusing initially on flows into and out of California.

The energy relationships between regions are frequently thought of and depicted solely in terms of interregional flows of the primary energy-resource commodities (oil, gas, and coal) and electricity. The picture that thereby emerges may be misleading since it neglects the less visible flows of energy embodied in the commodities processed and fabricated by the manufacturing in- 
dustries.

Our first attempt to estimate these flows for California was based on the 1967 Commodity Transportation Survey of the Census of Transportation. The tonnage flows presented by this Census had to be converted first to value terms and then to energy (Btu) terms. Problems of incomplete data and bridging between different commodity codes were difficult but manageable. What proved to be a more intractable problem was the lack of a consistent set of values per unit weight to match against the detailed commodity code of the Census material.

In the absence of such data, we attempted an alternate method of converting the tonnage flows into value terms. From the 1967 Census of Manufactures we were able to estimate the value of total shipments by California mamufacturers in each classification of the Standard Industrial Classification (SIC). To develop an estimate of the value of shipments sent out-of-state we multiplied each value total by the ratio of tonnage shipped out to total tonnage shipped. (These tonnages were derived from the Census of Transportation.)

Our estimate of the value of each commodity shipped out of California derived by this method turned out to be an unreasonably large proportion of the total value of that commodity manufactured in California. The reason for the overestimate is that the Commodity Transporatation survey samples only those shipments that travel more than 25 miles. Thus virtually all intracity shipments are excluded, which exaggerates the ratio used above.

We are now proceeding along somewhat different lines, using two new sources of data: the 1972 Commodity Transporation Survey, in machinereadable form, from the Bureau of the Census; and the results of a MIT study that gives values per pound, along with several other commodity attributes, for all five-digit classifications of the Standard Transportation Commodity code (STCC). Using these data, after overcoming various disclosure problems, we can compute values of commodities shipped for each class (or combination of classes) of STCC.

After aggregating the flows out of and into California to an appropriate level and bridging to a different industrial code, we will apply a set of energy coefficients developed at the Center for Advanced Computation at the University of I1linois. These coefficients give the number of Btu's embodied in a dollar output for each of some 80 industrial sectors. Applying this method to commodity flows in and out of California will allow us to estimate the net flow of energy embodied in manufactured commodities. The methodology and programing is being developed in a general form so that it can be applied to any state for which adequate data are presented in the Commodity Transportation Survey.

\title{
ENERGY BUDGETS AND MUTIPLIERS FOR THE ROCKY MOUNTAIN STATES
}

\author{
Jayant A. Sathaye and Leonard Kunin
}

In order to anticipate the likely impacts of energy-related developments, we have developed interindustry ( $\mathrm{I}-0$ ) tables for the economies of each of the Rocky Mountain states. This eightstate region contains much of the nation's energy reserves and thus will bear the brunt, over the next few decades, of increased energy activities. Using these I-0 tabies, we have simulated the economic structure of the state economies.

\section{REGIONAL INTERINDUSTRY ACCOUNTS}

There are two general methods for developing interindustry relationships: one relies on survey techniques; the other is based on secondary data. Although the survey method is usually considered to be the more accurate and reliable, there is a growing awareness that detailed national data that can be appropriately regionalized may afford sufficient insight for policy planning purposes.

The usefulness of an I-0 table is often related to the amount of sectoral disaggregation that is feasible. Where interest focuses on a particular sector, it is important that the production relations in this sector be defined as precisely as possible; the higher the degree of disaggregation, the smaller the errors stemming from varying "products mix" among regions. For this reason, our regional tables are at a level of 400 sectors; 44 or these represent minerals industries, which are important in the Rocky Mountain region.

To construct a regional interindustry matrix from the national matrix, 1 a set of regional vectors were developed independently of the national table by "scaling down" the national data and using proxies for actual measures. This method permits consistency checks on data and results in regional accounting frameworks that are comparable to and consistent with the national income and product account. The cost-effectiveness of this approach has been dealt with in a paper by Boster and Martin ${ }^{2}$. It has been tested and ranked as "the best of the purely non-survey approaches." 3

\section{REGIONAL ENERGY BUDGETS}

Having developed I-O tables for each of the 
state economies in the Rocky Mountain region, we jecided to use the information in them, together with other data on the energy content of the sectoral products and with the energy coefficients developed by R. Herendeen, 4 to construct state energy budgets. Our goal is to produce a sources account and a uses account for each of six energy sectors: coal, crude oil and natural gas, refined petroleum products, electric utilities, and gas utilities. The sources side of each account is derived by converting the net output (gross output minus imports) of each energy sector into Btu's. Deriving the uses side of the account in a similar manner fails to take into account the import of energy embodied in the various inputs to industry; it is better simply to calculate the.Btu's contributed by each of the six energy sectors to each of the producing sectors. We thus develop a clear picture of the distribution of energy use in each state by type of energy and industry.

\section{ENERGY MULTIPLIERS}

The economy of the Rocky Mountain region and that of the rest of the United States are inextricably linked through imports and exports of commodities. We would like to know what impact the expansion of demand for the products of one sector may have on the output of any other industry.

The changes in demand in any one industry diffuse throughout the other industries of the state and can be traced by means of the coefficient and flow matrices for each state. To determine the extent of this diffusion, we have made use of both the Type I and Type II regional epergy multipliers as defined by Moore and Petersen. 5

The initial impact of a decrease in final demand for any industry is a curtailment of output by that industry and therefore a drop in energy consumption for that industry. The energy change in such a case is described by a Type I energy multiplier; we have calculated Type I energy multipliers for each of the six energy forms.

A change in final demand also produces a change in consumer expenditures, which then generates changes in production and energy use in the economy. One method of estimating this effect is to include the value-added row and the personal consumption expenditure colum in the coefficient and inverse matrices. These changes are captured by Type II energy multipliers, which have been calculated for each of the six energy forms.

\section{REFERENCES}

1. The Input-Output Structure of the U.S. Economy: 1967, Survey of Current Business, Feb. 1974.

2. R.S. Boster and W.E. Martin, "The Value of Primary Versus Secondary Data in Interindustry Analysis: A Study in the Economics of the Economic Model" The Annals of Regional Science, Vo1. 6, No.2, Dec. 1972.

3. W.I. Morrison and P. Smith, 'Nonsurvey InputOutput Techniques at Smal1 Area Level: An Evaluation," Journal of Regional Science, Vo1. 14, No. 1, 1974 .

4. R.A. Herendeen, An Energy Input-Output Matrix for the U.S., 1963: User's Guide, Center for Advanced Computation, University of Illinois, Urbana, I11., March 1973.

5. F.T. Moore and J.W. Peterson, "Regional Analysis: An Interindustry Model of Utah," Review of Economics and Statistics, Nov. 1955, pp. 368-383.
An extremely useful tool in the analysis of the U.S. economy is the input-output (I/O) model, in wich the interdependence of all industry sectors is treated through a matrix formalism. Each industrial sector is considered to have inputs from all other sectors; these are assumed to vary linearly with activity level as measured by the sector's gross output. Final demand for the output of a given industry is taken to be its gross output less the sum of its contributions to a11 other industry sectors.

\section{APPLICATIONS OF I/O MODELING}

The input-output model has the ability, in principle at least, to predict changes in employment resulting from changes in economic activity. Considering labor as an additional input to each industrial sector, one may calculate for each industry a labor coefficient which is the ratio of employment to gross output in that industry. Then by hypothesizing a given change in final demand, the direct and indirect impacts upon employment in all sectors of the economy can be estimated. If separate employment coefficients for various occupational classifications are provided, the different impacts on different classes of workers (e.g., administrators, sales workers, laborers, etc.) may be estimated.

Input-output analyses of employment impacts, resulting, for example from the adoption of various possible energy alternatives, have been performed in various laboratories and institutions to answer questions of interest to ERDA and other government agencies. In all of these studies, there is an urgent need for reliable, self-consistent, timely, highly disaggregated employment 
data. These data must be available in a form that can be readily used with the input-output tables regularly published by the U.S. Bureau of Economic Analysis (BEA).1

In order to update or disaggregate U.S. employment data, one must use data from many different sources. But these data are presented for industrial sectors that do not correspond, even closely, to those of the BEA input-output tables or to each other's. Major differences occur in the treatment of employment in government enterprises, such as govermment-owned utilities and transportation facilities. Part-time workers, agricultural workers, household workers, self-employed workers, and unpaid family workers are not treated in a consistent fashion. Finally, employment is defined sometimes as jobs (including part-time), sometimes as persons employed, and sometimes as full-time equivalents.

\section{CURRENT WORK}

In this project we have developed estimates of 1972 U.S. employment by state, industry, and occupation. In their most highly disaggregated form, these estimates are available for 51 states (including D.C.), for 368 BEA input-output industry classifications, and for 422 occupational classifications. For purposes of comparison and cross-checking, the industrial disaggregation can also be expressed in terms of two-digit 1967 SIC (Standard Industrial Classification) codes, as we11 as in terms of the industrial classifications used in the 1970 U.S. Census of Population and in the monthly Current Population Survey. With some additional work, disaggregation to four-digit 1967 SIC codes can be performed.

In developing these employment estimates, we have used, as far as possible, only regularly published and widely available U.S. govermment data sources plus the well-established definitions and methodologies developed by the Bureau of Labor Statistics. Results were checked for consistency with other data sources.

Totals and subtotals were normalized to published BLS figures, down to the level of detail for which published data are available. Further disaggregation was performed with the use of other data; but the overriding consideration was that totals over states, over industries, and over occupations should agree with published BLS data.

The entire analysis was performed by computer, and the programs and data permanently stored and internally documented in the LBL Mass Storage System (MSS). The analysis may be duplicated, or modified for any other year, by any person having local or remote access to the LBL computer system.

\section{SUMMARY OF RESULTS} follows:

The results are provided in four parts, as

1. Estimates of total U.S. employment (jobs) for 368 input-output industry sectors, for 1963, 1967, and 1972. This portion of the analysis is described in detail in Ref. 2. Separate results are provided for wage and salary employees and for self-employed and unpaid family workers.

2. Estimates of total U.S. employment (persons employed) for 422 occupational classifications, for 368 input-output industry sectors, for 1972.

3. Estimates of U.S. employment by states (persons employed) for 368 input-output industry sectors, for 1972 .

4. Estimates of total U.S. employment for 368 input-output industry sectors, for 1972, for

(a) jobs, wage-and-salary workers

(b) jobs, self-employed and unpaid family workers

(c) jobs, total

(d) persons employed, total

(e) full-time equivalents, wage-andsalary workers

(f) full-time equivalents, self-employed and unpaid family workers

(g) full-time equivalents, total

The methodology and assumptions involved in obtaining the results 1 through 4 are discussed in Ref. 3. In addition, Ref. 3 provides instructions for accessing the computer-stored results, as we11 as the computer programs, input data, and detailed program documentation.

With the use of existing programs, any of the data can be comveniently aggregated to any smaller number of sectors, for example to the 80-odd twodigit BEA I/O industry sectors defined in Ref. 1.

The 1972 employment estimates developed in this project depend on many implicit and explicit assumptions not stated here. Some of these assumptions are known to be faulty; but they were necessary, in the absence of adequate data, in order to provide any estimates at all. We believe that enough checking and cross checking was performed to eliminate any outright gross blunders. The agreement of the results in part 1 above, with independent results from BLS 4 and Bezdek et a1,5 supports this belief. Most of the results presented are probably accurate to within 10 or 15 percent. An exception is in the agricultural sectors, where the various input data are not in agreement, and state-by-industry employment data are not available.

\section{FOOTNOTE AND REFERENCES}

* This project was performed in conjunction with work being done by Dr. Clark Bullard of the Center for Advanced Computation (CAC), University of I11inois at Urbana-Champaign. The CAC contract of which this work forms a part is "Energy, Employment, and National Product-A Linear Programming Analysis," funded in 1974 by the National Science Foundation. Drs. Everard Lofting, Esther Schroeder, Henry Ruderman, Jayant Sathaye, and Leonard Kunin made contributions to the early phase of this work.

1. U.S. Bureau of Economic Analysis, "The inputOutput Structure of the U.S. Economy- 1967", in Survey of Current Business, February 197', Vo1. 54, No. 2, pp. 24-56. 
2. D. Merrill, U.S. Employment for 368 InputOutput Sectors for 1963,1967 and 1972; Report UCID-3757; June 30, 1975; about 200 pages including appendices.

3. D. Merril1, Estimates of 1972 U.S. Employment for Use in Input/Output Applications, in preparation.
4. U.S. Bureau of Labor Statistics; Patterns of U.S. Economic Growth; BLS Bulletin No. 1672; published 1970 .

5. R. Bezdek, B. Hannon and S. Nakagama; Derivation of the 1963 and 1967 Total Employment Vector for 362 I/0 Sectors; CAC Document No. 63, Center for Advanced Computation, University of Illinois at Urbana-Champaign.

\author{
PROBABILISTIC AND STATISTICAL STUDIES \\ $\mathrm{OF}$ \\ DISEASE EFFECTS OF POLLUTANTS
}

\author{
J. Neyman and E. L. Scott*
}

The ultimate goal of this relatively new project is to contribute to the development of reliable statistical methodology for answering such questions as: Given a patterm of pollutants pre-existing in some specified locality, and given the additional pollution to be expected from an electrical power generating plant (either nuclear or conventional) contemplated for this locality, how many additional cancer cases, heart attacks, etc., are to be expected from normal functioning of the plant? Although definitive answers to such questions can be obtained only from a rather difficult multi-pollutant and multi-1ocality epidemiological study, requiring the cooperation of several government agencies, we hope to solve a number of preliminary relevant theoreticalstatistical problems.

\section{DETECTION OF SYNERGISTIC EFFECTS}

Synergistic effects (say, of radiation and some chemical agent) are very important from the practical point of view but rather difficult to handle theoretically. In fact, the statistical methodology universally used to evaluate experiments to discover synergisms is faulty to a remarkable extent. It was found in $1935^{1}$ that even when work is done with 1 s significant probabilities, erroneous conclusions may occur with frequencies of $30 \%$ in unfavorable conditions. Because of the practical importance of detecting synergisms, several individuals in our group - including $R$. Faith, R. Traxler, V. Joshi, and R. Bohrer - have made efforts to solve the problem. Yet the finding of an "optimal" procedure still lies in the future.

\section{COMPETING RISKS}

Two papers by A. Tsiatis ${ }^{2}$ and A. Peterson 3 have appeared from our Laboratory which suggest that the methodology used for handling the problem of competing risks is unreliable. Another paper by Tsiatis is forthcoming in which he i1lustrates how treacherous the traditional methodlogy may be-even though it continues to be used. in assessment of the problems was recently published by J. Neyman. 4

The proper approach to the problem of com- peting risks is through Markov chains, but unfortunately, there are difficulties. One is that the data must be from experiments with "serial sacrifices," which are expensive and cumbersome; another is connected with the necessarily large number of unknowns. A group of graduate students supervised by $\mathrm{Dr}$. Peter Clifford is now working to find an appropriate practical procedure, using data provided by Dr. John Storer of Oak Ridge National Laboratory.

\section{CARCINOGENESIS AND REPAIR}

The general direction of our efforts in the area of carcinogenesis and repair is indicated by the following problems:

- When mice are irradiated with ganma rays administered at a fixed dose-rate, the incidence of leukemia first increases as the dose is increased, reaches a maximum, then decreases; something of this sort also appears to be true with neutrons.

- The dose-rate shortening-of-1ife effect of irradiation with gamma rays is quite pronounced, but this is not true with neutrons.

Dr. Puri and one of us are working on a theory of these phenomena. The mechanism contemplated involves a combination of several stochastic processes, including clustering.

\section{ULTRAVIOLET RADIATION AND SKIN CANCER}

The subject of this study is the methodology of estimating the increase in skin cancer to be expected from an increasing intensity of ultraviolet radiation (due to depletion of stratospheric ozone). The usual method consists of estimating the relation between the probability of skin cancer at a given locality and the intensity of ultraviolet at the same locality. But both these quantities are subject to chance variation; therefore the least-squares estimate of their relations will be biased and the increase in skin cancer underestimated.

\section{CHROMOSOME ABERRATIONS}

We are also working toward a comprehensive 
stochastic model of observable chromosome aberrations in cells exposed to radiation and chemical agents. Although some such aberrations are relatively easy to observe directly, this is not necessarily the case with heritable mutations. Hopeful$1 y$, the model constructed to fit the data on aberrations will have implications for mutations that may be verified by genetic studies.

\section{FOOTNOTE AND REFERENCES}

*Associated with Statistical Laboratory, University of California, Berkeley

1. J. Neyman, "Complex Experiments. Contribution to the Discussion of the Paper by F. Yates,"
J. Roy. Statistical Society Supp1., 2 (1935), pp. 235-242. See also, A Seléction of Early Statistical Papers of $J$. Neyman, pp. 225-232. University of California Press, Berkeley, 1967.

2. Proceedings of the National Academy of Sciences, 72, January 1975, pp. 20-22.

3. Proceedings of the National Academy of Sciences, 73, January 1976, pp. 11-13.

4. J. Neyman, "Assessing the Chain: Energy Crisis Pollution and Health," International Statistical Review, 43, (1975), pp. 253-268. 


\title{
.2312 \\ Energy Use and Conservation
}

\author{
INTRODUCTION
}

Research in the Division's energy use and conservation program addresses a broad range of technical and institutional issues that influence the pattern of energy use. These include basic research into the physics of combustion processes, energy use in buildings and the investment behaviar of the electric utility industry.

The combustion research program has concentrated upon developing optical instrumentation techniques that can be used to study the fundamental nature of combustion and the formation of pollutants. Measurement techniques have been devised which use the scattering of laser light by combustion gases. These techniques are used to study the temperature of combustion, the concentration of nitrogen oxides, and the degree of catalytic reaction in a pre-mixed fuel-air stream.

The Division's work on energy use in buildings in 1975 was concentrated on a summer study held at the College of Environmental Design on the University of California campus. This study concentrated on the use of natural heat and light for thermal control and task illumination respectively. New designs which exploit solar energy in a passive rather than active mode allow for the conservation of fossil fuels and electricity. Among the techniques investigated were various approaches to window management, daylighting, and natural ventilation. Modeling energy flows in buildings with computer simulation was also investigated.

Research on the assessment of energy conservation policy alternatives was initiated along with a program to investigate specific conservation measures. The framework of this analysis emphasized the complex interrelationships between technical and institutional constraints on energy use. A procedure was developed for making short assessments of measures affecting industry, transportation, buildings, appliances, agriculture and energy supply systems. As part of this exercise, a model of investment behavior in the electric utility industry was developed to demonstrate the potential problems of uncoordinated energy supply and demand policies. The ultimate aim of our work is to aid in the formulation of energy policy that will address both the constraints on supply development as well as the efficiency of end use energy patterns.

\section{COMBUSTION RESEARCH}

\author{
F. Robben, Y. Agrowal, T. Hadeishi and R. MoLoughtin
}

\section{INTRODUCTION}

Combustion is probably the oldest and most important technological process supporting the modern industrial society. By far the largest percentage of energy needs are supplied by the combustion of fossil fuels, and this will continue into the next century. Fire was used by primitive societies to meet some of their energy needs, and there has been a steady progression of increased consumption of fuels as societies became more industrially, and presumably culturally, advanced.

The burning of fuels has also taken place in increasingly complex combustion systens, generally developed by skilled inventors and improved by a trial and error engineering process. Traditionally, there has been little contact between the practical engineering of combustion systems and fundamental research. In a way this is not surprising, since so much money and effort is spent on the practical engineering compared to what is spent on fundamental research. The basic research problems in combustion are not so simple that a few research projects can be expected to have any real impact on the practical engineering.
At present, it is recognized that considerable improvement in combustion systems is highly desirable. What is desired is more efficient combustion combined with lower air pollution, while at the same time burning dirtier fuels such as coal and high sulfur oil. The high technology approach to development, combining fundamental research with engineering, should progress much more efficiently towards these goals than the traditional engineering approach. A fairly complete engineering understanding of combustion processes is possible and can be used as the basis for development of combustion systems which are significantly improved over present standards.

The effort at LBL is directed partially at some advanced optical instrumentation techniques and partially at some fundamental studies of processes in premixed combustion systems. The instrumentation techniques are being developed primarily for our own laboratory research program but should be applicable and useful for other research purposes and perhaps for engineering development work. We have been concentrating on premixed combustion systems, partly because there is one less variable (degree of mixing) than in diffusion systems, and 
partly because there is considerable promise of high efficiency and low pollution from advanced systems of this type.1

\section{COMPARISON OF DENSITY AND TEMPERATURE MEASUREMENT USING RAMAN AND RAYLEIGH SCATTERING}

A theoretical comparison was made of the signal-to-noise ratio for Raman scattering measurements of species density and temperature. 2 These techniques depend on the scattering of laser light by the combustion gases and are capable of measurements in a very small volume within the flame zone itself without disturbing the combustion process. However, the scattering cross section is very small and the accuracy will often be limited by the small number of photons which can be collected.

The conclusions of these calculations can be summarized as follows. Starting with the highest signal-to-noise ratio, the measurement techniques can be ranked as 1) Rayleigh for total density, 2) Raman for $\mathrm{N}_{2}$ density, 3) Rayleigh for temperature, 4) Raman for temperature. Rayleigh density measurement, and to a lesser extent Raman $\mathrm{N}_{2}$ density measurement and Rayleigh temperature measurement have sufficient signal-to-noise ratio that direct measurement of nonsteady combustion on the millisecond time scale is feasible. It is also shown that the moments and correlations in steady turbulent combustion can be satisfactorily measured with good accuracy by time averaging techniques.

\section{TEMPERATURE AND DENSITY IN A HYDROGEN-AIR FLAME FROM RAYLEIGH SCATTERING}

A technique has been developed for obtaining spatially resolved measurements of temperature and density under combustion conditions. 3 The spectral structure of Rayleigh scattered laser light is determined using a Fabry-Perot interferometer. Spectra in the postcombustion zone exhibit simple Doppler broadening with a Gaussian line profile, however, spectra in the higher density, precombustion zone have appreciable Brillouin components. Radial and axial surveys of temperature and intensity in the premixed hydrogen-air flame issuing from a $3.2 \mathrm{~m}$ diameter welding torch tip are obtained. Measured temperatures in the postcombustion zone are in agreement with the adiabatic flame temperature. This technique, while rather complex, can be used with good precision in steady, particulate free flames. It may also be used for time resolved measurements in turbulent combustion under particulate free conditions.

\section{SPECTRAL STRUCTURE OF RAYLEIGH SCATTERED LIGHT}

The determination of the temperature of combustion gases from Rayleigh scattered laser light can be greatly simplified if the effect of Brillouin scattering on the Rayleigh spectra from nitrogen, oxygen, air, and argon have been made with a Fabry-Perot spectrometer. 4 These spectra were analyzed using a trimodal Gaussian model convoluted with the instrument function for the spectrometer. The results of this investigation indicate that if the value of parameter $y$, the ratio of the wavelength of the fluctuations producing the scattering divided by $2 \pi$ to the collision mean free path, is less than 0.1, Brillouin scattering is negligible. This criterion is shown to be satisfied in the post flame zone of an atmospheric pressure premixed hydrogen air flame.

\section{MEASUREMENT OF $\mathrm{NO}_{2}$ CONCENTRATION IN COMBUSTION} USING FLUORESCENCE EXCITED BY AN ARGON LASER

Preliminary results on the measurement of $\mathrm{NO}_{2}$ in a premixed hydrogen-air flame have been carried out. 5 The effective broadband fluorescence $(6700 \AA$ - 7500 A) cross sections for five laser lines were measured using $\mathrm{NO}_{2}$-air mixtures at atmospheric pressure and room temperature, giving values on the order of $10^{-25} \mathrm{~cm}^{2} / \mathrm{sr}$. Under these conditions the fluorescence intensity was linear with $\mathrm{NO}_{2}$ concentration up to $500 \mathrm{ppm}$. Measurements in heated mixtures up to $700^{\circ} \mathrm{K}$ indicated little change in fluorescence intensity. When measurements of $\mathrm{NO}_{2}$ in a 1.1 equivalence ratio (fuel rich) flame was attempted, no $\mathrm{NO}_{2}$ was found, within our estimated sensitivity limit of $20 \mathrm{ppm}$. Improved spatial discrimination and more extensive calibration should lower the sensitivity limit and help towards resolving the questions of existence and mechanisms for $\mathrm{NO}_{2}$ formation in combustion.

MEASUREMENT OF NO CONCENTRATION IN COMBUSTION USING RESONANCE ABSORPTION OF A ZEEMAN TUNED CADMIUM LAMP

This technique has been developed at LBL6 for trace concentration measurement of various atomic elements and promises to be a highly sensitive and selective method of determining NO concentration. In this case one of the Zeeman components of $2144.38 \AA$ Cd II line is shifted into resonance with the $K=13$ rotational transition of the $A^{2} \Sigma, 0-1$ vibrationa1 band of NO. The differential absorption of this Zeeman component with respect to the other component (which is not absorbed by NO) is measured using a lock-in technique. Preliminary measurements ${ }^{7}$ of absorption line shape as a function of magnetic field have been made with 0.5 torr NO, with and without 1 atm $\mathrm{N}_{2}$. Sensitivity of better than $1 \mathrm{ppm}$ is expected for combustion conditions.

\section{STUDY OF SURFACE CATALYZED COMBUSTION}

Work is just beginning on an experimental and computational study of combustion in the boundary layer of a catalytic flat plate in a premixed fue1air stream. 8 The velocity, gas density, and degree of reaction in the boundary layer will be measured using laser light scattering techniques. The computational study involves solution of the boundary layer equations with known homogeneous chemical reactions and modeled catalytic reactions at the surface. Comparison of experiment and computations should verify the analysis and give some parameters of the assumed catalytic reactions. The principal goals of this research are: 1) a fuller understanding of the relation between the catalyzed heterogeneous reactions at the wall and the homogeneous reactions in the boundary layer, 2) the development of a suit able computational model to enable these processes to be predicted in more complex geometry, and 3) the testing of suitable catalytic surfaces for combustion. 


\section{STRUCTURE OF A FLAME FRONT IN TURBULENT FLOW}

In this study, which is just beginning, a turbulent premixed fuel-air stream is created by use of a wire grid in the flow. A flame front is stabilized in this flow by a suitable flame holder, and the structure of the flame front is obtained by following the density with Rayleigh scattering and the velocity with a laser Doppler velocimeter. Good time and space resolution can be obtained, and statistical analysis will be used to obtain the probability density and power spectral density of the measured quantities.

The propagation of flame fronts in turbulent mixtures is a feature of many practical combustion systems, for instance in internal combustion engines. The present experiment represents a highly idealized situation, but this is exactly where one must begin if an improved fundamental understanding of the details of the effects of fluid mechanical turbulence on flame front propagation is to be obtained. Assuming that the experiment behaves as planned, several refinements as well as comparison with various theories can be used to gain valuable insight into this important problem.

\section{REFERENCES}

1. A.K. Oppenheim and F.J. Weinberg, "Combustion R and D : Key to our Energy Future," Astronautics and Aeronautics, (November, 1974) p. 22.

2. F. Robben, "Comparison of Density and Temperature Measurement Using Raman Scattering and Rayleigh Scattering," Proceedings of Workshop on Combustion Measurements in Jet Propulsion Systems, Purdue University, May 22-23, Ed. by R. Goulard, sponsored by Project Squid (1975).
3. R. Pitz, R. Cattolica, F. Robben, and L. Talbot, "Temperature and Density in a Hydrogen-Air Flame from Rayleigh Scattering," Combustion and Flame, 27, n. 3, p. 313-20 (1976).

4. R. Cattolica, F. Robben and L. Talbot, "The Interpretation of the Spectral Structure of Rayleigh Scattered Light from Combustion gases," AIAA preprint 76-31, presented at 14th Aerospace Sciences Meeting, January 26-30, Washington, D.C.; published in Progress in Astronautics and Aeronautics 39, volume on Measurements in Combustion Research (1976).

5. Y. Agrawal, T. Hadeishi, and F. Robben, "Measurement of $\mathrm{NO}_{2}$ Concentration in Combustion Using Fluorescence Excited by an ArgonIon Laser," AIAA preprint 76-136, presented at 14th Aerospace Sciences Meeting, January 26-30, Washington, D.C. ; published in Progress in Astronautics and Aeronautics 39 , volume on Measurements in Combustion Research (1976).

6. T. Hadeishi, Applied Physics Letters 21, 9 (1972) pp. 438-440.

7. T. Hadeishi, Y. Agrawal, R. McLaughlin and F. Robben, "Resonance Absorption Line Shape of NO using a Zeeman Tuned Cd 1amp: Application to Combustion," unpublished report, Lawrence Berkeley Laboratory (1976), LBL -5213 .

8. F. Robben, Y. Agrawal and R. Schefer, "A Study of Surface Catalyzed Combustion," summary of presentation at EPA Workshop on Catalytic Combustion, Raleigh, N.C., May $25-26,1976$.

ENERGY AND BUILDINGS-A REPORT ON THE 1975 BERKELEY SUMMER STUDY

E. T. Dean and A. H. Rosenfeld

Doing more with less may be the wave of the future in architecture and building design. In particular the conservation of depleting sources of energy will be emphasized along with maintaining comfortable and livable environments within buildings. The subject is so new that very little is really known, in a predictable and quantifiable way, of the actual effects on energy use of the various elements of building design.

The result has been a major effort on the part of the federal government in the area of building energy research, and the development of building research programs at the national laboratories to support that effort.

To launch the program at IBL, a Summer Study on the Efficient Use of Energy in Buildings was held at the College of Environmental Design on the University of California campus. Sponsored by $L B L$, the purpose of the study was to bring to- gether designers and researchers from the building profession, universities and government agencies for an intensive examination of the problems of improved efficiencies of energy use for the heating and cooling of buildings. The focus of the Study was the development of an understanding of the maximm potential and practical difficulties for the use of natural heat and light in what has become known as the "passive mode". Consequently much of the work centered on window systems, daylighting and ventilation.

Some 25 architects, engineers and scientists met during the four weeks starting Jume 23, 1975, in a regular schedule of seminars on the various topics and lengthy group-task research sessions. The product of the four-week Study is a report 1 containing the results of the work carried out by the participants, some highlights of which will be discussed here. 
UNDERSTANDING THE NEED

The motivation for this Study was the recognition that buildings in general are not designed, constructed or operated well from the point of view of energy use. The appropriate strategies for maximum energy efficiency are not well understood. An idea of the magnitude of the building energyuse problem can be obtained from Table 1, which shows a breakdown of energy consumption for space heating and lighting as a component of the total U.S. energy use (77 quads, or $77 \times 10^{15} \mathrm{Btu}$, in 1974). All buildings account for one-third of this total at the present time.

\section{UNDERSTANDING THE PROBLEM: COMMRCIAL}

Table 1 also gives some indication of the strategies to be adopted. Note that of the total amount of primary energy used by conmercial buildings, more than 438 goes into lighting. The primary energy consumption per square foot is about the same for heating residential and commercial buildings. It is clear therefore that the energy problem for commercial buildings is one of electrical power generation, primarily for lighting and secondarily for airconditioning.

1. Lighting. For large commercial buildings (comprising 2 billion square feet of the 24 billion square feet of commercial space), lighting is the major contributor to the large internal loads that essentially heat the building core in winter. Electrical heating in any form is the most energy wasteful because of the 3-to-1 ratio of energy consumed at the power plant to the electrical enerby delivered to the building, so any improved efficiencies in lighting are desirable. During the summer these same high internal loads create a constant demand for airconditioning. Consequently, during the Study some attention was paid to the problems and opportunities of daylighting.

One fact that was noted, for instance, is that sunlight, if distributed uniformly, contains enough lumens (a measure of light intensity) in

Table 1. National Statistics: Building energy use in U.S., $1973^{\mathrm{a}}$

\begin{tabular}{|c|c|c|c|c|c|c|c|}
\hline \multirow[b]{2}{*}{$\begin{array}{l}\text { Building } \\
\text { Category }\end{array}$} & \multirow[b]{2}{*}{$\begin{array}{c}\text { F1oor Space } \\
(\text { sq.ft.) }\end{array}$} & \multicolumn{3}{|c|}{ Heating } & \multicolumn{3}{|c|}{ Lighting $^{b}$} \\
\hline & & $\begin{array}{l}\text { Heating Rate- } \\
\text { End Use } \\
\text { (therms/sq.ft. } \\
\text { per year) }\end{array}$ & $\begin{array}{l}\text { Total Energy } \\
\text { for Heating } \\
\text { (Quads - } \\
\text { primary) }\end{array}$ & $\begin{array}{l}\text { Fraction of } \\
\text { U.S. Total } \\
\text { (77 Quads) }\end{array}$ & $\begin{array}{l}\text { Lighting Rate- } \\
\text { End Use } \\
\text { (Kwh/sq.ft. } \\
\text { per year) }\end{array}$ & $\begin{array}{l}\text { Total Energy } \\
\text { for Lighting } \\
\text { (Quads - } \\
\text { primary)c }\end{array}$ & $\begin{array}{l}\text { Fraction of } \\
\text { U.S. Total } \\
\text { (77 Quads) }\end{array}$ \\
\hline $\begin{array}{l}\text { Residential } \\
\text { Only }\end{array}$ & 70 million & 1.1 & $10.8^{\mathrm{d}}$ & 148 & 1.3 & 0.9 & 1.28 \\
\hline $\begin{array}{c}\text { Commercial } \\
\text { only }\end{array}$ & 24 billion & 1.3 & $3.1^{\mathrm{e}}$ & 48 & 8.0 & 2.4 & 3.18 \\
\hline $\begin{array}{l}\text { Residential } \\
\text { and } \\
\text { Commercial } \\
\text { Total }\end{array}$ & & & 13.9 & $18 \%$ & & 3.3 & 4.38 \\
\hline
\end{tabular}

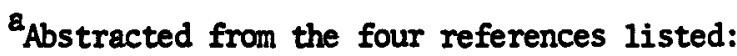

1. Project Independence Task Force Report, Federal Energy Agency, Vo1. 1, (Nov. 1974).

2. The Efficient Use of Energy - A Physics Perspective, American Institute of Physics Conference Proceedings, Vo1. 25, or NTIS Report PB 242-773 or 242-116 (1974).

3. Energy Conservation Applied to Office Lighting, Ross and Baruzzini, FEA Contract 14-01-0001-1845 (1975).

4. Energy Conservation in Public and Commercial Buildings, R. Salter and D. Morris, kand Report P-5093, Santa Monica, California 90406 (1973).

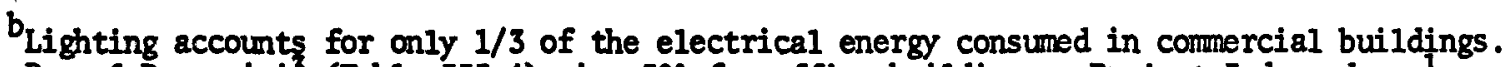
Ross \& Baruzzini ${ }^{3}$ (Table III-4) give $30 \%$ for office buildings. Project Independence 1

(Table A.5) gives 348. For residential, Ross \& Baruzzini use 16\%, while ceneral Electric Company reports that lighting uses 148 of the electricity consumed in houses.

Cased on 12,415 Btu/Kwh ; that is, 10,500 Btu for each Kwhe generated plus $1915 \mathrm{Btu} / \mathrm{Kwh}$ e for electrical distribution losses.

Based on 7 million electrically heated houses, using a heat factor of 3.4 for primary energy/Kwhe.

$e_{\text {Assumes electrical heating of comnercial buildings is negligible. }}$ 
a single square foot area to provide fifty footcandles (a measure of illumination) over an area of 180 square feet. (Standard lighting levels now recommended for office tasks are 50-75 footcandles). Unfortunately, an enormous amount of heat $(300 \mathrm{Btu} / \mathrm{hr}-\mathrm{sq} \mathrm{ft})$ accompanies this light, most of which must be removed to maintain a comfortable thermal environment. Artificial illumination, however, also produces large amounts of heat, and the "efficacy" (lumens/watt) is less than for natural daylight. Daylight produces about 100 lumens/watt as compared with neariy 70 lumens/watt for a new 48-inch flourescent bulb and ballast, or only 18 lumens/watt for incandescent bulbs.

, The strategy for direct natural illumination in the typical office buildings with reduced thermal loads, (either heating or cooling), therefore, is to minimize the intensity of direct sunlight while at the same time diffusing the direct beam uniformly throughout the space. One possible scheme is suggested in Fig. 1. It was estimated in terms of gross figures on a national scale, that peak electrical power demand can be reduced by 3 Gigawatts if direct beam daylighting were utilized in $20 \%$ of existing commercial floor space.

\section{South Facing Wall (schematic section)}

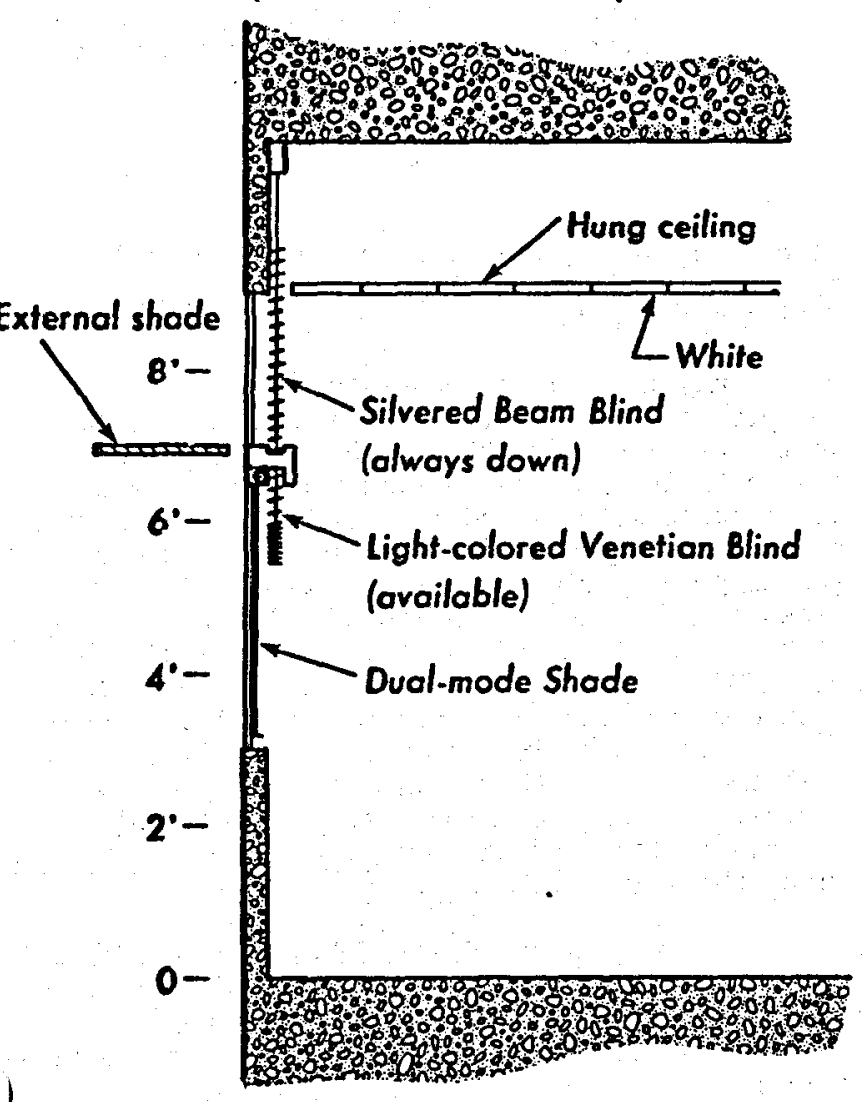

Fig. 1. Cross section of typical office space (south perimeter location) showing 10cation and schematic operation of beam daylight apparatus.
2. Ventilation. An overview of the ASHRAE literature and current building practice indicated that there is an unnecessary waste of about $15 \%$ of the total space heat consumption for office buildings and schools because of excess ventilation. A ventilation rate of 7-1/2 cfm of fresh air per person was not found to be perceptibly different for both comfort and odor control than the typical building specifications of $15 \mathrm{cfm}$ per person. Present standards are based on the inaccurate and energy wasteful idea that ventilation flow rate for odor control must increase as air space per occupant decreases.

In addition to the problem of outside air requirements during the winter heating season, we explored the question of utilizing natural ventilation to the maximum extent possible during the cooling season. 2 The purpose again, however, is not so much to reduce overall energy use during the summer, but to reduce peak electrical power demand. In fact, the rule for energy use in buildings that eventually evolved during the Study was "In winter, think energy; in summer, think peak-power." The major gains from natural ventilation occur for climates where the intermediate season is substantially long. During this season the windows can be opened to compensate for solar gains and no mechanical ventilation or cooling is used in the peripheral areas.

\section{UNDERSTANDING THE PROBLEM: RESIDENTIAL}

As Table 1 indicates, the primary problem for residential buildings is one of gross energy use for heating. With the movement toward new energy performance standards and proscriptive building codes, the Surmer Study focused in part on some of the aspects of residences that contribute to the heating problem. A number of tasks were undertaken.

A model for natural energy flow in houses was formulated using elements of the NBSLD load program and some algorithms for the building structure and operation. The model was used to evaluate the effects of the proposed code limitations on window size. Figures 2 and 3 show the effects on annual energy consumption for the model house of varying the amount of glass on south and west elevations, respectively. Increasing south-facing glass area was found to have little effect on annual energy use in most climates, thereby indicating that the codes are unnecessarily restrictive in this regard. However, there doesn't appear to be any inherent advantage to adding south-glass, contrary to popular belief, for the typical American house. Increasing west-facing (and east-facing) glass does mean an increase in energy consumption, as the figure shows. Restricting non-south-facing glass area, therefore, is an effective energy-conserving strategy.

As part of the follow-up research from the Summer Study results, we have begun an investigation of modifications or new ideas in the design and construction of houses that lead to an increased "energy-value" of windows. Such new designs have been generally categorized under the name of "passive solar" design, and in this sense the studies in energy conservation overlap those in solar energy. 

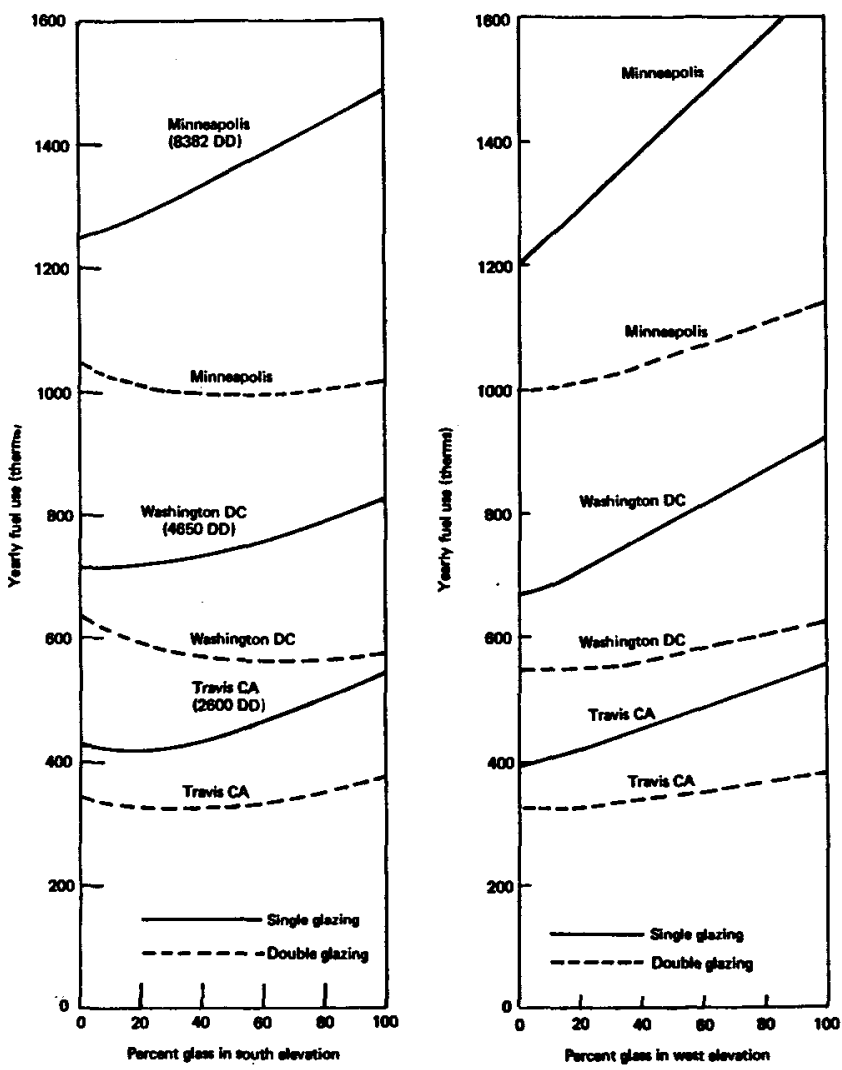

Fig. 2. Detached residence Fig. 3. Detached residence fuel use for sample house envelope in three climates, with moderate temperature setback, for variations in south glazing.

(XBL 7512-10093)

fuel use for sample house envelope in three climates, with moderate temperature setback, for variations in west glazing.

(XBL 7512-10090)

The Summer Study work on modeling typical houses assumed no special participation of the user in the energy management of the dwelling. Another task undertaken during the Study was to examine the effect of window management (by means of shades, blinds, insulating shutters, etc.). It was found, of course, that such management results in substantial savings during the heating season, and that a well-managed single-glazed window has the same energy performance as a doubleglazed window. The management device, however, will not be cost effective unless it is a simple manual device. Automated systems are much more expensive than the cost of the fuel saved.

Another study undertaken during the summer was an investigation of the various metallic films used in solar control retrofit applications. Figure 4 shows a device that was proposed as an energy-conserving window management system, using a particular type of this material. The results of some numerical modeling of this device show that this "dual shade"1) will be approximately $90 \%$ as energy efficient as an unshaded window while intercepting direct sunlight, which is considered undesirable because of glare or fading. The shade will 2) save $40 \%$ on energy losses through a single pane window during the winter

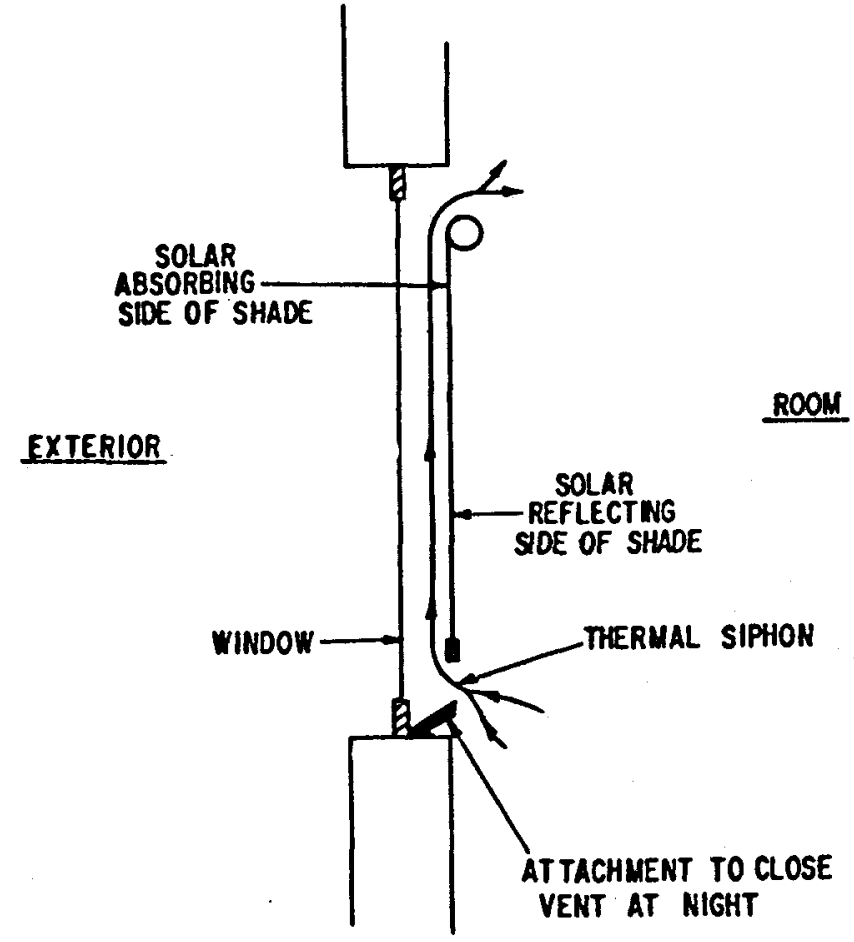

Fig. 4. Heating season daytime configuration of dual shade. At nighttime the shade is attached at the bottom to close lower vent. During the summer the shade is reversed so solar reflecting surface faces the exterior.

nighttime, and 3) will reduce solar heat gain by about $70 \%$ during peak-power summer air conditioning periods:

A major effort to understand and deal with the problem of predicting building energy requirements was also undertaken. The advantages and drawbacks in practical office situations of both approximate methods and the more precise computer methods were discussed. The widespread use of the computer for these calculations will be necessary for implementation of a National Energy Conservation Standard for building design. The new ASHRAE Standard 90-75 essentially requires such computer methods. Several proprietary programs exist, but give no option of dealing with building systems other than standard types, are expensive to use (no simplifying default options are available), and have outputs that do not agree among themselves (see Fig. 5).

Since the Summer Study, LBL has been funded to develop public-domain software for the analysis of energy consumption in buildings, with built-in options for simplified calculations, system optimization routines based on system components rather than whole system-types. This major research effort is the result of one of the principal proposed goals of the Summer Study, namely to pinpoint major areas of work that could be undertaken at LBL and to develop proposals for such research. 


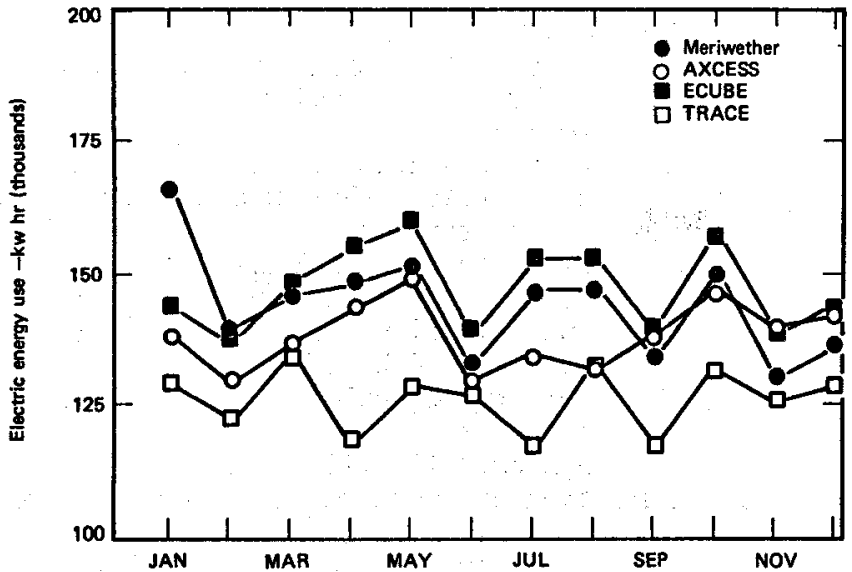

REFERENCE

1. The Summer Study Proceedings, LBL-4411, are available as a special issue of the new journal "Energy and Buildings," Vol. 1, No. $1,1977$.

2. Air-conditioned buildings need almost $50 \%$ more primary energy than naturally ventilated buildings.

Fig. 5. Electrical consumption of Building Mode1 1 as computed by four proprietary energy consumption computer programs.

(XBL 7512-10156)

\title{
ENERGY CONSERVATION ASSESSMENT
}

\author{
S. M. Berman, C. Blumstein, P. Caesar,
}

M. Davidson, E. P. Kahn, and A. Makhijani

\section{INTRODUCTION}

In September of 1975 ERDA's Division of Interprogram Applications, under the Assistant Administrator for Conservation, initiated a program for the analysis of policy alternatives in energy conservation. The seven ERDA general purpose National Laboratories participated in the program, which was coordinated by LBL. Among the topics examined by the program were objectives for a national energy conservation policy, the role of the federal government in energy conservation, and objectives for ERDA's energy conservation $R \& D$ efforts. In addition, a procedure was developed for making short assessments of specific energy conservation measures and a number of such assessments were initiated.

\section{OBJECTIVES OF ENERGY CONSERVATION}

When the Energy Conservation Assessment project was initiated, it was felt that a better understanding of the objectives of an energy conservation program would be essential in the analysis of policy alternatives. For this reason, a significant part of our effort in the early phase of this project was devoted to an analysis of the ends which could be achieved by an effective energy conservation program. A list of important objectives for energy conservation which emerged from our analysis is given below.

\section{Fulfillment of High Priority National Goals}

Energy conservaton will contribute to decreasing our dependence on foreign suppliers of energy resources.

Energy conservation, by reducing demand, will decrease the likelihood of energy shortages and the economic disruption they produce.
Energy conservation could help the oil importing developing nations by reducing those upward pressures on oil prices that are caused by increasing oil demand in the developed countries.

\section{Expansion of Energy Supply Options}

Energy conservation will enable us to conserve our supply of high quality, but non-renewable, fossil fuels for a longer period of time.

Energy conservation will help provide the lead time necessary for the development of additional energy sources.

Energy conservation in buildings and buildingrelated end-uses increases the economic feasibility of the currently available systems for directly utilizing the energy of the sum and wind.

Energy conservation increases the economic and technical feasibility of local satisfaction of local energy requirements.

\section{Reduction of Environmental Impacts}

Energy conservation, by reducing energy consumption per unit of goods and services, will reduce the pollution and other environmental costs associated with the production and use of fuels and electricity.

Energy conservation, through recycling and waste utilization, will reduce waste disposal problems and their attendant environmental costs.

Energy conservation contributes to more equitable regional development. 
Strengthening our Economy

Energy conservation is, in many instances, a more productive use of our capital resources than a corresponding investment in energy supply.

Energy conservation will reduce the lifecycle cost of many goods and services.

Energy conservation can, in many instances, result in increased employment.

Energy conservation can increase the accessibility to lower income people of many necessary resources.

\section{Preservation of Policy Choices}

By facilitating the achievement of the objectives listed above, energy conservation will help preserve energy policy choices for current and future consideration.

ANALYTICAL FRAMEWORK FOR THE ANALYSIS OF SPECIFIC ENERGY OONSERVATION MEASURES

While the broad objectives set the direction, an energy conservation program must be built from specific measures. There are a large number of possible measures from which a selection must be made. Therefore, we together with the other participating laboratories undertook to develop a systematic framework in which energy conservation measures could be analysed in order to establish priorities. This framework is given below in outline form.

I. TITLE (should identify measure and affected subsector clearly)

II. RECOMMENDATIONS (as briefly as possible should indicate what, if any, actions would be useful - and at what funding levels and time scale)

III. SUMMARY (should summarize data in IV below in such a manner as to document recommendations in II above)

IV. DATA

A. Savings (efficiency improvement and reduction of petroleum imports)

1. Subsector size

a. Present

b. Projections

2. Maximum theoretical savings

3. Restrictions and other considerations

4. Alternative strategies that affect this subsector

B. Technical assessment (of one or several technologies or policies)

1. Savings possible with present technologies or policies

2. Savings possible from future technology or policy
3. Cost of $R \& D$

4. Cost of necessary demonstrations

5. Technical risk and associated problem areas

6. Current status of research

a. Present ERDA programs and their locations

b. Other federal and state programs

c. Private industry

7. Justification for federal involvement in $R$ \& $D$ efforts

8. Foreign technology and technology transfers

9. System reliability

C. Marketing and commercialization (barriers to adoption)

1. Profitability and marketability

2. Capital requirements

3. Financing modes

4. Industrial resistance

5. Implementation time

6. Implementation cost

D. Social and economic impacts

1. Employment-size and distribution

2. "Quality of 1ife"

a. Consumer (housing and living patterns, urbanization, etc.)

b. Industry (quality, nature and location of employment, etc.)

3. Resource requirements

4. Foreign trade

5. Regional factors

E. Environment, health and safety

1. Health and safety

a. Consumer

b. Industry.

2. Environmental impacts

F. Public policy issues

1. Institutional and organizational constraints

2. Subsidies and tax policy

3. Regulation and legislation

4. Public perception and acceptance

5. Alteration of social and economic patterns

6. Anticipated implementation difficulties (other than direct costs)

G. Program recommendation (one for each recommendation for technology or policy)

1. Type of program (systems analysis, RED, demonstration, or technology utilization)

2. Scope, size, degree of federal involvement

3. Problems

4. Time scale

5. Participation

6. Estimate of probability of success

Using this framework, a number of analyses of specific measures have been initiated by LBL and the other participating laboratories. Energy conservation measures affecting industry, transportation, buildings, appliances, agriculture, and energy supply systems are being examined. 
E. P. Kahn, M. Davidson, A Makhijani, P. Caesar, and $S$. M. Berman

As a part of the Division's Energy Conservation Assessment Program, we initiated a study of the role that investments play in influencing energy consumption. Any investment plan is based on a forecast of future demand for the cormodity in question. We examine the possibility that in the area of electricity generation, forecasts may tend to be selffulfilling. One reason for this is the amount of capital involved in utility expansion plans. This is so large that allocation of funds for rapid growth in capacity will tend to foreclose the possibility of alternate energy strategies. If, moreover, electricity demand does not reach the forecasted levels, financial instability may result for the electric utility industry. In this scenario we may end up with a large investment in power plants that are not utilized to their capacity.

To examine the electric utility industry in detail we construct a simple mathematical model that incorporates its basic features. Our model consists of two equations, one for electricity demand and one for its average cost. The demand equation (1) states that the quantity of electricity consumed in a region is a function of the average cost of electricity (R) and the gross product (G) of the region. The influence of these independent variables is determined by a price elasticity $\varepsilon$ and an income elasticity $\alpha$.

$$
D=\delta R^{\varepsilon} G^{\alpha}, \quad \delta=\text { constant }
$$

Our expression for average cost is an identity that derives from balance sheet information. It says that total revenues (which is demand $D$ times average cost $R$ ) are the sum of two terms, one which is proportional to demand, (namely the variable costs, especially fuel) and fixed capital costs B.

$$
\begin{aligned}
& \mathrm{DR}=\mathrm{A}(\mathrm{D})+\mathrm{B} \\
& \mathrm{R}=\mathrm{A}+\mathrm{B} / \mathrm{D}
\end{aligned}
$$

The final feature that we incorporate in our model is lag time. The demand adjustments which Eq. (1) models do not occur instantaneously. To incorporate the lag time we write a first order linear differential to express the changes in demand due to the level of average rates and gross regional product.

$$
\frac{d D}{d t}=-K\left(D-\delta G^{\alpha} R^{\varepsilon}\right), K>0
$$

We specify the mode1 (2a) - (3) by values of the parameters $\alpha, \varepsilon, \delta$ and $K$. For simplicity we choose $\alpha=1, \varepsilon=-1$ which are close to the mean values for these parameters in other utility models. The parameters $\mathrm{K}$ and $\delta$ are fitted to historical data for the particular systems studied. $\delta$ is a parameter measuring the technology of a region, roughly it converts unit outputs in dollars into demand for electricity. $K$ is a lag time parameter, $1 / \mathrm{K}$ being the time required for demand to adjust to a new rate.

With our specification for $\alpha$ and $\varepsilon$ we study different systems, the Pacific Gas and Electric Company, the aggregate of all private utilities in the nation, and the aggregate of all utilities, public and private. We use Eq. (3) to determine $\mathrm{K}$ and $\delta$. The rate model (2a) is specified with financial data from FPC statistics. In both demand and rate equations the fit with historical data is good.

Having verified our model with historical data, we then study the effect of various investment schedules and federal tax policies on all private utilities. Large investments in new capacity increase the utilities' rate base upon which their prices are determined. This can have the effect of lowering demand through price elasticity. The investment tax credit that utilities receive mitigates the price increase due to new capacity. We study these variables under different GNP scenarios. The results are presented in Table 1. We offer two investment plans. The high investment program is based on the Electrical World 26th Annual Forecast, which projects 309 gigawatts of new capacity in the period 1975-85. As an alternative we analyze a cutback construction schedule of 129 gigawatts in the same period.

Several conclusions may be drawn from Table 1. First, economic growth does not necessarily imply

Table 1. Percent annual growth for various scenarios with three assumed rates of growth in real GNP.

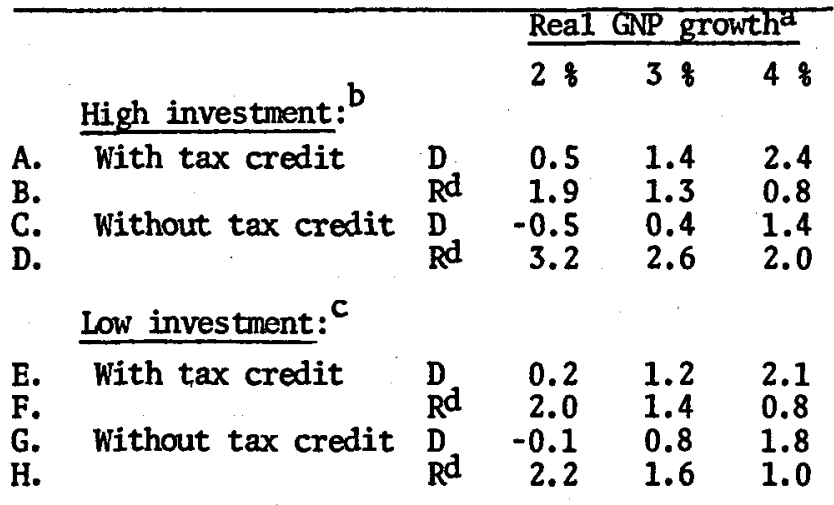

a. Annual percent growth in real GNP assumed in the model.

b. Based on Electrical World construction schedule.

c. Based on cutback construction schedule.

d. Percent increases are for real rates. 
increased energy consumption. Even though GNP may grow at a healthy rate in the future, electricity demand may not grow at historical rates of 6 to 7 annually. The reason for this is increasing fuel and capital costs. Roughly, the model suggests that the rate of increase in demand is equal to the rate of increase of GNP minus the rate of increase of average rates. Thus, a growth in GNP can be canceled by a simultaneous growth in rates, yielding a demand which does not grow, or does not grow as fast as GNP.

An examination of Table 1 shows that tax policy is a major factor in investment planning. The investment tax credit and accelerated depreciation allowance give a stimulus to investment. Tax policy also effects the demand for electricity. The tax credit subsidizes the production of power, resulting in lower rates to the user in the high investment case. These lower rates stimulate demand. By comparing lines A and E of Table 1, we see that demand grows $.3 \%$ faster for high investment. When the tax credit is removed, however, the situation reverses. Without the tax credit the direct user, rather than the indirect taxpayer, bears the cost of potential overcapacity. If high investment occurred without the tax credit, then demand would be reduced relative to a cutback construction schedule. By comparing lines C and G of Table 1 , we see that demand in the cutback case increases about .48 faster than the high investment case, and rates increase about $1 \%$ slower. Finally reduction or removal of the tax credit would favor a cutback construction schedule and would reduce electricity demand. Within a given investment plan, it should be noted, the tax credit increases demand. For the high investment case this is a 18 effect (compare A with $C$ in
Table 1). The effect on demand is smaller for a lower investment program (compare $\mathrm{E}$ with $\mathrm{G}$ ).

The type of model we have constructed neglects shock effects in the energy supply sector. In particular, the impact of natural gas curtailments will tend to increase the demand for electricity. We estimate the size of this effect in the residential and industrial sectors, factoring in conservation potentials as well. The influence of a trend toward industrial power generation is alşo estimated. Rising energy prices will tend to encourage industrial users to lower their demand for utility produced electricity in favor of generating their own power on the industrial site. While all of these factors are subject to a good deal of uncertainty, we conclude that the increases in electricity demand due to a gas shortage will be canceled by the decline in the industrial electricity demand from the utilities.

This work has several natural extensions. Regional variations are significant. Applying (2a) and (3) to different regions will yield different estimates of the lagtime parameter $K$ and technology parameter $\delta$. Our Table 1 only presents analysis of a single tax policy, the in vestment tax credit. In our projections we treat all new generating capacity uniformly. In fact, the financial impact of differing supply technologies varies. Nuclear fuel, for example, is treated as a capital cost. Pollution control equipment is subject to special tax rules. The effect of various mixes of generating equipment can be studied in our framework.

FOOTNOTE

*Condensed from LBL-4474 


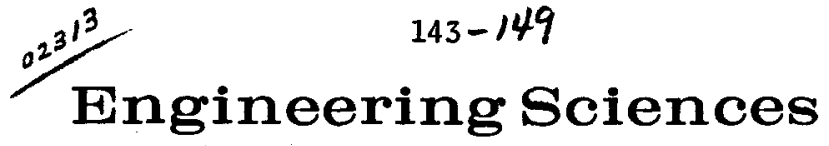

\author{
INTRODUCTION
}

The Engineering Sciences Program focuses specialized engineering resources from both the Lawrence Berkeley Laboratory and the University of California campus onto certain energy and environment-related problems. At present, emphasis is placed on problems in two areas - transportation and earthquake studies.

The Transportation Studies area includes two projects that are currently underway. The first of these, Mass Transit Analysis, is concerned primarily with studies of the Bay Area Rapid Transit District (BART) equipment and operations. These studies are to provide an independent appraisal of the operational capability, reliability and safety of the transit system. Certain critical areas have been identified. Task forces have been organized to study these areas, make recommendations, and, in some cases, participate in the design of improved components and facilities.

The second transportation project is the Roadway Power System for Full-Performance Electric Cars. In this project, a method is being investigated to safely, efficiently and economically transfer power to personal automobiles while they are traveling on highways. The purpose is to make the use of electric vehicles a practical reality, and to aid in reducing reliance on gasoline powered vehicles.
The Earthquake Studies area also has two projects. The first is concerned with a longstanding problem, namely, how to predict the occurrence of earthquakes. In this project, a potential method for predicting earthquakes through electrical resistivity methods is being investigated. Field experiments in the Hollister area are being set up to study the feasibility of the method.

In the second earthquake project, the design techniques used to provide the earthquake resistance of components for nuclear power plants are being studied through theoretical analysis and through experiment using the Earthquake Simulator at the UC Earthquake Engineering Research Center. The object is to inprove the state-of-the-art of these design techniques. Such improvements will facilitate the process of evaluating the seismic qualities of proposed power plant structures while they are still in the design stage.

These projects are individually described in more detail in the following sections: (1) Mass Transit Analysis, (2) Roadway Power System for Ful1Performance Electric Cars, (3) Earthquake Prediction, and (4) Earthquake Reactor Safety Research.

\title{
MASS TRANSIT ANALYSIS*
}

D. Theodore Scalise, AlZan A. Arthur, David Bereznai, J.G. Bolger, V.P. Elischer, D.M. Evans, Martin Graham, J. Gunn, Raymond Louis, Frank Olken, A. Roberts, David B. Turner, Kenneth G. Wiley

\section{INTRODUCTION}

The goals of the LBL Rapid Transit Study Program are to make an independent evaluation of the Automatic Train Control (ATC) systems currently in use and to engage in the research and design of such systems in order to improve their effectiveness, safety, and reliability.

LBL's association with the rapid transit industry began in 1974 when the California Public Utilities Commission (PUC) and the Bay Area Rapid Transit District (BART) came to an impasse over certification to begin full operation. The Chairman of the State Senate Committee on Public Utilities, Transit and Energy (Senate Committee) asked LBL to give technical advice on BART's readiness to begin safe service and on the economic impact of the safety problems. The BART District also asked LBL for technical help to improve the reliability of BART's ATC system.
Since then LBL has made important contributions to the increased safety level of the BART system and has been formally acknowledged by the BART Board of Directors for its role in aiding BART to achieve successful. Trans-Bay service.

LBL has also contributed to the BART system in perhaps an even more significant, but umexpected way, in that the Laboratory has identified many incipient problems that might otherwise have gone unaddressed with possibly serious consequences. An example is the identification of the lower-thandesigned-for braking rates in trains and the resultant changes required in speed codes in the track circuits throughout the system. Another example is the early identification of the requirement for extensive modification (or replacement) of the Central Computer system before the design level of revenue-service can be achieved. 


\section{ANNUAL ACTIVITIES}

The planned LBL activities for the year are described in agreements with the BART District and with the Senate Committee. In the IBL/BART agreement, two principal areas of work are defined: Equipment Reliability Improvements and Long-Range Computer System Improvements. The first area required $\mathrm{LBL}$ assistance for:

- the determination of theoretical failure modes,

- the development of special test instrumentation and performance of the necessary dynamic testing to experimentally identify equipment failure modes, and

- the formulation of feasible engineering prototype solutions.

The second area of the BART agreement provided for LBL assistance to investigate and formulate longterm plans for more effective utilization of BART's thirteen independent computer systems. In the LBL/ Senate Committee agreement, LBL is charged in part with the evaluation and monitoring of the verification process for the BART Sequential Occupancy Release (SOR) backup system to the ATC system.

During 1975 in pursuance of the above agreements, LBL recommended that task forces be established to study the reliability problems in three areas: BART Vehicles, Central Control Computer System, and Wayside and Local Station Equipment. Accordingly, these task forces were organized and consisted of BART, LBL, and PUC engineers and computer scientists. They were to assume a rigorous diagnostic approach, to develop instrumentation for identifying the root causes of the problems, and to seek the solution of the identified problems. The first three activities described below cover those of the reliability task forces; the last two cover other areas.

Vehicle Reliability. The vehicle reliability group studied problems in traction motors, braking systems and train control electronics. LBL helped identify causes for friction brake failures, catastrophic traction motor flashovers and $10 \mathrm{w}$ reliability in control electronics. For this project, extensive instrumentation was assembled aboard a test train. As a result of the tests, numerous prototype test modifications were made on certain vehicles. These are now undergoing reliability improvement evaluation.

Central Computer Reliability. This effort was concerned with the reliability of the Central Computer system and the problem of restoring normal operation quickly after a failure. Both software and hardware modifications were developed to improve the operations. While measuring performance, instruments and techniques were developed for evaluating the system's ability to schedule and control a greater number of revenue trains than are presently in operation. Consequently, the modifications required to achieve full service are now recognized.
Wayside and Local Station Reliability. This effort was designed to identify unreliable elements in the train control system that are situated at fixed locations throughout the BART system. The causes of a number of incidents of both false and lost occupancy conditions in the primary train detection system were determined.

Long Term Computer Systems Investigation. This task was to identify the reasons for the limited success in integrating computers into the overall train control system. Each of the 13 computer systems was analyzed for individual support requirements, interdependency on each of the other systems, and effectiveness in performing its functions. This effort has led to an understanding of the problems faced by the District in reaching various service level goals. A plan has been presented to BART which discusses the present deficiencies and improvement needed to achieve an improved, more cost-effective, maintainable and integrated computer network.

Safety-Oriented Senate Task. A permanent backup addition to the primary train detection system was designed in 1974 and was implemented during 1975. LBL's role has been to monitor the implementation of this backup system, which is called the Sequential Occupancy Release System (SOR). In this role, LBL has made recommendations to BART, the PUC and the Senate Committee both to insure a high-integrity final product and to provide techniques and criteria for testing the system. In this context, LBL introduced Failure-Mode-Analyses to circumvent the consequences and time delays normally required in the transit industry to collect failure data from actual operating experience. The failure-modes identified in the analyses were used in conjunction with Integrity Tests for determining the effectiveness of the SOR system - a system essential for achieving increased revenue service.

In addition to the above planned activities, a number of unplanned activities were undertaken in response to changing BART requirements during the year. These unplanned activities required the redirection of the available LBL effort. Two such activities are described below.

Maintenance Vehicle Detection System. At the request of the Senate Committee, a substantial portion of the LBL electronics engineering effort was diverted to the design and development of an automatic system for detecting the presence of maintenance vehicles on the BART tracks. This effort resulted in the production of a prototype, which demonstrated the feasibility of such a detection system. Such detection systems do not exist in other transit districts or in other railroads.

PUC Hearings. Another unplanned activity came from a PUC request for the loan of $\mathrm{LBL}$ services to advise the PUC commissioners on certain safety practices at BART. As a result, LBL alerted them to the significance of manual interventions in the operation of an automatic system. The vulnerability of such manual operations to human error can lead to unsafe conditions for which the automatic system provided no protection. LBL also recormended a rigorous approach to the preparation of operating 
rules and procedures to reduce failure-modes of the uman operators and provide an appreciably higher ofety leve1. Suggestions regarding organizational and operational changes were also made.

\section{FUTURE ACTIVITIES}

The BART agreement includes six categories of tasks for future activities in the LBL investigation of the BART Train Control System. Three of these categories are described below.

Equipment Reliability Improvement Tasks. These tasks are those required to continue the present level of BART revenue service operations (approximately 30 trains, 3-1ines) in a more reliable manner, i.e., with a reduced number of equipment failures and a reduced level of intermptions to scheduled service. As reliability and safety are interrelated, solving reliability problems should also improve safety.

Medium-term tasks. These are required to reach BART revenue-service medium-term goals, including operations with four lines (adding the Richmond-SF line) and 40 trains, and the removal of the present CABS one-station-train-separation constraint.
Longer-term "Full-Service" Oriented Tasks. These tasks are those which should be started immediately and must be completed before reaching the longer-term goals of BART revenue-service operations: (a) at significantly higher levels ( 80 trains or more, full weekend service) than the medium-term levels; (b) with the capability to handle deviations in normal passenger demands and to minimize interruptions caused by equipment failures; and (c) with the capacity to accommodate eventual BART line extensions. The BART Central Train Control System must be developed to achieve these goals; it must be flexible in order to handle both the present transit line system and the possible future transit line extensions; and it must provide solutions to the problems posed by the existing system.

\section{FOOTNOTE}

*Condensed, in part, from LBL report nos. UCID-3838 and UCID-3856. This work was supported by the State of California Senate Committee on Pub1ic Utilities, Transit and Energy and by the San Francisco Bay Area Rapid Transit District.

ROADWAY POWER SYSTEM FOR FULL-PERFORMANCE ELECTRIC CARS (ROLEC)

\author{
J.G. Bolger and D.T. Scalize
}

\section{INTRODUCTION}

The relative importance of the factors that influence the design of automobiles has been changing rapidly. Major concerns of the sixties were emissions and safety, while major concerns projected for the next decade are the consumption of critical energy and material resources. These concerns become especially important since the automobile and industries peripheral to it form the largest single segment of the national economy. 1

The Roadway Power System for Full-Performance Electric Cars (ROLEC) under development at LBL can have significant impact on these concerns. This system is intended to make the electric car an efficient, viable commercial product. The utility of electric vehicles is currently handicapped by the limited performance and limited range imposed by the high weight and cost of available batteries.?

Even for the long-range, projections for advanced batteries ${ }^{3}$ and flywhee $1 s^{4}$ show that their energy density will be less than that of gasoline by more than an order of magnitude. The efficacy of the LBL concept is based upon its use of rea1time energy supply to vehicles. This means that energy is transferred to the vehicle as it is used; it avoids the need for large amounts of stored energy with all its concomitant weight, efficiency, performance, and cost penalties.

\section{SYSTEM DESCRIPTION}

The ROLEC system consists of an electromagnetic power source installed in arterial roadway lanes. Electric cars will carry a power pickup that can be lowered to a height of approximately an inch above the source. Power can then be transferred from source to pickup by means of magnetic coupling. Figures 1 and 2 illustrate the arrangement of components. The source conductor (single-turn of stranded wire) provides sufficient ampere turns at approximately $180 \mathrm{~Hz}$ to couple the magnetic flux necessary to transfer about $20 \mathrm{~kW}$ of power to the vehicle. Much of this power will be used for moving the car via an electric motor.

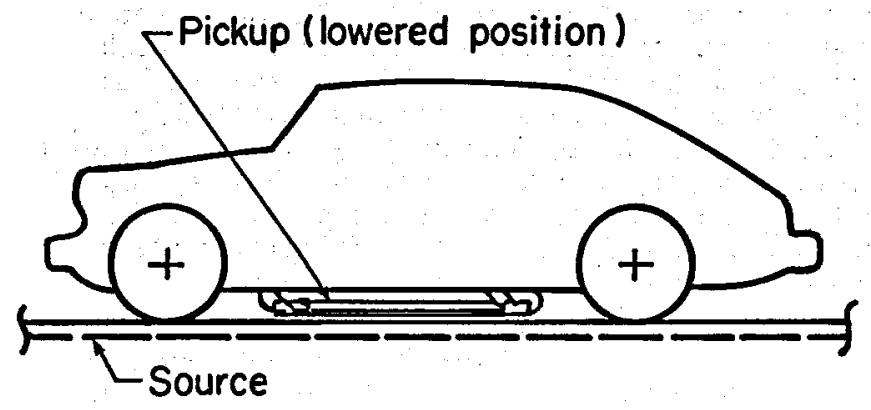

Fig. 1. Vehicle with Power Pickup. 


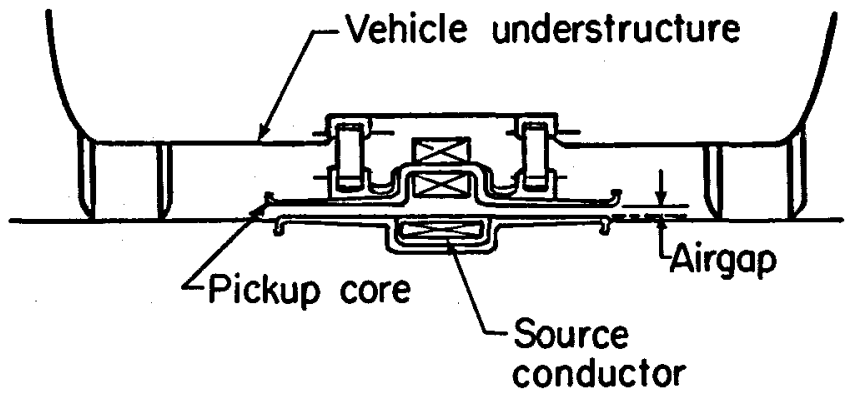

Fig. 2. Vehicle Understructure with Power Pickup. (XBL 771-240)

The rest can be used to charge the car's battery, except when operating at maximum power. The poles of the source structure are wide, and the pickup long enough so that the flux density in the airgap is relatively low. It is important to restrict the flux density to a fraction of a kilogauss so that downward forces on the pickup are tractably 10w, and so that the source's field without a pickup present will not preclude the operation of present automobiles on the lane. Losses are kept low by designing for low densities of current and flux in the source and pickup structures.

For off-arterial roadways, the cars will carry a small battery pack to supply the propulsion power. This use is typically at low speeds and for limited distances, for which ordinary leadacid batteries (or advanced batteries) are suited. The pack also will serve as an effective load leveling device for the car - it will provide a tolerance for the guidance and airgap variations which must be expected in normal service.

Power supplies will be designed to provide constant current to the roadway source conductor. A densely populated lane-mile of arterial system will represent a power load of approximately one megawatt. A virtue of this inductive system is that power can be supplied without significant hazard since there are no exposed conductors (this is not possible in 'third rail' systems). The source conductor is protected by a thick insulation board. Solid state fault detectors/ interrupters can provide an additional level of protection.

\section{TRANSITION CONSIDERATIONS}

An important feature of the ROLEC concept is that its implementation need not interfere with the use of the road by existing internal-combustion-engine (ICE) cars. The source assembly is flush with the roadway surface, and the magnetic field above it is low. Because of this compatibility, the transition to electric cars could be accomplished at gradual rates of implementation and minimum inconvenience to the public.

Arterial traffic loads are usually severely peaked in the early morning and late afternoon. Since normal metropolitan electric loads peak just after midday, the additional highway loads will require much less than a proportionate increase in generating capacity.

\section{POTENTIAL BENEFITS}

The ROLEC system offers potential benefits with respect to the diversification and reduced consumption of primary energy sources. Since electric power is a common denominator for energy conversion processes, the use of coal or nuclear energy by automobiles becomes feasible. The energy-saving potential of the system stems from the higher efficiency of stationary power plants as compared to internal combustion engines. Compact electric cars using the roadway-supplied electric power should be capable of achieving efficiencies equivalent to ICE cars obtaining 30 miles per gallon of gasoline in urban/suburban driving cycles.

Although primarily aimed at energy and petroleum conservation, the ROLEC system provides a useful foundation for advanced automatic highway control systems. The magnetic field of the roadway source is a ready reference for automatic guidance. Advanced traffic-control systems are now under study and development elsewhere. Such systems will increase the traffic capacity and effective use of roadways.

\section{CAPITAL COSTS}

Preliminary economic studies indicate that the capital cost of the roadway power system can be returned to the public within a short time in the form of lessened energy costs. The amortization of the roadway system costs over a twentyyear period at normal freeway traffic density would result in a distributed cost of about two mils (\$.002) per vehicle mile. The estimated investment in equipping all freeways in Los Angeles (637 miles) with two lanes of system would be less than one-quarter the investment that was required for constructing the 75-mile BART system.

\section{PLANNED ACTIVITIES}

Although the ERDA-funded project has just begun, a functional small-scale system model was constructed in 1975 for use in demonstrating the concept to the funding agency. The work during FY 1976 will focus on the interrelationship of design parameters with regard to function, efficiency, and cost. Interfacing requirements with power grids and roads will be explored.

Institutional problems, industrial participation, consumer acceptance, and other problems associated with implementation of the ROLEC system need to be explored. These problems will be studied in FY 1977. FY 1977 work also will optimize the design of system components, and will include the construction and testing of full-scale prototype components.

The development of system components could be sufficiently advanced in FY 1978 to begin the construction, with industrial and Caltrans participation, of a full-scale car and track for testing and demonstrating the ROLEC system. 
REFERENCES

1. B. Sternlicht and G.M. Thur, "Alternative Prime Movers for Future Automobiles," Second Symposium on Low Pollution Power Systems Development.

2. R.R. Stephenson, et al., "Should We Have a New Engine: An Automotive Power Systems Evaluation," JPL for Ford Motor Company.
3. W.F. Hamilton, "Impacts of Electric Car Use in Los Angeles."

4. F. Biggs, "Flywhee1 Energy Systems," Sandia 74-0113 UC-13.

EARTHQUAKE PREDICTION: IN SITU STRESS MONITORING-RESISTIVITY*

H.F. Morrison

\section{INTRODUCTION}

Changes in the electrical resistivity of the earth have been observed prior to earthquakes on the San Andreas Fault south of Hollister, California and in the Garm region of the USSR. The magnitude of the apparent resistivity change observed on the surface is large, up to $20 \%$, and is difficult to explain in terms of existing data relating strain, porosity, and electrical resistivity.

Theoretical analysis has shown that resistivity variations are to be expected in association with porosity changes especially during rock dilatational stages prior to fracturing. Unfortunately, laboratory data are confined to studies on small homogeneous rock samples and the effect of large scale fracture porosity is not included. The behavior of the fracture porosity is not understood and perhaps can only be studied indirectly by the resistivity measurements. The earthquake prediction experiments thus require some sort of calibration; measurements must be made of resistivity as a function of known strain on a scale large enough to include bulk fracture effects.

\section{ANNUAL ACTIVITIES}

Earth tides provide a naturally occurring strain signal of order $10^{-8}$ in amplitude with a periodic variation of 12 and 24 hours related to lunar and solar times. The strains are theoretically predictable to better than $10 \%$ so that resistivity changes associated with this required known strain can be obtained if changes in resistivity of sufficient accuracy can be detected. Theoretical analysis and some small scale experimental work suggests that resistivity changes $\Delta \rho / \rho$ are a factor of $10^{3}$ or $10^{4}$ times the strain so that measurements of resistivity to one part in $10^{5}$ should provide the desired results.

Such a system has been designed and is now being built. A current transmitter has been built and a receiver based on a quasi-digital signal averager is being tested.

\section{PLANNED ACTIVITIES}

First the instruments will be tested in the laboratory. Then preliminary field tests will be made at the University of California Richmond Field Station at a site 250-350 meters from the San Francisco Bay shoreline, where, because of this proximity to the ocean and the ocean tidal loads, $\Delta \rho / \rho$ will be of the magnitude $10^{-4}$. This should prove useful for evaluating the system's capabilities before more sensitive field measurements are made. Finally, field experiments will be conducted in the Hollister area with the system placed alongside existing strain meters.

\section{FOOTNOTE}

*This work was supported by the U.S. Nuclear Regulatory Commission, through the U.S. Energy Research and Development Administration.

EARTHQUAKE REACTOR-SAFETY RESEARCH*

\author{
M. Aslam, W.G. Godden, L. Ruby and D.T. Scalise
}

\section{INTRODUCTION}

The general purpose of this project is to evaluate, improve, or verify analytical design methods used in the seismic qualification of structures, systems, and components important to the safety of nuclear power plants; and to conduct tests of selected items on the Earthquake Simulator Facility at the Earthquake Engineering Research Center of the University of California.

This seismic-testing facility includes a unique 20-foot shaking table capable of handling up to 60-ton loads. This table can provide simul- 
taneous motions in both the vertical and horizontal planes, and is computer controlled to generate anything from simple harmonic motions to complex displacement pattems that reproduce actual recorded (or postulated) earthquake motions.

The emphasis of this program is on fundamental studies rather than on proof-testing of actual reactor components. The intent is to produce results of value both to the Nuclear Regulatory Commission and to industry. Therefore the program entails coordination with industry to identify items or classes of items for which a research testing program to develop and verify analytic methods may advance the state-of-the-art in seismic qualification.

\section{ANNUAL ACTIVITIES}

The area selected for the initial investigation is the sloshing response of water in annular circular tanks under arbitrary horizontal ground motions. This study will assist in the prediction and control of earthquake-driven sloshing phenomena in the pressure-suppression pool of a BWR reactor.

A linearized theory for the phenomenon has been developed to derive the velocity potential for irrotational flow from which the water surface displacements, pressures, and velocities can be determined anywhere in the fluid. Comparisons with test results have shown that this linear solution has a sufficient range for practical problems such as those encountered in annular tanks with an outer diameter of 120 feet, an inner diameter of $80 \mathrm{feet}$, and a depth of water of 20 feet.

For the mathematical model the following assumptions were made:
1. tank walls are rigid,

2. fluid displacements are small,

3. fluid is incompressible and inviscid,

4. flow is irrotational.

Since the flow is irrotational, the velocity potential $\phi$ must satisfy the Laplace equation. Laplace's equation for $\phi$ was solved with appropriate boundary conditions, noting that the boundary conditions are time-dependent. A computer program was written to obtain numerical solutions for arbitrary ground motions applied to the tank.

Once the velocity potential is known, the displacement of the fluid, the pressure, and the velocity at any point in the fluid can be derived from the velocity potential. The computer program determines the sloshing frequencies, mode shapes, and water surface profile. Distribution of the pressure variation with depth along the inner and outer boundaries is also determined.

Tests to determine sloshing frequencies and water surface displacements were made on a small scale model (outer radius $=9$ in., inner radius $=$ 6 in., height of water $=3$ in.). These tests were carried out under harmonic motions. The agreement between analytical and test results was within $10 \%$ for water surface displacement as high as $12 q$ of the water depth, indicating a good range for the linear solution.

Test data for a 12-foot diameter simple circular tank under actual earthquake ground accelerations were made available to us by Douglas Clough from his doctoral research project. Figure 1 shows the comparison between our theory and these test data for the E1 Centro (1940) earthquake. The analysis was carried out by letting

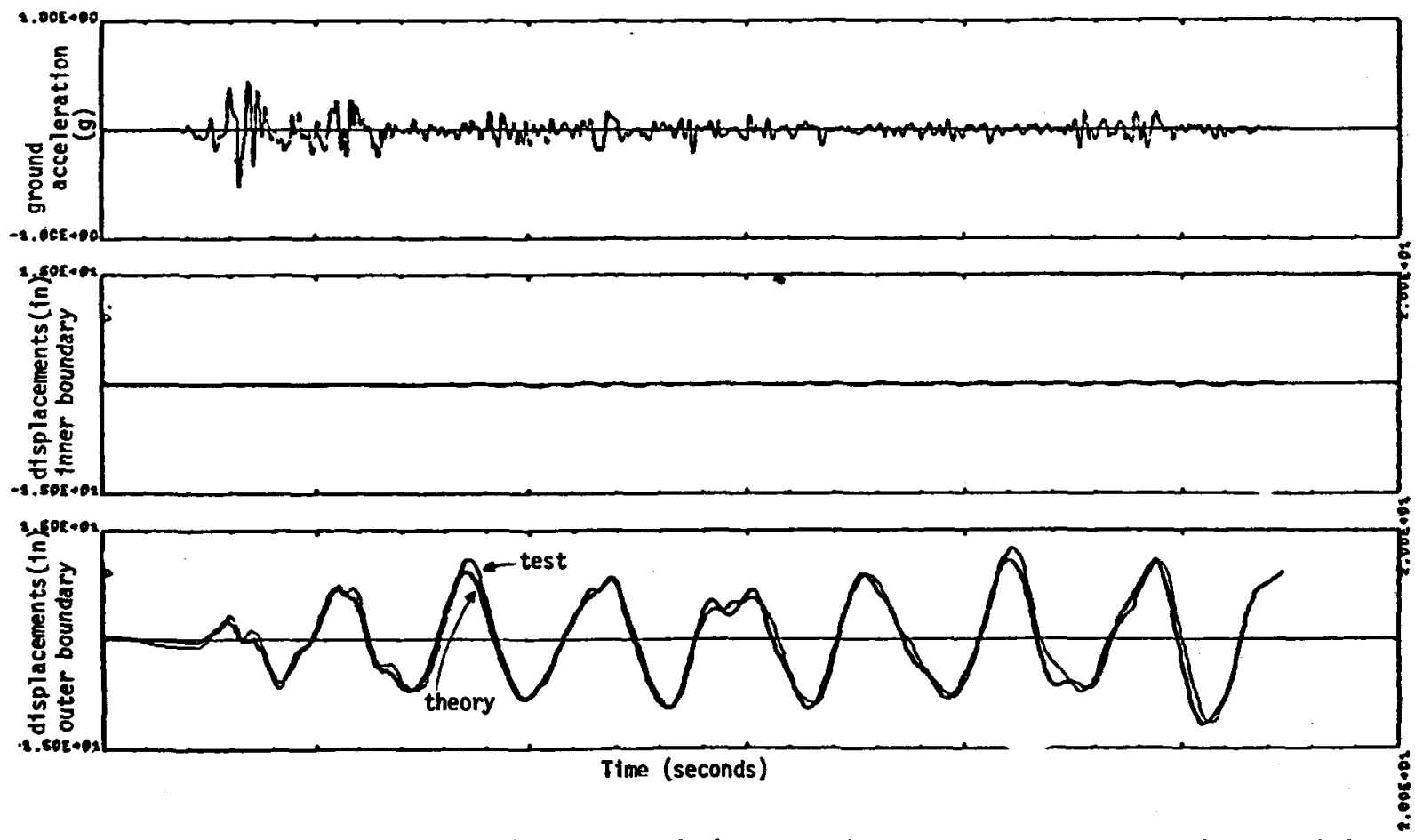

Fig. 1. Sloshing response of water in annular tank (inner radius $=0.1$ in., outer radius $=72.0$ in., depth of water $=60.0$ in.) under El Centro earthquake $(1940)$ time scale $=1.73$, maximu acceleration $=0.56$. 
the inner radius approach zero. The depth of vater in the tank was 5 feet, and the maximum vertical displacement of the water surface was about 12.7 inches. It should be noted that for surface water displacements as high as $21 \%$ of the water depth, the agreement between the test and analytical results was remarkably good, and the differences were within $10 \%$. It should also be noted that the analytical model is good not only for the annular tanks, but also for the simple circular tanks. The accuracy of dynamic pressure was checked against a known analytical solution for a simple circular tank and was found to agree within 18 .

\section{PLANNED ACTIVITIES}

The small-scale sloshing tests are yielding fundamental information that will be important to large-scale tests to be conducted at the Earthquake Engineering Research Center at the U.C. Richmond Field Station. A BWR manufacturer will loan us an 8-foot tank (which represents a 1/15-scale model of a BWR pressure-suppression pool tank) for testing at the Shaking Table at the field station. The special instrumentation, analysis, and similitude experiments will yield information enabling us to prepare a report that will include:

1. the theoretical analysis;

2. a computer code of a mathematical model, validated by experiment, to predict the seismic response of the fluid in waterfilled toroidal vessels;

3. a comparison of the theoretical predictions with the experimental results obtained with the 20-foot Shaking Table at the Richmond Field Station; and

4. a description of the effectiveness of various measures to control the amount of sloshing of the exposed water surface.
In addition to this analysis of the sloshing phenomena, other individual projects will be selected for investigation and will include the following phases:

1. continuing coordination with manufacturers, designers, and builders of plants and components to identify test requirements and classes of items for which testing is recormended;

2. evaluating test requirements and conditions for recommended test items;

3. performing theoretical analyses and conducting tests on recommended and approved items to validate the analyses;

4. investigating theoretical assumptions used in the derivation of similitude hypotheses through actual testing.

As with the sloshing phenomena, the theoretical analyses will include the development of mathematical models to predict the response to earthquake motions of components or systems. The mathematical models with be improved and validated through the comparison with experimental results with hardware models on the shaking table. The experiments will be aimed at determining the structural response and (where applicable) the functional performance of vital equipment and systems.

\section{FOOTNOTE}

*Condensed in part from LBL-5225. This work was supported by the U.S. Nuclear Regulatory Commission, through the U.S. Energy Research and Development Administration. 


\title{
Special Research Projects
}

\author{
INTRODUCTION
}

This section includes reports on research that has been made possible by capabilities developed in other division projects but which is not necessarily related to energy or the enviroment. This year, the research topics in this category include sickle cell anemia, archaeology, and the physics of solids and gases.

The sickle cell anemia work grew out of an attempt to preserve parts of the photochenical system in chloroplasts with the aim of constructing an in vitro system for the production of hydrogen by solar energy. In the course of these investigations it was hypothesized that methods similar to those used to stabilize chloroplasts might be useful in preventing the sickling of human erythrocytes.

The archeological studies were made possible by the development of sophisticated instrumentation for neutron activation analysis. This capability has been developed and improved at the Laboratory over a period of many years and is now being used extensively in the division's geothermal energy and geosciences program.

The studies on the physics of solids and gases were made possible by the development of laser spectroscopy instrumentation for environmental applications. The work reported involves studies of crystalline selenium and nitrogen dioxide gas. While the results are primarily of interest in basic physics and chemistry, they may have practical applications. Selenium has many technological applications and nitrogen dioxide is a significant air pollutant. Increased understanding of these materials may be important for their use and/or control.

\section{MECHANISM OF SICKLE CELL HEMOGLOBIN MODIFICATION BY ETHYL ACETIMIDATE}

\author{
AN ANTISICKLING AGENT
}

Lester Packer, Edwin N. Bymun and Gabriel Ogunmola*

\section{INTRODUCTION}

Sicklemia is a homozygous human disease which afflicts about 50,000 Americans and a larger number of persons in Africa and Asia. An abnormal gene results in a substitution of a single amino acid in the sixth position of the $B$ chain of hemoglobin (valine instead of glutamic acid). The most significant difference in physical properties between sickle cell hemoglobin (HbS) and normal adult hemoglobin (HbA) is the reduced solubility of deoxy-Hbs when compared with the oxygenated HbS. Normal adult hemoglobin shows no difference in solubility in the deoxy and oxygenated forms. The solubility of oxy-HbS is identical to that of normal adult hemoglobin. It is this property of reduced solubility that may account for the aggregation of sickle cell hemoglobin within the erythrocyte in the deaxygenated condition. Sickling is believed to arise from an abnormal interaction of the chains of the hemoglobin. In the network, deoxygenated hemoglobin molecules form long fibrous-like configuration which has many consequences leading to sicklemia. Most of the problems of the sickle cell patient, therefore, arise when the erythrocyte cells are starved of oxygen.

A potent antisickling agent would probably have to be able to improve the solubility of the deoxy-HbS. On this basis oxygen would be the most potent substance to inhibit sickling but unfortunately we have no means of getting oxygen down to the tissue except through the red cell itself.
Thus, the main strategy toward prevention of sicklemia has been directed towards preventing the abnormal physical changes of HbS by chemical modification of the erythrocyte in vivo and in vitro. Another approach is to switch on the synthesis of fetal hemoglobin (HbF) in place of $\mathrm{HbS}$, but efforts to genetically engineer such modifications in hemoglobin biosynthesis have not yet been successful. Both approaches are a formidable task when one considers that approximately 6 million new erythrocytes are formed every second in the human body.

Our work has concentrated on the chemical modification approach. Our early work showed that treatment of sickle erythrocytes with bifunctional imidoesters like dimethyladipimidate (DMA) result in prevention of sickling of human erythrocytes under deoxygenated conditions in vitro. 1 Subsequently we found that monofunctional imidoesters like ethyl acetimidate (EA) were capable of inhibiting in vitro sickling of erythrocytes with equal effectiveness to that of the bifunctional reagents?,3 This finding suggested that cross-linking was most likely not the major factor by which anti-sickling action occurred.

In the past year we have continued our investigation of the effects of EA and IMA on erythro cytes in vitro with the aim of learning more about the mechanism by which these reagents inhibit sickling. We have also begun toxicological studies of these reagents as an initial step in the development of clinical applications. 


\section{CURRENT RESEARCH}

Since monofunctional reagents should not crosslink, they should be less harmful to erythrocyte functions and physical properties. It is necessary for erythrocytes to retain their ability to easily deform themselves for this is a manner in which they squeeze through the small capillary systems. Bifunctional imidates have been shown to modify red cells in a way that changes their osmotic properties and this might confer greater rigidity. 4 our research has now shown that monofunctional reagents do not affect the osmotic properties of the red cel1.5 Examination of osmotic fragility of sickle erythrocytes after treatment with monofunctional and bifunctional reagents clearly show such differences (Fig. 1). Resistance to hemolysis is detectable with $1 \mathrm{mM}$. DMA, but no such effect is seen with EA even if the cells are treated with a 200 times higher concentration of the monofunctional reagent. The range in which sickling is inhibited is $1 \mathrm{mM}$.

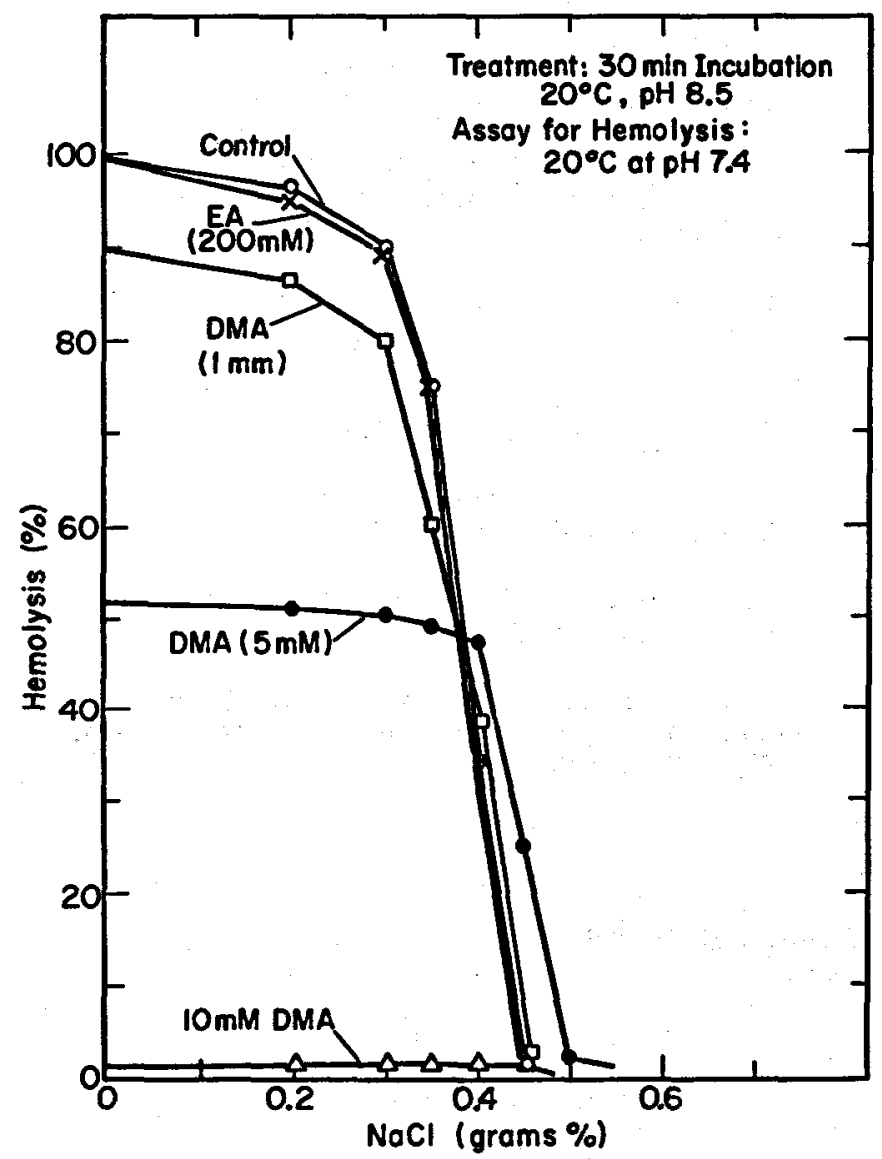

Fig. 1 Effect of EA and DMA treatment on osmotic fragility.

(XBL 765-5460)

Also, since old erythrocytes need to be recycled in the spleen, cross-linked molecules might be more difficult to digest. In view of this, it seemed of interest to carry out the red cell survival studies of DMA- and EA-treated erythrocyte cells as compared with those of untreated cells. Experiments with Cr51-1abeled DMA- and EA-treated rat erythrocytes, reinjected into theše animals, indicated that the survival rates are not significantly affected by the imidate treatment. This suggests that the imidate-treated erythrocytes would survive for the same length of time as normal cells.

Animal toxicity experiments carried out by injecting EA and DMA intraperitoneally into rats and mice indicated an LD50 of 800 and $725 \mathrm{mg} / \mathrm{kg}$ for EA and DMA respectively. Intravenous injection of EA and DMA into rats and mice gave an $\mathrm{LD}_{50}$ of 400 and $200 \mathrm{mg} / \mathrm{kg}$ for EA and DMA respectively. The D5 $_{50}$ increases with the weight of the animal and there was no difference in the $\mathrm{LD}_{50}$ values for male and female animals. Thus, the mono-imidate is somewhat less toxic than the bifunctional imidate in rodents. Similarly, EA is much less inhibitory than DMA to mitochondrial enzymes. 6

In the past year we have also shown that imidoesters inhibit sicklemia, through amidination of the hemoglobin, but not necessarily by modification of the oxygen affinity. Table 1 shows that DMA and EA both decrease hemoglobin oxygen affinities when cells are treated with these reagents under oxy conditions, while sickle cells treated under deoxygenated conditions show an increased hemoglobin oxygen affinity with EA and DMA. However, sickling is equally inhibited by both treatments. Thus, amidination of $\mathrm{HbS}$, rather than changes in oxygen affinity, are important for antisickling properties of EA and DMA (cf. also 7,8).

We have also investigated gelation, another umusual phenomenon observed with $\mathrm{HbS}^{9}$. We have found that the minimum gelling concentration of $\mathrm{HbS}$ at $37^{\circ}$ is $13 \mathrm{~g} \%$, and that after treatment of sickle erythrocytes with $5 \mathrm{mM}$ EA or DMA the minimum gelling concentrations of $\mathrm{HbS}$ is increased to 19 and $21 \mathrm{~g}:$ respectively. Our results are in agreement with the observations of Waterman et a1. 10 for DMA.

\section{PLANNED ACTIVITIES}

We intend to establish collaborative arrangements with clinical hematologists, either in the Donner Laboratory or in San Francisco to carry out survival studies of human sickle erythrocytes following chemical modification with EA and DMA. We believe such experiments are promising because our preliminary studies on rat erythrocytes which were chemically modified with these reagents indicate that erythrocyte cell survival is unaltered after reinjection into rodents.

Also, development of a suitable animal model for testing the antisickling agents will be undertaken in collaboration with Professor Gabriel. Ogumnola of the University of Ibadan in Nigeria. our collaborative program will be based upon primates from malaria infested areas.

We shall examine $\mathrm{HbS}$ solubility curves after EA and DMA treatment. Physiochemical studies relating solubility to gelation will also be pursued.

We also intend to study whether trapping of charged monofunctional imidates within lipid 
Table 1. The effect of amidination on hemoglobin-oxygen affinity.

\begin{tabular}{lccc}
\hline \multirow{2}{*}{ Sample } & $\begin{array}{c}\text { Imidate } \\
\text { concentration }\end{array}$ & $\mathrm{P}_{50}(\mathrm{~mm} \mathrm{Hg})^{\mathrm{a}}$ \\
\hline Oxygenated & Deoxygenated \\
Normal cells & 0 & 27.0 & -- \\
Sickle cells & 0 & 31.0 & - \\
Normal cells & EA (1 mM) & 25.0 & 28.0 \\
Sickle cells & EA (1 mM) & 27.0 & 35.0 \\
Normal cells & DMA (1 mM) & 25.0 & 29.0 \\
Sickle cells & DMA (1 mM) & 26.5 & 35.0 \\
& & & \\
\hline
\end{tabular}

$a_{\text {The }} P_{50}$ values of whole blood were determined in a tonometer.

vesicles might provide a means to deliver the antisickling action to erythrocytes by fusion of the vesicles with the blood cell membrane.

\section{FOOTNOTE AND REFERENCES}

On leave from the Department of Chemistry, University of Ibadan, Ibadan, Nigeria.

1. B.H. Lubin, V. Pena, W.C. Mentzer, E. Bymun, T.B. Bradley and L. Packer, Proc. Natl. Acad. Sci. USA 72, (1975) 43-46.

2. E.N. Bymm, The Prevention of Sickling Human Erythrocytes in vitro by Imidoester Treatment, MA thesis, University of California, Berkeley, CA (1975).

3. L. Packer and E.N. Bymun, Energy and Environment Annual Report 1974, Lawrence Berkeley Laboratory Rept. LBL 3253 (1975).
4. N.I. Krinsky, E.N. Bymun and L. Packer, Arch. Biochem. Biophys. 160, (1974) 350-352.

5. L. Packer, E.N. Bymun, H.M. Tinberg and G. Ogunnola, (1976) Arch. Biochem. Biophys. (in press).

6. H.M. Tinberg, P.R.V. Nayudu, and L. Packer, Arch. Biochem. Biophys. 172, (1975) 734-740.

7. D.T. Zolock and W.G. Niehaus Jr., J. Biol. Chem. 250, (1975) 3080-3088.

8. T.L. Chao, M.R. Berenfeld and T.G. Gabuzda, FEBS Letters 62 (1976) 57-59.

9. R.M. Bookchin and R.L. Nage1, J. Mol. Biol., 60, (1971) 263-270.

10. M.R. Waterman, K. Yamaoka, A.H. Chuang and G.L. Cottam, Biochem. Biophys. Res. Commun. 63 , (1975) 580-587.

\section{APPLICATIONS OF SCIENIIFIC TECHNIQUES TO ARCHEOLOGY}

Frank Asaro and Helen V. Michel

This program is currently concerned with studies of ancient ceramics, studies of the origin of obsidian artifacts and studies of desert varnish aimed at the dating of petroglyphs.

\section{ANCIENT CERAMICS}

In a collaborative study with M. Artzy (LBL) the usefulness of $x$-ray fluorescencel is being investigated as a complement, supplement or replacement for neutron activation analysis in the determination of the origin of Bronze Age pottery from the Eastern Mediteranean. Soft x-ray fluorescence measurements have been shown to be adequate in the most pertinent cases to distinguish whether a type of pottery which was wide-spread in the Eastern Mediterranean, Bichrome Ware, was imported from Cyprus or locally-made in Palestine. A second type of pottery, Tell e1-Yahudiyah ware found in several areas of the Eastern Mediterranean was shown by neutron activation analysis to have originated in Egypt.

In a collaborative study ${ }^{2}$ with Allan Dobel and Professors George Dales and Anne Kilmer of the Department of Near Eastern Studies (U.C. Berkeley), 
the site of the capital city of the ancient Hurrians, Wassukanni, is being searched for by neutron activation analysis techniques. The site is expected to be in the region of northern Syria or perhaps southern Turkey. One of the Hurrian Kings, Tusratta, wrote letters from Wassukanni to the Egyptian Pharaoh on clay tablets. We have tested a number of these tablets by precise measurements of the abundance of trace elements, and found a large proportion of these tested to be rather homogeneous chemically. If we can find locally-made pottery from one of the ancient mounds or Tells which matches chemically the pattern of the Tusratta tablets, and if no other Tell has locally-made pottery which corresponds to this composition; then we probably will have found Wassukanni and will also have developed a new method of archeological prospecting. In partially testing pottery and tablets from one of the most discussed sites thought to be Wassukanni, Tél1 Fe-heriyeh, we found all samples to be distinctly different in composition from the Tusratta tablets. The strong implication is that Tell Feheriyeh is not Wassukanni and we will now search elsewhere.

In a collaborative study ${ }^{3}$ with Professors J.D. Frierman (U.C.L.A.) and David Whitehouse (Rome) we have investigated the chemical composition of glazed pottery from Siraf, a prominent medieval port on the eastern side of the Persian Gulf. We found that essentially all of the glazed pottery that we analyzed was imported, and in particular a very abundant blue-green ware was found to have been imported from Mesopotamia. The amounts of this ware found in Siraf were sufficiently large to indicate that it represented a very important item of commerce in the Mesopotamian export trade and was one of the cornerstones of the Mesopotamian economy.

\section{OBSIDIAN}

Previous studies by precise methods of neutron activation analysis have shown that the origin of obsidian artifacts can be determined with certainty from their chemical compositon. Other studies by less expensive techniques, such as $x$-ray fluorescence, have shown that origins can be determined but with more uncertainty. In the present work, methods were developed for first determing with high precision the chemical composition of a limited muber of samples from (or representing) source areas. Then for a given archeological problem, the least expensive method of chemical measurement is used to distinguish the likely sites. One test of the method was made in collaboration with Richard Burger and Professor Rowe of the Department of Anthropology (U.C. Berkeley) on obsidian artifacts from Peru and Ecuador that previously could not be adequately separated into groups by $x$-ray fluorescence measurements. Large scale obsidian analysis usually had previously amounted to several hundred samples. We analyzed about 50 samples of Peruvian obsidian by neutron activation analysis in order to determine the main chemical groups. After determining that the elements that best distinguished these groups were $\mathrm{Ba}$ and $\mathrm{Sr}$, approximately 800 samples were analyzed' by nondestructive $x$-ray fluorescence measurements. About $10 \%$ of these were subsequently analyzed by neutron activation analysis because of uncertain- ties in the XRF measurements or the discovery of new chemical groups. By these methods it was possible to classify essentially all of the obsidian artifacts and determine trade patterns as a function of time with minimm expense. Further archeological studies in this area involving obsidian could be made even less expensively as the source areas in Peru have already been classified to a considerable extent.

A sma11 study ${ }^{4}$ in collaboration with F. Stross (LBL) and Prof. Ruth Gruhn (University of Alberta) was made on obsidian artifacts from the Paleoindian site of Los Tapiales in Guatemala. The likely sources had already been classified to a small extent by neutron activation analysis. It was determined that a relatively inexpensive abbreviated neutron activation analysis would be sufficient to ascertain the origin of most of the artifacts. From these measurements three different sources were detected, all within $50-75 \mathrm{~km}$ of Los Tapiales. Two assignments were certain and one somewhat uncertain as only $x$-ray fluorescence measurements had been made on the source material.

\section{ROCK PATINA STUDIES}

The possible relative dating of petroglyphs by nuclear measurements in desert varnish is being explored 5 in collaboration with James C. Bard and Prof. Robert F. Heizer of the Department of Anthropology (U.C. Berkeley). The abundances of 28 trace and major elements in desert varnish from the Grimes Point Petroglyph Site in Western Nevada were measured by precise neutron activation analysis techniques. Samples of both desert varnish and heart rock from a test boulder (not an artifact) of andesite were studied. The amount of desert varnish presumably varies with the exposure to the environment. The elements manganese, uranium, thorium and cerium exhibited the greatest enhancements in the varnish fraction and they offer the best possibilities for dating purposes by these methods.

\section{REFERENCES}

1. M. Artzy, An Archaeologist Looks at X-ray Fluorescence vs Neutron Activation Analysis, Proceedings of conference of Application of Physical Sciences to Medieval Ceramics, (LBL 5017) (1976).

2. F. Asaro, Determination of the Location of Wassukanni. Utilizing Neutron Activation Analysis, NEH Proposal (1976).

3. H.V. Michel, F. Asaro, J.D. Frierman, Proceedings of Conference of Application of Physical Sciences to Medieval Ceramics (LBL 4061) (1975).

4. F.H. Stross, F. Asaro, H.V. Miche1, R. Gruhn, Sources of Some Obsidian Flakes from a Paleoindian Site in Guatemala, American Antiquity, in press (LBL 4483) (1976).

5. J.C. Bard, F. Heizer, R.F. Heizer, Perspective on Dating of Great Basin Petroglyphs by Neutron Activation Analysis of Patinated Surfaces, (LBL 4475) (1976). 


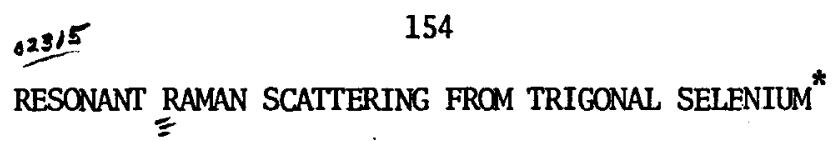

Nabiz M. Amer

\section{INTRODUCTION}

Recently resonant Raman scattering (RRS) has been shown to be a powerful new spectroscopic technique that encompasses both traditional optical and Raman spectroscopies and in addition can provide information on electron-phonon interaction in semiconductors.1 Selenium, a semiconductor whose properties have been studied extensively 2 because of its technological applications, is well suited to resonant Raman studies. This is because trigonal Se has strong anisotropic excitonic absorption peaks lying within the tuning range of available dye lasers. Presently very little is known about the interactions of these excitons with phonons.

Richter ${ }^{3}$ has studied RRS in Se at room temperature over a wide range of incident photon frequencies using the discrete lines of various ion lasers, augmented by a CW dye laser. Because of the high temperature and discrete tuning nature of that experiment only broad structures in the dispersion of the Raman cross-section were observed. Previously 4 we reported low temperature RRS in trigonal Se for incident photon frequencies above its direct absorption edge. Now we have extended our measurements below Se's indirect absorption edge. We have also obtained new results which confirm our earlier identification of the $\mathrm{E}(1)(\mathrm{L})$ mode in Se.

\section{RESULTS}

our experiments were performed in a backscattering configuration using a conventional Raman scattering system. The Raman spectra were excited by a $\mathrm{CW}$ dye laser pumped by an $\mathrm{Ar}^{+}$laser.
The samples we used were single crystalline trigonal Se and were immersed in superfluid He. It is important to point out that by keeping the incident laser power low $(10 \mathrm{~mW})$, as well as by using a cylindrical lens for focusing the laser on the sample and by immersing the sample in superfluid $\mathrm{He}\left(\mathrm{T}<2^{\circ} \mathrm{K}\right)$ we were able to avoid any damage to the samples during the course of our experiments.

We have studied RRS in Se for three scattering configurations:

a. The electric field vectors of both the incident and scattered laser light are parallel to each other and perpendicular to the optic axis of the crystal (E $\| \mathrm{E}_{\mathrm{s}}^{\perp} \mathrm{c}$-axis).

b. The electric field vector of the incident light is parallel to the c-axis while that of the scattered light is perpendicular to it $\left(\hat{\mathrm{E}}_{Q} \| \mathrm{c}\right.$-axis $\left.\perp \mathrm{E}_{\mathrm{s}}\right)$.

c. The electric field vectors of both the incident and the scattered light are both parallel to the c-axis of the crystal ( $\hat{\mathrm{E}}_{\ell}\left\|\hat{\mathrm{E}}_{\mathrm{s}}\right\| \mathrm{c}$-axis).

According to Table 1 , only two types of onephonon Raman modes, $E$ and $A_{1}$, are allowed in the configurations $\mathrm{E}_{\ell} \| \mathrm{E}_{S} \perp \mathrm{c}$-axis. Since the $\mathrm{A}_{1}$ and $E(2)$ modes are separated by only $3 \mathrm{~cm}^{-1}$ and we have selected a spectral slit width of $24 \mathrm{~cm}^{-1}$ to obtain the best combination of signal-to-noise ratio and resolution, we were not able to resolve these two modes. As a result we label the unresolved $233 \mathrm{~cm}^{-1}$ mode in this configuration as the $\left(A_{1}, E(2)\right)$ mode.

Table 1. Symmetry, frequency and Raman selection rules of zone center Optical phonons of trigonal Se.

\begin{tabular}{|c|c|c|c|}
\hline \multirow{2}{*}{ Mode } & \multicolumn{2}{|c|}{ Frequency $\left(\mathrm{cm}^{-}\right)$} & \multirow{2}{*}{ Raman Selection Rules } \\
\hline & Previous work & Present work & \\
\hline$A_{2}(\mathrm{TO})$ & 112 & & \\
\hline $\mathrm{A}_{2}(\mathrm{LO})$ & & 114 & \\
\hline $\mathrm{E}^{(1)}(\mathrm{TO})$ & 147 & 147 & $\hat{\mathrm{E}} \perp c$-axis \\
\hline $\mathrm{E}^{(1)}(\mathrm{L})$ & & 154 & and $E^{-} \perp E_{s}$ \\
\hline $\mathrm{E}^{(2)}$ & 232 & & \\
\hline$A_{1}$ & 235 & & $\hat{\mathrm{E}}_{\ell} \| \hat{\mathrm{E}}_{\mathrm{s}}$ \\
\hline
\end{tabular}


The Raman cross sections of the $233 \mathrm{~cm}^{-1}$ (A1, E(2) ) $147 \mathrm{~cm}^{-1}$ (E(1) (TO)); $154 \mathrm{~cm}^{-1}(\mathrm{E}(1)$ (LO) and $304 \mathrm{~cm}^{-1}$ (2E(1) (LO)) modes measured in the configuration $\hat{\mathrm{E}}_{\ell} \| \hat{\mathrm{E}} \perp \mathrm{c}$-axis are plotted in Fig. 1 as a function of the incident photon energy. The normally Raman inactive $112 \mathrm{~cm}^{-1}\left(\mathrm{~A}_{2}\right)$ mode begins to appear for incident photon energies above $1.94 \mathrm{eV}$. This mode exhibits resonant enhancement very similar to the $E(1)$ (LO) mode for incident photon energy between 1.94 and $2.03 \mathrm{eV}$.

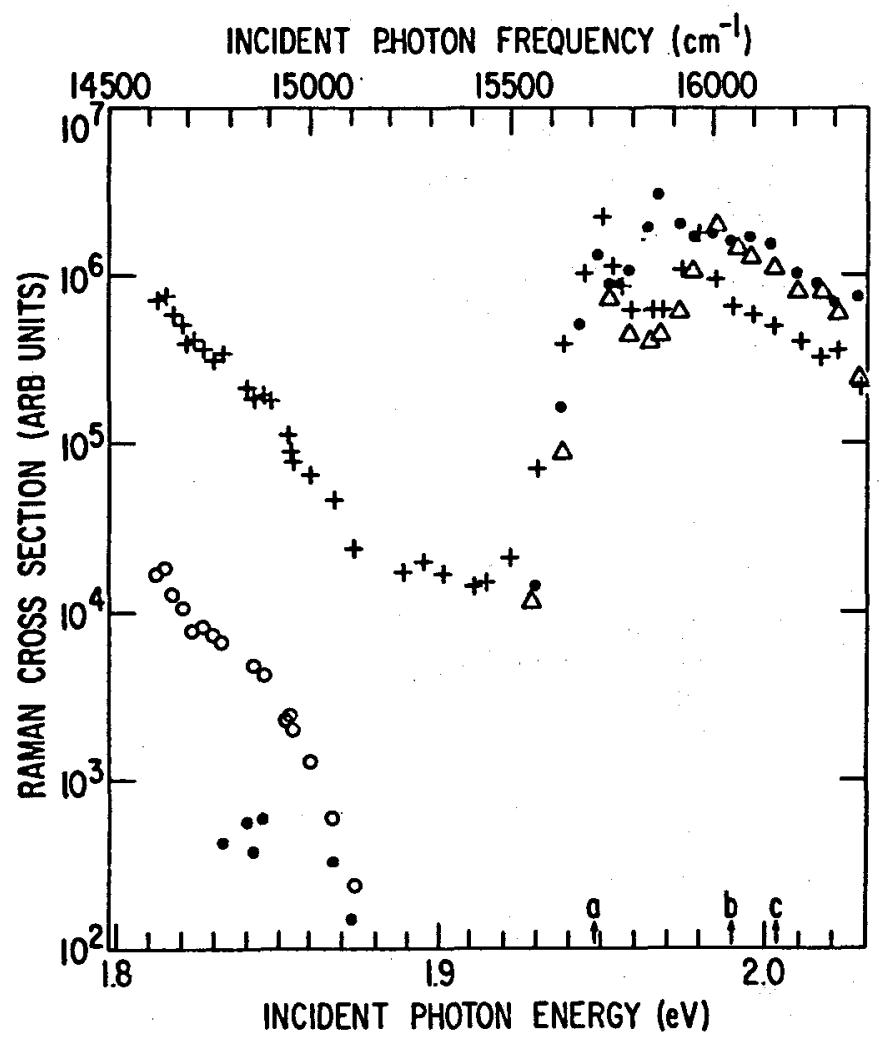

Fig. 1. Raman cross-section of the $\left(A_{1}, E^{(2)}\right)(+)$, $E(1)(T O)(0), E(1)(L O)(\bullet)$ and $Z E(1)(L O)(\Delta)$ modes plotted as a function of the incident photon energy. The scattering configuration is $\hat{E}_{\ell} \| \hat{E}_{S} \perp$ c-axis.

In the configuration $\hat{\mathrm{E}}_{\ell} \| \mathrm{c}$-axis $\perp \hat{\mathrm{E}}_{\mathrm{s}}$ only the $\mathrm{E}$ modes are allowed. In this configuration we found that none of the observed Raman modes shows any enhancement. As shown in Fig. 2 (a) the three E modes $=233 \mathrm{~cm}^{-1}(\mathrm{E}(2)) ; 147 \mathrm{~cm}^{-1}$ (TO)) and $154 \mathrm{~cm}^{-1}(\mathrm{E}(\mathrm{I})(\mathrm{LO}))$ decrease in intensity monotonically with increase in incident photon energy.

$\hat{E}_{l} \| \hat{E}_{S} \perp c$-axis is the only configuration in which we observed enhancement of Raman modes at the $d$ exciton. The modes enhanced are the $A_{1}$ and $E(1)(10)$ modes and their cross-sections are plotted in Fig. 2 (b) as a function of incident photon energies.

In obtaining the Raman cross sections in Figs. 1 and 2 the effect of the absorption coefficient on the scattering intensity has been corrected for in a manner described by Loudon. 5

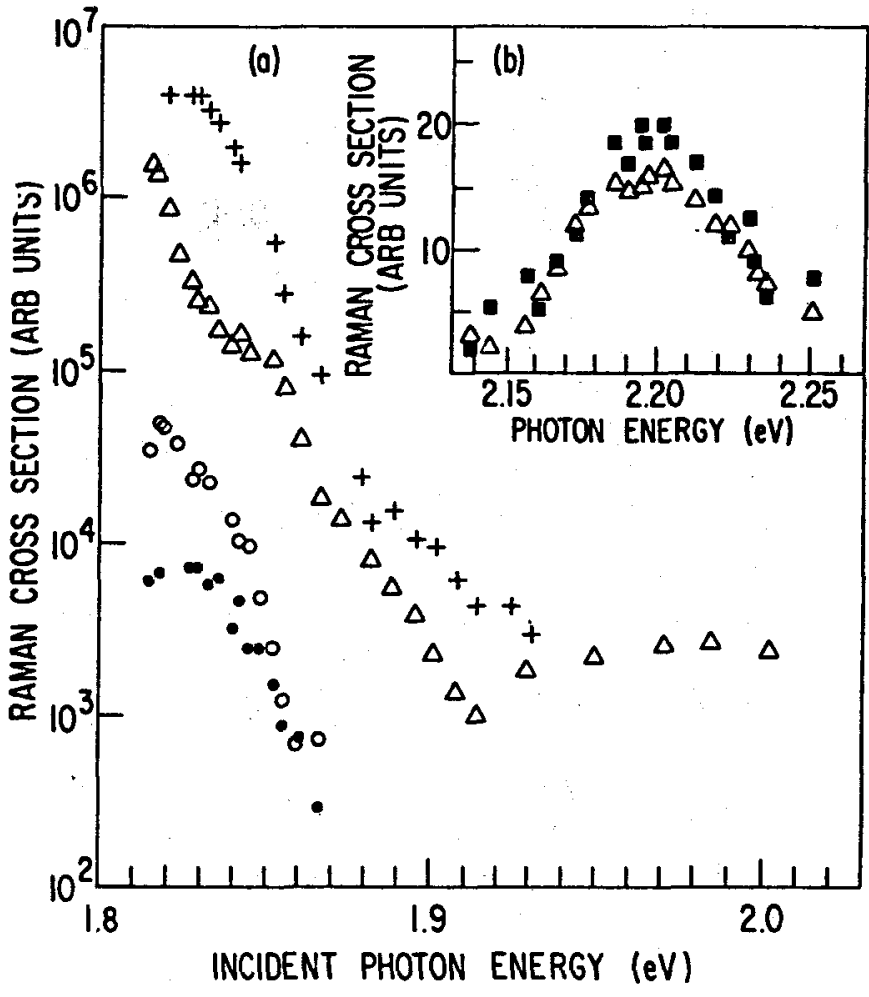

Fig. 2. Raman cross section of Se plotted against incident photon energy for two different energy ranges: (a) $1.8-2.0 \mathrm{eV}$ and (b) 2.14-2.25 eV. In (a) the scattering configuration is $\hat{E}_{\ell} \|$ c-axis. 1 E for the $E^{(2)}(+), E^{(1)}(T O)(0)$ and $E^{(1)}(L \delta)(\bullet)$ modes and $E_{l}\left\|\hat{E}_{s}\right\| c$-axis for the $A_{1}$ mode $(\Delta)$. In (b) the scattering configuration is $E$ \| $\hat{E}_{f} \|$ c-axis for both the $A_{1}(\Delta)$ and $E(1)(L \delta)(\square)$ modes.

We have also examined the theoretical implicatons of our results. We were able to give a qualitative explanation for the decrease in Raman cross sections above $1.8 \mathrm{eV}$ in terms of a resonant cancellation between a constant background and a dispersive contribution from the $2.20 \mathrm{eV}$ peak in the reflectivity spectra of Se. We were able to provide a quantitative explanation for the enhancements due to the direct excitons observed in the scattering configuration $\mathrm{E}_{\ell} \| \mathrm{E}_{S} \perp$-axis. In this case a simple model involving the 1.95 $\mathrm{eV}$ and $1.99 \mathrm{eV}$ excitons as resonant intermediate states provided a reasonable fit, (Fig. 3).

\section{FOOTNOTE AND REFERENCES}

* This work was done in collaboration with P.Y. Yu, Y.R. Shen, and Y. Petroff. A complete version has been accepted for publication in Physical Review B.

1. See recent reviews by J.L. Birman in Proceedings of the Twelfth International Conference on the Physics of Semiconductors ed. by M. Milhuhn, B.G. Teubner, Stuttgart (1974); P.Y. Yu in Proceedings of the Third International Conference on Light Scattering in Solids (to be published) and R.M. Martin 


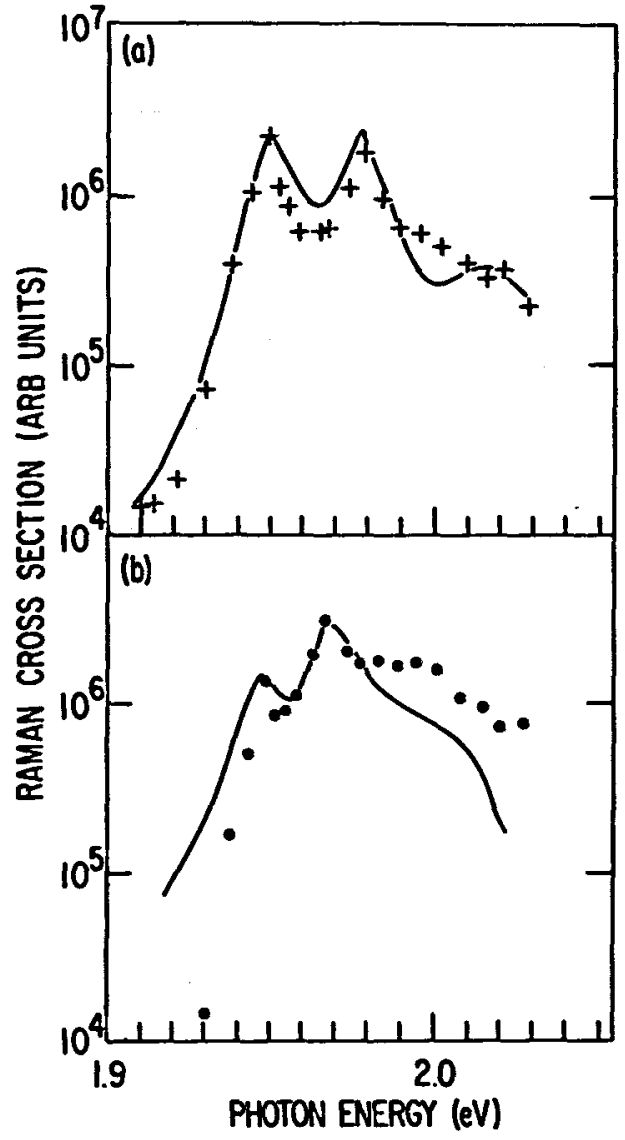

and L.M. Falicov, in Light Scattering in Solids ed: by M. Cardona, (Springer-Ver1ag, Heidelberg, 1975).

2. See articles in The Physics of Selenium and Tellurium, ed. by W.C. Cooper, (Pergamon Press, London, 1969).

3. W. Richter in The Proceedings of the Eleventh International Conference on the Physics of Semiconductors, (Polish Scientific Publishers, Warsaw, 1972) Vol. 2.

4. N. Amer, Y. Petroff, Y.R. Shen and P.Y. Yu, in Proceedings of the Twelfth International Conference on the Physics of Semiconductors, ed. by M. Pilkuhn, (B.G. Teubner, Stuttgart, 1974), p. 473.

5. R. Loudion, J. Phys, Radium 26, 677 (1965).
Fig. 3. Comparison between the theoretical curve (solid lines) and the experimental points for (a) $\left(A_{1}, E^{(2)}\right)$ modes and (b) $E(1)$ (L) mode.

$$
U \frac{.02116}{\text { TUNABLE LASER SPECTROSCOPY OF } \mathrm{NO}_{2}}
$$

P. Robrish and H. Rosen

The visible absorption spectrum of $\mathrm{NO}_{2}$ extends from about $4000-7000 \AA 1$ and seems to consist of mmerous overlapping lines forming a quasi-contimum, with a few recognizable bands 2 superimposed upon it. Although several limited regions, usually near fixed frequency laser wavelengths, have been successfully analyzed using laser induced fluorescence 3 and microwave-optical double resonance 4,5 techniques, there is still considerable uncertainty in the interpretation of the overall absorption spectrum, including the sharp line structures found by Douglas and Huber.2 There is also a contimuing problem of reconciling the short excited state lifetimes deduced from the absorption spectrum 6 with the long measured lifetimes, 6,7 although several workers have reported evidence of short lived intermediate states, 5,8 We have been investigating a technique which may fur. ther help to unravel the complex spectra of simple polyatomic molecules like $\mathrm{NO}_{2}$.

We have measured the excitation spectrum for the $v_{2}$ mode of $\mathrm{NO}_{2}$ using a narrow band $(0.1 \mathrm{~A})$ nitrogen-1aser-pumped dye laser. 9 This technique allows one not only to excite the $\mathrm{NO}_{2}$ molecule selectively, but also to monitor a particular re-emission mode. We tuned the laser through most of the visible absorption spectrum of $\mathrm{NO}_{2}(4200-6000 \AA)$ and observed the fluorescence through a spectrometer with a band- width of $25 \AA$. As the laser was tuned, the spectrometer was advanced so as to keep its bandpass centered on the $v_{2}$ mode located $\sim 750 \mathrm{~cm}^{-1}$ from the laser frequency. Our excitation spectra show several narrow regions where there are large enhancements in the intensity of the $v_{2}$ fluorescence mode. These narrow structures, which consist of sharp and intense lines, are shown in Fig. 1. For a given dye, other structures were observed, but they were either of lower intensity or did not consist of resolved sharp lines. The positions of five of the structures in Figure 1, whose leading edges are located at 18215, 19523, 21099, 21996, and $22989 \mathrm{~cm}^{-1}$, are given to within $10 \mathrm{~cm}^{-1}$ by the following formula:

$$
v=19215+576.22 \mathrm{~N}+36.38 \mathrm{~N}^{2}
$$

with $\mathrm{N}$ respectively equal to $0,2,4,5,6$. The frequencies corresponding to $\mathrm{N}=1$ and $\mathrm{N}=3$ occur at gaps between the dyes and we speculate that there are similar structures in these two regions with all seven forming a smooth progression. We are uncertain of the explanation for this progression, but if it is due to vibrational spacing in the upper slate, then it has a peculiar anharmonicity since the spacing between members of the progression increases with frequency. It should be noted that, of the six observed structures, only the one near $4545 \AA$ 

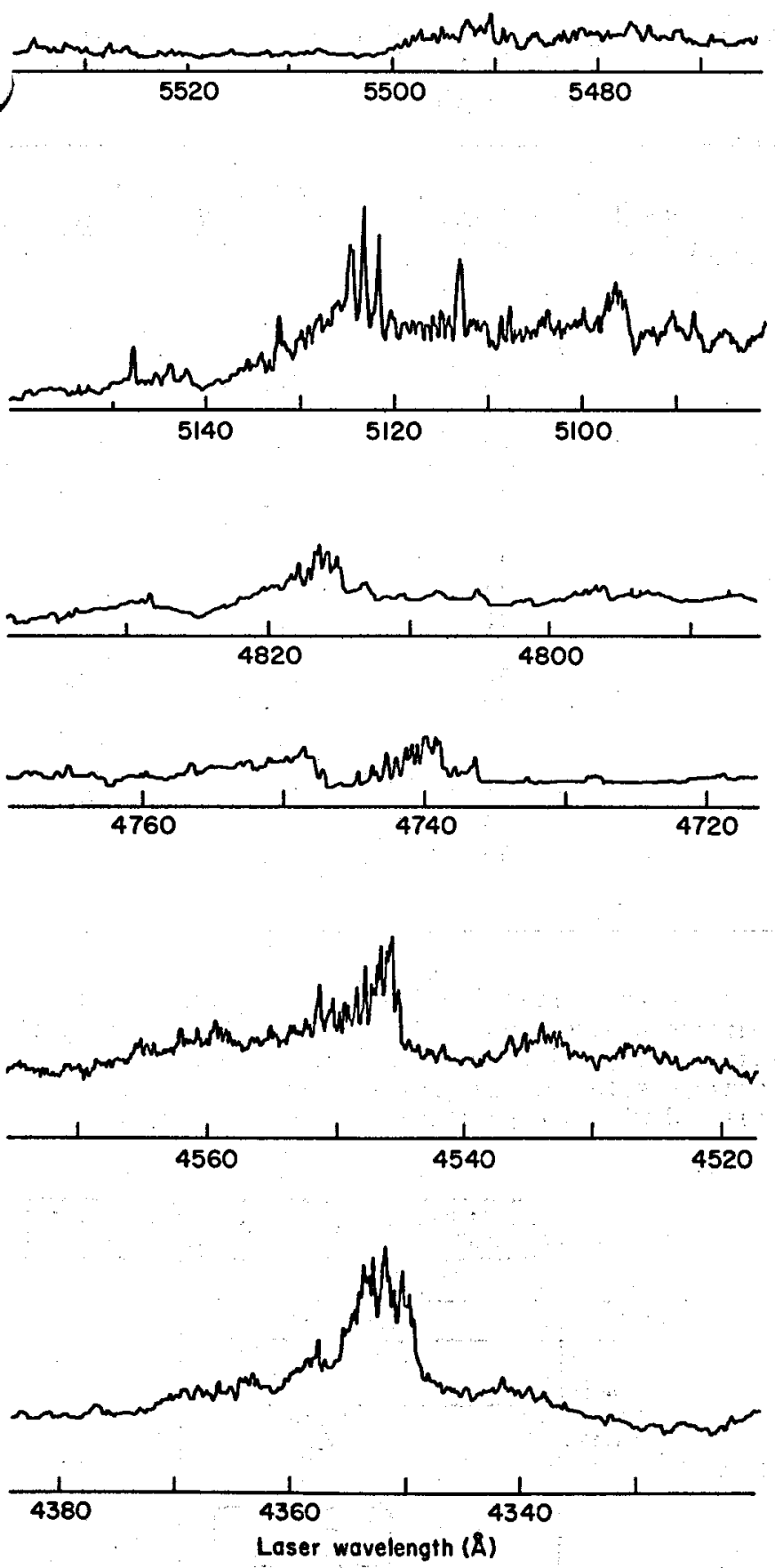

Fig. 1 Intensity in the $v_{2}$ mode as a function of laser wavelength over wavelength regions where sharp and intense structures were observed. The bandwidth of the laser was $\approx 0.1 \mathrm{~A}$ and the spectrometer bandwidth was set to $25 \AA$. The intensities in different scans can not be compared directly due to variations in laser power and detector sensitivity.

(XBL 7412-8438)

(21996 $\left.\mathrm{cm}^{-1}\right)$ corresponds to any of the bands reported by Douglas and Huber. 2 For this band, however, there is a one to one correspondence between the lines in the excitation spectrum and those in the absorption spectrum.
When the laser is narrowed to a bandwidth of $.01 \AA$ the excitation spectrum is more clearly resolved into individual lines as shown in Fig. 2.

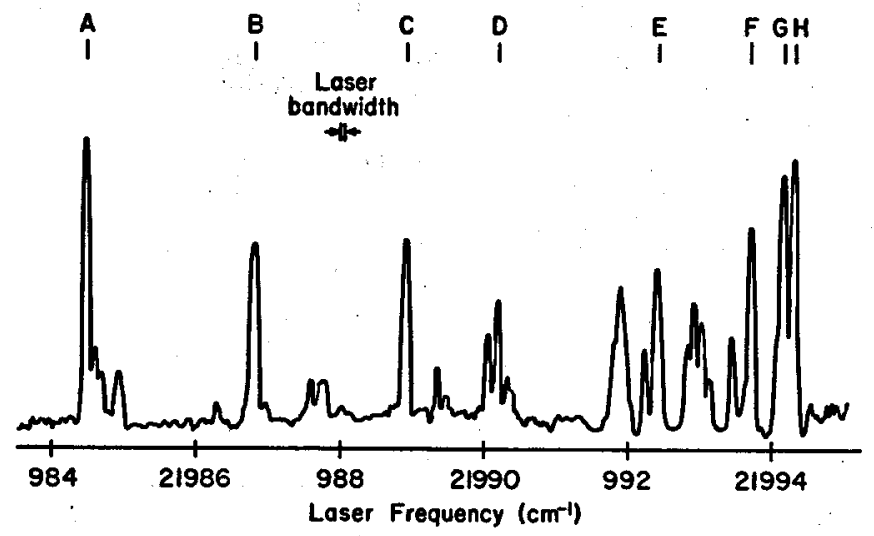

Fig. 2. Intensity in the $v_{2}$ mode as a function of laser frequency, near $22,000 \mathrm{~cm}^{-1}$ (4545 $\AA$ ), with narrow band laser excitation. The linewidth of the laser was $\sim .04 \mathrm{~cm}^{-1}$ and the bandwidth of the spectrometer $120 \mathrm{~cm}^{-1}$. The relative positions of the lines could be determined to $\pm 3 \%$ and the absolute positions were set using the wavelength determination of Douglas and Huber ${ }^{1}$ for the $Q(13)$ line which corresponds to line $B$ in our scan.

(XBL 7412-8437)

With the laser tuned to the peak of an intense line within this structure, the $v_{2}$ fluorescence crosssection is found to be about two orders of magnitude larger than those typically obtained using fixed frequency lasers. 10 This large cross section is particularly surprising given the fact that the absorption cross sections we have measured at lines near $4545 \AA$ are comparable to that of the background absorption. We have investigated the band near $4545 \mathrm{~A}$ in much greater detail than the other bands. We have attempted to make a partial rotational analysis of this region by tuning the laser to several of the most intense lines, designated by the letters A through $\mathrm{H}$ in Fig. 2, and resolving the $v_{2}$ fluorescence mode located at $749.8 \mathrm{~cm}^{-1}$. In Fig. 3 the resolved fluorescence for the laser tuned to lines $A, B, C, E$ and $G$ are presented. Since the narrow band laser selects a single initial to intermediate state transition, the observed triplet structures can be used in combination with the selection rules for a slightly asymetric top $(\Delta \mathrm{N}= \pm 1.0 ; \Delta \mathrm{K}= \pm 1.0)$ and the ground state spectroscopic constants, to assign the final state quantum numbers. This determination then allows one to make assignments for both the initial and intermediate states. These identifications are shown in Fig. 3 and a comparison of calculated and observed splittings is made in Table 1.

In collaboration with the Bradley Moore group of the Materials and Molecular Research Division, we have also made measurements of the fluorescent lifetimes of several lines within this structure near $4545 \AA$.

Neuberger and Duncan ${ }^{6}$ were the first to find that the fluorescence lifetime of $\mathrm{NO}_{2}$ is anomalously 

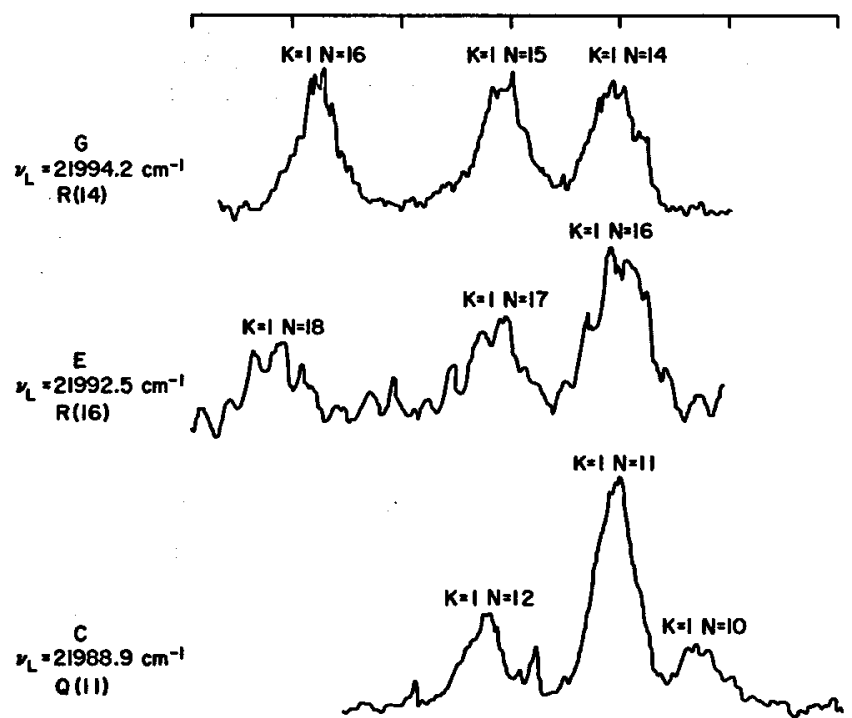
$1986.8 \mathrm{~cm}^{-1}$ 0 (13)

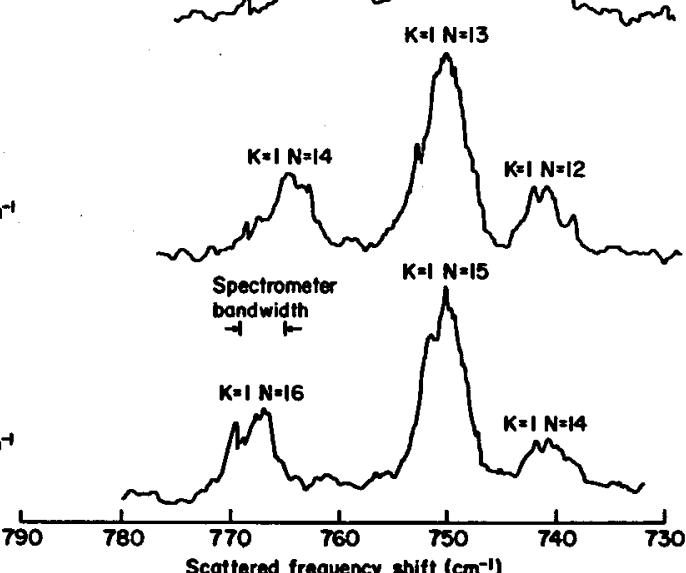

Fig. 3. High resolution sepctrometer scans of the $v_{2}$ mode located $\sim 750 \mathrm{~cm}^{-1}$ lower in frequency than the laser. The laser was tuned to the peaks of lines $A, B$, $C, E$ and $G$ of Fig. 2. The $K$ and $N$ values refer to the final state of the various transitions. The spectrometer bandwidth was $4 \mathrm{~cm}^{-1}$ and the laser bandwidth 0.04 $\mathrm{cm}^{-1}$.

(XBL 7412-8439)

long when compared to the lifetime obtained from the integrated absorption coefficient. This effect is believed to arise from interactions between the different electronic levels in the visible region. Despite much effort to observe short-lived states directly, only two such observations have been reported to date. The first of these involved $\mathrm{K}_{\mathrm{a}}=0$, ${ }_{31}$ lines $^{8}$ which form prominent features of the absorption spectrum 2 . However, its accuracy was severely limited by poor signal-to-noise and lack of shot-to-shot stability in the laser employed. The second of these observations 5 involved not the $\mathrm{K}_{\mathrm{a}}=0,2_{\mathrm{B}_{1}}$ lines, but rather a $5_{24}, 2_{\mathrm{B}_{2}}$ rotational level pumped by the $488 \mathrm{~mm}$ line of an argon ion laser. A lifetime of $3.39 \pm 0.36 \mu \mathrm{sec}$ was obtained from the linewidth of the microwave-optical double resonance signal.

Our investigation has demonstrated that $\mathrm{k}_{\mathrm{a}}=0$, $2 B_{1}$ lines near $454.8 \mathrm{~mm}$ do not have the short lifetimes expected from previous experimental results ${ }^{8}$ and perturbation analysis.
Table 1. Comparison of calculated and measured splittings between members of triplet structures shown in Fig. 3.

\begin{tabular}{|c|c|c|c|}
\hline Transition & $\begin{array}{c}\text { Final state } \\
\mathrm{N} \text { value } \\
\end{array}$ & $\begin{array}{l}\text { Frequency } \\
\text { central N } \\
\text { Calculated }\end{array}$ & $\begin{array}{l}\text { shift from } \\
\text { value }\left(\mathrm{cm}^{-1}\right) \\
\text { Measured }\end{array}$ \\
\hline A & $\begin{array}{l}16 \\
15 \\
14\end{array}$ & $\begin{array}{c}16.5 \\
- \\
-10.0\end{array}$ & $\begin{array}{c}17.5 \\
- \\
-9.3\end{array}$ \\
\hline B & $\begin{array}{l}14 \\
13 \\
12\end{array}$ & $\begin{array}{c}14.1 \\
- \\
-9.0\end{array}$ & $\begin{array}{c}13.8 \\
- \\
-8.8\end{array}$ \\
\hline C & $\begin{array}{l}12 \\
11 \\
10\end{array}$ & $\begin{array}{c}11.8 \\
- \\
-7.9\end{array}$ & $\begin{array}{c}11.7 \\
- \\
-7.2\end{array}$ \\
\hline E & $\begin{array}{l}18 \\
17 \\
16\end{array}$ & $\begin{array}{c}20.2 \\
- \\
-11.3\end{array}$ & $\begin{array}{c}19.0 \\
- \\
-11.0\end{array}$ \\
\hline G & $\begin{array}{l}16 \\
15 \\
14\end{array}$ & $\begin{array}{c}16.5 \\
- \\
-10.0\end{array}$ & $\begin{array}{c}16.7 \\
- \\
-10.1\end{array}$ \\
\hline
\end{tabular}

Figure 4 shows the experimental arrangement. A nitrogen-1aser-pumped dye laser of $1 \mathrm{GHz}$ bandwidth was used to excite $\mathrm{NO}_{2}$ in a 22-1iter bulb connected to a greaseless vacurm system. Pressures were measured with a capacitance manometer. The laser

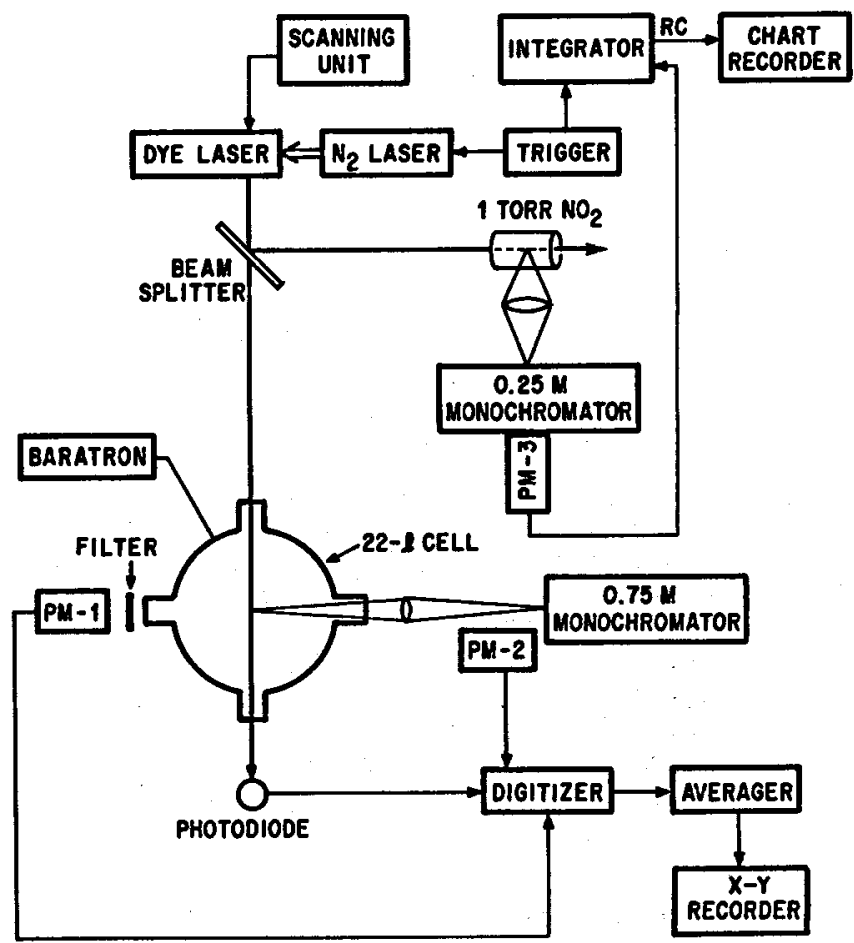

Fig. 4. Experimental apparatus for recording the fluorescence excitation spectrum and measuring the lifetimes of $\mathrm{NO}_{2}$.

(XBL 755-1358) 

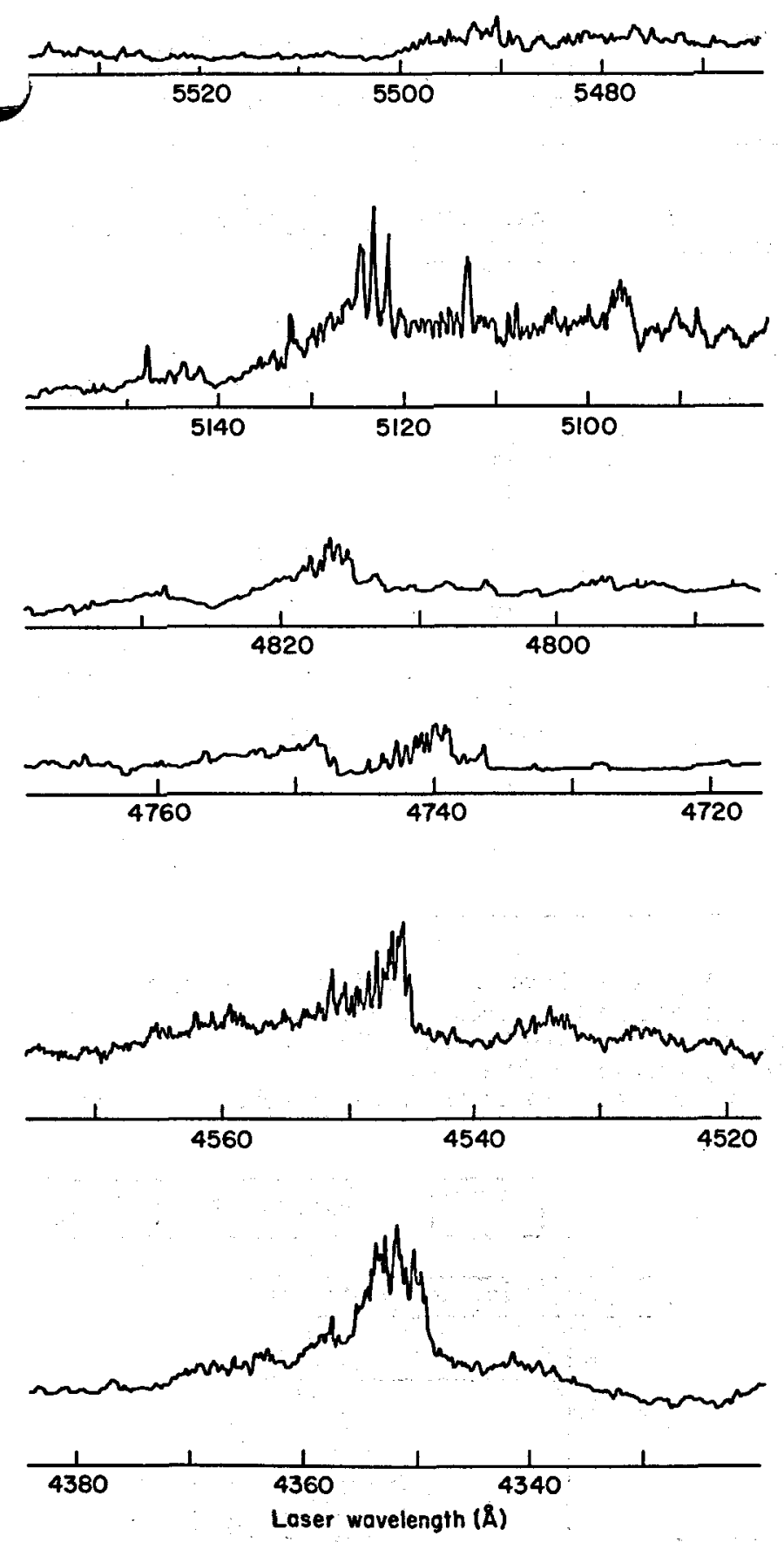

Fig. 1 Intensity in the $v_{2}$ mode as a function of laser wavelength over wavelength regions where sharp and intense structures were observed. The bandwidth of the laser was $\approx 0.1 \mathrm{~A}$ and the spectrometer bandwidth was set to $25 \mathrm{~A}$. The intensities in different scans can not be compared directly due to variations in laser power and detector sensitivity. $\ldots$ (XBL 7412-8438)

(21996 $\left.\mathrm{cm}^{-1}\right)$ corresponds to any of the bands reported by Douglas and Huber. 2 For this band, however, there is a one to one correspondence between the lines in the excitation spectrum and those in the absorption spectrum.
When the laser is narrowed to a bandwidth of $.01 \mathrm{~A}$ the excitation spectrum is more clearly resolved into individual lines as shown in Fig. 2.

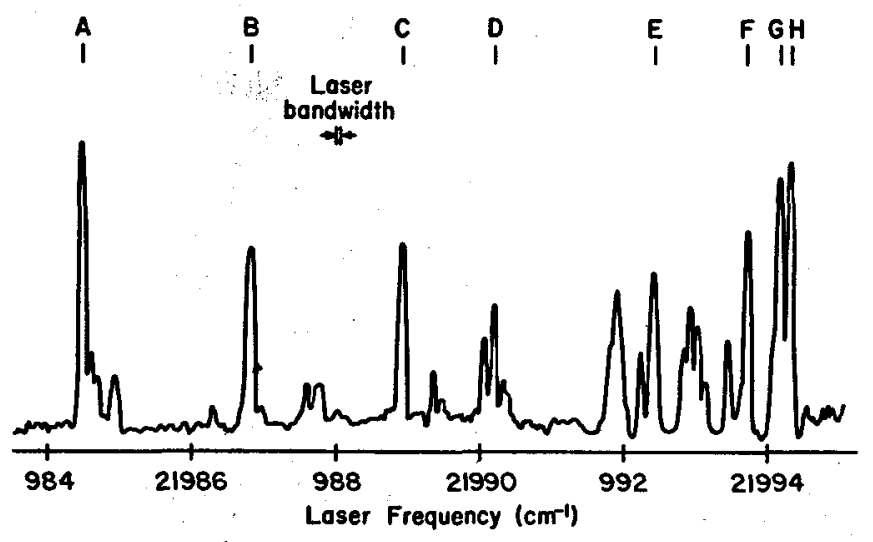

Fig. 2. Intensity in the $v_{2}$ mode as a function of laser frequency, near $22,000 \mathrm{~cm}^{-1}$ (4545 $\mathrm{\AA}$ ), with narrow band laser excitation. The linewidth of the laser was $\sim .04 \mathrm{~cm}^{-1}$ and the bandwidth of the spectrometer $120 \mathrm{~cm}^{-1}$. The relative positions of the lines could be determined to $\pm 3 \%$ and the absolute positions were set using the wavelength determination of Douglas and Huber 1 for the $Q(13)$ line which corresponds to line $B$ in our scan.

(XBL 7412-8437)

With the laser tuned to the peak of an intense line within this structure, the $v_{2}$ fluorescence crosssection is found to be about two orders of magnitude larger than those typically obtained using fixed frequency lasers. 10 This large cross section is particularly surprising given the fact that the absorption cross sections we have measured at lines near $4545 \AA$ are comparable to that of the background absorption. We have investigated the band near $4545 \mathrm{~A}$ in much greater detail than the other bands. We have attempted to make a partial rotational analysis of this region by tuning the laser to several of the most intense lines, designated by the letters $A$ through $H$ in Fig. 2, and resolving the $\nu_{2}$ fluorescence mode located at $749.8 \mathrm{~cm}^{-1}$. In Fig. 3 the resolved fluorescence for the laser tuned to lines A, B, C, E and G are presented. Since the narrow band laser selects a single initial to intermediate state transition, the observed triplet structures can be used in combination with the selection rules for a slightly asymmetric top $(\Delta N= \pm 1.0 ; \quad \Delta K= \pm 1.0)$ and the ground state spectroscopic constants, to assign the final state quantum numbers. This determination then allows one to make assignments for both the initial and intermediate states. These identifications are shown in Fig. 3 and a comparison of calculated and observed splittings is made in Table 1.

In collaboration with the Bradley Moore group of the Materials and Molecular Research Division, we have also made measurements of the fluorescent lifetimes of several lines within this structure near $4545 \AA$.

Neuberger and Duncan ${ }^{6}$ were the first to find that the fluorescence lifetime of $\mathrm{NO}_{2}$ is anomalously 

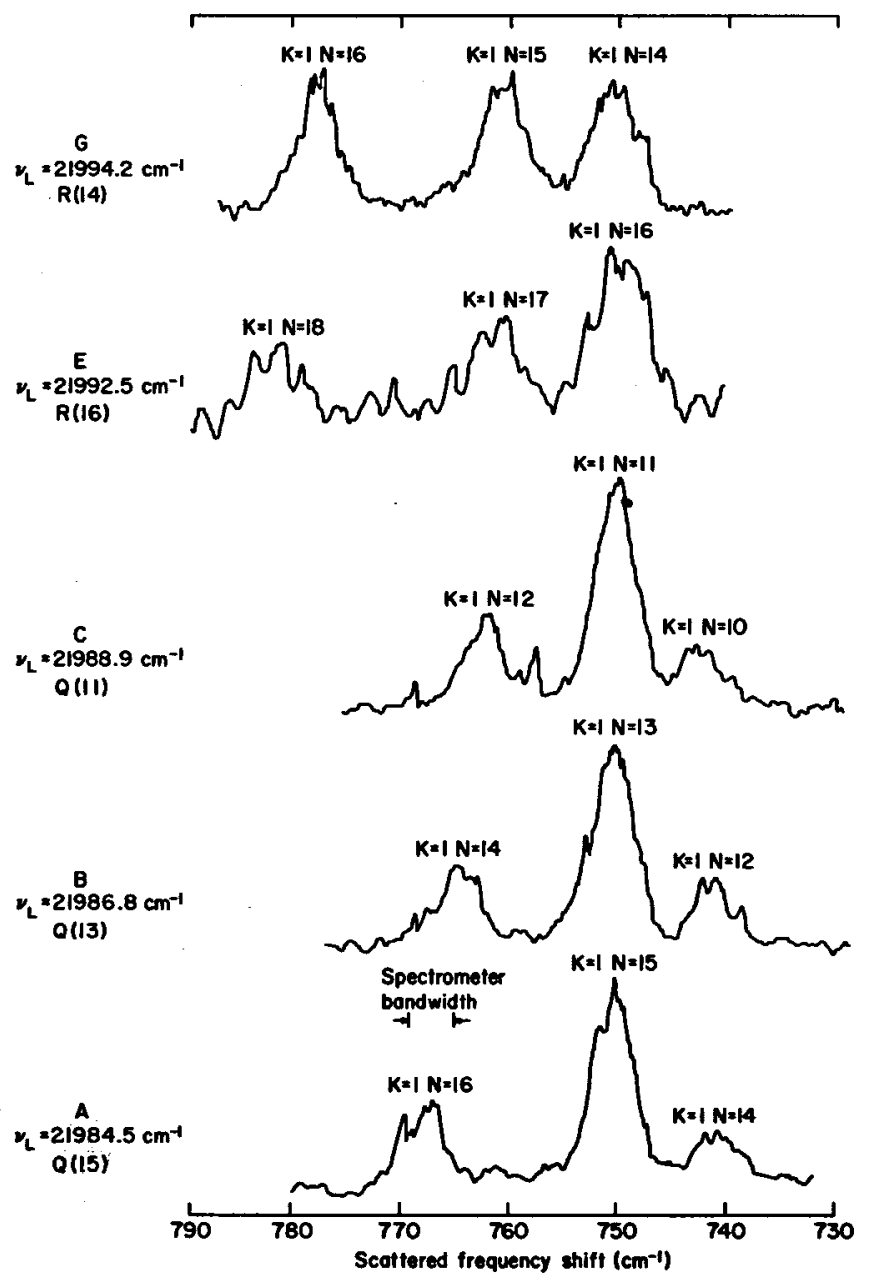

Fig. 3. High resolution sepctrometer scans of the $v_{2}$ mode located $\sim 750 \mathrm{~cm}^{-1}$ lower in frequency than the laser. The laser was tumed to the peaks of lines A, B, $\mathrm{C}, \mathrm{E}$ and $\mathrm{G}$ of Fig. 2. The $\mathrm{K}$ and $\mathrm{N}$ values refer to the final state of the various transitions. The spectrometer bandwidth was $4 \mathrm{~cm}^{-1}$ and the laser bandwidth 0.04 $\mathrm{cm}^{-1}$.

(XBL 7412-8439)

long when compared to the lifetime obtained from the integrated absorption coefficient. This effect is believed to arise from interactions between the different electronic levels in the visible region. Despite much effort to observe short-lived states directly, only two such observations have been reported to date. The first of these involved $\mathrm{K}_{\mathrm{a}}=0$, ${ }_{B}$ lines $^{8}$ which form prominent features of the absorption spectrum 2 . However, its accuracy was severely limited by poor signal-to-noise and lack of shot-to-shot stability in the laser employed. The second of these observations 5 involved not the $\mathrm{K}_{\mathrm{a}}=0,2_{\mathrm{B}_{1}}$ lines, but rather a $5_{24}, 2_{\mathrm{B}_{2}}$ rotational level pumped by the $488 \mathrm{~mm}$ line of an argon ion laser. A lifetime of $3.39 \pm 0.36 \mu \mathrm{sec}$ was obtained from the linewidth of the microwave-optical double resonance signal.

Our investigation has demonstrated that $k_{a}=0$, $2 \mathrm{~B}_{1}$ lines near $454.8 \mathrm{~nm}$ do not have the short lifetimes expected from previous experimental results 8 and perturbation analysis.
Table 1. Comparison of calculated and measured splittings between members of triplet structures shown in Fig. 3.

\begin{tabular}{|c|c|c|c|}
\hline Transition & $\begin{array}{c}\text { Final state } \\
\mathrm{N} \text { value }\end{array}$ & $\begin{array}{l}\text { Frequency } \\
\text { centra1 N } \\
\text { Calculated }\end{array}$ & $\begin{array}{l}\text { shift from } \\
\text { value }\left(\mathrm{cm}^{-1}\right) \\
\text { Measured }\end{array}$ \\
\hline A & $\begin{array}{l}16 \\
15 \\
14\end{array}$ & $\begin{array}{c}16.5 \\
- \\
-10.0\end{array}$ & $\begin{array}{r}17.5 \\
- \\
-9.3\end{array}$ \\
\hline B & $\begin{array}{l}14 \\
13 \\
12\end{array}$ & $\begin{array}{c}14.1 \\
- \\
-9.0\end{array}$ & $\begin{array}{c}13.8 \\
-8.8\end{array}$ \\
\hline C & $\begin{array}{l}12 \\
11 \\
10\end{array}$ & $\begin{array}{c}11.8 \\
- \\
-7.9\end{array}$ & $\begin{array}{c}11.7 \\
- \\
-7.2\end{array}$ \\
\hline E & $\begin{array}{l}18 \\
17 \\
16\end{array}$ & $\begin{array}{r}20.2 \\
- \\
-11.3\end{array}$ & $\begin{array}{c}19.0 \\
- \\
-11.0\end{array}$ \\
\hline G & $\begin{array}{l}16 \\
15 \\
14\end{array}$ & $\begin{array}{c}16.5 \\
- \\
-10.0\end{array}$ & $\begin{array}{c}16.7 \\
- \\
-10.1\end{array}$ \\
\hline
\end{tabular}

Figure 4 shows the experimental arrangement. A nitrogen-laser-pumped dye laser of $1 \mathrm{GHz}$ bandwidth was used to excite $\mathrm{NO}_{2}$ in a 22-1iter bulb connected to a greaseless vacurm system. Pressures were measured with a capacitance manometer. The laser

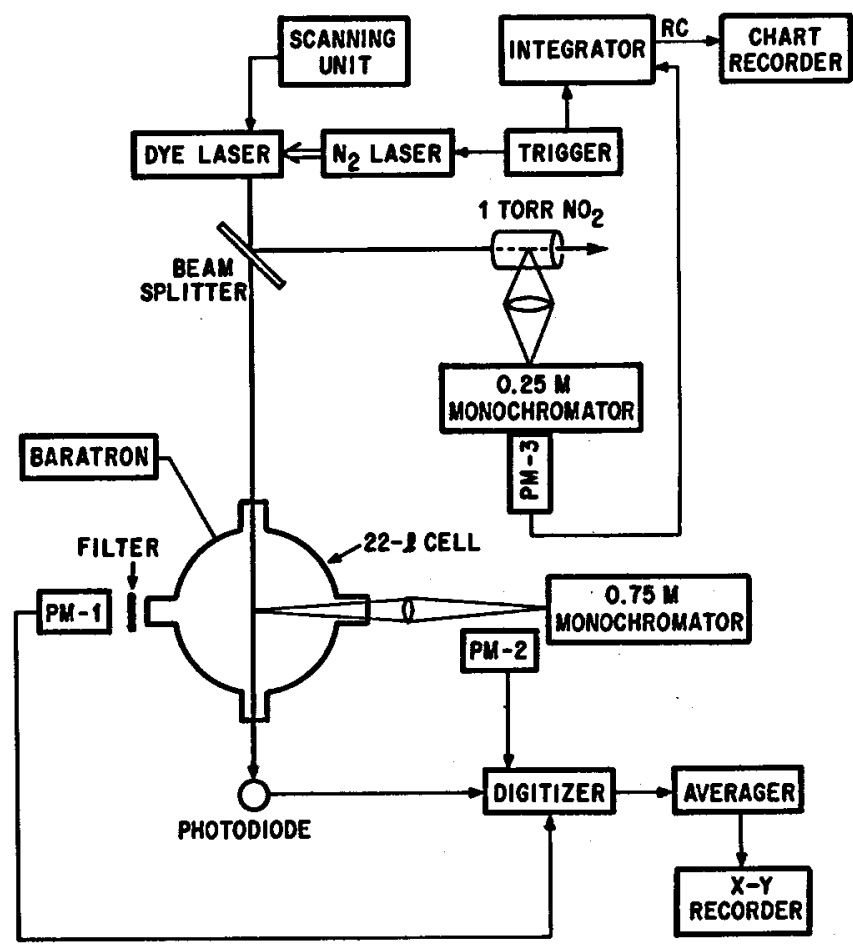

Fig. 4. Experimental apparatus for recording the fluorescence excitation spectrum and measuring the lifetimes of $\mathrm{NO}_{2}$.

(XBL 755-1358) 
frequency was scanned and fluorescence at right. angles to the beam was observed by two photomultipliers. One photomultiplier was equipped with a blue cutoff filter and observed "red" fluorescence with $\lambda_{\text {fluor }}>530 \mathrm{~mm}$. The second photomultiplier observed fluorescence which was focused using $f / 10$ optics and resolved by a monochromator. The monochromator was set to pass "blue" fluorescence 750 $\mathrm{cm}^{-1}$ below the excitation line with a bandpass of roughly $90 \mathrm{~cm}^{-1}$ (FWHM, $2 \mathrm{~mm}$ slits).

At specific lines in the excitation spectrum, the laser scan was stopped and the lifetime measurements were made using the 22-1iter bulb for both the red and the blue fluorescence. To obtain improved signal-to-noise many fluorescence traces were digitized and averaged. During the period in which the fluorescence was averaged, typically 2-5 min., the laser remained stable in frequency to $\sim \mathrm{GHz}$ as judged by the signal intensity of the excitation spectrum.

Fluorescence in either the blue or red region appeared to decay with a single exponential, and linear Stern-Volmer kinetics were obtained over nearly two orders of magnitude in pressure. Reciprocal lifetimes as a function of pressure are shown in Fig. 5 both for the red fluorescence and for the blue fluorescence excited at each of the $Q(11)$ and $Q(13)$ lines denoted by $E$ and $C$ in Fig. 2.

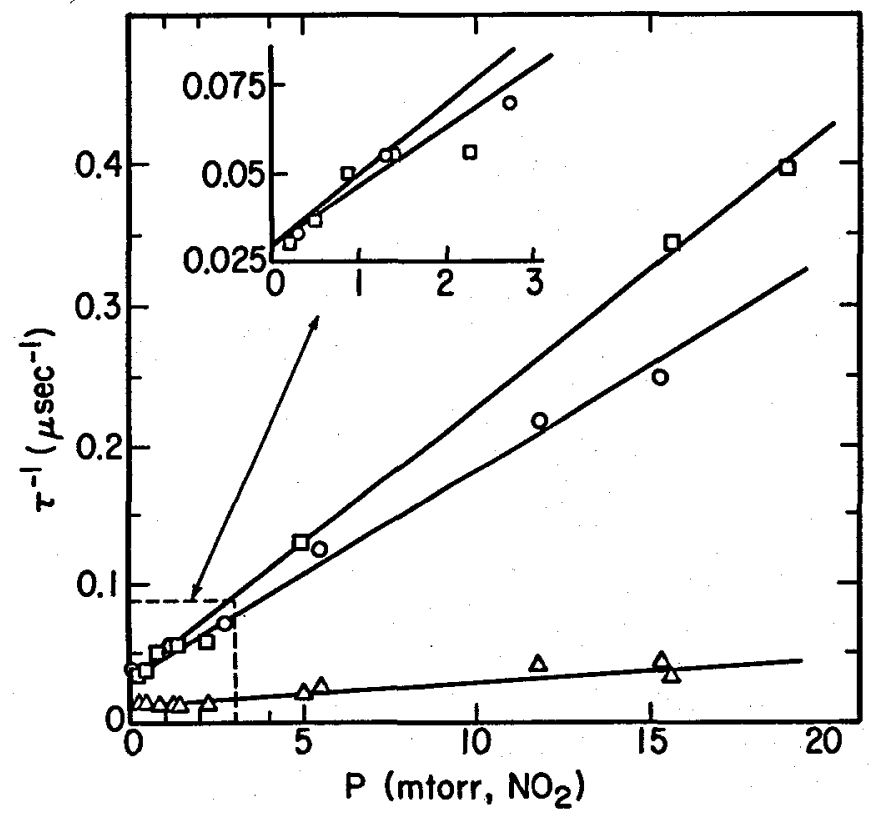

Fig. 5. Reciprocal lifetimes for $\mathrm{NO}_{2}$ fluorescence as a function of pressure. The circles and squares are for resolved blue fluorescence excited by the $Q(13)$ and $Q(11)$ lines, respectively. The triangles are for the unresolved red fluorescence. The insert shows an expansion of the lowpressure regions for the blue fluroescence. (XBL 755-1360)

The zero-pressure lifetime of the blue fluorescence excited by either the $Q(11)$ or $Q(13)$ lines is found to be $33 \pm 4 \mu \mathrm{sec}$. A careful examination of the low-pressure fluorescence traces for fast components with lifetimes on the order of 1-3 $\mu \mathrm{sec}$ has shown that such components, if present, form less than 5\% of the fluorescence amplitude. Previous investigators 8 have found short components for $\mathrm{K}_{\mathrm{a}}=0$ states whose amplitude at unspecified pressures was roughly $13 \%$ of the broad-band fluorescence. Since our experiment isolates fluorescence specifically from $K_{2}=0$ states, it should be extremely sensitive to such short components if present. Since no evidence for these components was found, we conclude that $\mathrm{K}_{\mathrm{a}}=0$ lines of the $\mathrm{Z}_{\mathrm{B}_{1}}$ electronic state do not have short lifetimes comparable to the integrated absorption lifetime of $0.3 \mu \mathrm{sec}$. This fact may indicate that such lines are still appreciably perturbed. However, the mechanism for this perturbation is not immediately obvigus. The Renner-Teller effect should not mix $K_{a}=0,{ }^{B_{1}}$ states with ${ }^{2} A_{1}$ states, and $\mathrm{NO}_{2}$ lacks the proper vibrational mode $\left(\mathrm{A}_{2}\right)$ to allow first-order vibronic coupling of the ${ }_{\mathrm{B}_{1}}$ and $2_{\mathrm{B}_{2}}$ states. Perhaps this implies a higher order coupling mechanism.

On the other hand, the fact that these states do stand out in the absorption spectrum and even more so in the excitation spectrum of the $\nu_{2}$ fluorescence mode suggests that they are, indeed, unperturbed. Since a lengthening of the lifetime by a factor of 100 is not compatible with essentially unperturbed states, perhaps the oscillator intensity from which a $0.3 \mu \mathrm{sec}$ lifetime is calculated is due primarily to transitions to states other than the ${ }^{2} B_{1}$. It has been previously suggested that the $0.5 \mathrm{usec}$ lifetime may be that of the $2_{\mathrm{B}_{2}}$ state. If, in fact, the $\mathrm{K}_{\mathrm{a}}=0$ levels are unperturbed, then the lifetime measured in this experiment may be the true lifetime of the ${ }^{2} B_{1}$ electronic state.

\section{ACKNOWLEDGEMENT}

We would like to thank Owen Chamberlain for his encouragement and helpful discussions throughout the course of this work.

REFERENCES

1. T.C. Hall Jr. and F.E. Blacet, J. Chem. Phys. 20 (1952) 1745.

2. A.E. Douglas and K.P. Huber, Can J. Phys. 43 (1965) 74 .

3. K. Abe, F. Myers, T.K. McCubbin and S.R. Polo, J. Mo1. Spect. 38 (1971) 552;

K. Abe, J. Mo1. Spect. 48 (1973) 395;

K. Abe, F. Myers, T.K. McCubbin, Jr. and S.R. Polo, J. Mo1. Spect. 50 (1974) 413;

J.C.D. Brand, J.L. Hardwick, R.J. Pirkle and C.J. Seliskar, Can J. Phys. 51 (1973) 2184;

W. Demtroder, F. Paech and R. Schmied1, Chem. Phys. Letts. 26 (1974) 381;

C.G. Stevens, M.W. Swagel, R. Wa1lace and R.N. Zare, Chem. Phys. Letts. 18 (1973) 465;

R.E. Smalley, B.L. Ramakrishna, R.H. Levy and L. Wharton, J. Chem. Phys. 61 (1974) 4363. 
4. R. Solarz and D.H. Levy, J. Chem. Phys. 58 (1973) 4026;

R. Solarz, D.H. Levy, K. Ave and R.F. Cur1, J. Chem. Phys. 60 (1974) 1158;

T. Tanada, A.D. English, R.W. Field, D.A. Jennings and D.0. Harris, J. Chem. Phys: 59 (1973) 5217 .

T. Tanaka, K. Abe and R.F. Curl, J. Mol. Spect. 49 (1974) 310 .

5. R. Solarz and D.H. Levy, J. Chem. Phys. 60 (1974) 842 .

6. D. Neuberger and A.B.F. Duncan, J. Chem. Phys. 22 (1954) 1693.

7. G.H. Myers, D.M. Silver and F. Kaufman, J. Chem. Phys. 44 (1966) 718;
K Sakurai and H.P. Broida, J. Chem. Phys. 50 (1969) 2404;

P.B. Sackett and J.T. Yardley, Chem. Phys. Letts: 6 (1970) 323;

L.F. Keyser, S.Z. Levine and F. Kaufman, J. Chem. Phys. 54 (1971) 355;

P.B. Sackett and J.T. Yardley, J. Chem. Phys. 57 (1972) 152 .

8. P.B. Sackett and J.T. Yardley, Chem. Phys. Letts. $\underline{9}$ (1971) 612 .

9. T.W. Hansch, Applied Optics 11 (1972) 895.

10. D.G. Fouche, A. Herzenberg and R.K. Chang, J. App1. Phys. 43 (1972) 3846;

C.M. Penny, W.W. Morey, R.L. St. Peters, S.D. Silverstein, M. Lapp and D.R. White, NASA Report number NASA-CR-132363. 